fis when simstes

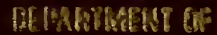

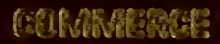
Perstchinos

\title{
NSRDS-MBS 36
}

NBS

\section{PUBLICATIONS}

DSEARTMERT

0

COHAHECE

Nationa!

Burean

of

Standards 



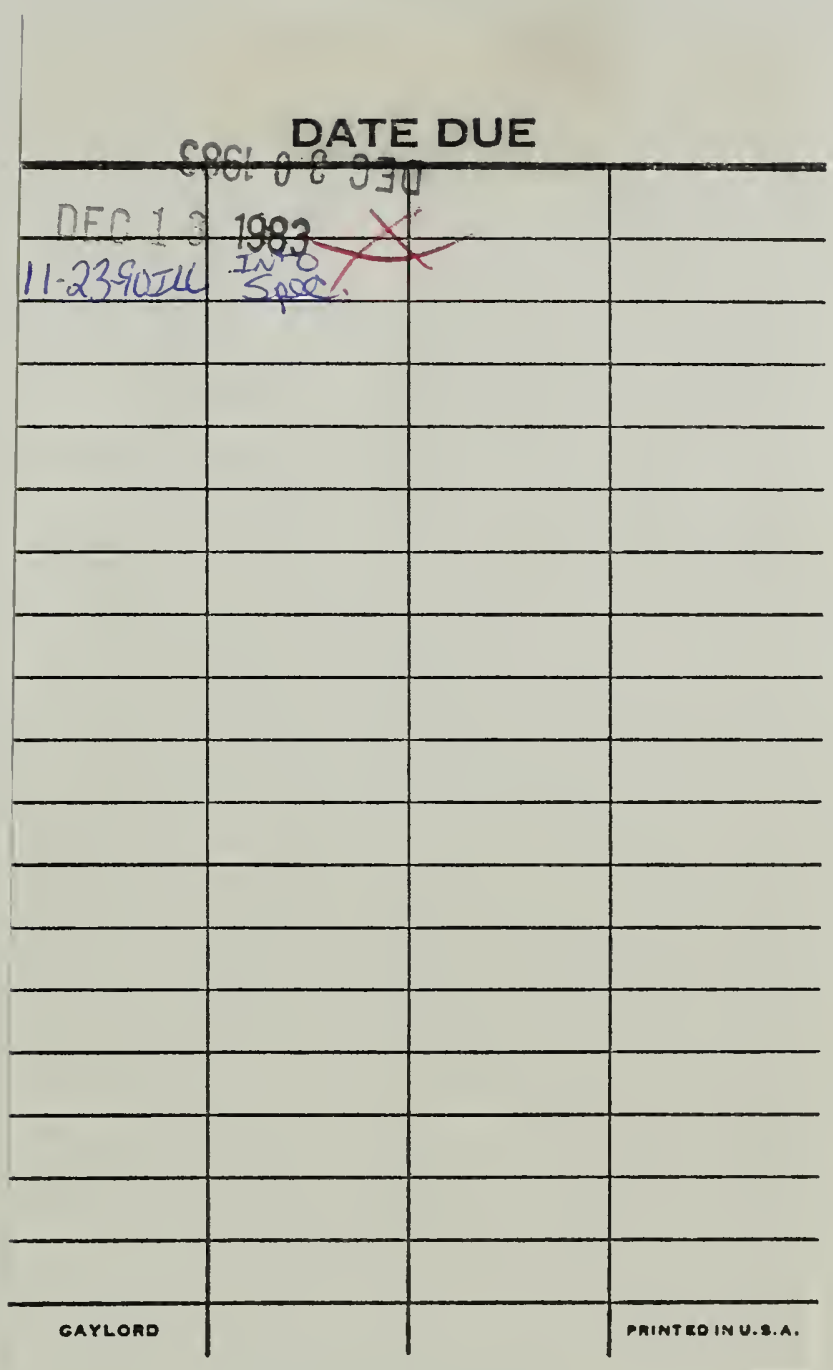





\title{
Critical Micelle Concentrations of Aqueous Surfactant Systems
}

\author{
Pasupati Mukerjee \\ University of Wisconsin \\ School of Pharmacy \\ Madison, Wisconsin 53706 \\ and \\ Karol J. Mysels \\ R. J. Reynolds Tobacco Co. \\ Winston-Salem, North Carolina 27102
}
This compilation was prepared under contract for the Office of Standard Reference Data
National Bureau of Standards
Washington, D.C. 20234

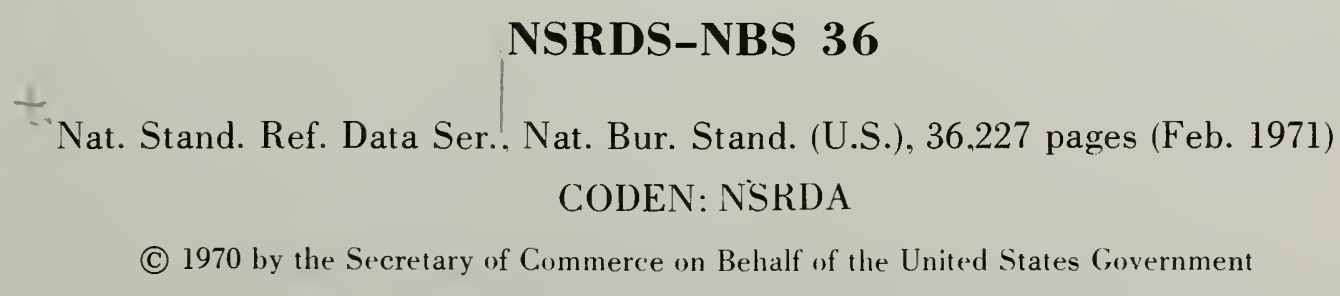

Issued February 1971 
SATIONAL BUREU OF STANDARDS

MAY 171971

$160 \%$ hi:

Q 100

. L 513

$N j+6$

1971

$\left[\sin y^{2}\right.$

Library of Congress Catalog Card Number: 70-603902 


\section{Contents}

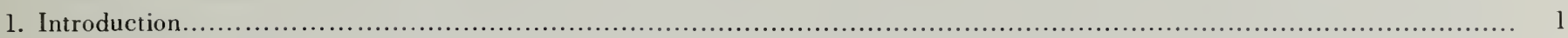

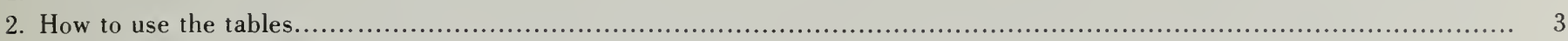

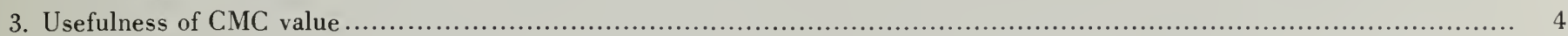

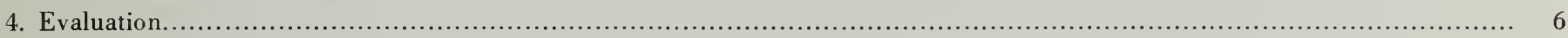

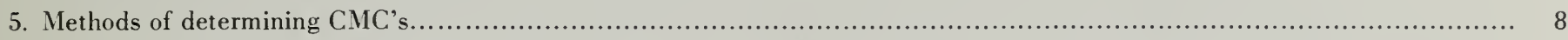

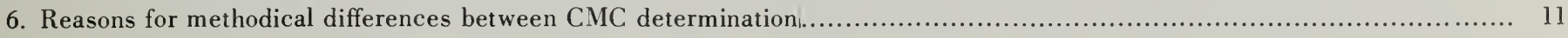

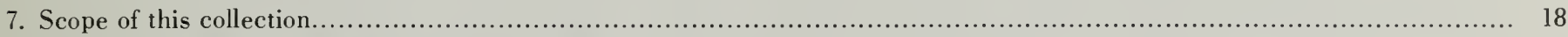

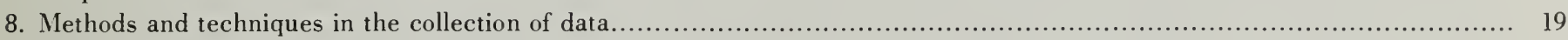

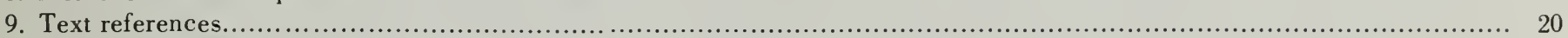

Structural indexes of compounds with keys to compound numbers............................................................. 23

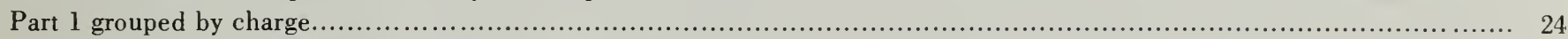

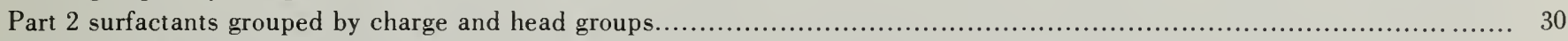

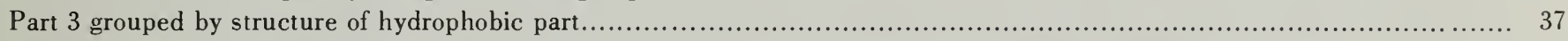

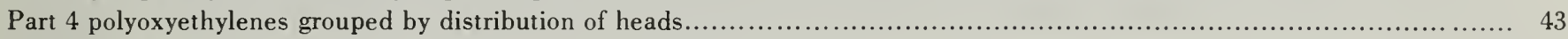

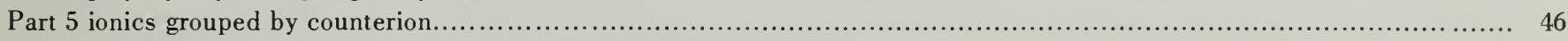

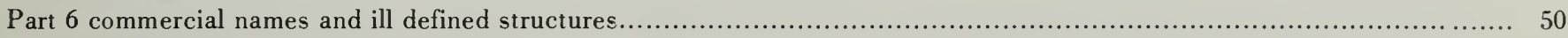

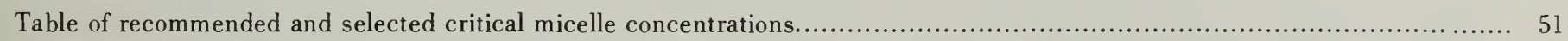

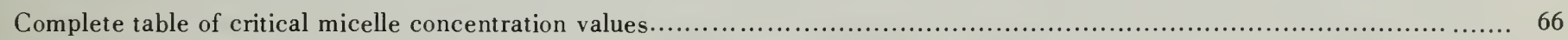

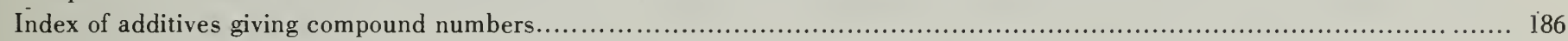

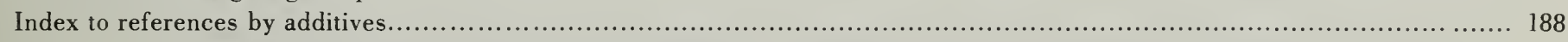

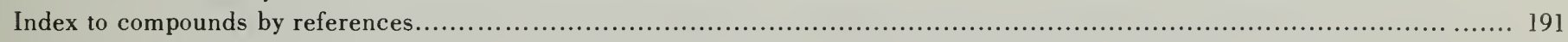

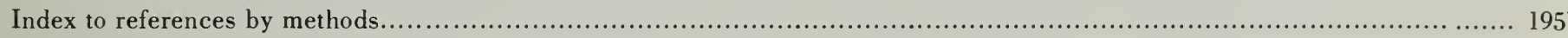

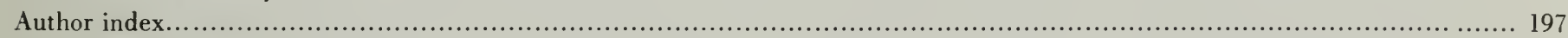

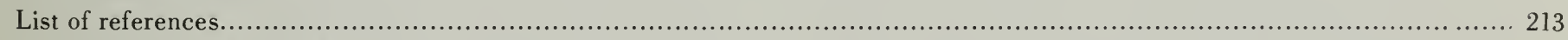

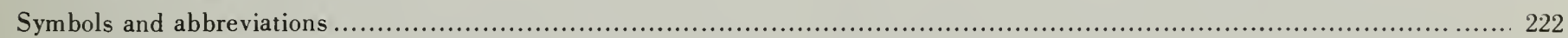





\section{FOREWORD}

The National Standard Reference Data System provides effective access to the quantitative data of physical science, critically evaluated and compiled for convenience, and readily accessible through a variety of distribution channels. The System was established in 1963 by action of the President's Office of Science and Technology and the Federal Council for Science and Technology, with responsibility to administer it assigned to the National Bureau of Standards.

The System now comprises a complex of data centers and other activities, carried on in academic institutions and other laboratories both in and out of government. The independent operational status of existing critical data projects is maintained and encouraged. Data centers that are components of the NSRDS produce compilations of critically evaluated data, critical reviews of the state of quantitative knowledge in specialized areas, and computations of useful functions derived from standard reference data. In addition, the centers and projects establish criteria for evaluation and compilation of data and make recommendations on needed improvements in experimental techniques. They are normally closely associated with active research in the relevant field.

The technical scope of the NSRDS is indicated by the principal categories of data compilation projects now active or being planned: nuclear properties, atomic and molecular properties, solid state properties, thermodynamic and transport properties, chemical kinetics, and colloid and surface properties.

The NSRDS receives advice and planning assistance from the National Research Council of the National Academy of Sciences-National Academy of Engineering. An overall Review Committee considers the program as a whole and makes recommendations on policy, long-term planning, and international collaboration. Advisory Panels, each concerned with a single technical area, meet regularly to examine major portions of the program, assign relative priorities, and identify specific key problems in need of further attention. For selected specific topics, the Advisory Panels sponsor subpanels which make detailed studies of users' needs, the present state of knowledge, and existing data resources as a basis for recommending one or more data compilation activities. This assembly of advisory services contributes greatly to the guidance of NSRDS activities.

The NSRDS-NBS series of publications is intended primarily to include evaluated reference data and critical reviews of long-term interest to the scientific and technical community.

Lewis M. BRanscomb, Director. 


\title{
Critical Micelle Concentrations of Aqueous Surfactant Systems
}

\author{
Pasupati Mukerjee* and Karol J. Mysels**
}

\begin{abstract}
Critical micelle concentrations (CMC's). have been collected, organized and evaluated. The literature has been scanned for numerical values from 1926 up to and including 1966. In addition. over 800 values. hitherto available only in graphical form or implied in experimental data. have been extracted from the publications and are included. Close to 5.000 entries. based on 333 references. dealing with 720 compounds are tabulated in the main tables. Whenever available. the temperature. any additives present, the method of determination and the literature source are given for each C.C value and an indication of the apparent quality of the preparation and method used are included. A shorter table gives selected values which are believed to be particularly reliable. including highly accurate ones. Among these, concordant values from at least two independent laboratories are emphasized.

Included in the Introduction is a general discussion of the importance and significance of C.IC values and of methods for their determination, as well as a summary of the procedures used in the collection, evaluation and presentation of these values in the present work. Extensive indexes are provided.
\end{abstract}

Key words: Association colloid; bibliography; CMC: colloid; colloidal electrolyte: critical concentration: critical micelle concentration: detergent; hydrophobic bonding: Krafft point: long chain compounds: micelle: paraffin chain salts: selected values: soap: solubilization: standard values: surface active agents: surface chemistry: surface tension: surfactant.

\section{Introduction}

Critical micelle concentrations are here to stay! This conclusion is evident from figures 1 to 3 which are based on the literature used in this work. They show a continuing growth since the middle thirties in the number of articles appearing each year which contribute new values and in the number of new values reported. The number of new values per article seems to have passed its peak, which suggests more careful and critical work in recent years.

The reason for this growth is that a critical micelle concentration (CMC) is probably the simplest means of characterizing the colloid and surface behavior of a surfactant solute, which in turn determines its industrial usefulness and biological activity, and gives a measure of the structurally interesting solute-solvent and solute-solute interactions. However, these published CMC values are widely scattered through the literature-we have consulted 87 different publications-and vary greatly in quality from clearly erroneous data to highly accurate values.

Furthermore, some of the existing values are clearly tabulated, but others - often the best onesare hidden in graphs. or even in tabulations of some

\footnotetext{
*Present address: Lniversity of $W$ isconsin. Madison. $W$ isconsin 53706 .

** Present address: Gulf General Atomic, Inc.. P.O. Box 608. San Diego. California 92112.
}

measured property slich as conductivity. These require considerable effort and judgment to retrieve. Frequently, the quality of the work cannot be judged without consultation of several references and intercomparison with other pertinent publications. Hence, much of the literature is not now readily accessible or useful to those interested in learning what has been established thus far.

The primary purpose of this publication is to provide a list of values in which the user can place high confidence. In the process of obtaining these, we had ta make a survey. as complete as possible, of all available values. To present the results of this survey so as to make both the literature and the results contained therein readily available became, therefore, a secondary objective. Perhaps the best evidence for the usefulness of this effort is that nearly two-thirds of the best data reported herein were not previously directly available in the literature but required at least some. and often quite extensive, interpretation of a publication or individual correspondence.

The book itself is divided into four parts:

(1) The Table of Recommended and Selected Values lists the values we believe to be most reliable. They contain further guides to the quality of the data. 


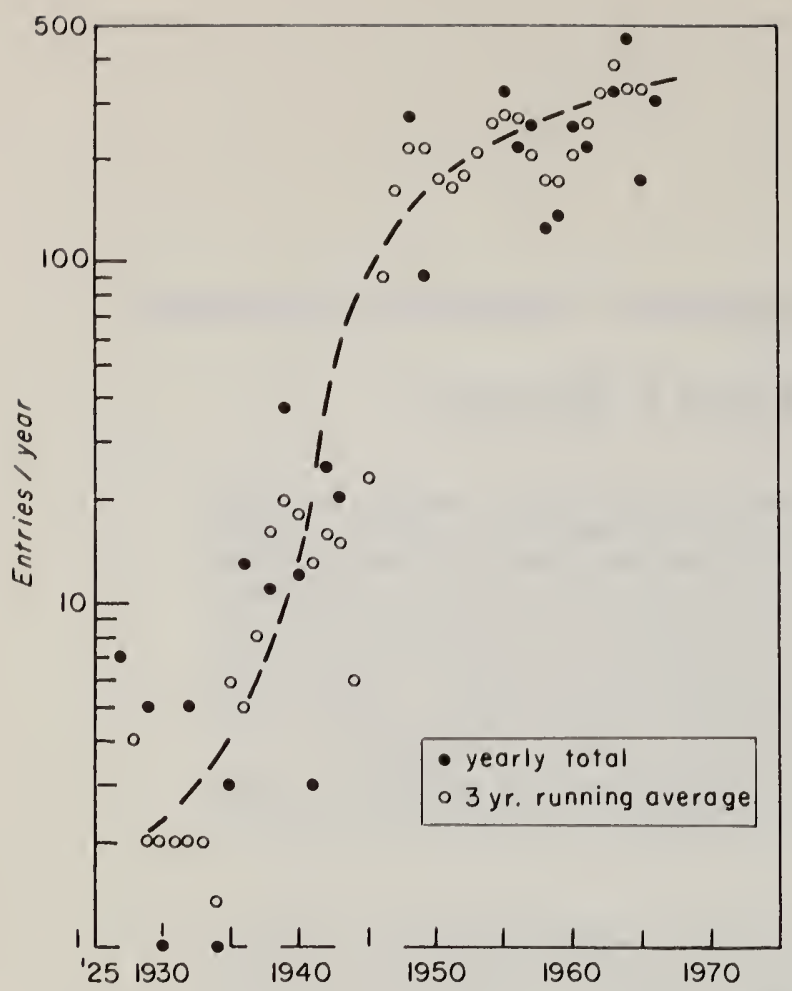

Ficule: l. Number of entries for the complete tables originating within each year from 1927 to 1966.

There are no entries in 1928, 1931, 1933, 1934, 1937 and 1945.

(2) The Complete Table contains all values found which were published through 1966.

(3) Several indexes and lists, particularly the compound indexes, should permit the reader to find any desired compound or its closest analogs, give him the meaning of any abbreviation or symbol, and also guide him to the pertinent literature.

(4) The Introduction discusses the thoughts that went into the collection, evaluation, and presentation of the data. A glance at "How to Use These Tables" may be helpful before consulting them.

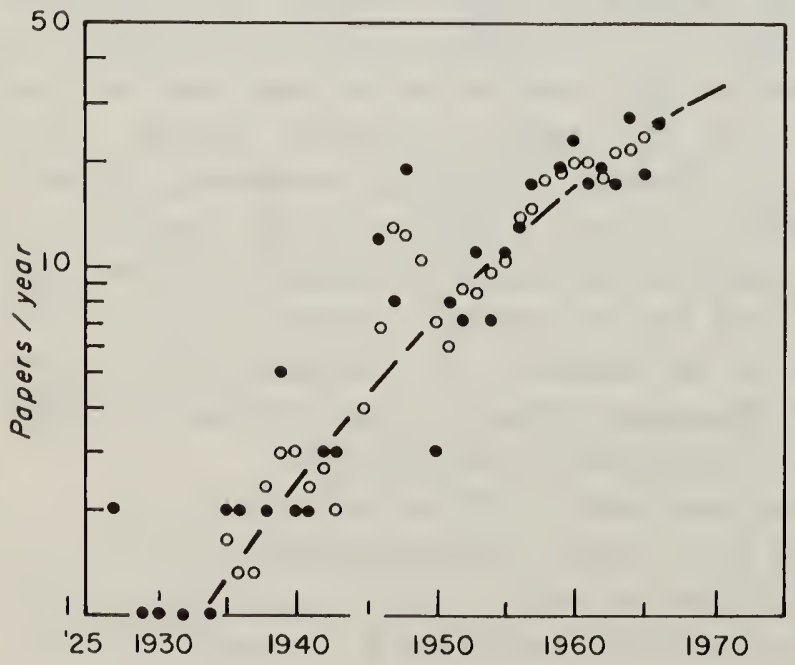

Ficure: 2. Number of papers containing at loust one entry for the complete tables originating within a given year.

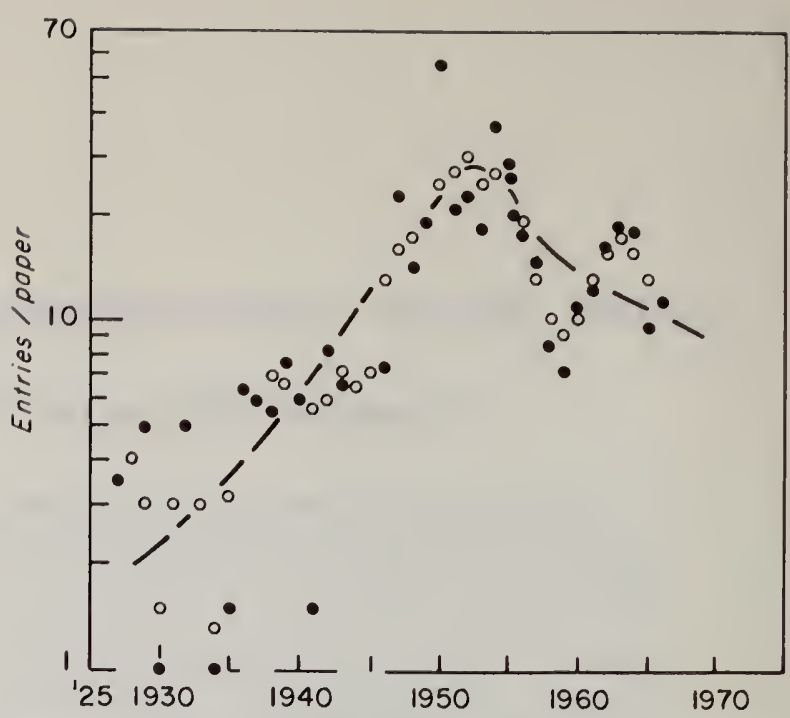

FiCURE 3. Average number of entries per paper containing at least one entry within a given year and within a three year period.

The largest number of entries from a single paper was 167, from reference 55004 .

We hope that this work will make us more friends than enemies. We tricd to be objective in the evaluation of the data, but some errors and personal prejudices are unavoidable. Our evaluation procedure of the individual data is described in the introduction, which contains also general considerations about the validity and significance of the various methods of determination.

We are gratcful to many who have helped us in this work. Close to a hundred authors have responded to our request for reprints and many have provided additional comments, data, and interpretations. Dr. Edward L. Brady was most helpful in getting us started properly in the task of handling this multitude of data. Dr. H. J. White's patience and cooperation are greatly appreciated. The extensive computer handling of the data was made possiblc by the free availability of the data processing facilities of the R. J. Reynolds Tobacco Co., and the programming skill and understanding of Mr. Bill Donovan. Several secretaries have been involved in the careful verification of the hundreds of thousands of bits of information recorded. Mrs. Jerry Wilson and Miss Judy Tate were particularly involved in the final stages. The work leading to this publication was begun in 1964 at the University of Southern California under contract with the National Bureau of Standards and continued there until September 1966 when the authors transferred to their present connections with the University of Wisconsin, where the support of the National Bureau of Standards continued, and the R. J. 
Reynolds Tobacco Co. Clearly, the resources of these three institutions made the completion of our work possible.

\section{How to Use the Tables}

This section presents a brief guide to the admittedly complicated arrangement of the tables. Space and computer requirements dictated much of this complexity; some is inherent in the dissimilarity of the compounds and the variety of conditions used for CMC determinations. The guide is arranged to answer a series of questions which may be raised by readers.

How do I find the compound I am interested in? In the tables the compounds are arranged in numerical order by arbitrary "Compound Numbers." To find this number you must go through the "Compound Index" in which the listed compounds are arranged by structure. There are five parts to this index (plus an alphabetical one for commercial names) and in each the compounds are listed according to different structural properties. These arrangements are described on the first page of the compound index (p. 23).

What can I do if my compound is not listed? The indexes will lead you to the most closely related compounds that are listed. These should permit you to make a good guess by interpolation and extrapolation.

How do I learn about the effect of an additive? The names of additives are abbreviated (if the abbreviation is not clear, its meaning can be found in the list on p. 222). Surfactant additives are indicated by their "Compound Number." For each compound, CMC values in the presence of additives are listed after the simple (surfactant-water) system in alphabetical order of the abbreviations. This is followed by systems with two additives and then by those with three additives.

What if the additive is not listed with my compound? The additive index shows all the compounds reported for any additive. You may find some useful analogies in this way.

What is the effect of temperature? Within each system (compound-additive(s)) the values are arranged by increasing temperature. By checking the author, or better the reference column, you can locate groups of values that were obtained specifically to show the effect of temperature (which is often small).
Which are the "good" CMC values? The shorter tables beginning on page 51 contain the "Selected" and "Recommended" values ( $15 \%$ of all reported values). Those that carry a "l" in the last column have been independently confirmed and should be highly reliable (to $1.5 \%$, keeping in mind that different methods can give significantly different values-cf. p. 11). Those marked " 2 " are of the same apparent quality but lack confirmation. Among those marked " $D$ " for each system, there is probably one that is as good as those of the preceding categories, but we do not know which. The many marked " 3 " do not seem to be in the same class but should be good to 10 percent.

What do I find in the long tables? These tables beginning on page 66 contain all the "Recommended and Selected" values plus all the others that we have located. In a number of cases, indicated by " $R$ " in the last column, we make references to the literature where additional data or calculated values may be found or to warn the reader that the values are duplicates of those already listed or are in error. The bulk of the values carry an "L" in the last column. These may be useful and some may be excellent but we could not "recommend" or "select" them for a variety of reasons. Some clue to these reasons may be found in the "quality" column.

What is the "quality" column? In this column the first letter refers to the material and the second to the measurement. The meaning of the letters may be found on page 6 . In general the quality decreases in alphabetical order. It represents our opinion after a careful study of the reference.

Are there more data in the literature? Our search does not cover anything published in 1967 or later (including the 1964 Congress of Surface Activity which did not appear in print until 1968). There are also older references that we may have overlooked. All the references within this field that we have scanned are listed in the literature index starting on page 213 whether they have yielded any entries or not. We would like to be informed of overlooked articles. Some of the literature scanned does contain data which, if properly interpreted, could lead to a CMC value which is not included. We have made such interpretations in many hundreds of cases, but not always. However, if a CMC value was mentioned as such in the article, we have tried to include it in all cases.

What are those various "methods"? The "method" column contains generally an abbreviation of the 
method by which the CMC value was obtained. These methods are discussed briefly (and their abbreviations given) on pages 8 to 11 . The "methods index" lists the references which have used each. These references should be consulted for details.

Occasionally the methods column contains information about the literature or a cross-reference. This is only the case when the entry does not give a CMC value.

In what units are the CMC's? We have followed the references except for order of magnitude conversions (e.g., from millimoles to moles) and as a result have a large number of units. The meaning of the abbreviations is given on page 222 and in the footnote to the table. For noncommercial compounds, for which a molecular weight is likely to have meaning, we have added a value in moles (per liter or $\mathrm{kg}$ of solution or $\mathrm{kg}$ of solvent) when the corresponding weight concentration of the compound was given. This was done by the computer on the basis of the molecular weight listed for the compound which in turn was also obtained by the computer from a structural or empirical formula of the compound. The value is printed by itself on a separate line below the value given by the author and is characterized by "M" in the "source" column.

What units are used for additives? The same units and symbols as for CMC's plus a number of others, including such peculiar ones as $\mathrm{pH}$, again following the authors. In addition, we have used the additive columns to record certain special conditions such as pressure. The meaning of the abbreviations is listed on page 222. For additives we have not made any conversions to mole units.

What compound nomenclature is used? We have generally followed the first author whom we encountered dealing with the particular compound in the hope that this will also be the most common and understandable name. In case of ambiguity or some exotic names, we have added an alternative name or a formula in parentheses.

Are there any values for solvents other than water? If the solvent is a mixed one including water, the other components have been considered as additives. Nonaqueous systems have not been included for reasons discussed on page 18 with the exception of $\mathrm{D}_{2} \mathrm{O}$ which is treated as an additive at 100 mole percent concentration!

What is the meaning of "source"? This column serves to indicate in what way the pertinent CMC value was obtained by us. The meaning of the abbreviations is listed on page 222 . In some cases the reader can check our listing directly or after carefully reading a graph or replotting some numerical data. In a few cases, however, our listing is based not only on what appears in the article but on correspondence or conversation with the authors. In this case an $\mathrm{L}$ in the source column is given. We have not included, however, values made available to us privately which did not have a basis in the published literature.

Where do these CMC's come from? The exact reference may be found in the Reference index starting on page 213 through the number in the "reference" column of each entry. However, much information can be obtained from this number itself since the first two digits give the year of publication and from the "authors" column which carries the first four letters of the name of the author or two of the authors of that publication. Particularly for those familiar with the field, this should often permit identification of the reference.

Are the numbers of digits really significant? Not in the great majority of entries. We have again followed the authors for the sake of the record and it is clear that most authors paid no attention whatsoever to the rules pertaining to significant figures. A better idea of the precision of the values is given by our "quality" rating of the method (second letter). See page 6 for the approximate meaning of these letters. When the value quoted is obtained by ourselves from published graphs, etc., the significant figures refer to how well these graphs could be read or interpreted without digging further into the uncertainties of the experiment.

\section{Usefulness of CMC Value}

The expression critical micelle concentration (CMC), as will be discussed later, is slightly misleading because of the use of the singular form of the noun "concentration." The formation of micelles from the constituent monomers involves a rapid, dynamic, association-dissociation equilibrium. Experimentally, it is found, in accord with the expectations from such equilibria, that micelles are undetectable in dilute solutions of the monomers, and become detectable over a narrow range of concentrations as the total concentration of solute is increased, above which nearly all additional solute material forms micelles. The concentration at which the micelles become first detectable depends on the sensitivity of the experimental probe used. The concentration range over which 
the fraction of additional solute which forms micelles changes from nearly zero to nearly unity depends on such factors as the number of monomers in the micelle, the chain length of the monomer, the properties of counterions and other details affecting the monomer-micelle equilibrium. An approximate rule is that the higher the CMC value, the broader is the concentration range over which this transition takes place, in absolute value as well as in relative value in comparison to the CMC. Since different experimental methods may reflect this transition to different extents, some systematic variations in operationally defined CMC's are expected, as discussed in more detail later (p. 11).

Nevertheless, in spite of these various sources of uncertainty in defining and pinpointing the CMC exactly, the range of uncertainty is often no more than \pm 1 to 2 percent of the CMC value. Thus, the CMC is a quantity which can be, and often is, determined experimentally to a much higher precision and accuracy than nearly any other property which is characteristic of solutions of surface-active agents, a point we would like to emphasize strongly. For comparative purposes, in careful work, the precision is often within \pm 1 percent.

The usefulness of CMC values in various qualitative and quantitative investigations involving surfactant solutions arises basically from the fact that the surface and interfacial activity of the amphipathic (polar-nonpolar) monomers is closely reflected in the value of the CMC. The tendency to form micelles arises mainly from the presence of a hydrophobic part in the amphipathic monomers. The role of the hydrophilic part, nonionic, zwitter-ionic, or ionic (with associated counterions), which is essential for conferring enough of a solubility to the hydrocarbon chain so that CMC values can be reached or exceeded, is essentially a negative one as far as the stability of micelles is concerned. The same factors are involved qualitatively in the surface activity of the monomers, irrespective of whether the surface is an air-water interface, oil-water interface, or a nonpolar solid-water interface. There is thus an excellent correspondence between the adsorbability of the monomers, their ability to reduce surface and interfacial tensions, and the value of the CMC $[1,2] .{ }^{1}$ The more surface active the monomer is, the higher is the tendency to form micelles and the lower the CMC value. Since above the CMC the monomer activity rises only very

'Italicized figures in brackets indicate the literature references on page 20. slowly, the CMC is also a measure of the concentration at which the thermodynamic activity of the monomer and, therefore, its net surface activity and adsorbability to various substrates, level off to nearly constant values $[1-3]$. In closely comparable systems, particularly if the hydrophilic moiety of the monomer is kept the same and the hydrophobic part is varied, there is a considerable similarity in the amount of adsorption to air-water and oil-water interfaces at concentrations close to the CMC. It is thus often possible to obtain rough estimates of equilibrium monolayer concentrations from the CMC values in homologous systems [1-3].

Since adsorption from surfactant solutions is involved in widely ranging systems of technical importance such as foams, froths, emulsions, suspensions, and surface coatings, CMC values are important in a wide variety of industrial operations $[4,5]$.

In striking contrast to monomers, the micelles, which have a hydrophilic exterior, are not surfaceactive. As a result, above the CMC, excepting in some cases where small micelles form and the monomer activity increases appreciably, the surface and interfacial tensions decrease very little $[1,2,6]$. The CMC, therefore, indicates the concentration at which surface and interfacial tensions reach, approximately, their lowest values. Characteristic values at room temperature are often about $35 \mathrm{dyn} / \mathrm{cm}$ for surface tensions and $5 \mathrm{dyn} / \mathrm{cm}$ for interfacial tensions.

The CMC, of course, is the concentration at which the micelles make their first appearance. Micelles provide in many ways one of the most convenient systems available to study in depth the properties of colloids. As the properties of micelles depend on micelle-medium interactions and also micelle-micelle interactions, to understand the former without the latter complication, it is necessary to extrapolate properties of micelles to a point where micelle-micelle interactions become negligible. The corresponding extrapolation of preformed colloidal systems, such as polymers or proteins, which do not dissociate on extensive dilution, is made to "infinite dilution." For micellar properties, the CMC serves as a convenient point for extrapolation, i.e., "infinite dilution" for micelles. Just as binary protein-protein interactions (i.e., those involved in second virial coefficients) are experimentally determined from the slopes of curves as they approach infinite dilution, so in micellar 
systems, the corresponding concentration range is the one just above the CMC [7-10].

In systems involving solubilization of an additional component or its distribution between the bulk solution and the micelle, the CMC again is a measure of the concentration at which such phenomena become first apparent. It will be discussed later that the addition of the third component may modify the CMC itself to some extent and, therefore, the CMC of the system in presence of the third component is the value to be used. The change in the CMC, however, is often small.

In situations where a quantitative estimate of the amount or concentration of micelles is desired, for example, in estimating solubilizing powers, or the effect of micelle concentrations on the chemical reactivities of constituent monomers or solubilized species, an area of research which is of considerable current interest [11-15], the CMC again serves the purpose of giving a rough estimate of the monomer concentration in the solution. The micelle concentration in equivalents, therefore, can be closely approximated as the total concentration minus the CMC.

For the quantitative study of the thermodynamics of the interactions involved in the monomer-micelle equilibrium, the CMC is of paramount importance [16-21]. Although considerable uncertainties still exist with regard to the proper means of estimating the charge effects in ionic micelles, for uncharged systems the CMC itself gives an approximate quantitative measure of the standard free energy of formation of micelles. These free energies and other derived thermodynamic quantities are of great potential and actual use in understanding hydrophobic interactions in general [22-24]. Such interactions are involved in a wide variety of biochemical phenomena, e.g., the stability, structure, conformation, and activity of proteins, enzymes, and membranes. With ionic micelles, as mentioned before, the calculation of thermodynamic quantities characterizing the various interactions is not on sure ground as yet. For comparison of related systems, however, e.g., in noting the effect of varying the chainlength, salt concentrations, or counterions, the CMC provides quite a good quantitative measure of the changes as they affect the monomer-micelle equilibrium $[8,18,25]$.

\section{Evaluation}

An important part of this work is the evaluation of the data presented. We hope to guide the reader to those data that are most useful and reliable in our considered judgment and also to show him other values that exist in the literature so that he may make more easily his own evaluation. We also tried to indicate the relevant literature and data that we have considered but not used in final listings.

We divided our evaluation into two steps: one, which we may call the individual or preliminary evaluation; the other, the comparative evaluation. The former represents our opinion on the basis of the individual paper (and its references or related papers of the same author); the latter is based on intercomparison of all the available data for a given compound under the same, or closely related, conditions. The former was done as the work progressed over a period of three years, 1965-68, and, therefore, is subject to the drift and development of our ideas and skills during that time. The latter was done in a short period of time after all the data had been collected and sorted by the computer.

Individual evaluation. A preliminary separation involved the question whether a given CMC value should be reported in detail or not. Values which are indicated in the article as being duplicates of other published values are omitted completely. Others, however, which are clearly duplicates but not explicitly indicated as such by the authors, are mentioned as "VALUES FROM REF IN CMC," with the article from which they are taken listed in the column in which the CMC is normally found.

Values which could not be retrieved profitably, e.g., those in the form of small-scale graphs or summarizing equations, are indicated as "GRAPH DATA NOT RETRIEVED" and "SUMMARIZING EQN ONLY" for the reader who wishes to examine them himself. There are 41 entries in the former category and 22 in the latter.

Once a value was included explicitly, we attempted to evaluate the purity of the materials and the accuracy and precision of the method used. These were noted separately as reported in the "Quality" Rating columns. The meaning of the symbols is as follows:

\section{MATERIAL}

A Highest purity-not likely to be significantly improved in the future

\section{METHOD}

precise to about $1 \%$ accurate to $1.5 \%$ 
B Very pure but may still contain significant traces of impurities

C Purified but likely to contain significant impurities

D Compounds purified without special precautions against surfactant impurities

E Good quality mixture of head groups

F Good quality mixture of homologues

G Not purified

H Commercial surfactant material

P High purity claimed without supporting data

Q

theoretically

calculated

$\mathrm{X}$ No information given

These ratings should be taken with certain reservations, both because of the possibility of personal bias and of the frequent lack of all the information required to form a definite opinion, and the unavoidable lack of consistency over the four-year span during which they were being assigned. All that can be said is that they represent our best opinion at the time of a detailed reading of the article cited. Because the evaluation was individual, it is not surprising that upon later comparative evaluation, some values which were initially ranked high appeared more questionable or vice versa. Hence, the ratings are at best meaningful to plus or minus one category. We may mention, however, that during the final evaluation we frequently referred to the original papers, and only in two cases did we feel the need to change the preliminary ratings.

Comparative Evaluation. This evaluation was performed after all the data were collected and classified by the computer into the form of the "Complete CMC Listing" of this report. This permitted easy intercomparison of values reported for the same or similar systems. Each value was then assigned to one of a set of categories. This assignment also formed the basis for obtaining the "con- firmed." "recommended," "disputed," and "selected" values for a separate listing.

The following categories were used:

1-Confirmed. - Values from at least two independent laboratories which are of good quality both with respect to materials and methods and which agree within the expected limit for a given method or between methods. These may be used with high confidence.

2-Recommended.-Values of the same quality as above but lacking independent confirmation.

D-Disputed.-These are values which seem as accurate as the preceding ones, but disagree by more than would permit them to be classified as "confirmed." It is likely that one of the values is correct but we did not have a firm basis for deciding which. In some cases correlation with values for other systems indicated a definite choice and the preferred value was assigned a "2," i.e., "recommended" rating, and remaining values were assigned the " $L$ " or "literature" rating.

3-Selected. - These are values which do not appear to have the accuracy of the above categories but should still be of considerable utility. We feel that they are probably within 10 percent of the "true" value, as measured by the same approach. Their total number is less than 10 percent of all the entries, and they represent the next most reliable group after (1) and (2).

L-Literature. - This category includes the great majority of the values. They are provided for bibliographical completeness and because some readers may prefer any value - no matter how unreliable - to no value at all. Some of these values may be accurate, but there is insufficient information to certify that this is the case. Some will turn out to be off by orders of magnitude. In some cases where the literature provided prima facie evidence that the CMC value was erroneously assigned, we have indicated this by the statement "QUESTIONABLE CRITERION" in the "method" column.

P-Preferred.-Occasionally, widely differing values have been reported for what should be a single CMC. The reader's choice can generally be guided by the quality rank assignment to the compound and method. In a few cases we thought it advisable to indicate the preferred value by a $P$. These data did not qualify for one of the "selected" or "recommended" categories. but seem to be clearly preferable to the others.

$\mathrm{R}$-Indicates a literature reference in which the reader may find a value not tabulated by us. A 
statement in the "methods" column indicates whether the reason for omission is that we considered it a duplication (without explicit reference) of a value from another publication, or a value which could not or should not be listed.

$\mathrm{X}$-Indicates a cross-reference within our tabulation and is used for mixed systems of surfactants which are reported only once under one compound but cross-referenced under the others.

In deciding about the probable accuracy of a CMC value we considered not only the details of the particular measurement but also the general validity and limitation of the method used. It may, therefore, be appropriate now to review briefly the multitude of these methods and then to consider some factors which guided us in judging their intrinsic accuracies.

\section{Methods of Determining CMC's}

Although abrupt changes in the concentration dependence of several properties of several surfactant solutions had been observed before the 1930's [26-28] and the concept of micelles in such solutions had been developed by McBain and coworkers $[29,30]$, the existence of a narrow concentration range, called "the critical concentration for micelles" [31], below which the solution contains negligible amounts of micelles and above which practically all additional surfactant is found in the form of additional micelles, was established by Bury and his coworkers [31,32] and Hartley and his coworkers [33] in the early 1930's. Since that time, CMC determinations have multiplied and the results have been used in a variety of ways. The importance of a definite CMC value to which micellar properties could be extrapolated so as to give the infinite dilution behavior of micelles was emphasized by Debye soon after World War II [34].

In the process of collecting the present data we have distinguished 71 methods of determining the CMC, ranging from a few widely used ones to a few reported only once. These may be grouped as follows, with the numbers in parentheses giving the number of CMC's in this report for each method or group of methods. Experimental details of these methods may be found by following the references given for each in the index to methods.

The entry "METHOD NOT CITED" (128) in most cases refers to just that, when a CMC value appears with no further indication. In a few cases, however, it results from the fact that the article is not clear as to which of two or three well-defined methods was used in determining each individual value. One or two of such cases deserved, in our opinion, enough confidence to be included among "selected" values.

Also in a special category is the THEORETICALLY ESTIMATED (5) entry which we included for completeness.

The other methods can be divided into two broad classes depending on whether another material is added to the system specifically for the purpose of the measurement (as opposed to an additive whose effect is being studied). They are at present all based on the study of a property of the system as some function of concentration and detection of a particularity, such as a change of slope or a discontinuity, at the CMC.

\section{Methods Requiring No Additive}

1. SURFACE TENSION (940). This method of increasing popularity involves the measurement of surface tensions of solutions by a variety of methods which we did not attempt to classify (such as the du Nouy ring detachment method, the Wilhelmy plate equilibrium or detachment, drop weight or volume or shape). The data are generally plotted against the logarithm of concentration - LOG PLOT (843) - as the abscissa and the transition between a descending line (often assumed to be straight) and another one close to the horizontal is taken as the CMC. The data can also be plotted directly against the concentration-LINEAR PLOT (18)-in which case the curvature of the descending portion is much more marked and the transition less sharp. A MINIMUM (15) in the curve is now known to be due to the presence of a third component (contamination or products of hydrolysis) which is more surface active but removed from the surface by solubilization in the micelles above the CMC. It is sometimes reported as the CMC of the system. We have also encountered the UNSPEC (64) where no details are given. Interfacial tension methods are considered later among methods involving an additive.

A related method is based on the FOAMING POWER (2) of the solution. It is not clear at present how the changes in this property are related to the association of monomers into micelles.

2. Electric conductivity-COND or COND. CTNCE (953)-is based on the measurement of the A-C electric resistances of the solutions. These can then be interpreted in terms of the specific 
conductivity-SPECFC (386)-and plottedGRAPH (343)-against concentration to give two almost straight lines whose intersection is the CMC or the data corresponding to each straight line can be summarized by equations - EQUATNS (43)and the CMC obtained analytically. The data can also be converted to equivalent conductivityEQUIV (352) - and the CMC obtained graphicallyGRAPH (319)-usually plotting against the square root of concentration. Occasionally linear or cube root plots have been encountered. In much early work it was the first detected deviation-1ST DEVIATION (18)-that was reported as the CMC. As the CMC thus assigned depended clearly on the sensitivity of the method, this approach seems to have been abandoned. A few surfactants having low CMC's show a maximum of equivalent conductance in the CMC region. The origin of this maximum is not yet clear $[34 a]$ and in some cases the beginning of the rise-MAX BEGINING (10)in others the MAXIMUM (5)-itself is reported as the CMC.

Some authors report averages-AVER (110)-of two of the above methods, namely specific and equivalent-SP EQUIV (104)-conductivities or of the beginning of the rise to the maximum and of specific conductance-COND BEGINING MAXIM (6). Some report results based on conductance without further details - UNSPEC (105).

The variation of electric conductance with frequency at high frequencies, the so-called WIEN EFFECT (1), is different for micelles and monomers and has been used to determine the CMC.

3. There is a variety of methods to investigate optical and spectroscopic properties of a solution, and they may be classified as follows:

(a) Measurements of scattered light-LITE SCATR or LIGHT SCATTER (317) depends on the measurement of the intensity of light at an angle, generally $90^{\circ}$, to the incident beam.

A plot of this intensity or of the turbidity of the solution (which is proportional to it) shows a low slope for dilute solutions and a steeper one above the CMC. The intersection of the two parts on a TURBIDITY PLT (291) gives the CMC. Debye has pointed out that the concentration above the CMC divided by the turbidity (or excess turbidity above that at the CMC) is close to a straight line. Conversely the CMC may be obtained by selecting the value which gives the best straight line on such a DEBYE PLT (25). In light scattering the exact procedure was not indicated only once-UNSPEC (1).

(b) The CMC can be obtained from the change of slope of the REFRACTIVE INDEX (134) when plotted against concentration.

(c) The absorption spectrum of some surfactants is different when they are in micellar and in free form. Hence, plotting the absorbancy at a suitable wavelength can give a change of slope corresponding to the CMC. This MICELLAR SPECTRAL CHANGE (30) method should be distinguished sharply from the multitude of other spectral change methods in that it does not require any additive.

(d) Others. A CMC has been obtained by the change in the X-RAY DIFFRACTION (1) pattern but this method has a very low sensitivity and, therefore, precision. Another method using a spectroscopic technique which has been introduced after the closing of this survey involves shifts of nmr peaks.

4. Calorimetric methods used to obtain CMC's are those of SPECIFIC HEAT (4) and HEAT OF DILUTION (24).

5. Two colligative properties have been used. FREEZING POINT (14) lowering which gives the CMC at a single temperature, generally close to zero, determined by the nature of the solute and VAPOR PRESSURE LOWERING (38) generally measured with a so-called vapor pressure osmometer based on the temperature comparison of two droplets, one of solution, the other of solvent.

6. The abrupt increase of solubility with increasing temperature which occurs once the solubility reaches the CMC region is the basis of the KRAFT POINT SOLUBILITY (21) method. This change is sharpest when the logarithm of the solubility is plotted against the inverse absolute temperature. Often other plots or even single point experiments are used which have little or no value.

7. Several of what may be called transport properties have been used. They include:

(a) Measurements of the DIFFUSION COEFFICIENT (2) of the surfactant which gives, of course, an average of the very different mobilities of micelles and monomers and leads to a change of slope in a $\mathrm{D}$ versus $\mathrm{C}$ plot to give the CMC.

(b) The VISCOSITY (9) of the solution plotted as the specific viscosity $\left[\left(\eta-\eta_{0}\right) / \eta_{0}\right]$ or as the reduced viscosity $\left(\eta_{\mathrm{sp}} / c\right)$ shows a change of slope at the CMC. A related method depends on an observed VISCOSITY MINIMUM (14) in the CMC region. This minimum probably results from a combination 
of surface tension, contact angle, and viscosity effects in the capillary instrument used, so that its relation to the micelle formation is not clear.

(c) Measurements of STREAMING CURRENT (6) have been also used for determining the CMC and should perhaps be classified among methods requiring an additive since these electrokinetic phenomena depend on the adsorption on the solid involved which is specific to the surface. They also depend, though only in a secondary way, on changes occurring in the bulk of the solution which involves the CMC.

(d) Changes in the concentration of the filtrate in ULTRAFILTRATION (9) and in the sedimentation coefficient in ULTRACENTRIFUGATION (1) also lead to CMC values.

8. Potentiometric measurements use several approaches:

(a) ELECTROMOTIVE FORCE (32) methods involve measurements of potential of either specific ion electrodes other than $\mathrm{pH}$ ones or of an insoluble mercury-surfactant salt electrode against a salt bridge-reference electrode. It may be noted that the presence of the salt bridge often causes local precipitation of the surfactant and that insoluble salts can be solubilized by micelles. These complications are often overlooked but should, perhaps, cause these methods to be considered with those involving additives.

(b) EMF ALONG CONC GRADIENT (1) uses two closely spaced identical electrodes moved through a solution having a known concentration gradient. Hence, it depends on a change of the slope of the emf-versus - concentration curve.

(c) PH AND HYDROLYSIS (9) method involve $\mathrm{pH}$ measurements interpreted either directly or after conversion to a degree of hydrolysis. They are grouped together since they often involve problems of carbon dioxide contamination as well as salt bridges.

9. Other bulk properties that have been used to determine CMC's are density (23) based generally on magnetic float methods plotted directly as DENSITY (17) or after conversion to PARTIAL VOLUME (6) and ultrasonic VELOCITY OF SOUND (10).

\section{Methods Involving the Use of an Additive in the Bulk of the Solution}

1. Spectral change-SPCTR CHNGE (1602)methods.

This family of methods whose limitation (which we consider quite severe) will be discussed below (p. 12) is by far the most fertile one as far as supplying CMC values. It is based on the fact that the spectra of many dyes added in very small amounts to a surfactant solution are very different in the region below and in that above the CMC. Pinacyanole-PNCN (1176)-is also by far the most popular among these dyes. The CMC may be determined, for example, by titration of a solution above the CMC by one below the CMC, both containing the same concentration of dye. Some definite shade is chosen as the end point corresponding to the CMC. Alternatively, solutions having concentrations bracketing the CMC are prepared containing the same concentration of the dye and their color examined visually, or their spectrum or their absorbancy at a specific wavelength measured. In any case, the concentration at which some sharp change occurs is taken as the CMC. In some cases the values are extrapolated to zero dye concentration. For one dye Rhodamine 6G-RHD6 (74) in addition to the spectral change, the change in fluorescence FLUOR CHNGE (19) was used and is based on the same principles.

We have noted whether the technique used was VISUAL (1277) photometric-FOTOMTR (305)-or UNSPEC $(20)-$ as this is related to the precision expected.

Other dyes which have been used in this method are Fluorescein-FL (2), Erythrosin-ERTS (7), dichlorofluorescein-DCLF (9), Benzopurpurine 4B-BZP4 (11), Bromphenol Blue-BRPB (21), Skyblue FF-SKYB (43), Eosin-EOSN (51), Indophenol-INPX (129) and, included because it performs the same function although not a dye in the strict sense, Iodine-12 (52). There is also unspecified VISUAL SPECTR CHNGE (27).

2. The fact that many water-insoluble substances dissolve significantly in the presence of micelles, i.e., are solubilized, has been used in the determination of CMC's by solubilization - SOLUBLZTN (293) - methods. The solubility of dyes has always been determined photometrically-FOTOMTR (274) - unless it is UNSPEC (12), that of TOLUENE (4) has been determined volumetrically, and the limit of solubility of lauryl alcohol has been es. tablished turbidimetrically-TURBIDMTR LOH (3) - . The most popular dye is the so-called orange OT-OROT (158) - which is always 1-0-tolyl-azo-2naphthol, CI Solvent Orange 2, m.p. 128-9 ${ }^{\circ}$, and not the complicated pigment which was once available under that name which is CI Pigment Orange 13, 
m.p. $332^{\circ}$. Other solubilizates used are paradimethylaminoazobenzene-PDMAB (59), Sudan 4-SDN4 (46), azobenzene-AZBA (11), Yellow OB-YLOB (9), 2-nitro-diphenylamine-2NPA (2) and dimethyl yellow-DMYL (1).

3. Methods based on liquid-liquid interface phenomena.

(a) Measurements of INTERFACIAL TNSN or TENSION (52) between an aqueous solution of a surfactant and an immiscible liquid have been used in the determination of the CMC. Most values were obtained from semilogarithmic plots, LOGM (46) but we also found UNSPEC (6). These methods are classified among those requiring an additive because of the always finite solubility of the other liquid in the aqueous phase especially above the CMC. A frequently present source of error in this method is the solution of some of the surfactant in the nonaqueous phase which can radically change its real concentration.

(b) We are placing in the same category methods based on the suppression of the POLAROGRAPHIC MAXIMUM (45) since this suppression is related to an increase in surface (dilational) viscosity of the mercury-solution interface which reduces the convection currents responsible for the maximum in the "diffusion" current. These surface viscosity changes are in turn related to the adsorption of the surfactant and thus depend on its activity. However, it is not clear at present how the final polarographic criterion is related to the CMC. These measurements require also the presence of a very high concentration of an inert electrolyte and that of an electrochemically active indicator ion. This makes the composition of the solutions rather unique and prevents comparison with other data. Although we are quite skeptical about the significance of data obtained by this method, we have reported them in view of lack of any definite evidence of their invalidity. A further disturbing fact is that sometimes two CMC's are reported for a given system. One of them is, therefore, certainly wrong. However, for the sake of completeness we had to record both.

4. Other methods in this class include a result obtained from changes in the FLOCCULATION RATE (1) of a suspension and those from changes in the (reaction) REACTN RATE OF A SOLU. BILIZATE (2) as it becomes solubilized above the CMC.

\section{Reasons for Methodical Differences Between CMC Determinations}

As shown by the above review of methods used, the determination of a CMC involves a series of measurements of some property of solutions of surfactant, alone or with an indicator, as a function of concentration followed by the detection of some characteristic point which is called the CMC. Methodical differences may originate from the choice of the characteristic point, the kind of plot on which this point is chosen, the kind of data which are plotted, and the effect of the indicator, if any, upon these data. We use here the term "plot" in a general sense to include any serial representation and even titration, although in the great majority of cases it is a real graphical plot that is involved.

If the CMC were a sharply defined point. such as a melting point, above which some properties were qualitatively different from those below it, methodical differences would be nonexistent or greatly reduced. In fact, however, all properties of a solution in the CMC region vary in a continuous manner and so do all their derivatives. There is, nevertheless, a relatively narrow region of concentration in which these changes are most marked. This is illustrated by precise measurements on pure systems such as those of figure 4 (and is, of course, exaggerated on impure ones) but is perhaps best

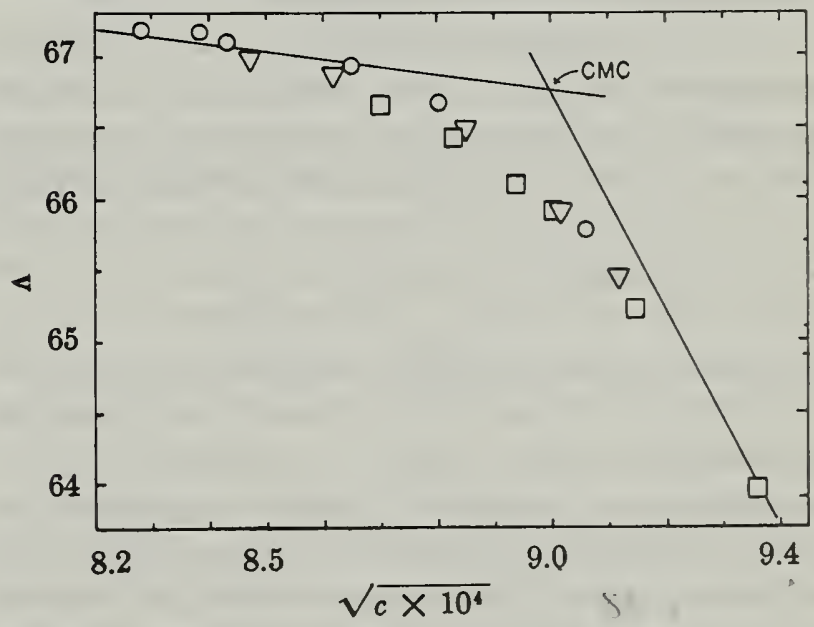

Figure 4. Detail of the CMC region for sodium dodecyl sulfate.

The straight lines are based on many points beyond the limits of the figure. The points were obtained by two investigators on two different preparations of the compound. From reference 58013, reprinted by permission of the Journal of Physical Chemistry.

grounded on broad theoretical considerations. One is that a micelle is by definition a reversible aggregate of a large but not infinite number of monomers and that the micelle-forming reaction must obey the laws of chemical equilibrium. As such, the 
concentration dependence of the degree of micellization has to change gradually although it may change rapidly $[32,33,35]$. A truly abrupt, discontinuous, transition is excluded. Consequently, all properties of the solution must show similarly rapid but gradual changes. Another argument which is less self-evident but seems to be well supported is that there is not a unique number of monomers which can form a micelle but a range with relatively wide limits. Hence, micelles are polydisperse. This is in agreement with the fact that (the average) micellar size varies continuously with conditions such as temperature, concentration of surfactant, concentration of counterion or other additives, nature of counterion, chain length and structure, and also that micelles are good solvents both for other materials as shown by solubilization, and for each other as shown by the frequent continuous formation of mixed micelles. Reasonable assumptions about forces determining the size of the micelle also lead to the conclusion that they must be polydisperse [35a]. Hence, in the CMC region not one kind of micelle, but many kinds appear, each with a slightly different concentration dependence which further spreads and complicates the changes of bulk properties occurring in this region.

In light of these considerations, we shall now review in more detail the sources of methodical discrepancies in CMC determinations and their effect upon our critical evaluation.

1. Choice of the characteristic point. As already mentioned, in some early work, it was the first detectable deviation from monomeric properties that was taken as the CMC. This depended greatly on the sensitivity of the method and has generally been replaced by an extrapolation from below and from above to a point of intersection. If the two lines are straight and differ markedly in slope, this is a simple procedure. Unfortunately, such is seldom the case. Some of the procedures, therefore, involve a treatment of the data to obtain a straight line. Thus, equivalent conductivity of strong electrolytes is plotted against the square root of the concentration in accordance with the Onsager limiting slope.

It also seems true that those properties which give the best straight lines, because they are rather insensitive to interparticle interactions, also give small differences of slope for the same reason. Density and refractive index or specific conductance in the presence of high salt concentrations are good examples. Hence, they require precise meas- urements to give useful results. This implies keeping constant all factors other than the concentration of surfactant, such as the temperature, and also the concentration of other components such as added salts. The latter often requires extreme precautions against evaporation. In the interpretation of such precise measurements, difference plots (i.e., experimental value minus a straight line value) are useful. A few methods, however, give good straight lines of very different slopes, e.g., conductance in the absence of high salt concentrations, solubilization of some dyes, or micellar spectral changes of some pyridinium compounds.

Closely related to the extrapolation method is that of the point of maximum rate of change of slope (i.e., zero value of third derivative) which is useful in theoretical calculations [16] but is so difficult to apply experimentally that it does not seem to have been used.

In the spectral change methods using indicator dyes there is generally a change of absorbancy at any given wavelength from one characteristic value below the CMC to another above the CMC. Some authors take the beginning, some the end, and most the midpoint of that change. The latter is by far the most objectively defined but, as we shall see later, is a function of concentration of the indicator. In visual methods there is much subjectivity and the result can depend markedly on local illumination because of the very complicated absorption spectrum of certain dyes, particularly the most frequently used one, pinacyanole.

Clearly, depending on which point is defined as the CMC, values covering a considerable range may be obtained. We have given weight only to those methods which defined a point in the middle part of the range, particularly those using an extrapolation procedure, and much of the following discussion will be restricted to those.

2. The kind of plot chosen. A point which is not often appreciated is that the same experimental data can give different values for the CMC by extrapolation procedures depending on how they are plotted. The best known example of this is the difference between CMC's obtained by plotting conductance versus concentration and the corresponding equivalent conductance versus the square root of concentration. Figure 5 shows an example of the same set of experimental data plotted in these two ways. Very reasonable linear extrapolations give a well-defined CMC in each case, but the two values 


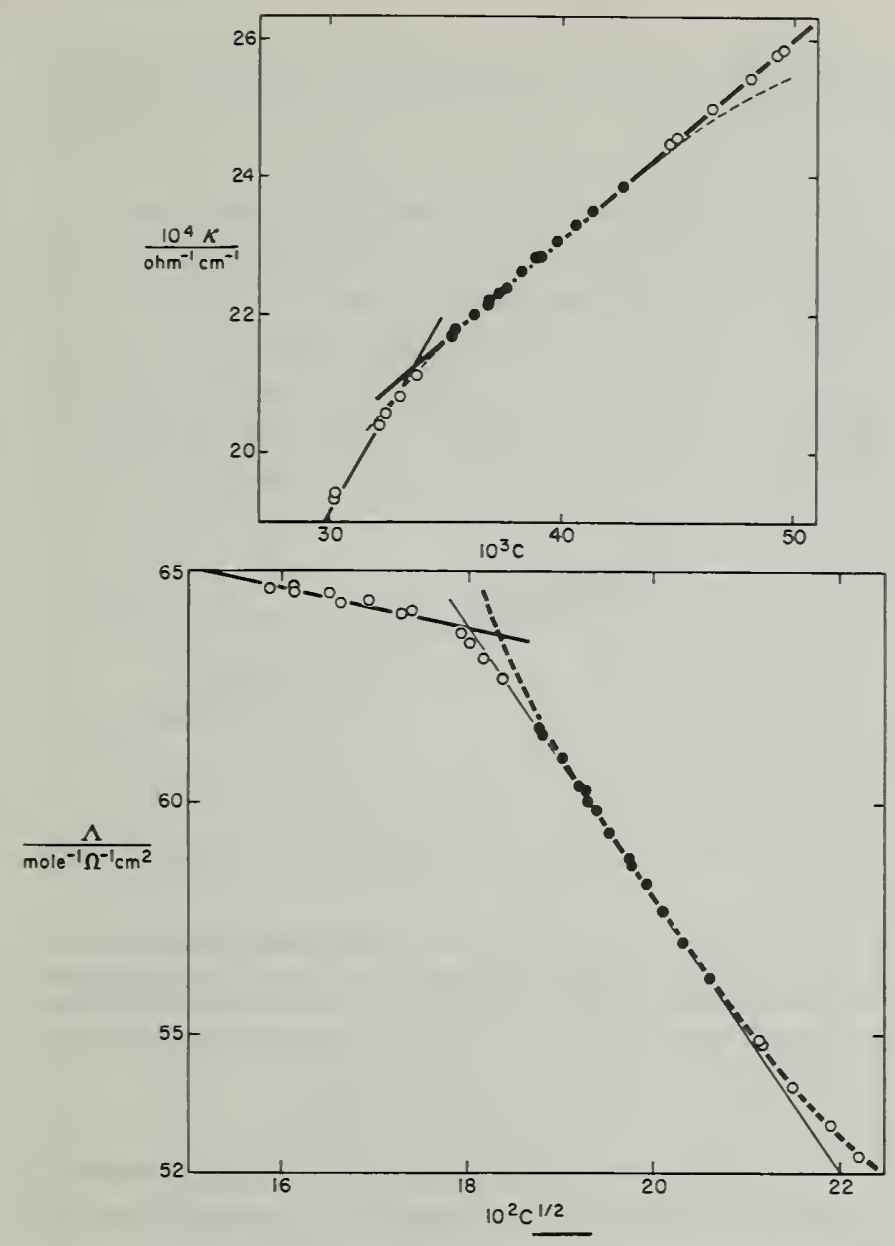

Figure 5. The effect of the way of plotting upon the value of the CMC obtained from the same data.

Above: conductivity versus concentration; below: equivalent conductivity versus square root of concentration. The dotted curves on each graph correspond to the straight line of the other graph. The filled points are common to both lines. Data for sodium decyl sulfate obtained bv Dr. R. J. Otter. From the 1tth Technical Report, Office of Naval Research. Contract Nonr-27400) by K. J. Mysels. R. J. Otter and P. Kapauan, University of Southern California, October. 1960. (Cf. Ref. 61017).

differ by about 3 percent, which is much more than the uncertainty in each. The discrepancy is due in large part to the fact that. a straight line on one plot is a curve on the other so that different points are chosen as the basis for extrapolation and the intersection is shifted.

3. The kind of data. If micelles were monodisperse then, for CMC determinations. the molecular weight or charge dependence of a property would not matter as long as it was linear with some function of the concentration. However, if one accepts the premise that polydispersity is possible. it is clear that different physical properties will give differently weighted measures of micellar concentrations and, therefore, different extrapolated values of zero micelle concentration, i.e., different CMC's [18]. The most accessible experimental variable is the total concentration of surfactant conveniently ex- pressed in equivalents. We will estimate the kind of average that is plotted against this variable in some popular methods without attempting to discuss this subject fully.

Solubilization. As a first approximation we may assume that the surfactant chains have a constant solvent power independent of their configuration, i.e., independent of the micellar size. The quantity measured-the concentration of solubilizate-is then directly proportional to the equivalent concentration of micellized surfactant and independent of polydispersity. More formally, we call $n_{i}$ the number of monomers per micelle of a given size. $M_{i}$ the molar concentration of these micelles, and $C_{i}$ their equivalent concentration. Each group of micelles then dissolves an amount of solubilizate proportional to $M_{i} n_{i}$ and the measured total give us

$$
\sum M_{i} n_{i}=\sum C_{i}=C_{m}
$$

where $C_{m}$ is the total equivalent concentration of micelles. Thus, the result is unweighted.

Colligative measurements. These give the sum of molar concentrations of micelles (for the simple nonionic case). Hence, we get

$$
\sum M_{i}=\sum C_{i} / n_{i}
$$

the inverse-size weighted equivalent concentration of micelles.

Turbidity. The total turbidity is the sum of the turbidities of the individual micelles and these in turn are proportional to the square of their mass (again neglecting charge effects and other interactions). Hence, we measure

$$
\sum \tau_{i}=\sum M_{i} n_{i}^{2}=\sum C_{i} n_{i}
$$

or the size weighted equivalent concentration of micelles.

Thus, it is clear that these different methods must yield different results. Yet, when the polydispersity is small. the micelles large, and the transition region narrow. the differences may be small. often smaller than experimental uncertainties. Thus, depending on the precision involved and the particular system under consideration. the term CMC may have a definite meaning or may have to be tempered by a specification of the experimental method used and also of the way in which the results are interpreted. Unfortunately, there is little definitive information about the final effect that these factors have on the CMC values determined by various methods. 
In the final evaluation of the CMC values, we had to face the question whether two numbers obtained under allegedly identical conditions and by apparently reliable measurements did agree sufficiently to confirm each other. In making the decision, we tried to take into account the differences expected between methods in addition to differences due to the unavoidable experimental uncertainty on which we placed an arbitrary limit of 1.5 percent. Hence, two CMC values differing by 4 percent may be taken as comfirming each other, if the methods used would be expected to differ by 3 percent (in the proper direction), as is indeed the case for conductance and equivalent conductivity measurements.

4. The effect of the concentration range used. Extrapolation generally uses straight lines. Few physical properties vary, however, exactly linearly. Generally, some curvature is present in reality. Hence, the line drawn tends to be a chord and its direction and position are a function of the portion of the curve that is being approximated. This remains true whether a line is drawn by inspection or after a least square calculation. In terms of CMC determinations, it means that the value obtained depends generally on the range of concentrations above and below the CMC over which the extrapolation is taken and, therefore, the number and spacing of points. There is no general agreement as to what these ranges should be and herein lies another source of discrepancies. Figure 6 shows the effect of the range of data used upon the CMC.

In dilute solutions there are some theoretical guides to the expected curvature which suggest the kind of graph which is likely to make the experimental points fall close to a straight line. The square root for conductivity, the logarithm for surface tension are good concentration scales for monomers, and micellar concentration divided by turbidity is a good one for light scattering. In more concentrated solutions the systems depart more and more from ideality and all plots become nonlinear. Extrapolation then becomes more and more a question of judgment and of the range and spacing of available data, and the CMC value becomes highly subjective. Since compounds with shorter chain lengths tend to have higher CMC's, this is an important factor in reducing the accuracy of $\mathrm{CMC}$ determinations as the chain length decreases below 10 carbon atoms.

The CMC region is itself a region of curvature of any measured property. This means that experi-

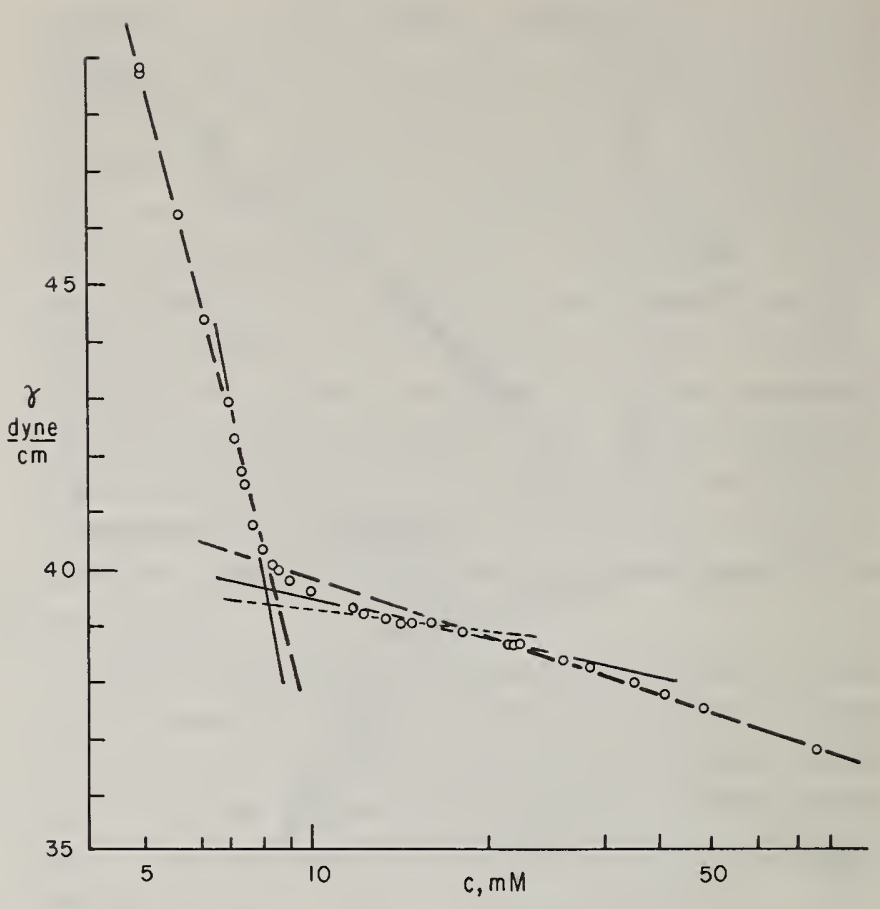

FigURE 6. The effect of the range of values used upon the CMC. Surface tension data for highly purified sodium dodecyl sulfate from reference 66007 . The various straight lines represent extrapolation based on points within a certain range of concentrations. If points outside this range were not available, each of these extrapolations could be considered as quite reasonable.

mental points very close to the CMC cannot be used for its determination because they deviate from both lines used in the extrapolation. The sharper the curvature the smaller the unusable concentration span and the simpler the extrapolation. Theoretical calculations show clearly $[33,35]$ and it is easily seen physically, that the larger the micelle the more cooperative is micelle formation and the sharper the curvature. Polydispersity of micelles can only smear out the transition region. Hence, pure systems show much better defined and more easily measured CMC's because of reduced polydispersity. For space-filling reasons, long-chain monomers can form larger spherical micelles, and do form larger micelles in general according to available experimental evidence [8]. Short-chain compounds, therefore, present an additional difficulty because of the spread of their CMC region not only in absolute terms but also in relative ones.

The combined effects of curvature at higher concentrations and of the relative width of the transition region account for the fact that we have not been able to include any $\mathrm{C}-8$ compounds among those for which confirmed or recommended values are available. This is despite the fact that these compounds are intrinsically easier to prepare in a state of high purity, that the measurement of the physical properties of their solutions can be made 
more precisely and that any impurities in the solvent are less disturbing just because the CMC's are higher.

Long-chain compounds present different but no less serious difficulties because their CMC's are so low. Here the actual measurement of the physical property becomes difficult because it often differs little from that of the solvent, the control of concentration may become a problem because surfactants tend to adsorb at interfaces, and trace impurities in the solvent play a larger role. Furthermore, the compounds themselves are intrinsically less easily purified because certain operations, such as distillation of intermediates, become more difficult, and also because there is an increased number of isomers and because properties of homologues and isomers become more similar. In our collection only one value for a $\mathrm{C}-16$ compound became recommended and an inordinate fraction is marked " $\mathrm{D}$ " for disputed, reflecting the difficulties involved.

Thus, the bulk of reliable CMC data deals with compounds in the $\mathrm{C}-10$ to $\mathrm{C}-14$ range. It is clear that better experimental methods are needed to extend the upper limit and better interpretation to overcome the lower one.

5. The effect of indicators. A very large fraction of all reported CMC values was obtained by methods involving the deliberate addition of an indicator, generally a dye which is solubilized and presents an easily measurable difference in the solubilized and not-solubilized state. This requires a discussion of the role of these indicators and of the reasons which led us to accept certain of these methods as valid and to reject the great majority as likely to be biased by significant methodical errors.

Solubilization is the marked increase of solubility associated with the formation of micelles. It is of special interest for substances which are sparingly soluble in water but is not limited to these. To a first approximation the excess solubility is directly proportional to the concentration of micelles [36] showing that these act as a solvent having a limited solvent power for the solubilizate. We can consider the saturated solution as an equilibrium system in which both the true solvent (water) and the micelles are saturated with the solubilizate. In undersaturated systems there are good reasons to believe [36, 37] that a distribution equilibrium between water and micelles exists.

It is a well-known rule of physical chemistry that the activity of the solvent is lower in a solution than in the pure state. Hence, in the macroscopic world, the liquid phase forms at a lower vapor pressure in the presence of a solute than in its absence. In fact, at equilibrium the first drop always forms at the vapor pressure of the saturated solution. In the microscopic world of the micelle considered as a solvent, the analogy is clear: the activity of the surfactant in the micelle is lower in the presence of a solubilizate. Hence, micelles form at a lower concentration of monomers in the presence of the solubilizate. In fact, the first micelles exist at the equilibrium monomer concentration corresponding to a micelle saturated with the solubilizate under the conditions of the experiment. Two conclusions can, therefore, be drawn: the presence of a solubilized indicator always lowers the CMC, and this lowering reaches a maximum if the micelle is saturated with the indicator during the determination.

If the solubilizate is assumed to form an ideal solution in the micelle and related simplifying assumptions are made, it has been shown by Shinoda [38] that the CMC is changed by a factor $(1-1.36 x)$ for an ionic surfactant where $x$ is the mole fraction of additive in the micelle. For the nonionic case, the factor becomes simply $(1-x)$. The value of $x$ depends, of course, on the concentration of micelles at the point taken as the CMC, the total available amount of indicator, and its solubility in water and in the micelle.

An additional and often neglected complication occurs when the indicator is a high molecular weight organic ion such as most of the dyes used in the spectral change methods, in particular pinacyanole. If the charge of the dye is the opposite of that of the surfactant, an insoluble salt may form. In fact, such a salt generally does form [39-41] as is evident from the color change produced by subCMC amounts of surfactant. This precipitate often remains finely dispersed by an excess of the surfactant and escapes detection. It is this precipitate which then becomes solubilized by micelles when the concentration of surfactant is further increased, causing a second color change that is generally reported as the CMC. The indicators are mostly used in small concentrations $\left(10^{-4}-10^{-5} M\right)$ so that the amount of surfactant consumed by precipitation is often negligible compared to the CMC but can become significant for low CMC's.

The presence of this water-insoluble precipitate has, however, a more profound effect on the determination of the CMC: it maintains a saturated solution until the last of the precipitate is dissolved. This also 
corresponds to the end of at least one absorbancy change, the disappearance of the color of the precipitate (the $\gamma$ band for pinacyanole) [39]. Hence, it has the effect of producing the maximum lowering of the CMC through the actual determination range in many of these methods. Lowering the dye concentration reduces the fraction of surfactant used up in precipitating the dye but does not affect significantly the degree of saturation of the micelles at the color change.

Saturation by the indicator in case of oppositely charged dyes is particularly objectionable because of their frequently high solubility in the micelles. Thus, the solubility of pinacyanole was estimated at 15 to 20 mole percent, in the first mixed micelles that formed [39].

There are other methods of CMC determinations in which the situation is more favorable, either because saturation of the micelle is avoided or because the mole fraction at saturation is much lower. In the former category falls the use of similarly charged dyes. The formation of a water-insoluble, micelle-soluble salt is then avoided; the dye remains in aqueous undersaturated solution and its mole fraction in the micelle is only a corresponding fraction of saturation. Unfortunately, no methodical studies on this approach are known to us. In the same class are dye or iodine methods used with nonionic surfactants.

Some of the solubilization methods use nonionic, water-insoluble dyes whose solubility in the micelles is known to be low. "Orange OT" is the most popular of these, and here it is known [42-44] that the mole fraction at saturation is about 0.01 , thus producing a generally negligible error. Some of the other solubilized indicators such as lauryl alcohol or toluene are likely to lead to mole fractions, and hence CMC changes, of the order of 10 percent or more.

The above discussion makes clear our objection to accepting values, obtained in the presence of easily solubilized indicators under conditions favoring saturation, as valid CMC measurements for the surfactant itself. They are classified as $G$ as far as "Method Quality" is concerned, indicating that a substantial methodical error is likely to be present. On the other hand, such data could be, in principle, excellent values for the mixed system (surfactant + indicator + water). In the great majority of the cases this is not so because the conditions are not sufficiently specified and the proportion of additive often unstated. In some cases, however, we were able to treat the data in just this way and some of the indicators may be found among the additives.

6. The effect of impurities. What has been said above about the effect of indicators applies to the effect of any impurities with the important provision that indicators are added consciously and generally specified in an article, whereas impurities are included inadvertently and their nature is uncertain. We have always scanned an article for clues to the presence and nature of impurities. Such clues can be found in details of the method of preparation, of analyses, and of other physical constants or properties reported. The results are included in our "quality rating" of the compound. In general we have been more skeptical of the older measurements performed before concern about the effect of impurities became widespread and modern analytical techniques were developed. We have given great weight to foam purification at a concentration below the CMC and to chromatographic methods.

The effect of impurities upon the CMC value depends both on the nature of the impurities and on the method used in the determination. We can learn something about the effects of different classes of impurities from their effects upon the CMC when they are added deliberately as "additives." Some have little effect unless present in concentrations too high ever to be reached by impurities. Hydrophilic organic compounds such as sugar or ethanol are in this class as are "inert" salts. On the other hand, oleophilic impurities and salts containing ions forming water-insoluble easily solubilized precipitates can have large effects as we have seen in connection with indicators because they tend to saturate the few micelles present at the CMC and lower the activity of surfactant in them. Higher homologues or incompletely reacted organic intermediates such as alcohols are among objectionable impurities likely to be encountered.

The case of true soaps, i.e., salts of higher carboxylic acids, merits a special mention. Here the acid itself, highly water-insoluble and easily solubilized, is the most important impurity. Some of it is normally formed by hydrolysis and must be considered as a normal constituent of the (watersurfactant) system. Additional substantial amounts are readily formed unless the presence of atmospheric carbon dioxide is carefully excluded. If precautions to this effect were not explicitly mentioned, we assumed that the system was impure and gave it a " $\mathrm{D}$ " rating. The effect of $\mathrm{CO}_{2}$ can be largely neutralized by operating in the presence of a slight 
excess of base. The CMC is not likely to differ substantially from that of the pure system but we have always reported such experiments among systems with additives, specifying the nature and concentration of the base used whenever possible.

The effect of an impurity upon different methods of determining the CMC is largely unknown. Some of our unpublished experiments suggest that traces have a larger effect on values obtained by plotting the equivalent conductivity than those obtained by plotting the specific conductance for the same systems. Dye indicator methods seem to give particularly low results in the presence of higher homologues or intermediates because both tend to concentrate in the first mixed micelles formed. It is likely that pinacyanole may change color by dissolving in droplets of emulsified higher alcohol far below the CMC [45].

The best studied effect is that of a surface active impurity upon the surface tension method. It is now well understood that such impurities may lower the surface tension significantly below the CMC and then become solubilized sufficiently in micelles to be more or less completely removed from the surface $[6,46-48]$. This leads then to a higher surface tension after an intermediate minimum. As already mentioned, such minima have been often used as CMC values but we now take them as prima facie evidence of the presence of easily solubilized-and therefore significantimpurities or hydrolysis products.

An impurity leading to a minimum must have a surface activity at least comparable with that of the surfactant. If the surfactant is very surface active, the same impurity may remain unnoticed. This is shown by experiments of Harrold [49] who found that addition of salt (which increases the surface activity of the ionic surfactant much more than that of the alcohol) leads to a disappearance of the minimum along with a lowering of surface tension and of the CMC. Hence, the more surface active the surfactant, the less significant is the absence of a minimum as a criterion of purity.

When a minimum is present it becomes difficult if not impossible to obtain an accurate value for the CMC from surface tension measurements. However, even when a minimum is absent it seems that the surface tension method is particularly strongly affected by traces of impurities. This is a point which became apparent gradually in the course of our evaluations. Initially, we rated careful surface tension measurements showing no minima as BB. Later,
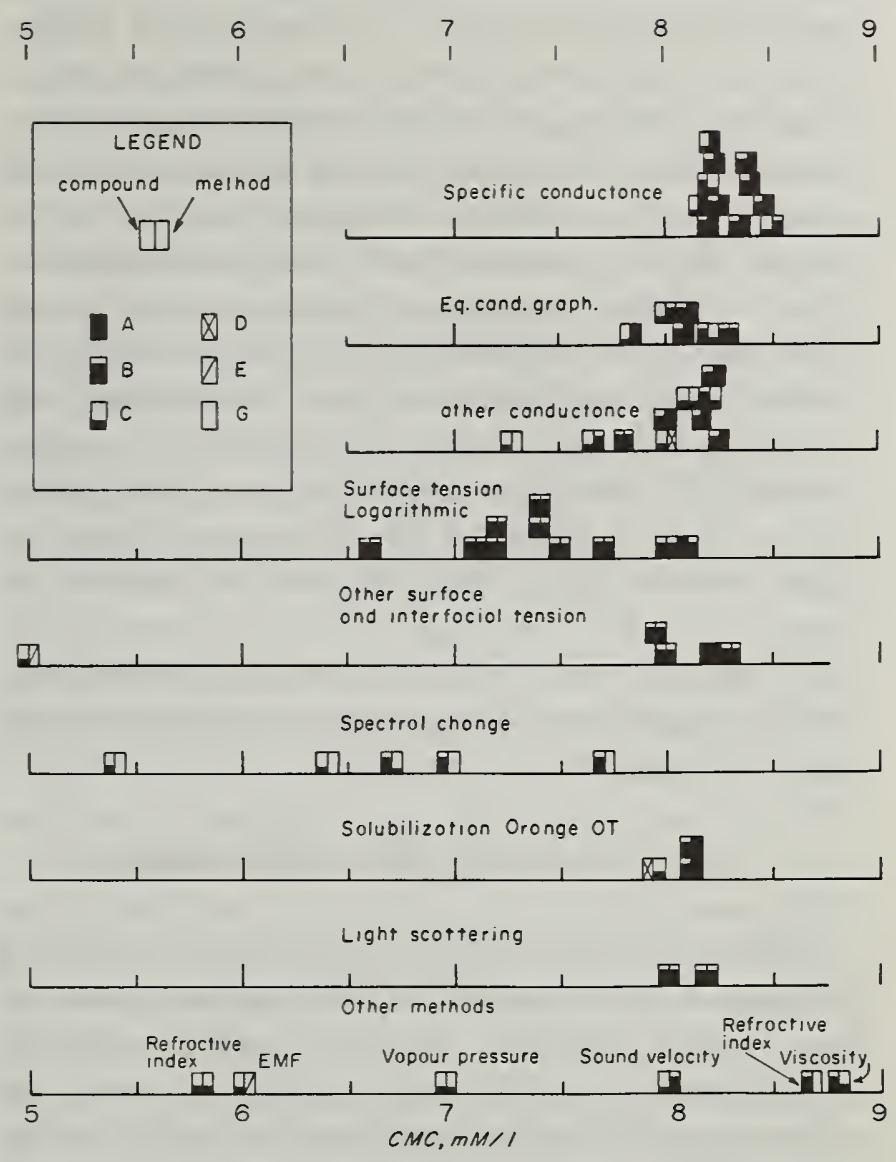

FIGURE 7. All the critical micelle concentrations for sodium dodecyl sulfate at temperatures between 20 and $30^{\circ} \mathrm{C}$, carrying a quality rating of $C-G$ or better, obtained by various experimental methods.

Each square represents a determination and as shown in detail in the legend. the amount of black increases with the quality of the determination. It may be seen that CMC's determined by surface tension from a logarithmic plot scatter much more than other high-quality data obtained by a single method.

we found that this was often an overestimate of the accuracy of the method, although the details of the source of error remain obscure. Figure 7 illustrates our case. It shows all the measurements rated CC or better, and CG for sodium dodecyl sulfate at $25 \pm 5^{\circ}$. Values obtained by different methods are shown on separate lines and the shading of the points indicates their individual quality rating. It may be seen that for conductance methods and for the miscellaneous ones the better values cluster together and the methodical difference mentioned earlier between equivalent and specific conductance plots is visible. The spectral change methods give, as would be expected, widely scattered results. However, the surface tension values rated BB, although not nearly as bad as the spectral change ones. cover a surprisingly wide range and show no clustering near the CMC expected for the pure compound. Part of this may be due to differences in extrapolation. but measurements on an especially 
carefully purified sample (foamed in situ) yielded much more acceptable values using different ranges of points. This suggests that residual impurities are to blame. It also suggests that for surfactants having greater surface activity the effect may well be reduced, but there are no data to test this hypothesis.

As a result of these considerations we did not depend on surface tension values alone for any "confirmed" result although we have used them occasionally to confirm results obtained by another method. We have also used them more liberally for the nonionic compounds both because these are more surface active and because alternatives are fewer.

For the sake of consistency, we have maintained the $\mathrm{BB}$ rating for this type of data throughout in the individual quality evaluations.

\section{Scope of this Collection}

Micelle-forming systems cover a wide variety of molecular species which have in common one feature often described as amphipathy, a built-in asymmetry in the molecule which is composed of a nonpolar, hydrophobic portion, and a polar, hydrophilic part. The systems we have covered include monomers containing straight or branched aliphatic chains, sometimes partly or completely halogenated, and sometimes partly aromatic, with a wide variety of head groups, nonionic, zwitterionic, or ionic, including a variety of counterions in the last case. The solvents are aqueous, partially or completely; in a few systems the medium is heavy water. The variables which are explicitly cataloged, are temperature, pressure (except when it is atmospheric), the nature and the concentration of additives to the medium, the experimental method used, and the type and source of the CMC data themselves.

Certain types of systems were excluded intentionally. The reasons for such exclusions are given.

The association of monomers to produce oligomers and multimers in solution is a frequently observed phenomenon. The existence of a critical micelle concentration, however, requires fairly large multimers (containing roughly 20 or more monomers) in preference to small oligomers. Thus, for example, if monomers associate to produce dimers. trimers, and higher multimers with approximately equal stepwise association constants (the association constant describing the equilibrium between the $N$ th mer, the $(N+1)$ th mer and the monomer), the degree of aggregation is a mild function of the total concentration; the aggregates are very polydisperse, and the system does not show any pronounced critical concentration.

The difference between systems which exhibit fairly sharp critical concentrations and those which do not is obviously a matter of degree. It seems, however, that the requirement of relatively greater stability of large multimers as compared to small oligomers is met mainly by monomeric systems which contain some flexible aliphatic chains. Those monomers which contain fused aromatic ring systems, such as dyes and other flat organic molecules, or fused alicyclic systems such as bile-salts, are expected to have, and seem to have in fact, rather diffuse and extended concentration ranges over which the degree of association increases from low to high values. For many such systems, CMC values have been reported in the literature [50]. Because of the relatively greater uncertainty of defining and determining the CMC values in such systems, particularly when the average degree of association is small, we have not extended our compilation to such systems. We have, however, recorded CMC values for many systems where the monomer is partly aromatic and partly aliphatic.

The phenomenon of micelle formation in aqueous systems is primarily a result of hydrophobic interactions. Hydrophobic interactions are a net result of a number of factors involving structural rearrangements of water molecules and solute-solvent, solvent-solvent, and solute-solute van der Waal's interactions. It is, therefore, not unlikely that in solvents other than water, similar interactions, perhaps on a reduced scale, can occur to produce other kinds of "solvophobic" bonding. In our compilation, we have many examples of mixed aqueousnonaqueous systems including such media as 96 percent sulfuric acid. It is expected that future research will provide examples of micellization in many other completely nonaqueous systems where the micelles will have essentially the same kind of structure as in aqueous media, namely a hydrocarbonoid core and a polar interface.

When the medium is nonpolar, however, e.g., benzene or other hydrocarbon-like solvents, it is relatively improbable that the hydrophobic moiety of the monomers will have any great tendency to aggregate. For such systems, any aggregation that occurs is more likely to be due to the association of the hydrophilic groups of the monomers, which are now "solvophobic," to produce micelles of the 
opposite kind of structure, i.e., "invert micelles," having a core composed of the hydrophilic groups of the monomers with the hydrocarbon groups remaining outside. Although such associations have been studied, and CMC values have been quoted [51-54], considerable uncertainty remains about their significance. We have, therefore, excluded such systems from our compilation.

Well above the CMC, in aqueous media, there occur extensive interactions between micelles, which are often difficult to unravel, particularly if the micelles are ionic. In such concentrated solutions, many physical properties of solutions exhibit breaks or kinks somewhat similar to the ones that occur at the CMC where micelles first form. The concentrations where such breaks or kinks occur are often described as "the second CMC" $[55,56]$. The second CMC is not well understood, but it clearly involves changes in inter-micellar interactions as also monomer-micelle interactions. These "higher CMC" values have also been excluded from our compilation.

While the above restrictions imposed on our compilation are voluntary, there are some involuntary omissions and probable errors which must be mentioned. Of foremost concern, of course, is that many publications must have been missed altogether. A balance has had to be struck between completeness of coverage (up to December 1966) and inordinate delays in producing this report. It is hoped that not many high quality data on well-characterized systems have been omitted. It is probably unwise to hazard a guess as to what percentage of available CMC values in the literature has been missed, but we hope that it is below 10 percent. It is possible, also, that some CMC values in the publications we have examined have been unintentionally overlooked. We hope that readers of this report who note either of such omissions will draw them to our attention.

\section{Methods and Techniques in the Collection of Data}

This section deals with some of the problems we encountered in the retrieval of the literature pertaining to CMC measurements and in obtaining numerical values of the CMC.

Because of the wide-spread importance of and interest in surfactants in solution, CMC values appear in a wide variety of journals. As the values themselves are often of auxilliary and secondary in- terest to the main purposes of the investigation, neither the expression "critical micelle concentration" nor the word "micelle" may appear in the title of the articles. For the standard methods of literature search using Chemical Abstracts and other such publications, we, therefore, had to use a variety of key words for searching purposes. Use was made whenever possible of published books and review articles for the entries they provide into the literature. It was found necessary, however, to make extensive use of personal appeals to various investigators in the field of surfactant solutions to provide references to published work.

To obtain numerical values of the CMC, several procedures had to be used, as indicated by the source symbols. All numerical values quoted in the publications were given the source symbol T. The majority of these values were found in tables, but a substantial number were found dispersed in the written part of the text of the papers. It was necessary, therefore, to scrutinize the whole paper carefully. Of about 4700 numerical values reported in this compilation, 3207 , or 68 percent, were obtained from this "direct" source. Numerical data were thus not available in the published literature for about $1500 \mathrm{CMC}$ values which had to be obtained from various "indirect" sources described below.

In many publications, CMC values themselves are presented in graphical form, or some measured physical property of surfactant solutions is plotted as some function of the concentration, so that the CMC can be obtained from the "breaks" in the curves. A large number of numerical values of this type, i.e., values which are not given in the papers, were obtained by indirect means. A total of 598 values, given the source symbol $\mathrm{L}$, involve, in addition to the published data, a private communication from an author. 515 values were "read" from published graphs and are given the source symbol G. 122 values used combined sources, GL. 90 CMC values were obtained from the kinks of published graphs of some physical property vs. concentration and are given the source symbol K.

In many publications, numerical values of some physical property measured, e.g., conductance, are tabulated as a function of concentration. When such measurements seemed to be of high quality, we obtained CMC values by making plots ourselves of the physical property measured. These CMC values, 96 in all, were given the source symbol $P$.

A small number of values, 21, were obtained by solving published equations relating numerical 
values of some physical property, particularly conductance, with concentration, below and above the CMC. These have the source symbol E.

$43 \mathrm{CMC}$ values have combined source symbols, e.g., TL, KL, indicating dual sources of the values. These include 13 containing the symbol $\mathrm{A}$, which denotes values which have been corrected for obvious misprints.

A substantial number of entries in the compilation do not report numerical CMC values because the entries either give a cross-reference to mixed surfactant systems, involve references to published graphical data for which retrieval was uncertain, or relate to numerical values obtained using criteria which we consider questionable.

The private correspondence from several investigators contained numerical data and references to unpublished work. We have not used these data in our present compilation.

A partial justification of the use of the various "indirect" sources referred to above is that their contributions to the categories of all selected data are high. Out of 620 data included in the categories $1,2,3$, and D, only 270 were obtained from tabulated sources, T. Thus, 56 percent of all selected data are from "indirect" sources. Similarly, of the 106 confirmed (1) and recommended (2) CMC values, 67 are from "indirect" sources, not readily available from the literature.

\section{References}

[1] Kling, W., and Lange, H., Proc. 2nd Inter. Cong. Surf. Activity, 1, 295 (Butterworths, London, 1957).

[2] Shinoda, K., Yamaguchi, T., and Hori, R., Bull. Chem. Soc. Japan 34, 237 (1961).

[3] Benton, D. P., and Sparks, B. D., Trans. Faraday Soc. 62, 3244 (1966).

[4] Ginn, M. E., and Harris, J. C., J. Am. Oil Chem. Soc. 38, 605 (1961).

[5] Preston, W. C., J. Phys. Coll. Chem. 52, 84 (1948).

[6] Elworthy, P. H., and Mysels, K. J., J. Colloid Sci. 21, 331 (1966).

[7] Stigter, D., and Mysels, K. J., J. Phys. Chem. 59, 45 (1955).

[8] Huisman, F., Proc. Konink. Ned. Akad. Wetenschap. B67, 367, 376, 388, 407 (1964).

[9] Mysels, K. J., and Princen, L. H., J. Phys. Chem. 63, 1696 (1959).

[10] Mukerjee, P., and Banerjee, K., J. Phys. Chem. 68, 3567 (1964).

[11] Dunlap, R. B., and Cordes, E. H., J. Phys. Chem. 73, 361 (1969).

[12] Tong, L. K. J., Reeves, R. L., and Andrus, R. W., J. Phys. Chem. 69, 2357 (1965).

[13] Bruice, T. C., Katzhendler, J., and Fedor, L. R., J. Am. Chem. Soc. 90, 1333 (1968).
[14] Ochoa-Solano, A., Romero, G., and Gitler, C., Science 156 , 1243 (1967).

[15] Winters, L. J., and Grunwald, E., J. Am. Chem. Soc. 87, 4608 (1965).

[16] Phillips, J. N., Trans. Faraday Soc. 51, 561 (1955).

[17] Overbeek, J. Th. G., Stigter. D., Rec. Trav. Chim. 75, 1263 (1956).

[18] Mukerjee, P., Adv. Coll. Interf. Sci. 1, 241 (1967).

[19] Shinoda, K., and Hutchinson, E., J. Phys. Chem. 66, 577 (1962).

[20] Aranow, R. H., J. Phys. Chem. 67, 556 (1963).

[21] Emerson, M. F., and Holtzer, A., J. Phys. Chem. 69, 3718 (1965).

[22] Goddard, E. D., Hoeve, C. A. J., and Benson, G. C.. J. Phys. Chem. 61, 593 (1957).

[23] Mukerjee, P., and Ray, A., J. Phys. Chem. 67, 190 (1963).

[24] Emerson, M. F., and Holtzer, A., J. Phys. Chem. 71, 3320 (1967).

[25] Mukerjee, P., Mysels, K. J., and Kapauan, P., J. Phys. Chem. 71, 4166 (1967).

[26] McBain, J. W., Laing, M. E., and Titley, A. F., J. Chem. Soc. 115, 1279 (1919).

[27] Jones, E. R., and Bury, C. R., Phil. Mag. 4, 841 (1927).

[28] Ekwall, P., Acta Acad. Aboensis (Math. et Phys.), 4, 1 (1927).

[29] McBain, J. W., and Salmon, C. S., J. Am. Chem. Soc. 42 , $426(1920)$.

[30] McBain, J. W., Colloid Science (D. C. Heath and Co., Boston, 1950).

[31] Davies, D. G., and Bury, C. R., J. Chem. Soc. 2263 (1930).

[32] Grindley, J., and Bury, C. R., J. Chem. Soc. 679 (1929).

[33] Hartley, G. S., Aqueous Solutions of Paraffin-Chain Salts, Hermann, Paris (1936).

[34] Debye, P., Ann. N. Y. Acad. Sci. 5 1, 575 (1949).

[34a] Kraus, C. A., Proc. Nat. Acad. Sci. 39, 1213 (1953); Stigter, D., Rec. Trav. Chim. Pays-Bas 73, 611 (1954).

[35] Mysels, K. J., J. Colloid Sci. 10, 507 (1955).

[35a] Stigter, D., and Overbeek, J. Th. G., Proc. 2nd Congress of Surface Activity, London, 1957, Vol. 1, p. 311.

[36] McBain, M. E. L., and Hutchinson, E., Solubilization and Related Phenomena (Academic. New York, 1955).

[37] McBain, J. W., and O'Connor, J. J., J. Am. Chem. Soc. 62, 2853 (1940).

[38] Shinoda, K., Nakagawa, T., Tamamushi, B., and Isemura, T., Colloidal Surfactants, p. 73 (Academic, New York. 1963).

[39] Mukerjee, P., and Mysels, K. J., J. Am. Chem. Soc. 77, 2937 (1955).

[40] Hiskey, C. F., and Downey, T. A., J. Phys. Chem. 58, 835 (1954).

[41] Jones, J. H., J. Assoc. Offic. Agr. Chemists 28, 398 (1945).

[42] Williams, R. J., Phillips, J. N., and Mysels, K. J., Trans. Faraday Soc. 51, 728 (1955).

[43] Schott, H., J. Phys. Chem. 70, 2966 (1966).

[44] Kolthoff, I. M., and Stricks, W., J. Phys. Coll. Chem. 53, 424. (1949).

[45] Ginn, M. E., and Harris, J. C., J. Phys. Chem. 62, 1554 (1958).

[46] Miles, G. D., and Shedlovsky, L., J. Phys. Chem. 48, 57 (1944).

[47] Brady, A. P., J. Phys. Chem. 53, 56 (1949).

[48] Williams, E. F., Woodberry, N. T., and Dixon, J. K.. J. Colloid Sci. 12, 452 (1957). 
[49] Harrold, S. P., J. Phys. Chem. 63, 317 (1959).

[50] Hofmann, A. F., and Small, D. M., Ann. Rev. of Medicine 18, 333 (1967).

[51] Becher, P., J. Phys. Chem. 64, 1221 (1960).

[52] Debye, P., and Cole, H., J. Colloid Sci. 17, 220 (1962).

[53] Kitahara, A. Kobayashi, T., and Tachibana, T., J. Phys. Chem. 66, 363 (1962).
[54] Fowkes, F. M., article in Solvent Properties of Surfactant Solutions, p. 65, Ed. K. Shinoda (Marcel Dekker, New York, 1967).

[55] Ekwall, P., Eikrem, H., and Mandell, L., Acta Chem. Scand. 17, 111 (1963).

[56] Ekwall, P., and Holmberg, P., Acta Chem. Scand. 19, 573 (1965). 


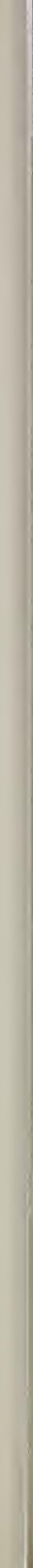




\section{STRUCTURAL INDEXES OF COMPOUNDS WITH KEYS TO COMPOUND NUMBERS}

PART 1. GROUPED BY CHARGE (Arranged by number of carbon atoms in longest hydrophobic tail and by heads)
A. Anionics
B. Cationics
C. Nonionics

PART 2. GROUPED BY CHARGE AND HEAD GROUPS (Arranged by number of carbon atoms in longest hydrophobic tail and by heads)
A. Anionics
B. Cationics
C. Nonionics

PART 3. GROUPED BY STRUCTURE OF HYDROPHOBIC PART (Arranged by number of carbon atoms in longest hydrophobic tail)
A. Alkanes by Branching and Number of Carbon Atoms
B. Alkyl Aryl
C. Unsaturated and Substituted
D. Heterocyclic

PART 4. POLYOXYETHYLENES GROUPED BY DISTRIBUTION OF HEADS (Arranged by number of carbon atoms in longest hydrophobic tail and by heads)
A. Homogeneous Head Groups
B. Reduced Polydispersity of Head Groups
C. Natural Distribution of Head Groups

PART 5. IONICS GROUPED BY COUNTERION (Arranged by number of carbon atoms in longest hydrophobic tail and by heads)
A. Anionics
B. Cationics

PART 6. COMMERCIAL NAMES AND ILL DEFINED STRUCTURES (Arranged alphabetically) 


\section{Structural Indexes of Compounds with Keys to Compound Numbers}

\section{PART 1. Grouped by charge and arranged by number of carbon atoms in longest hydrophobic tail and by}

heads.

\section{IA. ANIONICS BY NUMBER OF CARBON ATOMS}

$\mathrm{C}-1$

428 PERFLUORO ACETIC ACID

$\mathrm{C}-2$

429 PERFLUORO PROPIONIC ACID

$\mathrm{C}-3$

43 BUTYRIC ACID

430 PERFLUORO BUTYRIC ACID

452 3-4-DICHLOROPERFLUORO BUTYRIC/KEL-F/ACID

699 SODIUM BUTYRATE

272 TRI-ISOPROPYL BENZENE SULFONIC ACID

271 SODIUM TRI-ISO-PROPYL BENZENE SULFONATE

$\mathrm{C}-4$

484 SODIUM PENTANOATE/VALERATE/

257 SODIUM DI-N-BUTYL SULFOSUCCINATE

261 SODIUM DI-ISOBUTYL SULFOSUCCINATE

681 SODIUM DIBUTYL BENZENE SULFONATE

445 SODIUM PARA-BIS-/N-BUTYL/ BENZENE SULFONATE

682 SODIUM DIBUTYL NAPHTHALENE SULFONATE /NEKAL/

$\mathrm{C}-5$

700 HEXANOIC ACID

416 PERFLUORO HEXANOIC ACID

453 3-5-6-TRICHLOROPERFLUORO HEXANOIC/KEL-F/ACID

188 POTASSIUM HEXANOATE

701 POTASSIUM PERFLUROHEXANOATE

485 SODIUM HEXANOATE/CAPROATE/

343 SODIUM PENTANE SULFONATE

258 SODIUM DI-N-AMYL SULFOSUCCINATE

C-6

296 POTASSIUM HEPTANOATE

374 DODECAFLUOROHEPTANOIC ACID H/CF2/6COOH

486 SODIUM HEPTANOATE

355 AMMONIUM DODECAFLUOROHEPTANOATE H/CF2/6COONH4

352 SODIUM DI-1-METHYLISOAMYL SULFOSUCCINATE

259 SODIUM DI-N-HEXYL SULFOSUCCINATE

344 SODIUM HEXANE SULFONATE

339 MAGNESIUM HEXANE SULFONATE

501 SODIUM HEXYL BENZENE SULFONATE

446 SODIUM PARA-BIS-/N-HEXYL/ BENZENE SULFONATE

704 POTASSIUM 4-HEXYL RESORCINOLATE

$\mathrm{C}-7$

529 OCTANOIC ACID

417 PERFLUORO OCTANOIC ACID

454 3-5-7-8-TETRACHLOROPERFLUORO OCTANOIC/KEL-F/ACID

476 SODIUM OCTANOATE

44 POTASSIUM OCTANOATE

456 POTASSIUM PERFLUORO OCTANOATE

284 HEXANOLAMINE- $\mathrm{CH} 3 \mathrm{CH} / \mathrm{OH} / \mathrm{CH} 2 \mathrm{C} / \mathrm{CH} 3 / 2 \mathrm{NH} 2-O C T A N O A T E$

303 DI-ISOPROPYLAMONIUM CAPRYLATE

502 SODIUM HEPTYL BENZENE SULFONATE

$\mathrm{C}-8$

375 HEXADECAFLUORONONANOIC ACID $\mathrm{H} / \mathrm{CF} 2 / 8 \mathrm{COOH}$

487 SODIUM NONANOATE

350 POTASSIUM NONANOATE

372 AMMONIUM HEXADECAFLUORONONANOATE H/CF2/8 $\mathrm{COO} \mathrm{NH4}$

420 POTASSIUM 1-1-2-OCTANE TRICARBOXYLATE

6 SODIUM ALPHA SULFOPELARGONIC ACID

25 SODIUM ETHYL ALPHA SULFOPELARGONATE

26 SODIUM AMYL ALPHA SULFOPELARGOIJATE

27 SODIUM HEXYL ALPHA SULFOPELARGONATE

28 SODIUM HEPTYL ALPHA SULFOPELARGONATE

7 SODIUM OCTYL ALPHA SULFOPELARGONATE

31 SODIUM 2 OCTYL ALPHA SULFOPELARGONATE

32 SODIUM 2 ETHYLHEXYL ALPHA SULFOPELARGONATE

35 SODIUM H/CF2CF2/3CH2 ALPHA SULFOPELARGONATE

611 ALPHAPHOSPHONO PELARGONIC ACID
605 SODIUM AMYL ALPHAPHOSPHONO PELARGONATE

260 SODIUM DI-N-OCTYL SULFOSUCCINATE

262 SODIUM DI-2-ETHYL-HEXYL SULFOSUCCINATE

286 AMMONIUM DI-2-ETHYL-HEXYL SULFOSUCCINATE

2 SODIUM OCTYL 1 SULFATE

66 SODIUM OCTYL 2 SULFATE

347 OCTYL TRIMETHYLAMMOMIUM OCTYL SULFATE

643 DODECYL TRIMETHYLAMMONIUM OCTANE SULFATE

181 SODIUM OCTYL 1-SULFONATE

340 MAGNESIUM OCTANE SULFONATE

287 OCTYL TRIMETHYLAMMONIUM OCTANE SULFONATE

503 SODIUM OCTYL BENZENE SULFONATE

49 SODIUM P OCTYL BENZENE SULFONATE

172 SODIUM 2-N-OCTYL BENZENE SULFONATE

510 SODIUM 2-ETHYL-HEXYL BENZENE SULFONATE

676 SODIUM 1, 1,3,3-TETRAMETHYL BUTYL BENZENE SULFONATE

447 SODIUM PARA-BIS-/N-OCTYL/ BENZENE SULFONATE

677 SODIUM DI-/1,1,3,3-TETRAMETHYL BUTYL/BENZENESULFONATE

C-9

530 DECANOIC ACID

455 PERFLUORO DECANOIC ACID

299 SODIUM DECANOATE

90 POTASSIUM DECANOATE

702 POTASSIUM PERFLUORODECANOATE

668 DIPOTASSIUM OCTYL MALONATE

29 SODIUM NONYL ALPHA SULFOPELARGONATE

612 ALPHAPHOSPHONO DECANOIC ACID

616 MONOSODIUM ALPHAPHOSPHONO DECANOATE

606 SODIUM AMYL ALPHAPHOSPHONO CAPRATE

136 SODIUM ALPHA DIMETHYL AMINO CAPRATE

295 SODIUM NONYL 1-SULFATE

536 NONYL SULFONIC ACID

504 SODIUM NONYL BENZENE SULFONATE

493 SODIUM P-NONYL BENZENE SULFONATE

138 SODIUM NONYL BENZENE SULFONATE BRANCHED HYDROCARBON CHAIN

$\mathrm{C}-10$

297 POTASSIUM UNDECANOATE

373 AMMONIUM EICOSAFLUOROUNDECANOATE H/CF2/10 COO NH4

POTASSIUM 1-1-2-DECANE TRICARBOXYLATE

SODIUM DECYL ALPHA SULFOPELARGONATE

SODIUM /OXO/ DECYL ALPHA SULFOPELARGONATE

SODIUM DECYL 1 SULFATE

SODIUM DECYL 2 SULFATE

OCTYL TRIMETHYLAMMONIUM DECANE SULFATE

DECYL TRIMETHYLAMMONIUM DECYL SULFATE

SODIUM DECYL 1-SULFONATE

MAGNESIUM DECANE SULFONATE

OCTYL TRIMETHYLAMMONIUM DECANE SULFONATE

DECYL TRIMETHYLAMMONIUM DECANE SULFONATE

SODIUM DECYL BENZENE SULFONATE

SODIUM P DECYL BENZENE SULFONATE

SODIUM 2-N-DECYL BENZENE SULFONATE

SEDUM DECYL BENZENE SULFONATE BRANCHED HYDROCARBON CHAIN

511 SODIUM 2-PROPYL-HEPTYL BENZENE SULFONATE

$\mathrm{C}-11$

531 DODECANOIC ACID

273 SODIUM DODECANOATE

91 POTASSIUM DODECANOATE

627 CESIUM DODECANOATE

277 BENZYL TRIMETHYL AMMONIUM DODECANOATE

527 SODIUM UNDECANE-3-CARBOXYLATE

669 DIPOTASSIUM DECYL MALONATE

602 ALPHA SULFO LAURIC ACID

235 SODIUM ALPHA SULFO LAURIC ACID

603 SODIUM PROPYL ALPHA SULFO LAURATE

613 ALPHAPHOSPHONO DODECANOIC ACID

617 MONOSODIUM ALPHAPHOSPHONO DODECANOATE

620 DISODIUM ALPHAPHOSPHONO DODECANOATE

607 SODIUM ISOPROPYL ALPHAPHOSPHONO LAURATE

311 SODIUM UNDECYL 1-SULFATE

72 SODIUM UNDECYL 3 SULFAT 
PART 1. Grouped by charge and arranged by number of carbon atoms in longest hydrophobic tail and by heads-Continued

80 SODIUM UNDECYL 6 SULFATE

462 SODIUM MONOLAURIN SULFATE

639 SODIUM UNDECYL THIOSULFATE

537 UNDECYL SULFONIC ACID

418 SODIUM UNDECYL SULFONATE

45 SODIUM P I METHYL DECYL BENZENE SULFONATE

678 SODIUM 1,1-DIMETHYLNONYL BENZENE SULFONATE

679 SODIUM 3-BUTYL HEPTYL BENZENE SULFONATE

$\mathrm{C}-12$

351 POTASSIUM TRIDECANOATE

421 POTASSIUM 1-1-2-DODECANE TRICARBOXYLATE

34 SODIUM DODECYL ALPHA SULFOPELARGONATE

632 POTASSIUM N-DODECYL BETA-ALANINATE

705 DODECYL SULFURIC ACID

1 SODIUM DODECYL 1 SULFATE

67 SODIUM DODECYL 2 SULFATE

634 POTASSIUM DODECYL SULFATE

111 LITHIUM DODECYL 1 SULFATE

23 SILVER DODECYL I SULFATE

568 MAGNESIUM DODECYL SULFATE

24 CALCIUM DODECYL 1 SULFATE

569 STRONTIUM DODECYL SULFATE

572 COBALTOUS DODECYL SULFATE

573 CUPRIC DODECYL SULFATE

570 LEAD DODECYL SULFATE

571 MANGANESE DODECYL SULFATE

575 NICKEL DODECYL SULFATE

574 ZINC DODECYL SULFATE

386 AMMONIUM DODECYL SULFATE

387 METHYLAMMONIUM DODECYL SULFATE

388 ETHYLAMMONIUM DODECYL SULFATE

389 BUTYLAMMONIUM DODECYL SULFATE

112 TETRAMETHYL AMMONIUM DODECYL I SULFATE

383 BUTYL TRIMETHYLAMMONIUM DODECYL SULFATE

382 ETHYL TRIMETHYLAMMONIUM DODECYL SULFATE

718 TETRAETHYLAMMONIUM DODECYL SULFATE

719 TETRABUTYLAMMONIUM DODECYL SULFATE

720 1-6-DITRIMETHYLAMMONIUM-HEXANE/DODECYL SULFATE/2

409 TRIETHANOLAMMONIUM DODECYL SULFATE

410 MORPHOLINIUM DODECYL SULFATE

391 OCTYLAMMONIUM DODECYL SULFATE

385 OCTYL TRIMETHYLAMMONIUM DODECYL SULFATE

280 DECYL TRIMETHYL AMMONIUM DODECYL SULFATE

281 DODECYL TRIMETHYLAMMONIUM DODECYL SULFATE

562 SODIUM DODECENYL SULFATE

636 SODIUM DODECYL THIOSULFATE

541 SODIUM DODECYL MONO-OXYETHYLENE SULFATE

542 SODIUM DODECYL. DIOXYETHYLENE SULFATE

113 SODIUM DODECYL TRI-OXYETHYLENE SULFATE

543 SODIUM DODECYL TETRA-OXYETHYLENE SULFATE

114 SODIUM DODECYL /OXYETHYLENE/17.5 SULFATE

597 SODIUM DODECYL MONO-OXYPROPYL SULFATE

200 DODECYL SULFONIC ACID

179 SODIUM DODECANE 1-SULFONATE

175 SODIUM DODECANE 2-SULFONATE

40 POTASSIUM DODECYL I SULFONATE

635 LITHIUM. DODECYL SULFONATE

342 MAGNESIUM DODEGANE SULFONATE

238 SODIUM DODECANE 1-HYDROXY 2-SULFONATE

247 DODECANE 1-HYDROXY 2-SULFONIC ACID

506 SODIUM DODECYL BENZENE SULFONATE

51 SODIUM $P$ DODECYL BENZENE SULFONATE

171 SODIUM 2-N-DODECYL BENZENE SULFONATE

301 SODIUM 3-N-DODECYL BENZENE SULFONATE

302 SODIUM 4-N-DODECYL BENZENE SULFONATE

514 SODIUM 6-N-DODECYL BENZENE SULFONATE

512 SODIUM 2-BUTYL-OCTYL BENZENE SULFONATE

680 SODIUM 2-PROPYL-4-METHYL-OCTYL BENZENE SULFONATE

492 SODIUM TETRAPROPYLENE/1-3-5-7-TETRAMETHYLOCTYL/BENZENE SULFONATE

SODIUM DODECYL BENZENE SULFONATE BRANCHED HYDROCARBON CHAIN

$\mathrm{C}-13$

532 TETRADECANOIC ACID

298 SODIUM TETRADECANOATE

92 POTASSIUM TETRADECANOATE

670 DIPOTASSIUM DODECYL MALONATE

189 ALPHA SULFOMYRISTIC ACID

236 SODIUM ALPHA SULFO MYRISTIC ACID

604 . SODIUM METHYL ALPHA SULFO MYRISTATE

233 DISODIUM ALPHA SULFO MYRISTATE

614 ALPHAPHOSPHONO TETRADECANOIC ACID
618 MONOSODIUM ALPHAPHOSPHONO TETRADECANOATE

621 DISODIUM ALPHAPHOSPHONO TETRADECANOATE

624 TRISODIUM ALPHAPHOSPHONO TETRADECANOATE

608 SODIUM METHYL ALPHAPHOSPHONO MYRISTATE

68 SODIUM TRIDECYL 2 SULFATE

83 SODIUM TRIDECYL 7 SULFATE

242 TRIDECANE 1-SULFONIC ACID

229 SODIUM TRIDECANE 1-SULFONATE

46 SODIUM $P$ I METHYL DODECYL BENZENE SULFONATE

141 SODIUM TRIDECYL BENZENE SULFONATE BRANCHED HYDROCARBON CHAIN

$\mathrm{C}-14$

426 POTASSIUM 1-1-2-TETRADECANE TRICARBOXYLATE

4 SODIUM TETRADECYL 1 SULFATE

16 SODIUM TETRADECYL 2 SULFATE

73 SODIUM TETRADECYL 3 SULFATE

17 SODIUM TETRADECYL 4 SULFATE

77 SODIUM TETRADECYL 5 SULFATE

525 SODIUM TETRADECYL 6-SULFATE

84 SODIUM TETRADECYL 7 SULFATE

526 SODIUM 2-DI-N-HEXYL ETHYL SULFATE

637 LITHIUM TETRADECYL SULFATE

576 CUPRIC TETRADECYL SULFATE

544 SODIUM TETRADECYL MONO-OXYETHYLENE SULFATE

545 SODIUM TETRADECYL DI-OXYETHYLENE SULFATE

546 SODIUM TETRADECYL TRI-OXYETHYLENE SULFATE

598 SODIUM TETRADECYL MONO-OXYPROPYL SULFATE

599 SODIUM TETRADECYL DI-OXYPROPYL SULFATE

243 TETRADECANE 1-SULFONIC ACID

183 SODIUM TETRADECYL 1-SULFONATE

176 SODIUM TETRADECANE 2-SULFONATE

248 TETRADECANE 1-HYDROXY 2-SULFONIC ACID

239 SODIUM TETRADECANE 1-HYDROXY 2-SULFONATE

507 SODIUM TETRADECYL BENZENE SULFONATE

174 SODIUM 2-N-TETRADECYL BENZENE SULFONATE

513 SODIUM 2-AMYL-NONYL BENZENE SULFONATE

$\mathrm{C}-15$

533 HEXADECANOIC ACID

300 SODIUM HEXADECANOATE

185 POTASSIUM HEXADECANOATE

571 DIPOTASSIUM TETRADECYL MALONATE

190 ALPHA SULFOPALMITIC ACID

237 SODIUM ALPHA SULFO PALMITIC ACID

234 DISODIUM ALPHA SULFO PALMITATE

36 SODIUM METHYL ALPHA SULFOPALMITATE

192 SODIUM ETHYL ALPHA SULFOPALMITATE

193 SODIUM PROPYL ALPHA SULFOPALMITATE

197 DISODIUM 2 SULFOETHYL ALPHA SULFOPALMITATE

615 ALPHAPHOSPHONO HEXADECANOIC ACID

619 MONOSODIUM ALPHAPHOSPHONO HEXADECANOATE

622 DISODIUM ALPHAPHOSPHONO HEXADECANOATE

625 TRISODIUM ALPHAPHOSPHONO HEXADECANOATE

609 SODIUM ISOPROPYL ALPHAPHOSPHONO PALMITATE

69 SODIUM PENTADECYL 2 SULFATE

74 SODIUM PENTADECYL 3 SULFATE

78 SODIUM PENTADECYL 5 SULFATE

85 SODIUM PENTADECYL 8 SULFATE

244 PENTADECANE 1-SULFONIC ACID

230 SODIUM PENTADECANE I-SULFONATE

47 SODIUM P I METHYL TETRADECYL BENZENE SULFONATE

142 SODIUM PENTADECYL BENZENE SULFONATE BRANCHED HYDROCARBON CHAIN

596 TETRAHYDROANACARDOL AMMONIUM MONOSULFONATE

C-16

422 POTASSIUM 1-1-2-HEXADECANE TRICARBOXYLATE

5 SODIUM HEXADECYL 1 SULFATE

75 SODIUM HEXADECYL 4 SULFATE

81 SODIUM HEXADECYL 6 SUL

86 SODIUM HEXADECYL 8 SULFATE

638 LITHIUM HEXADECYL SULFATE

577 CUPRIC HEXADECYL SULFATE

60 TRIETHANOL AMMONIUM HEXADECYL SULFATE

52 SODIUM HEXADECYL MONO OXYETHYLENE SULFATE

53 SODIUM HEXADECYL DI OXYETHYLENE SULFATE

54 SODIUM HEXADECYL TRI OXYETHYLENE SULFATE

55 SODIUM HEXADECYL TETRA OXYETHYLENE SULFATE

600 SODIUM HEXADECYL MONO-OXYPROPYL SULFATE

245 HEXADECANE 1-SULFONIC ACID

184 SODIUM HEXADECYL 1-SULFONATE

177 SODIUM HEXADECANE 2-SULFONATE

408 POTASSIUM HEXADECANE 1-SULFONATE 
PART 1. Grouped by charge and arranged by number of carbon atoms in longest hydrophobic tail and by heads - Continued

249 HEXADECANE 1-HYDROXY 2-SULFONIC ACID

240 SODIUM HEXADECANE 1-HYDROXY 2-SULFONATE

508 SODIUM HEXADECYL BENZENE SULFONATE

$\mathrm{C}-17$

534 OCTADECANOIC ACID

448 SODIUM OCTADECANOATE /STEARATE/

256 POTASSIUM STEARATE

672 DIPOTASSIUM HEXADECYL MALONATE

263 SODIUM OLEATE /CIS-9-OCTADECENOATE/

305 POTASSIUM OLEATE/ CIS-9-OCTADECENOATE/

285 HEXANOLAMINE-CH3CH/OH/CH2C/CH3/2NH2-OLEATE

264 SODIUM ELAIDATE/TRANS-9-OCTADECENOATE/

629 POTASSIUM ELAIDATE/TRANS-9-OCTADECENOATE/

283 HEXANOLAMINE-CH3CH/OH/CH2C/CH3/2NH2-ELAIDATE

630 POTASSIUM RICINOLEATE/12 HYDROXY OLEATE/

631 POTASSIUM RICINELAIDATE/I2 HYDROXY ELAIDATE/

255 POTASSIUM 9,10 DIHYDROXY STEARATE

191 ALPHA SULFOSTEARIC ACID

553 XYLYL SULFOSTEARIC ACID

9 SODIUM ALPHA SULFOSTEARIC ACID

10 DISODIUM ALPHA SULFOSTEARATE

194 SODIUM METHYL ALPHA SULFOSTEARATE

195 SODIUM ETHYL ALPHA SULFOSTEARATE

196 SODIUM PROPYL ALPHA SULFOSTEARATE

199 SODIUM ISOPROPYL ALPHA SULFOSTEARATE

198 DISODIUM 2 SULFOETHYL ALPHA SULFOSTEARATE

551 PHENYL SULFOSTEARIC ACID

11 SODIUM ALPHA SULFO PHENYL STEARIC ACID

8 DISODIUM ALPHA SULFOPHENYLSTEARATE

552 TOLYL SULFOSTEARIC ACID

14 DISODIUM 9,10 DIHYDROXY ALPHA SULFOSTEARATE

12 SODIUM 9,10 DICHLORO ALPHA SULFOSTEARIC ACID

13 DISODIUM 9,10 DICHLORO ALPHA SULFOSTEARATE

623 DISODIUM ALPHAPHOSPHONO OCTADECANOATE

626 TRISODIUM ALPHAPHOSPHONO OCTADECANOATE

610 SODIUM METHYL ALPHAPHOSPHONO STEARATE

70 SODIUM HEPTADECYL 2 SULFATE

87 SODIUM HEPTADECYL 9 SULFATE

246 HEPTADECANE 1-SULFONIC ACID

231 SODIUM HEPTADECANE 1-SULFONATE

48 SODIUM $P$ I METHYL HEXADECYL BENZENE SULFONATE

C-18

64 SODIUM OCTADECYL I SULFATE

71 SODIUM OCTADECYL 2 SULFATE

76 SODIUM OCTADECYL 4 SULFATE

82 SODIUM OCTADECYL 6 SULFATE

65 TRIETHANOLAMMONIUM OCTADECYL 1 SULFATE

61 SODIUM OLEYL/CIS 9 OCTADECENOYL/SULFATE

62 SODIUM ELAIDYL/TRANS 9 OCTADECENOYL/SULFATE

250 OCTADECANE 1-HYDROXY 2-SULFONIC ACID

63 SODIUM 910 DICHLORO OCTADECYL SULFATE

56 SODIUM OCTADECYL MONO OXYETHYLENE SULFATE

57 SODIUM OCTADECYL DI OXYETHYLENE SULFATE

58 SODIUM OCTADECYL TRI OXYETHYLENE SULFATE

59 SODIUM OCTADECYL TETRA OXYETHYLENE SULFATE

547 SODIUM OLEYL MONO-OXYETHYLENE SULFATE

548 SODIUM OLEYL DI-OXYETHYLENE SULFATE

549 SODIUM OLEYL TRI-OXYETHYLENE SULFATE

601 SODIUM OCTADECYL MONO-OXYPROPYL SULFATE

232 OCTADECANE 1-SULFONIC ACID

349 SODIUM OCTADECANE 1-SULFONATE

178 SODIUM OCTADECANE 2-SULFONATE

419 SODIUM ALPHA-HEPTYL UNDECYL SULFONATE

241 SODIUM OCTADECANE 1-HYDROXY 2-SULFONATE

509 SODIUM OCTADECYL BENZENE SULFONATE

C -19

673 DIPOTASSIUM OCTADECYL MALONATE

79 SODIUM NONADECYL 5 SULFATE

88 SODIUM 1 NONYL DECYL SULFATE

$\mathrm{C}-20$

683 SODIUM EICOSYLBENZENE SULFONATE

C-29

89 SODIUM 1 TETRADECYL PENTADECYL SULFATE

1B. CATIONICS BY NUMBER OF CARBON ATOMS

$\mathrm{C}-3$

706 PERFLUORO PROPYLAMINE

707 PERFLUORO PROPYLAMINE HYDROCHLORIDE
C-6

708 HEXYLAMINE

709 HEXYLAMINE HYDROCHLORIDE

390 HEXYLAMMONIUM DODECYL SULFATE

640 HEXYL TRIMETHYLAMMONIUM HEXANE SULFATE

644 HEXYL TRIMETHYLAMMONIUM HEXANE SULFONATE

641 HEXYL TRIMETHYLAMMONIUM OCTANE SULFATE

384 HEXYL TRIMETHYLAMMONIUM DODECYL SULFATE

354 HEXYL BENZYL DIMETHYLAMMONIUM CHLORIDE

C-8

392 OCTYLAMMONIUM CHLORIDE

391 OCTYLAMMONIUM DODECYL SULFATE

93 OCTYL TRIMETHYL AMMONIUM BROMIDE

347 OCTYL TRIMETHYLAMMOMIUM OCTYL SULFATE

287 OCTYL TRIMETHYLAMMONIUM OCTANE SULFONATE

642 OCTYL TRIMETHYLAMMONIUM DECANE SULFATE

353 OCTYL TRIMETHYLAMMONIUM DECANE SULFONATE

385 OCTYL TRIMETHYLAMMONIUM DODECYL SULFATE

483 DIOCTYL DIMETHYL AMMONIUM CHLORIDE

135 OCTYL C BETAINE HYDROCHLORIDE

359 OCTYL 4-NITROBENZYL DIMETHYLAMMONIUM CHLORIDE

100 OCTYL PYRIDINIUM BROMIDE

451 PARA DI-ISOBUTYLPHENOXYETHOXYETHYL DIMETHYLBENZYL AMMONIUM CHLORIDE/HYAMINE 1622/

C-9

94 NONYL TRIMETHYL AMMONIUM BROMIDE

ALPHA DIMETHYLAMINO CAPRIC ACID HYDROCHLORIDE

C-10

37 DECYLAMMONIUM CHLORIDE

411 DECYLAMMONIUM ACETATE

203 DECYL TRIMETHYL AMMONIUM CHLORIDE

95 DECYL TRIMETHYL AMMONIUM BROMIDE

306 DECYL TRIMETHYLAMMONIUM SULFATE

346 DECYL TRIMETHYLAMMONIUM DECYL SULFATE

288 DECYL TRIMETHYLAMMONIUM DECANESULFONATE

280 DECYL TRIMETHYL AMMONIUM DODECYL SULFATE

356 DECYL BENZYL DIMETHYLAMMONIUM CHLORIDE

360 DECYL 4-NITROBENZYL DIMETHYLAMMONIUM CHLORIDE

458 DECYL PYRIDINIUM IODIDE

$\mathrm{C}-11$

96 UNDECYL TRIMETHYL AMMONIUM BROMIDE

101 UNDECYL PYRIDINIUM BROMIDE

304 CATOL 607 (N-/LAURYL-COLAMINO-FORMYL-METHYL/ PYRIDINIUM CHLORIDE)

560 EMULSOL 607L (N-(2-KETO-2-(2,-LAUROYLOXYETHYL AMINO) ETHYL) PYRIDINIUM CHLORIDE

$\mathrm{C}-12$

38 DODECYL AMMONIUM CHLORIDE

628 DODECYLAMMONIUM BROMIDE

482 DODECYL AMMONIUM NITRATE

412 DODECYLAMMONIUM ACETATE

449 DODECYLMETHYL AMMONIUM CHLORIDE

450 DODECYLDIMETHYL AMMONIUM CHLGRIDE

399 DODECYL DIMETHYL ETHYLAMMONIUM CHLORIDE

345 DIDODECYL DIMETHYLAMMONIUM CHLORIDE

41 DODECYL TRIMETHYL AMMONIUM CHLORIDE

97 DODECYL TRIMETHYL. AMMONIUM BROMIDE

126 DODECYL TRIMETHYLAMMONIUM IODIDE

130 DODECYL TRIMETHYL AMMONIUM FLUORIDE

131 DODECYL TRIMETHYLAMMONIUM NITRATE

129 DODECYL TRIMETHYL AMMONIUM BROMATE

127 DODECYL TRIMETHYL AMMONIUM IODATE

307 DODECYL TRIMETHYLAMMONIUM SULFATE

128 DODECYL TRIMETHYL AMMONIUM FORMATE

643 DODECYL TRIMETHYLAMMONIUM OCTANE SULFATE

281 DODECYL TRIMETHYLAMMONIUM DODECYL SULFATE

400 DODECYL METHYL DIETHYLAMMONIUM CHLORIDE

401 DODECYL TRIETHYLAMMONIUM CHLORIDE

22 DIMETHYL DODECYL AMINE OXIDE HYDROCHLORIDE

293 DODECYL DIMETHYL AMINE OXIDE HYDROBROMIDE

633 N-DODECYL BETA-ALANINE HYDROCHLORIDE

124 DODECYL N BETAINE HYDROCHLORIDE

500 DODECYL TRI/2-HYDROXYETHYL/AMMONIUM CHLORIDE

279 DODECYL BENZYL DIMETHYLAMMONIUM CHLORIDE

403 DODECYL DIMETHYLPHENYLAMMONIUM CHLORIDE

404 DODECYL DIMETHYL 2-PHENYLETHYLAMMONIUM CHLORIDE $\mathrm{C} 6 \mathrm{H} 5 \mathrm{CH} 2 \mathrm{CH} 2 / \mathrm{N} / \mathrm{CH} 3 / 2 / \mathrm{C} 12 \mathrm{H} 25$ 
PART 1. Grouped by charge and arranged by number of carbon atoms in longest hydrophobic tail and by heads-Continued

407 DODECYL DIMETHYL 3-PHENYLPROPYLAMMONIUM CHLORIDE $\mathrm{C} 6 \mathrm{H} 5 \mathrm{CH} 2 \mathrm{CH} 2 / \mathrm{N} / \mathrm{CH} 3 / 2 / \mathrm{C} 12 \mathrm{H} 25$

405 DODECYL METHYLETHYLBENZYLAMMONIUM CHLORIDE

371 DODECYL 3-4-DIMETHOXYBENZ,YL DIMETHYLAMMONIUIM CHLORIDE

370 DODECYL 3-4-METHYLENEDIOXYBENZYL DIMETHYLAMMONIUM CHLORIDE

365 DODECYL 2-CHLOROBENZYL DIMETHYLAMMONIUM CHLORIDE

367 DODECYL 4-CHLOROBENZYL DIMETHYLAMMONIUM CHLORIDE

366 DODECYL 2-4-DICHLOROBENZYL DIMETHYLAMMONIUM CHLORIDE

369 DODECYL 3-4-DICHLOROBENZYL DIMETHYLAMMONIUM CHLORIDE

406 DODECYL DIMETHYL META-TRIFLUOROMETHYLBENZYL AMMONIUM CHLORIDE CF3C6H4CH2/N/CH3/2/C12H 25

361 DODECYL 4-NITROBENZYL DIMETHYL AMMONIUM CHLORIDE

368 DODECYL 2-HYDROXY-5-NITROBENZYL DIMETHYLAMMONIUM CHLORIDE

278 DODECYL PYRIDINIUM CHLORIDE

290 DODECYL PYRIDINIUM BROMIDE

376 DODECYL PYRIDINIUM IODIDE

717 DODECYLQUINOLINIUM BROMIDE

528 DODECYL TROPYLIUM PERCHLORATE

460 DODECYL TROPYLIUM MONOPHOSPHATE

491 DODECYL TROPYLIUM BISULFATE

C-13

402 TRIDECYL TRIMETHYLAMONIUM CHLORIDE

$\mathrm{C}-14$

39 TETRADECYL AMMONIUM CHLORIDE

413 TETRADECYLAMMONIUM ACETATE

42 TETRADECYL TRIMETHYL AMMONIUM CHLORIDE

98 TETRADECYL TRIMETHYL AMMONIUM BROMIDE

308 TETRADECYL TRIMETHYLAMMONIUM SULFATE

291 TETRADECYL TRIPROPYLAMMONIUM BROMIDE

125 TETRADECYL N BETAINE HYDROCHLORIDE

357 TETRADECYL BENZYL DIMETHYLAMMONIUM CHLORIDE

362 TETRADECYL 4-NITROBENZYL DIMETHYLAMMONIUM CHLORIDE

102 TETRADECYL PYRIDINIUM BROMIDE

479 TETRADECYL PYRIDINIUM IODIDE

$\mathrm{C}-16$

186 HEXADECYL AMMONIUM CHLORIDE

414 HEXADECYLAMMONIUM ACETATE

478 HEXADECYL DIMETHYLETHYLAMMONIUM BROMIDE

265 HEXADECYL TRIMETHYL AMMONIUM CHLORIDE

99 HEXADECYL TRIMETHYLAMMONIUM BROMIDE

309 HEXADECYL TRIMETHYLAMMONIUM SULFATE

666 HEXADECYL TRIBUTYLAMMONIUM BROMATE

292 HEXADECYL TRIPROPYLAMMONIUM BROMIDE

266 HEXADECYL DIMETHYL 2-HYDROXYETHYL AMMONIUM CHLORIDE

267 HEXADECYL DI-/2-HYDROXYETHYL/METHYL AMMONIUMCHLORIDE

268 HEXADECYL TRI-/2-HYDROXYETHYL/ AMMONIUM CHLORIDE

269 HEXADECYLDIMETHYL 2,3-DIHYDROXY PROPYLAMMONIUMCHLOR IDE

275 HEXADECYL DIMETHYLBENZYLAMMONIUM CHLORIDE

363 HEXADECYL 4-NITROBENZYL DIMETHYLAMMONIUM CHLORIDE

274 HEXADECYL PYRIDINIUM CHLORIDE

427 HEXADECYL PYRIDINIUM BRONIDE

480 HEXADECYL PYRIDINIUM IODIDE

660 HEXADECYL PYRIDINIUM IODATE

693 N-CETYL 2-METHYL PYRIDINIUM CHLORIDE

696 N-CETYL 2-METHYL PYRIDINIUM IODIDE

694 N-CETYL 3-METHYL PYRIDINIUM CHLORIDE

697 N-CETYL-3-METHYL PYRIDINIUM IODIDE

695 N-CETYL 4-METHYL PYRIDINIUM CHLORIDE

698 N-CETYL-4-METHYL PYRIDINIUM IODIDE

$\mathrm{C}-18$

187 OCTADECYL AMMONIUM CHLORIDE

415 OCTADECYLAMMONIUM ACETATE

270 OCTADECYL TRIMETHYLAMMONIUM CHLORIDE

477 OCTADECYL TRIMETHYLAMMONIUM BROMIDE

654 OCTADECYL TRIMETHYLAMMONIUM NITRATE

658 OCTADECYL TRIMETHYLAMMONIUM BROMATE

659 OCTADECYL TRIMETHYLAMMONIUM FORMATE

667 OCTADECYL TRIMETHYLAMMONIUM OXALATE

662 OCTADECYL TRIETHYLAMMONIUM BROMATE

663 OCTADECYL TRIPROPYLAMMONIUM BROMATE

664 OCTADECYL TRIBUTYLAMMONIUM BROMATE

665 OCTADECYL TRIAMYLAMMONIUM BROMATE

358 OCTADECYL BENZYL DIMETHYLAMMONIUM CHLORIDE

364 OCTADECYL 4-NITROBENZYL DIMETHYLAMMONIUM CHLORIDE

655 OCTADECYL PYRIDINIUM CHLORIDE

657 OCTADECYL PYRIDINIUM BROMIDE

481 OCTADECYL PYRIDINIUM IODIDE

656 OCTADECYL PYRIDINIUM NITRATE

661 OCTADECYL PYRIDINIUM IODATE

\section{C. NONIONICS BY NUMBER OF CARBON ATOMS}

C-2

645 PARA/BETA-D-GLUCOSYL/ETHYLBENZENE

$\mathrm{C}-3$

43 BUTYRIC ACID

706 PERFLURO PROPYLAMINE

646 PARA/BETA-D-GLUCOSYL/PROPYL BENZENE

$\mathrm{C}-4$

647 PARA/BETA-D-GLUCOSYL/BUTYLBENZENE

650 PARA/BETA-D-XYLOSYL/BUTYL BENZENE

461 BUTYL/OXYETHYLENE/I ALCOHOL--BUTYL GLYCOL ETHER HOMOGENEOUS HEAD GROUP

393 BUTYL/OXYETHYLENE/6 ALCOHOL HOMOGENEOUS HEAD GROUP

394 1-METHYL PROPYL/OXYE'THYLENE/6 ALCOHOL HOMOGENEOUS HEAD GROUP

674 DIISOBUTYL BENZENE/OXYETHYLENE/6 ALCOHOL (DB) BRANCHED CHAIN, NATURAL OE DISTRIBUTION

C-5

700 HEXANOIC ACID

$\mathrm{C}-6$

708 HEXYLAMINE

714 HEXYL DIMETHYL AMTNE OXIDE

686 HEXYL SULFINYLBUTANOL

684 HEXYL SULFINYLETHANOL

685 HEXYL SULFINYLPROPANOL

687 HEXYL SULFINYLPENTANOL

457 1-4-HEXANEDIOL

703 4-HEXYL RESORCINOL

103 HEXYL /OXYETHYLENE/ 3 ALCOHOL HOMOGENOUS HEAD GROUP

380 HEXYL/OXYETHYLENE/4 ALCOHOL HOMOGENEOUS HEAD GROUP

381 HEXYL/OXYETHYLENE/5 ALCOHOL HOWOGENEOUS HEAD GROUP

294 HEXYL/OXYETHYLENE/6 ALCOHOL HOMOGENOUS HEAD GROUP

395 2-ETHYL BUTYL/OXYETHYLENE/6 ALCOHOL HOMOGENEOUS HEAD GROUP

$\mathrm{C}-7$

529 OCTANOIC ACID

715 HEPTYL DIMETHYL AMINE OXIDE

586 METHYL /OXYETHYLENE/ 7.6 OCTANOATE REDUCED POLYDISPERSITY OF HEAD GROUPS

$\mathrm{C}-8$

118 OCTYL N BETAINE

132 OCTYL C BETAINE

251 OCTYL DIMETHYL AMINE OXIDE

348 NN-DIMETHYL 1-1-DIHYDROPENTADECAFLUORO OCTYL AMINE NOXIDE /C7F15CH2N/CH3/20/

710 OCTYL DIMETHYL PHOSPHINE OXIDE

688 OCTYL SULFINYLETHANOL

689 OCTYL SULFINYLPROPANOL

690 OCTYL SULFINYLBUTANOL

691 OCTYL METHYL SULFOXIDE

648 ALPHA-D-GLUCOSYL OCTANE

424 OCTYL ALPHA-GLYCERYL ETHER

18 OCTYL BETA D GLUCOSIDE

423 OCTYL/OXYETHYLENE/I ALCOHOL--OCTYL GLYCOL ETHER HOMOGENEOUS HEAD GROUP

104 OCTYL /OXYETHYLENE/ 3 ALCOHOL HOMOGENOUS HEAD GROUP

105 OCTYL /OXYETHYLENE/ 6 ALCOHOL HOMOGENOUS HEAD GROUP

106 OCTYL /OXYETHYLENE/ 9 ALCOHOL HOMOGENOUS HEAD GROUP

515 ISO-OCTYL/OXYETHYLENE/4 ALCOHOL BRANCHED CHAIN, NATURAL OE DISTRIBUTION

396 2-PROPYL PENTYL/OXYETHYLENE/6 ALCOHOL HOMOGENEOUS HEAD GROUP

335 OCTYL BENZENE/OXYETHYLENE/8.5 ALCOHOL BRANCHED CHAIN, NATURAL OE DISTRIBUTION

675 OCTYL BENZENE /OXYETHYLENE/10 ALCOHOL NATURAL DISTRIBUTION OF HEAD GROUPS

317 T-OCTYL BENZENE/OXYETHYLENE/10 ALCOHOL REDUCED POLYDISPERSITY OF HEAD GROUPS

318 T-OCTYL BENZENE/OXYETHYLENE/20 ALCOHOL REDUCED POLYDISPERSITY OF HEAD GROUPS

319 T-OCTYL BENZENE/OXYETHYLENE/30 ALCOHOL REDUCED POLYDISPERSITY OF HEAD GROUPS

207 P-T-OCTYL BENZENE /OXYETHYLENE/I ALCOHOL HOMOGENOUS HEAD GROUP

217 P-T-OCTYL BENZENE /OXYETHYLENE/I ALCOHOL NATURAL DISTRIBUTION OF HEAD GROUPS 
PART 1. Grouped by charge and arranged by number of carbon atoms in longest hydrophobic tail and by heads - Continued

208 P-T-OCTYL BENZENE /OXYETHYLENE/2 ALCOHOL HOMOGENOUS HEAD GROUP

218 P-T-OCTYL BENZENE /OXYETHYLENE/2 ALCOHOL NATURAL DISTRIBUTION OF HEAD GROUPS

209 P-T-OCTYL BENZENE /OXYETHYLENE/3 ALCOHOL HOMOGENOUS HEAD GROUP

219 P-T-OCTYL BENZENE /OXYETHYLENE/3 ALCOHOL NATURAL DISTRIBUTION OF HEAD GROUPS

210 P-T-OCTYL BENZENE /OXYETHYLENE/4 ALCOHOL HOMOGENOUS HEAD GROUP

220 P-T-OCTYL BENZENE /OXYETHYLENE/4 ALCOHOL NATURAL DISTRIBUTION OF HEAD GROUPS

211 P-T-OCTYL BENZENE /OXYETHYLENE/5 ALCOHOL HOMOGENOUS HEAD GROUP

221 P-T-OCTYL BENZENE /OXYETHYLENE/5 ALCOHOL NATURAL DISTRIBUTION OF HEAD GROUPS

212 P-T-OCTYL BENZENE /OXYETHYLENE/6 ALCOHOL HOMOGENOUS HEAD GROUP

222 P-T-OCTYL BENZENE /OXYETHYLENE/6 ALCOHOL NATURAL DISTRIBUTION OF HEAD GROUPS

213 P-T-OCTYL BENZENE /OXYETHYLENE/7 ALCOHOL HOMOGENOUS HEAD GROUP

223 P-T-OCTYL BENZENE /OXYETHYLENE/7 ALCOHOL NATURAL DISTRIBUTION OF HEAD GROUPS

214 P-T-OCTYL BENZENE /OXYETHYLENE/8 ALCOHOL HOMOGENOUS HEAD GROUP

224 P-T-OCTYL BENZENE /OXYETHYLENE/8 ALCOHOL NATURAL DISTRIBUTION OF HEAD GROUPS

215 P-T-OCTYL BENZENE /OXYETHYLENE/9 ALCOHOL HOMOGENOUS HEAD GROUP

223 P-T-OCTYL BENZENE /OXYETHYLENE/7 ALCOHOL NATURAL DISTRIBUTION OF HEAD GROUPS

214 P-T-OCTYL BENZENE /OXYETHYLENE/8 ALCOHOL HOMOGENOUS HEAD GROUP

224 P-T-OCTYL BENZENE /OXYETHYLENE/8 ALCOHOL NATURAL DISTRIBUTION OF HEAD GROUPS

215 P-T-OCTYL BENZENE /OXYETHYLENE/9 ALCOHOL HOMOGENOUS HEAD GROUP

225 P-T-OCTYL BENZENE /OXYETHYLENE/9 ALCOHOL NATURAL DISTRIBUTION OF HEAD GROUPS

206 TRITON X-1OO(P-T-OCTYL BENZENE/OXYETHYLENE/9NATURAL DISTRIBUTION OF HEAD GROUPS

216 P-T-OCTYL BENZENE /OXYETHYLENE/1O ALCOHOL HOMOGENOUS HEAD GROUP

226 P-T-OCTYL BENZENE /OXYETHYLENE/10 ALCOHOL NATURAL DISTRIBUTION OF HEAD GROUPS

227 P-T-OCTYL BENZENE /OXYETHYLENE/16 ALCOHOL NATURAL DISTRIBUTION OF HEAD GROUPS

228 P-T-OCTYL BENZENE /OXYETHYLENE/40 ALCOHOL NATURAL DISTRIBUTION OF HEAD GROUPS

C-9

530 DECANOIC ACID

254 NONYL DIMETHYL AMINE OXIDE

580 METHYL /OXYETHYLENE/ 7.0 DECANOATE REDUCED POLYDISPERSITY OF HEAD GROUPS

581 METHYL /OXYETHYLENE/ 10.3 DECANOATE REDUCED POLYDISPERSITY OF HEAD GROUPS

578 METHYL /OXYETHYLENE/ 11.9 DECANOATE REDUCED POLYDISPERSITY OF HEAD GROUPS

582 METHYL /OXYETHYLENE/ 16.0 DECANOATE NATURAL DISTRIBUTION OF HEAD GROUPS

516 NONYL/OXYETHYLENE/4 ALCOHOL BRANCHED CHAIN, NATURAL OE DISTRIBUTION

153 NONYL BENZENE /OXYETHYLENE/ 5.0 ALCOHOL BRANCHED CHAIN, NATURAL OE DISTRIBUTION

336 NONYL BENZENE/OXYETHYLENE/9.5 ALCOHOL BRANCHED CHAIN, NATURAL OE DISTRIBUTION

154 NONYL BENZENE /OXYETHYLENE/ 9.8 ALCOHOL BRANCHED CHAIN, NATURAL OE DISTRIBUTION

320 NONYL BENZENE/OXYETHYLENE/10 ALCOHOL BRANCHED CHAIN. REDUCED OE DISTRIBUTION

167 NONYL BENZENE /OXYETHYLENE/ 10 ALCOHOL BRANCHED CHAIN NATURAL OE DISTRIBUTION

337 NONYL BENZENE/OXYETHYLENE/10.5 ALCOHOL BRANCHED CHAIN NATURAL OE DISTRIBUTION

463 NONYL BENZENE/OXYETHYLENE/II ALCOHOL NATURAL DISTRIBUTION OF HEAD GROUPS

321 NONYL BENZENE/OXYETHYLENE/15 ALCOHOL BRANCHED CHAIN, REDUCED OE DISTRIBUTION

464 NONYL BENZENE/OXYETHYLENE/15 ALCOHOL NATURAL DISTRIBUTION OF HEAD GROUPS

168 NONYL BENZENE /OXYETHYLENE/ 15 ALCOHOL BRANCHED CHAIN NATURAL OE DISTRIBUTION
155 NONYL BENZENE /OXYETHYLENE/ 15.4 ALCOHOL BRANCHED CHAIN, NATURAL OE DISTRIBUTION

322 NONYL BENZENE/OXYETHYLENE/2O ALCOHOL BRANCHED CHAIN REDUCED OE DISTRIBUTION

156 NONYL BENZENE /OXYETHYLENE/ 20.0 ALCOHOL BRANCHED CHAIN, NATURAL OE DISTRIBUTION

323 NONYL BENZENE/OXYETHYLENE/30 ALCOHOL BRANCHED CHAIN REDUCED OE DISTRIBUTION

721 NONYL BENZENE /OXYETHYLENE/30 ALCOHOL BRANCHED CHAIN DIALYSED

169 NONYL BENZENE /OXYETHYLENE/ 30 ALCOHOL BRANCHED CHAIN NATURAL OE DISTRIBUTION

157 NONYL BENZENE /OXYETHYLENE/ 30.2 ALCOHOL BRANCHED CHAIN, NATURAL OE DISTRIBUTION

324 NONYL BENZENE/OXYETHYLENE/50 ALCOHOL BRANCHED CHAIN, REDUCED OE DISTRIBUTION

338 NONYL BENZENE/OXYETHYLENE/100 ALCOHOL BRANCHED CHAIN. NATURAL OE DISTRIBUTION

$\mathrm{C}-10$

119 DECYL N BETAINE

133 DECYL C BETAINE

252 DECYL DIMETHYL AMINE OXIDE

711 DECYL DIMETHYL PHOSPHINE OXIDE

587 DECYL DIMETHYLAMMONIOPROPANE SULFONATE

692 DECYL SULFINYLETHANOL

19 DECYL BETA D GLUCOSIDE

201 DECYL /OXYETHYLENE/8 OXYMETHYL REDUCED POLYDISPERSION OF HEAD GROUPS

202 DECYL /OXYETHYLENE/11 OXYMETHYL REDUCED POLYDISPERSION OF HEAD GROUPS

204 DECYL /OXYETHYLENE/12 OXYMETHYL REDUCED POLYDISPERSION

107 DECYL /OXYETHYLENE/ 3 ALCOHOL HOMOGENOUS HEAD GROUP

379 DECYL/OXYETHYLENE/4 ALCOHOL HOMOGENEOUS HEAD GROUP

519 DECYL/OXO-PROCESS/ /OXYETHYLENE/4 ALCOHOL BRANCHED CHAIN, NATURAL OE DISTRIBUTION

378 DECYL/OXYETHYLENE/5 ALCOHOL HOMOGENEOUS HEAD GROUP

143 DECYL /OXYETHYLENE/ 5.0 ALCOHOL BRANCHED CHAIN NATURAL OE DISTRIBUTION

108 DECYL /OXYETHYLENE/ 6 ALCOHOL HOMOGENOUS HEAD GROUP

109 DECYL /OXYETHYLENE/ 9 ALCOHOL HOMOGENOUS HEAD GROUP

144 DECYL /OXYETHYLENE/ 10.0 ALCOHOL BRANCHED CHAIN. NATURAL OE DISTRIBUTION

517 DECYL/OXO-PROCESS/ /OXYETHYLENE/1O ALCOHOL BRANCHED CHAIN, NATURAL OE DISTRIBUTION

145 DECYL /OXYETHYLENE/ 14.9 ALCOHOL BRANCHED CHAIN, NATURAL OE DISTRIBUTION

146 DECYL /OXYETHYLENE/ 20.0 ALCOHOL BRANCHED CHAIN, NATURAL OE DISTRIBUTION

147 DECYL /OXYETHYLENE/ 29.4 ALCOHOL BRANCHED CHAIN, NATURAL OE DISTRIBUTION

397 2-BUTYL HEXYL/OXYETHYLENE/6 ALCOHOL HOMOGENEOUS HEAD GROUP

398 2-BUTYL HEXYL/OXYETHYLENE/9 ALCOHOL HOMOGENEOUS HEAD GROUP

523 DECYL BENZENE PARA/OXYETHYLENE/I1.O ALCOHOL NATURAL DISTRIBUTION OF HEAD GROUPS

522 DECYL BENZENE ORTHO/OXYETHYLENE/11.2 ALCOHOL NATURAL DISTRIBUTION OF HEAD GROUPS

C -11

531 DODECANOIC ACID

120 UNDECYL N BETAINE

713 UNDECYL DIMETHYL AMINE OXIDE

495 SUCROSE MONOLAURATE

276 NONAETHYLENE GLYCOL MONODODECANOATE NATURAL DISTRIBUTION OF HEAD GROUPS

583 METHYL /OXYETHYLENE/ 6.0 DODECANOATE REDUCED POLYDISPERSITY OF HEAD GROUPS

584 METHYL /OXYETHYLENE/ 8.4 DODECANOATE REDUCED POLYDISPERSITY OF HEAD GROUPS

585 METHYL /OXYETHYLENE/ 11.2 DODECANOATE REDUCED POLYDISPERSITY OF HEAD GROUPS

579 METHYL /OXYETHYLENE/ 12.5 DODECANOATE REDUCED POLYDISPERSITY OF HEAD GROUPS

440 /OXYETHYLENE/4 SORBITAN MONOLAURATE ISOMERS AND DISTRIBUTED MULTIPLE OE CHAINS

441 /OXYETHYLENE/8 SORBITAN MONOLAURATE ISOMERS AND DISTRIBUTED MULTIPLE OE CHAINS

442 /OXYETHYLENE/IO SORBITAN MONOLAURATE ISOMERS AND DISTRIBUTED MULTIPLE OE CHAINS

443 /OXYETHYLENE/2O SORBITAN MONOLAURATE ISOMERS AND DISTRIBUTED MULTIPLE OE CHAINS

444 /OXYETHYLENE/25 SORBITAN MONOLAURATE ISOMERS AND DISTRIBUTED MULTIPLE OE CHAINS 
PART 1. Grouped by charge and arranged by number of carbon atoms in longest hydrophobic tail and by heads - Continued

520 UNDECYL/OXYETHYLENE/8 ALCOHOL NATURAL DISTRIBUTION OF HEAD GROUPS

550 LAURIC ACID DIETHANOLAMINE CONDENSATE

$\mathrm{C}-12$

121 DODECYL N BETAINE

592 DODECYL N-DIETHYL N-BETAINE

134 DODECYL C BETAINE

21 DIMETHYL DODECYL AMINE OXIDE

712 DODECYL DIMETHYL PHOSPHINE OXIDE

590 DODECYL DIMETHYLAMMONIOPROPANE CARBOXYLATE

588 DODECYL DIMETHYLAMMONIOPROPANE SULFONATE

593 DIMETHYL DODECYLAMMONIOPROPANE HYDROXY SULFONATE

594 DODECYL DIPROPYL AMMONIOPROPANE SULFONATE

595 DODECYL DIMETHYL AMMONIOPROPANE SULFATE

591 DODECYL DIMETHYLPHOSPHONIOPROPANE SULFONATE

649 ALPHA-D-GLUCOSYL DODECANE

20 DODECYL BETA D GLUCOSIDE

205 DODECYL /OXYETHYLENE/12 OXYMETHYL REDUCED POLYDISPERSION OF HEAD GROUPS

325 DODECYL/OXYETHYLENE/4 ALCOHOL REDUCED POLYDISPERSITY OF HEAD GROUPS

377 DODECYL/OXYETHYLENE/ 5 ALCOHOL HOMOGENEOUS HEAD GROUP

110 DODECYL /OXYETHYLENE/ 6 ALCOHOL HOMOGENOUS HEAD GROUP

468 DODECYL/OXYETHYLENE/6.2 ALCOHOL NATURAL DISTRIBUTION OF HEAD GROUPS

488 DODECYL/OXYETHYLENE/7 ALCOHOL HOMOGENEOUS HEAD GROUP

115 DODECYL /OXYETHYLENE/7 ALCOHOL REDUCED POLYDISPERSION OF HEAD GROUPS

469 DODECYL/OXYETHYLENE/7.6 ALCOHOL NATURAL DISTRIBUTION OF HEAD GROUPS

163 DODECYL /OXYETHYLENE/ 8 ALCOHOL NATURAL DISTRIBUTICN OF HEAD GROUPS

489 DODECYL/OXYETHYLENE/9 ALCOHOL HOMOGENEOUS HEAD GROUP

310 DODECYL/OXYETHYLENE/10 ALCOHOL NATURAL DISTRIBUTION OF HEAD GROUPS

470 DODECYL/OXYETHYLENE/10.6 ALCOHOL NATURAL DISTRIBUTION OF HEAD GROUPS

490 DODECYL/OXYETHYLENE/12 ALCOHOL HOMOGENEOUS HEAD GROUP

164 DODECYL /OXVETHYLENE/ 12 ALCOHOL NATURAL DISTRIBUTION OF HEAD GROUPS

326 DODECYL/OXYETHYLENE/14 ALCOHOL REDUCED POLYDISPERSITY OF HEAD GROUPS

471 DODECYL/OXYETHYLENE/15.8 ALCOHOL NATURAL DISTRIBUTION OF HEAD GROUPS

716 DODECYL /OXYETHYLENE/16 ALCOHOL REDUCED POLYDISPERSITY OF HEAD GROUPS

165 DODECYL /OXYETHYLENE/ 18 ALCOHOL NATURAL DISTRIBUTION OF HEAD GROUPS

472 DODECYL/OXYETHYLENE/20.4 ALCOHOL NATURAL DISTRIBUTION OF HEAD GROUPS

327 DODECYL/OXYETHYLENE/23 ALCOHOL REDUCED POLYDISPERSITY OF HEAD GROUPS

166 DODECYL /OXYETHYLENE/ 23 ALCOHOL NATURAL DISTRIBUTION OF HEAD G OUPS

473 DODECYL/OXYETHYLENE/27. I ALCOHOL NATURAL DISTRIBUTION OF HEAD GROUPS

170 DODECYL /OXYETHYLENE/ 28 ALCOHOL REDUCED DISTRIBUTION OF HEAD GROUPS

116 DODECYL /OXYETHYLENE/30 ALCOHOL REDUCED POLYDISPERSION OF HEAD GROUPS

474 DODECYL/OXYETHYLENE/38.6 ALCOHOL NATURAL DISTRIBUTION OF HEAD GROUPS

475 DODECYL/OXYETHYLENE/67.3 ALCOHOL NATURAL DISTRIBUTION OF HEAD GROUPS

518 TRIMETHYLNONYL/OXYETHYLENE/6 ALCOHOL BRANCHED CHAIN, NATURAL OE DISTRIBUTION

158 DODECYL BENZENE /OXYETHYLENE/ 5.0 ALCOHOL BRANCHED CHAIN, NATURAL OE DISTRIBUTION

159 DODECYL BENZENE /OXYETHYLENE/ 10.2 ALCOHOL BRANCHED CHAIN, NATURAL OE DISTRIBUTION

160 DODECYL BENZENE /OXYETHYLENE/ 15.1 ALCOHOL BRANCHED CHAIN, NATURAL OE DISTRIBUTION

161 DODECYL BENZENE /OXYETHYLENE/ 20.1 ALCOHOL BRANCHED CHAIN, NATURAL OE DISTRIBUTION

162 DODECYL BENZENE/OXYETHYLENE/ 30.6 ALCOHOL BRANCHED CHAIN, NATURAL OE DISTRIBUTION

C-13

532 TETRADECANOIC ACID

497 SUCROSE MONOMYRISTATE

312 TRIDECYL/OXYETHYLENE/5 ALCOHOL BRANCHED CHAIN, REDUCED OE DISTRIBUTION
148 TRIDECYL /OXYETHYLENE/ 5.2 ALCOHOL BRANCHED CHAIN, NATURAL OE DISTRIBUTION

431 TRIDECYL/OXO-PROCESS/ /OXYETHYLENE/8.5 ALCOHOL BRANCHED CHAIN, NATURAL OE DISTRIBUTION

465 TRILECYL/OXYETHYLENE/9 ALCOHOL NATURAL DISTRIBUTION OF HEAD GROUPS

466 TRIDECYL/SECONDARY//OXYETHYLENE/9 ALCOHOL NATURAL DISTRIBUTION OF HEAD GROUPS

313 TRIDECYL/OXYETHYLENE/9.5 ALCOHOL BRANCHED CHAIN REDUCED OE DISTRIBUTION

521 TRIDECYL/OXYETHYLENE/10 ALCOHOL BRANCHED CHAIN, NATURAL OE DISTRIBUTION

432 TRIDECYL/OXO PROCESS/ /OXYETHYLENE/IO ALCOHOL BRANCHED CHAIN, NATURAL OE DISTRIBUTION

149 TRIDECYL/OXYETHYLENE/ 10.1 ALCOHOL BRANCHED CHAIN NATURAL OE DISTRIBUTION

467 TRIDECYL/SECONDARY//OXYETHYLENE/12 ALCOHOL NATURAL DISTRIBUTION OF HEAD GROUPS

314 TRIDECYL/OXYETHYLENE/14 ALCOHOL BRANCHED CHAIN REDUCED OE DISTRIBUTION

433 TRIDECYL/OXO-PROCESS//OXYETHYLENE/15 ALCOHOL BRANCHED CHAIN, NATURAL OE DISTRIBUTION

150 TRIDECYL /OXYETHYLENE/ 15.2 ALCOHOL BRANCHED CHAIN NATURAL OE DISTRIBUTION

315 TRIDECYL/OXYETHYLENE/20 ALCOHOL BRANCHED CHAIN, REDUCED OE DISTRIBUTION

151 TRIDECYL /OXYETHYLENE/ 20.0 ALCOHOL BRANCHED CHAIN NATURAL OE DISTRIBUTION

434 TRIDECYL/OXO-PROCESS/ /OXYETHYLENE/22 ALCOHOL BRANCHED CHAIN, NATURAL OE DISTRIBUTION

316 TRIDECYL/OXYETHYLENE/30 ALCOHOL BRANCHED CHAIN, REDUCED OE DISTRIBUTION

152 TRIDECYL /OXYETHYLENE/ 30.6 ALCOHOL BRANCHED CHAIN, NATURAL OE DISTRIBUTION

C -14

122 TETRADECYL N BETAINE

253 TETRADECYL DIMETHYL AMINE OXIDE

289 TETRADECYL/OXYETHYLENE/ 6 ALCOHOL HOMOGENOUS HEAD GROUP

C-15

533 HEXADECANOIC ACID

498 SUCROSE MONOPALMITATE

C-16

123 HEXADECYL $N$ BETAINE

589 HEXADECYLDIMETHYLAMMONIOPROPANE SULFONATE

499 SUCROSE DI-PALMITATE

282 HEXADECYL /OXYETHYLENE/ 6 ALCOHOL HOMOGENOUS HEAD GROUP

330 HEXADECYL/OXYETHYLENE/7 ALCOHOL HOMOGENEOUS HEAD GROUP

331 HEXADECYL/OXYETHYLENE/9 ALCOHOL HOMOGENEOUS HEAD GROUP

332 HEXADECYL/OXYETHYLENE/12 ALCOHOL HOMOGENEOUS HEAD GROUP

524 HEXADECYL/OXYETHYLENE/14 ALCOHOL BRANCHED CHAIN, NATURAL OE DISTRIBUTION

333 HEXADECYL/OXYETHYLENE/15 ALCOHOL HOMOGENEOUS HEAD GROUP

535 HEXADECYL/OXYETHYLENE/18 ALCOHOL

334 HEXADECYL/OXYETHYLENE/21 ALCOHOL HOMOGENEOUS HEAD GROUP

17 HEXADECYL /OXYETHYLENE/30 ALCOHOL REDUCED POLYDISPERSION OF HEAD GROUPS

C-17

534 OCTADECANOIC ACID

496 SUCROSE MONOSTEARATE

553 XYLYL SULFOSTEARIC ACID

C- 18

435 OCTADECYL/OXYETHYLENE/10 ALCOHOL NATURAL DISTRIBUTION OF HEAD GROUPS

328 OCTADECYL/OXYETHYLENE/14 ALCOHOL REDUCED POLYDISPERSITY OF HEAD GROUPS

436 OCTADECYL/OXYETHYLENE/20 ALCOHOL NATURAL DISTRIBUTION OF HEAD GROUPS

329 OCTADECYL/OXYETHYLENE/100 ALCOHOL REDUCED POLYDISPERSITY OF HEAD GROUPS

437 OLEYL/CIS-9-OCTADECENOYL/ /OXYETHYLENE/12 ALCOHOL NATURAL DISTRIBUTION OF HEAD GROUPS

438 OLEYL/CIS-9-OCTADECENOYL/ /OXYETHYLENE/16 ALCOHOL NATURAL DISTRIBUTION OF HEAD GROUPS

439 OLEYL/CIS-9-OCTADECENOYL/ /OXYETHYLENE/2O ALCOHOL NATURAL DISTRIBUTION OF HEAD GROUP 


\section{Structural Indexes of Compounds with Keys to Compound Numbers - Continued}

PART 2. Surfactants grouped by charge and by head group and arranged by number of carbon atoms in longest hydrophobic tail.

\section{A. ANIONICS BY HEAD GROUP}

\section{CARBOXYLIC ACID}

428 PERFLUORO ACETIC ACID

429 PERFLUORO PROPIONIC ACID

43 BUTYRIC ACID

430 PERFLUORO BUTYRIC ACID

452 3-4-DICHLOROPERFLUORO BUTYRIC/KEL-F/ACID

700 HEXANOIC ACID

416 PERFLUORO HEXANOIC ACID

453 3-5-6-TRICHLOROPERFLUORO HEXANOIC/KEL-F/ACID

374 DODECAFLUOROHEPTANOIC ACID H/CF $2 / 6 \mathrm{COOH}$

529 OCTANOIC ACID

$4 I 7$ PERFLUORO OCTANOIC ACID

454 3-5-7-8-TETRACHLOROPERFLUORO OCTANOIC/KEL-F/ACID

375 HEXADECAFLUORONONANOIC ACID H/CF2/8COOH

530 DECANOIC ACID

455 PERFLUORO DECANOIC ACID

531 DODECANOIC ACID

532 TETRADECANOIC ACID

533 HEXADECANOIC ACID

534 OCTADECANOIC ACID

\section{MONOCARBOXYLATE}

699 SODIUM BUTYRATE

484 SODIUM PENTANOATE/VALERATE/

485 SODIUM HEXANOATE/CAPROATE/

188 POTASSIUM HEXANOATE

701 POTASSIUM PERFLUROHEXANOATE

486 SODIUM HEPTANOATE

296 POTASSIUM HEPTANOATE

355 AMMONIUM DODECAFLUOROHEPTANOATE H/CF2/6COONH4

476 SODIUM OCTANOATE

44 POTASSIUM OCTANOATE

456 POTASSIUM PERFLUURO OCTANOATE

303 DI-ISOPROPYLAMONIUM CAPRYLATE

284 HEXANOLAMINE-CH3CH/OH/CH2C/CH3/2NH2-OCTANOATE

487 SODIUM NONANOATE

350 POTASSIUM NONANOATE

372 AMMONIUM HEXADECAFLUORONONANOATE H/CF2/8 COO NH4

299 SODIUM DECANOATE

90 POTASSIUM DECANOATE

702 POTASSIUM PERFLUORODECANOATE

297 POTASSIUM UNDECANOATE

527 SODIUM UNDECANE-3-CARBOXYLATE

373 AMMONIUM EICOSAFLUOROUNDECANOATE H/CF2/10 $\mathrm{COO}$ NH4

273 SODIUM DODECANOATE

91 POTASSIUM DODECANOATE

627 CESIUM DODECANOATE

277 BENZYL TRIMETHYL AMMONIUM DODECANOATE

351 POTASSIUM TRIDECANOATE

298 SODIUM TETRADECANOATE

92 POTASSIUM TETRADECANOATE

300 SODIUM HEXADECANOATE

185 POTASSIUM HEXADECANOATE

448 SODIUM OCTADECANOATE /STEARATE/

256 POTASSIUM STEARATE

263 SODIUM OLEATE /CIS-9-OCTADECENOATE/

305 POTASSIUM OLEATE/ CIS-9-OCTADECENOATE/

285 HEXANOLAMINE-CH3CH/OH/CH2C/CH3/2NH2-OLEATE

264 SODIUM ELAIDATE /TRANS-9-OCTADECENOATE/

629 POTASSIUM ELAIDATE/TRANS-9-OCTADECENOATE/

283 HEXANOLAMINE-CH3CH/OH/CH2C/CH3/2NH2-ELAIDATE

255 POTASSIUM 9,10 DIHYDROXY STEARATE

630 POTASSIUM RICINOLEATE/12 HYDROXY OLEATE/

631 POTASSIUM RICINELAIDATE/12 HYDROXY ELAIDATE/

\section{POLYCARBOXYLATE}

668 DIPOTASSIUM OCTYL MALONATE

669 DIPOTASSIUM DECYL MALONATE

670 DIPOTASSIUM DODECYL MALONATE

671 DIPOTASSIUM TETRADECYL MALONATE

672 DIPOTASSIUM HEXADECYL MALONATE

673 DIPOTASSIUM OCTADECYL MALONATE

420 POTASSIUM 1-1-2-OCTANE TRICARBOXYLATE

425 POTASSIUM 1-1-2-DECANE TRICARBOXYLATE
421 POTASSIUM 1-1-2-DODECANE TRICARBOXYLATE

426 POTASSIUM 1-1-2-TETRADECANE TRICARBOXYLATE

422 POTASSIUM 1-1-2-HEXADECANE TRICARBOXYLATE

SULFO CARBOXYLIC ACID

6 SODIUM ALPHA SULFOPELARGONIC ACID

602 ALPHA SULFO LAURIC ACID

235 SODIUM ALPHA SULFO LAURIC ACID

189 ALPHA SULFOMYRISTIC ACID

236 SODIUM ALPHA SULFO MYRISTIC ACID

190 ALPHA SULFOPALMITIC ACID

237 SODIUM ALPHA SULFO PALMITIC ACID

191 ALPHA SULFOSTEARIC ACID

551 PHENYL SULFOSTEARIC ACID

552 TOLYL SULFOSTEARIC ACID

553 XYLYL SULFOSTEARIC ACID

9 SODIUM ALPHA SULFOSTEARIC ACID

11 SODIUM ALPHA SULFO PHENYL STEARIC ACID

12 SODIUM 9,10 DICHLORO ALPHA SULFOSTEARIC ACID

SULFO CARBOXYLATE

233 DISODIUM ALPHA SULFO MYRISTATE

234 DISODIUM ALPHA SULFO PALMITATE

197 DISODIUM 2 SULFOETHYL ALPHA SULFOPALMITATE

8 DISODIUM ALPHA SULFOPHENYLSTEARATE

10 DISODIUM ALPHA SULFOSTEARATE

198 DISODIUM 2 SULFOETHYL ALPHA SULFOSTEARATE

13 DISODIUM 9,10 DICHLORO ALPHA SULFOSTEARATE

14 DISODIUM 9,10 DIHYDROXY ALPHA SULFOSTEARATE

PHOSPHONO CARBOXYLIC ACID

611 ALPHAPHOSPHONO PELARGONIC ACID

612 ALPHAPHOSPHONO DECANOIC ACID

613 ALPHAPHOSPHONO DODECANOIC ACID

614 ALPHAPHOSPHONO TETRADECANOIC ACID

615 ALPHAPHOSPHONO HEXADECANOIC ACID

PHOSPHONO CARBOXYLATE

616 MONOSODIUM ALPHAPHOSPHONO DECANOATE

617 MONOSODIUM ALPHAPHOSPHONO DODECANOATE

618 MONOSODIUM ALPHAPHOSPHONO TETRADECANOATE

619 MONOSODIUM ALPHAPHOSPHONO HEXADECANOATE

620 DISCDIUM ALPHAPHOSPHONO DODECANOATE

621 DISODIUM ALPHAPHOSPHONO TETRADECANOATE

622 DISODIUM ALPHAPHOSPHONO HEXADECANOATE

623 DISODIUM ALPHAPHOSPHONO OCTADECANOATE

624 TRISODIUM ALPHAPHOSPHONO TETRADECANOATE

625 TRISODIUM ALPHAPHOSPHONO HEXADECANOATE

626 TRISODIUM ALPHAPHOSPHONO OCTADECANOATE

SULFO CARBOXYLIC ESTER

25 SODIUM ETHYL ALPHA SULFOPELARGONATE

26 SODIUM AMYL ALPHA SULFOPELARGONATE

27 SODIUM HEXYL ALPHA SULFOPELARGONATE

28 SODIUM HEPTYL ALPHA SULFOPELARGONATE

35 SODIUM H/CF2CF2/3CH2 ALPHA SULFOPELARGONATE

7 SODIUM OCTYL ALPHA SULFOPELARGONATE

31 SODIUM 2 OCTYL ALPHA SULFOPELARGONATE

32 SODIUM 2 ETHYLHEXYL ALPHA SULFOPELARGONATE

29 SODIUM NONYL ALPHA SULFOPELARGONATE

30 SODIUM DECYL ALPHA SULFOPELARGONATE

33 SODIUM /OXO/ DECYL ALPHA SULFOPELARGONATE

34 SODIUM DODECYL ALPHA SULFOPELARGONATE

603 SODIUM PROPYL ALPHA SULFO LAURATE

604 SODIUM METHYL ALPHA SULFO MYRISTATE

36 SODIUM METHYL ALPHA SULFOPALMITATE

192 SODIUM ETHYL ALPHA SULFOPALMITATE

193 SODIUM PROPYL ALPHA SULFOPALMITATE

194 SODIUM METHYL ALPHA SULFOSTEARATE

195 SODIUM ETHYL ALPHA SULFOSTEARATE

196 SODIUM PROPYL ALPHA SULFOSTEARATE

199 SODIUM ISOPROPYL ALPHA SULFOSTEARATE

PHOSPHONO ESTER

605 SODIUM AMYL ALPHAPHOSPHONO PELARGONATE 
PART 2. Surfactants grouped by charge and by head group and arranged by number of carbon atoms in longest hydrophobic tail - Continued

606 SODIUM AMYL ALPHAPHOSPHONO CAPRATE

607 SODIUM ISOPROPYL ALPHAPHOSPHONO LAURATE

608 SODIUM METHYL ALPHAPHOSPHONO MYRISTATE

610 SODIUM METHYL ALPHAPHOSPHONO STEARATE

609 SODIUM ISOPROPYL ALPHAPHOSPHONO PALMITATE

SULFATE

2 SODIUM OCTYL 1 SULFATE

347 OCTYL TRIMETHYLAMMOMIUM OCTYL SULFATE

643 DODECYL TRIMETHYLAMMONIUM OCTANE SULFATE

66 SODIUM OCTYL 2 SULFATE

295 SODIUM NONYL 1-SULFATE

3 SODIUM DECYL 1 SULFATE

642 OCTYL TRIMETHYLAMMONIUM DECANE SULFATE

79 SODIUM NONADECYL 5 SULFATE

346 DECYL TRIMETHYLAMMONIUM DECYL SULFATE

15 SODIUM DECYL 2 SULFATE

311 SODIUM UNDECYL 1-SULFATE

72 SODIUM UNDECYL 3 SULFATE

80 SODIUM UNDECYL 6 SULFATE

$\mathrm{C}-12$

705 DODECYL SULFURIC ACID

1 SODIUM DODECYL 1 SULFATE

634 POTASSIUM DODECYL SULFATE

111 LITHIUM DODECYL 1 SULFATE

23 SILVER DODECYL 1 SULFATE

568 MAGNESIUM DODECYL SULFATE

24 CALCIUM DODECYL I SULFATE

569 STRONTIUM DODECYL SULFATE

575 NICKEL DODECYL SULFATE

572 COBALTOUS DODECYL SULFATE

574 ZINC DODECYL SULFATE

570 LEAD DODECYL SULFATE

571 MANGANESE DODECYL SULFATE

573 CUPRIC DODECYL SULFATE

386 AMMONIUM DODECYL SULFATE

387 METHYLAMMONIUM DODECYL SULFATE

388 ETHYLAMMONIUM DODECYL SULFATE

389 BUTYLAMMONIUM DODECYL SULFATE

112 TETRAMETHYL AMMONIUM DODECYL 1 SULFATE

382 ETHYL TRIMETHYLAMMONIUM DODECYL SULFATE

383 BUTYL TRIMETHYLAMMONIUM DODECYL SULFATE

718 TETRAETHYLAMMONIUM DODECYL SULFATE

719 TETRABUTYLAMMONIUM DODECYL SULFATE

720 1-6-DITRIMETHYLAMMONIUM-HEXANE/DODECYL SULFATE/2

409 TRIETHANOLAMMONIUM DODECYL SULFATE

410 MORPHOLINIUM DODECYL SULFATE

391 OCTYLAMMONIUM DODECYL SULFATE

385 OCTYL TRIMETHYLAMMONIUM DODECYL SULFATE

280 DECYL TRIMETHYL AMMONIUM DODECYL SULFATE

281 DODECYL TRIMETHYLAMMONIUM DODECYL SULFATE

67 SODIUM DODECYL 2 SULFATE

562 SODIUM DODECENYL SULFATE

68 SODIUM TRIDECYL 2 SULFATE

83 SODIUM TRIDECYL 7 SULFATE

4 SODIUM TETRADECYL 1 SULFATE

526 SODIUM 2-DI-N-HEXYL ETHYL SULFATE

637 LITHIUM TETRADECYL SULFATE

576 CUPRIC TETRADECYL SULFATE

16 SODIUM TETRADECYL 2 SULFATE

73 SODIUM TETRADECYL 3 SULFATE

17 SODIUM TETRADECYL 4 SULFATE

77 SODIUM TETRADECYL 5 SULFATE

525 SODIUM TETRADECYL 6-SULFATE

84 SODIUM TETRADECYL 7 SULFATE

69 SODIUM PENTADECYL 2 SULFATE

74 SODIUM PENTADECYL 3 SULFATE

78 SODIUM PENTADECYL 5 SULFATE

85 SODIUM PENTADECYL 8 SULFATE

5 SODIUM HEXADECYL I SULFATE

638 LITHIUM HEXADECYL SULFATE

577 CUPRIC HEXADECYL SULFATE

60 TRIETHANOL AMMONIUM HEXADECYL SULFATE

75 SODIUM HEXADECYL 4 SULFATE

81 SODIUM HEXADECYL 6 SULFATE

86 SODIUM HEXADECYL 8 SULFATE

70 SODIUM HEPTADECYL 2 SULFATE

87 SODIUM HEPTADECYL 9 SULFATE

$\mathrm{C}-18$

64 SODIUM OCTADECYL 1 SULFATE

65 TRIETHANOLAMMONIUM OCTADECYL 1 SULFATE

61 SODIUM OLEYL/CIS 9 OCTADECENOYL/ SULFATE
62 SODIUM ELAIDYL/TRANS 9 OCTADECENOYL/SULFATE

63 SODIUM 910 DICHLORO OCTADECYL SULFATE

71 SODIUM OCTADECYL 2 SULFATE

76 SODIUM OCTADECYL 4 SULFATE

82 SODIUM OCTADECYL 6 SULFATE

88 SODIUM 1 NONYL DECYL SULFATE

89 SODIUM 1 TETRADECYL PENTADECYL SULFATE

POLYOL SULFATE

462 SODIUM MONOLAURIN SULFATE

\section{THIOSULFATE}

639 SODIUM UNDECYL THIOSULFATE

636 SODIUM DODECYL THIOSULFATE

ALKYL SULFONATE

343 SODIUM PENTANE SULFONATE

344 SODIUM HEXANE SULFONATE

339 MAGNESIUM HEXANE SULFONATE

181 SODIUM OCTYL 1-SULFONATE

340 MAGNESIUM OCTANE SULFONATE

287 OCTYL TRIMETHYLAMMONIUM OCTANE SULFONATE

536 NONYL SULFONIC ACID

288 DECYL TRIMETHYLAMMONIUM DECANESULFONATE

182 SODIUM DECYL 1-SULFONATE

341 MAGNESIUM DECANE SULFONATE

353 OCTYL TRIMETHYLAMMONIUM DECANE SULFONATE

537 UNDECYL SULFONIC ACID

418 SODIUM UNDECYL SULFONATE

200 DODECYL SULFONIC ACID

179 SODIUM DODECANE 1-SULFONATE

40 POTASSIUM DODECYL 1 SULFONATE

635 LITHIUM DODECYL SULFONATE

342 MAGNESIUM DODECANE SULFONATE

175 SODIUM DODECANE 2-SULFONATE

242 TRIDECANE 1-SULFONIC ACID

229 SODIUM TRIDECANE 1-SULFONATE

243 TETRADECANE 1-SULFONIC ACID

183 SODIUM TETRADECYL 1-SULFONATE

176 SODIUM TETRADECANE 2-SULFONATE

244 PENTADECANE 1-SULFONIC ACID

230 SODIUM PENTADECANE 1-SULFONATE

245 HEXADECANE 1-SULFONIC ACID

184 SODIUM HEXADECYL 1-SULFONATE

408 POTASSIUM HEXADECANE 1-SULFONATE

177 SODIUM HEXADECANE 2-SULFONATE

246 HEPTADECANE 1-SULFONIC ACID

231 SODIUM HEPTADECANE 1-SULFONATE

232 OCTADECANE 1-SULFONIC ACID

349 SODIUM OCTADECANE 1-SULFONATE

419 SODIUM ALPHA-HEPTYL UNDECYL SULFONATE

178 SODIUM OCTADECANE 2-SULFONATE

HYDROXY ALKYL SULFONATE

247 DODECANE 1-HYDROXY 2-SULFONIC ACTD

238 SODIUM DODECANE 1-HYDROXY 2-SULFONATE

248 TETRADECANE 1-HYDROXY 2-SULFONIC ACID

239 SODIUM TETRADECANE 1-HYDROXY 2-SULFONATE

249 HEXADECANE 1-HYDROXY 2-SULFONIC ACID

240 SODIUM HEXADECANE 1-HYDROXY 2-SULFONATE

250 OCTADECANE 1-HYDROXY 2-SULFONIC ACID

241 SODIUM OCTADECANE 1-HYDROXY 2-SULFONATE

\section{SULFOSUCCINATE DIESTER}

257 SODIUM DI-N-BUTYL SULFOSUCCINATE

261 SODIUM DI-ISOBUTYL SULFOSUCCINATE

258 SODIUM DI-N-AMYL SULFOSUCCINATE

259 SODIUM DI-N-HEXYL SULFOSUCCINATE

352 SODIUM DI-1-METHYLISOAMYL SULFOSUCCINATE

260 SODIUM DI-N-OCTYL SULFOSUCCINATE

262 SODIUM DI-2-ETHYL-HEXYL SULFOSUCCINATE

286 AMMONIUM DI-2-ETHYL-HEXYL SULFOSUCCINATE

PARA ALKARYL SULFONATE

445 SODIUM PARA-BIS-/N-BUTYL/ BENZENE SULFONATE

446 SODIUM PARA-BIS-/N-HEXYL/ BENZENE SULFONATE

49 SODIUM P OCTYL BENZENE SULFONATE

447 SODIUM PARA-BIS-/N-OCTYL/ BENZENE SULFONATE

493 SODIUM P-NONYL BENZENE SULFONATE

50 SODIUM $P$ DECYL BENZENE SULFONATE

45 SODIUM $P$ I METHYL DECYL BENZENE SULFONATE

51 SODIUM $P$ DODECYL BENZENE SULFONATE

46 SODIUM $P$ I METHYL DODECYL BENZENE SULFONATE 
PART 2. Surfactants grouped by charge and by head group and arranged by number of carbon atoms in longest hydrophobic tail-Continued

47 SODIUM $P$ I METHYL TETRADECYL BENZENE SULFONATE

48 SODIUM $P$ I METHYL HEXADECYL BENZENE SULFONATE

ALKARYL SULFONATE (UNSPECIFIED)

682 SODIUM DIBUTYL NAPHTHALENE SULFONATE /NEKAL/

681 SODIUM DIBUTYL BENZENE SULFONATE

501 SODIUM HEXYL BENZENE SULFONATE

502 SODIUM HEPTYL BENZENE SULFONATE

$\mathrm{C}-8$

503 SODIUM OCTYL BENZENE SULFONATE

510 SODIUM 2-ETHYL-HEXYL BENZENE SULFONATE

676 SODIUM $1,1,3,3$-TETRAMETHYL BUTYL BENZENE SULFONATE

677 SODIUM DI- $/ 1,1,3,3$-TETRAMETHYL BUTYL/BENZENESULFONATE

172 SODIUM 2-N-OCTYL BENZENE SULFONATE

C-9

504 SODIUM NONYL BENZENE SULFONATE

138 SODIUM NONYL BENZENE SULFONATE BRANCHED HYDROCARBON CHAIN

272 TRI-ISOPROPYL BENZENE SULFONIC ACID

271 SODIUM TRI-ISO-PROPYL BENZENE SULFONATE

$\mathrm{C}-10$

505 SODIUM DECYL BENZENE SULFONATE

173 SODIUM 2-N-DECYL BENZENE SULFONATE

511 SODIUM 2-PROPYL-HEPTYL BENZENE SULFONATE

678 SODIUM 1,1-DIMETHYLNONYL BENZENE SULFONATE

679 SODIUM 3-BUTYL HEPTYL BENZENE SULFONATE

140 SODIUM DECYL BENZENE SULFONATE BRANCHED HYDROCARBON CHAIN

561 SANTOMERSE D /SODIUM DECYLBENZENE SULFONATE/

$\mathrm{C}-12$

680 SODIUM 2-PROPYL-4-METHYL-OCTYL BENZENE SULFONATE

492 SODIUM TETRAPROPYLENE/1-3-5-7-TETRAMETHYLOCTYL/BENZENE SULFONATE

506 SODIUM DODECYL BENZENE SULFONATE

139 SODIUM DODECYL BENZENE SULFONATE BRANCHED HYDROCARBON CHAIN

171 SODIUM 2-N-DODECYL BENZENE SULFONATE

301 SODIUM 3-N-DODECYL BENZENE SULFONATE

302 SODIUM 4-N-DODECYL BENZENE SULFONATE

514 SODIUM 6-N-DODECYL BENZENE SULFONATE

512 SODIUM 2-BUTYL-OCTYL BENZENE SULFONATE

141 SODIUM TRIDECYL BENZENE SULFONATE BRANCHED HYDROCARBON CHAIN

554 SANTOMERSE 3 /SODIUM DODECYL BENZENE SULFONATE/

$\mathrm{C}-14$

507 SODIUM TETRADECYL BENZENE SULFONATE

174 SODIUM 2-N-TETRADECYL BENZENE SULFONATE

513 SODIUM 2-AMYL-NONYL BENZENE SULFONATE

596 TETRAHYDROANACARDOL AMMONIUM MONOSULFONATE

142 SODIUM PENTADECYL BENZENE SULFONATE BRANCHED HYDROCARBON CHAIN

508 SODIUM HEXADECYL BENZENE SULFONATE

509 SODIUM OCTADECYL BENZENE SULFONATE

683 SODIUM EICOSYLBENZENE SULFONATE

557 ÁRESKET 300 /MONOBUTYL BIPHENYL SODIUM MONOSULFONATE/

556 ARESKAP 100 /MONOBUTYL PHENYLPHENOL SODIUM MONOSULFONATE

558 ARESKLENE 400 /DIBUTYL PHENYLPHENOL DISODIUMDISULFONATE

OXYPROPYL SULFATE

597 SODIUM DODECYL MONO-OXYPROPYL SULFATE

598 SODIUM TETRADECYL MONO-OXYPROPYL SULFATE

600 SODIUM HEXADECYL MONO-OXYPROPYL SULFATE

601 SODIUM OCTADECYL MONO-OXYPROPYL SULFATE

599 SODIUM TETRADECYL DI-OXYPROPYL SULFATE

OXYETHYLENE SULFATE

541 SODIUM DODECYL MONO-OXYETHYLENE SULFATE

544 SODIUM TETRADECYL MONO-OXYETHYLENE SULFATE

52 SODIUM HEXADECYL MONO OXYETHYLENE SULFATE

56 SODIUM OCTADECYL MONO OXYETHYLENE SULFATE

547 SODIUM OLEYL MONO-OXYETHYLENE SULFATE

542 SODIUM DODECYL DIOXYETHYLENE SULFATE

545 SODIUM TETRADECYL DI-OXYETHYLENE SULFATE

53 SODIUM HEXADECYL DI OXYETHYLENE SULFATE

57 SODIUM OCTADECYL DI OXYETHYLENE SULFATE

548 SODIUM OLEYL DI-OXYETHYLENE SULFATE
113 SODIUM DODECYL TRI-OXYETHYLENE SULFATE

546 SODIUM TETRADECYL TRI-OXYETHYLENE SULFATE

54 SODIUM HEXADECYL TRI OXYETHYLENE SULFATE

58 SODIUM OCTADECYL TRI OXYETHYLENE SULFATE

549 SODIUM OLEYL TRI-OXYETHYLENE SULFATE

543 SODIUM DODECYL TETRA-OXYETHYLENE SULFATE

55 SODIUM HEXADECYL TETRA OXYETHYLENE SULFATE

59 SODIUM OCTADECYL TETRA OXYETHYLENE SULFATE

114 SODIUM DODECYL /OXYETHYLENE/17.5 SULFATE

OTHER

136 SODIUM ALPHA DIMETHYL AMINO CAPRATE

704 POTASSIUM 4-HEXYL RESORCINOLATE

632 POTASSIUM N-DODECYL BETA-ALANINATE

\section{B. CATIONICS BY HEAD GROUP}

AMINE

708 HEXYLAMINE

706 PERFLUORO PROPYLAMINE

$\mathrm{R}-\mathrm{H} 3$

707 PERFLUORO PROPYLAMINE HYDROCHLORIDE

709 HEXYLAMINE HYDROCHLORIDE

390 HEXYLAMMONIUM DODECYL SULFATE

392 OCTYLAMMONIUM CHLORIDE

391 OCTYLAMMONIUM DODECYL SULFATE

37 DECYLAMMONIUM CHLORIDE

411 DECYLAMMONIUM ACETATE

38 DODECYL AMMONIUM CHLORIDE

628 DODECYLAMMONIUM BROMIDE

482 DODECYL AMMONIUM NITRATE

412 DODECYLAMMONIUM ACETATE

39 TETRADECYL AMMONIUM CHLORIDE

413 TETRADECYLAMMONIUM ACETATE

186 HEXADECYL AMMONIUM CHLORIDE

414 HEXADECYLAMMONIUM ACETATE

187 OCTADECYL AMMONIUM CHLORIDE

415 OCTADECYLAMMONIUM ACETATE

$\mathrm{RR}-\mathrm{H} 2$

449 DODECYLMETHYL AMMONIUM CHLORIDE

RRR-H

450 DODECYLDIMETHYL AMMONIUM CHLORIDE

$\mathrm{R}-(\mathrm{CH} 3) 3$

644 HEXYL TRIMETHYLAMMONIUM HEXANE SULFONATE

640 HEXYL TRIMETHYLAMMONIUM HEXANE SULFATE

641 HEXYL TRIMETHYLAMMONIUM OCTANE SULFATE

93 OCTYL TRIMETHYL AMMONIUM BROMIDE

$\mathrm{C}-8$

347 OCTYL TRIMETHYLAMMOMIUM OCTYL SULFATE

287 OCTYL TRIMETHYLAMMONIUM OCTANE SULFONATE

642 OCTYL TRIMETHYLAMMONIUM DECANE SULFATE

353 OCTYL TRIMETHYLAMMONIUM DECANE SULFONATE

385 OCTYL TRIMETHYLAMMONIUM DODECYL SULFATE

C-9

94 NONYL TRIMETHYL AMMONIUM BROMIDE

C-10

203 DECYL TRIMETHYL AMMONIUM CHLORIDE

95 DECYL TRIMETHYL AMMONIUM BROMIDE

306 DECYL TRIMETHYLAMMONIUM SULFATE

346 DECYL TRIMETHYLAMMONIUM DECYL SULFATE

280 DECYL TRIMETHYL AMMONIUM DODECYL SULFATE

288 DECYL TRIMETHYLAMMONIUM DECANESULFONATE

$\mathrm{C}-11$

96 UNDECYL TRIMETHYL AMMONIUM BROMIDE

C-12

41 DODECYL TRIMETHYL AMMONIUM CHLORIDE

126 DODECYL TRIMETHYLAMMONIUM IODIDE

97 DODECYL TRIMETHYL AMMONIUM BROMIDE

130 DODECYL TRIMETHYL AMMONIUM FLUORIDE

131 DODECYL TRIMETHYLAMMONIUM NITRATE

127 DODECYL TRIMETHYL AMMONIUM IODATE

129 DODECYL TRIMETHYL AMMONIUM BROMATE 
PART 2. Surfactants grouped by charge and by head group and arranged by number of carbon atoms in longest hydrophobic tail-Continued

307 DODECYL TRIMETHYLAMMONIUM SULFATE

128 DODECYL TRIMETHYL AMMONIUM FORMATE

643 DODECYL TRIMETHYLAMMONIUM OCTANE SULFATE

281 DODECYL TRIMETHYLAMMONIUM DODECYL SULFATE

384 HEXYL TRIMETHYLAMMONIUM DODECYL SULFATE

$\mathrm{C}-13$

402 TRIDECYL TRIMETHYLAMMONIUM CHLORIDE

C -14

42 TETRADECYL TRIMETHYL AMMONIUM CHLORIDE

98 TETRADECYL TRIMETHYL AMMONIUM BROMIDE

308 TETRADECYL TRIMETHYLAMMONIUM SULFATE

$\mathrm{C}-16$

265 HEXADECYL TRIMETHYL AMMONIUM CHLORIDE

99 HEXADECYL TRIMETHYLAMMONIUM BROMIDE

309 HEXADECYL TRIMETHYLAMMONIUM SULFATE

559 CATOL $605 /(\mathrm{N}-(2-$ KETO-2-(2,-LAUROYL

OXYETHYLAMINO) ) ETHYL TRIMETHYLAMMONIUM CHLORIDE/

C-18

270 OCTADECYL TRIMETHYLAMMONIUM CHLORIDE

477 OCTADECYL TRIMETHYLAMMONIUM BROMIDE

654 OCTADECYL TRIMETHYLAMMONIUM NITRATE

658 OCTADECYL TRIMETHYLAMMONIUM BROMATE

659 OCTADECYL TRIMETHYLAMMONIUM FORMATE

667 OCTADECYL TRIMETHYLAMMONIUM OXALATE

$\mathrm{RR}-(\mathrm{CH} 3) 2$

399 DODECYL DIMETHYL ETHYLAMMONIUM CHLORIDE

403 DODECYL DIMETHYLPHENYLAMMONIUM CHLORIDE

478 HEXADECYL DIMETHYLETHYLAMMONIUM BROMIDE

266 HEXADECYL DIMETHYL 2-HYDROXYETHYL AMMONIUM CHLORIDE

269 HEXADECYLDIMETHYL 2,3-DIHYDROXYPROPYLAMMONIUM CHLORIDE

483 DIOCTYL DIMETHYL AMMONIUM CHLORIDE

345 DIDODECYL DIMETHYLAMMONIUM CHLORIDE

BENZYL

354 HEXYL BENZYL DIMETHYLAMMONIUM CHLORIDE

356 DECYL BENZYL DIMETHYLAMMONIUM CHLORIDE

279 DODECYL BENZYL DIMETHYLAMMONIUM CHLORIDE

357 TETRADECYL BENZYL DIMETHYLAMMONIUM CHLORIDE

275 HEXADECYL DIMETHYLBENZYLAMMONIUM CHLORIDE

358 OCTADECYL BENZYL DIMETHYLAMMONIUM CHLORIDE

406 DODECYL DIMETHYL META-TRIFLUOROMETHYLBENZYL AMMONIUM

CHLORIDE CF3C6H4CH2/N/CH3/2/C12H25

359 OCTYL 4-NITROBENZYL DIMETHYLAMMONIUM CHLORIDE

360 DECYL 4-NITROBENZYL DIMETHYLAMMONIUM CHLORIDE

361 DODECYL 4-NITROBENZYL DIMETHYL AMMONIUM CHLORIDE

362 TETRADECYL 4-NITROBENZYL DIMETHYLAMMONIUM CHLORIDE

363 HEXADECYL 4-NITROBENZYL DIMETHYLAMMONIUM CHLORIDE

364 OCTADECYL 4-NITROBENZYL DIMETHYLAMMONIUM CHLORIDE

365 DODECYL 2-CHLOROBENZYL DIMETHYLAMMONIUM CHLORIDE

367 DODECYL 4-CHLOROBENZYL DIMETHYLAMMONIUM CHLORIDE

366 DODECYL 2-4-DICHLOROBENZYL DIMETHYLAMMONIUM CHLORIDE

369 DODECYL 3-4-DICHLOROBENZYL DIMETHYLAMMONIUM CHLORIDE

371 DODECYL 3-4-DIMETHOXYBENZYL DIMETHYLAMMONIUM CHLORIDE

370 DODECYL 3-4-METHYLENEDIOXYBENZYL DIMETHYLAMMONIUM CHLORIDE

368 DODECYL 2-HYDROXY-5-NITROBENZYL DIMETHYLAMMONIUM CHLORIDE

451 PARA DI-ISOBUTYLPHENOXYETHOXYETHYL DIMETHYLBENZYL AMMONIUM CHLORIDE/HYAMINE 1622/

404 DODECYL DIMETHYL 2-PHENYLETHYLAMMONIUM CHLORIDE $\mathrm{C} 6 \mathrm{H} 5 \mathrm{CH} 2 \mathrm{CH} 2 / \mathrm{N} / \mathrm{CH} 3 / 2 / \mathrm{C} 12 \mathrm{H} 25$

407 DODECYL DIMETHYL 3-PHENYLPROPYLAMMONIUM CHLORIDE $\mathrm{C} 6 \mathrm{H} 5 \mathrm{CH} 2 \mathrm{CH} 2 / \mathrm{N} / \mathrm{CH} 3 / 2 / \mathrm{Cl} 2 \mathrm{H} 25$

$\mathrm{RRR}-\mathrm{CH} 3$

400 DODECYL METHYL DIETHYLAMMONIUM CHLORIDE

405 DODECYL METHYLETHYLBENZYLAMMONIUM CHLORIDE

267 HEXADECYL DI-/2-HYDROXYETHYL/METHYL AMMONIUMCHLORIDE

$\mathrm{R}-\mathrm{R} 3$

662 OCTADECYL TRIETHYLAMMONIUM BROMATE

401 DODECYL TRIETHYLAMMONIUM CHLORIDE

663 OCTADECYL TRIPROPYLAMMONIUM BROMATE

292 HEXADECYL TRIPROPYLAMMONIUM BROMIDE

291 TETRADECYL TRIPROPYLAMMONIUM BROMIDE

664 OCTADECYL TRIBUTYLAMMONIUM BROMATE

666 HEXADECYL TRIBUTYLAMMONIUM BROMATE

665 OCTADECYL TRIAMYLAMMONIUM BROMATE
268 HEXADECYL TRI-/2-HYDROXYETHYL/ AMMONIUM CHLORIDE

500 DODECYL TRI/2-HYDROXYETHYL/AMMONIUM CHLORIDE

RRR-0

293 DODECYL DIMETHYL AMINE OXIDE HYDROBROMIDE

22 DIMETHYL DODECYL AMINE OXIDE HYDROCHLORIDE

BETAINE

135 OCTYL C BETAINE HYDROCHLORIDE

124 DODECYL N BETAINE HYDROCHLORIDE

125 TETRADECYL N BETAINE HYDROCHLORIDE

AMINO ACID

633 N-DODECYL BETA-ALANINE HYDROCHLORIDE

137 ALPHA DIMETHYLAMINO CAPRIC ACID HYDROCHLORIDE

\section{PYRIDINIUM}

100 OCTYL PYRIDINIUM BROMIDE

458 DECYL PYRIDINIUM IODIDE

101 UNDECYL PYRIDINIUM BROMIDE

278 DODECYL PYRIDINIUM CHLORIDE

290 DODECYL PYRIDINIUM BROMIDE

376 DODECYL PYRIDINIUM IODIDE

102 TETRADECYL PYRIDINIUM BROMIDE

479 TETRADECYL PYRIDINIUM IODIDE

304 CATOL 607 (N-/LAURYL-COLAMINO-FORMYL-METHYL/ PYRIDINIUM CHLORIDE

C-16

274 HEXADECYL PYRIDINIUM CHLORIDE

427 HEXADECYL PYRIDINIUM BROMIDE

480 HEXADECYL PYRIDINIUM IODIDE

660 HEXADECYL PYRIDINIUM IODATE

693 N-CETYL 2-METHYL PYRIDINIUM CHLORIDE

696 N-CETYL 2-METHYL PYRIDINIUM IODIDE

694 N-CETYL 3-METHYL PYRIDINIUM CHLORIDE

697 N-CETYL-3-METHYL PYRIDINIUM IODIDE

695 N-CETYL 4-METHYL PYRIDINIUM CHLORIDE

698 N-CETYL-4-METHYL PYRIDINIUM IODIDE

560 EMULSOL 607L (N-(2-KETO-2-(2,-LAUROYLOXETHY LAMINO) ETHYL) PYRIDINIUM CHLORIDE

655 OCTADECYL PYRIDINIUM CHLORIDE

657 OCTADECYL PYRIDINIUM BROMIDE

481 OCTADECYL PYRIDINIUM IODIDE

656 OCTADECYL PYRIDINIUM NITRATE

661 OCTADECYL PYRIDINIUM IODATE

QUINOLINIUM

717 DODECYLQUINOLINIUM. BROMIDE

TROPYLIUM

528 DODECYL TROPYLIUM PERCHLORATE

460 DODECYL TROPYLIUM MONOPHOSPHATE

491 DODECYL TROPYLIUM BISULFATE

\section{C. NONIONICS BY HEAD GROUP}

CARBOXYLIC ACID

43 BUTYRIC ACID

700 HEXANOIC ACID

529 OCTANOIC ACID

530 DECANOIC ACID

531 DODECANOIC ACID

532 TETRADECANOIC ACID

533 HEXADECANOIC ACID

534 OCTADECANOIC ACID

AMINE

706 PERFLURO PROPYLAMINE

708 HEXYLAMINE

N-BETAINE

118 OCTYL N BETAINE

119 DECYL N BETAINE

120 UNDECYL N BETAINE

121 DODECYL N BETAINE

592 DODECYL N-DIETHYL N-BETAINE

122 TETRADECYL N BETAINE

123 HEXADECYL N BETAINE

C-BETAINE

132 OCTYL C BETAINE 
PART 2. Surfactants grouped by charge and by head group and arranged by number of carbon atoms in longest hydrophobic tail-Continued

133 DECYL C BETAINE

134 DODECYL C BETAINE

AMINE OXIDE

714 HEXYL DIMETHYL AMINE OXIDE

715 HEPTYL DIMETHYL AMINE OXIDE

251 OCTYL DIMETHYL AMINE OXIDE

254 NONYL DIMETHYL AMINE OXIDE

252 DECYL DIMETHYL AMINE OXIDE

713 UNDECYL DIMETHYL AMINE OXIDE

21 DIMETHYL DODECYL AMINE OXIDE

253 TETRADECYL DIMETHYL AMINE OXIDE

348 NN-DIMETHYL 1-1-DIHYDROPENTADECAFLUORO OCTYL AMINE NOXIDE /C7F15CH2N/CH3/20/

PHOSPHINE OXIDE

710 OCTYL DIMETHYL PHOSPHINE OXIDE

711 DECYL DIMETHYL PHOSPHINE OXIDE

712 DODECYL DIMETHYL PHOSPHINE OXIDE

-ONIO -ATE

590 DODECYL DIMETHYLAMMONIOPROPANE CARBOXYLATE

587 DECYL DIMETHYLAMMONIOPROPANE SULFONATE

588 DODECYL DIMETHYLAMMONIOPROPANE SULFONATE

594 DODECYL DIPROPYL AMMONIOPROPANE SULFONATE

589 HEXADECYLDIMETHYLAMMONIOPROPANE SULFONATE

595 DODECYL DIMETHYL AMMONIOPROPANE SULFATE

593 DIMETHYL DODECYLAMMONIOPROPANE HYDROXY SULFONATE

591 DODECYL DIMETHYLPHOSPHONIOPROPANE SULFONATE

SULFINYL OL

684 HEXYL SULFINYLETHANOL

688 OCTYL SULFINYLETHANOL

692 DECYL SULFINYLETHANOL

685 HEXYL SULFINYLPROPANOL

689 OCTYL SULFINYLPROPANOL

686 HEXYL SULFINYLBUTANOL

690 OCTYL SULFINYLBUTANOL

687 HEXYL SULFINYLPENTANOL

SUGAR ALKYLATE

648 ALPHA-D-GLUCOSYL OCTANE

649 ALPHA-D-GLUCOSYL DODECANE

645 PARA/BETA-D-GLUCOSYL/ETHYLBENZENE

646 PARA/BETA-D-GLUCOSYL/PROPYL BENZENE

647 PARA/BETA-D-GLUCOSYL/BUTYLBENZENE

650 PARA/BETA-D-XYLOSYL/BUTYL BENZENE

SUGAR ESTER

495 SUCROSE MONOLAURATE

497 SUCROSE MONOMYRISTATE

498 SUCROSE MONOPALMITATE

499 SUCROSE DI-PALMITATE

496 SUCROSE MONOSTEARATE

POLYOL ETHER

424 OCTYL ALPHA-GLYCERYL ETHER

18 OCTYL BETA D GLUCOSIDE

19 DECYL BETA D GLUCOSIDE

20 DODECYL BETA D GLUCOSIDE

OTHER

457 1-4-HEXANEDIOL

703 4-HEXYL RESORCINOL

691 OCTYL METHYL SULFOXIDE

METHYL-OXYETHYLENE ESTER

583 METHYL /OXYETHYLENE/ 6.0 DODECANOATE REDUCED POLYDISPERSITY OF HEAD GROUPS

580 METHYL /OXYETHYLENE/ 7.0 DECANOATE REDUCED POLYDISPERSITY OF HEAD GROUPS

586 METHYL /OXYETHYLENE/ 7.6 OCTANOATE REDUCED POLYDISPERSITY OF HEAD GROUPS

584 METHYL /OXYETHYLENE/ 8.4 DODECANOATE REDUCED POLYDISPERSITY OF HEAD GROUPS

581 METHYL /OXYETHYLENE/ 10.3 DECANOATE REDUCED POLYDISPERSITY OF HEAD GROUPS

585 METHYL /OXYETHYLENE/ 11.2 DODECANOATE REDUCED POLYDISPERSITY OF HEAD GROUPS

578 METHYL /OXYETHYLENE/ 11.9 DECANOATE REDUCED POLYDISPERSITY OF HEAD GROUPS

579 METHYL /OXYETHYLENE/ 12.5 DODECANOATE REDUCED POLYDISPERSITY OF HEAD GROUPS
582 METHYL /OXYETHYLENE/ 16.0 DECANOATE NATURAL DISTRIBUTION OF HEAD GROUPS

METHYL-OXYETHYLENE ETHER

201 DECYL /OXYETHYLENE/8 OXYMETHYL REDUCED POLYDISPERSION OF HEAD GROUPS

202 DECYL /OXYETHYLENE/11 OXYMETHYL REDUCED POLYDISPERSIOI OF HEAD GROUPS

204 DECYL /OXYETHYLENE/12 OXYMETHYL REDUCED POLYDISPERSIO' OF HEAD GROUPS

205 DODECYL /OXYETHYLENE/12 OXYMETHYL REDUCED POLYDISPERSION OF HEAD GROUPS

OXYETHYLENE SORBITAN

440 /OXYETHYLENE/4 SORBITAN MONOLAURATE ISOMERS AND DISTRIBUTED MULTIPLE OE CHAINS

441 /OXYETHYLENE/8 SORBITAN MONOLAURATE ISOMERS AND DISTRIBUTED MULTIPLE OF CHAINS

442 /OXYETHYLENE/1O SORBITAN MONOLAURATE ISOMERS AND DISTRIBUTED MULTIPLE OE CHAINS

443 /OXYETHYLENE/2O SORBITAN MONOLAURATE ISOMERS AND DISTRIBUTED MULTIPLE OE CHAINS

444 /OXYETHYLENE/25 SORBITAN MONOLAURATE ISOMERS AND DISTRIBUTED MULTIPLE OE CHAINS

OXYETHYLENE DIOL ESTER

276 NONAETHYLENE GLYCOL MONODODECANOATE NATURAL DISTRIBUTION OF HEAD GROUPS

\section{OXYETHYLENE ALCOHOL}

OE 1

461 BUTYL/OXYETHYLENE/1 ALCOHOL--BUTYL GLYCOL ETHER HOMOGENEOUS HEAD GROUP

423 OCTYL/OXYETHYLENE/1 ALCOHOL--OCTYL GLYCOL ETHER HOMOGENEOUS HEAD GROUP

207 P-T-OCTYL BENZENE /OXYETHYLENE/I ALCOHOL HOMOGENOUS HEAD GROUP

217 P-T-OCTYL BENZENE /OXYETHYLENE/I ALCOHOL NATURAL DISTRIBUTION OF HEAD GROUPS

OE 2

208 P-T-OCTYL BENZENE /OXYETHYLENE/2 ALCOHOL HOMOGENOUS HEAD GROUP

218 P-T-OCTYL BENZENE /OXYETHYLENE/2 ALCOHOL NATURAL DISTRIBUTION OF HEAD GROUPS

OE 3

103 HEXYL /OXYETHYLENE/ 3 ALCOHOL HOMOGENOUS HEAD GROUP

104 OCTYL /OXYETHYLENE/ 3 ALCOHOL HOMOGENOUS HEAD GROUP

209 P-T-OCTYL BENZENE /OXYETHYLENE/3 ALCOHOL HOMOGENOUS HEAD GROUP

219 P-T-OCTYL BENZENE /OXYETHYLENE/3 ALCOHOL NATURAL DISTRIBUTION OF HEAD GROUPS

107 DECYL /OXYETHYLENE/ 3 ALCOHOL HOMOGENOUS HEAD GROUP

OE 3

380 HEXYL/OXYETHYLENE/4 ALCOHOL HOMOGENEOUS HEAD GROUP

515 ISO-OCTYL/UXYETHYLENE/4 ALCOHOL BRANCHED CHAIN, NATURAL OE DISTRIBUTION

210 P-T-OCTYL BENZENE /OXYETHYLENE/4 ALCOHOL HOMOGENOUS HEAD GROUP

220 P-T-OCTYL BENZENE /OXYETHYLENE/4 ALCOHOL NATURAL DISTRIBUTION OF HEAD GROUPS

516 NONYL/OXYETHYLENE/4 ALCOHOL BRANCHED CHAIN, NATURAL OE DISTRIBUTION

379 DECYL/OXYETHYLENE/4 ALCOHOL HOMOGENEOUS HEAD GROUP

519 DECYL/OXO-PROCESS/ /OXYETHYLENE/4 ALCOHOL BRANCHED CHAIN, NATURAL OE DISTRIBUTION

325 DODECYL/OXYETHYLENE/4 ALCOHOL REDUCED POLYDISPERSITY OF HEAD GROUPS

\section{OE 5}

381 HEXYL/OXYETHYLENE/5 ALCOHOL HOMOGENEOUS HEAD GROUP

211 P-T-OCTYL BENZENE /OXYETHYLENE/5 ALCOHOL HOMOGENOUS HEAD GROUP

221 P-T-OCTYL BENZENE /OXYETHYLENE/5 ALCOHOL NATURAL DISTRIBUTION OF HEAD GROUPS

153 NONYL BENZENE /OXYETHYLENE/ 5.0 ALCOHOL BRANCHED CHAIN, NATURAL OE DISTRIBUTION

378 DECYL/OXYETHYLENE/5 ALCOHOL HOMOGENEOUS HEAD GROUP 
PART 2. Surfactants grouped by charge and by head group and arranged by number of carbon atoms in longest hydrophobic tail-Continued

143 DECYL /OXYETHYLENE/ 5.0 ALCOHOL BRANCHED CHAIN, NATURAL OE DISTRIBUTION

377 DODECYL/OXYETHYLENE/ 5 ALCOHOL HOMOGENEOUS HEAD GROUP

158 DODECYL BENZENE /OXYETHYLENE/ 5.0 ALCOHOL BRANCHED CHAIN, NATURAL OE DISTRIBUTION

312 TRIDECYL/OXYETHYLENE/5 ALCOHOL BRANCHED CHAIN, REDUCED OE DISTRIBUTION

148 TRIDECYL /OXYETHYLENE/ 5.2 ALCOHOL BRANCHED CHAIN, NATURAL OE DISTRIBUTION

\section{OE 6}

393 BUTYL/OXYETHYLENE/ 6 ALCOHOL HOMOGENEOUS HEAD GROUP

394 1-METHYL PROPYL/OXYETHYLENE/6 ALCOHOL HOMOGENEOUS HEAD GROUP

674 DIISOBUTYL BENZENE/OXYETHYLENE/ 6 ALCOHOL /DBBRANCHED CHAIN, NATURAL OE DISTRIBUTION

294 HEXYL/OXYETHYLENE/6 ALCOHOL HOMOGENOUS HEAD GROUP

395 2-ETHYL BUTYL/OXYETHYLENE/6 ALCOHOL HOMOGENEOUS HEAD GROUP

105 OCTYL /OXYETHYLENE/ 6 ALCOHOL HOMOGENOUS HEAD GROUP

396 2-PROPYL PENTYL/OXYETHYLENE/6 ALCOHOL HOMOGENEOUS HEAD GROUP

212 P-T-OCTYL BENZENE /OXYETHYLENE/6 ALCOHOL HOMOGENOUS HEAD GROUP

222 P-T-OCTYL BENZENE /OXYETHYLENE/6 ALCOHOL NATURAL DISTRIBUTION OF HEAD GROUPS

108 DECYL /OXYETHYLENE/ 6 ALCOHOL HOMOGENOUS HEAD GROUP

397 2-BUTYL HEXYL/OXYETHYLENE/6 ALCOHOL HOMOGENEOUS HEAD GROUP

110 DODECYL /OXYETHYLENE/ 6 ALCOHOL HOMOGENOUS HEAD GROUP

468 DODECYL/OXYETHYLENE/6.2 ALCOHOL NATURAL DISTRIBUTION OF HEAD GROUPS

518 TRIMETHYLNONYL/OXYETHYLENE/6 ALCOHOL BRANCHED CHAIN, NATURAL OE DISTRIBUTION

289 TETRADECYL/OXYETHYLENE/6 ALCOHOL HOMOGENOUS HEAD GROUP

282 HEXADECYL/OXYETHYLENE/ 6 ALCOHOL HOMOGENOUS HEAD GROUP

OE 7

213 P-T-OCTYL BENZENE /OXYETHYLENE/7 ALCOHOL HOMOGENOUS HEAD GROUP

223 P-T-OCTYL BENZENE /OXYETHYLENE/7 ALCOHOL NATURAL DISTRIBUTION OF HEAD GROUPS

488 DODECYL/OXYETHYLENE/7 ALCOHOL HOMOGENEOUS HEAD GROUP

115 DODECYL /OXYETHYLENE/7 ALCOHOL REDUCED POLYDISPERSION OF HEAD GROUPS

469 DODECYL/OXYETHYLENE/7.6 ALCOHOL NATURAL DISTRIBUTION OF HEAD GROUPS -

330 HEXADECYL/OXYETHYLENE/7 ALCOHOL HOMOGENEOUS HEAD GROUP

\section{OE 8}

335 OCTYL BENZENE/OXYETHYLENE/8.5 ALCOHOL BRANCHED CHAIN, NATURAL OE DISTRIBUTION

214 P-T-OCTYL BENZENE /OXYETHYLENE/8 ALCOHOL HOMOGENOUS HEAD GROUP

224 P-T-OCTYL BENZENE /OXYETHYLENE/8 ALCOHOL NATURAL DISTRIBUTION OF HEAD GROUPS

520 UNDECYL/OXYETHYLENE/8 ALCOHOL NATURAL DISTRIBUTION OF HEAD GROUPS

163 DODECYL /OXYETHYLENE/ 8 ALCOHOL NATURAL DISTRIBUTION OF HEAD GROUPS

431 TRIDECYL/OXO-PROCESS/ /OXYETHYLENE/8.5 ALCOHOL BRANCHED CHAIN. NATURAL OE DISTRIBUTION

OE 9

106 OCTYL /OXYETHYLENE/ 9 ALCOHOL HOMOGENOUS HEAD GROUP

215 P-T-OCTYL BENZENE /OXYETHYLENE/9 ALCOHOL HOMOGENOUS HEAD GROUP

225 P-T-OCTYL BENZENE /OXYETHYLENE/9 ALCOHOL NATURAL DISTRIBUTION OF HEAD GROUPS

206 TRITON X-100(P-T-OCTYL BENZENE/OXYETHYLENE/9NATURAL DISTRIBUTION OF HEAD GROUPS

336 NONYL BENZENE/OXYETHYLENE/9.5 ALCOHOL BRANCHED CHAIN, NATURAL OE DISTRIBUTION

154 NONYL BENZENE /OXYETHYLENE/ 9.8 ALCOHOL BRANCHED CHAIN, NATURAL OE DISTRIBUTION

109 DECYL /OXYETHYLENE/ 9 ALCOHOL HOMOGENOUS HEAD GROUP

398 2-BUTYL HEXYL/OXYETHYLENE/9 ALCOHOL HOMOGENEOUS HEAD GROUP

489 DODECYL/OXYETHYLENE/9 ALCOHOL HOMOGENEOUS HEAD GROUP

465 TRIDECYL/OXYETHYLENE/9 ALCOHOL NATURAL DISTRIBUTION OF HEAD GROUPS

466 TRIDECYL/SECONDARY/ /OXYETHYLENE/9 ALCOHOL NATURAL DISTRIBUTION OF HEAD GROUPS
313 TRIDECYL/OXYETHYLENE/9.5 ALCOHOL BRANCHED CHAIN, REDUCED OE DISTRIBUTION

331 HEXADECYL/OXYETHYLENE/9 ALCOHOL HOMOGENEOUS HEAD GROUP

OE 10

675 OCTYL BENZENE /OXYETHYLENE/10 ALCOHOL NATURAL DISTRIBUTION OF HEAD GROUPS

216 P-T-OCTYL BENZENE /OXYETHYLENE/10 ALCOHOL HOMOGENOUS HEAD GROUP

317 T-OCTYL BENZENE/OXYETHYLENE/10 ALCOHOL REDUCED POLYDISPERSITY OF HEAD GROUPS

226 P-T-OCTYL BENZENE /OXYETHYLENE/10 ALCOHOL NATURAL DISTRIBUTION OF HEAD GROUPS

320 NONYL BENZENE/OXYETHYLENE/10 ALCOHOL BRANCHED CHAIN, REDUCED OE DISTRIBUTION

167 NONYL BENZENE /OXYETHYLENE/ 10 ALCOHOL BRANCHED CHAIN NATURAL OE DISTRIBUTION

337 NONYL BENZENE/OXYETHYLENE/10.5 ALCOHOL BRANCHED CHAIN NATURAL OE DISTRIBUTION

144 DECYL /OXYETHYLENE/ 10.0 ALCOHOL BRANCHED CHAIN, NATURAL OE DISTRIBUTION

517 DECYL/OXO-PROCESS/ /OXYETHYLENE/IO ALCOHOL BRANCHED CHAIN, NATURAL OE DISTRIBUTION

310 DODECYL/OXYETHYLENE/10 ALCOHOL NATURAL DISTRIBUTION OF HEAD GROUPS

470 DODECYL/OXYETHYLENE/10.6 ALCOHOL NATURAL DISTRIBUTION OF HEAD GROUPS

159 DODECYL BENZENE /OXYETHYLENE/ 10.2 ALCOHOL BRANCHED CHAIN, NATURAL OE DISTRIBUTION

521 TRIDECYL/OXYETHYLENE/10 ALCOHOL BRANCHED CHAIN, NATURAL OE DISTRIBUTION

149 TRIDECYL /OXYETHYLENE/ 10.1 ALCOHOL BRANCHED CHAIN, NATURAL OE DISTRIBUTION

432 TRIDECYL/OXO-PROCESS/ /OXYETHYLENE/1O ALCOHOL BRANCHED CHAIN, NATURAL OE DISTRIBUTION

435 OCTADECYL/OXYETHYLENE/10 ALCOHOL NATURAL DISTRIBUTION OF HEAD GROUPS

OE 11

463 NONYL BENZENE/OXYETHYLENE/11 ALCOHOL NATURAL DISTRIBUTION OF HEAD GROUPS

523 DECYL BENZENE PARA/OXYETHYLENE/11.0 ALCOHOL NATURAL DISTRIBUTION OF HEAD GROUPS

522 DECYL BENZENE ORTHO/OXYETHYLENE/11.2 ALCOHOL NATURAL DISTRIBUTION OF HEAD GROUPS

OE 12

490 DODECYL/OXYETHYLENE/12 ALCOHOL HOMOGENEOUS HEAD GROUP

164 DODECYL /OXYETHYLENE/ 12 ALCOHOL NATURAL DISTRIBUTION OF HEAD GROUPS

467 TRIDECYL/SECONDARY / /OXYETHYLENE/12 ALCOHOL NATURAL DISTRIBUTION OF HEAD GROUPS

332 HEXADECYL/OXYETHYLENE/12 ALCOHOL HOMOGENEOUS HEAD GROUP

437 OLEYL/CIS-9-OCTADECENOYL/ /OXYETHYLENE/12 ALCOHOL NATURAL, DISTRIBUTION OF HEAD GROUPS

OE 14

145 DECYL /OXYETHYLENE/ 14.9 ALCOHOL BRANCHED CHAIN, NATURAL OE DISTRIBUTION

326 DODECYL/OXYETHYLENE/14 ALCOHOL REDUCED POLYDISPERSITY OF HEAD GROUPS

314 TRIDECYL/OXYETHYLENE/14 ALCOHOL BRANCHED CHAIN. REDUCED OE DISTTRIBUTION

524 HEXADECYL/OXYETHYLENE/14 ALCOHOL BRANCHED CHAIN, NATURAL OE DISTRIBUTION

328 OCTADECYL/OXYETHYLENE/14 ALCOHOL REDUCED POLYDISPERSITY OF HEAD GROIPS

OE 15

321 NONYL BENZENE/OXYETHYLENE/15 ALCOHOL BRANCHED CHAIN, REDUCED OE DISTRIBUTION

464 NONYL BENZENE/OXYETHYLENE/15 ALCOHOL NATURAL DISTRIBUTION OF HEAD GROUPS

168 NONYL BENZENE /OXYETHYLENE/ 15 ALCOHOL BRANCHED CHAIN, NATURAL OE DISTRIBUTION

155 NONYL BENZENE /OXYETHYLENE/ 15.4 ALCOHOL BRANCHED CHAIN, NATURAL OE DISTRIBUTION

471 DODECYL/OXYETHYLENE/15.8 ALCOHOL NATURAL DISTRIBUTION OF HEAD GROUPS

160 DODECYL BENZENE /OXYETHYLENE/ 15.1 ALCOHOL BRANCHED CHAIN, NATURAL OE DISTRIBUTION

150 TRIDECYL /OXYETHYLENE/ 15.2 ALCOHOL BRANCHED CHAIN, NATURAL OE DISTRIBUTION 
PART 2. Surfactants grouped by charge and by head group and arranged by number of carbon atoms in longest hydrophobic tail-Continued

433 TRIDECYL/OXO-PROCESS/ /OXYETHYLENE/15 ALCOHOL BRANCHED CHAIN, NATURAL OE DISTRIBUTION

333 HEXADECYL/OXYETHYLENE/15 ALCOHOL HOMOGENEOUS HEAD GROUP

OE 16

227 P-T-OCTYL BENZENE /OXYETHYLENE/16 ALCOHOL NATURAL DISTRIBUTION OF HEAD GROUPS

716 DODECYL /OXYETHYLENE/16 ALCOHOL REDUCED POLYDISPERSITY OF HEAD GROUPS

438 OLEYL/CIS-9-OCTADECENOYL/ /OXYETHYLENE/16 ALCOHOL NATURAL DISTRIBUTION OF HEAD GROUPS

OE 18

165 DODECYL /OXYETHYLENE/ 18 ALCOHOL NATURAL DISTRIBUTION OF HEAD GROUPS

535 HEXADECYL/OXYETHYLENE/18 ALCOHOL

OE 20

318 T-OCTYL BENZENE/OXYETHYLENE/20 ALCOHOL REDUCED POLYDISPERSITY OF HEAD GROUPS

322 NONYL BENZENE/OXYETHYLENE/20 ALCOHOL BRANCHED CHAIN. REDUCED OE DISTRIBUTION

156 NONYL BENZENE /OXYETHYLENE/20.0 ALCOHOL BRANCHED CHAIN, NATURAL OE DISTRIBUTION

146 DECYL /OXYETHYLENE/ 20.0 ALCOHOL BRANCHED CHAIN, NATURAL OE DISTRIBUTION

472 DODECYL/OXYETHYLENE/20.4 ALCOHOL NATURAL DISTRIBUTION OF HEAD GROUPS

161 DODECYL BENZENE /OXYETHYLENE/ 20.1 ALCOHOL BRANCHED CHAIN, NATURAL OE DISTRIBUTION

315 TRIDECYL/OXYETHYLENE/20 ALCOHOL BRANCHED CHAIN REDUCED OE DISTRIBUTION

151 TRIDECYL /OXYETHYLENE/ 20.0 ALCOHOL BRANCHED CHAIN NATURAL OE DISTRIBUTION

436 OCTADECYL/OXYETHYLENE/20 ALCOHOL NATURAL DISTRIBUTION OF HEAD GROUPS

439 OLEYL/CIS-9-OCTADECENOYL/ /OXYETHYLENE/2O ALCOHOL NATURAL DISTRIBUTION OF HEAD GROUP

OE $21-25$

334 HEXADECYL/OXYETHYLENE/21 ALCOHOL HOMOGENEOUS HEAD GROUP

434 TRIDECYL/OXO-PROCESS/ /OXYETHYLENE/22 ALCOHOL BRANCHED CHAIN, NATURAL OE DISTRIBUTION

327 DODECYL/OXYETHYLENE/23 ALCOHOL REDUCED POLYDISPERSITY OF HEAD GROUPS
166

DDECYL /OXYETHYLENE/ 23 ALCOHOL NATURAL DISTRIBUTION OF HEAD GROUPS

OE $26-30$

473 DODECYL/OXYETHYLENE/27. I ALCOHOL NATURAL DISTRIBUTION OF HEAD GROUPS

170 DODECYL /OXYETHYLENE/ 28 ALCOHOL REDUCED DISTRIBUTION OF HEAD GROUPS

147 DECYL /OXYETHYLENE/ 29.4 ALCOHOL BRANCHED CHAIN, NATURAL OE DISTRIBUTION

319 T-OCTYL BENZENE/OXYETHYLENE/30 ALCOHOL REDUCED POLYDISPERSITY OF HEAD GROUPS

323 NONYL BENZENE/OXYETHYLENE/30 ALCOHOL BRANCHED CHAIN REDUCED OE DISTRIBUTION

721 NONYL BENZENE /OXYETHYLENE/30 ALCOHOL BRANCHED CHAIN DIALYSED

169 NONYL BENZENE /OXYETHYLENE/ 30 ALCOHOL BRANCHED CHAIN, NATURAL OE DISTRIBUTION

157 NONYL BENZENE /OXYETHYLENE/ 30.2 ALCOHOL BRANCHED CHAIN, NATURAL OE DISTRIBUTION

116 DODECYL /OXYETHYLENE/30 ALCOHOL REDUCED POLYDISPERSION OF HEAD GROUPS

162 DODECYL BENZENE /OXYETHYLENE/ 30.6 ALCOHOL BRANCHED CHAIN, NATURAL OE DISTRIBUTION

316 TRIDECYL/OXYETHYLENE/30 ALCOHOL BRANCHED CHAIN REDUCED OE DISTRIBUTION

152 TRIDECYL /OXYETHYLENE/ 30.6 ALCOHOL BRANCHED CHAIN, NATURAL OE DISTRIBUTION

117 HEXADECYL/OXYETHYLENE/30 ALCOHOL REDUCED POLYDISPERSION OF HEAD GROUPS

OE $31-40$

474 DODECYL/OXYETHYLENE/38.6 ALCOHOL NATURAL DISTRIBUTION OF HEAD GROUPS

228

P-T-OCTYL BENZENE/OXYETHYLENE/40 ALCOHOL NATURAL DISTRIBUTION OF HEAD GROUPS

OE $41+$

324 NONYL BENZENE/OXYETHYLENE/5O ALCOHOL BRANCHED CHAIN. REDUCED OE DISTRIBUTION

475 DODECYL/OXYETHYLENE/67.3 ALCOHOL NATURAL DISTRIBUTION OF HEAD GROUPS

338 NONYL BENZENE/OXYETHYLENE/100 ALCOHOL BRANCHED CHAIN. NATURAL OE DISTRIBUTION

329 OCTADECYL/OXYETHYLENE/100 ALCOHOL REDUCED POLYDISPERSITY OF HEAD •GROUPS 


\section{Structural Indexes of Compounds with Keys to Compound Numbers - Continued}

PART 3. Grouped by structure of hydrophobic part and arranged by number of carbon atoms in longest hydrophobic tail and by charge.

\section{A. ALKANES}

\section{N-ALKANES}

$\mathrm{C}-3$

699 SODIUM BUTYRATE

43 BUTYRIC ACID

707 PERFLUORO PROPYLAMINE HYDROCHLORIDE

706 PERFLURO PROPYLAMINE

C-4

484 SODTUM PENTANOATE/VALERATE

257 SODIUM DI-N-BUTYL SULFOSUCCINATE

461 BUTYL/OXYETHYLENE/I ALCOHOL--BUTYL GLYCOL ETHER HOMOGENEOUS HEAD GROUP

393 BUTYL/OXYETHYLENE/6 ALCOHOL HOMOGENEOUS HEAD GROUP

$\mathrm{C}-5$

700 HEXANOIC ACID

485 SODIUM HEXANOATE/CAPROATE/

188 POTASSIUM HEXANOATE

258 SODIUM DI-N-AMYL SULFOSUCCINATE

343 SODIUM PENTANE SULFONATE

700 HEXANOIC ACID

C-6

486 SODIUM HEPTANOATE

296 POTASSIUM HEPTANOATE

344 SODIUM HEXANE SULFONATE

339 MAGNESIUM HEXANE SULFONATE

259 SODIUM DI-N-HEXYL SULFOSUCCINATE

704 POTASSIUM 4-HEXYL RESORCINOLATE

708 HEXYLAMINE

709 HEXYLAMINE HYDROCHLORIDE

640 HEXYL TRIMETHYLAMMONIUM HEXANE SULFATE

644 HEXYL TRIMETHYLAMMONIUM HEXANE SULFONATE

641 HEXYL TRIMETHYLAMMONIUM OCTANE SULFATE

708 HEXYLAMINE

390 HEXYLAMMONIUM DODECYL SULFATE

714 HEXYL DIMETHYL AMINE OXIDE

684 HEXYL SULFINYLETHANOL

685 HEXYL SULFINYLPROPANOL

686 HEXYL SULFINYLBUTANOL

687 HEXYL SULFINYLPENTANOL

457 1-4-HEXANEDIOL

103 HEXYL /OXYETHYLENE/ 3 ALCOHOL HOMOGENOUS HEAD GROUP

380 HEXYL/OXYETHYLENE/4 ALCOHOL HOMOGENEOUS HEAD GROUP

381 HEXYL/OXYETHYLENE/5 ALCOHOL HOMOGENEOUS HEAD GROUP

294 HEXYL/OXYETHYLENE/6 ALCOHOL HOMOGENOUS HEAD GROUP

703 4-HEXYL RESORCINOL

C-7

529 OCTANOIC ACID

476 SODIUM OCTANOATE

44 POTASSIUM OCTANOATE

284 HEXANOLAMINE-CH3CH/OH/CH2C/CH3/2NH2-OCTANOATE

303 DI-ISOPROPYLAMONIUM CAPRYLATE

529 OCTANOIC ACID

715 HEPTYL DIMETHYL AMINE OXIDE

586 METHYL /OXYETHYLENE/ 7.6 OCTANOATE REDUCED POLYDISPERSITY OF HEAD GROUPS

$\mathrm{C}-8$

487 SODIUM NONANOATE

350 POTASSIUM NONANOATE

420 POTASSIUM 1-1-2-OCTANE TRICARBOXYLATE

6 SODIUM ALPHA SULFOPELARGONIC ACID

25 SODIUM ETHYL ALPHA SULFOPELARGONATE

26 SODIUM AMYL ALPHA SULFOPELARGONATE SODIUM HEXYL ALPHA SULFOPELARGONATE SODIUM HEPTYL ALPHA SULFOPELARGONATE SODIUM OCTYL ALPHA SULFOPELARGONATE

SODIUM 2 OCTYL ALPHA SULFOPELARGONATE

SODIUM 2 ETHYLHEXYL ALPHA SULFOPELARGONATE

35 SODIUM H/CF2CF2/3CH2 ALPHA SULFOPELARGONATE

611 ALPHAPHOSPHONO PELARGONIC ACID

605 SODIUM AMYL ALPHAPHOSPHONO PELARGONATE

2 SODIUK OCTYL 1 SULFATE

66 SODIUM OCTYL 2 SULFATE

347 OCTYL TRIMETHYLAMMOMIUM OCTYL SULFATE

643 DODECYL TRIMETHYLAMMONIUM OCTANE SULFATE

181 SODIUM OCTYL I-SULFONATE

340 MAGNESIUM OCTANE SULFONATE

287 OCTYL TRIMETHYLAMMONIUM OCTANE SULFONATE

260 SODIUM DI-N-OCTYL SULFOSUCCINATE

392 OCTYLAMMONIUM CHLORIDE

391 OCTYLAMMONIUM DODECYL SULFATE

93 OCTYL TRIMETHYL AMMNIUM BROMTDE

347 OCTYL TRIMETHYLAMMOMIUM OCTYL SULFATE

642 OCTYL TRIMETHYLAMMONIUM DECANE SULFATE

385 OCTYL TRIMETHYLAMONIUM DODECYL SULFATE

287 OCTYL TRIMETHYLAMMONIUM OCTANE SULFONATE

353 OCTYL TRIMETHYLAMMONIUM DECANE SULFONATE

483 DIOCTYL DIMETHYL AMMONIUM CHLORIDE

135 OCTYL C BETAINE HYDROCHLORIDE

132 OCTYL C BETAINE

118 OCTYL N BETAINE

251 OCTYL DIMETHYL AMINE OXIDE

710 OCTYL DIMETHYL PHOSPHINE OXIDE

688 OCTYL SULFINYLETHANOL

689 OCTYL SULFINYLPROPANOL

690 OCTYL SULFINYLBUTANOL

648 ALPHA-D-GLUCOSYL OCTANE

424 OCTYL ALPHA-GLYCERYL ETHER

18 OCTYL BETA D GLUCOSIDE

691 OCTYL KETHYL SULFOXIDE

423

OCTYL/OXYETHYLENE/I ALCOHOL--OCTYL GLYCOL ETHER

HOMOGENEOUS HEAD GROUP

104 OCTYL /OXYETHYLENE/ 3 ALCOHOL HOMOGENOUS HEAD GROUP

105 OCTYL /OXYETHY

106 OCTYL/OXYETHYLENE/ 9 ALCOHOL HOMOGENOUS HEAD GROUP

C-9

530 DECANOIC ACID

299 SODIUM DECANOATE

90 POTASSIUM DECANOATE

668 DIPOTASSIUM OCTYL MALONATE

29 SODIUM NONYL ALPHA SULFOPELARGONATE

606 SODIUM AMYL ALPHAPHOSPHONO CAPRATE

612 ALPHAPHOSPHONO DECANOIC ACID

616 MONOSODIUM ALPHAPHOSPHONO DECANOATE

136 SOLIUM ALPHA DIMETHYL AMINO CAPRATE

295 SODIUM NONYL 1-SULFATE

536 NONYL SULFONIC ACID

94 NONYL TRIMETHYL AMMONIUM BROMIDE

137 ALPHA DIMEMHYLAMINO CAPRIC ACID HYDROCHLORIDE

530 DECANOIC ACID

254 NONYL DIMETHYL AMINE OXIDE

580 METHYL /OXYETHYLENE/ 7.0 DECANOATE REDUCED POLYDISPERSITY OF HEAD GROUPS

581 METHYL /OXYETHYLENE/ 10.3 DECANOATE REDUCED POLYDISPERSITY OF HEAD GROUPS

578 METHYL /OXYETHYLENE/ 11.9 DECANOATE REDUCED POLYDISPERSITY OF HEAD GROUPS

582 METHYL /OXYETHYLENE/ 16.0 DECANOATE NATURAL DISTRIBUTION OF HEAD GROUPS

C-10

297 POTASSIUM UNDECANOATE

425 POTASSIUM 1-1-2-DECANE TRICARBOXYLATE

30 SODIUM DECYL ALPHA SULFOPELARGONATE

33 SODIUM /OXO/ DECYL ALPHA SULFOPELARGONATE

3 SODIUM DECYL 1 SULFATE

15 SODIUM DECYL 2 SULFATE

346 DECYL TRIMETHYLAMMONIUM DECYL SULFATE

642 OCTYL TRIMETHYLAMMONIUM DECANE SULFATE

182 SODIUM DECYL I-SULFONATE

341 MAGNESIUM DECANE SULFONATE

353 OCTYL TRIMETHYLAMMONIUM DECANE SULFONATE 
PART 3. Grouped by structure of hydrophobic part and arranged by number of carbon atoms in longest hydrophobic tail and by charge-Continued

288 DECYL TRIMETHYLAMMONIUM DECANESULFONATE

37 DECYLAMMONIUM CHLORIDE

411 DECYLAMMONIUM ACETATE

203 DECYL TRIMETHYL AMMONIUM CHLORIDE

95 DECYL TRIMETHYL AMMONIUM BROMIDE

306 DECYL TRIMETHYLAMMONIUM SULFATE

346 DECYL TRIMETHYLAMMONIUM DECYL SULFATE

288 DECYL TRIMETHYLAMMONIUM DECANESULFONATE

280 DECYL TRIMETHYL AMMONIUM DODECYL SULFATE

133 DECYL C BETAINE

119 DECYL N BETAINE

252 DECYL DIMETHYL AMINE OXIDE

711 DECYL DIMETHYL PHOSPHINE OXIDE

587 DECYL DIMETHYLAMMONIOPROPANE SULFONATE

692 DECYL SULFINYLETHANOL

19 DECYL BETA D GLUCOSIDE

201 DECYL /OXYETHYLENE/8 OXYMETHYL REDUCED POLYDISPERSION OF HEAD GROUPS

202 DECYL /OXYETHYLENE/II OXYMETHYL REDUCED POLYDISPERSION OF HEAD GROUPS

204 DECYL /OXYETHYLENE/12 OXYMETHYL REDUCED POLYDISPERSION OF HEAD GROUPS

107 DECYL /OXYETHYLENE/ 3 ALCOHOL HOMOGENOUS HEAD GROUP

379 DECYL/OXYETHYLENE/4 ALCOHOL HOMOGENEOUS HEAD GROUP

378 DECYL/OXYETHYLENE/5 ALCOHOL HOMOGENEOUS HEAD GROUP

108 DECYL /OXYETHYLENE/ 6 ALCOHOL HOMOGENOUS HEAD GROUP

109 DECYL /OXYETHYLENE/ 9 ALCOHOL HOMOGENOUS HEAD GROUP

C-11

531 DODECANOIC ACID

273 SODIUM DODECANOATE

91 POTASSIUM DODECANOATE

627 CESIUM DODECANOATE

277 BENZYL TRIMETHYL AMMONIUM DODECANOATE

527 SODIUM UNDECANE-3-CARBOXYLATE

669 DIPOTASSIUM DECYL MALONATE

602 ALPHA SULFO LAURIC ACID

235 SODIUM ALPHA SULFO LAURIC ACID

603 SODIUM PROPYL ALPHA SULFO LAURATE

613 ALPHAPHOSPHONO DODECANOIC ACID

617 MONOSODIUM ALPHAPHOSPHONO DODECANOATE

620 DISODIUM ALPHAPHOSPHONO DODECANOATE

607 SODIUM ISOPROPYL ALPHAPHOSPHONO LAURATE

311 SODIUM UNDECYL 1-SULFATE

72 SODIUM UNDECYL 3 SULFATE

80 SODIUM UNDECYL 6 SULFATE

639 SODIUM UNDECYL THIOSULFATE

537 UNDECYL SULFONIC ACID

418 SODIUM UNDECYL SULFONATE

96 UNDECYL TRIMETHYL AMMONIUM BROMIDE

531 DODECANOIC ACID

120 UNDECYL N BETAINE

713 UNDECYL DIMETHYL AMINE OXIDE

495 SUCROSE MONOLAURATE

276 NONAETHYLENE GLYCOL MONODODECANOATE NATURAL DISTRIBUTION OF HEAD GROUPS

FTHYL /OXYETHYLENE/ 6.0 DODECANOATE REDUCED POLYDISPERSITY OF HEAD GROUPS

584 METHYL /OXYETHYLENE/ 8.4 DODECANOATE REDUCED POLYDISPERSITY OF HEAD GROUPS

585 METHYL /OXYETHYLENE/ 11.2 DODECANOATE REDUCED POLYDISPERSITY OF HEAD GROUPS

579 METHYL /OXYETHYLENE/ 12.5 DODECANOATE REDUCED POLYDISPERSITY OF HEAD GROUPS

440 /OXYETHYLENE/4 SORBITAN MONOLAURATE ISOMERS AND DISTRIBUTED MULTIPLE OE CHAINS

441 /OXYETHYLENE/8 SORBITAN MONOLAURATE ISOMERS AND DISTRIBUTED MULTIPLE OE CHAINS

442 /OXYETHYLENE/1O SORBITAN MONOLAURATE ISOMERS AND DISTRIBUTED MULTIPLE OE CHAINS

443 /OXYETHYLENE/2O SORBITAN MONOLAURATE ISOMERS AND DISTRIBUTED MULTIPLE OE CHAINS

444 /OXYETHYLENE/25 SORBITAN MONOLAURATE ISOMERS AND DISTRIBUTED MULTIPLE OE CHAINS

520 UNDECYL/OXYETHYLENE/8 ALCOHOL NATURAL DISTRIBUTION OF HEAD GROUPS

550 LAURIC ACID DIETHANOLAMINE CONDENSATE

\section{$\mathrm{C}-12$}

ANIONIC

351 POTASSIUM TRIDECANOATE

421 POTASSIUM 1-1-2-DODECANE TRICARBOXYLATE
34 SODIUM DODECYL ALPHA SULFOPELARGONATE

632 POTASSIUM N-DODECYL BETA-ALANINATE

705 DODECYL SULFURIC ACID

1 SODIUM DODECYL I SULFATE

67 SODIUM DODECYL 2 SULFATE

634 POTASSIUM DODECYL SULFATE

111 LITHIUM DODECYL I SULFATE

23 SILVER DODECYL I SULFATE

568 MAGNESIUM DODECYL SULFATE

24 CALCIUM DODECYL 1 SULFATE

569 STRONTIUM DODECYL SULFATE

571 MANGANESE DODECYL SULFATE

572 COBALTOUS DODECYL SULFATE

575 NICKEL DODECYL SULFATE

573 CUPRIC DODECYL SULFATE

570 LEAD DODECYL SULFATE

574 ZINC DODECYL SULFATE

386 AMMONIUM DODECYL SULFATE

720 1-6-DITRIMETHYLAMMONIUM-HEXANE/DODECYL SULFATE/2

410 MORPHOLINIUM DODECYL SULFATE

391 OCTYLAMMONIUM DODECYL SULFATE

385 OCTYL TRIMETHYLAMMONIUM DODECYL SULFATE

280 DECYL TRIMETHYL AMMONIUM DODECYL SULFATE

281 DODECYL TRIMETHYLAMMONIUM DODECYL SULFATE

636 SODIUM DODECYL THIOSULFATE

462 SODIUM MONOLAURIN SULFATE

541 SODIUM DODECYL MONO-OXYETHYLENE SULFATE

542 SODIUM DODECYL DIOXYETHYLENE SULFATE

113 SODIUM DODECYL TRI-OXYETHYLENE SULFATE

543 SODIUM DODECYL TETRA-OXYETHYLENE SULFATE

114 SODIUM DODECYL/OXYETHYLENE/17.5 SULFATE

597 SODIUM DODECYL MONO-OXYPROPYL SULFATE

200 DODECYL SULFONIC ACID

179 SODIUM DODECANE 1-SULFONATE

175 SODIUM DODECANE 2-SULFONATE

40 POTASSIUM DODECYL 1 SULFONATE

635 LITHIUM DODECYL SULFONATE

342 MAGNESIUM DODECANE SULFONATE

\section{CATIONIC}

38 DODECYL AMMONIUM CHLORIDE

628 DODECYLAMMONIUM BROMIDE

482 DODECYL AMMONIUM NITRATE

412 DODECYLAMMONIUM ACETATE

449 DODECYLMETHYL AMMONIUM CHLORIDE

450 DODECYLDIMETHYL AMMONIUM CHLORIDE

41 DODECYL TRIMETHYL AMMONIUM CHLORIDE

307 DODECYL TRIMETHYLAMMONIUM SULFATE

97 DODECYL TRIMETHYL AMMONIUM BROMIDE

126 DODECYL TRIMETHYLAMMONIUM IODIDE

130 DODECYL TRIMETHYL AMMONIUM FLUORIDE

131 DODECYL TRIMETHYLAMMONIUM NITRATE

129 DODECYL TRIMETHYL AMMONIUM BROMATE

127 DODECYL TRIMETHYL AMMONIUM IODATE

128 DODECYL TRIMETHYL AMMONIUM FORMATE

643 DODECYL TRIMETHYLAMMONIUM OCTANE SULFATE

281 DODECYL TRIMETHYLAMMONIUM DODECYL SULFATE

384 HEXYL TRIMETHYLAMMONIUM DODECYL. SULFATE

399 DODECYL DIMETHYL ETHYLAMMONIUM CHLORIDE

345 DIDODECYL DIMETHYLAMMONIUM CHLORIDE

400 DODECYL METHYL DIETHYLAMMONIUM CHLORIDE

401 DODECYL TRIETHYLAMMONIUM CHLORIDE

500 DODECYL TRI/2-HYDROXYETHYL/AMMONIUM CHLORIDE

22 DIMETHYL DODECYL AMINE OXIDE HYDROCHLORIDE

293 DODECYL DIMETHYL AMINE OXIDE HYDROBROMIDE

124 DODECYL N BETAINE HYDROCHLORIDE

633 N-DODECYL BETA-ALANINE HYDROCHLORIDE

NONIONIC

121 DODECYL $N$ BETAINE

592 DODECYL N-DIETHYL N-BETAINE

134 DODECYL C BETAINE

21 DIMETHYL DODECYL AMINE OXIDE

712 DODECYL DIMETHYL PHOSPHINE OXIDE

590 DODECYL DIMETHYLAMMONIOPROPANE CARBOXYLATE

588 DODECYL DIMETHYLAMMONIOPROPANE SULFONATE 
PART 3. Grouped by structure of hydrophobic part and arranged by number of carbon atoms in longest hydrophobic tail and by charge-Continued

591 DODECYL DIMETHYLPHOSPHONIOPROPANE SULFONATE

595 DODECYL DIMETHYL AMMONIOPROPANE SULFATE

594 DODECYL DIPROPYL AMMONIOPROPANE SULFONATE

593 DIMETHYL DODECYLAMMONIOPROPANE HYDROXY SULFONATE

20 DODECYL BETA D GLUCOSIDE

649 ALPHA-D-GLUCOSYL DODECANE

205 DODECYL /OXYETHYLENE/12 OXYMETHYL REDUCED POLYDISPERSION OF HEAD GROUPS

325 DODECYL/OXYETHYLENE/4 ALCOHOL REDUCED POLYDISPERSITY OF HEAD GROUPS

377 DODECYL/OXYETHYLENE/ 5 ALCOHOL HOMOGENEOUS HEAD GROUP 110 DODECYL /OXYETHYLENE/ 6 ALCOHOL HOMOGENOUS HEAD GROUP 468 DODECYL/OXYETHYLENE/6.2 ALCOHOL NATURAL DISTRIBUTION OF HEAD GROUPS 115 DODECYL /OXYETHYLENE/7 ALCOHOL REDUCED POLYDISPERSION
OF HEAD GROUPS

488 DODECYL/OXYETHYLENE/7 ALCOHOL HOMOGENEOUS HEAD GROUP

469 DODECYL/OXYETHYLENE/7.6 ALCOHOL NATURAL DISTRIBUTION OF HEAD GROUPS

163 DODECYL /OXYETHYLENE/ 8 ALCOHOL NATURAL DISTRIBUTION OF HEAD GROUPS

489 DODECYL/OXYETHYLENE/9 ALCOHOL HOMOGENEOUS HEAD GROUP

310 DODECYL/OXYETHYLENE/10 ALCOHOL NATURAL DISTRIBUTION OF HEAD GROUPS

470 DODECYL/OXYETHYLENE/10.6 ALCOHOL NATURAL DISTRIBUTION OF HEAD GROUPS

490 DODECYL/OXYETHYLENE/12 ALCOHOL HOMOGENEOUS HEAD GROUP

164 DODECYL /OXYETHYLENE/ 12 ALCOHOL NATURAL DISTRIBUTION OF HEAD GROUPS

326 DODECYL/OXYETHYLENE/14 ALCOHOL REDUCED POLYDISPERSITY OF HEAD GROUPS

471 DODECYL/OXYETHYLENE/15.8 ALCOHOL NATURAL DISTRIBUTION OF HEAD GROUPS

716 DODECYL /OXYETHYLENE/16 ALCOHOL REDUCED POLYDISPERSITY OF HEAD GROUPS

165 DODECYL /OXYETHYLENE/ 18 ALCOHOL NATURAL DISTRIBUTION OF HEAD GROUPS

472 DODECYL/OXYETHYLENE/20.4 ALCOHOL NATURAL DISTRIBUTION OF HEAD GROUPS

327 DODECYL/OXYETHYLENE/23 ALCOHOL REDUCED POLYDISPERSITY OF HEAD GROUPS

166 DODECYL /OXYETHYLENE/ 23 ALCOHOL NATURAL DISTRIBUTION OF HEAD GROUPS

473 DODECYL/OXYETHYLENE/27. I ALCOHOL NATURAL DISTRIBUTION OF HEAD GROUPS

170 DODECYL /OXYETHYLENE/ 28 ALCOHOL REDUCED DISTRIBUTION OF HEAD GROUPS

116 DODECYL /OXYETHYLENE/30 ALCOHOL REDUCED POLYDISPERSION OF HEAD GROUPS

474 DODECYL/OXYETHYLENE/38.6 ALCOHOL NATURAL DISTRIBUTION OF HEAD GROUPS

475 DODECYL/OXYETHYLENE/67.3 ALCOHOL NATURAL DISTRIBUTION OF HEAD GROUPS

$\mathrm{C}-13$

532 TETRADECANOIC ACID

298 SODIUM TETRADECANOATE

92 POTASSIUM TETRADECANOATE

670 DIPOTASSIUM DODECYL MALONATE

189 ALPHA SULFOMYRISTIC ACID

236 SODIUM ALPHA SULFO MYRISTIC ACID

604 SODIUM METHYL ALPHA SULFO MYRISTATE

618 MONOSODIUM ALPHAPHOSPHONO TETRADECANOATE

608 SODIUM METHYL ALPHAPHOSPHONO MYRISTATE

233 DISODIUM ALPHA SULFO MYRISTATE

614 ALPHAPHOSPHONO TETRADECANOIC ACID

621 DISODIUM ALPHAPHOSPHONO TETRADECANOATE

624 TRISODIUM ALPHAPHOSPHONO TETRADECANOATE

68 SODIUM TRIDECYL 2 SULFATE

83 SODIUM TRIDECYL 7 SULFATE

242 TRIDECANE 1-SULFONIC ACID

229 SODIUM TRIDECANE I-SULFONATE

402 TRIDECYL TRIMETHYLAMMONIUM CHLORIDE

532 TETRADECANOIC ACID

497 SUCROSE MONOMYRISTATE

465 TRIDECYL/OXYETHYLENE/9 ALCOHOL NATURAL DISTRIBUTION OF HEAD GROUPS

466 TRIDECYL/SECONDARY//OXYETHYLENE/9 ALCOHOL NATURAL DISTRIBUTION OF HEAD GROUPS

C-14

426 POTASSIUM 1-1-2-TETRADECANE TRICARBOXYLATE

4 SODIUM TETRADECYL I SULFATE

16 SODIUM TETRADECYL 2 SULFATE
73 SODIUM TETRADECYL 3 SULFATE

17 SODIUM TETRADECYL 4 SULFATE

77 SODIUM TETRADECYL 5 SULFATE

525 SODIUM TETRADECYL 6-SULFATE

84 SODIUM TETRADECYL 7 SULFATE

637 LITHIUM TETRADECYL SULFATE

576 CUPRIC TETRADECYL SULFATE

544 SODIUM TETRADECYL MONO-OXYETHYLENE SULFATE

545 SODIUM TETRADECYL DI-OXYETHYLENE SULFATE

546 SODIUM TETRADECYL TRI-OXYETHYLENE SULFATE

598 SODIUM TETRADECYL MONO-OXYPROPYL SULFATE

599 SODIUM TETRADECYL DI-OXYPROPYL SULFATE

243 TETRADECANE 1-SULFONIC ACID

183 SODIUM TETRADECYL 1-SULFONATE

176 SODIUM TETRADECANE 2-SULFONATE

39 TETRADECYL AMMONIUM CHLORIDE

413 TETRADECYLAMMONIUM ACETATE

42 TETRADECYL TRIMETHYL AMMONIUM CHLORIDE

98 TETRADECYL TRIMETHYL AMMONIUM BROMIDE

308 TETRADECYL TRIMETHYLAMMONIUM SULFATE

291 TETRADECYL TRIPROPYLAMMONIUM BROMIDE

125 TETRADECYL N BETAINE HYDROCHLORIDE

122 TETRADECYL N BETAINE

253 TETRADECYL DIMETHYL AMINE OXIDE

289 TETRADECYL/OXYETHYLENE/6 ALCOHOL HOMOGENOUS HEAD GROUP

C- 15

533 HEXADECANOIC ACID

300 SODIUM HEXADECANOATE

185 POTASSIUM HEXADECANOATE

671 DIPOTASSIUM TETRADECYL MALONATE

190 ALPHA SULFOPALMITIC ACID

237 SODIUM ALPHA SULFO PALMITIC ACID

36 SODIUM METHYL ALPHA SULFOPALMITATE

192 SODIUM ETHYL ALPHA SULFOPALMITATE

193 SODIUM PROPYL ALPHA SULFOPALMITATE

234 DISODIUM ALPHA SULFO PALMITATE

197 DISODIUM 2 SULFOETHYL ALPHA SULFOPALMITATE

615 ALPHAPHOSPHONO HEXADECANOIC ACID

619 MONOSODIUM ALPHAPHOSPHONO HEXADECANOATE

609 SODIUM ISOPROPYL ALPHAPHOSPHONO PALMITATE

622 DISODIUM ALPHAPHOSPHONO HEXADECANOATE

625 TRISODIUM ALPHAPHOSPHONO HEXADECANOATE

69 SODIUM PENTADECYL 2 SULFATE

74 SODIUM PENTADECYL 3 SULFATE

78 SODIUM PENTADECYL 5 SULFATE

85 SODIUM PENTADECYL 8 SULFATE

244 PENTADECANE 1-SULFONIC ACID

230 SODIUM PENTADECANE 1-SULFONATE

533 HEXADECANOIC ACID

498 SUCROSE MONOPALMITATE

C -16

422 POTASSIUM 1-1-2-HEXADECANE TRICARBOXYLATE

5 SODIUM HEXADECYL 1 SULFATE

75 SODIUM HEXADECYL 4 SULFATE

81 SODIUM HEXADECYL 6 SULFATE

86 SODIUM HEXADECYL 8 SULFATE

638 LITHIUM HEXADECYL SULFATE

577 CUPRIC HEXADECYL SULFATE

60 TRIETHANOL AMMONIUM HEXADECYL SULFATE

52 SODIUM HEXADECYL MONO OXYETHYLENE SULFATE

53 SODIUM HEXADECYL DI OXYETHYLENE SULFATE

54 SODIUM HEXADECYL TRI OXYETHYLENE SULFATE

55 SODIUM HEXADECYL TETRA OXYETHYLENE SULFATE

600 SODIUM HEXADECYL MONO-OXYPROPYL SULFATE

245 HEXADECANE 1-SULFONIC ACID

184 SODIUM HEXADECYL 1-SULFONATE

177 SODIUM HEXADECANE 2-SULFONATE

408 POTASSIUM HEXADECANE I-SULFONATE

186 HEXADECYL AMMONIUM CHLORIDE

414 HEXADECYLAMMONIUM ACETATE

265 HEXADECYL TRIMETHYL AMMONIUM CHLORIDE

99 HEXADECYL TRIMETHYLAMMONIUM BROMIDE

309 HEXADECYL TRIMETHYLAMMONIUM SULFATE

478 HEXADECYL DIMETHYLETHYLAMMONIUM BROMIDE

266 HEXADECYL DIMETHYL 2-HYDROXYETHYL AMMONIUM CHLORIDE

269 HEXADECYLDIMETHYL2,3-DIHYDROXYPROPYLAMMONIUM CHLORIDE

267 HEXADECYL DI-/2-HYDROXYETHYL/METHYL AMMONIUM CHLORIDE

268 HEXADECYL TRI-/2-HYDROXYETHYL/ AMMONIUM CHLORIDE

292 HEXADECYL TRIPROPYLAMMONIUM BROMIDE

666 HEXADECYL TRIBUTYLAMMONIUM BROMATE

123 HEXADECYL N BETAINE

589 HEXADECYLDIMETHYLAMMONIOPROPANE SULFONATE

499 SUCROSE DI-PALMITATE 
PART 3. Grouped by structure of hydrophobic part and arranged by number of carbon atoms in longest hydrophobic tail and by charge-Continued

282 HEXADECYL /OXYETHYLENE/ 6 ALCOHOL HOMOGENOUS HEAD GROUP

330 HEXADECYL/OXYETHYLENE/7 ALCOHOL HOMOGENEOUS HEAD GROUP

331 HEXADECYL/OXYETHYLENE/9 ALCOHOL HOMOGENEOUS HEAD GROUP

332 HEXADECYL/OXYETHYLENE/12 ALCOHOL HOMOGENEOUS HEAD GROUP

333 HEXADECYL/OXYETHYLENE/15 ALCOHOL HOMOGENEOUS HEAD GROUP

535 HEXADECYL/OXYETHYLENE/18 ALCOHOL

334 HEXADECYL/OXYETHYLENE/21 ALCOHOL HOMOGENEOUS HEAD GROUP

117 HEXADECYL /OXYETHYLENE/30 ALCOHOL REDUCED POLYDISPERSION OF HEAD GROUPS

C -17

534 OCTADECANOIC ACID

448 SODIUM OCTADECANOATE /STEARATE/

256 POTASSIUM STEARATE

672 DIPOTASSIUM HEXADECYL MALONATE

191 ALPHA SULFOSTEARIC ACID

9 SODIUM ALPHA SULFOSTEARIC ACID

194 SODIUM METHYL ALPHA SULFOSTEARATE

195 SODIUM ETHYL ALPHA SULFOSTEARATE

196 SODIUM PROPYL ALPHA SULFOSTEARATE

199 SODIUM ISOPROPYL ALPHA SULFOSTEARATE

10 DISODIUM ALPHA SULFOSTEARATE

198 DISODIUM 2 SULFOETHYL ALPHA SULFOSTEARATE

553 XYLYL SULFOSTEARIC ACID

610 SODIUM METHYL ALPHAPHOSPHONO STEARATE

623 DISODIUM ALPHAPHOSPHONO OCTADECANOATE

626 TRISODIUM ALPHAPHOSPHONO OCTADECANOATE

70 SODIUM HEPTADECYL 2 SULFATE

87 SODIUM HEPTADECYL 9 SULFATE

246 HEPTADECANE 1-SULFONIC ACID

231 SODIUM HEPTADECANE 1-SULFONATE

534 OCTADECANOIC ACID

496 SUCROSE MONOSTEARATE

C-18

673 DIPOTASSIUM OCTADECYL MALONATE

64 SODIUM OCTADECYL 1 SULFATE

71 SODIUM OCTADECYL 2 SULFATE

76 SODIUM OCTADECYL 4 SULFATE

82 SODIUM OCTADECYL 6 SULFATE

65 TRIETHANOLAMMONIUM OCTADECYL 1 SULFATE

56 SODIUM OCTADECYL MONO OXYETHYLENE SULFATE

57 SODIUM OCTADECYL DI OXYETHYLENE SULFATE

58 SODIUM OCTADECYL TRI OXYETHYLENE SULFATE

59 SODIUM OCTADECYL TETRA OXYETHYLENE SULFATE

601 SODIUM OCTADECYL MONO-OXYPROPYL SULFATE

232 OCTADECANE 1-SULFONIC ACID

349 SODIUM OCTADECANE 1-SULFONATE

178 SODIUM OCTADECANE 2-SULFONATE

419 SODIUM ALPHA-HEPTYL UNDECYL SULFONATE

187 OCTADECYL AMMONIUM CHLORIDE

415 OCTADECYLAMMONIUM ACETATE

270 OCTADECYL TRIMETHYLAMMONIUM CHLORIDE

477 OCTADECYL TRIMETHYLAMMONIUM BROMIDE

654 OCTADECYL TRIMETHYLAMMONIUM NITRATE

658 OCTADECYL TRIMETHYLAMMONIUM BROMATE

659 OCTADECYL TRIMETHYLAMMONIUM FORMATE

667 OCTADECYL TRIMETHYLAMMONIUM OXALATE

662 OCTADECYL TRIETHYLAMMONIUM BROMATE

663 OCTADECYL TRIPROPYLAMMONIUM BROMAT

664 OCTADECYL TRIBUTYLAMMONIUM BROMATE

665 OCTADECYL TRIAMYLAMMONIUM BROMATE

435 OCTADECYL/OXYETHYLENE/10 ALCOHOL NATURAL DISTRIBUTION OF HEAD GROUPS

328 OCTADECYL/OXYETHYLENE/14 ALCOHOL REDUCED POLYDISPERSITY OF HEAD GROUPS

436 OCTADECYL/OXYETHYLENE/20 ALCOHOL NATURAL DISTRIBUTION OF HEAD GROUPS

329 OCTADECYL/OXYETHYLENE/100 ALCOHOL REDUCED POLYDISPERSITY OF HEAD GROUPS

C-19

79 SODIUM NONADECYL 5 SULFATE

88 SODIUM 1 NONYL DECYL SULFATE

$C-20$

683 SODIUM EICOSYLBENZENE SULFONATE

C-29

89 SODIUM 1 TETRADECYL PENTADECYL SULFATE
ISO-ALKANES

261 SODIUM DI-ISOBUTYL SULFOSUCCINATE

394 1-METHYL PROPYL/OXYETHYLENE/6 ALCOHOL HOMOGENEOUS HEAD GROUP

352 SODIUM DI-1-METHYLISOAMYL SULFOSUCCINATE

395 2-ETHYL BUTYL/OXYETHYLENE/6 ALCOHOL HOMOGENEOUS HEAD GROUP

262 SODIUM DI-2-ETHYL-HEXYL SULFOSUCCINATE

286 AMMONIUM DI-2-ETHYL-HEXYL SULFOSUCCINATE

396 2-PROPYL PENTYL/OXYETHYLENE/6 ALCOHOL HOMOGENEOUS HEAD GROUP

515 ISO-OCTYL/OXYETHYLENE/4 ALCOHOL BRANCHED CHAIN, NATURAL OE DISTRIBUTION

516 NONYL/OXYETHYLENE/4 ALCOHOL BRANCHED CHAIN, NATURAL OE DISTRIBUTION

397 2-BUTYL HEXYL/OXYETHYLENE/6 ALCOHOL HOMOGENEOUS HEAD GROUP

398 2-BUTYL HEXYL/OXYETHYLENE/9 ALCOHOL HOMOGENEOUS HEAD GROUP

517 DECYL/OXO-PROCESS/ /OXYETHYLENE/10 ALCOHOL BRANCHED CHAIN, NATURAL OE DISTRIBUTION

519 DECYL/OXO-PROCESS/ /OXYETHYLENE/4 ALCOHOL BRANCHED CHAIN, NATURAL OE DISTRIBUTION

143 DECYL /OXYETHYLENE/ 5.0 ALCOHOL BRANCHED CHAIN, NATURAL OE DISTRIBUTION

144 DECYL /OXYETHYLENE/ 10.0 ALCOHOL BRANCHED CHAIN, NATURAL OE DISTRIBUTION

145 DECYL /OXYETHYLENE/ 14.9 ALCOHOL BRANCHED CHAIN NATURAL OE DISTRIBUTION

146 DECYL /OXYETHYLENE/ 20.0 ALCOHOL BRANCHED CHAIN NATURAL OE DISTRIBUTION

147 DECYL /OXYETHYLENE/ 29.4 ALCOHOL BRANCHED CHAIN, NATURAL OE DISTRIBUTION

518 TRIMETHYLNONYL/OXYETHYLENE/6 ALCOHOL BRANCHED CHAIN NATURAL OE DISTRIBUTION

$\mathrm{C}-13$

312 TRIDECYL/OXYETHYLENE/5 ALCOHOL BRANCHED CHAIN, REDUCED OE DISTRIBUTION

148 TRIDECYL /OXYETHYLENE/ 5.2 ALCOHOL BRANCHED CHAIN, NATURAL OE DISTRIBUTION

313 TRIDECYL/OXYETHYLENE/9.5 ALCOHOL BRANCHED CHAIN REDUCED OE DISTRIBUTION

521 TRIDECYL/OXYETHYLENE/1O ALCOHOL BRANCHED CHAIN, NATURAL OE DISTRIBUTION

149 TRIDECYL /OXYETHYLENE/ 10.1 ALCOHOL BRANCHED CHAIN, NATURAL OE DISTRIBUTION

314 TRIDECYL/OXYETHYLENE/I4 ALCOHOL BRANCHED CHAIN, REDUCED OE DISTRIBUTION

150 TRIDECYL /OXYETHYLENE/ 15.2 ALCOHOL BRANCHED CHAIN NATURAL OE DISTRIBUTION

315 TRIDECYL/OXYETHYLENE/2O ALCOHOL BRANCHED CHAIN, REDUCED OE DISTRIBUTION

151 TRIDECYL /OXYETHYLENE/ 20.0 ALCOHOL BRANCHED CHAIN, NATURAL OE DISTRIBUTION

316 TRIDECYL/OXYETHYLENE/30 ALCOHOL BRANCHED CHAIN, REDUCED OE DISTRIBUTION

152 TRIDECYL /OXYETHYLENE/ 30.6 ALCOHOL BRANCHED CHAIN, NATURAL OE DISTRIBUTION

431 TRIDECYL/OXO-PROCESS/ /OXYETHYLENE/8.5 ALCOHOL BRANCHED CHAIN, NATURAL OE DISTRIBUTION

432 TRIDECYL/OXO-PROCESS/ /OXYETHYLENE/1O ALCOHOL BRANCHED CHAIN, NATURAL OE DISTRIBUTION

433 TRIDECYL/OXO-PROCESS/ /OXYETHYLENE/I5 ALCOHOL BRANCHED CHAIN, NATURAL OE DISTRIBUTION

434 TRIDECYL/OXO-PROCESS/ /OXYETHYLENE/22 ALCOHOL BRANCHED CHAIN, NATURAL OE DISTRIBUTION

526 SODIUM 2-DI-N-HEXYL ETHYL SULFATE

524 HEXADECYL/OXYETHYLENE/I4 ALCOHOL BRANCHED CHAIN, NATURAL OE DISTRIBUTION

3B. ALKYL ARYL

ALKYL AND ARYL

354 HEXYL BENZYL DIMETHYLAMMONIUM CHLORIDE

356 DECYL BENZYL DIMETHYLAMMONIUM CHLORIDE

403 DODECYL DIMETHYLPHENYLAMMONIUM CHLORIDE

279 DODECYL BENZYL DIMETHYLAMMONIUM CHLORIDE

404 DODECYL DIMETHYL 2-PHENYLETHYLAMMONIUM CHLORIDE $\mathrm{C} 6 \mathrm{H} 5 \mathrm{CH} 2 \mathrm{CH} 2 / \mathrm{N} / \mathrm{CH} 3 / 2 / \mathrm{C} 12 \mathrm{H} 25$

407 DODECYL DIMETHYL 3-PHENYLPROPYLAMMONIUM CHLORIDE $\mathrm{C} 6 \mathrm{H} 5 \mathrm{CH} 2 \mathrm{CH} 2 / \mathrm{N} / \mathrm{CH} 3 / 2 / \mathrm{C} 12 \mathrm{H} 25$

405 DODECYL METHYLETHYLBENZYLAMMONIUM CHLORIDE

357 TETRADECYL BENZYL DIMETHYLAMMONIUM CHLORIDE 
PART 3. Grouped by structure of hydrophobic part and arranged by number of carbon atoms in longest hydrophobic tail and by charge-Continued

275 HEXADECYL DIMETHYLBENZYLAMMONIUM CHLORIDE

358 OCTADECYL BENZYL DIMETHYLAMMONIUM CHLORIDE

1-N-ALKYL ARYL

645 PARA/BETA-D-GLUCOSYL/ETHYLBENZENE

646 PARA/BETA-D-GLUCOSYL/PROPYL BENZENE

445 SODIUM PARA-BIS-/N-BUTYL/ BENZENE SULFONATE

681 SODIUM DIBUTYL BENZENE SULFONATE

647 PARA/BETA-D-GLUCOSYL/BUTYLBENZENE

650 PARA/BETA-D-XYLOSYL/BUTYL BENZENE

501 SODIUM HEXYL BENZENE SULFONATE

446 SODIUM PARA-BIS-/N-HEXYL/ BENZENE SULFONATE

502 SODIUM HEPTYL BENZENE SULFONATE

49 SODIUM P OCTYL BENZENE SULFONATE

503 SODIUM OCTYL BENZENE SULFONATE

447 SODIUM PARA-BIS-/N-OCTYL/ BENZENE SULFONATE

675 OCTYL BENZENE/OXYETHYLENE/10 ALCOHOL NATURAL DISTRIBUTION OF HEAD GROUPS

493 SODIUM P-NONYL BENZENE SULFONATE

504 SODIUM NONYL BENZENE SULFONATE

463 NONYL BENZENE/OXYETHYLENE/11 ALCOHOL NATURAL DISTRIBUTION OF HEAD GROUPS

464 NONYL BENZENE/OXYETHYLENE/15 ALCOHOL NATURAL DISTRIBUTION OF HEAD GROUPS

50 SODIUM P DECYL BENZENE SULFONATE

505 SODIUM DECYL BENZENE SULFONATE

522 DECYL BENZENE ORTHO/OXYETHYLENE/11.2 ALCOHOLNATURAL DISTRIBUTION OF HEAD GROUPS

523 DECYL BENZENE PARA/OXYETHYLENE/11.0 ALCOHOL NATURAL DISTRIBUTION OF HEAD GROUPS

51 SODIUM P DODECYL BENZENE SULFONATE

506 SODIUM DODECYL BENZENE SULFONATE

507 SODIUM TETRADECYL BENZENE SULFONATE

596 TETRAHYDROANACARDOL AMMONIUM MONOSULFONATE

508 SODIUM HEXADECYL BENZENE SULFONATE

552 TOLYL SULFOSTEARIC ACID

509 SODIUM OCTADECYL BENZENE SULFONATE

OTHER N-ALKYL ARYL

172 SODIUM 2-N-OCTYL BENZENE SULFONATE

173 SODIUM 2-N-DECYL BENZENE SULFONATE

45 SODIUM $P$ I METHYL DECYL BENZENE SULFONATE

171 SODIUM 2-N-DODECYL BENZENE SULFONATE

301 SODIUM 3-N-DODECYL BENZENE SULFONATE

302 SODIUM 4-N-DODECYL BENZENE SULFONATE

514 SODIUM 6-N-DODECYL BENZENE SULFONATE

46 SODIUM $P$ I METHYL DODECYL BENZENE SULFONATE

174 SODIUM 2-N-TETRADECYL BENZENE SULFONATE

47 SODIUM $P$ I METHYL TETRADECYL BENZENE SULFONATE

48 SODIUM $P$ I METHYL HEXADECYL BENZENE SULFONATE

551 PHENYL SULFOSTEARIC ACID

ISO-ALKYL ARYL - KNOWN BRANCHING

674 DIISOBUTYL BENZENE/OXYETHYLENE/6 ALCOHOL (DB) BRANCHED CHAIN, NATURAL OE DISTRIBUTION

510 SODIUM 2-ETHYL-HEXYL BENZENE SULFONATE

676 SODIUM $1,1,3,3$-TETRAMETHYL BUTYL BENZENE SULFONATE

677 SODIUM DI-/1,1,3,3-TETRAMETHYL BUTYL/BENZENESULFONATE

P-TERTIARY OCTYL BENZENE

207 P-T-OCTYL BENZENE /OXYETHYLENE/1 ALCOHOL HOMOGENOUS HEAD GROUP

217 P-T-OCTYL BENZENE /OXYETHYLENE/I ALCOHOL NATURAL DISTRIBUTION OF HEAD GROUPS

208 P-T-OCTYL BENZENE /OXYETHYLENE/2 ALCOHOL HOMOGENOUS HEAD GROUP

218 P-T-OCTYL BENZENE /OXYETHYLENE/2 ALCOHOL NATURAL DISTRIBUTION OF HEAD GROUPS

209 P-T-OCTYL BENZENE/OXYETHYLENE/3 ALCOHOL HOMOGENOUS HEAD GROUP

219 P-T-OCTYL BENZENE /OXYETHYLENE/3 ALCOHOL NATURAL DISTRIBUTION OF HEAD GROUPS

210 P-T-OCTYL BENZENE /OXYETHYLENE/4 ALCOHOL HOMOGENOUS HEAD GROUP

220 P-T-OCTYL BENZENE/OXYETHYLENE/4 ALCOHOL NATURAL DISTRIBUTION OF HEAD GROUPS

211 P-T-OCTYL BENZENE /OXYETHYLENE/5 ALCOHOL HOMOGENOUS HEAD GROUP

221 P-T-OCTYL BENZENE/OXYETHYLENE/5 ALCOHOL NATURAL DISTRIBUTION OF HEAD GROUPS

212 P-T-OCTYL BENZENE /OXYETHYLENE/6 ALCOHOL HOMOGENOUS HEAD GROUP

222 P-T-OCTYL BENZENE /OXYETHYLENE/6 ALCOHOL NATURAL DISTRIBUTION OF HEAD GROUPS
213 P-T-OCTYL BENZENE/OXYETHYLENE/7 ALCOHOL HOMOGENOUS HEAD GROUP

223 P-T-OCTYL BENZENE /OXYETHYLENE/7 ALCOHOL NATURAL DISTRIBUTION OF HEAD GROUPS

214 P-T-OCTYL BENZENE /OXYETHYLENE/8 ALCOHOL HOMOGENOUS HEAD GROUP

224 P-T-OCTYL BENZENE/OXYETHYLENE/8 ALCOHOL NATURAL DISTRIBUTION OF HEAD GROUPS

215 P-T-OCTYL BENZENE /OXYETHYLENE/9 ALCOHOL HOMOGENOUS HEAD GROUP

225 P-T-OCTYL BENZENE /OXYETHYLENE/9 ALCOHOL NATURAL DISTRIBUTION OF HEAD GROUPS

206 TRITON X-100(P-T-OCTYL BENZENE/OXYETHYLENE/9NATURAL DISTRIBUTION OF HEAD GROUPS

216 P-T-OCTYL BENZENE /OXYETHYLENE/1O ALCOHOL HOMOGENOUS HEAD GROUP

317 T-OCTYL BENZENE/OXYETHYLENE/10 ALCOHOL REDUCED POLYDISPERSITY OF HEAD GROUPS

226 P-T-OCTYL BENZENE /OXYETHYLENE/10 ALCOHOL NATURAL DISTRIBUTION OF HEAD GROUPS

227 P-T-OCTYL BENZENE /OXYETHYLENE/16 ALCOHOL NATURAL DISTRIBUTION OF HEAD GROUPS

318 T-OCTYL BENZENE/OXYETHYLENE/20 ALCOHOL REDUCED POLYDISPERSITY OF HEAD GROUPS

319 T-OCTYL BENZENE/OXYETHYLENE/30 ALCOHOL REDUCED POLYDISPERSITY OF HEAD GROUPS

228 P-T-OCTYL BENZENE /OXYETHYLENE/40 ALCOHOL NATURAL DISTRIBUTION OF HEAD GROUPS

272 TRI-ISOPROPYL BENZENE SULFONIC ACID

271 SODIUM TRI-ISO-PROPYL BENZENE SULFONATE

678 SODIUM 1,1-DIMETHYLNONYL BENZENE SULFONATE

511 SODIUM 2-PROPYL-HEPTYL BENZENE SULFONATE

679 SODIUM 3-BUTYL HEPTYL BENZENE SULFONATE

680 SODIUM 2-PROPYL-4-METHYL-OCTYL BENZENE SULFONATE

492 SODIUM TETRAPROPYLENE/1-3-5-7-TETRAMETHYLOCTYL/BENZENE SULFONATE

512 SODIUM 2-BUTYL-OCTYL BENZENE SULFONATE

513 SODIUM 2-AMYL-NONYL BENZENE SULFONATE

ISO-ALKYL ARYL - BRANCHING UNSPECIFIED

335 OCTYL BENZENE/OXYETHYLENE/8.5 ALCOHOL BRANCHED CHAIN NATURAL OE DISTRIBUTION

138 SODIUM NONYL BENZENE SULFONATE BRANCHED HYDROCARBON

ISO-NONYL BENZENE

153 NONYL BENZENE /OXYETHYLENE/ 5 O ALCOHOL BRANCHED CHAIN, NATURAL OE DISTRIBUTION

154 NONYL BENZENE /OXYETHYLENE/ 9.8 ALCOHOL BRANCHED CHAIN, NATURAL OE DISTRIBUTION

336 NONYL BENZENE/OXYETHYLENE/9.5 ALCOHOL BRANCHED CHAIN NATURAL OE DISTRIBUTION

320 NONYL BENZENE/OXYETHYLENE/10 ALCOHOL BRANCHED CHAIN, REDUCED OE DISTRIBUTION

167 NONYL BENZENE /OXYETHYLENE/ 10 ALCOHOL BRANCHED CHAIN, NATURAL OE DISTRIBUTION

337 NONYL BENZENE/OXYETHYLENE/10.5 ALCOHOL BRANCHED CHAIN NATURAL OE DISTRIBUTION

168 NONYL BENZENE /OXYETHYLENE/ 15 ALCOHOL BRANCHED CHAIN NATURAL OE DISTRIBUTION

321 NONYL BENZENE/OXYETHYLENE/15 ALCOHOL BRANCHED CHAIN, REDUCED OE DISTRIBUTION

155 NONYL BENZENE /OXYETHYLENE/ 15.4 ALCOHOL BRANCHED CHAIN, NATURAL OE DISTRIBUTION

322 NONYL BENZENE/OXYETHYLENE/2O ALCOHOL BRANCHED CHAIN REDUCED OE DISTRIBUTION

156 NONYL BENZENE /OXYETHYLENE/20.0 ALCOHOL BRANCHED CHAIN, NATURAL OE DISTRIBUTION

323 NONYL BENZENE/OXYETHYLENE/3O ALCOHOL BRANCHED CHAIN. REDUCED OE DISTRIBUTION

721 NONYL BENZENE /OXYETHYLENE/30 ALCOHOL BRANCHED CHAIN DIALYSED

169 NONYL BENZENE /OXYETHYLENE/ 30 ALCOHOL BRANCHED CHAIN NATURAL OE DISTRIBUTION

157 NONYL BENZENE /OXYETHYLENE/ 30.2 ALCOHOL BRANCHED CHAIN. NATURAL OE DISTRIBUTION

324 NONYL BENZENE/OXYETHYLENE/50 ALCOHOL BRANCHED CHAIN, REDUCED OE DISTRIBUTION

338 NONYL BENZENE/OXYETHYLENE/100 ALCOHOL BRANCHED CHAIN NATURAL OE DISTRIBUTION

140 SODIUM DECYL BENZENE SULFONATE BRANCHED HYDROCARBON CHAIN

139 SODIUM DODECYL BENZENE SULFONATE BRANCHED HYDROCARBON CHAIN 
PART 3. Grouped by structure of hydrophobic part and arranged by number of carbon atoms in longest hydrophobic tail and by charge-Continued

158 DODECYL BENZENE /OXYETHYLENE/ 5.0 ALCOHOL BRANCHED CHAIN, NATURAL OE DISTRIBUTION

159 DODECYL BENZENE /OXYETHYLENE/ 10.2 ALCOHOL BRANCHED CHAIN, NATURAL OE DISTRIBUTION

160 DODECYL BENZENE /OXYETHYLENE/ 15.1 ALCOHOL BRANCHED CHAIN, NATURAL OE DISTRIBUTION

161 DODECYL BENZENE /OXYETHYLENE/ 20.1 ALCOHOL BRANCHED CHAIN, NATURAL OE DISTRIBUTION

162 DODECYL BENZENE /OXYETHYLENE/ 30.6 ALCOHOL BRANCHED CHAIN, NATURAL OE DISTRIBUTION

141 SODIUM TRIDECYL BENZENE SULFONATE BRANCHED HYDROCARBON CHAIN

142 SODIUM FENTADECYL BENZENE SULFONATE BRANCHED HYDROCARBON CHAIN

ALKYL-ARYL - PRESENCE OF BRANCHING UNCERTAIN

682 SODIUM DIBUTYL NAPHTHALENE SULFONATE /NEKAL/

11 SODIUM ALPHA SULFO PHENYL STEARIC ACID

8 DISODIUM ALPHA SULFOPHENYLSTEARATE

\section{C. UNSATURATED AND SUBSTITUTED}

\section{UNSATURATED HYDROCARBON}

562 SODIUM DODECENYL SULFATE

263 SODIUM OLEATE /CIS-9-OCTADECENOATE/

305 POTASSIUM OLEATE/ CIS-9-OCTADECENOATE/

285 HEXANOLAMINE-CH3CH/OH/CH2C/CH3/2NH2-OLEATE

61 SODIUM OLEYL/CIS 9 OCTADECENOYL/ SULFATE

547 SODIUM OLEYL MONO-OXYETHYLENE SULFATE

548 SODIUM OLEYL DI-OXYETHYLENE SULFATE

549 SODIUM OLEYL TRI-OXYETHYLENE SULFATE

437 OLEYL/CIS-9-OCTADECENOYL/ /OXYETHYLENE/12 ALCOHOL NATURAL DISTRIBUTION OF HEAD GROUPS

438 OLEYL/CIS-9-OCTADECENOYL/ /OXYETHYLENE/16 ALCOHOL NATURAL DISTRIBUTION OF HEAD GROUPS

439 OLEYL/CIS-9-OCTADECENOYL/ /OXYETHYLENE/2O ALCOHOL NATURAL DISTRIBUTION OF HEAD GROUP

264 SODIUM ELAIDATE /TRANS-9-OCTADECENOATE/

629 POTASSIUM ELAIDATE/TRANS-9-OCTADECENOATE/

283 HEXANOLAMINE-CH3CH/OH/CH2C/CH3/2NH2-ELAIDATE

62 SODIUM ELAIDYL/TRANS 9 OCTADECENOYL/SULFATE

\section{OXYGEN}

451 PARA DI-ISOBUTYLPHENOXYETHOXYETHYL DIMETHYLBENZYL AMMONIUM CHLORIDE/HYAMINE 1622/

238 SODIUM DODECANE 1-HYDROXY 2-SULFONATE

247 DODECANE 1-HYDROXY 2-SULFONIC ACID

370 DODECYL 3-4-METHYLENEDIOXYBENZYL DIMETHYLAMMONIUM CHLORIDE

371 DODECYL 3-4-DIMETHOXYBENZYL DIMETHYLAMMONIUM CHLORIDE

248 TETRADECANE 1-HYDROXY 2-SULFONIC ACID

239 SODIUM TETRADECANE 1-HYDROXY 2-SULFONATE

249 HEXADECANE 1-HYDROXY 2-SULFONIC ACID

240 SODIUM HEXADECANE 1-HYDROXY 2-SULFONATE

255 POTASSIUM 9,10 DIHYDROXY STEARATE

630 POTASSIUM RICINOLEATE/12 HYDROXY OLEATE/

631 POTASSIUM RICINELAIDATE/12 HYDROXY ELAIDATE/

14 DISODIUM 9,10 DIHYDROXY ALPHA SULFOSTEARATE

250 OCTADECANE 1-HYDROXY 2-SULFONIC ACID

241 SODIUM OCTADECANE 1-HYDROXY 2-SULFONATE

\section{NITROBENZYL}

359 OCTYL 4-NITROBENZYL DIMETHYLAMMONIUM CHLORIDE

360 DECYL 4-NITROBENZYL DIMETHYLAMMONIUM CHLORIDE

361 DODECYL 4-NITROBENZYL DIMETHYL AMMONIUM CHLORIDE
368 DODECYL 2-HYDROXY-5-NITROBENZYL DIMETHYLAMMONIUM CHLORIDE

362 TETRADECYL 4-NITROBENZYL DIMETHYLAMMONIUM CHLORIDE

363 HEXADECYL 4-NITROBENZYL DIMETHYLAMMONIUM CHLORIDE

364 OCTADECYL 4-NITROBENZYL DIMETHYLAMMONIUM CHLORIDE

\section{HALOGEN}

428 PERFLUORO ACETIC ACID

429 PERFLUORO PROPIONIC ACID

430 PERFLUORO BUTYRIC ACID

706 PERFLUORO PROPYLAMINE

416 PERFLUORO HEXANOIC ACID

701 POTASSIUM PERFLUROHEXANOATE

417 PERFLUORO OCTANOIC ACID

702 POTASSIUM PERFLUORODECANOATE

452 3-4-DICHLOROPERFLUORO BUTYRIC/KEL-F/ACID

453 3-5-6-TRICHLOROPERFLUORO HEXANOIC/KEL-F/ACID

454 3-5-7-8-TETRACHLOROPERFLUORO OCTANOIC/KEL-F/ACID

374 DODECAFLUOROHEPTANOIC ACID H/CF2/6COOH

355 AMMONIUM DODECAFLUOROHEPTANOATE H/CF2/6COONH4

375 HEXADECAFLUORONONANOIC ACID H/CF2/8COOH

372 AMMONIUM HEXADECAFLUORONONANOATE H/CF2/8 COO NH4

373 AMMONIUM EICOSAFLUOROUNDECANOATE H/CF2/10 COO NH4

348 NN-DIMETHYL 1-1-DIHYDROPENTADECAFLUORO OCTYL AMINE NOXIDE /C7F $15 \mathrm{CH} 2 \mathrm{~N} / \mathrm{CH} 3 / 20 /$

365 DODECYL 2-CHLOROBENZYL DIMETHYLAMMONIUM CHLORIDE

367 DODECYL 4-CHLOROBENZYL DIMETHYLAMMONIUM CHLORIDE

366 DODECYL 2-4-DICHLOROBENZYL DIMETHYLAMMONIUM CHLORIDE

369 DODECYL 3-4-DICHLOROBENZYL DIMETHYLAMMONIUM CHLORIDE

406 DODECYL DIMETHYL META-TRIFLUOROMETHYLBENZYL AMMONIUM CHLORIDE $\mathrm{CF} 3 \mathrm{C} 6 \mathrm{H} 4 \mathrm{CH} 2 / \mathrm{N} / \mathrm{CH} 3 / 2 / \mathrm{Cl} 2 \mathrm{H} 25$

12 SODIUM 9,10 DICHLORO ALPHA SULFOSTEARIC ACID

13 DISODIUM 9,10 DICHLORO ALPHA SULFOSTEARATE

63 SODIUM 910 DICHLORO OCTADECYL SULFATE

\section{D. HETEROCYCLIC}

100 OCTYL PYRIDINIUM BROMIDE

458 DECYL PYRIDINIUM IODIDE

101 UNDECYL PYRIDINIUM BROMIDE

278 DODECYL PYRIDINIUM CHLORIDE

290 DODECYL PYRIDINIUM BROMIDE

376 DODECYL PYRIDINIUM IODIDE

102 TETRADECYL PYRIDINIUM BROMIDE

479 TETRADECYL PYRIDINIUM IODIDE

274 HEXADECYL PYRIDINIUM CHLORIDE

427 HEXADECYL PYRIDINIUM BROMIDE

480 HEXADECYL PYRIDINIUM IODIDE

660 HEXADECYL PYRIDINIUM IODATE

655 OCTADECYL PYRIDINIUM CHLORIDE

657 OCTADECYL PYRIDINIUM BROMIDE

481 OCTADECYL PYRIDINIUM IODIDE

656 OCTADECYL PYRIDINIUM NITRATE

661 OCTADECYL PYRIDINIUM IODATE

693 N-CETYL 2-METHYL PYRIDINIUM CHLORIDE

696 N-CETYL 2-METHYL PYRIDINIUM IODIDE

694 N-CETYL 3-METHYL PYRIDINIUM CHLORIDE

697 N-CETYL-3-METHYL PYRIDINIUM IODIDE

695 N-CETYL 4-METHYL PYRIDINIUM CHLORIDE

698 N-CETYL-4-METHYL PYRIDINIUM IODIDE

304 CATOL 607 (N-/LAURYL-COLAMINO-FORMYL-METHYL/ PYRIDINIUM CHLORIDE)

560 EMULSOL 607L (N-(2-KETO-2-(2,-LAUROYLOXYETHYL AMINO $)$ ETHYL) PYRIDINIUM CHLORIDE

717 DODECYLQUINOLINIUM BROMIDE

491 DODECYL TROPYLIUM BISULFATE

528 DODECYL TROPYLIUM PERCHLORATE

460 DODECYL TROPYLIUM MONOPHOSPHATE 


\title{
Structural Indexes of Compounds with Keys to Compound Numbers - Continued
}

\author{
PART 4. Polyoxyethylenes grouped by distribution of heads arranged by number of carbon atoms in longest \\ hydrophobic tail and by heads.
}

4A. POLYOXYETHYLENES - HOMOGENEOUS HEAD GROUPS

OE 1

461 BUTYL/OXYETHYLENE/1 ALCOHOL--BUTYL GLYCOL ETHER HOMOGENEOUS HEAD GROUP

423 OCTYL/OXYETHYLENE/1 ALCOHOL--OCTYL GLYCOL ETHER HOMOGENEOUS HEAD GROUP

207 P-T-OCTYL BENZENE /OXYETHYLENE/1 ALCOHOL HOMOGENOUS HEAD GROUP

208 P-T-OCTYL BENZENE /OXYETHYLENE/2 ALCOHOL HOMOGENOUS HEAD GROUP

103 HEXYL /OXYETHYLENE/ 3 ALCOHOL HOMOGENOUS HEAD GROUP

104 OCTYL /OXYETHYLENE/ 3 ALCOHOL HOMOGENOUS HEAD GROUP

209 P-T-OCTYL BENZENE /OXYETHYLENE/3 ALCOHOL HOMOGENOUS HEAD GROUP

107 DECYL /OXYETHYLENE/ 3 ALCOHOL HOMOGENOUS HEAD GROUP

OE 4

380 HEXYL/OXYETHYLENE/4 ALCOHOL HOMOGENEOUS HEAD GROUP

210 P-T-OCTYL BENZENE /OXYETHYLENE/4 ALCOHOL HOMOGENOUS HEAD GROUP

379 DECYL/OXYETHYLENE/4 ALCOHOL HOMOGENEOUS HEAD GROUP

381 HEXYL/OXYETHYLENE/5 ALCOHOL HOMOGENEOUS HEAD GROUP

211 P-T-OCTYL BENZENE /OXYETHYLENE/5 ALCOHOL HOMOGENOUS HEAD GROUP

378 DECYL/OXYETHYLENE/5 ALCOHOL HOMOGENEOUS HEAD GROUP

377 DODECYL/OXYETHYLENE/ 5 ALCOHOL HOMOGENEOUS HEAD GROUP

OE 6

393 BUTYL/OXYETHYLENE/6 ALCOHOL HOMOGENEOUS HEAD GROUP

394 1-METHYL PROPYL/OXYETHYLENE/6 ALCOHOL HOMOGENEOUS HEAD GROUP

294 HEXYL/OXYETHYLENE/ 6 ALCOHOL HOMOGENOUS HEAD GROUP

395 2-ETHYL BUTYL/OXYETHYLENE/6 ALCOHOL HOMOGENEOUS HEAD GROUP

105 OCTYL /OXYETHYLENE/ 6 ALCOHOL HOMOGENOUS HEAD GROUP

396 2-PROPYL PENTYL/OXYETHYLENE/6 ALCOHOL HOMOGENEOUS HEAD GROUP

212 P-T-OCTYL BENZENE /OXYETHYLENE/6 ALCOHOL HOMOGENOUS HEAD GROUP

108 DECYL /OXYETHYLENE/ 6 ALCOHOL HOMOGENOUS HEAD GROUP

397 2-BUTYL HEXYL/OXYETHYLENE/6 ALCOHOL HOMOGENEOUS HEAD GROUP

110 DODECYL /OXYETHYLENE/ 6 ALCOHOL HOMOGENOUS HEAD GROUP

289 TETRADECYL/OXYETHYLENE/ 6 ALCOHOL HOMOGENOUS HEAD GROUP

282 HEXADECYL /OXYETHYLENE/ 6 ALCOHOL HOMOGENOUS HEAD GROUP

OE 7

213 P-T-OCTYL BENZENE /OXYETHYLENE/7 ALCOHOL HOMOGENOUS HEAD GROUP

488 DODECYL/OXYETHYLENE/7 ALCOHOL HOMOGENEOUS HEAD GROU?

330 HEXADECYL/OXYETHYLENE/7 ALCOHOL HOMOGENEOUS HEAD GROUP

214 P-T-OCTYL BENZENE /OXYETHYLENE/8 ALCOHOL HOMOGENOUS HEAD GROUP

106 OCTYL /OXYETHYLENE/ 9 ALCOHOL HOMOGENOUS HEAD GROUP

215 P-T-OCTYL BENZENE /OXYETHYLENE/9 ALCOHOL HOMOGENOUS HEAD GROUP

109 DECYL /OXYETHYLENE/ 9 ALCOHOL HOMOGENOUS HEAD GROUP

398 2-BUTYL HEXYL/OXYETHYLENE/9 ALCOHOL HOMOGENEOUS HEAD GROUP

331 HEXADECYL/OXYETHYLENE/9 ALCOHOL HOMOGENEOUS HEAD GROUP

489 DODECYL/OXYETHYLENE/9 ALCOHOL HOMOGENEOUS HEAD GROUP

OE 10

216 P-T-OCTYL BENZENE /OXYETHYLENE/1O ALCOHOL HOMOGENOUS HEAD GROUP

490 DODECYL/OXYETHYLENE/12 ALCOHOL HOMOGENEOUS HEAD GROUP

332 HEXADECYL/OXYETHYLENE/12 ALCOHOL HOMOGENEOUS HEAD GROUP

333 HEXADECYL/OXYETHYLENE/15 ALCOHOL HOMOGENEOUS HEAD GROUP

334 HEXADECYL/OXYETHYLENE/21 ALCOHOL HOMOGENEOUS HEAD GROUP
4B. POLYOXYETHYLENES - REDUCED POLYDISPERSITY OF HEAD GROUPS

OE 4

325 DODECYL/OXYETHYLENE/4 ALCOHOL REDUCED POLYDISPERSITY OF HEAD GROUPS

312 TRIDECYL/OXYETHYLENE/5 ALCOHOL BRANCHED CHAIN, REDUCED OE DISTRIBUTION

583 METHYL /OXYETHYLENE/ 6.0 DODECANOATE REDUCED POLYDISPERSITY OF HEAD GROUPS

586 METHYL /OXYETHYLENE/ 7.6 OCTANOATE REDUCED POLYDISPERSITY OF HEAD GROUPS

580 METHYL /OXYETHYLENE/ 7.0 DECANOATE REDUCED POLYDISPERSITY OF HEAD GROUPS

115 DODECYL /OXYETHYLENE/7 ALCOHOL REDUCED POLYDISPERSION OF HEAD GROUPS

201 DECYL /OXYETHYLENE/8 OXYMETHYL REDUCED POLYDISPERSION OF HEAD GROUPS

584 METHYL /OXYETHYLENE/ 8.4 DODECANOATE REDUCED POLYDISPERSITY OF HEAD GROUPS

313 TRIDECYL/OXYETHYLENE/9.5 ALCOHOL BRANCHED CHAIN, REDUCED OE DISTRIBUTION

OE 10

317 T-OCTYL BENZENE/OXYETHYLENE/10 ALCOHOL REDUCED POLYDISPERSITY OF HEAD GROUPS

320 NONYL BENZENE/OXYETHYLENE/10 ALCOHOL BRANCHED CHAIN REDUCED OE DISTRIBUTION

581 METHYL /OXYETHYLENE/ 10.3 DECANOATE REDUCED POLYDISPERSITY OF HEAD GROUPS

202 DECYL /OXYETHYLENE/1I OXYMETHYL REDUCED POLYDISPERSION OF HEAD GROUPS

585 METHYL /OXYETHYLENE/ 11.2 DODECANOATE REDUCED POLYDISPERSITY OF HEAD GROUPS

578 METHYL /OXYETHYLENE/ 11.9 DECANOATE REDUCED POLYDISPERSITY OF HEAD GROUPS

204 DECYL /OXYETHYLENE/12 OXYMETHYL REDUCED POLYDISPERSION OF HEAD GROUPS

205 DODECYL /OXYETHYLENE/12 OXYMETHYL REDUCED POLYDISPERSION OF HEAD GROUPS

579 METHYL /OXYETHYLENE/ 12.5 DODECANOATE REDUCED POLYDISPERSITY OF HEAD GROUPS

OE 14

326 DODECYL/OXYETHYLENE/14 ALCOHOL REDUCED POLYDISPERSITY OF HEAD GROUPS

328 OCTADECYL/OXYETHYLENE/14 ALCOHOL REDUCED POLYDISPERSITY OF HEAD GROUPS

314 TRIDECYL/OXYETHYLENE/14 ALCOHOL BRANCHED CHAIN, REDUCED OE DISTRIBUTION

321 NONYL BENZENE/OXYETHYLENE/I5 ALCOHOL BRANCHED CHAIN, REDUCED OE DISTRIBUTION

716 DODECYL /OXYETHYLENE/16 ALCOHOL REDUCED POLYDISPERSITY OF HEAD GROUPS

535 HEXADECYL/OXYETHYLENE/18 ALCOHOL

OE 20

318 T-OCTYL BENZENE/OXYETHYLENE/2O ALCOHOL REDUCED POLYDISPERSITY OF HEAD GROUPS

322 NONYL BENZENE/OXYETHYLENE/2O ALCOHOL BRANCHED CHAIN, REDUCED OE DISTRIBUTION

327 DODECYL/OXYETHYLENE/23 ALCOHOL REDUCED POLYDISPERSITY OF HEAD GROUPS

315 TRIDECYL/OXYETHYLENE/20 ALCOHOL BRANCHED CHAIN REDUCED OE DISTRIBUTION

319 T-OCTYL BENZENE/OXYETHYLENE/30 ALCOHOL REDUCED POLYDISPERSITY OF HEAD GROUPS

323 NONYL BENZENE/OXYETHYLENE/3O ALCOHOL BRANCHED CHAIN REDUCED OE DISTRIBUTION

721 NONYL BENZENE /OXYETHYLENE/30 ALCOHOL BRANCHED CHAIN DIALYSED

116 DODECYL /OXYETHYLENE/3O ALCOHOL REDUCED POLYDISPERSION OF HEAD GROUPS 
PART 4. Polyoxyethylenes grouped by distribution of heads arranged by number of carbon atoms in longest hydrophobic tail and by heads - Continued

316 TRIDECYL/OXYETHYLENE/30 ALCOHOL BRANCHED CHAIN, REDUCED OE DISTRIBUTION

117 HEXADECYL /OXYETHYLENE/30 ALCOHOL REDUCED POLYDISPERSION OF HEAD GROUPS

324 NONYL BENZENE/OXYETHYLENE/5O ALCOHOL BRANCHED CHAIN, REDUCED OE DISTRIBUTION

329 OCTADECYL/OXYETHYLENE/100 ALCOHOL REDUCED POLYDISPERSITY OF HEAD GROUPS

\section{C. POLYOXYETHYLENES - NATURAL DISTRIBUTION OF} HEAD GROUPS

OE 1

217 P-T-OCTYL BENZENE /OXYETHYLENE/1 ALCOHOL NATURAL DISTRIBUTION OF HEAD GROUPS

218 P-T-OCTYL BENZENE /OXYETHYLENE/2 ALCOHOL NATURAL DISTRIBUTION OF HEAD GROUPS

219 P-T-OCTYL BENZENE /OXYETHYLENE/3 ALCOHOL NATURAL DISTRIBUTION OF HEAD GROUPS

220 P-T-OCTYL BENZENE /OXYETHYLENE/4 ALCOHOL NATURAL DISTRIBUTION OF HEAD GROUPS

515 ISO-OCTYL/OXYETHYLENE/4 ALCOHOL BRANCHED CHAIN NATURAL OE DISTRIBUTION

516 NONYL/OXYETHYLENE/4 ALCOHOL BRANCHED CHAIN, NATURAL OE DISTRIBUTION

519 DECYL/OXO-PROCESS/ /OXYETHYLENE/4 ALCOHOL BRANCHED CHAIN, NATURAL OE DISTRIBUTION

440 /OXYETHYLENE/4 SORBITAN MONOLAURATE ISOMERS AND DISTRIBUTED MULTIPLE OE CHAINS

$O E 5$

221 P-T-OCTYL BENZENE /OXYETHYLENE/5 ALCOHOL NATURAL DISTRIBUTION OF HEAD GROUPS

153 NONYL BENZENE /OXYETHYLENE/ 5.0 ALCOHOL BRANCHED CHAIN, NATURAL OE DISTRIBUTION

143 DECYL /OXYETHYLENE/ 5.0 ALCOHOL BRANCHED CHAIN, NATURAL OE DISTRIBUTION

158 DODECYL BENZENE /OXYETHYLENE/ 5.0 ALCOHOL BRANCHED CHAIN, NATURAL OE DISTRIBUTION

148 TRIDECYL /OXYETHYLENE/ 5.2 ALCOHOL BRANCHED CHAIN NATURAL OE DISTRIBUTION

674 DIISOBUTYL BENZENE/OXYETHYLENE/6 ALCOHOL (DB) BRANCHED CHAIN, NATURAL OE DISTRIBUTION

222 P-T-OCTYL BENZENE /OXYETHYLENE/6 ALCOHOL NATURAL DISTRIBUTION OF HEAD GROUPS

468 DODECYL/OXYETHYLENE/6.2 ALCOHOL NATURAL DISTRIBUTION OF HEAD GROUPS

518 TRIMETHYLNONYL/OXYETHYLENE/ 6 ALCOHOL BRANCHED CHAIN, NATURAL OE DISTRIBUTION

223 P-T-OCTYL BENZENE /OXYETHYLENE/7 ALCOHOL NATURAL DISTRIBUTION OF HEAD GROUPS

469 DODECYL/OXYETHYLENE/7.6 ALCOHOL NATURAL DISTRIBUTION OF HEAD GROUPS

OE 8

335 OCTYL BENZENE/OXYETHYLENE/8.5 ALCOHOL BRANCHED CHAIN NATURAL OE DISTRIBUTION

224 P-T-OCTYL BENZENE /OXYETHYLENE/8 ALCOHOL NATURAL DISTRIBUTION OF HEAD GROUPS

520 -UNDECYL/OXYETHYLENE/8 ALCOHOL NATURAL DISTRIBUTION OF HEAD GROUPS

441 /OXYETHYLENE/8 SORBITAN MONOLAURATE ISOMERS AND DISTRIBUTED MULTIPLE OE CHAINS

163 DODECYL /OXYETHYLENE/ 8 ALCOHOL NATURAL DISTRIBUTION OF HEAD GROUPS

431 TRIDECYL/OXO-PROCESS/ /OXYETHYLENE/8.5 ALCOHOL BRANCHED CHAIN, NATURAL OE DISTRIBUTION

206 TRITON X-100(P-T-OCTYL BENZENE/OXYETHYLENE/9NATURAL DISTRIBUTION OF HEAD GROUPS

225 P-T-OCTYL BENZENE /OXYETHYLENE/9 ALCOHOL NATURAL DISTRIBUTION OF HEAD GROUPS

336 NONYL BENZENE/OXYETHYLENE/9.5 ALCOHOL BRANCHED CHAIN NATURAL OE DISTRIBUTION

154 NONYL BENZENE /OXYETHYLENE/ 9.8 ALCOHOL BRANCHED CHAIN, NATURAL OE DISTRIBUTION

276 NONAETHYLENE GLYCOL MONODODECANOATE NATURAL DISTRIBUTION OF HEAD GROUPS

465 TRIDECYL/OXYETHYLENE/9 ALCOHOL NATURAL DISTRIBUTION OF HEAD GROUPS

466 TRIDECYL/SECONDARY/ /OXYETHYLENE/9 ALCOHOL NATURAL DISTRIBUTION OF HEAD GROUPS
OE 10

675 OCTYL BENZENE /OXYETHYLENE/10 ALCOHOL NATURAL DISTRIBUTION OF HEAD GROUPS

226 P-T-OCTYL BENZENE /OXYETHYLENE/10 ALCOHOL NATURAL DISTRIBUTION OF HEAD GROUPS

167 NONYL BENZENE /OXYETHYLENE/ 10 ALCOHOL BRANCHED CHAIN. NATURAL OE DISTRIBUTION

337 NONYL BENZENE/OXYETHYLENE/10.5 ALCOHOL BRANCHED CHAIN NATURAL OE DISTRIBUTION

144 DECYL /OXYETHYLENE/ 10.0 ALCOHOL BRANCHED CHAIN, NATURAL OE DISTRIBUTION

517 DECYL/OXO-PROCESS/ /OXYETHYLENE/10 ALCOHOL BRANCHED CHAIN, NATURAL OE DISTRIBUTION

442 /OXYETHYLENE/1O SORBITAN MONOLAURATE ISOMERS AND DISTRIBUTED MULTIPLE OE CHAINS

310 DODECYL/OXYETHYLENE/10 ALCOHOL NATURAL DISTRIBUTION OF HEAD GROUPS

159 DODECYL BENZENE /OXYETHYLENE/ 10.2 ALCOHOL BRANCHED CHAIN, NATURAL OE DISTRIBUTION

470 DODECYL/OXYETHYLENE/10.6 ALCOHOL NATURAL DISTRIBUTION OF HEAD GROUPS

521 TRIDECYL/OXYETHYLENE/10 ALCOHOL BRANCHED CHAIN NATURAL OE DISTRIBUTION

432 TRIDECYL/OXO-PROCESS//OXYETHYLENE/10 ALCOHOL BRANCHED CHAIN, NATURAL OE DISTRIBUTION

149 TRIDECYL /OXYETHYLENE/ 10.1 ALCOHOL BRANCHED CHAIN NATURAL OE DISTRIBUTION

435 OCTADECYL/OXYETHYLENE/10 ALCOHOL NATURAL DISTRIBUTION OF HEAD GROUPS

OE 11

463 NONYL BENZENE/OXYETHYLENE/11 ALCOHOL NATURAL DISTRIBUTION OF HEAD GROUPS

523 DECYL BENZENE PARA/OXYETHYLENE/11.0 ALCOHOL NATURAL DISTRIBUTION OF HEAD GROUPS

522 DECYL BENZENE ORTHO/OXYETHYLENE/11.2 ALCOHOLNATURAL DISTRIBUTION OF HEAD GROUPS

164 DODECYL /OXYETHYLENE/ 12 ALCOHOL NATURAL DISTRIBUTION OF HEAD GROUPS

467 TRIDECYL/SECONDARY//OXYETHYLENE/12 ALCOHOL NATURAL DISTRIBUTION OF HEAD GROUPS

437 OLEYL/CIS-9-OCTADECENOYL/ /OXYETHYLENE/12 ALCOHOL NATURAL DISTRIBUTION OF HEAD GROUPS

OE 14

145 DECYL /OXYETHYLENE/ 14.9 ALCOHOL BRANCHED CHAIN NATURAL OE DISTRIBUTION

524 HEXADECYL/OXYETHYLENE/14 ALCOHOL BRANCHED CHAIN NATURAL OE DISTRIBUTION

464 NONYL BENZENE/OXYETHYLENE/15 ALCOHOL NATURAL DISTRIBUTION OF HEAD GROUPS

168 NONYL BENZENE /OXYETHYLENE/ I5 ALCOHOL BRANCHED CHAIN NATURAL OE DISTRIBUTION

155 NONYL BENZENE /OXYETHYLENE/ 15.4 ALCOHOL BRANCHED CHAIN, NATURAL OE DISTRIBUTION

160 DODECYL BENZENE /OXYETHYLENE/ 15.1 ALCOHOL BRANCHED CHAIN, NATURAL OE DISTRIBUTION

150 TRIDECYL /OXYETHYLENE/ 15.2 ALCOHOL BRANCHED CHAIN NATURAL OE DISTRIBUTION

433 TRIDECYL/OXO-PROCESS/ /OXYETHYLENE/15 ALCOHOL BRANCHED CHAIN, NATURAL OE DISTRIBUTION

OE 16

227 P-T-OCTYL BENZENE /OXYETHYLENE/16 ALCOHOL NATURAL DISTRIBUTION OF HEAD GROUPS

582 METHYL /OXYETHYLENE/ 16.0 DECANOATE NATURAL DISTRIBUTION OF HEAD GROUPS

471 DODECYL/OXYETHYLENE/15.8 ALCOHOL NATURAL DISTRIBUTION OF HEAD GROUPS

438 OLEYL/CIS-9-OCTADECENOYL/ /OXYETHYLENE/16 ALCOHOL NATURAL DISTRIBUTION OF HEAD GROUPS

165 DODECYL /OXYETHYLENE/ 18 ALCOHOL NATURAL DISTRIBUTION OF HEAD GROUPS

OE 20

151 TRIDECYL /OXYETHYLENE/20.0 ALCOHOL BRANCHED CHAIN NATURAL OE DISTRIBUTION

436 OCTADECYL/OXYETHYLENE/20 ALCOHOL NATURAL DISTRIBUTION OF HEAD GROUPS

439 OLEYL/CIS-9-OCTADECENOYL/ /OXYETHYLENE/2O ALCOHOL NATURAL DISTRIBUTION OF HEAD GROUP

156 NONYL BENZENE /OXYETHYLENE/ 20.0 ALCOHOL BRANCHED CHAIN, NATURAL OE DISTRIBUTION 
PART 4. Polyoxyethylenes grouped by distribution of heads arranged by number of carbon atoms in longest hydrophobic tail and by heads - Continued

146 DECYL /OXYETHYLENE/ 20.0 ALCOHOL BRANCHED CHAIN, NATURAL OE DISTRIBUTION

443 /OXYETHYLENE/2O SORBITAN MONOLAURATE ISOMERS AND DISTRIBUTED MULTIPLE OE CHAINS

472 DODECYL/OXYETHYLENE/20.4 ALCOHOL NATURAL DISTRIBUTION OF HEAD GROUPS

161 DODECYL BENZENE /OXYETHYLENE/ 20.1 ALCOHOL BRANCHED CHAIN, NATURAL OE DISTRIBUTION

434 TRIDECYL/OXO-PROCESS/ /OXYETHYLENE/22 ALCOHOL BRANCHED CHAIN, NATURAL OE DISTRIBUTION

166 DODECYL /OXYETHYLENE/ 23 ALCOHOL NATURAL DISTRIBUTION OF HEAD GROUPS

\section{$\mathrm{OE}-25$}

444 /OXYETHYLENE/25 SORBITAN MONOLAURATE ISOMERS AND DISTRIBUTED MULTIPLE OE CHAINS

473 DODECYL/OXYETHYLENE/27.1 ALCOHOL NATURAL DISTRIBUTION OF HEAD GROUPS
170 DODECYL /OXYETHYLENE/ 28 ALCOHOL REDUCED DISTRIBUTION OF HEAD GROUPS

147 DECYL /OXYETHYLENE/ 29.4 ALCOHOL BRANCHED CHAIN NATURAL OE DISTRIBUTION

169 NONYL BENZENE /OXYETHYLENE/ 30 ALCOHOL BRANCHED CHAIN, NATURAL OE DISTRIBUTION

157 NONYL BENZENE /OXYETHYLENE/ 30.2 ALCOHOL BRANCHED CHAIN, NATURAL OE DISTRIBUTION

162 DODECYL BENZENE /OXYETHYLENE/ 30.6 ALCOHOL BRANCHED CHAIN, NATURAL OE DISTRIBUTION

152 TRIDECYL /OXYETHYLENE/ 30.6 ALCOHOL BRANCHED CHAIN NATURAL OE DISTRIBUTION

474 DODECYL/OXYETHYLENE/38.6 ALCOHOL NATURAL DISTRIBUTION OF HEAD GROUPS

228 P-T-OCTYL BENZENE /OXYETHYLENE/40 ALCOHOL NATURAL DISTRIBUTION OF HEAD GROUPS

475 DODECYL/OXYETHYLENE/67.3 ALCOHOL NATURAL DISTRIBUTION OF HEAD GROUPS

338 NONYL BENZENE/OXYETHYLENE/100 ALCOHOL BRANCHED CHAIN, NATURAL OE DISTRIBUTION 


\section{Structural Indexes of Compounds with Keys to Compound Numbers - Continued}

PART 5. Ionics grouped by counterion and arranged by number of carbon atoms in longest hydrophobic tail and by heads.

\section{A. ANIONICS BY COUNTERION}

\section{HYDROGEN}

428 PERFLUORO ACETIC ACID

429 PERFLUORO PROPIONIC ACID

43 BUTYRIC ACID

452 3-4-DICHLOROPERFLUORO BUTYRIC/KEL-F/ACID

430 PERFLUORO BUTYRIC ACID

700 HEXANOIC ACID

453 3-5-6-TRICHLOROPERFLUORO HEXANOIC/KEL-F/ACID

416 PERFLUORO HEXANOIC ACID

374 DODECAFLUOROHEPTANOIC ACID H/CF2/6COOH

529 OCTANOIC ACID

417 PERFLUORO OCTANOIC ACID

375 HEXADECAFLUORONONANOIC ACID H/CF2/8COOH

611 ALPHAPHOSPHONO PELARGONIC ACID

530 DECANOIC ACID

455 PERFLUORO DECANOIC ACID

612 ALPHAPHCSPHONO DECANOIC ACID

536 NONYL SULFONIC ACID

272 TRI-ISOPROPYL BENZENE SULFONIC ACID

531 DODECANOIC ACID

602 ALPHA SULFO LAURIC ACID

613 ALPHAPHOSPHONO DODECANOIC ACID

537 UNDECYL SULFONIC ACID

\section{C-12}

705 DODECYL SULFURIC ACID

200 DODECYL SULFONIC ACID

247 DODECANE 1-HYDROXY 2-SULFONIC ACID

532 TETRADECANOIC ACID

614 ALPHAPHOSPHONO TETRADECANOIC ACID

189 ALPHA SULFOMYRISTIC ACID

242 TRIDECANE I-SULFONIC ACID

243 TETRADECANE 1-SULFONIC ACID

248 TETRADECANE 1-HYDROXY 2-SULFONIC ACID

533 HEXADECANOIC ACID

190 ALPHA SULFOPALMITIC ACID

615 ALPHAPHOSPHONO HEXADECANOIC ACID

244 PENTADECANE 1-SULFONIC ACID

245 HEXADECANE 1-SULFONIC ACID

249 HEXADECANE 1-HYDROXY 2-SULFONIC ACID

534 OCTADECANOIC ACID

191 ALPHA SULFOSTEARIC ACID

551 PHENYL SULFOSTEARIC ACID

552 TOLYL SULFOSTEARIC ACID

553 XYLYL SULFOSTEARIC ACID

246 HEPTADECANE 1-SULFONIC ACID

232 OCTADECANE 1-SULFONIC ACID

250 OCTADECANE 1-HYDROXY 2-SULFONIC ACID

SODIUM

699 SODIUM BUTYRATE

681 SODIUM DIBUTYL BENZENE SULFONATE

682 SODIUM DIBUTYL NAPHTHALENE SULFONATE /NEKAL/

484 SODIUM PENTANOATE/VALERATE/

257 SODIUM DI-N-BUTYL SULFOSUCCINATE

261 SODIUM DI-ISOBUTYL SULFOSUCCINATE

445 SODIUM PARA-BIS-/N-BUTYL/ BENZENE SULFONATE

556 ARESKAP 100 /MONOBUTYL PHENYLPHENOL SODIUM MONOSULFONATE/

557 ARESKET 300 /MONOBUTYL BIPHENYL SODIUM MONOSULFONATE/

558 ARESKLENE 400 /DIBUTYL PHENYLPHENOL DISODIUMDISULFONATE

485 SODIUM HEXANOATE/CAPROATE/

258 SODIUM DI-N-AMYL SULFOSUCCINATE

343 SODIUM PENTANE SULFONATE

C-6

486 SODIUM HEPTANOATE

259 SODIUM DI-N-HEXYL SULFOSUCCINATE

352 SODIUM DI-1-METHYLISOAMYL SULFOSUCCINATE

344 SODIUM HEXANE SULFONATE

501 SODIUM HEXYL BENZENE SULFONATE
446 SODIUM PARA-BIS-/N-HEXYL/ BENZENE SULFONATE

476 SODIUM OCTANOATE

502 SODIUM HEPTYL BENZENE SULFONATE

$\mathrm{C}-8$

487 SODIUM NONANOATE

260 SODIUM DI-N-OCTYL SULFOSUCCINATE

262 SODIUM DI-2-ETHYL-HEXYL SULFOSUCCINATE

6 SODIUM ALPHA SULFOPELARGONIC ACID

25 SODIUM ETHYL ALPHA SULFOPELARGONATE

26 SODIUM AMYL ALPHA SULFOPELARGONATE

27 SODIUM HEXYL ALPHA SULFOPELARGONATE

28 SODIUM HEPTYL ALPHA SULFOPELARGONATE

7 SODIUM OCTYL ALPHA SULFOPELARGONATE

31 SODIUM 2 OCTYL ALPHA SULFOPELARGONATE

32 SODIUM 2 ETHYLHEXYL ALPHA SULFOPELARGONATE

35 SODIUM H/CF2CF2/3CH2 ALPHA SULFOPELARGONATE

605 SODIUM AMYL ALPHAPHOSPHONO PELARGONATE

181 SODIUM OCTYL 1-SULFONATE

2 SODIUM OCTYL I SULFATE

66 SODIUM OCTYL 2 SULFATE

49 SODIUM P OCTYL BENZENE SULFONATE

503 SODIUM OCTYL BENZENE SULFONATE

172 SODIUM 2-N-OCTYL BENZENE SULFONATE

510 SODIUM 2-ETHYL-HEXYL BENZENE SULFONATE

676 SODIUM $1,1,3,3$-TETRAMETHYL BUTYL BENZENE SULFONATE

677 SODIUM DI- $/ 1,1,3,3$-TETRAMETHYL BUTYL/BENZENESULFONATE

447 SODIUM PARA-BIS-/N-OCTYL/ BENZENE SULFONATE

C-9

299 SODIUM DECANOATE

29 SODIUM NONYL ALPHA SULFOPELARGONATE

616 MONOSODIUM ALPHAPHOSPHONO DECANOATE

606 SODIUM AMYL ALPHAPHOSPHONO CAPRATE

136 SODIUM ALPHA DIMETHYL AMINO CAPRATE

295 SODIUM NONYL 1-SULFATE

493 SODIUM P-NONYL BENZENE SULFONATE

504 SODIUM NONYL BENZENE SULFONATE

271 SODIUM TRI-ISO-PROPYL BENZENE SULFONATE

138 SODIUM NONYL BENZENE SULFONATE BRANCHED HYDROCARBON CHAIN

C-10

30 SODIUM DECYL ALPHA SULFOPELARGONATE

33 SODIUM /OXO/ DECYL ALPHA SULFOPELARGONATE

3 SODIUM DECYL 1 SULFATE

15 SODIUM DECYL 2 SULFATE

182 SODIUM DECYL I-SULFONATE

50 SODIUM P DECYL BENZENE SULFONATE

505 SODIUM DECYL BENZENE SULFONATE

173 SODIUM 2-N-DECYL BENZENE SULFONATE

511 SODIUM 2-PROPYL-HEPTYL BENZENE SULFONATE

140 SODIUM DECYL BENZENE SULFONATE BRANCHED HYDROCARBON CHAIN

561 SANTOMERSE D /SODIUM DECYLBENZENE SULFONATE/

C-11

273 SODIUM DODECANOATE

527 SODIUM UNDECANE-3-CARBOXYLATE

235 SODIUM ALPHA SULFO LAURIC ACID

603 SODIUM PROPYL ALPHA SULFO LAURATE

607 SODIUM ISOPROPYL ALPHAPHOSPHONO LAURATE

620 DISODIUM ALPHAPHOSPHONO DODECANOATE

617 MONOSODIUM ALPHAPHOSPHONO DODECANOATE

311 SODIUM UNDECYL 1-SULFATE

72 SODIUM UNDECYL 3 SULFATE

80 SODIUM UNDECYL 6 SULFATE

639 SODIUM UNDECYL THIOSULFATE

462 SODIUM MONOLAURIN SULFATE

418 SODIUM UNDECYL SULFONATE

45 SODIUM P I METHYL DECYL BENZENE SULFONATE

678 SODIUM 1,1-DIMETHYLNONYL BENZENE SULFONATE

679 SODIUM 3-BUTYL HEPTYL BENZENE SULFONATE 
PART 5. Ionics grouped by counterion and arranged by number of carbon atoms in longest hydrophobic tail and by heads - Continued

C-12

34 SODIUM DODECYL ALPHA SULFOPELARGONATE

1 SODIUM DODECYL I SULFATE

67 SODIUM DODECYL 2 SULFATE

562 SODIUM DODECENYL SULFATE

636 SODIUM DODECYL THIOSULFATE

541 SODIUM DODECYL MONO-OXYETHYLENE SULFATE

542 SODIUM DODECYL DIOXYETHYLENE SULFATE

113 SODIUM DODECYL TRI-OXYETHYLENE SULFATE

543 SODIUM DODECYL TETRA-OXYETHYLENE SULFATE

114 SODIUM DODECYL /OXYETHYLENE/17.5 SULFATE

597 SODIUM DODECYL MONO-OXYPROPYL SULFATE

179 SODIUM DODECANE 1-SULFONATE

175 SODIUM DODECANE 2-SULFONATE

238 SODIUM DODECANE 1-HYDROXY 2-SULFONATE

51 SODIUM $P$ DODECYL BENZENE SULFONATE

506 SODIUM DODECYL BENZENE SULFONATE

171 SODIUM 2-N-DODECYL BENZENE SULFONATE

301 SODIUM 3-N-DODECYL BENZENE SULFONATE

302 SODIUM 4-N-DODECYL BENZENE SULFONATE

514 SODIUM 6-N-DODECYL BENZENE SULFONATE

512 SODIUM 2-BUTYL-OCTYL BENZENE SULFONATE

492 SODIUM TETRAPROPYLENE/1-3-5-7-TETRAMETHYLOCTYL/BENZENE SULFONATE

680 SODIUM 2-PROPYL-4-METHYL-OCTYL BENZENE SULFONATE

139 SODIUM DODECYL BENZENE SULFONATE BRANCHED HYDROCARBON CHAIN

554 SANTOMERSE 3 /SODIUM DODECYL BENZENE SULFONATE/

$\mathrm{C}-13$

298 SODIUM TETRADECANOATE

236 SODIUM ALPHA SULFO MYRISTIC ACID

604 SODIUM METHYL ALPHA SULFO MYRISTATE

233 DISODIUM ALPHA SULFO MYRISTATE

618 MONOSODIUM ALPHAPHOSPHONO TETRADECANOATE

608 SODIUM METHYL ALPHAPHOSPHONO MYRISTATE

621 DISODIUM ALPHAPHOSPHONO TETRADECANOATE

624 TRISODIUM ALPHAPHOSPHONO TETRADECANOATE

68 SODIUM TRIDECYL 2 SULFATE

83 SODIUM TRIDECYL 7 SULFATE

229 SODIUM TRIDECANE 1-SULFONATE

46 SODIUM $P$ I METHYL DODECYL BENZENE SULFONATE

141 SODIUM TRIDECYL BENZENE SULFONATE BRANCHED HYDROCARBON CHAIN

C-14

4 SODIUM TETRADECYL 1 SULFATE

16 SODTUM TETRADECYL 2 SULFATE

73 SODIUM TETRADECYL 3 SULFATE

17 SODIUM TETRADECYL 4 SULFATE

77 SODIUM TETRADECYL 5 SULFATE

525 SODIUM TETRADECYL 6-SULFATE

84 SODIUM TETRADECYL 7 SULFATE

526 SODIUM 2-DI-N-HEXYL ETHYL SULFATE

544 SODIUM TETRADECYL MONO-OXYETHYLENE SULFATE

545 SODIUM TETRADECYL DI-OXYETHYLENE SULFATE

546 SODIUM TETRADECYL TRI-OXYETHYLENE SULFATE

598 SODIUM TETRADECYL MONO-OXYPROPYL SULFATE

599 SODIUM TETRADECYL DI-OXYPROPYL SULFATE

183 SODIUM TETRADECYL 1-SULFONATE

176 SODIUM TETRADECANE 2-SULFONATE

239 SODIUM TETRADECANE 1-HYDROXY 2-SULFONATE

507 SODIUM TETRADECYL BENZENE SULFONATE

174 SODIUM 2-N-TETRADECYL BENZENE SULFONATE

513 SODIUM 2-AMYL-NONYL BENZENE SULFONATE

C-15

300 SODIUM HEXADECANOATE

237 SODIUM ALPHA SULFO PALMITIC ACID

36 SODIUM METHYL ALPHA SULFOPALMITATE

192 SODIUM ETHYL ALPHA SULFOPALMITATE

193 SODIUKS PROPYL ALPHA SULFOPALMITATE

234 DISODIUM ALPHA SULFO PALMITATE

197 DISODIUM 2 SULFOETHYL ALPHA SULFOPALMITATE

619 MONOSODIUM ALPHAPHOSPHONO HEXADECANOATE

609 SODIUM ISOPROPYL ALPHAPHOSPHONO PALMITATE

622 DISODIUM ALPHAPHOSPHONO HEXADECANOATE

625 TRISODIUM ALPHAPHOSPHONO HEXADECANOATE

69 SODIUM PENTADECYL 2 SULFATE

74 SODIUM PENTADECYL 3 SULFATE

78 SODIUM PENTADECYL 5 SULFATE

85 SODIUM PENTADECYL 8 SULFATE

230 SODIUM PENTADECANE 1-SULFONATE

47

SODIUM $P$ I METHYL TETRADECYL BENZENE SULFONATE
142 SODIUM PENTADECYL BENZENE SULFONATE BRANCHED HYDROCARBON CHAIN

$\mathrm{C}-16$

5 SODIUM HEXADECYL 1 SULFATE

75 SODIUM HEXADECYL 4 SULFATE

81 SODIUM HEXADECYL 6 SULFATE

86 SODIUM HEXADECYL 8 SULFATE

52 SODIUM HEXADECYL MONO OXYETHYLENE SULFATE

53 SODIUM HEXADECYL DI OXYETHYLENE SULFATE

54 SODIUM HEXADECYL TRI OXYETHYLENE SULFATE

55 SODIUM HEXADECYL TETRA OXYETHYLENE SULFATE

600 SODIUM HEXADECYL MONO-OXYPROPYL SULFATE

184 SODIUM HEXADECYL 1-SULFONATE

177 SODIUM HEXADECANE 2-SULFONATE

240 SODIUM HEXADECANE 1-HYDROXY 2-SULFONATE

508 SODIUM HEXADECYL BENZENE SULFONATE

$\mathrm{C}-17$

448 SODIUM OCTADECANOATE/STEARATE/

263 SODIUM OLEATE /CIS-9-OCTADECENOATE/

264 SODIUM ELAIDATE /TRANS-9-OCTADECENOATE/

9 SODIUM ALPHA SULFOSTEARIC ACID

194 SODIUM METHYL ALPHA SULFOSTEARATE

195 SODIUM ETHYL ALPHA SULFOSTEARATE

196 SODIUM PROPYL ALPHA SULFOSTEARATE

199 SODIUM ISOPROPYL ALPHA SULFOSTEARATE

10 DISODIUM ALPHA SULFOSTEARATE

198 DISODIUM 2 SULFOETHYL ALPHA SULFOSTEARATE

11 SODIUM ALPHA SULFO PHENYL STEARIC ACID

8 DISODIUM ALPHA SULFOPHENYLSTEARATE

12 SODIUM 9,10 DICHLORO ALPHA SULFOSTEARIC ACID

13 DISODIUM 9,10 DICHLORO ALPHA SULFOSTEARATE

14 DISODIUM 9,10 DIHYDROXY ALPHA SULFOSTEARATE

610 SODIUM METHYL ALPHAPHOSPHONO STEARATE

623 DISODIUM ALPHAPHOSPHONO OCTADECANOATE

626 TRISODIUM ALPHAPHOSPHONO OCTADECANOATE

70 SODIUM HEPTADECYL 2 SULFATE

87 SODIUM HEPTADECYL 9 SULFATE

231 SODIUM HEPTADECANE 1-SULFONATE

48 SODTUM P I METHYL HEXADECYL BENZENE SULFONATE

$\mathrm{C}-18$

64 SODIUM OCTADECYL 1 SULFATE

71 SODIUM OCTADECYL 2 SULFATE

76 SODIUM OCTADECYL 4 SULFATE

82 SODIUM OCTADECYL 6 SULFATE

61 SODIUM OLEYL/CIS 9 OCTADECENOYL/ SULFATE

62 SODIUM ELAIDYL/TRANS 9 OCTADECENOYL/SULFATE

63 SODIUM 910 DICHLORO OCTADECYL SULFATE

56 SODIUM OCTADECYL MONO OXYETHYLENE SULFATE

547 SODIUM OLEYL MONO-OXYETHYLENE SULFATE

57 SODIUM OCTADECYL DI OXYETHYLENE SULFATE

548 SODIUM OLEYL DI-OXYETHYLENE SULFATE

58 SODIUM OCTADECYL TRI OXYETHYLENE SULFATE

549 SODIUM OLEYL TRI-OXYETHYLENE SULFATE

59 SODIUM OCTADECYL TETRA OXYETHYLENE SULFATE

601 SODIUM OCTADECYL MONO-OXYPROPYL SULFATE

349 SODIUM OCTADECANE 1-SULFONATE

178 SODIUM OCTADECANE 2-SULFONATE

419 SODIUM ALPHA-HEPTYL UNDECYL SULFONATE

241 SODIUM OCTADECANE 1-HYDROXY 2-SULFONATE

509 SODIUM OCTADECYL BENZENE SULFONATE

79 SODIUM NONADECYL 5 SULFATE

88 SODIUM 1 NONYL DECYL SULFATE

683 SODIUM EICOSYLBENZENE SULFONATE

89 SODIUM 1 TETRADECYL PENTADECYL SULFATE

POTASSIUM

188 POTASSIUM HEXANOATE

701 POTASSIUM PERFLUROHEXANOATE

296 POTASSIUM HEPTANOATE

704 POTASSIUM 4-HEXYL RESORCINOLATE

44 POTASSIUM OCTANOATE

456 POTASSIUM PERFLUORO OCTANOATE

350 POTASSIUM NONANOATE

420 POTASSIUM 1-1-2-OCTANE TRICARBOXYLATE

90 POTASSIUM DECANOATE

702 POTASSIUM PERFLUORODECANOATE

668 DIPOTASSIUM OCTYL MALONATE

297 POTASSIUM UNDECANOATE

425 POTASSIUM 1-1-2-DECANE TRICARBOXYLATE

91 POTASSIUM DODECANOATE

669 DIPOTASSIUM DECYL MALONATE 
PART 5. Ionics grouped by counterion and arranged by number of carbon atoms in longest hydrophobic tail and by heads-Continued

$\mathrm{C}-12$

351 POTASSIUM TRIDECANOATE

421 POTASSIUM 1-1-2-DODECANE TRICARBOXYLATE

632 POTASSIUM N-DODECYL BETA-ALANINATE

634 POTASSIUM DODECYL SULFATE

40 POTASSIUM DODECYL 1 SULFONATE

92 POTASSIUM TETRADECANOATE

670 DIPOTASSIUM DODECYL MALONATE

426 POTASSIUM 1-1-2-TETRADECANE TRICARBOXYLATE

185 POTASSIUM HEXADECANOATE

671 DIPOTASSIUM TETRADECYL MALONATE

422 POTASSIUM 1-1-2-HEXADECANE TRICARBOXYLATE

408 POTASSIUM HEXADECANE 1-SULFONATE

256 POTASSIUM STEARATE

305 POTASSIUM OLEATE/ CIS-9-OCTADECENOATE/

629 POTASSIUM ELAIDATE/TRANS-9-OCTADECENOATE/

255 POTASSIUM 9,10 DIHYDROXY STEARATE

630 POTASSIUM RICINOLEATE/12 HYDROXY OLEATE/

631 POTASSIUM RICINELAIDATE/12 HYDROXY ELAIDATE/

672 DIPOTASSIUM HEXADECYL MALONATE

673 DIPOTASSIUM OCTADECYL MALONATE

494 POTASSIUM DILINOLEATE

OTHER MONOVALENT METALS

111 LITHIUM DODECYL 1 SULFATE

635 LITHIUM DODECYL SULFONATE

637 LITHIUM TETRADECYL SULFATE

638 LITHIUM HEXADECYL SULFATE

627 CESIUM DODECANOATE

23 SILVER DODECYL 1 SULFATE

POLYVALENT METALS

339 MAGNESIUM HEXANE SULFONATE

340 MAGNESIUM OCTANE SULFONATE

341 MAGNESIUM DECANE SULFONATE

568 MAGNESIUM DODECYL SULFATE

342 MAGNESIUM DODECANE SULFONATE

24 CALCIUM DODECYL 1 SULFATE

569 STRONTIUM DODECYL SULFATE

573 CUPRIC DODECYL SULFATE

576 CUPRIC TETRADECYL SULFATE

577 CUPRIC HEXADECYL SULFATE

572 COBALTOUS DODECYL SULFATE

574 ZINC DODECYL SULFATE

571 MANGANESE DODECYL SULFATE

570 LEAD DODECYL SULFATE

575 NICKEL DODECYL SULFATE

AMMONIUM

355 AMMONIUM DODECAFLUOROHEPTANOATE H/CF2/6COONH4

286 AMMONIUM DI-2-ETHYL-HEXYL SULFOSUCCINATE

372 AMMONIUM HEXADECAFLUORONONANOATE H/CF2/8 COO NH4

373 AMMONIUM EICOSAFLUOROUNDECANOATE H/CF2/10 COO NH4

386 AMMONIUM DODECYL SULFATE

387 METHYLAMMONIUM DODECYL SULFATE

388 ETHYLAMMONIUM DODECYL SULFATE

389 BUTYLAMMONIUM DODECYL SULFATE

303 DI-ISOPROPYLAMMONIUM CAPRYLATE

596 TETRAHYDROANACARDOL AMMONIUM MONOSULFONATE

409 TRIETHANOLAMMONIUM DODECYL SULFATE

60 TRIETHANOL AMMONIUM HEXADECYL SULFATE

65 TRIETHANOLAMMONIUM OCTADECYL 1 SULFATE

284 HEXANOLAMINE-CH3CH/OH/CH2C/CH3/2NH2-OCTANOATE

285 HEXANOLAMINE-CH3CH/OH/CH2C/CH3/2NH2-OLEATE

283 HEXANOLAMINE- $\mathrm{CH} 3 \mathrm{CH} / \mathrm{OH} / \mathrm{CH} 2 \mathrm{C} / \mathrm{CH} 3 / 2 \mathrm{NH} 2-\mathrm{ELAIDATE}$

\section{QUATERNARIES}

112 TETRAMETHYL AMMONIUM DODECYL 1 SULFATE

382 ETHYL TRIMETHYLAMMONIUM DODECYL SULFATE

383 BUTYL TRIMETHYLAMMONIUM DODECYL SULFATE

277 BENZYL TRIMETHYL AMMONIUM DODECANOATE

720 1-6-DITRIMETHYLAMMONIUM-HEXANE/DODECYL SULFATE/2

718 TETRAETHYLAMMONIUM DODECYL SULFATE

719 TETRABUTYLAMMONIUM DODECYL SULFATE

410 MORPHOLINIUM DODECYL SULFATE

SURFACTANT

390 HEXYLAMMONIUM DODECYL SULFATE

391 OCTYLAMMONIUM DODECYL SULFATE

640 HEXYL TRIMETHYLAMMONIUM HEXANE SULFATE

644 HEXYL TRIMETHYLAMMONIUM HEXANE SULFONATE

641 HEXYL TRIMETHYLAMMONIUM OCTANE SLLFATE

384 HEXYL TRIMETHYLAMMONIUM DODECYL SULFATE
347 OCTYL TRIMETHYLAMMOMIUM OCTYL SULFATE

287 OCTYL TRIMETHYLAMMONIUM OCTANE SULFONATE

642 OCTYL TRIMETHYLAMMONIUM DECANE SULFATE

353 OCTYL TRIMETHYLAMMONIUM DECANE SULFONATE

385 OCTYL TRIMETHYLAMMONIUM DODECYL SULFATE

346 DECYL TRIMETHYLAMMONIUM DECYL SULFATE

288 DECYL TRIMETHYLAMMONIUM DECANESULFONATE

280 DECYL TRIMETHYL AMMONIUM DODECYL SULFATE

643 DODECYL TRIMETHYLAMMONIUM OCTANE SULFATE

281 DODECYL TRIMETHYLAMMONIUM DODECYL SULFATE

\section{B. CATIONICS BY COUNTERIONS}

HYDROXYL

706 PERFLUORO PROPYLAMINE

708 HEXYLAMINE

\section{CHLORIDE}

707 PERFLUORO PROPYLAMINE HYDROCHLORIDE

709 HEXYLAMINE HYDROCHLORIDE

354 HEXYL BENZYL DIMETHYLAMMONIUM CHLORIDE

392 OCTYLAMMONIUM CHLORIDE

483 DIOCTYL DIMETHYL AMMONIUM CHLORIDE

135 OCTYL C BETAINE HYDROCHLORIDE

451 PARA DI-ISOBUTYLPHENOXYETHOXYETHYL DIMETHYLBENZYL AMMONIUM CHLORIDE/HYAMINE 1622/

359 OCTYL 4-NITROBENZYL DIMETHYLAMMONIUM CHLORIDE

137 ALPHA DIMETHYLAMINO CAPRIC ACID HYDROCHLORIDE

$\mathrm{C}-10$

37 DECYLAMMONIUM CHLORIDE

203 DECYL TRIMETHYL AMMONIUM CHLORIDE

356 DECYL BENZYL DIMETHYLAMMONIUM CHLORIDE

360 DECYL 4-NITROBENZYL DIMETHYLAMMONIUM CHLORIDE

304 CATOL 607 (N-/LAURYL-COLAMINO-FORMYL-METHYL/ PYRIDINIUM CHLORIDE)

$\mathrm{C}-12$

38 DODECYL AMMONIUM CHLORIDE

449 DODECYLMETHYL AMMONIUM CHLORIDE

450 DODECYLDIMETHYL AMMONIUM CHLORIDE

41 DODECYL TRIMETHYL AMMONIUM CHLORIDE

399 DODECYL DIMETHYL ĖTHYLAMMONIUM CHLORIDE

345 DIDODECYL DIMETHYLAMMONIUM CHLORIDE

403 DODECYL DIMETHYLPHENYLAMMONIUM CHLORIDE

279 DODECYL BENZYL DIMETHYLAMMONIUM CHLORIDE

404 DODECYL DIMETHYL 2-PHENYLETHYLAMMONIUM CHLORIDE $\mathrm{C} 6 \mathrm{H} 5 \mathrm{CH} 2 \mathrm{CH} 2 / \mathrm{N} / \mathrm{CH} 3 / 2 / \mathrm{Cl} 2 \mathrm{H} 25$

407 DODECYL DIMETHYL 3-PHENYLPROPYLAMMONIUM CHLORIDE $\mathrm{C} 6 \mathrm{H} 5 \mathrm{CH} 2 \mathrm{CH} 2 / \mathrm{N} / \mathrm{CH} 3 / 2 / \mathrm{Cl} 2 \mathrm{H} 25$

370 DODECYL 3-4-METHYLENEDIOXYBENZYL DIMETHYLAMMONIUM CHLORIDE

371 DODECYL 3-4-DIMETHOXYBENZYL DIMETHYLAMMONIUM CHLORIDE

361 DODECYL 4-NITROBENZYL DIMETHYL AMMONIUM CHLORIDE

368 DODECYL 2-HYDROXY-5-NITROBENZYL DIMETHYLAMMONIUM CHLORIDE

365 DODECYL 2-CHLOROBENZYL DIMETHYLAMMONIUM CHLORIDE

367 DODECYL 4-CHLOROBENZYL DIMETHYLAMMONIUM CHLORIDE

366 DODECYL 2-4-DICHLOROBENZYL DIMETHYLAMMONIUM CHLORIDE

369 DODECYL 3-4-DICHLOROBENZYL DIMETHYLAMMONIUM CHLORIDE

406 DODECYL DIMETHYL META-TRIFLUOROMETHYLBENZYL AMMONIUM CHLORIDE CF3C6H4CH2/N/CH3/2/C12H25

400 DODECYL METHYL DIETHYLAMMONIUM CHLORIDE

405 DODECYL METHYLETHYLBENZYLAMMONIUM CHLORIDE

401 DODECYL TRIETHYLAMMONIUM CHLORIDE

22 DIMETHYL DODECYL AMINE OXIDE HYDROCHLORIDE

633 N-DODECYL BETA-ALANINE HYDROCHLORIDE

124 DODECYL N BETAINE HYDROCHLORIDE

278 DODECYL PYRIDINIUM CHLORIDE

528 DODECYL TROPYLIUM PERCHLORATE

500 DODECYL TRI/2-HYDROXYETHYL/AMMONIUM CHLORIDE

$\mathrm{C}-13$

402 TRIDECYL TRIMETHYLAMMONIUM CHLORIDE

39 TETRADECYL AMMONIUM CHLORIDE

42 TETRADECYL TRIMETHYL AMMONIUM CHLORIDE

357 TETRADECYL BENZYL DIMETHYLAMMONIUM CHLORIDE

362 TETRADECYL 4-NITROBENZYL DIMETHYLAMMONIUM CHLORIDE

125 TETRADECYL N BETAINE HYDROCHLORIDE

C-16

186 HEXADECYL AMMONIUM CHLORIDE 
PART 5. Ionics grouped by counterion and arranged by number of carbon atoms in longest hydrophobic tail and by heads - Continued

275 HEXADECYL DIMETHYLBENZYLAMMONIUM CHLORIDE

265 HEXADECYL TRIMETHYL AMMONIUM CHLORIDE

266 HEXADECYL DIMETHYL 2-HYDROXYETHYL AMMONIUM CHLORIDE

269 HEXADECYLDIMETHYL2, 3-DIHYDROXYPROPYLAMMONIUM CHLORIDE

363 HEXADECYL 4-NITROBENZYL DIMETHYLAMMONIUM CHLORIDE

267 HEXADECYL DI-/2-HYDROXYETHYL/METHYL AMMONIUMCHLORIDE

268 HEXADECYL TRI-/2-HYDROXYETHYL/ AMMONIUM CHLORIDE

274 HEXADECYL PYRIDINIUM CHLORIDE

693 N-CETYL 2-METHYL PYRIDINIUM CHLORIDE

694 N-CETYL 3-METHYL PYRIDINIUM CHLORIDE

695 N-CETYL 4-METHYL PYRIDINIUM CHLORIDE

$\mathrm{C}-18$

187 OCTADECYL AMMONIUM CHLORIDE

270 OCTADECYL TRIMETHYLAMMONIUM CHLORIDE

358 OCTADECYL BENZYL DIMETHYLAMMONIUM CHLORIDE

364 OCTADECYL 4-NITROBENZYL DIMETHYLAMMONIUM CHLORIDE

655 OCTADECYL PYRIDINIUM CHLORIDE

\section{BROMIDE}

93 OCTYL TRIMETHYL AMMONIUM BROMIDE

100 OCTYL PYRIDINIUM BROMIDE

94 NONYL TRIMETHYL AMMONIUM BROMIDE

95 DECYL TRIMETHYL AMMONIUM BROMIDE

96 UNDECYL TRIMETHYL AMMONIUM BROMIDE

101 UNDECYL PYRIDINIUM BROMIDE

628 DODECYLAMMONIUM BROMIDE

97 DODECYL TRIMETHYL AMMONIUM BROMIDE

293 DODECYL DIMETHYL AMINE OXIDE HYDROBROMIDE

290 DODECYL PYRIDINIUM BROMIDE

717 DODECYLQUINOLINIUM BROMIDE

\section{C-14}

98 TETRADECYL TRIMETHYL AMMONIUM BROMIDE

291 TETRADECYL TRIPROPYLAMMONIUM BROMIDE

102 TETRADECYL PYRIDINIUM BROMIDE

99 HEXADECYL TRIMETHYLAMMONIUM BROMIDE

478 HEXADECYL DIMETHYLETHYLAMMONIUM BROMIDE

292 HEXADECYL TRIPROPYLAMMONIUM BROMIDE

427 HEXADECYL PYRIDINIUM BROMIDE

477 OCTADECYL TRIMETHYLAMMONIUM BROMIDE

657 OCTADECYL PYRIDINIUM BROMIDE

IODIDE

458 DECYL PYRIDINIUM IODIDE

126 DODECYL TRIMETHYLAMMONIUM IODIDE

376 DODECYL PYRIDINIUM IODIDE

479 TETRADECYL PYRIDINIUM IODIDE

480 HEXADECYL PYRIDINIUM IODIDE

696 N-CETYL 2-METHYL PYRIDINIUM IODIDE

697 N-CETYL-3-METHYL PYRIDINIUM IODIDE

698 N-CETYL-4-METHYL PYRIDINIUM IODIDE

481 OCTADECYL PYRIDINIUM IODIDE

\section{FLUORIDE}

130 DODECYL TRIMETHYL AMMONIUM FLUORIDE
NITRATE

482 DODECYL AMMONIUM NITRATE

131 DODECYL TRIMETHYLAMMONIUM NITRATE

654 OCTADECYL TRIMETHYLAMMONIUM NITRATE

656 OCTADECYL PYRIDINIUM NITRATE

\section{BROMATE}

129 DODECYL TRIMETHYL AMMONIUM BROMATE

666 HEXADECYL TRIBUTYLAMMONIUM BROMATE

658 OCTADECYL TRIMETHYLAMMONIUM BROMATE

662 OCTADECYL TRIETHYLAMMONIUM BROMATE

663 OCTADECYL TRIPROPYLAMMONIUM BROMATE

664 OCTADECYL TRIBUTYLAMMONIUM BROMATE

665 OCTADECYL TRIAMYLAMMONIUM BROMATE

IODATE

127 DODECYL TRIMETHYL AMMONIUM IODATE

660 HEXADECYL PYRIDINIUM IODATE

661 OCTADECYL PYRIDINIUM IODATE

\section{SULFATE}

306 DECYL TRIMETHYLAMMONIUM SULFATE

307 DODECYL TRIMETHYLAMMONIUM SULFATE

491 DODECYL TROPYLIUM BISULFATE

308 TETRADECYL TRIMETHYLAMMONIUM SULFATE

309 HEXADECYL TRIMETHYLAMMONIUM SULFATE

PHOSPHATE

460 DODECYL TROPYLIUM MONOPHOSPHATE

\section{CARBOXYLATE}

128 DODECYL TRIMETHYL AMMONIUM FORMATE

659 OCTADECYL TRIMETHYLAMMONIUM FORMATE

415 OCTADECYLAMMONIUM ACETATE

411 DECYLAMMONIUM ACETATE

412 DODECYLAMMONIUM ACETATE

413 TETRADECYLAMMONIUM ACETATE

414 HEXADECYLAMMONIUM ACETATE

667 OCTADECYL TRIMETHYLAMMONIUM OXALATE

SURFACTANT

391 OCTYLAMMONIUM DODECYL SULFATE

347 OCTYL TRIMETHYLAMMONIUM OCTYL SULFATE

287 OCTYL TRIMETHYLAMMONIUM OCTANE SULFONATE

642 OCTYL TRIMETHYLAMMONIUM DECANE SULFATE

353 OCTYL TRIMETHYLAMMONIUM DECANE SULFONATE

385 OCTYL TRIMETHYLAMMONIUM DODECYL SULFATE

346 DECYL TRIMETHYLAMMONIUM DECYL SULFATE

280 DECYL TRIMETHYL AMMONIUM DODECYL SULFATE

288 DECYL TRIMETHYLAMMONIUM DECANESULFONATE

643 DODECYL TRIMETHYLAMMONIUM OCTANE SULFATE

281 DODECYL TRIMETHYLAMMONIUM DODECYL SULFATE 


\section{Structural Indexes of Compounds with Keys to Compound Numbers - Continued}

PART 6. Commercial names and ill defined structures arranged alphabetically.

\section{COMMERCIAL AND ILL-DEFINED}

556

557

558

566

559

304

459

565

674

494

651
ARESKAP 100 /MONOBUTYL PHENYLPHENOL SODIUM MONOSULFONATE/

ARESKET 300 /MONOBUTYL BIPHENYL SODIUM MONOSULFONATE/ ARESKLENE 400 /DIBUTYL PHENYLPHENOL

DISODIUMDISULFONATE

AQUAREX D

CATOL $605 /(\mathrm{N}-12-\mathrm{KETO}-2-(2,-$ LAUROYL

OXYETHYLAMINO))ETHYL TRIMETHYLAMMONIUM CHLORIDE/

CATOL 607 (N-/LAURYL-COLAMINO-FORMYL-METHYL/ -

PYRIDINIUM CHLORIDE)

ALKYL/15-17/ /OXYETHYLENE/ /19-23/ALCOHOL /

CETOMACROGOL 1000/

DAXAD 11

DIISOBUTYL BENZENE/OXYETHYLENE/6 ALCOHOL (DB) BRANCHED CHAIN, NATURAL OE DISTRIBUTION

POTASSIUM DILINOLEATE
652 EMASOL 1130 /ALKYL POLYOXYETHYLENE SORBITAN ESTER/

653 EMULGEN 120 /ALKYL POLYOXYETHYLENE ETHER/

560 EMULSOL 607L (N-(2-KETO-2-(2,-LAUROYLOXYETHYL AMINO)) ETHYL) PYRIDINIUM CHLORIDE

451 PARA DI-ISOBUTYLPHENOXYETHOXYETHYL DIMETHYLBENZYL AMMONIUM CHLORIDE/HYAMINE 1622/

452 3-4-DICHLOROPERFLUORO BUTYRIC/KEL-F/ACID

453 3-5-6-TRICHLOROPERFLUORO HEXANOIC/KEL-F/ACID

454 3-5-7-8-TETRACHLOROPERFLUORO OCTANOIC/KEL-F/ACID

550 LAURIC ACID DIETHANOLAMINE CONDENSATE

564 NA OSR

682 SODIUM DIBUTYL NAPHTHALENE SULFONATE /NEKAL/

538 PLURONIC L62

539 RENEX 698

561 SANTOMERSE D /SODIUM DECYLBENZENE SULFONATE/

554 SANTOMERSE 3 /SODIUM DODECYL BENZENE SULFONATE/

567 SA-178

540 SIPONIC BC

555 TERGITOL TMN

563 ZEPHIRAN /COCONUT DIMETHYLBENZYL AMMONIUM CHLORIDE/ 


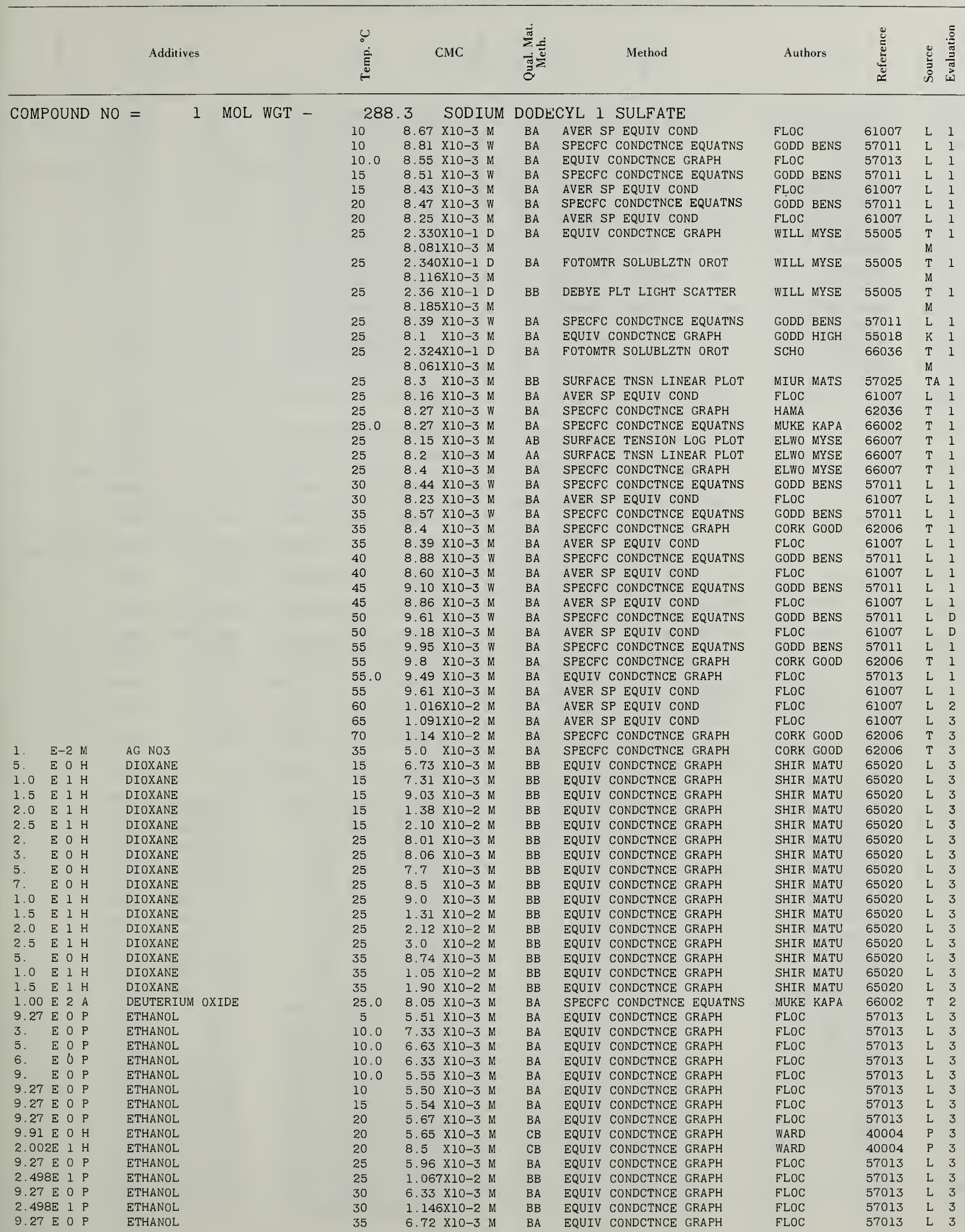

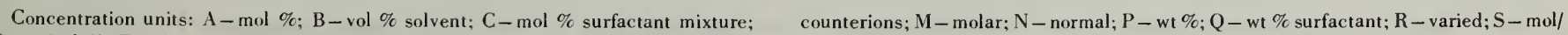

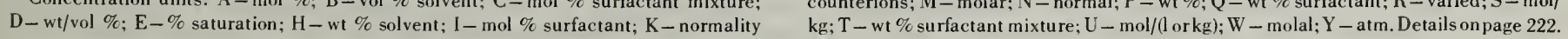




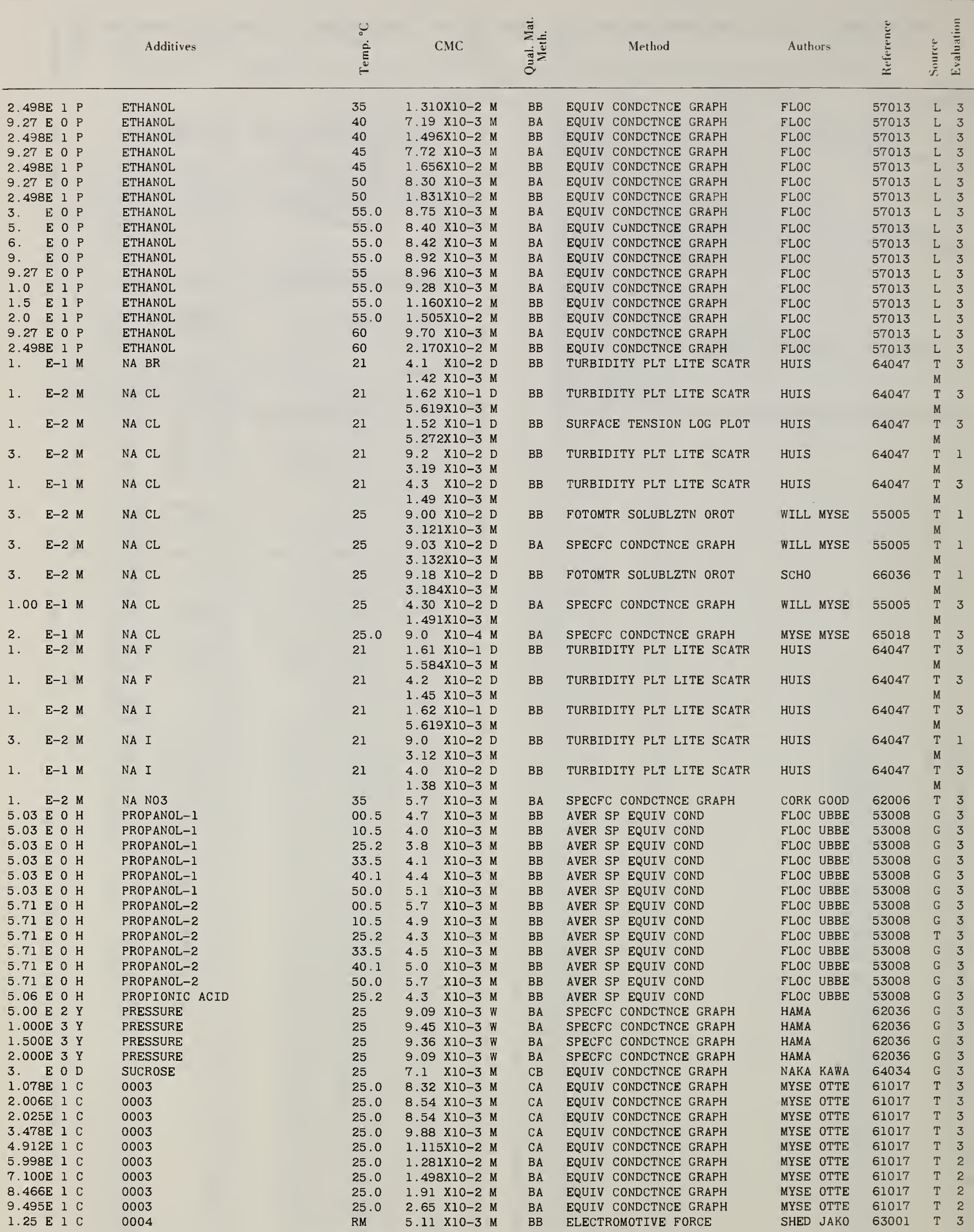

Concentration units: $\mathrm{A}-$ mol $\% ; \mathrm{B}-\mathrm{vol} \%$ solvent: $\mathrm{C}-$ mol $\%$ surfactant mixture; $\mathrm{D}-\mathrm{wt} / \mathrm{vol} \%$; E-\% saturation; $\mathrm{H}-\mathrm{wt} \%$ solvent; I-mol \% surfactant: $\mathrm{K}$-normality counterions; N-molar; N-normal; P - wt \%: Q - wt \% surfactant; R-varied: S-mol! kg; $\mathrm{T}-$ wt $\%$ surfactant mixture; $\mathrm{U}-\mathrm{mol} /(\mathrm{l}$ or $\mathrm{kg}) ; \mathrm{W}-$ molal: $\mathrm{Y}-\mathrm{atm}$. Detailson vage 222 


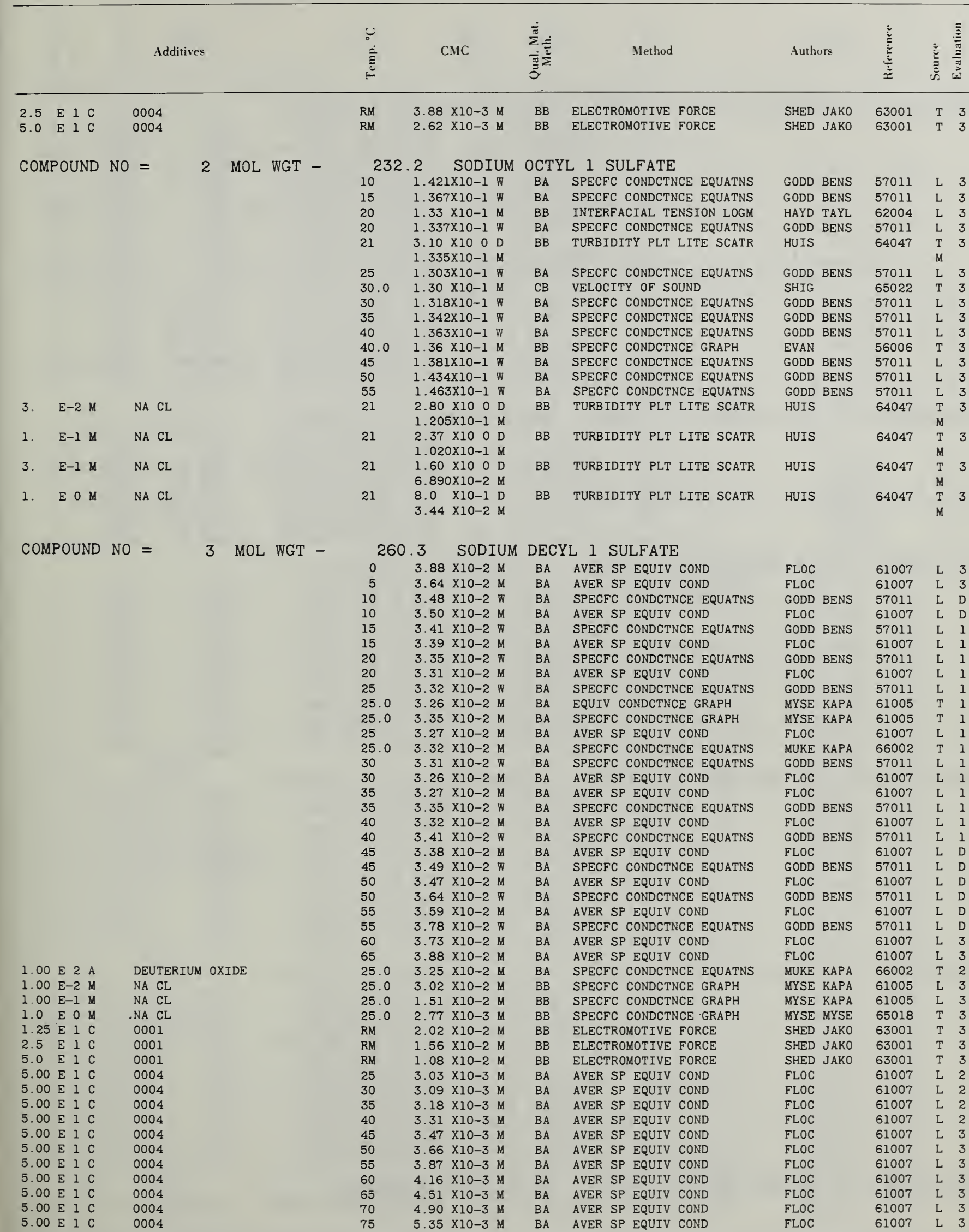

Concentration units: $\mathrm{A}-$ mol $\%: \mathrm{B}-\mathrm{vol} \%$ solvent; $\mathrm{C}-$ mol $\%$ surfactant mixture; $\mathrm{D}-\mathrm{wt} / \mathrm{vol} \%$; E- $\%$ saturation; $\mathrm{H}-\mathrm{wt} \%$ solvent; I- mol \% surfactant; K-normality counterions; $\mathrm{I}-$ molar; $\mathrm{N}$-normal; $\mathrm{P}-$ wt $\% ; \mathrm{Q}$ - wt $\%$ surfactant; $\mathrm{R}$-varied; $\mathrm{S}$-mol/ kg: T-wt $\%$ surfactant mixture: $\mathrm{C}-\mathrm{mol} /(\mathrm{l}$ or $\mathrm{kg})$; $\mathrm{W}-$ molal; $\mathrm{Y}$ - atm. Details on page 222. 


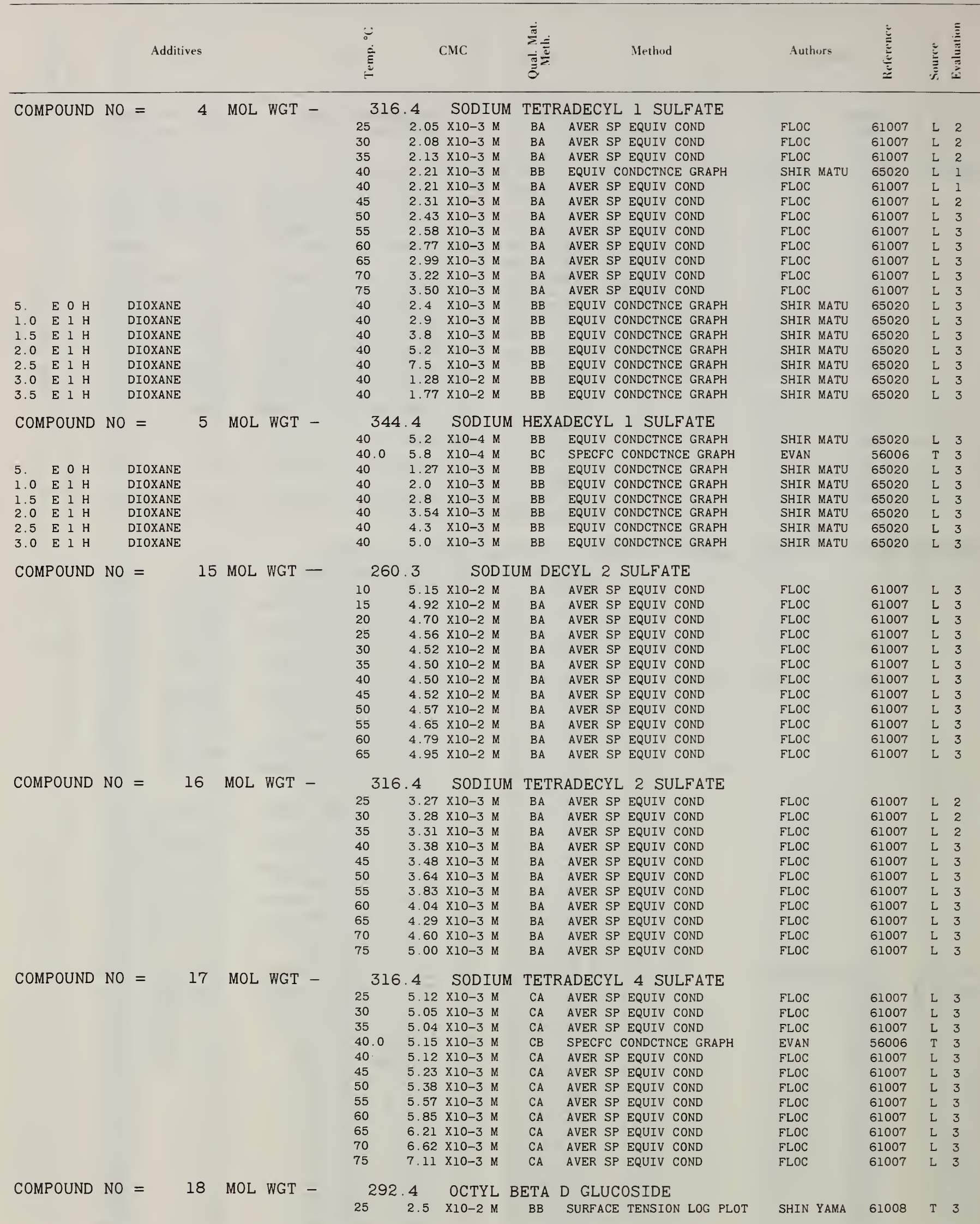

Concentration units: $\mathrm{A}-\mathrm{mol} \% ; \mathrm{B}-\mathrm{vol} \%$ solvent; $\mathrm{C}-$ mol \% surfactant mixture $\mathrm{D}-\mathrm{wt} / \mathrm{vol} \%$; $\mathrm{E}-\%$ saturation; $\mathrm{H}-\mathrm{wt} \%$ solvent; $\mathrm{I}-\mathrm{mol} \%$ surfactant; $\mathrm{K}$ - normality counterions; $\mathrm{N}$-molar; $\mathrm{N}$ - normal; $\mathrm{P}$ - wt \%; $\mathrm{Q}$ - wt \% surfactant: $\mathrm{R}$-varied: $\mathrm{S}-$ mol/ kg: T-wt \% surfactant mixture: $\mathrm{U}-\mathrm{mol} /(\mathrm{l}$ or $\mathrm{kg}): \mathrm{W}-$ molal: $\mathrm{Y}$ - atm. Detailson page 222. 


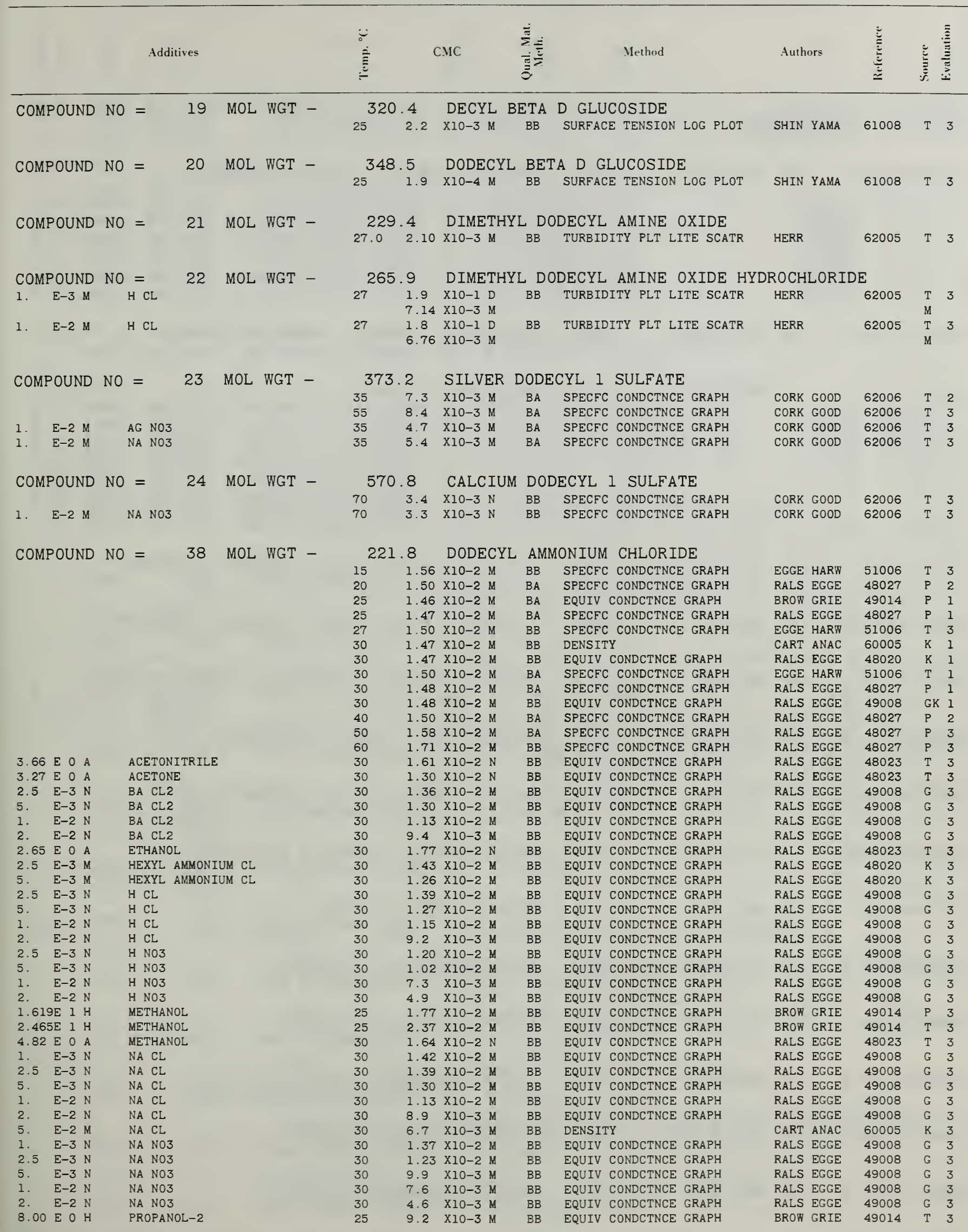

Concentration units: $\mathrm{A}-\mathrm{mol} \% ; \mathrm{B}-\mathrm{vol} \%$ solvent: $\mathrm{C}-$ mol $\%$ surfactant mixture: $\mathrm{D}-\mathrm{w} t / \mathrm{vol} \%$; E- \% saturation; $\mathrm{H}-\mathrm{wt} \%$ solvent: $1-$ mol $\%$ surfactant: $\mathrm{K}-$ normality counterions: II-molar: N-normal; P-wt \%; Q-wt \% surfactant; R-varied; S-mol/ kg: $\mathrm{T}-\mathrm{wt} \%$ surfactant mixture; $\mathrm{C}-\mathrm{mol} /(\mathrm{l}$ or kg); $\mathrm{W}$ - molal; $\mathrm{Y}$ - atm. Details on page 222. 


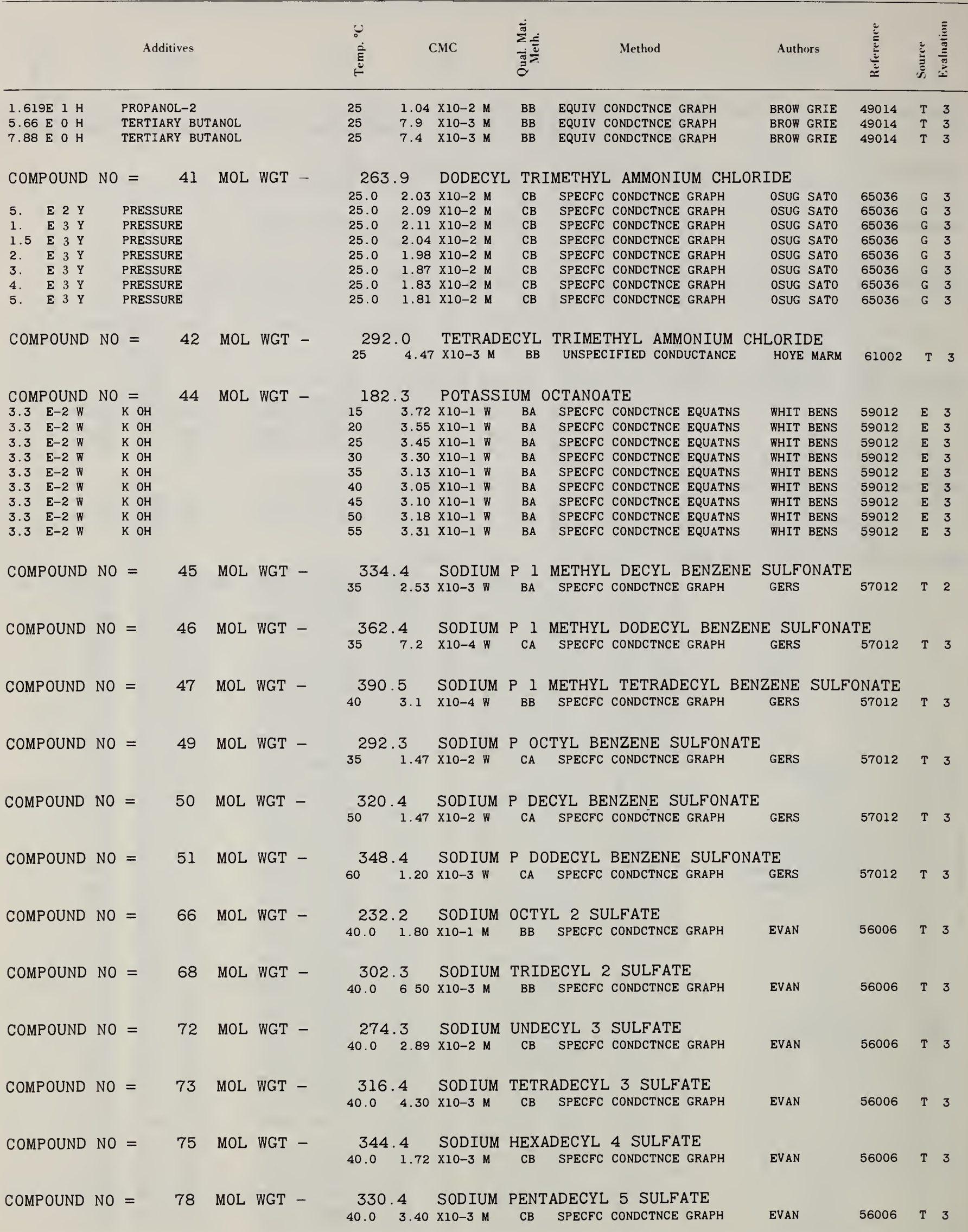

Concentration units: A-mol \%; B-vol \% solvent; $\mathrm{C}-$ mol \% surfactant mixture; $\mathrm{D}-\mathrm{wt} / \mathrm{vol} \%$; E-\% saturation; $\mathrm{H}-$ wt \% solvent; I- mol \% surfactant; $\mathrm{K}$-normality counterions; $\mathrm{M}$ - molar; $\mathrm{N}$-normal; $\mathrm{P}$ - wt \%; $\mathrm{Q}-$ wt \% surfactant; $\mathrm{R}$-varied; $\mathrm{S}-\mathrm{mol} /$ kg; T - wt \% surfactant mixture; $\mathrm{U}-\mathrm{mol} /(\mathrm{l}$ or kg); W - molal; $\mathrm{Y}$ - atm. Details on page 222. 


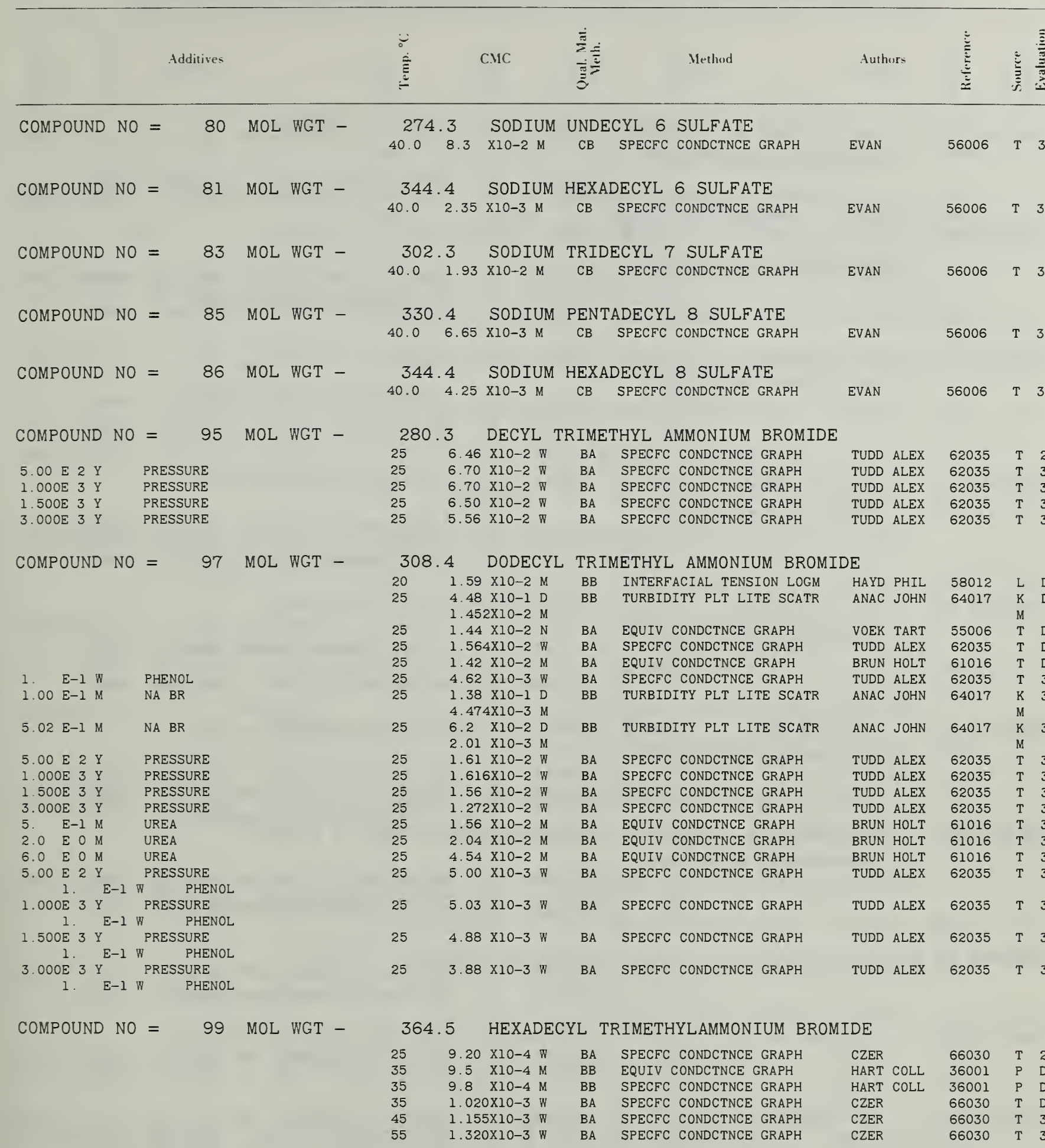
$\begin{aligned} & \text { COMPOUND NO }= 103 \text { MOL WGT }-\quad 234.3 \quad \text { HEXYL /OXYETHYLENE/ } 3 \text { ALCOHOL } \\ & \text { HOMOGENEOUS HEAD GROUP }\end{aligned}$

$\begin{array}{llllllllll}15 & 1.07 & \text { XIO-3 M } & \text { BB } & \text { SURFACE TENSION LOG PLOT } & \text { CORK GOOD } & 64003 & \text { T } & 3 \\ 25 & 1.00 & \text { XIO-1 } & \text { M } & \text { BB } & \text { SURFACE TENSION LOG PLOT } & \text { CORK GOOD } & 64003 & \text { T } & 3 \\ 35 & 7.8 & \text { XIO-2 } & \text { M } & \text { BB } & \text { SURFACE TENSION LOG PLOT } & \text { CORK GOOD } & 64003 & \text { T } & 3\end{array}$

\footnotetext{
Concentration units: $\mathrm{A}-$ mol $\%: \mathrm{B}-$ vol $\%$ solvent: $\mathrm{C}-$ mol $\%$ surfactant mixture: 


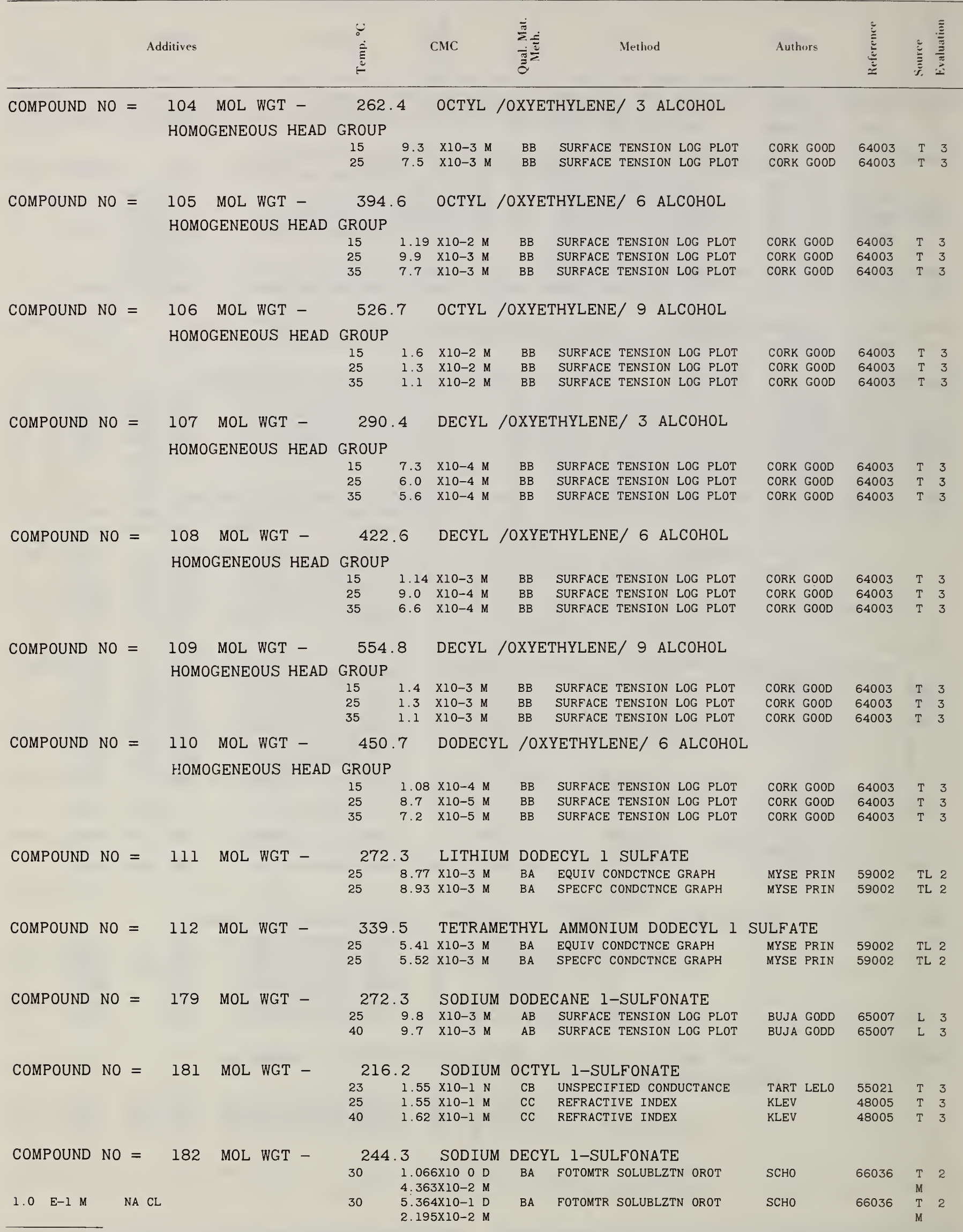

Concentration units: $\mathrm{A}-\mathrm{mol} \% ; \mathrm{B}-\mathrm{vol} \%$ solvent; $\mathrm{C}-$ mol $\%$ surfactant mixture; 


\begin{tabular}{|c|c|c|c|c|c|c|}
\hline Additives & 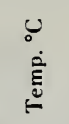 & CMC & 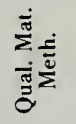 & Method & Authors & 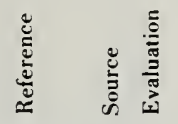 \\
\hline
\end{tabular}

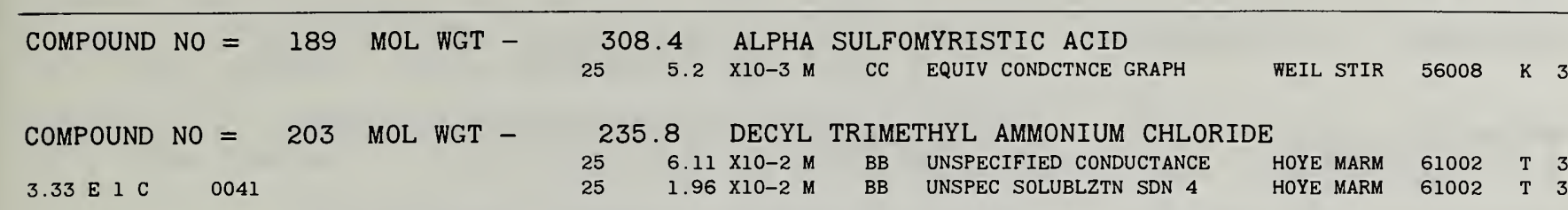

COMPOUND NO $=208$ MOL WGT $-\quad 294.4$ P-T-OCTYL BENZENE /OXYETHYLENE/2 ALCOHOL HOMOGENEOUS HEAD GROUP

$\begin{array}{lllllll}25 & 1.32 \times 10-4 & M & \text { BC } & \text { SURFACE TENSION LOG PLOT CROO FORD } 63017 & \text { GL } 3\end{array}$

COMPOUND NO $=209$ MOL WGT - 338.5 P-T-OCTYL BENZENE /OXYETHYLENE/3 ALCOHOL HOMOGENEOUS HEAD GROUP

$\begin{array}{llllllll}25 & 9.7 & \text { XIO-5 M } & \text { BC SURFACE TENSION LOG PLOT CROO FORD } 63017 & \text { GL } 3\end{array}$

COMPOUND NO $=210$ MOL WGT - 382.5 P-T-OCTYL BENZENE /OXYETHYLENE/4 ALCOHOL HOMOGENEOUS HEAD GROUP

$\begin{array}{lllllll}25 & 1.25 \times 10-4 \mathrm{M} & \mathrm{BC} & \text { SURFACE TENSION LOG PLOT } & \text { CROO FORD } 63017 & \text { GL } 3\end{array}$

COMPOUND NO $=211$ MOL WGT - 426.6 P-T-OCTYL BENZENE /OXYETHYLENE/5 ALCOHOL HOMOGENEOUS HEAD GROUP

$25 \quad 1.54$ X10-4 M BC SURFACE TENSION LOG PLOT CROO FORD 63017 GL 3

COMPOUND NO $=212$ MOL WGT $-\quad 470.7$ P-T-OCTYL BENZENE /OXYETHYLENE/6 ALCOHOL HOMOGENEOUS HEAD GROUP

$\begin{array}{lllllll}25 & 2.05 \times 10-4 \mathrm{M} & \text { BC } & \text { SURFACE TENSION LOG PLOT } & \text { CROO FORD } & 63017 & \text { GL } 3\end{array}$

COMPOUND NO $=213$ MOL WGT - 514.7 P-T-OCTYL BENZENE /OXYETHYLENE/7 ALCOHOL HOMOGENEOUS HEAD GROUP $25 \quad 2.46 \times 10-4 \mathrm{M} \quad$ BC SURFACE TENSION LOG PLOT CROO FORD 63017 GL 3

COMPOUND NO $=214$ MOL WGT $-\quad 558.8$ P-T-OCTYL BENZENE /OXYETHYLENE/8 ALCOHOL HOMOGENEOUS HEAD GROUP

$25 \quad 2.80 \times 10-4 \mathrm{M} \quad$ BC SURFACE TENSION LOG PLOT CROO FORD 63017 GL 3

COMPOUND NO $=215$ MOL WGT $-\quad 602.8$ P-T-OCTYL BENZENE /OXYETHYLENE/9 ALCOHOL HOMOGENEOUS HEAD GROUP

$25 \quad 3.35 \times 10-4 \mathrm{M} \quad$ BC SURFACE TENSION LOG PLOT CROO FORD 63017 GL 3

COMPOUND NO $=216$ MOL WGT - 646.9 P-T-OCTYL BENZENE /OXYETHYLENE/1O ALCOHOL HOMOGENEOUS HEAD GROUP

$25 \quad 3.35 \times 10-4 \mathrm{M} \quad$ BC SURFACE TENSION LOG PLOT CROO FORD 63017 GL 3

COMPOUND NO $=254$ MOL WGT -187.3 NONYL DIMETHYL AMINE OXIDE

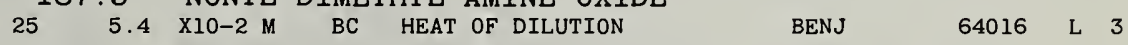

COMPOUND NO $=255$ MOL WGT - 354.6 POTASSIUM 9,10 DIHYDROXY STEARATE

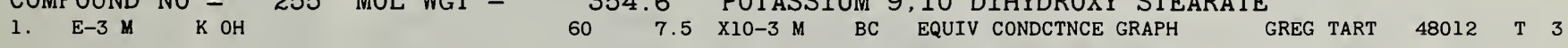

COMPOUND NO $=258$ MOL WGT - 360.3 SODIUM DI-N-AMYL SULFOSUCCINATE

$25 \quad 5.3$ X10-2 M BC SURFACE TENSION LOG PLOT WILL DIXO 57009 AL 3

COMPOUND NO $=259$ MOL WGT $-\quad 388.4$ SODIUM DI-N-HEXYL SULFOSUCCINATE

$25 \quad 1.24$ X10-2 M BC SURFACE TENSION LOG PLOT WILL DIXO 57009 TL 3

COMPOUND NO $=260$ MOL WGT -

444.5 SODIUM DI-N-OCTYL SULFOSUCCINATE

256.8 X10-4 M BC SURFACE TENSION LOG PLOT WILL DIXO 57009 TL 3

Concentration units: A-mol \%; B-vol \% solvent; $\mathrm{C}-$ mol \% surfactant mixture; $\mathrm{D}-$ wt/vol \%; E-\% saturation; $\mathrm{H}-$ wt \% solvent; I-mol \% surfactant; K-normality 


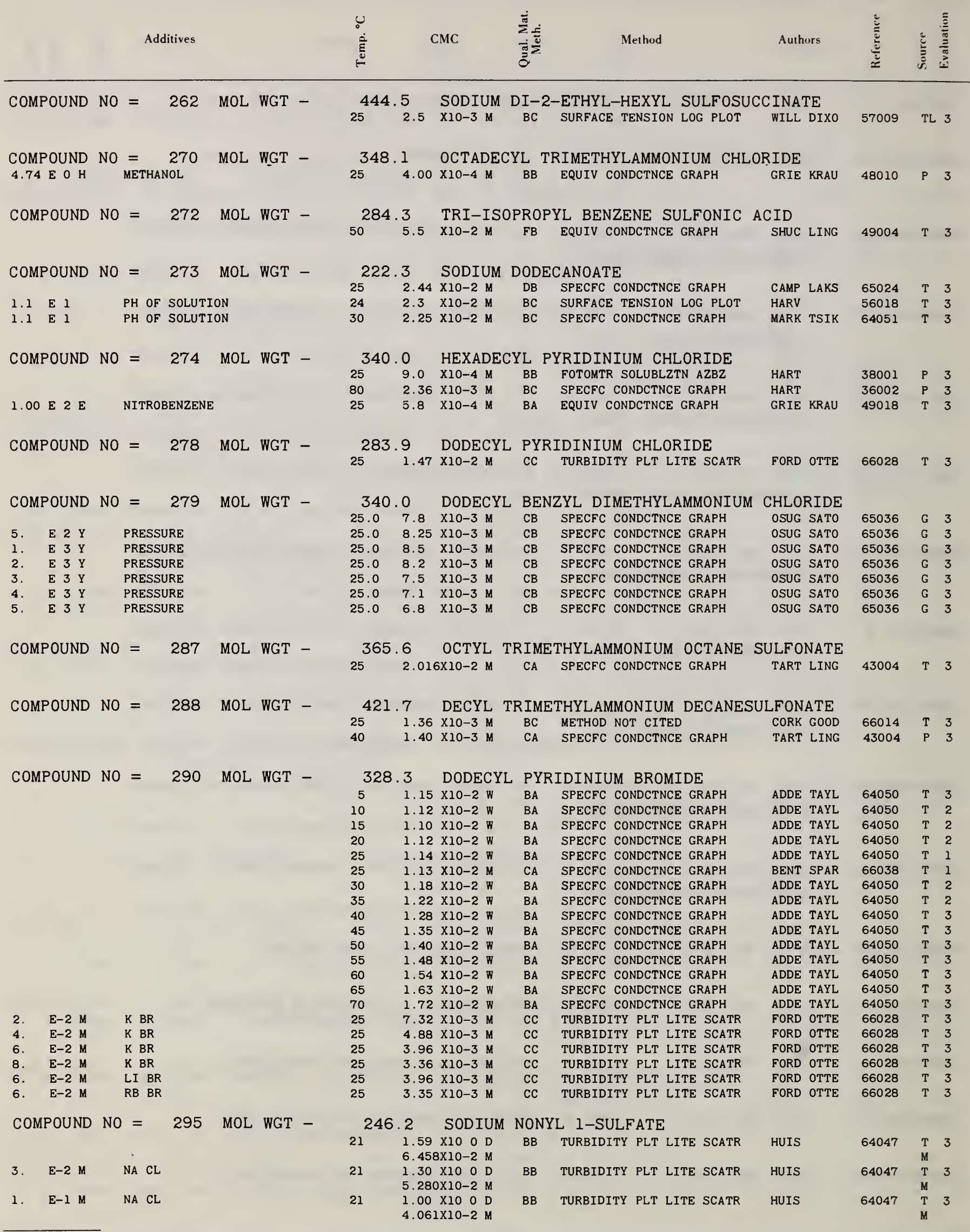

Concentration units: $\mathrm{A}-\mathrm{mol} \% ; \mathrm{B}-\mathrm{vol} \%$ solvent; $\mathrm{C}-$ mol \% surfactant mixture; $\mathrm{D}-\mathrm{wt} / \mathrm{vol} \% ; \mathrm{E}-\%$ saturation; $\mathrm{H}-$ wt $\%$ solvent; 1 - mol \% surfactant; $\mathrm{K}-$ normality counterions: $\mathrm{M}$ - molar; $\mathrm{N}$ - normal; $\mathrm{P}$-wt \%: $\mathrm{Q}$ - wt \% surfactant; $\mathrm{R}$-varied: $\mathrm{S}$ - mol/ kg: $\mathrm{T}-\mathrm{wt} \%$ surfactant mixture; $\mathrm{U}-\mathrm{mol} /(\mathrm{l}$ or $\mathrm{kg}) ; \mathrm{W}$ - Inolal; $\mathrm{Y}$ - at m. Details on page 222. 


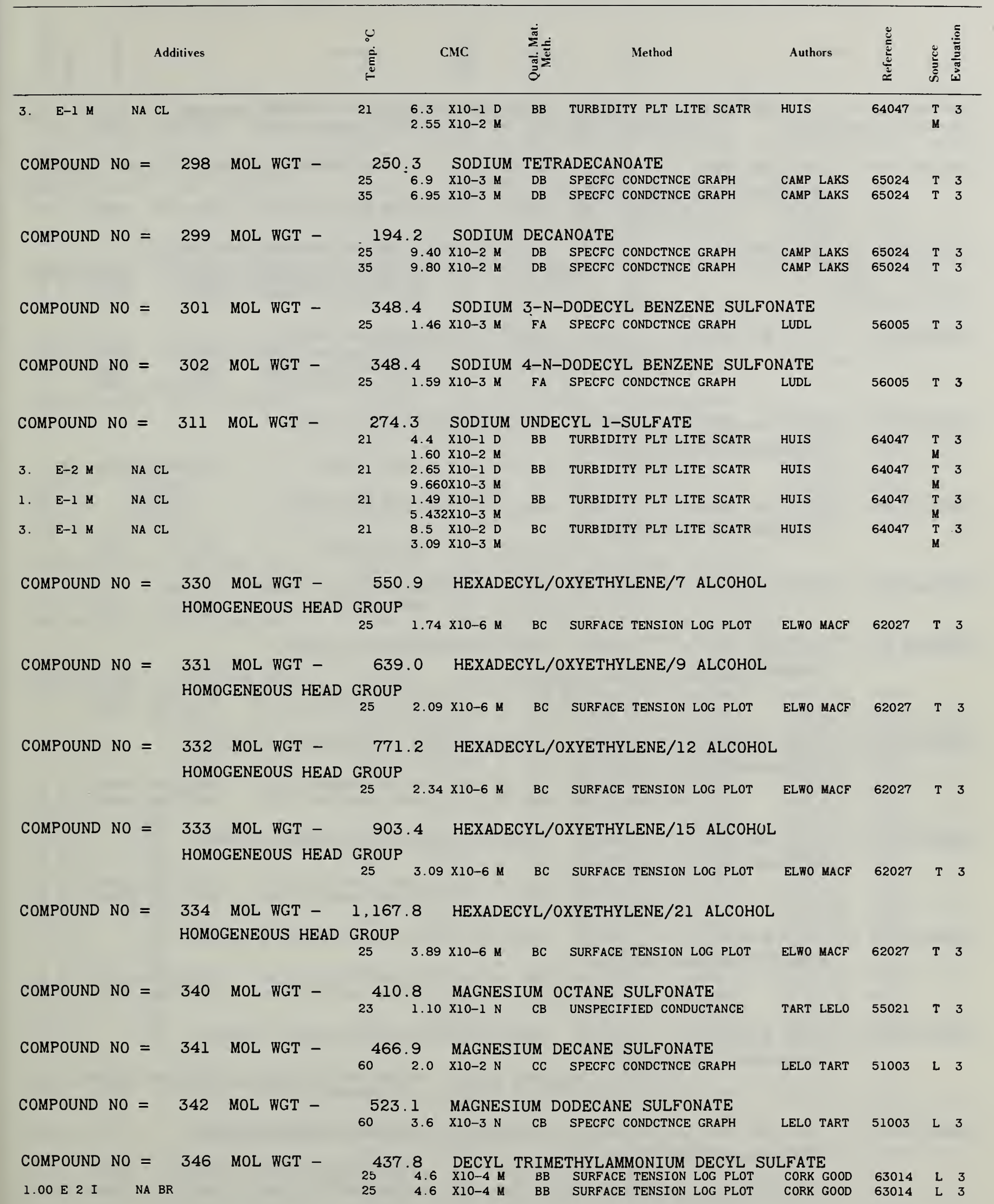

Concentration units: $\mathrm{A}-$ mol \%; $\mathrm{B}-$ vol \% solvent: $\mathrm{C}-$ mol \% surfactant mixture;

counterions; $\mathbf{M}$-molar; $\mathrm{N}$-normal; $\mathrm{P}$ - wt \%; $\mathrm{Q}$-wt \% surfactant; $\mathrm{R}$-varied; $\mathbf{S}$-mol/ D-wt/vol \%: E-\% saturation: $\mathrm{H}-$ wt \% solvent; I- mol \% surfactant; $\mathrm{K}$-normality 


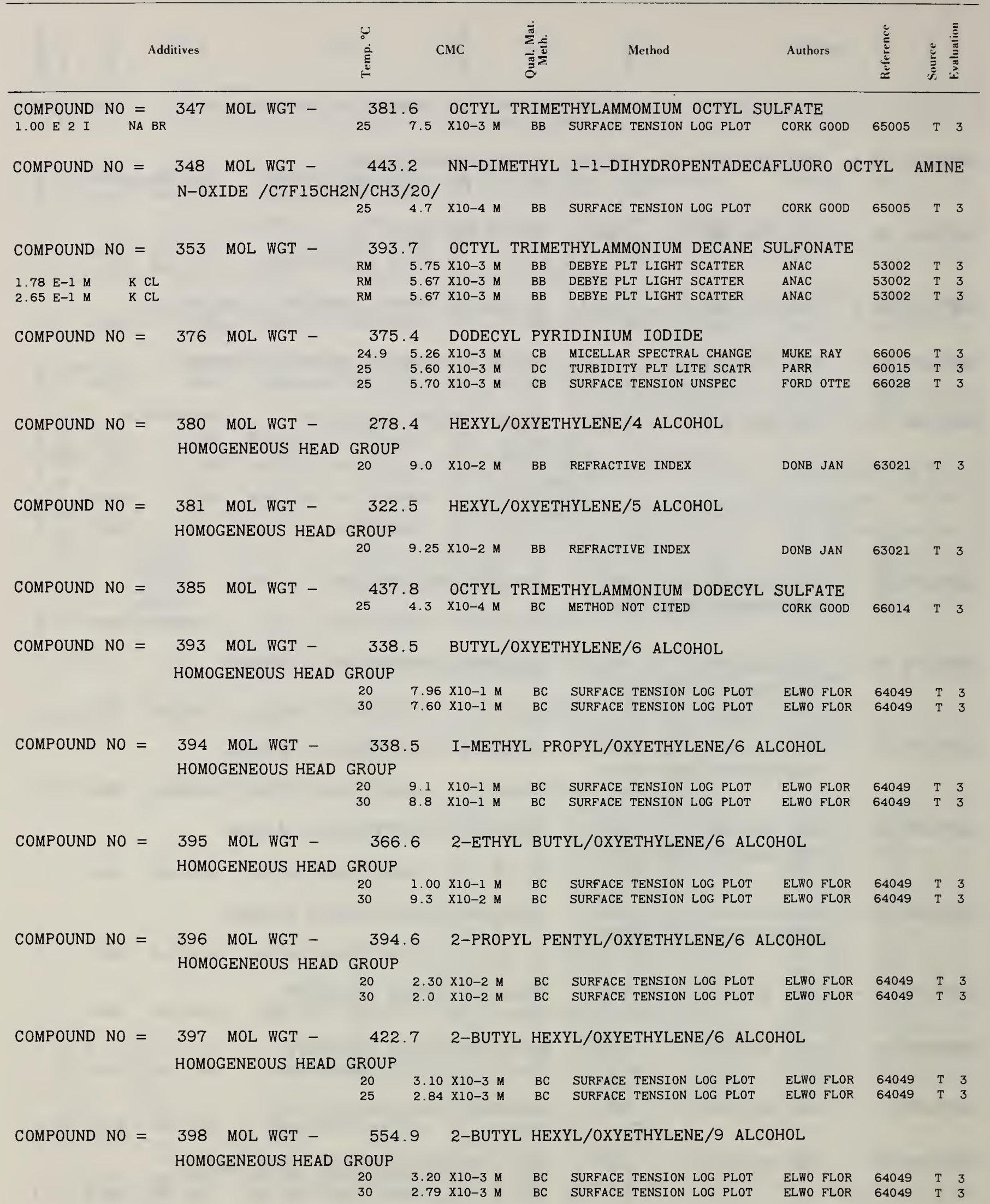

Concentration units: $\mathrm{A}-\operatorname{mol} \% ; \mathrm{B}-\mathrm{vol} \%$ solvent; $\mathrm{C}-$ mol \% surfactant mixture; D-wt/vol \%; E-\% saturation; $\mathrm{H}-$ wt \% solvent; I-mol \% surfactant; $\mathrm{K}$-normality counterions; $\mathrm{M}$ - molar; $\mathrm{N}$-normal; $\mathrm{P}$ - wt \%; $\mathrm{Q}$ - wt \% surfactant; $\mathrm{R}$-varied; $\mathrm{S}-$ mol/ kg; T - wt \% surfactant mixture; $\mathrm{U}-\mathrm{mol} /(\mathrm{l}$ or kg); $\mathrm{W}$ - molal; $\mathrm{Y}$ - atm. Details on page 222. 


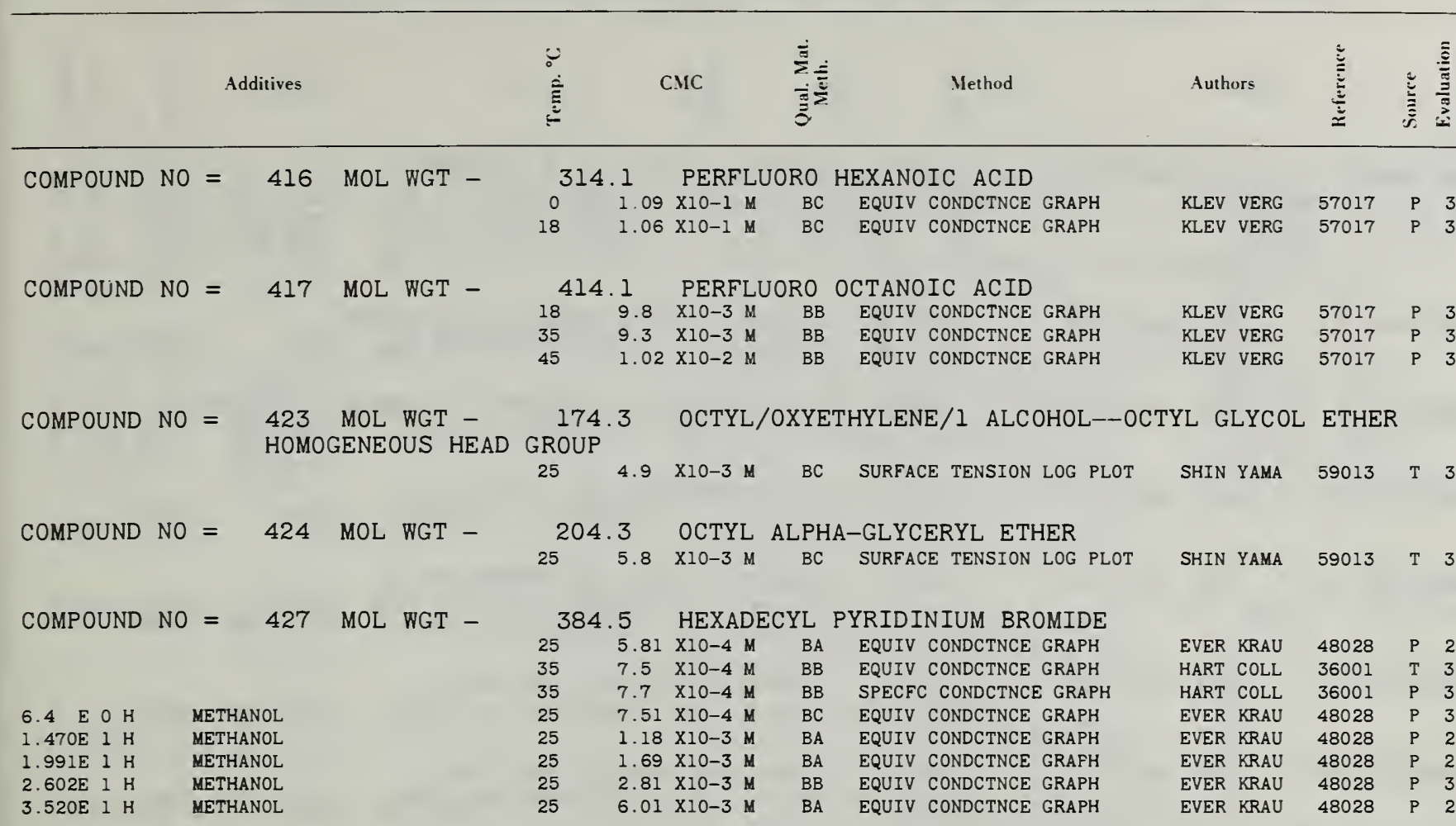

COMPOUND NO $=449$ MOL WGT $-\quad \begin{aligned} & 235.9 \\ & 30 \\ & 1.46 \times 10-2 \mathrm{M} \text { BC EQUIV CONDCTNCE GRAPH }\end{aligned}$

COMPOUND NO $=450$ MOL WGT -

COMPOUND NO $=456$ MOL WGT -

\subsection{POTASSIUM PERFLUORO OCTANOATE}

249.9 DODECYLDIMETHYL AMMONIUM CHLORIDE

$\begin{array}{lllllllll}30 & 1.61 & \text { X10-2 M } & \text { BC EQUIV CONDCTNCE GRAPH } & \text { RALS BRO0 } & 49013 & \text { T } & 3\end{array}$

\begin{tabular}{|c|c|c|c|}
\hline 9.4 & $E-3$ & T & $\mathrm{K} \mathrm{N03}$ \\
\hline 1.82 & $E-2$ & T & K N03 \\
\hline 2.96 & $E-2$ & $\pi$ & K N03 \\
\hline 8.3 & $E-3$ & T & K NO3 \\
\hline 2.39 & $E-2$ & $\pi$ & K N03 \\
\hline 4.08 & $E-2$ & T & K NO3 \\
\hline 7.31 & $E-2$ & T & $\mathrm{K} \mathrm{NO3}$ \\
\hline 1.13 & E-2 & T & K NO3 \\
\hline 2.30 & E-2 & T & K NO3 \\
\hline 3.54 & $E-2$ & $\mathrm{~T}$ & K NO3 \\
\hline 4.65 & $E-2$ & T & K NO3 \\
\hline 6.01 & $E-2$ & $\mathrm{~T}$ & $\mathrm{~K} \mathrm{NO3}$ \\
\hline 7.77 & $E-2$ & $T$ & K NO3 \\
\hline 1.28 & E-2 & T & K NO3 \\
\hline 3.77 & $E-2$ & T & $\mathrm{K} \mathrm{NO3}$ \\
\hline 8.22 & $E-2$ & T & $\mathrm{K} \mathrm{NO3}$ \\
\hline 4.65 & $E-2$ & $T$ & $\mathrm{~K} \mathrm{NO}$ \\
\hline 77 & $E-2$ & 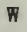 & $\mathrm{K}$ NO \\
\hline
\end{tabular}

COMPOUND NO $=458$ MOL WGT -

COMPOUND NO $=482$ MOL WGT -

COMPOUND NO $=483$ MOL WGT -
$25 \quad 2.88 \times 10-2 \mathbb{T} \quad$ BB UNSPECIFIED CONDUCTANCE

$30 \quad 2.74 \times 10-2 \%$ BB UNSPECIFIED CONDUCTANCE

$2.65 \times 10-2$ T BB UNSPECIFIED CONDUCTANCE

$2.76 \times 10-2$ T BB UNSPECIFIED CONDUCTANCE

$3.07 \times 10-2$ \% BB UNSPECIFIED CONDUCTANCE

$3.54 \times 10-2$ พ $B$ B UNSPECIFIED CONDUCTANCE

$2.43 \times 10-2$ W BB UNSPECIFIED CONDUCTANCE

$2.22 \times 10-2 \mathrm{~W}$ BB UNSPECIFIED CONDUCTANCE

$2.01 \times 10-2$ T BB UNSPECIFIED CONDUCTANCE

$2.40 \times 10-2$ T $B B$ UNSPECIFIED CONDUCTANCE

$2.07 \times 10-2 \mathrm{~W}$ BB UNSPECIFIED CONDUCTANCE

$1.79 \times 10-2$ T BB UNSPECIFIED CONDUCTANCE

$1.46 \times 10-2$ \% $B$ B UNSPECIFIED CONDUCTANCE

$2.42 \times 10-2$ W BB UNSPECIFIED CONDUCTANCE

$2.17 \times 10-2 \mathrm{~W}$ BB UNSPECIFIED CONDUCTANCE

$1.93 \times 10-2 \pi$ BB UNSPECIFIED CONDUCTANCE

$1.82 \times 10-2 \mathrm{~T}$ BB UNSPECIFIED CONDUCTANCE

$1.65 \times 10-2 \mathrm{~W}$ BB UNSPECIFIED CONDUCTANCE

$1.49 \times 10-2$ \% BB UNSPECIFIED CONDUCTANCE

$2.75 \times 10-2 \%$ BB UNSPECIFIED CONDUCTANCE

$2.22 \times 10-2 \mathrm{~W}$ BB UNSPECIFIED CONDUCTANCE

$1.82 \times 10-2 \mathrm{~W}$ BB UNSPECIFIED CONDUCTANCE

$2.59 \times 10-2 \pi$ BB UNSPECIFIED CONDUCTANCE

$2.27 \times 10-2 \pi$ BB UNSPECIFIED CONDUCTANCE
SHIN KATS 64011 T 3

SHIN KATS 64011 T 3

SHIN KATS 64011 T 3

SHIN KATS 64011 T 3

SHIN KATS 64011 T 3

SHIN KATS 64011 T 3

SHIN KATS 64011 L 3

SHIN KATS 64011 L 3

SHIN KATS 64011 L 3

SHIN KATS 64011 L 3

SHIN KATS 64011 L 3

SHIN KATS 64011 L 3

SHIN KATS 64011 L 3

SHIN KATS 64011 L 3

SHIN KATS 64011 L 3

SHIN KATS 64011 L 3

SHIN KATS 64011 L 3

SHIN KATS 64011 L 3

SHIN KATS 64011 L 3

SHIN KATS 64011 L 3

SHIN KATS 64011 L 3

SHIN KATS 64011 L 3

SHIN KATS 64011 L 3

SHIN KATS 64011 L 3

347.3 DECYL PYRIDINIUM IODIDE

RM $2.25 \times 10-2 \mathrm{M} \quad \mathrm{CB}$ MICELLAR SPECTRAL CHANGE HARK KRIZ 51010 T 3

248.4 DODECYL AMMONIUM NITRATE

$\begin{array}{lllllllll}30 & 9.9 & \text { XIO-3 M } & \text { BC } & \text { EQUIV CONDCTNCE GRAPH } & \text { RALS EGGE } & 49008 & \mathrm{~K} & 3\end{array}$

306.0 DIOCTYL DIMETHYL AMMONIUM CHLORIDE

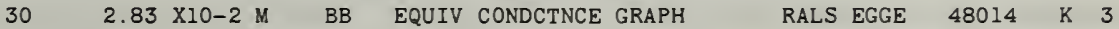

Concentration units: $\mathrm{A}-\operatorname{mol} \%: \mathrm{B}-\mathrm{vol} \%$ solvent: $\mathrm{C}-$ mol $\%$ surfactant mixture

counterions: $\mathrm{M}-$ molar: $\mathrm{N}$ - normal: $\mathrm{P}-\mathrm{wt} \%: \mathrm{Q}-\mathrm{wt} \%$ surfactant: $\mathrm{R}$-varied; $\mathrm{S}-$ mol/

D-wt/vol \%: E- \% saturation; $\mathrm{H}-$ wt $\%$ solvent; I - mol \% surfactant: $\mathrm{K}-$ normality

kg: $\mathrm{T}-w \mathrm{~F} \%$ surfactant mixture: $\mathrm{U}-\mathrm{mol} /(\mathrm{l}$ or $\mathrm{kg})$ : $\overline{\mathrm{W}}-$ molal; $\mathrm{Y}-\mathrm{atm}$. Details on page 222 


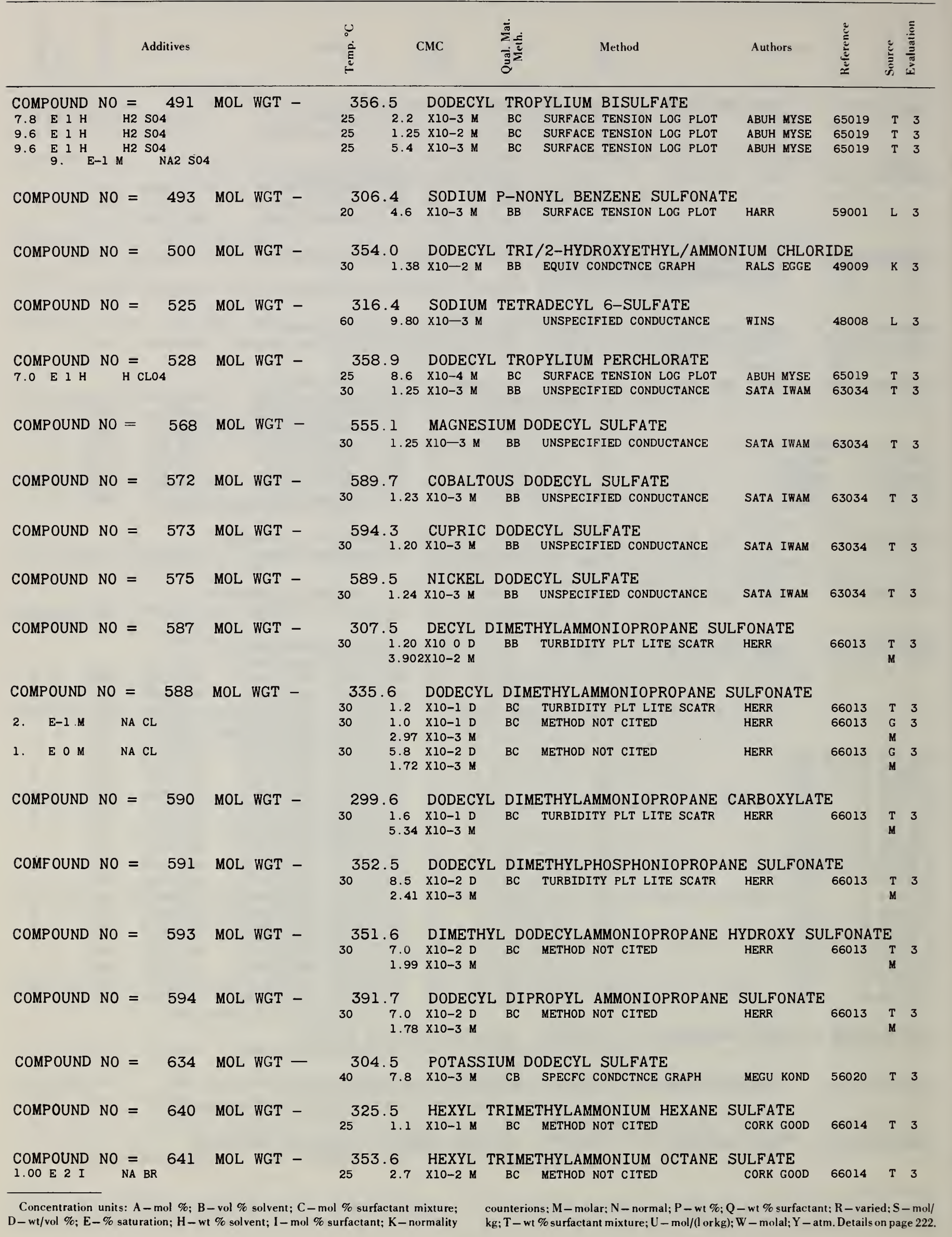




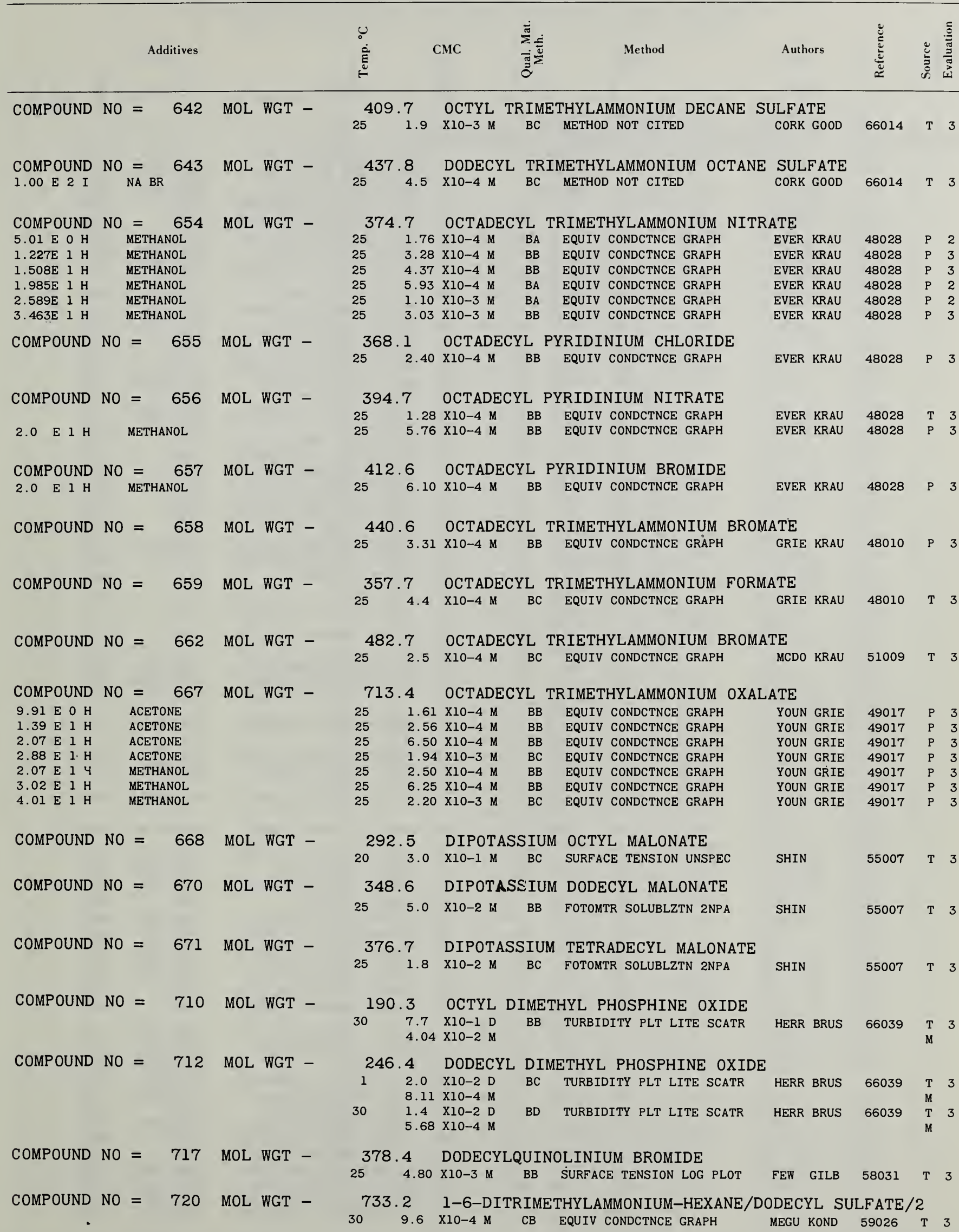

Concentration units: $\mathrm{A}-\mathrm{mol} \% ; \mathrm{B}-\mathrm{vol} \%$ solvent; $\mathrm{C}-$ mol \% surfactant mixture; counterions; M-molar; $\mathrm{N}$ - normal; $\mathrm{P}-$ wt \%; $\mathrm{Q}$ - wt \% surfactant; $\mathrm{R}$-varied; $\mathrm{S}-\mathrm{mol} /$ $\mathrm{D}-\mathrm{wt} / \mathrm{vol} \% ; \mathrm{E}-\%$ saturation; $\mathrm{H}-$ wt $\%$ solvent; $\mathrm{I}-\mathrm{mol} \%$ surfactant; $\mathrm{K}$-normality 


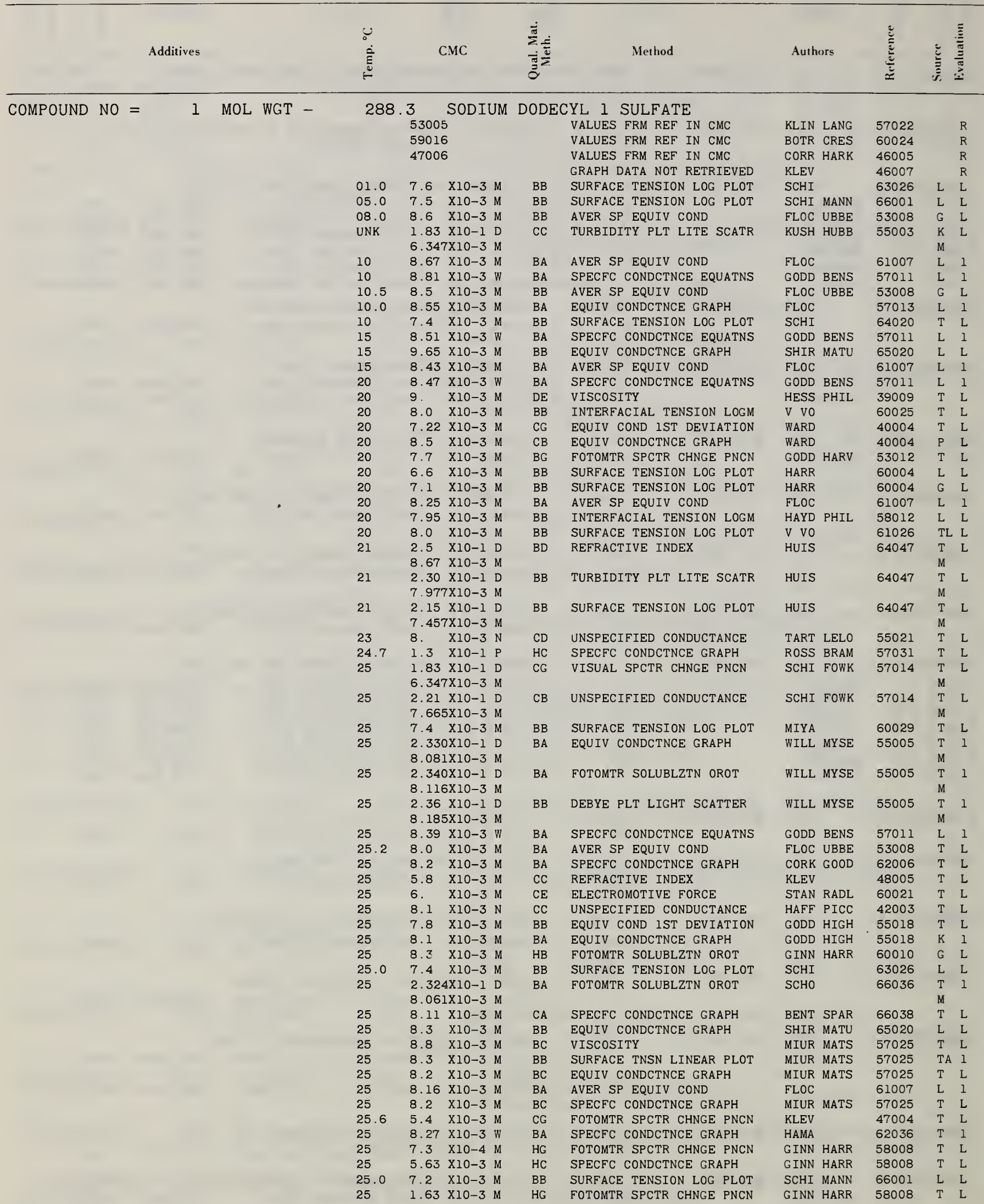

Concentration units: $\mathrm{A}-\mathrm{mol} \% ; \mathrm{B}-\mathrm{vol} \%$ solvent; $\mathrm{C}-$ mol $\%$ surfactant mixture: D-w1/vol \%; E- \% saturation; $\mathrm{H}-$ wt $\%$ solvent; I-mol \% surfactant; $\mathrm{K}$-normality

counterions: $\mathrm{I}$ - molar: N-normal: P-wit \%; Q-wt \% surfactant: $R$-varied: $S-$ mol/ kg: T - wt \% surfactant mixture; $U-$ mol/(l or kg): $W$ - molal; $Y$ - atm. Detailson page 222. 


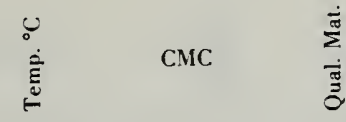

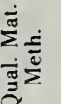

Method

Authors

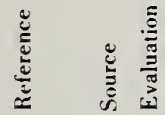

$4.03 \times 10-3 \mathrm{M}$

$7.2 \times 10-2 \mathrm{D}$

$7.2 \times 10-3 \mathrm{M}$

2. $29 \times 10-1 \mathrm{D}$

7. $943 \times 10-3 \mathrm{M}$

$25.0 \quad 8.22 \times 10-3 \mathrm{M}$

$25.07 .83 \times 10-3 \mathrm{M}$

8. $2 \times 10-3 \mathrm{M}$

$8.27 \times 10-3 \mathrm{M}$

$8.0 \times 10-3 \mathrm{M}$

8. $15 \times 10-3 \mathrm{M}$

$8.2 \times 10-3 \mathrm{M}$

8. $4 \mathrm{X} 10-3 \mathrm{M}$

$6.0 \times 10-3 \mathrm{M}$

$6.02 \times 10-3 \mathrm{M}$

$6.12 \times 10-3 \mathrm{M}$

$6.02 \times 10-3 \mathrm{M}$

$7.7 \times 10-3 \mathrm{M}$

$1.00 \times 10-2 \mathrm{M}$

8. $0 \times 10-3 \mathrm{M}$

$8.44 \times 10-3 \mathrm{~W}$

5. $\mathrm{X} 10-3 \mathrm{M}$

$7.0 \times 10-3 \mathrm{~W}$

$8.23 \times 10-3 \mathrm{M}$

$8.57 \times 10-3 \mathrm{~W}$

8. $4 \times 10-3 \mathrm{M}$

$9.18 \times 10-3 \mathrm{~N}$

8. $39 \times 10-3 \mathrm{M}$

$5.0 \times 10-3 \mathrm{M}$

$7.2 \times 10-3 \mathrm{M}$

$1.2 \times 10-2 \mathrm{M}$

$8.88 \times 10-3$

$40.18 .1 \times 10-3 \mathrm{M}$

$6.1 \times 10-3 \mathrm{M}$

$6.1 \mathrm{X} 10-3 \mathrm{M}$

$7.00 \times 10-3 \mathrm{M}$

$8.60 \times 10-3 \mathrm{M}$

$8.65 \times 10-3 \mathrm{M}$

$7.2 \times 10-3 \mathrm{M}$

8. $\mathrm{X} 10-3 \mathrm{M}$

$8.9 \times 10-3 \mathrm{M}$

9.10 X10-3 W

$8.86 \times 10-3 \mathrm{M}$

4. $6 \mathrm{X10}-3 \mathrm{M}$

$7.6 \times 10-3 \mathrm{M}$

$7.6 \times 10-3 \mathrm{M}$

$7.2 \mathrm{X} 10-3 \mathrm{M}$

$8.1 \times 10-3 \mathrm{M}$

$9.3 \times 10-3 \mathrm{M}$

$8.1 \times 10-3 \mathrm{M}$

$8.1 \mathrm{X} 10-3 \mathrm{M}$

$9.61 \times 10-3 \mathrm{~W}$

$8.00 \times 10-3 \mathrm{M}$

$7.3 \times 10-3 \mathrm{~W}$

$8.9 \times 10-3 \mathrm{M}$

$9.18 \times 10-3 \mathrm{M}$

$5.0 \times 10-3 \mathrm{M}$

$6.8 \mathrm{X} 10-3 \mathrm{M}$

$6.8 \times 10-3 \mathrm{M}$

$9.2 \times 10-3 \mathrm{M}$

$9.95 \times 10-3 \mathrm{~W}$

$9.8 \times 10-3 \mathrm{M}$

$9.3 \times 10-3 \mathrm{M}$

$9.49 \times 10-3 \mathrm{M}$

$2.26 \times 10-1 \mathrm{D}$

$7.839 \times 10-3 \mathrm{M}$

$7.8 \times 10-3 \mathrm{M}$

$9.61 \times 10-3 \mathrm{M}$

$4.5 \mathrm{X} 10-3 \mathrm{M}$

$7.3 \times 10-3 \mathrm{M}$

1. $10 \times 10-2 \mathrm{M}$

1. $016 \times 10-2 \mathrm{M}$

2. $25 \times 10-1 \mathrm{D}$

7.804X10-3 M

$65 \quad 1.091 \times 10-2 \mathrm{M}$

1. $4 \mathrm{X} 10-2 \mathrm{M}$

HC SPECFC CONDCTNCE GRAPH

\section{HE METHOD NOT CITED}

SURFACE TENSION LOG PLOT

FOTOMTR SOLUBLZTN OROT

SPECFC CONDCTNCE GRAPH

EQUIV CONDCTNCE GRAPH

UNSPECIFIED CONDUCTANCE

SPECFC CONDCTNCE EQUATNS

EQUIV CONDCTNCE GRAPH

SURFACE TENSION LOG PLOT

SURFACE TNSN LINEAR PLOT

SPECFC CONDCTNCE GRAPH

REFRACTIVE INDEX

VISUAL SPCTR CHNGE PNCN

VISUAL SPCTR CHNGE RHD6

VISUAL SPCTR CHNGE PNCN

SURFACE TENSION LOG PLOT

SPECFC CONDCTNCE GRAPH

VELOCITY OF SOUND

SPECFC CONDCTNCE EQUATNS

SURFACE TENSION MINIMUM

VAPR PRESURE LOWERING

AVER SP EQUIV COND

SPECFC CONDCTNCE EQUATNS

SPECFC CONDCTNCE GRAPH

EQUIV CONDCTNCE GRAPH

AVER SP EQUIV COND

FOTOMTR SPCTR CHNGE PNCN

TURBIDMTR SOLUBLZTN LOH

VISCOSITY MINIMUM

SPECFC CONDCTNCE EQUATNS

AVER SP EQUIV COND

VISUAL SPCTR CHNGE PNCN

VISUAL SPCTR CHNGE RHD6

FOTOMTR SPCTR CHNGE PNCN

AVER SP EQUIV COND

SPECFC CONDCTNCE GRAPH

VELOCITY OF SOUND

VISCOSITY

SPECFC CONDCTNCE GRAPH

SPECFC CONDCTNCE EQUATNS

AVER SP EQUIV COND

FOTOMTR SPCTR CHNGE PNCN

SURFACE TENSION LOG PLOT

SURFACE TENSION LOG PLOT

TURBIDMTR SOLUBLZTN LOH

EQUIV COND IST DEVIATION

EQUIV CONDCTNCE GRAPH

INTERFACIAL TENSION LOGM

SURFACE TENSION LRG PLOT

SPECFC CONDCTNCE EQUATNS

SURFACE TENSION UNSPEC

VAPR PRESURE LOWERING

FOTOMTR SOLUBLZTN OROT

AVER SP EQUIV COND

VISUAL SPCTR CHNGE PNCN

VISUAL SPCTR CHNGE PNCN

VISUAL SPCTR CHNGE PNCN

SURFACE TENSION LOG PLOT

SPECFC CONDCTNCE EQUATNS

SPECFC CONDCTNCE GRAPH

AVER SP EOUIV COND

EQUIV CONDCTNCE GRAPH

UNSPECIFIED CONDUCTANCE

BB SURFACE TENSION LOG PLOT

BA AVER SP EQUTV COND

CG FOTOMTR SPCTR CHNGE PNCN

TURBIDMTR SOLUBLZTN LOH

SPECFC CONDCTNCE GRAPH

AVER SP EQUIV COND

FOTOMTR SOLUBLZTN OROT

BA AVER SP EQUIV COND

XG VISCOSITY MINIMUM
Authors

2008

GINN HARR

GINN HARR

SCHI

61014 T $\mathrm{L}$

GINN KINN

MYSE OTTE

MYSE OTTE

MIUR ARIS

MUKE KAPA

NAKA KAWA

ELWO MYSE

ELWO MYSE

ELWO MYSE

LIN

CORR HARK

CORR HARK

CORR HARK

BRAD

MARK TSIK

SHIG

GODD BENS

KLEV RAIS

HUFF MCBA

GODD BENS

CORK GOOD

SHIR MATU

FLOC

KLEV

MIUR ARIS

SATA TYUZ

GODD BENS

FLOC UBBE

MIYA

MIYA

GODD HARV

FLOC

KUPP SURY

NAKA NINO

MEGU KOND

GODD BENS

FLOC

KLEV

SCHI MANN

SCHI

MIUR ARIS

LANG

LANG

KLIN LANG

KLIN LANC

GODD BENS

GOTT

HUFF MCBA

GINN HARR

FLOC

RAIS

WEIL STIR

WEIL STIR

MIYA

GODD BENS

CORK GOOD

FLOC UBBE

FLOC

SCHT FOWK

57014

SCHI $\quad 63026$

FLOC

KLEV

MIUR ARIS 58023

MARK TSIK

FLOC

GINN KINN 61015

$\begin{array}{lll}\text { FLOC } & 61007 \\ \text { SATA } & \text { TYUZ } & 53006\end{array}$ 


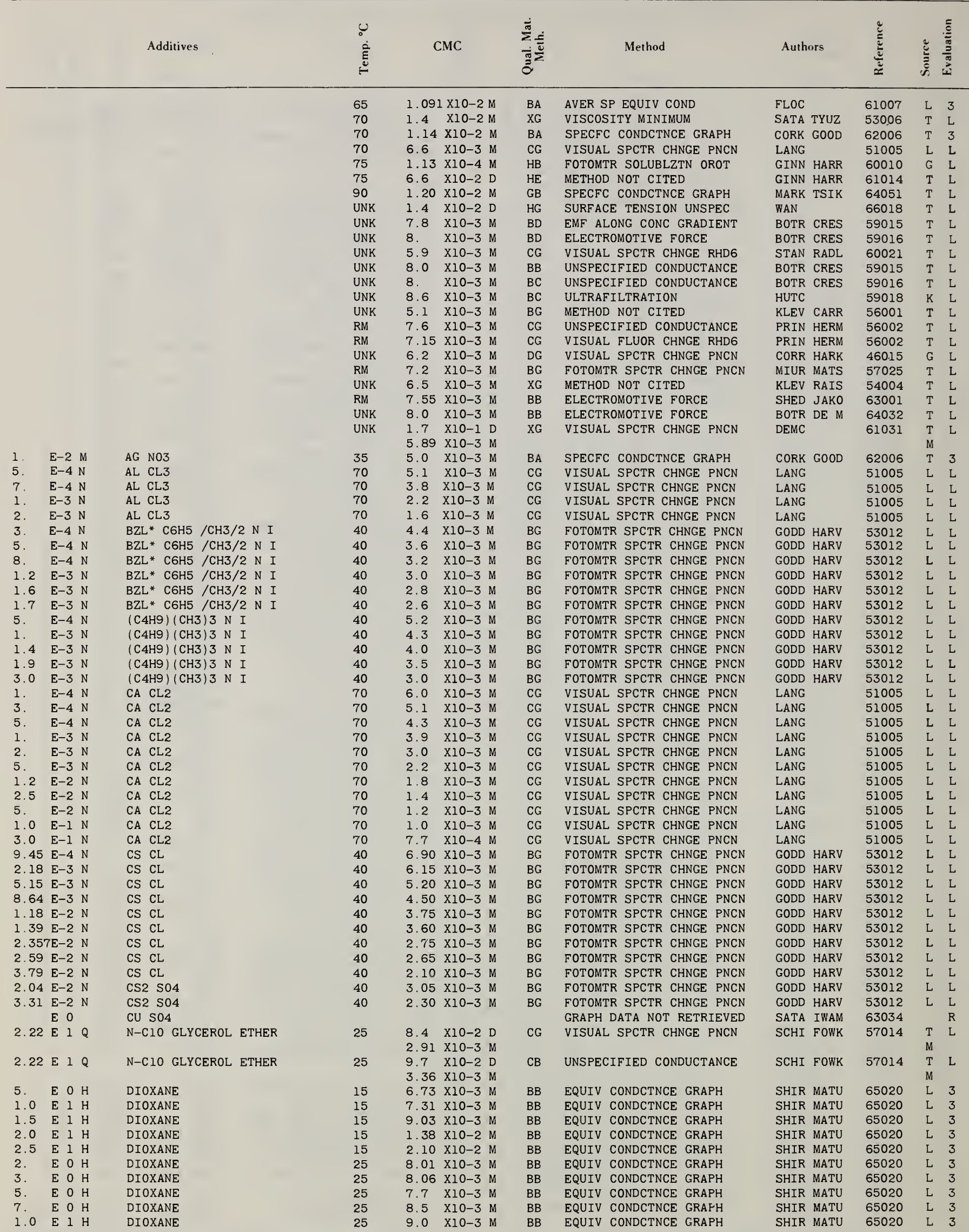

Concentration units: $\mathrm{A}-$ mol \%; $\mathrm{B}-\mathrm{vol} \%$ solvent: $\mathrm{C}-$ mol \% surfactant mixture; 


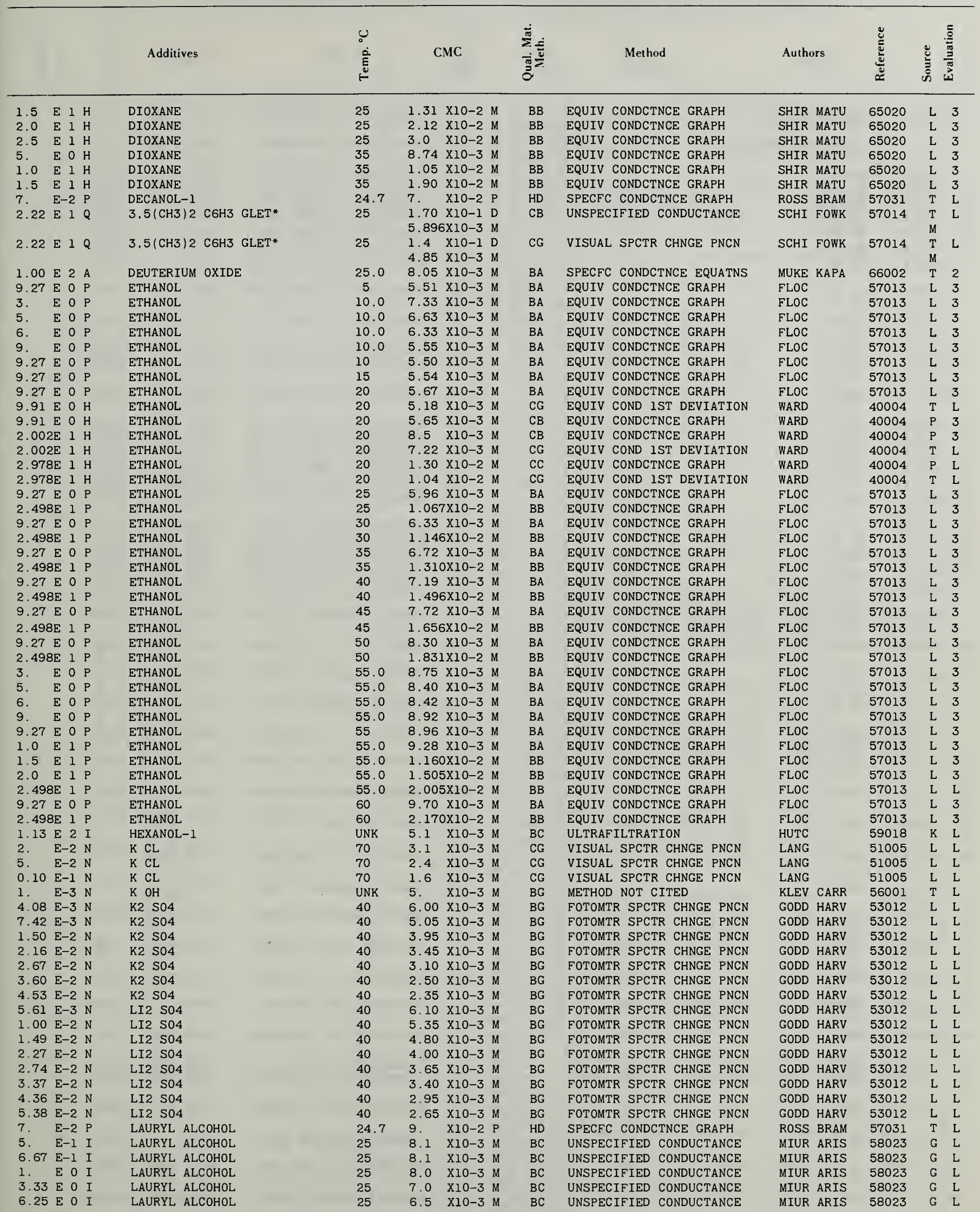

Concentration units: $\mathrm{A}-\mathrm{mol} \%$; $\mathrm{B}-\mathrm{vol} \%$ solvent; $\mathrm{C}-$ mol \% surfactant mixture; 


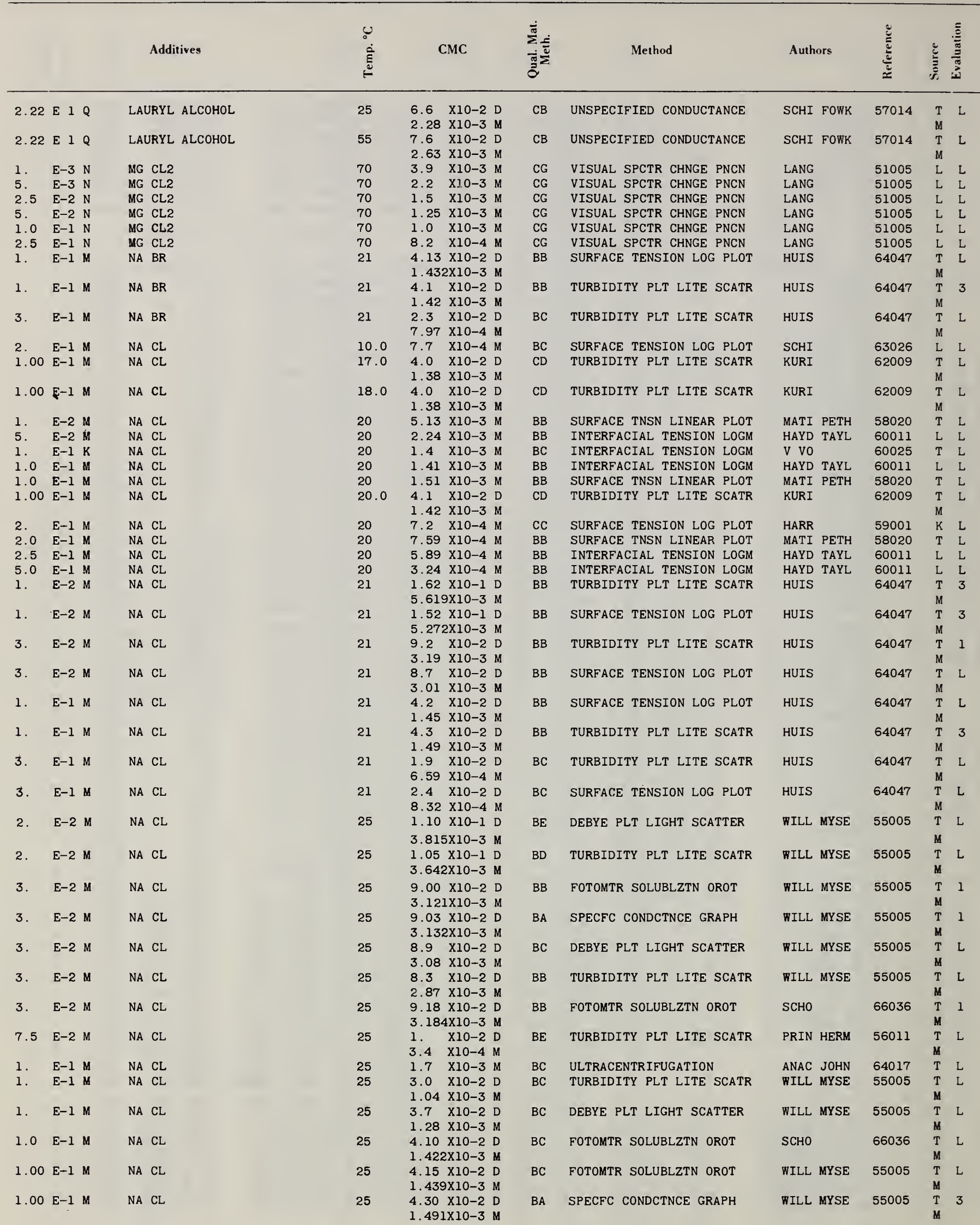

Concentration units: $\mathrm{A}-\mathrm{mol} \% ; \mathrm{B}-$ vol \% solvent; $\mathrm{C}-$ mol \% surfactant mixture; 
CMC

Authors

\begin{tabular}{|c|c|c|c|c|c|c|c|c|c|c|c|c|c|c|c|}
\hline 2. & $E-1$ & M & NA & $\mathrm{CL}$ & 25 & $\begin{array}{l}2.8 \\
9.71\end{array}$ & $\begin{array}{l}X 10-2 \\
\times 10-4\end{array}$ & $\begin{array}{l}D \\
M\end{array}$ & $\mathrm{BD}$ & DEBYE PLT LIGHT SCATTER & WILL & MYSE & 55005 & $\begin{array}{l}\mathrm{T} \\
\mathrm{M}\end{array}$ & L \\
\hline 2. & E-1 & M & NA & CL & 25 & $\begin{array}{l}2.5 \\
8.67\end{array}$ & $\begin{array}{l}\times 10-2 \\
\times 10-4\end{array}$ & $\begin{array}{l}D \\
M\end{array}$ & $\mathrm{BC}$ & TURBIDITY PLT LITE SCATR & WILL & MYSE & 55005 & $\begin{array}{l}\mathrm{T} \\
\mathrm{M}\end{array}$ & $\mathrm{L}$ \\
\hline 2. & $\mathrm{E}-1$ & M & NA & CL & 25.0 & 7.5 & $\mathrm{X} 10-4$ & M & $\mathrm{BC}$ & SURFACE TENSION LOG PLOT & SCHI & & 63026 & L & $\mathrm{L}$ \\
\hline 2. & $\mathrm{E}-1$ & M & NA & CL & 25.0 & 9.0 & $\times 10-4$ & M & BA & SPECFC CONDCTNCE GRAPH & MYSE & MYSE & 65018 & $\mathrm{~T}$ & 3 \\
\hline 3. & E-1 & M & NA & CL & 25.0 & 7. & $\mathrm{X} 10-4$ & M & $\mathrm{BD}$ & SPECFC CONDCTNCE GRAPH & MYSE & MYSE & 65018 & $\mathrm{~T}$ & L \\
\hline 4. & E-1 & M & NA & $\mathrm{CL}$ & 25 & $\begin{array}{l}1.7 \\
5.89\end{array}$ & $\begin{array}{l}X 10-2 \\
X 10-4\end{array}$ & $\begin{array}{l}\mathrm{D} \\
\mathrm{M}\end{array}$ & $\mathrm{BD}$ & DEBYE PLT LIGHT SCATTER & WILL & MYSE & 55005 & $\begin{array}{l}\mathrm{T} \\
\mathrm{M}\end{array}$ & L \\
\hline 4. & $E-1$ & M & NA & $\mathrm{CL}$ & 25 & $\begin{array}{l}1.6 \\
5.54\end{array}$ & $\begin{array}{l}X 10-2 \\
\times 10-4\end{array}$ & $\begin{array}{l}\text { D } \\
\text { M }\end{array}$ & $\mathrm{BD}$ & TURBIDITY PLT LITE SCATR & WILL & MYSE & 55005 & $\begin{array}{l}\mathrm{T} \\
\mathrm{M}\end{array}$ & $\mathbf{L}$ \\
\hline 4.0 & $\mathrm{E}-1$ & M & NA & CL & 25 & $\begin{array}{l}1.894 \\
6.569\end{array}$ & $\begin{array}{l}4 \times 10-2 \\
9 \times 10-4\end{array}$ & $\begin{array}{l}D \\
M\end{array}$ & $\mathrm{BC}$ & FOTOMTR SOLUBLZTN OROT & SCHO & & 66036 & $\begin{array}{l}\mathrm{T} \\
\mathrm{M}\end{array}$ & $\mathrm{L}$ \\
\hline 2.05 & $\mathrm{E}-3$ & M & NA & CL & 26 & 5.11 & $\mathrm{x} 10-3$ & M & DG & VISUAL SPCTR CHNGE PNCN & CORR & HARK & 47010 & $\mathrm{~T}$ & $\mathrm{~L}$ \\
\hline 4.88 & $\mathrm{E}-3$ & M & NA & CL & 26 & 4.47 & $\times 10-3$ & $M$ & DG & VISUAL SPCTR CHNGE PNCN & CORR & HARK & 47010 & $\mathrm{~T}$ & L \\
\hline 9.36 & $\mathrm{E}-3$ & M & NA & CL & 26 & 4.19 & $x 10-3$ & M & DG & VISUAL SPCTR CHNGE PNCN & CORR & HARK & 47010 & $\mathrm{~T}$ & L \\
\hline 1.85 & $\mathrm{E}-2$ & M & NA & CL & 26 & 3.40 & $\mathrm{x} 10-3$ & $\mathbf{M}$ & DG & VISUAL SPCTR CHNGE PNCN & CORR & HARK & 47010 & $\mathbf{T}$ & L \\
\hline 3.11 & $\mathrm{E}-2$ & $\mathbf{M}$ & NA & $\mathrm{CL}$ & 26 & 2.75 & $\mathrm{x} 10-3$ & M & DG & VISUAL SPCTR CHNGE PNCN & CORR & HARK & 47010 & $\mathrm{~T}$ & L \\
\hline 4.60 & $E-2$ & M & NA & CL & 26 & 2.11 & $\mathrm{X} 10-3$ & $\mathbf{M}$ & DG & VISUAL SPCTR CHNGE PNCN & CORR & HARK & 47010 & $\mathrm{~T}$ & L \\
\hline 7.53 & $\mathrm{E}-2$ & M & NA & CL & 26 & 1.73 & $x 10-3$ & M & DG & VISUAL SPCTR CHNGE PNCN & CORR & HARK & 47010 & $\mathbf{T}$ & L \\
\hline 1.47 & $\mathrm{E}-1$ & $\mathbf{M}$ & NA. & $\mathrm{CL}$ & 26 & 1.35 & $x 10-3$ & M & DG & VISUAL SPCTR CHNGE PNCN & CORR & HARK & 47010 & $\mathrm{~T}$ & L \\
\hline 2.43 & $E-1$ & M & NA & CL & 26 & 1.11 & $\times 10-3$ & $M$ & DG & VISUAL SPCTR CHNGE PNCN & CORR & HARK & 47010 & $\mathrm{~T}$ & L \\
\hline 2.69 & E-1 & M & NA & CL & 26 & 1.10 & $\times 10-3$ & M & DG & VISUAL SPCTR CHNGE PNCN & CORR & HARK & 47010 & $\mathrm{~T}$ & L \\
\hline 3.16 & $\mathrm{E}-1$ & M & NA & CL & 26 & 9.73 & $\mathrm{X} 10-4$ & M & DG & VISUAL SPCTR CHNGE PNCN & CORR & HARK & 47010 & $\mathrm{~T}$ & L \\
\hline 3.54 & $\mathrm{E}-1$ & M & NA & CL & 26 & 8.66 & $\mathrm{X} 10-4$ & M & DG & VISUAL SPCTR CHNGE PNCN & CORR & HARK & 47010 & $\mathrm{~T}$ & L \\
\hline 5. & $\mathrm{E}-2$ & M & NA & CL & 30 & 2.34 & $\mathrm{X} 10-3$ & M & $\mathrm{CC}$ & VELOCITY OF SOUND & SHIG & & 66010 & G & $\mathrm{L}$ \\
\hline 1 . & E-1 & M & NA & CL & 30 & 1.63 & $\times 10-3$ & M & $\mathrm{CC}$ & VELOCITY OF SOUND & SHIG & & 66010 & G & L \\
\hline 1.00 & $E-1$ & M & NA & CL & 30.0 & $\begin{array}{l}4.8 \\
1.66\end{array}$ & $\begin{array}{l}\times 10-2 \\
\times 10-3\end{array}$ & $\begin{array}{l}\text { D } \\
M\end{array}$ & $\mathrm{CD}$ & TURBIDITY PLT LITE SCATR & KURI & & 62009 & $\begin{array}{l}\mathrm{T} \\
M\end{array}$ & $\mathrm{~L}$ \\
\hline 2. & $E-1$ & M & NA & CL & 30 & $\begin{array}{l}3.0 \\
1.04\end{array}$ & $\begin{array}{l}\times 10-2 \\
\times 10-3\end{array}$ & $\begin{array}{l}\mathrm{D} \\
\mathrm{M}\end{array}$ & $\mathrm{CD}$ & TURBIDITY PLT LITE SCATR & KURI & NAKA & 62010 & $\begin{array}{l}T \\
M\end{array}$ & L \\
\hline 5. & $E-1$ & M & NA & CL & 30 & 5.9 & $\times 10-4$ & M & $\mathrm{CD}$ & VELOCITY OF SOUND & SHIG & & 66010 & G & L \\
\hline 1. & $E-2$ & M & NA & CL & 40 & 5.37 & $x 10-3$ & $M$ & BB & SURFACE TNSN LINEAR PLOT & MATI & PETH & 58020 & $\mathrm{~T}$ & L \\
\hline 6.75 & $E-2$ & M & NA & CL & 40 & 1.6 & $\times 10-3$ & M & CG & FOTOMTR SPCTR CHNGE RHD6 & MEGU & KOND & 56020 & $\mathrm{~T}$ & L \\
\hline 1.0 & $E-1$ & M & NA & CL & 40 & 1.62 & $\times 10-3$ & $M$ & BB & SURFACE TNSN LINEAR PLOT & MATI & PETH & 58020 & $\mathrm{~T}$ & $\mathrm{~L}$ \\
\hline 2.0 & $E-1$ & $\mathbf{M}$ & NA & CL & 40 & 8.71 & $X 10-4$ & M & BB & SURFACE TNSN LINEAR PLOT & MATI & PETH & 58020 & $\mathrm{~T}$ & $\mathrm{~L}$ \\
\hline 1.00 & $E-1$ & M & NA & CL & 50.2 & $\begin{array}{l}7.2 \\
2.49\end{array}$ & $\begin{array}{l}\times 10-2 \\
\times 10-3\end{array}$ & $\begin{array}{l}\mathrm{D} \\
\mathbf{M}\end{array}$ & $C D$ & TURBIDITY PLT LITE SCATR & KURI & & 62009 & $\begin{array}{l}\mathrm{T} \\
\mathrm{M}\end{array}$ & $L$ \\
\hline 2. & $E-1$ & M & NA & CL & 50 & $\begin{array}{l}4.0 \\
1.38\end{array}$ & $\begin{array}{l}\times 10-2 \\
\times 10-3\end{array}$ & $\begin{array}{l}\mathrm{D} \\
\mathrm{M}\end{array}$ & $\mathrm{CD}$ & TURBIDITY PLT LITE SCATR & KURI & NAKA & 62010 & $\begin{array}{l}\mathrm{T} \\
\mathrm{M}\end{array}$ & L \\
\hline 2. & $E-1$ & $M$ & NA & CL & 55.0 & 1.1 & $\mathrm{X} 10-3$ & M & $\mathrm{BD}$ & SURFACE TENSION LOG PLOT & SCHI & & 63026 & L & L \\
\hline 1. & $E-2$ & M & NA & CL & 60 & 6.17 & $x 10-3$ & $M$ & BB & SURFACE TNSN LINEAR PLOT & MATI & PETH & 58020 & $\mathrm{~T}$ & $\mathrm{~L}$ \\
\hline 1.0 & $E-1$ & M & NA & CL & 60 & 2.04 & $\mathrm{X} 10-3$ & $\mathbf{M}$ & BB & SURFACE TNSN LINEAR PLOT & MATI & PETH & 58020 & $\mathrm{~T}$ & $\mathbf{L}$ \\
\hline 2.0 & E-1 & M & NA & CL & 60 & 1.45 & $x 10-3$ & $\mathbf{M}$ & BB & SURFACE TNSN LINEAR PLOT & MATI & PETH & 58020 & $\mathrm{~T}$ & $\mathrm{~L}$ \\
\hline 1.00 & $E-1$ & M & NA & CL & 69.8 & $\begin{array}{l}1.10 \\
3.815\end{array}$ & $\begin{array}{r}\times 10-1 \\
5 \times 10-3\end{array}$ & $\begin{array}{l}D \\
M\end{array}$ & $C D$ & TURBIDITY PLT LITE SCATR & KURI & & 62009 & $\begin{array}{l}\mathrm{T} \\
\mathrm{M}\end{array}$ & L \\
\hline 1. & $E-2$ & $\mathrm{~N}$ & NA & CL & 70 & 4.5 & $\mathrm{X} 10-3$ & $\mathbf{M}$ & CG & VISUAL SPCTR CHNGE PNCN & LANG & & 51005 & L & L \\
\hline 2. & $\mathrm{E}-2$ & $\mathrm{~N}$ & NA & CL & 70 & 3.2 & $\mathrm{x} 10-3$ & M & CG & VISUAL SPCTR CHNGE PNCN & LANG & & 51005 & L & L \\
\hline 5. & $\mathrm{E}-2$ & N & NA & CL & 70 & 2.3 & $x 10-3$ & M & CG & VISUAL SPCTR CHNGE PNCN & LANG & & 51005 & L & L \\
\hline 1.0 & $\mathrm{E}-1$ & $\mathrm{~N}$ & NA & CL & 70 & 1.6 & $\mathrm{x} 10-3$ & M & CG & VISUAL SPCTR CHNGE PNCN & LANG & & 51005 & L & $\mathbf{L}$ \\
\hline 2. & $E-1$ & $M$ & NA & CL & 70 & $\begin{array}{l}6.0 \\
2.08\end{array}$ & $\begin{array}{l}\times 10-2 \\
\times 10-3\end{array}$ & $\begin{array}{l}D \\
M\end{array}$ & $\mathrm{CD}$ & TURBIDITY PLT LITE SCATR & KURI & NAKA & 62010 & $\begin{array}{l}T \\
M\end{array}$ & L \\
\hline 2.0 & $\mathrm{E}-1$ & N & NA & CL & 70 & 1.2 & $x 10-3$ & M & CG & VISUAL SPCTR CHNGE PNCN & LANG & & 51005 & L & $\mathrm{L}$ \\
\hline 5.0 & $\mathrm{E}-1$ & N & NA & CL & 70 & 7.5 & $\mathrm{X} 10-4$ & $M$ & CG & VISUAL SPCTR CHNGE PNCN & LANG & & 51005 & L & L \\
\hline 6.1 & $\mathrm{E}-3$ & $M$ & NA & CL & RM & 5.7 & $\mathrm{X} 10-3$ & $M$ & CG & VISUAL FLUOR CHNGE RHD6 & PRIN & HERM & 56002 & $\mathrm{~T}$ & $\mathrm{~L}$ \\
\hline 1.09 & $\mathrm{E}-2$ & $\mathrm{M}$ & NA & CL & $\mathrm{RM}$ & 5.0 & $\times 10-3$ & M & CG & VISUAL FLUOR CHNGE RHDG & PRIN & HERM & 56002 & $T$ & $\mathrm{~L}$ \\
\hline 1.83 & $E-2$ & M & NA & CL & $\mathrm{RM}$ & 4.1 & $x 10-3$ & $M$ & CG & VISUAL FLUOR CHNGE RHD6 & PRIN & HERM & 56002 & $\mathrm{~T}$ & $\mathrm{~L}$ \\
\hline 2. & $\mathrm{E}-2$ & M & NA & CL & UNK & $\begin{array}{l}7.1 \\
2.46\end{array}$ & $\begin{array}{l}\times 10-2 \\
\times 10-3\end{array}$ & $\begin{array}{l}\mathrm{D} \\
\mathrm{M}\end{array}$ & $\mathrm{CD}$ & TURBIDITY PLT LITE SCATR & KUSH & HUBB & 55003 & $\begin{array}{l}K \\
M\end{array}$ & $\mathrm{~L}$ \\
\hline 3.0 & $\mathrm{E}-2$ & M & NA & CL & RM & 3.5 & $\mathrm{x} 10-3$ & M & CG & VISUAL FLUOR CHNGE RHD6 & PRIN & HERM & 56002 & G & $\mathrm{L}$ \\
\hline 3.21 & $\mathrm{E}-2$ & $M$ & NA & CL & RM & 3.4 & $\mathrm{x} 10-3$ & M & CG & VISUAL FLUOR CHNGE RHD6 & PRIN & HERM & 56002 & $\mathrm{~T}$ & $\mathrm{~L}$ \\
\hline 4. & $\mathrm{E}-2$ & M & NA & CL & UNK & $\begin{array}{l}6.0 \\
2.08\end{array}$ & $\begin{array}{l}X 10-2 \\
\times 10-3\end{array}$ & $\begin{array}{l}\mathrm{D} \\
\mathrm{M}\end{array}$ & $\mathrm{CE}$ & TURBIDITY PLT LITE SCATR & KUSH & HUBB & 55003 & $\begin{array}{l}K \\
M\end{array}$ & $\mathrm{~L}$ \\
\hline 5.59 & $\mathrm{E}-2$ & M & NA & CL & RM & 3.0 & $\mathrm{X} 10-3$ & M & CG & VISUAL FLUOR CHNGE RHD6 & PRIN & HERM & 56002 & $\mathrm{~T}$ & L \\
\hline 6. & $\mathrm{E}-2$ & M & NA & CL & UNK & $\begin{array}{l}4.8 \\
1.66\end{array}$ & $\begin{array}{l}\times 10-2 \\
\times 10-3\end{array}$ & $\begin{array}{l}D \\
M\end{array}$ & $\mathrm{CE}$ & TURBIDITY PLT LITE SCATR & KUSH & HUBB & 55003 & $\begin{array}{l}\mathrm{K} \\
\mathrm{M}\end{array}$ & L \\
\hline 1.2 & $E-1$ & M & NA & CL & UNK & $\begin{array}{l}2.6 \\
9.01\end{array}$ & $\begin{array}{l}\times 10-2 \\
\times 10-4\end{array}$ & $\begin{array}{l}\mathrm{D} \\
\mathrm{M}\end{array}$ & $\mathrm{CE}$ & TURBIDITY PLT LITE SCATR & KUSH & HUBB & 55003 & $\begin{array}{l}K \\
M\end{array}$ & $\mathrm{~L}$ \\
\hline 2.0 & $E-1$ & M & NA & CL & UNK & $\begin{array}{l}1.1 \\
3.81\end{array}$ & $\begin{array}{l}\times 10-2 \\
\times 10-4\end{array}$ & $\begin{array}{l}\mathrm{D} \\
\mathrm{M}\end{array}$ & $\mathrm{CE}$ & TURBIDITY PLT LITE SCATR & KUSH & HUBB & 55003 & $\begin{array}{l}K \\
M\end{array}$ & L \\
\hline 1 . & $\mathrm{E}-2$ & M & NA & $F$ & 21 & $\begin{array}{l}1.61 \\
5.584\end{array}$ & $\begin{array}{r}\times 10-1 \\
4 \times 10-3\end{array}$ & $\begin{array}{l}\mathrm{D} \\
\mathrm{M}\end{array}$ & $\mathrm{BB}$ & TURBIDITY PLT LITE SCATR & HUIS & & 64047 & $\begin{array}{l}\mathrm{T} \\
\mathrm{M}\end{array}$ & 3 \\
\hline 3. & $\mathrm{E}-2$ & M & NA & $F$ & 21 & $\begin{array}{l}8.5 \\
2.94\end{array}$ & $\begin{array}{l}\times 10-2 \\
\times 10-3\end{array}$ & $\begin{array}{l}\mathrm{D} \\
\mathrm{M}\end{array}$ & $\mathrm{BB}$ & TURBIDITY PLT LITE SCATR & HUIS & & 64047 & $\begin{array}{l}\mathrm{T} \\
\mathrm{M}\end{array}$ & L \\
\hline
\end{tabular}

Concentration units: $\mathrm{A}-$ mol $\%$; $\mathrm{B}-$ vol \% solvent; $\mathrm{C}-$ mol $\%$ surfactant mixture 


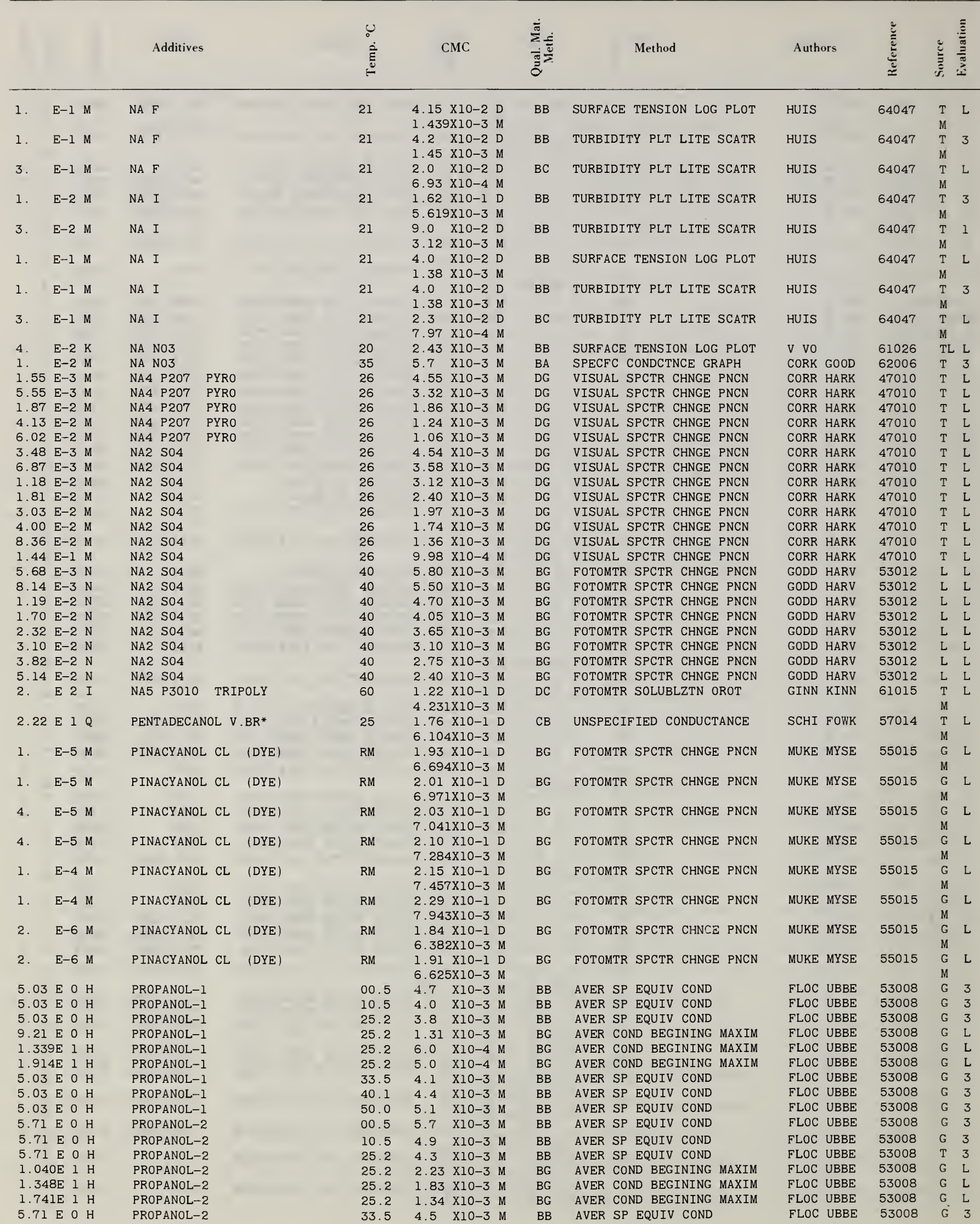

Concentration units: $\mathrm{A}-$ mol $\% ; \mathrm{B}-$ vol \% solvent; $\mathrm{C}-$ mol \% surfactant mixture; $\mathrm{D}-\mathrm{wt} / \mathrm{vol} \%$; E-\% saturation; $\mathrm{H}-\mathrm{wt} \%$ solvent; $\mathrm{I}-$ mol \% surfactant; $\mathrm{K}-$ normality counterions; $\mathrm{M}$ - molar; $\mathrm{N}$ - normal; $\mathrm{P}$ - wt \%; $\mathrm{Q}$ - wt \% surfactant; $\mathrm{R}$ - varied; $\mathrm{S}-$ mol/ $\mathrm{kg} ; \mathrm{T}-\mathrm{wt} \%$ surfactant mixture; $\mathrm{U}-\mathrm{mol} /(\mathrm{l}$ or $\mathrm{kg}) ; \mathrm{W}-$ molal; $\mathrm{Y}-\mathrm{atm}$. Detailson page 222. 


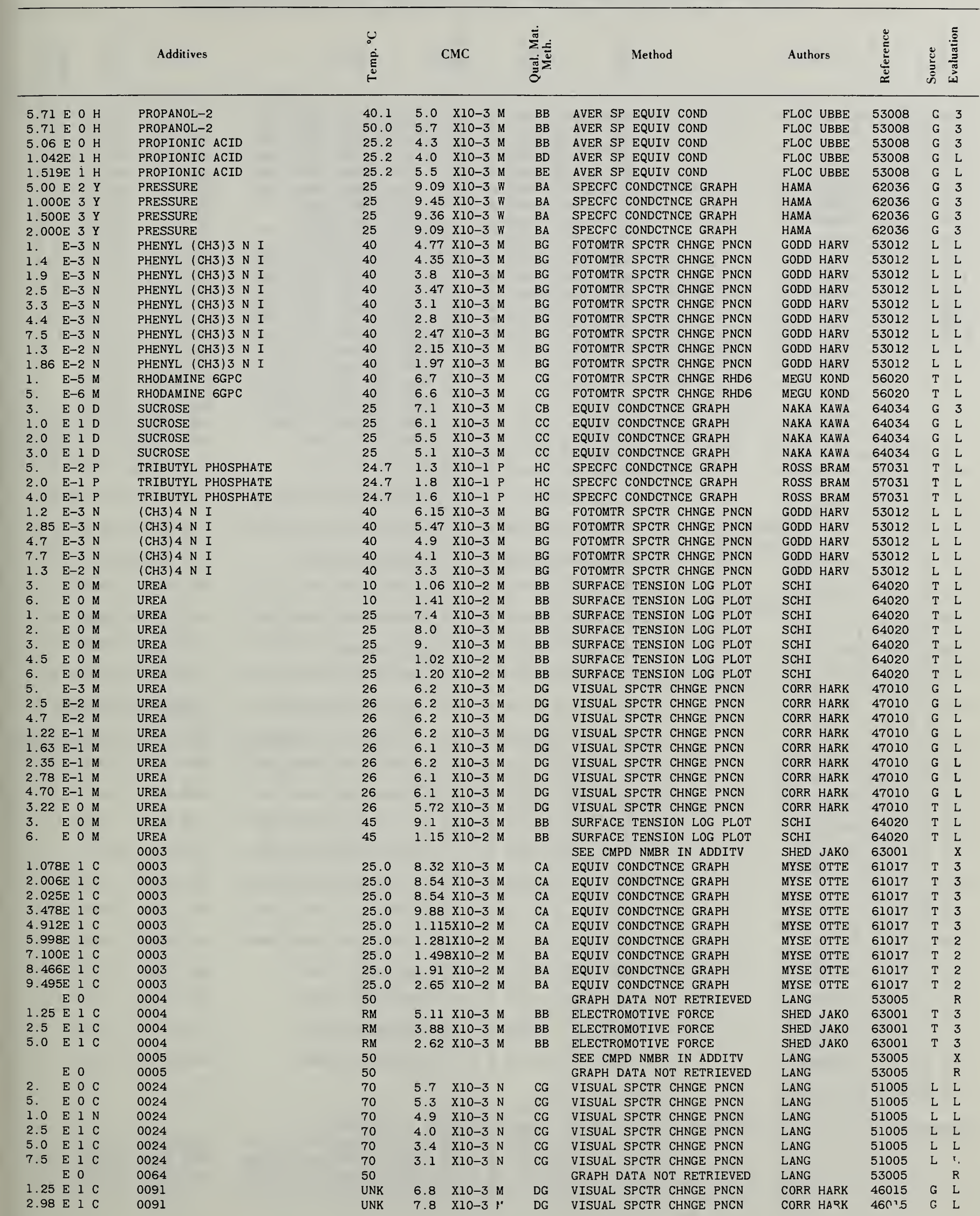

Concentration units: $\mathrm{A}-\mathrm{mol} \% ; \mathrm{B}-\mathrm{vol} \%$ solvent; $\mathrm{C}-\mathrm{mol} \%$ surfactant mixture; 


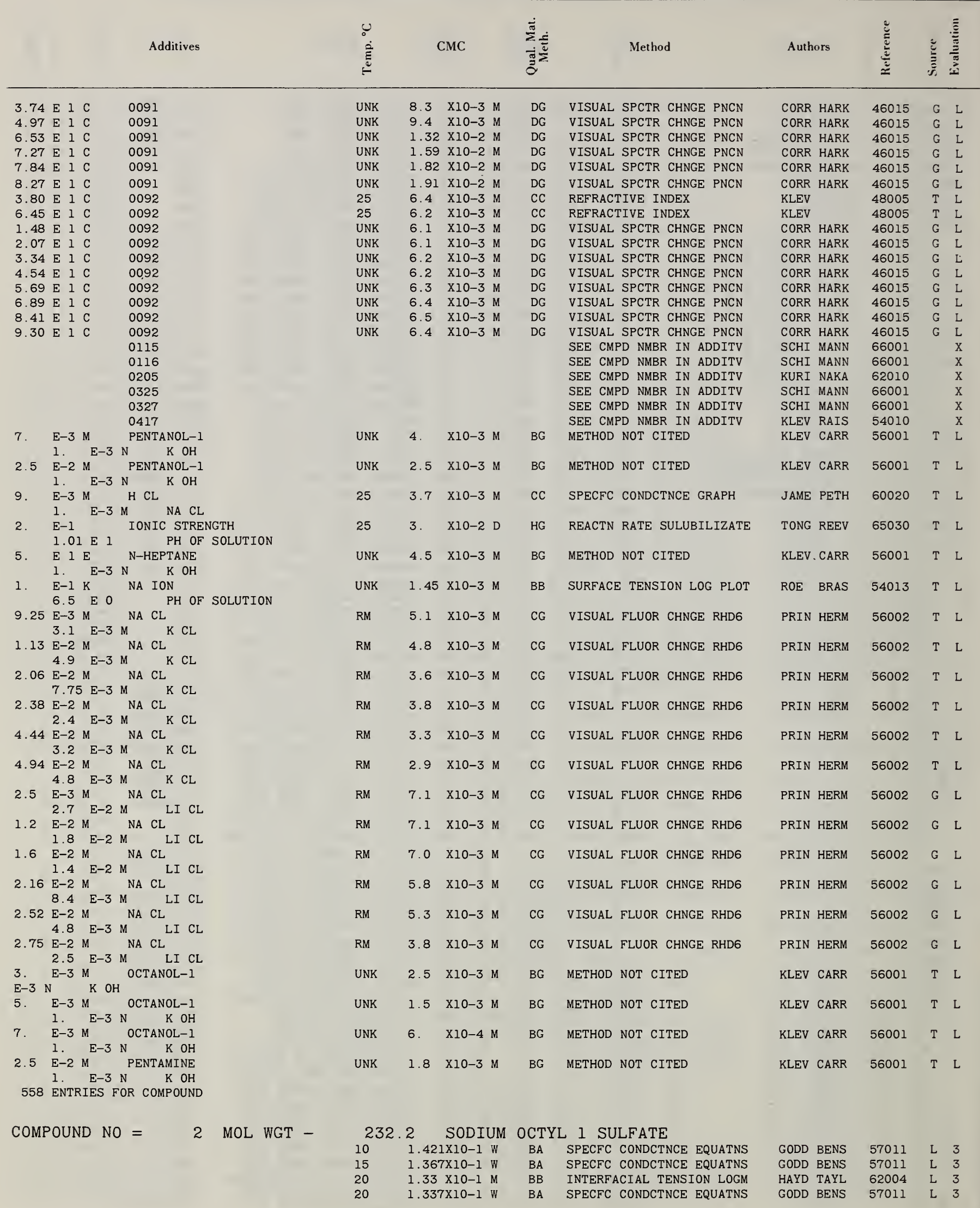

Concentration units: $\mathrm{A}-\mathrm{mol} \%$; $\mathrm{B}-\mathrm{vol} \%$ solvent; $\mathrm{C}-$ mol \% surfactant mixture; D-wt/vol \%; E-\% saturation; $\mathrm{H}-$ wt \% solvent; $\mathrm{I}-$ mol \% surfactant; $\mathrm{K}-$ normality counterions; $\mathrm{M}-$ molar; $\mathrm{N}$-normal; $\mathrm{P}-\mathrm{wt} \%$; $\mathrm{Q}-\mathrm{wt} \%$ surfactant; $\mathrm{R}$ - varied; $\mathrm{S}-$ mol/ $\mathrm{kg} ; \mathrm{T}-\mathrm{wt} \%$ surfactant mixture; $\mathrm{U}-\mathrm{mol} /(\mathrm{l}$ orkg); $\mathrm{W}-$ molal; $\mathrm{Y}-$ atm. Details on page 222. 
CMC

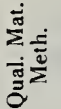

Method

Authors

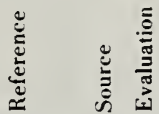

21
21
25
25
25
25

$2.96 \times 1000$

$.274 \times 10-1 \mathrm{M}$

$3.10 \times 100 \mathrm{D}$

1.335X10-1 M

7. $\quad \mathrm{X} 10-2 \mathrm{~N}$

$1.3 \times 10-1 \mathrm{~N}$

1.303X10-1 W

$3.0 \times 100$

1. $29 \times 10-1 \mathrm{M}$

$30.0 \quad 1.30 \times 10-1 \mathrm{M}$

$30 \quad 1.318 \times 10-1 \mathrm{~W}$

1.342X10-1 W

1. 363X10-1 W

$40.0 \quad 1.36 \times 10-1 \mathrm{M}$

1.381X10-1 W

$9.8 \times 10-2 \mathrm{M}$

1. $434 \times 10-1 \mathrm{~W}$

1. $463 \times 10-1 \mathrm{~W}$

2. $30 \times 101 \mathrm{D}$

9.905X10-1 M

$1.12 \times 10-1 \mathrm{M}$

$9.75 \times 10-2 \mathrm{M}$

$7.08 \times 10-2 \mathrm{M}$

$5.01 \times 10-2 \mathrm{M}$

$2.90 \times 100 \mathrm{D}$

1. 248X10-1 M

$2.70 \times 100 \mathrm{D}$

1.162X10-1 $\mathrm{M}$

$2.80 \times 100 \mathrm{D}$

$1.205 \times 10-1 \mathrm{M}$

$2.37 \times 100 \mathrm{D}$

$1.020 \times 10-1 \mathrm{M}$

$2.21 \times 100 \mathrm{D}$

9.517X10-2 M

1. $49 \times 100 \mathrm{D}$

6. $416 \times 10-2 \mathrm{M}$

$1.60 \times 100$

6.890X10-2

$8.0 \mathrm{X10-1} \mathrm{D}$

$3.44 \times 10-2 \mathrm{M}$

\section{BB}

BB

CD

$\mathrm{CC}$

BA

BD

CB

$B A$

BA

$\mathrm{BB}$

BA

$B B$
$B A$

$B A$

$\mathrm{XG}$

BB

$\mathrm{BB}$

$\mathrm{BB}$

$\mathrm{BB}$

$\mathrm{BB}$

BB

BB

BB

BB

BB

BB
SURFACE TENSION LOG PLOT TURBIDITY PLT LITE SCATR

SPECFC CONDCTNCE GRAPH UNSPECIFIED CONDUCTANCE SPECFC CONDCTNCE EQUATNS TURBIDITY PLT LITE SCATR

VELOCITY OF SOUND

SPECFC CONDCTNCE EOUATNS SPECFC CONDCTNCE EQUATNS SPECFC CONDCTNCE EQUATNS SPECFC CONDCTNCE GRAPH SPECFC CONDCTNCE EQUATNS INTERFACIAL TENSION LOGM SPECFC CONDCTNCE EQUATNS SPECFC CONDCTNCE EQUATNS VISUAL SPCTR CHNGE PNCN

INTERFACIAL TENSION LOGM INTERFACIAL TENSION LOGM INTERFACIAL TENSION LOGM INTERFACIAL TENSION LOGM SURFACE TENSION LOG PLOT

SURFACE TENSION LOG PLOT

TURBIDITY PLT LITE SCATR

TURBIDITY PLT LITE SCATR

SURFACE TENSION LOG PLOT

SURFACE TENSION LOG PLOT

TURBIDITY PLT LITE SCATR

TURBIDITY PLT LITE SCATR

32 ENTRIES FOR COMPOUND
260.3 SODIUM DECYL 1 SULFATE

61005

$3.88 \times 10-2 \mathrm{M}$

$3.64 \times 10-2 \mathrm{M}$

$3.48 \times 10-2$

$3.50 \times 10-2 \mathrm{M}$

$3.41 \times 10-2 \mathrm{~W}$

$3.39 \times 10-2 \mathrm{M}$

$3.35 \times 10-2$

$3.31 \times 10-2 \mathrm{M}$

$7.8 \times 10-1 \mathrm{D}$

$2.99 \times 10-2$

$7.9 \times 10-1 \mathrm{D}$

$3.03 \times 10-2 \mathrm{M}$

$3.1 \times 10-2 \mathrm{~N}$

$3.1 \times 10-2 \mathrm{~N}$

$3.32 \times 10-2 \mathrm{~W}$

$3.26 \times 10-2 \mathrm{M}$

$3.35 \times 10-2 \mathrm{M}$

$3.27 \times 10-2$

$8.4 \times 10-1 \mathrm{D}$

$3.22 \times 10-2 \mathrm{M}$

$8.5 \times 10-1 \mathrm{D}$

$3.26 \times 10-2 \mathrm{M}$

$3.1 \times 10-2 \mathrm{M}$

$25.0 \quad 3.32 \times 10-2 \mathrm{M}$

$3.31 \times 10-2$

$3.26 \times 10-2 \mathrm{M}$

$3.27 \times 10-2 \mathrm{M}$

$3.35 \times 10-2$

$3.32 \times 10-2$

$3.41 \times 10-2 \mathrm{~W}$

$3.38 \times 10-2 \mathrm{M}$
VALUES FRM REF IN CMC

BA AVER SP EQUIV COND

BA AVER SP EQUIV COND

BA SPECFC CONDCTNCE EQUATNS

BA AVER SP EQUIV COND

BA SPECFC CONDCTNCE EQUATNS

AVER SP EQUIV COND

SPECFC CONDCTNCE EQUATNS

AVER SP EQUIV COND

SURFACE TENSION LOG PLOT

TURBIDITY PLT LITE SCATR

UNSPECIFIED CONDUCTANCE UNSPECIFIED CONDUCTANCE

SPECFC CONDCTNCE EQUATNS EQUIV CONDCTNCE GRAPH

SPECFC CONDCTNCE GRAPH

AVER SP EQUIV COND

TURBIDITY PLT LITE SCATR

REFRACTIVE INDEX

SURFACE TENSION LOG PLOT SPECFC CONDCTNCE EQUATNS SPECFC CONDCTNCE EQUATNS AVER SP EQUIV COND AVER SP EQUIV COND SPECFC CONDCTNCE EQUATNS AVER SP EQUIV COND

SPECFC CONDCTNCE EQUATNS

AVER SP EQUIV COND

\section{HUIS \\ HUIS}

64047

T L

HAFF PICC

HAFF PICC

GODD BENS

PRIN HERM

SHIG

GODD BENS

GODD BENS

GODD BENS

EVAN

GODD BENS

KLIN LANG

GODD BENS

GODD BENS

DEMC

64047

$\begin{array}{ll}\mathrm{M} & \\ \mathrm{T} & 3\end{array}$

42003

57011 L 3

$56011 \mathrm{~T}$.

65022 T 3

57011 L 3

57011 L 3

57011 L 3

56006 T 3

57011 L 3

57022 T L

57011 L 3

57011 L 3

61031 T L

HAYD TAYL

62004

HAYD TAYL

HAYD TAYL

HAYD TAYL

HUIS

62004

62004

62004

64047

HUIS

64047

HUIS

HUIS

64047

64047

HUIS

HUIS

HUIS

HUIS

64047

64047

64047

64047

L L

L

$\begin{array}{ll}\text { L } & \text { L }\end{array}$

T L

M

T L

3

3

T L

$\begin{array}{llll}\text { MYSE OTTE } & 61017 & & \text { R } \\ \text { FLOC } & 61007 & \text { L } & 3 \\ \text { FLOC } & 61007 & \text { L } & 3 \\ \text { GODD BENS } & 57011 & \text { L } & \text { D } \\ \text { FLOC } & 61007 & \text { L } & \text { D } \\ \text { GODD BENS } & 57011 & \text { L } & 1 \\ \text { FLOC } & 61007 & \text { L } & 1 \\ \text { GODD BENS } & 57011 & \text { L } & 1 \\ \text { FLOC } & 61007 & \text { L } & 1 \\ \text { HUIS } & 64047 & \text { T } & \text { L } \\ & & \text { M } & \\ \text { HUIS } & 64047 & \text { T } & \text { L } \\ & & \text { M } & \\ \text { TART LELO } & 55021 & \text { T } & \text { L } \\ \text { HAFF PICC } & 42003 & \text { T } & \text { L } \\ \text { GODD BENS } & 57011 & \text { L } & 1 \\ \text { MYSE KAPA } & 61005 & \text { T } & 1 \\ \text { MYSE KAPA } & 61005 & \text { T } & 1 \\ \text { FLOC } & 61007 & \text { L } & 1 \\ \text { PRIN HERM } & 56011 & \text { T } & \text { L } \\ & & \text { M } & \\ \text { PRIN HERM } & 56011 & \text { T } & \text { L } \\ \text { SCHI } & 64020 & \text { T } & \text { L } \\ \text { MUKE KAPA } & 66002 & \text { T } & 1 \\ \text { GODD BENS } & 57011 & \text { L } & 1 \\ \text { FLOC } & 61007 & \text { L } & 1 \\ \text { FLOC } & 61007 & \text { L } & 1 \\ \text { GODD BENS } & 57011 & \text { L } & 1 \\ \text { FLOC } & 61007 & \text { L } & 1 \\ \text { GODD BENS } & 57011 & \text { L } & 1 \\ \text { FLOC } & 61007 & \text { L } & \text { D }\end{array}$

Concentration units: $\mathrm{A}-\mathrm{mol} \%$; $\mathrm{B}-\mathrm{vol} \%$ solvent; $\mathrm{C}-\mathrm{mol} \%$ surfactant mixture; $\mathrm{D}-\mathrm{wt} / \mathrm{vol} \%$; $\mathrm{E}-\%$ saturation; $\mathrm{H}-$ wt \% solvent; I-mol \% surfactant; $\mathrm{K}$-normality counterions; $\mathrm{M}$-molar; $\mathrm{N}$-normal; $\mathrm{P}$ - wt \%; $\mathrm{Q}$ - wt \% surfactant; $\mathrm{R}$-varied; $\mathrm{S}$-mol/ kg; $\mathrm{T}-$ wt \% surfactant mixture; $\mathrm{U}-\mathrm{mol} /(\mathrm{lor} \mathrm{kg}) ; \mathrm{W}-$ molal; $\mathrm{Y}-$ atm. Details on page 222. 


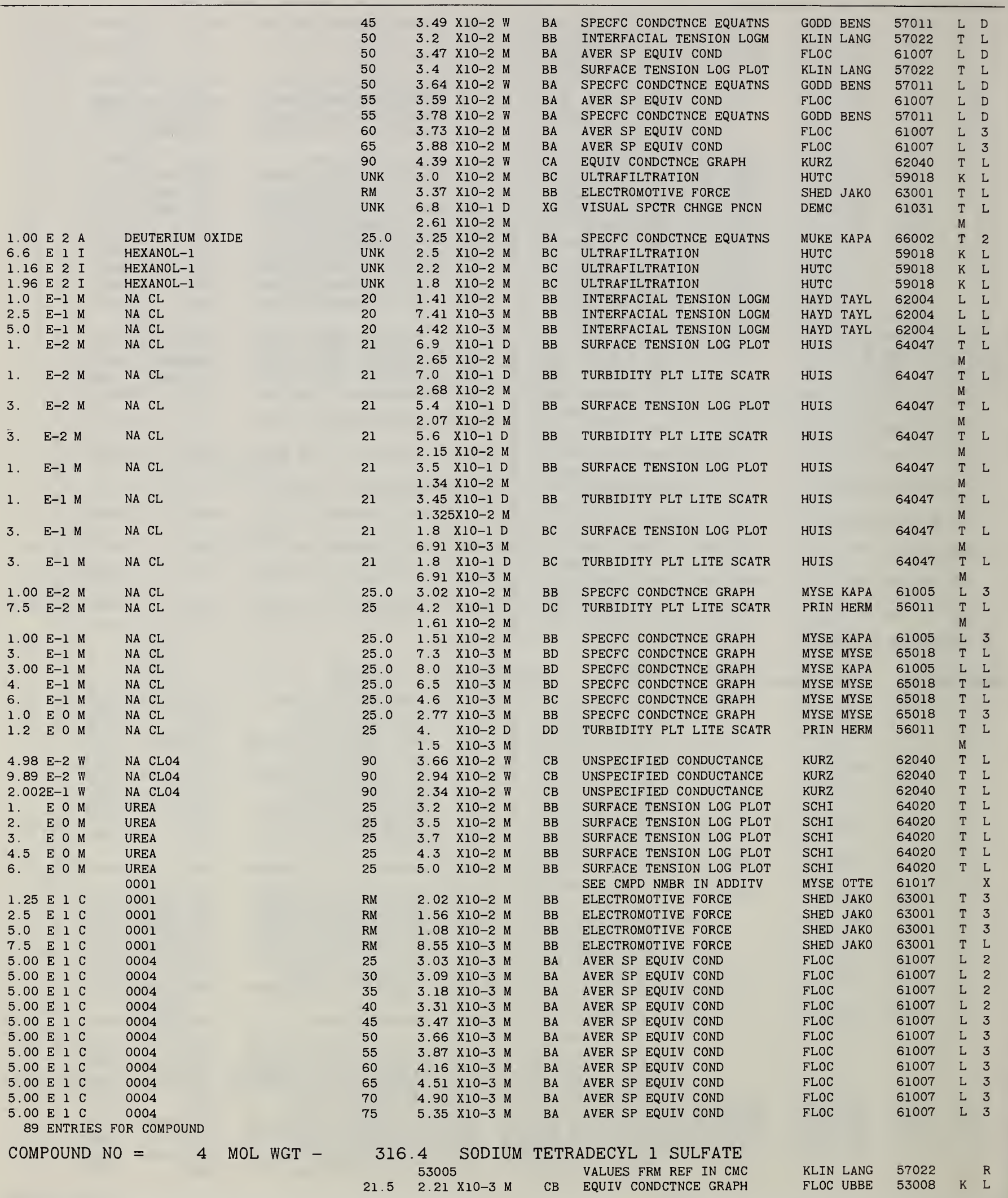

Concentration units: $\mathrm{A}-\mathrm{mol} \% ; \mathrm{B}-\mathrm{vol} \%$ solvent; $\mathrm{C}-\mathrm{mol} \%$ surfactant mixture: 


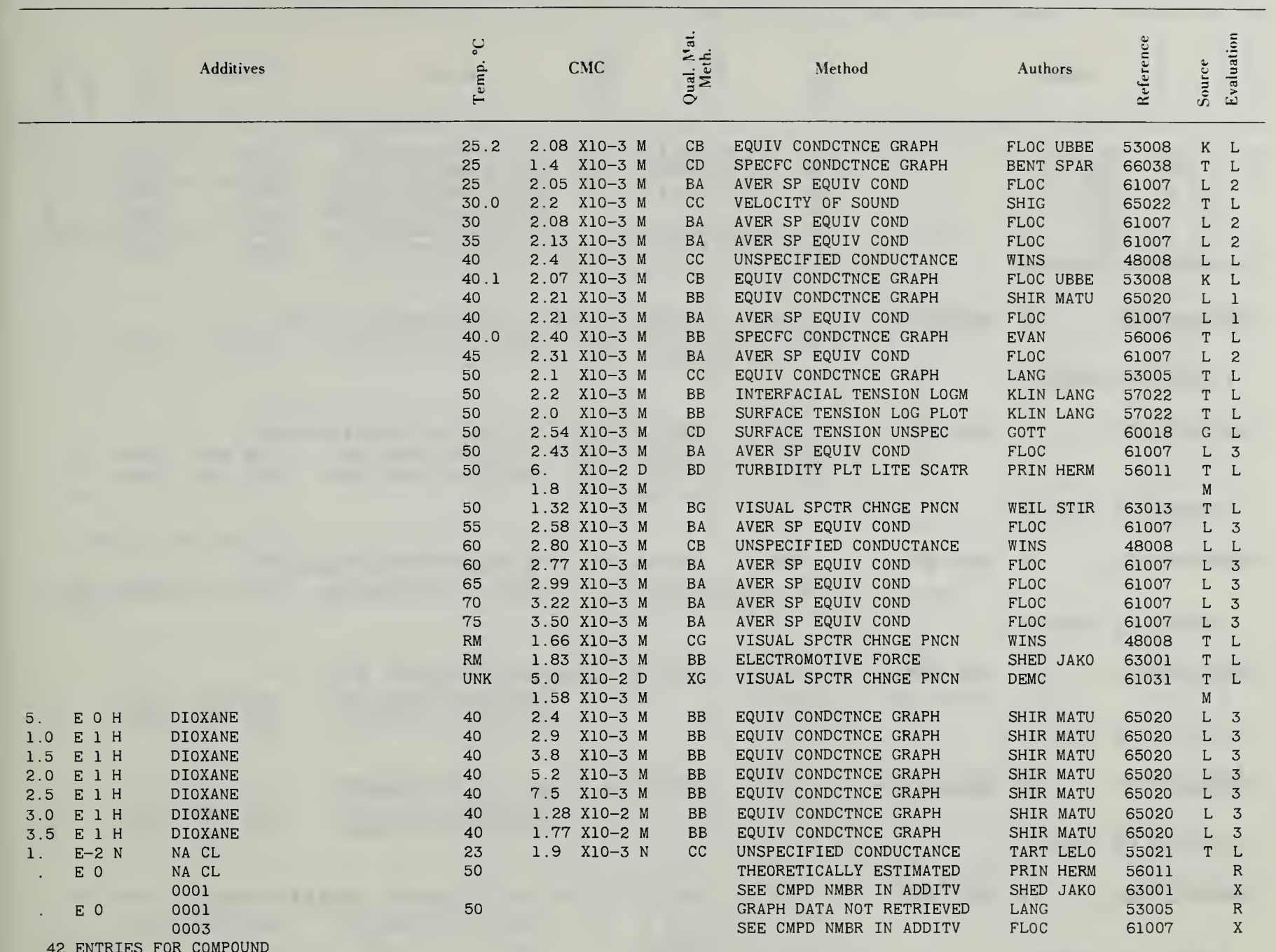

\section{COMPOUND NO $=$ \\ 5 MOL WGT -}

344.4 SODIUM HEXADECYL I SULFATE

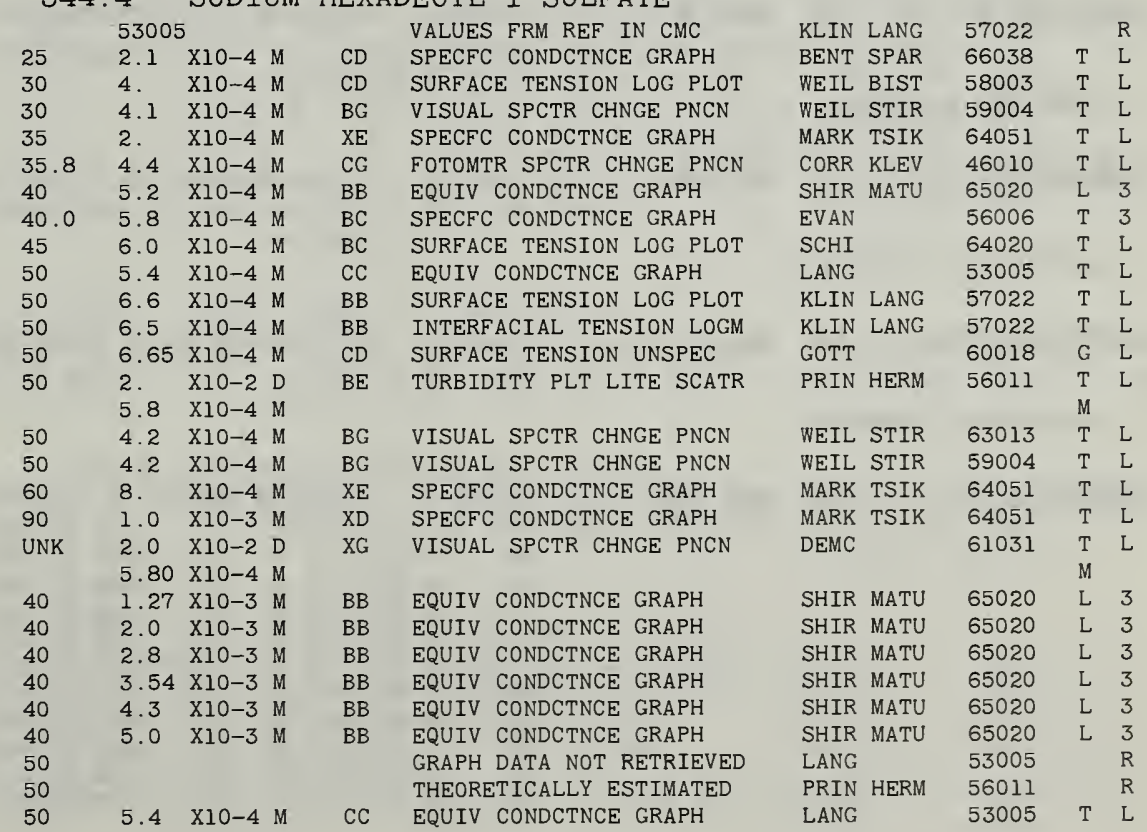

\begin{tabular}{|c|c|c|}
\hline & $\mathrm{EOH}$ & DIOXANE \\
\hline 1.0 & $\mathrm{E} \perp \mathrm{H}$ & DIOXANE \\
\hline 1.5 & $\mathrm{E} \perp \mathrm{H}$ & DIOXANE \\
\hline 2.0 & $\mathrm{E} \perp \mathrm{H}$ & DIOXANE \\
\hline 2.5 & $\mathrm{E} I \mathrm{H}$ & DIOXANE \\
\hline 3.0 & & $\begin{array}{l}\text { DIOXANE } \\
\text { NA CL }\end{array}$ \\
\hline & & NA CL \\
\hline
\end{tabular}

Concentration units: $\mathrm{A}-\mathrm{mol} \%$; $\mathrm{B}-$ vol \% solvent: $\mathrm{C}-$ mol $\%$ surfactant mixture; D-wt/vol \%; E-\% saturation; H-wt \% solvent; I-mol \% surfactant; K-normality counterions; $\mathrm{M}$ - molar; $\mathrm{N}$ - normal; $\mathrm{P}-\mathrm{wt} \%$; $\mathrm{Q}-\mathrm{wt} \%$ surfactant; $\mathrm{R}$ - varied; $\mathrm{S}-$ mol/ kg: T-wt \% surfactant mixture; $\mathrm{U}-\mathrm{mol} /(\mathrm{lorkg}) ; \mathrm{W}$-molal; $\mathrm{Y}$ - atm. Details on page 222. 


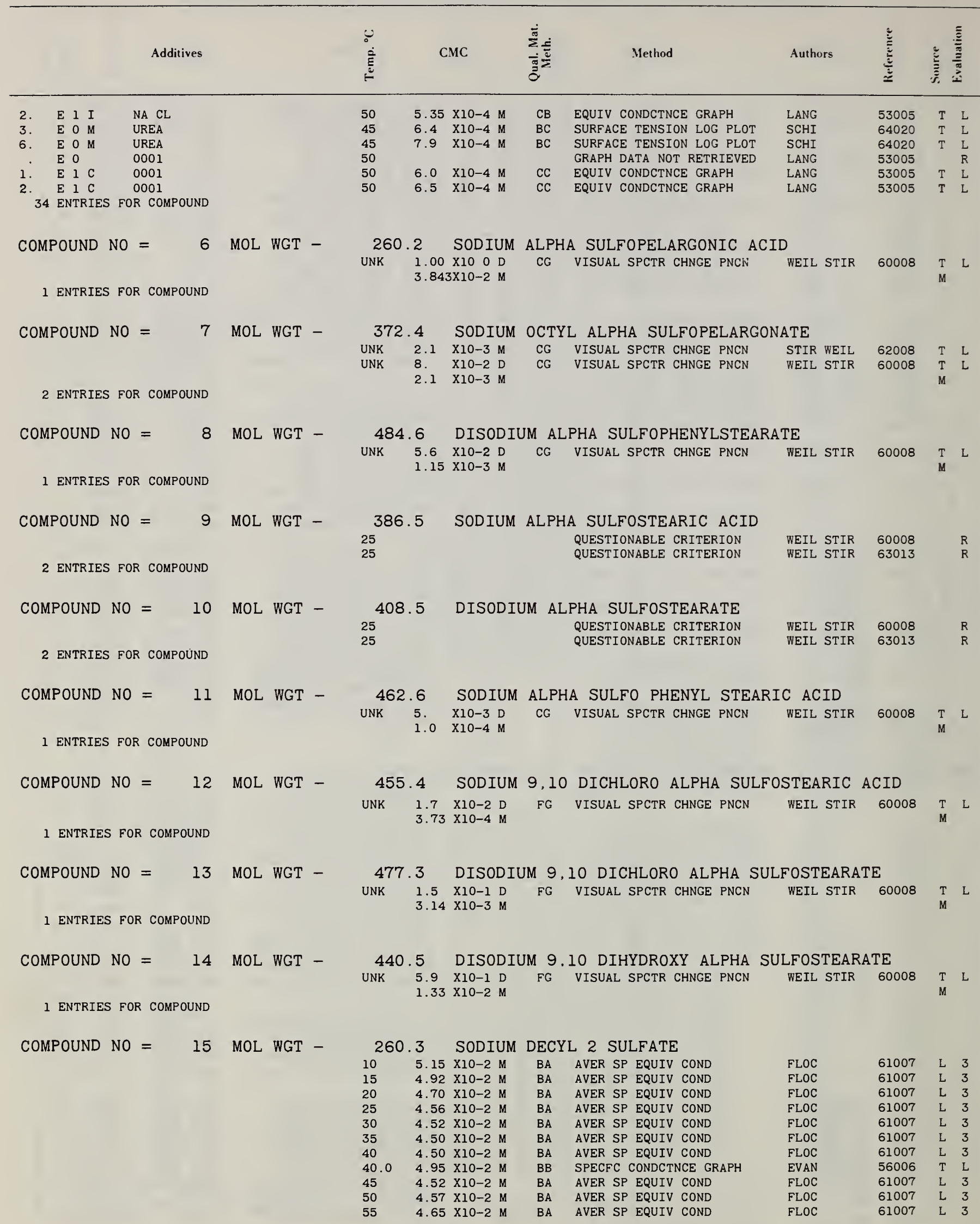

Concentration units: $\mathrm{A}-\mathrm{mol} \% ; \mathrm{B}-$ vol \% solvent; $\mathrm{C}-$ mol \% surfactant mixture; D-wt/vol \%; E-\% saturation; $\mathrm{H}-$ wt \% solvent; $\mathrm{I}-$ mol \% surfactant; $\mathrm{K}$ - normality counterions; $\mathrm{M}-$ molar: N-normal: $\mathrm{P}-\mathrm{wt} \%: \mathrm{Q}-\mathrm{wt} \%$ surfactant; $\mathrm{R}$-varied; $\mathrm{S}-\mathrm{mol} /$ kg: T- wt \% surfactant mixture; $\mathrm{U}-\mathrm{mol} /(\mathrm{l}$ or $\mathrm{kg})$; $\mathrm{W}-$ molal; $\mathrm{Y}-\mathrm{atm}$. Detailson page 222. 


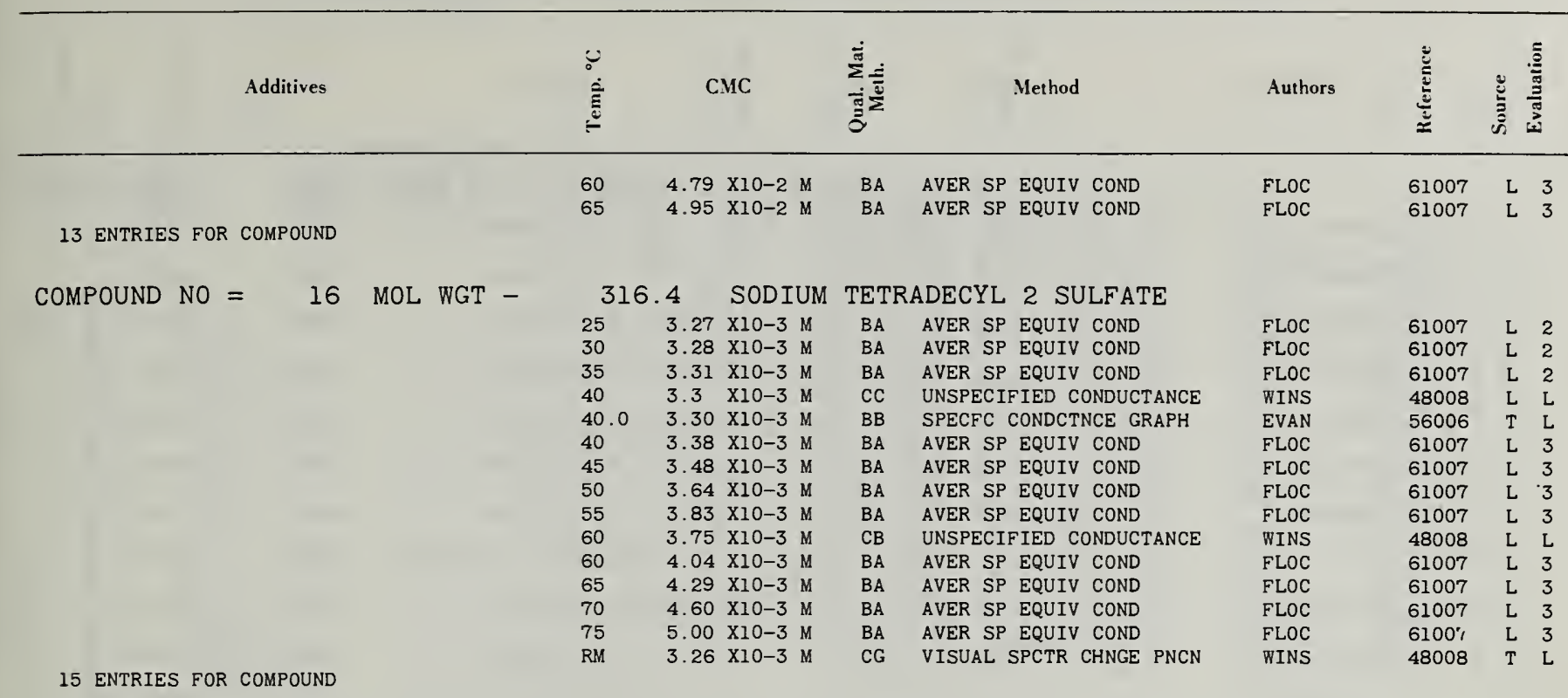

\section{COMPOUND NO $=17$ MOL WGT -}

15 ENTRIES FOR COMPOUND
316. 4 SODIUM TETRADECYL 4 SULFATE

$\begin{array}{lll}25 & 5.12 \times 10-3 \mathrm{M} & \mathrm{CA} \\ 30 & 5.05 \times 10-3 \mathrm{M} & \mathrm{CA} \\ 35 & 5.04 \times 10-3 \mathrm{M} & \mathrm{CA} \\ 40 & 5.2 \times 10-3 \mathrm{M} & \mathrm{CB} \\ 40.0 & 5.15 \times 10-3 \mathrm{M} & \mathrm{CB} \\ 40 & 5.12 \times 10-3 \mathrm{M} & \mathrm{CA} \\ 45 & 5.23 \times 10-3 \mathrm{M} & \mathrm{CA} \\ 50 & 5.38 \times 10-3 \mathrm{M} & \mathrm{CA} \\ 55 & 5.57 \times 10-3 \mathrm{M} & \mathrm{CA} \\ 60 & 6.10 \times 10-3 \mathrm{M} & \mathrm{CB} \\ 60 & 5.85 \times 10-3 \mathrm{M} & \mathrm{CA} \\ 65 & 6.21 \times 10-3 \mathrm{M} & \mathrm{CA} \\ 70 & 6.62 \times 10-3 \mathrm{M} & \mathrm{CA} \\ 75 & 7.11 \times 10-3 \mathrm{M} & \mathrm{CA} \\ \mathrm{RM} & 4.76 \times 10-3 \mathrm{M} & \mathrm{CC}\end{array}$

AVER SP EQUIV COND

AVER SP EQUIV COND

AVER SP EQUIV COND

UNSPECIFIED CONDUCTANCE

SPECFC CONDCTNCE GRAPH

AVER SP EQUIV COND

AVER SP EQUIV COND

AVER SP EQUIV COND

AVER SP EQUIV COND

UNSPECIFIED CONDUCTANCE

AVER SP EQUIV COND

AVER SP EQUIV COND

AVER SP EQUIV COND

AVER SP EQUIV COND

VISUAL SPCTR CHNGE PNCN
FLOC
FLOC
FLOC
WINS
EVAN
FLOC
FLOC
FLOC
FLOC
WINS
FLOC
FLOC
FLOC
FLOC
WINS

61007

61007

61007

48008

56006

61007

61007

61007

61007

48008

61007

61007

61007

61007

48008 $\begin{array}{ll}\text { L } & 3 \\ \text { L } & 3 \\ \text { L. } & 3 \\ \text { L } & \text { L } \\ \text { T } & 3 \\ \text { L } & 3 \\ \text { L } & 3 \\ \text { L } & 3 \\ \text { L } & 3 \\ \text { L } & \text { L } \\ \text { L } & 3 \\ \text { L } & 3 \\ \text { L } & 3 \\ \text { L } & 3 \\ \text { T } & \text { L }\end{array}$

292.4 OCTYL BETA D GLUCOSIDE

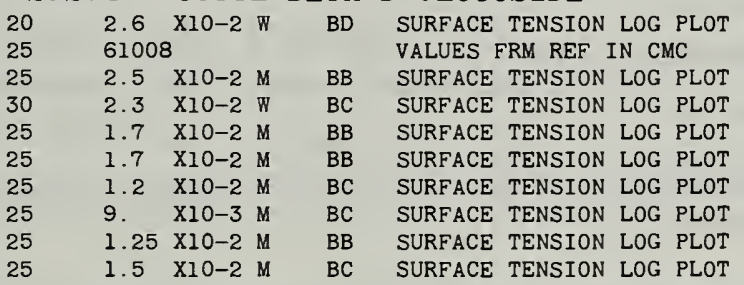

BURY BROW SHIN YAMA SHIN YAMA BURY BROW SHIN YAMA SHIN YAMA SHIN YAMA SHIN YAMA SHIN YAMA

SHIN YAMA

$\begin{array}{lll}52011 & \text { T } & \text { L } \\ 59013 & & \text { R } \\ 61008 & \text { T } & 3 \\ 52011 & \text { T } & \text { L } \\ 61008 & \text { T } & \text { L } \\ 61008 & \text { T } & \text { L } \\ 61008 & \text { T } & \text { L } \\ 61008 & \text { T } & \text { L } \\ 61008 & \text { T } & \text { L } \\ 61008 & \text { T } & \text { L }\end{array}$

320.4 DECYL BETA D GLUCOSIDE

$25 \quad 2.2 \quad \mathrm{X} 10-3 \mathrm{M} \quad \mathrm{BB}$ SURFACE TENSION LOG PLOT SEE CMPD NMBR IN ADDITV $\begin{array}{llll}\text { SHIN YAMA } & 61008 & \text { T } & 3 \\ \text { SHIN YAMA } & 61008 & & \mathrm{X}\end{array}$

SHIN YAMA 61008 T 3 SHIN YAMA $61008 \quad \mathrm{X}$

2 ENTRIES FOR COMPOUND

348.5 DODECYL BETA D GLUCOSIDE

$25 \quad 1.9 \quad$ X10-4 M $\quad$ BB SURFACE TENSION LOG PLOT SEE CMPD NMBR IN ADDITV
COMPOUND NO $=21$ MOL WGT -
229.4 DIMETHYL DODECYL AMINE OXIDE 62005 VALUES FRM REF IN CMC

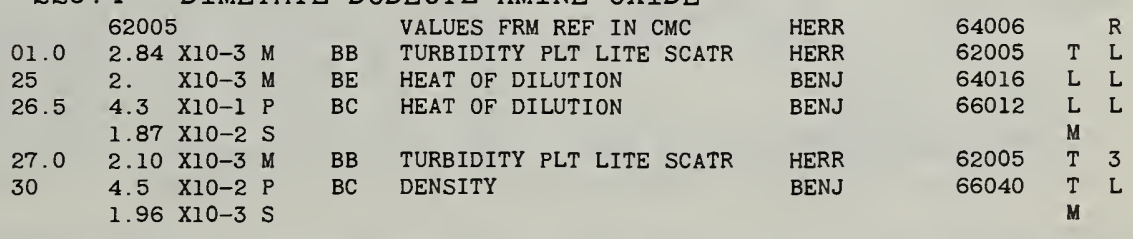

Concentration units: $\mathrm{A}-\mathrm{mol} \%$; $\mathrm{B}-$ vol \% solvent: $\mathrm{C}-\mathrm{mol} \%$ surfactant mixture; D-wt/vol \%; E- \% saturation; $\mathrm{H}-$ wt \% solvent; I-mol \% surfactant; $\mathrm{K}$ - normality counterions; $\mathrm{M}$ - molar; $\mathrm{N}$ - normal; $\mathrm{P}$ - wt \%; $\mathrm{Q}$ - wt \% surfactant; $\mathrm{R}$-varied; $\mathrm{S}$ - mol/ kg; $T$ - wt \% surfactant mixture; $U-m o l /($ or kg); $W$ - molal; $Y$ - atm. Detailson page 222. 


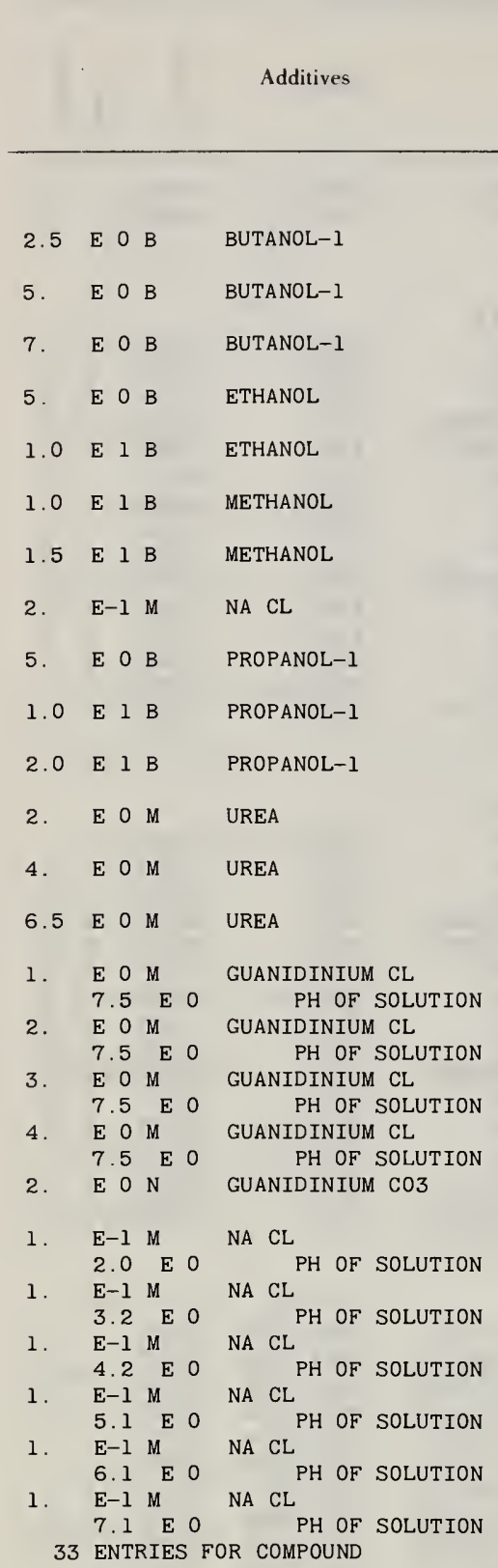

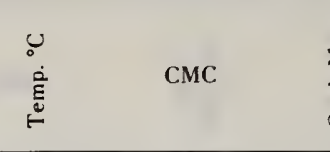

$40.0 \quad 1.83 \times 10-3 \mathrm{M}$

$50.0 \quad 1.75 \times 10-3 \mathrm{M}$

$26.52 .2 \times 10-1 \mathrm{P}$

$9.59 \times 10-3 \mathrm{~S}$

$\begin{array}{llll}26.5 & 1.2 \times 10-1 & P \\ & 5.23 \times 10-3 & 5\end{array}$

$\begin{array}{llll} & 5.23 & \times 10-3 & S \\ 26.5 & 6 . & \times 10-2 & P\end{array}$

$2.6 \quad \mathrm{X} 10-3 \mathrm{~S}$

$26.53 .9 \times 10-1 \mathrm{P}$

1.70 X10-2 S

$26.53 .4 \times 10-1 \mathrm{P}$

1. $48 \times 10-2 \mathrm{~S}$

$26.54 .5 \mathrm{X} 10-1 \mathrm{P}$

$1.96 \times 10-2 \mathrm{~S}$

$26.54 .0 \quad \times 10-1 \quad \mathrm{P}$

1. $74 \times 10-2 \mathrm{~S}$

$27 \quad 3.4 \times 10-2 \mathrm{D}$

$1.48 \times 10-3 \mathrm{M}$

26.52 .8 X10-1 P

1. $22 \times 10-2 \mathrm{~S}$

$26.5 \quad 1.7 \quad \times 10-1 \quad P$

$7.41 \times 10-3 \mathrm{~S}$

$26.51 .1 \times 10-1 \mathrm{P}$

4. $79 \times 10-3 \mathrm{~S}$

$26.5 \quad 5.4 \times 10-1 \mathrm{P}$

2. $35 \times 10-2 \mathrm{~S}$

26.56 .9 X10-1 P

3. $00 \times 10-2 \mathrm{~S}$

$26.51 .04 \times 100 \mathrm{P}$

4. $533 \times 10-2$ S

$26.54 .8 \times 10-1 \mathrm{P}$

$2.09 \times 10-2 \mathrm{~S}$

$26.5 \quad 6.2 \quad \times 10-1 \mathrm{P}$

2.70 X10-2 S

26.57 .8 X10-1 $\mathrm{P}$

$3.40 \times 10-2 \mathrm{~S}$

$26.58 .8 \times 10-1 \mathrm{P}$

$3.83 \times 10-2 \mathrm{~S}$

$26.5 \quad 1.9 \times 10-1 \mathrm{P}$

$8.28 \times 10-3 \mathrm{~S}$

$25 \quad 3.50 \times 10-3^{2} \mathrm{M}$

$253.10 \times 10-3 \mathrm{M} \quad \mathrm{BC}$

$25 \quad 1.95 \times 10-3 \mathrm{M} \quad \mathrm{BC}$

$25 \quad 1.25 \times 10-3 \mathrm{M} \quad \mathrm{BC}$

$25 \quad 1.01 \times 10-3 \mathrm{M} \quad \mathrm{BC}$

$25 \quad 1.00 \times 10-3 \mathrm{M} \quad \mathrm{BC}$

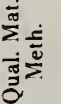

Method

Authors
BB TURBIDITY PLT LITE SCATR

BB TURBIDITY PLT LITE SCATR

HEAT OF DILUTION

HEAT OF DILUTION

BE HEAT OF DILUTION

BC HEAT OF DILUTION

HEAT OF DILUTION

HEAT OF DILUTION

HEAT OF DILUTION

TURBIDITY PLT LITE SCATR

HEAT OF DILUTION

HEAT OF DILUTION

HEAT OF DILUTION

HEAT OF DILUTION

HEAT OF DILUTION

HEAT OF DILUTION

HEAT OF DILUTION

HEAT OF DILUTION

HEAT OF DILUTION

HEAT OF DILUTION

HEAT OF DILUTION

FOTOMTR SOLUBLZTN YLOB

FOTOMTR SOLUBLZTN YLOB

FOTOMTR SOLUBLZTN YLOB

FOTOMTR SOLUBLZTN YLOB

FOTOMTR SOLUBLZTN YLOB

FOTOMTR SOLUBLZTN YLOB

\begin{tabular}{|c|c|c|c|c|}
\hline HERR & & 62005 & $\mathrm{~T}$ & $\mathrm{~L}$ \\
\hline HERR & & 62005 & $\mathrm{~T}$ & L \\
\hline BENJ & & 66012 & $\begin{array}{l}\mathrm{L} \\
\mathrm{M}\end{array}$ & $\mathrm{L}$ \\
\hline BENJ & & 66012 & $\begin{array}{l}\mathrm{L} \\
\mathrm{M}\end{array}$ & $\mathrm{L}$ \\
\hline BENJ & & 66012 & $\begin{array}{l}\mathrm{L} \\
\mathrm{M}\end{array}$ & $\mathrm{L}$ \\
\hline BENJ & & 66012 & $\begin{array}{l}\mathrm{L} \\
\mathrm{M}\end{array}$ & $\mathrm{L}$ \\
\hline BENJ & & 66012 & $\begin{array}{l}\mathrm{L} \\
\mathrm{M}\end{array}$ & $\mathrm{L}$ \\
\hline BENJ & & 66012 & $\begin{array}{l}\mathrm{L} \\
\mathrm{M}\end{array}$ & $\mathrm{L}$ \\
\hline BENJ & & 66012 & $\begin{array}{l}\text { L } \\
\text { M }\end{array}$ & L \\
\hline HERR & & 62005 & $\begin{array}{l}\mathrm{T} \\
\mathrm{M}\end{array}$ & $\mathrm{L}$ \\
\hline BENJ & & 66012 & $\begin{array}{l}\mathrm{L} \\
\mathrm{M}\end{array}$ & L \\
\hline BENJ & & 66012 & $\begin{array}{l}\mathrm{L} \\
\mathrm{M}\end{array}$ & $\mathrm{L}$ \\
\hline BENJ & & 66012 & $\begin{array}{l}\mathrm{L} \\
\mathrm{M}\end{array}$ & L \\
\hline BENJ & & 66012 & $\begin{array}{l}L \\
M\end{array}$ & $\mathrm{~L}$ \\
\hline BENJ & & 66012 & $\begin{array}{l}L \\
M\end{array}$ & $\mathrm{~L}$ \\
\hline BENJ & & 66012 & $\begin{array}{l}L \\
M\end{array}$ & L \\
\hline BENJ & & 66012 & $\begin{array}{l}\mathrm{L} \\
\mathrm{M}\end{array}$ & $\mathrm{L}$ \\
\hline BENJ & & 66012 & $\begin{array}{l}\mathrm{L} \\
\mathrm{M}\end{array}$ & $\mathrm{L}$ \\
\hline BENJ & & 66012 & $\begin{array}{l}\mathrm{L} \\
\mathrm{M}\end{array}$ & $\mathrm{L}$ \\
\hline BENJ & & 66012 & $\begin{array}{l}\mathrm{L} \\
\mathrm{M}\end{array}$ & $\mathrm{L}$ \\
\hline BENJ & & 66012 & $\begin{array}{l}\mathrm{L} \\
\mathrm{M}\end{array}$ & $\mathrm{L}$ \\
\hline TOKI & OHKI & 66027 & G & L \\
\hline TOKI & OHKI & 66027 & G & L \\
\hline TOKI & OHKI & 66027 & G & $\mathrm{L}$ \\
\hline TOKI & OHKI & 66027 & G & $\mathrm{L}$ \\
\hline TOKI & OHKI & 66027 & G & $\mathrm{L}$ \\
\hline TOKI & OHKI & 66027 & G & $\mathrm{L}$ \\
\hline
\end{tabular}

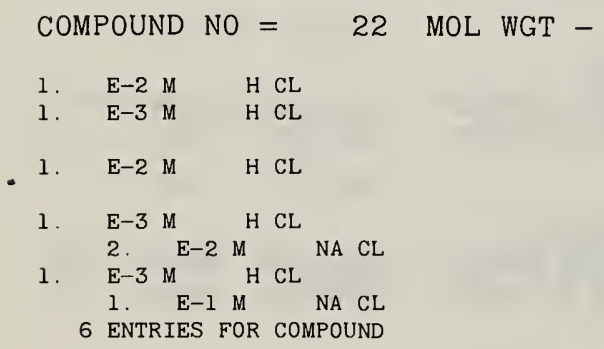

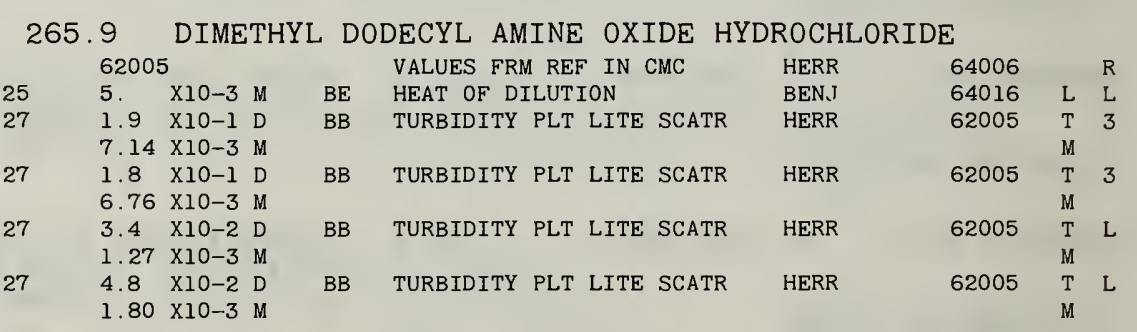

\subsection{SILVER DODECYL 1 SULFATE}

$\begin{array}{llllll}35 & 7.3 & \text { X10-3 } & \text { M } & \text { BA } & \text { SPECFC CONDCTNCE GRAPH } \\ 55 & 8.4 & \text { X10-3 } & \text { M } & \text { BA } & \text { SPECFC CONDCTNCE GRAPH } \\ 35 & 4.7 & \text { X10-3 } & \text { M } & \text { BA } & \text { SPECFC CONDCTNCE GRAPH } \\ 35 & 5.4 & \text { X10-3 } & \text { M } & \text { BA } & \text { SPECFC CONDCTNCE GRAPH }\end{array}$

CORK GOOD CORK GOOD CORK GOOD CORK GOOD
62006 T 2

62006 T 3

62006 T 3 62006 T 3
Concentration units: $\mathrm{A}-\mathrm{mol} \% ; \mathrm{B}-\mathrm{vol} \%$ solvent; $\mathrm{C}-\mathrm{mol} \%$ surfactant mixture $\mathrm{D}-\mathrm{wt} / \mathrm{vol} \% ; \mathrm{E}-\%$ saturation; $\mathrm{H}-\mathrm{wt} \%$ solvent; I-mol \% surfactant; $\mathrm{K}$-normality counterions; M-molar; N-normal; P-wt \%; Q-wt \% surfactant; R-varied; S-mol/ $\mathrm{kg} ; \mathrm{T}-\mathrm{wt} \%$ surfactant mixture; $\mathrm{U}-\mathrm{mol} /(\mathrm{lor} \mathrm{kg}) ; \mathrm{W}-$ molal; $Y$ - atm. Details on page 222. 


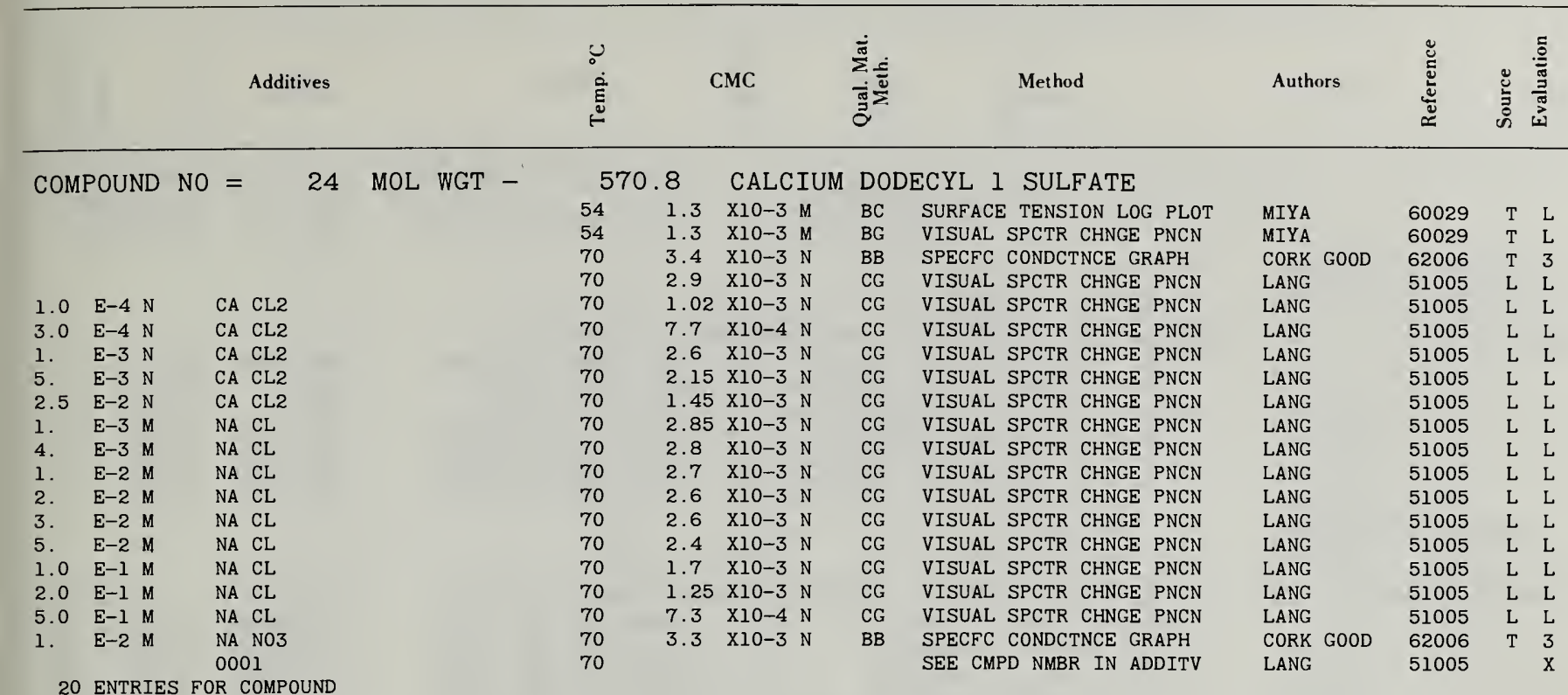

\section{COMPOUND NO $=25$ MOL WGT - \\ 1 ENTRIES FOR COMPOUND}

COMPOUND NO $=26$ MOL WGT -

2 ENTRIES FOR COMPOUND

COMPOUND NO $=27$ MOL WGT -

1 ENTRIES FOR COMPOUND

COMPOUND NO $=28$ MOL WGT -

1 ENTRIES FOR COMPOUND

COMPOUND NO $=29$ MOL WGT -

1 ENTRIES FOR COMPOUND

COMPOUND NO $=30$ MOL WGT -

1 ENTRIES FOR COMPOUND

COMPOUND NO $=31$ MOL WGT -

1 ENTRIES FOR COMPOUND

COMPOUND NO $=32$ MOL WGT -

1 ENTRIES FOR COMPOUND

COMPOUND NO $=33$ MOL WGT -

1 ENTRIES FOR COMPOUND

COMPOUND NO $=34$ MOL WGT -

1 ENTRIES FOR COMPOUND

COMPOUND NO $=35$ MOL WGT -

1 ENTRIES FOR COMPOUND
288.3 SODIUM ETHYL ALPHA SULFOPELARGONATE

UNK $9.80 \times 10-2 \mathrm{M}$ CG VISUAL SPCTR CHNGE PNCN STIR WEIL 62008 T $\mathrm{L}$

330.4 SODIUM AMYL ALPHA SULFOPELARGONATE

UNK $1.56 \times 10-2 \mathrm{M} \quad$ CG VISUAL SPCTR CHNGE PNCN MAUR STIR 64002 T UNK $1.60 \times 10-2 \mathrm{M}$ CG VISUAL SPCTR CHNGE PNCN STIR WEIL 62008 T

344. 4 SODIUM HEXYL ALPHA SULFOPELARGONATE

UNK 7.0 XI0-3 M CG VISUAL SPCTR CHNGE PNCN STIR WEIL 62008 $T$ T

358.4 SODIUM HEPTYL ALPHA SULFOPELARGONATE

$\begin{array}{llllllll} & \\ \text { UNK } & 4.5 & \text { XIO-3 M } & \text { CG VISUAL SPCTR CHNGE PNCN STIR WEIL } & 62008 & \text { T } & \text { L }\end{array}$

386.5 SODIUM NONYL ALPHA SULFOPELARGONATE

UNK $1.2 \times 10-3 \mathrm{M}$ CG VISUAL SPCTR CHNGE PNCN STIR WEIL 62008 T $\mathrm{L}$

400.5 SODIUM DECYL ALPHA SULFOPELARGONATE

UNK 5. X10-4 M CG VISUAL SPCTR CHNGE PNCN STIR WEIL 62008 T L

372.4 SODIUM 2 OCTYL ALPHA SULFOPELARGONATE

$\begin{array}{lllllllll}\text { UNK } & 3.6 & \text { X10-3 M } & \text { CG VISUAL SPCTR CHNGE PNCN STIR WEIL } 62008 & T & \mathrm{~T}\end{array}$

372.4 SODIUM 2 ETHYLHEXYL ALPHA SULFOPELARGONATE

UNK $\quad 1.9 \quad$ XIO-3 M CG VISUAL SPCTR CHNGE PNCN STIR WEIL 62008 T \begin{tabular}{lllllll}
\multicolumn{1}{l}{400.5} & SODIUM /OXO/ DECYL ALPHA SULFOPELARGONATE \\
UNK & 5. & XIO-4 M HG VISUAL SPCTR CHNGE PNCN STIR WEIL 62008 & T & L
\end{tabular}

428.6 SODIUM DODECYL ALPHA SULFOPELARGONATE

UNK 1. XIO-4 M CG VISUAL SPCTR CHNGE PNCN STIR WEIL 62008 T

574.3 SODIUM H/CF2CF2/3CH2 ALPHA SULFOPELARGONATE UNK $2.4 \quad \mathrm{XI0-3} M \quad$ HG VISUAL SPCTR CHNGE PNCN STIR WEIL 62008 T $\mathrm{L}$

Concentration units: $\mathrm{A}-\mathrm{mol} \%$; $\mathrm{B}-\mathrm{vol} \%$ solvent; $\mathrm{C}-\mathrm{mol} \%$ surfactant mixture; counterions; $\mathrm{M}-$ molar; $\mathrm{N}$-normal; $\mathrm{P}-$ wt \%; $\mathrm{Q}-$ wt \% surfactant; $\mathrm{R}-$ varied; $\mathrm{S}-$ mol/ 


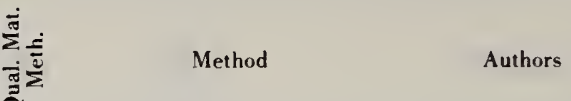

4 ENTRIES FOR COMPOUND

COMPOUND NO $=37$ MOL WGT -

7 ENTRIES FOR COMPOUND

$\begin{array}{llll}\mathrm{RM} & 1.4 & \mathrm{X} 10-2 & \mathrm{D} \\ & 3.75 & \mathrm{X} 10-4 & \mathrm{M} \\ \mathrm{RM} & 1.5 & \mathrm{X} 10-2 & \mathrm{D} \\ & 4.02 & \mathrm{X} 10-4 & \mathrm{M}\end{array}$

CG

CG VISUAL SPCTR CHNGE PNCN

WEIL STIR

56008 $1.5 \times 10-2 \mathrm{D}$ $4.02 \times 10-4 \mathrm{M}$

193.7 DECYLAMMONIUM CHLORIDE

$\begin{array}{llll}20 & 3.2 & \times 10-2 & M \\ 25 & 4 . & X 10-2 & M \\ 25 & 4.8 & X 10-2 & M \\ 25.0 & 5.40 & \times 10-2 & M \\ 40 & 3.8 & \text { X10-2 } & M \\ 60 & 3.8 & \text { X10-2 } & M \\ \text { RM } & 4 . & \text { X10-2 } & M\end{array}$

$\begin{array}{ll}\text { CC } & \text { EQUIV CONDCTNCE GRAPH } \\ \text { CE } & \text { REFRACTIVE INDEX } \\ \text { XC } & \text { REFRACTIVE INDEX } \\ \text { CB } & \text { SPECFC CONDCTNCE GRAPH } \\ \text { CC } & \text { EQUIV CONDCTNCE GRAPH } \\ \text { CC } & \text { EQUIV CONDCTNCE GRAPH } \\ \text { BD } & \text { PH OR HYDROLYSIS }\end{array}$

RALS HOER KLEV KLEV HOYE GREE RALS HOER RALS HOER VEIS HOER

$\begin{array}{lll}42002 & \text { P } & \text { L } \\ 48005 & \text { T } & \text { L } \\ 53010 & \text { T } & \text { L } \\ 57004 & \text { T } & \text { L } \\ 42002 & \text { P } & \text { L } \\ 42002 & \text { P } & \text { L } \\ 60001 & \text { T } & \text { L }\end{array}$

\section{8}

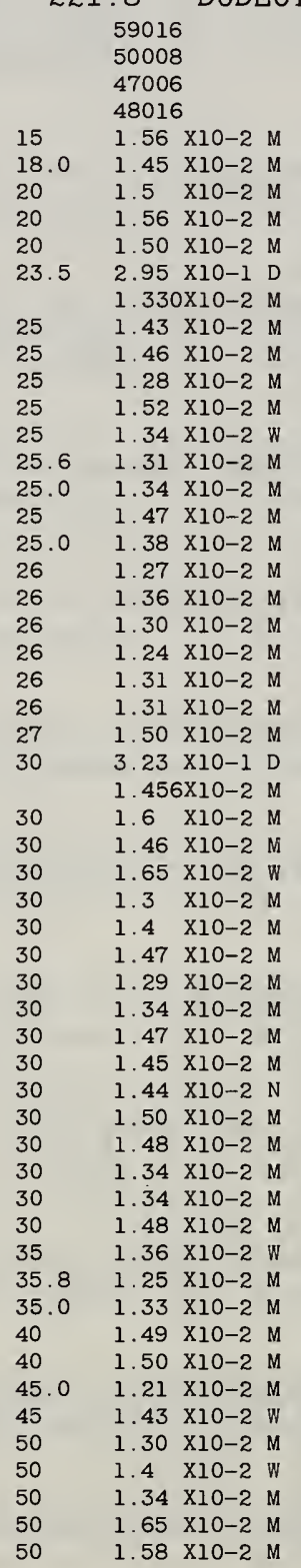

A-mol $\%$; B-vol\% solvent; C-mol $\%$ surfactant; D-wt/vol $\%$; $-\%$ saturation; H-wt solvent; I-mol\% surfactant; K-normality counterions:

\section{AMMONIUM CHLORIDE}

VALUES FRM REF IN CMC VALUES FRM REF IN CMC VALUES FRM REF IN CMC VALUES FRM REF IN CMC BB SPECFC CONDCTNCE GRAPH CB SPECFC CONDCTNCE GRAPH $X G$ VISCOSITY MINIMUM BB SPECFC CONDCTNCE GRAPH BA SPECFC CONDCTNCE GRAPH BB REFRACTIVE INDEX

BG EQUIV COND IST DEVIATION BA EQUIV CONDCTNCE GRAPH BG VISUAL SPCTR CHNGE INPX $\begin{array}{lll}\text { BG } & \text { VISUAL SPCTR CHNGE INPX } \\ \text { BB SPECFC CONDCTNCE GRAPH }\end{array}$ BB SPECFC CONDCTNCE GRAPH CG FOTOMTR SPCTR CHNGE INPX CB SPECFC CONDCTNCE GRAPH BA SPECFC CONDCTNCE GRAPH CB SPECFC CONDCTNCE GRAPH BG VISUAL SPCTR CHNGE SKYB BG VISUAL SPCTR CHNGE EOSN BG VISUAL SPCTR CHNGE FL BG VISUAL SPCTR CHNGE INPX BG VISUAL SPCTR CHNGE SKYB BG VISUAL SPCTR CHNGE INPX BB SPECFC CONDCTNCE GRAPH

CC TURBIDITY PLT LITE SCATR

XG VISCOSITY MINIMUM

BC EQUIV CONDCTNCE GRAPH XB VAPR PRESURE LOWERING CD REFRACTIVE INDEX

XG VISUAL SPCTR CHNGE BB DENSITY

CB SPECFC CONDCTNCE GRAPH CB FOTOMTR SOLUBLZTN PDMAB BB EQUIV CONDCTNCE GRAPH BC EQUIV CONDCTNCE GRAPH BB EQUIV CONDCTNCE GRAPH BA SPECFC CONDCTNCE GRAPH BA SPECFC CONDCTNCE GRAPH CB FOTOMTR SOLUBLZTN AZBZ CB FOTOMTR SOLUBLZTN OROT BB EQUIV CONDCTNCE GRAPH BB SPECFC CONDCTNCE GRAPH CG FOTOMTR SPCTR CHNGE INPX CB SPECFC CONDCTNCE GRAPH BA SPECFC CONDCTNCE GRAPH BA SPECFC CONDCTNCE GRAPH CG FOTOMTR SPCTR CHNGE INPX BB SPECFC CONDCTNCE GRAPH HB SPECFC CONDCTNCE GRAPH XD VAPR PRESURE LOWERING CB FOTOMTR SOLUBLZTN PDMAB BB SPECFC CONDCTNCE GRAPH BA SPECFC CONDCTNCE GRAPH

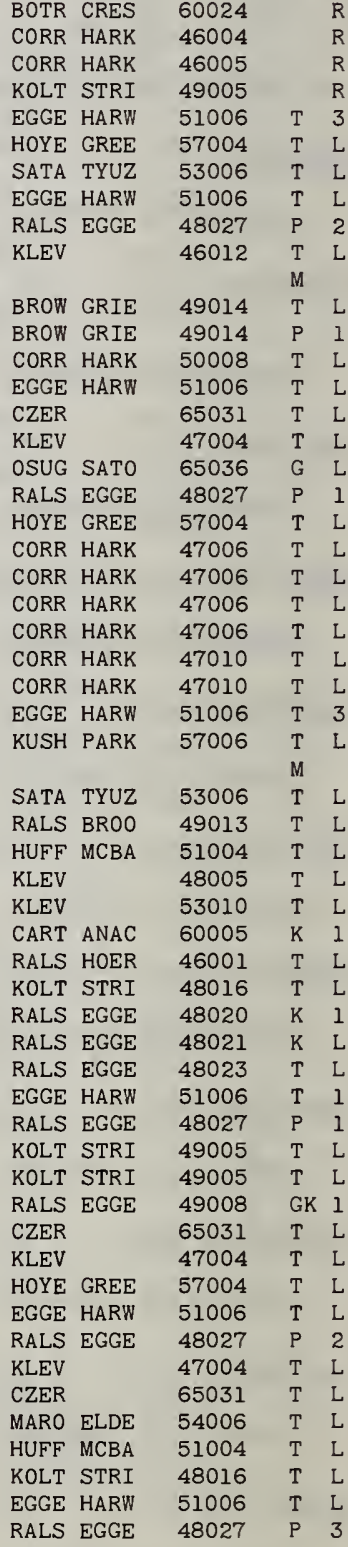




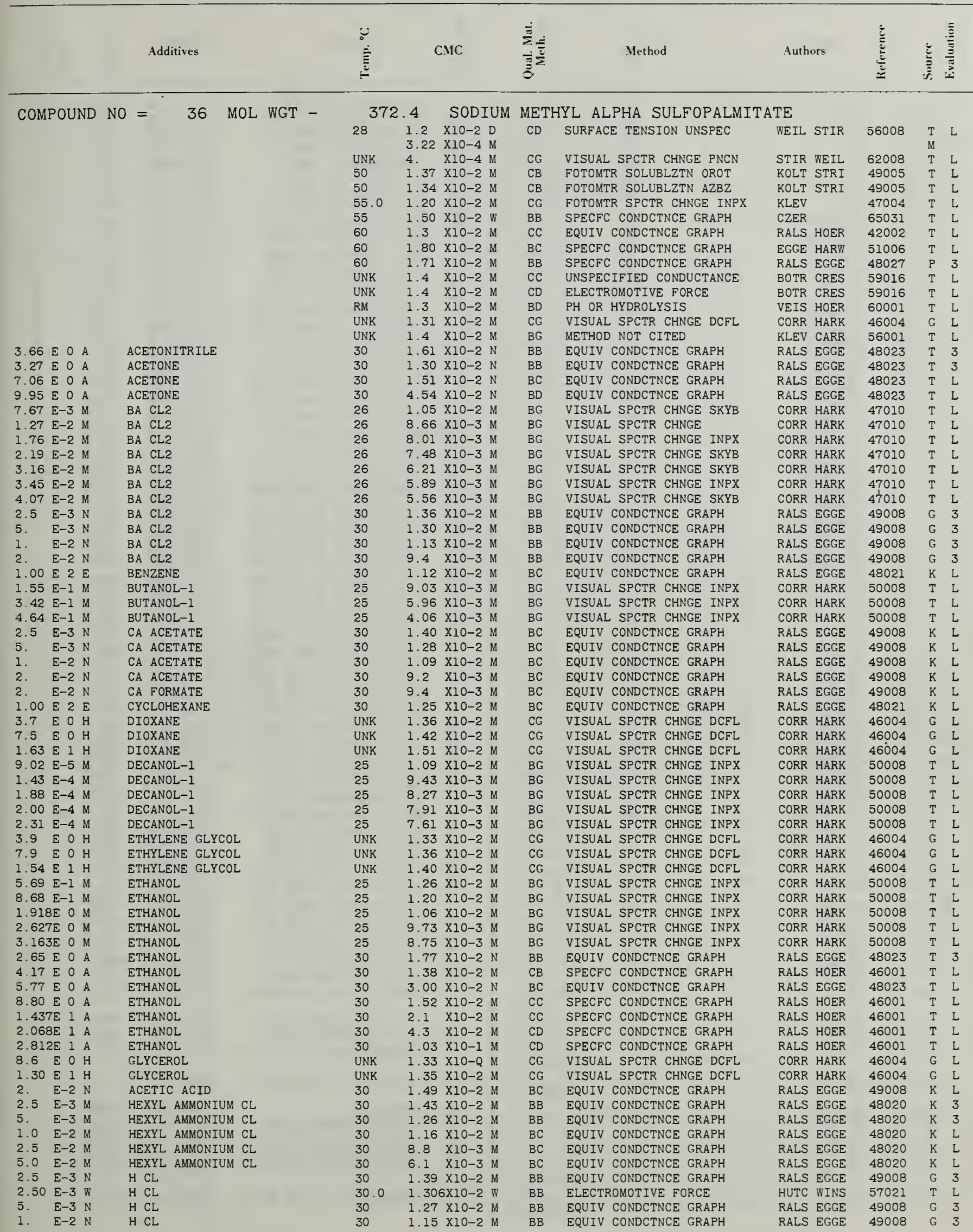

Concentration units: $\mathrm{A}-\mathrm{mol} \%$; $\mathrm{B}-$ vol $\%$ solvent; $\mathrm{C}-$ mol $\%$ surfactant mixture; counterions: $\mathrm{M}-$ molar: $\mathrm{N}$-normal; $\mathrm{P}-$ wt $\%$; $\mathrm{Q}-$ wt $\%$ surfactant; $\mathrm{R}$-varied; $\mathrm{S}-$ mol/ $\mathrm{D}-\mathrm{wt} / \mathrm{vol} \% ; \mathrm{E}-\%$ saturation; $\mathrm{H}-$ wt $\%$ solvent: $\mathrm{I}-$ mol \% surfactant; $\mathrm{K}-$ normality 


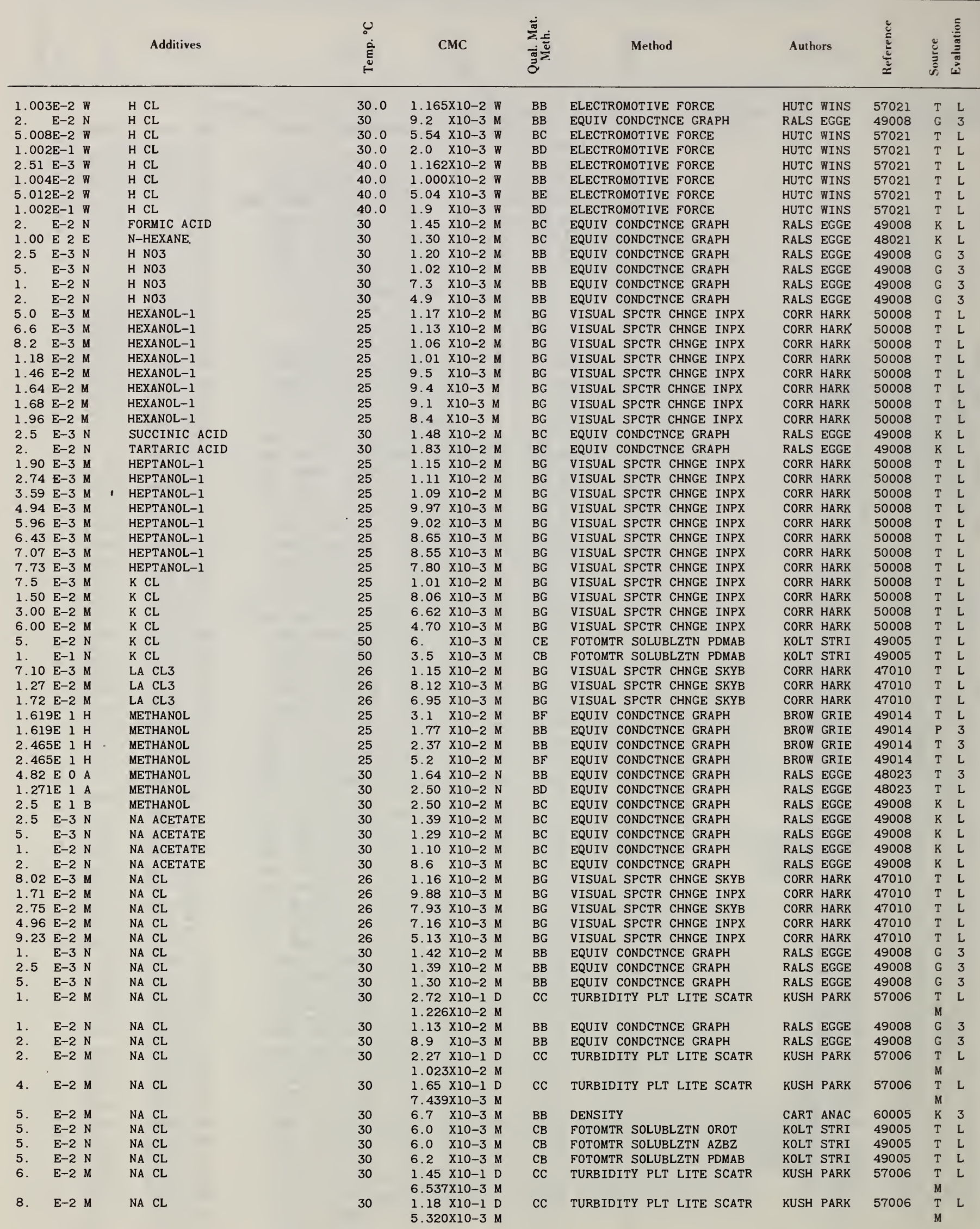

Concentration units: $\mathrm{A}-\mathrm{mol} \%$; $\mathrm{B}-\mathrm{vol} \%$ solvent; $\mathrm{C}-$ mol \% surfactant mixture; D-wt/vol \%; E-\% saturation; $\mathrm{H}-$ wt \% solvent; I-mol \% surfactant; $\mathrm{K}-$ normality counterions; $\mathrm{M}-$ molar; $\mathrm{N}$ - normal; $\mathrm{P}$ - wt \%; $\mathrm{Q}$ - wt \% surfactant; $\mathrm{R}$ - varied; $\mathrm{S}-$ mol/ $\mathrm{kg} ; \mathrm{T}-\mathrm{wt} \%$ surfactant mixture; $\mathrm{U}-\mathrm{mol} /(\mathrm{l}$ orkg); $\mathrm{W}-$ molal; $\mathrm{Y}-$ atm. Details on page 222. 


\begin{tabular}{|c|c|c|c|c|c|c|c|c|c|c|c|c|c|c|}
\hline & & \multicolumn{2}{|r|}{ Additives } & $\begin{array}{l}\cup \\
\stackrel{\dot{\delta}}{E} \\
\stackrel{-}{-}\end{array}$ & \multicolumn{3}{|c|}{$\mathrm{CMC}$} & 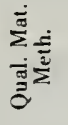 & Method & \multicolumn{2}{|c|}{ Authors } & $\frac{\mathscr{U}}{\check{E}}$ & $\stackrel{\check{\Sigma}}{\grave{n}}$ & 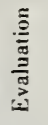 \\
\hline 1.0 & E-1 M & NA & CL & 30 & $\begin{array}{l}1.09 \\
4.914\end{array}$ & $\begin{array}{r}\times 10-1 \\
4 \times 10-3\end{array}$ & & $\mathrm{CD}$ & TURBIDITY PLT LITE SCATR & KUSH & PARK & 57006 & $\begin{array}{l}\mathrm{T} \\
\mathrm{M}\end{array}$ & L \\
\hline 1.5 & $E-1 \quad M$ & NA & CL & 30 & $\begin{array}{l}1.01 \\
4.553\end{array}$ & $\begin{array}{r}\times 10-1 \\
3 \times 10-3\end{array}$ & $\begin{array}{l}\mathrm{D} \\
\mathrm{M}\end{array}$ & $\mathrm{CD}$ & TURBIDITY PLT LITE SCATR & KUSH & PARK & 57006 & $\begin{array}{l}\mathrm{T} \\
\mathrm{M}\end{array}$ & $\mathrm{L}$ \\
\hline 2.0 & E-1 M & NA & CL & 30 & $\begin{array}{l}9.3 \\
4.19\end{array}$ & $\begin{array}{l}\times 10-2 \\
\times 10-3\end{array}$ & $\begin{array}{l}\mathrm{D} \\
\mathrm{M}\end{array}$ & CE & TURBIDITY PLT LITE SCATR & KUSH & PARK & 57006 & $\begin{array}{l}\mathrm{T} \\
\mathrm{M}\end{array}$ & $\mathrm{L}$ \\
\hline 2.5 & E-1 M & NA & $\mathrm{CL}$ & 30 & $\begin{array}{l}9.3 \\
4.19\end{array}$ & $\begin{array}{l}\times 10-2 \\
\times 10-3\end{array}$ & $\begin{array}{l}\mathrm{D} \\
\mathrm{M}\end{array}$ & $\mathrm{CE}$ & TURBIDITY PLT LITE SCATR & KUSH & PARK & 57006 & $\begin{array}{l}\mathrm{T} \\
\mathrm{M}\end{array}$ & $\mathrm{L}$ \\
\hline 3.0 & $E-1 M$ & NA & $\mathrm{CL}$ & 30 & $\begin{array}{l}8.5 \\
3.83\end{array}$ & $\begin{array}{l}\times 10-2 \\
\times 10-3\end{array}$ & $\begin{array}{l}\mathrm{D} \\
\mathrm{M}\end{array}$ & $\mathrm{CE}$ & TURBIDITY PLT LITE SCATR & KUSH & PARK & 57006 & $\begin{array}{l}\mathrm{T} \\
\mathrm{M}\end{array}$ & L \\
\hline 5. & $E-2 N$ & NA & $\mathrm{CL}$ & 50 & 6.5 & $\mathrm{x} 10-3$ & M & $\mathrm{CB}$ & FOTOMTR SOLUBLZTN PDMAB & KOLT & STRI & 49005 & $\mathrm{~T}$ & L \\
\hline 5. & $E-2 N$ & NA & $\mathrm{CL}$ & 50 & 6.0 & $\mathrm{x} 10-3$ & $M$ & $\mathrm{CB}$ & FOTOMTR SOLUBLZTN OROT & KOLT & STRI & 49005 & $\mathrm{~T}$ & L \\
\hline 1. & $\mathrm{E}-1 \mathrm{~N}$ & NA & $\mathrm{CL}$ & 50 & 3.6 & $x 10-3$ & $M$ & $\mathrm{CB}$ & FOTOMTR SOLUBLZTN PDMAB & KOLT & STRI & 49005 & $\mathrm{~T}$ & L \\
\hline 1 . & E-1 N & NA & CL & 50 & 3.5 & $\mathrm{x} 10-3$ & $M$ & $\mathrm{CB}$ & FOTOMTR SOLUBLZTN OROT & KOLT & STRI & 49005 & $\mathrm{~T}$ & $\mathrm{~L}$ \\
\hline 2. & $E-1 N$ & NA & CL & 50 & 2.6 & $\mathrm{X} 10-3$ & M & $\mathrm{CC}$ & FOTOMTR SOLUBLZTN PDMAB & KOLT & STRI & 49005 & $\mathrm{~T}$ & $\mathrm{~L}$ \\
\hline 2. & $E-2 N$ & $\mathrm{NA}$ & HCO2 FORMATE & 30 & 9.1 & $\mathrm{x} 10-3$ & M & $\mathrm{BC}$ & EQUIV CONDCTNCE GRAPH & RALS & EGGE & 49008 & $\mathrm{~K}$ & L \\
\hline 1. & $\mathrm{E}-3 \mathrm{~N}$ & NA & N03 & 30 & 1.37 & $\mathrm{x} 10-2$ & M & BB & EQUIV CONDCTNCE GRAPH & RALS & EGGE & 49008 & G & 3 \\
\hline 2.5 & $\mathrm{E}-3 \mathrm{~N}$ & NA & No3 & 30 & 1.23 & $\mathrm{x} 10-2$ & M & BB & EQUIV CONDCTNCE GRAPH & RALS & EGGE & 49008 & G & 3 \\
\hline 5. & $\mathrm{E}-3 \mathrm{~N}$ & NA & N03 & 30 & 9.9 & $\mathrm{x} 10-3$ & M & $\mathrm{BB}$ & EQUIV CONDCTNCE GRAPH & RALS & EGGE & 49008 & G & 3 \\
\hline 1. & $E-2 N$ & NA & N03 & 30 & 7.6 & $\mathrm{x} 10-3$ & M & $\mathrm{BB}$ & EQUIV CONDCTNCE GRAPH & RALS & EGGE & 49008 & G & 3 \\
\hline 2. & $E-2 N$ & NA & No3 & 30 & 4.6 & $\mathrm{x} 10-3$ & M & $\mathrm{BB}$ & EQUIV CONDCTNCE GRAPH & RALS & EGGE & 49008 & G & 3 \\
\hline 2.5 & $\mathrm{E}-3 \mathrm{~N}$ & NA & SUCCINATE & 30 & 6.1 & $x 10-3$ & M & $\mathrm{BC}$ & EQUIV CONDCTNCE GRAPH & RALS & EGGE & 49008 & $\mathrm{~K}$ & $\mathrm{~L}$ \\
\hline 3.85 & E-1 M & PRO & OPANOL-1 & 25 & 1.09 & $\mathrm{x} 10-2$ & M & BG & VISUAL SPCTR CHNGE INPX & CORR & HARK & 50008 & $\mathrm{~T}$ & $\mathrm{~L}$ \\
\hline 6.31 & E-1 M & PRO & PAANOL-1 & 25 & 8.99 & $\mathrm{x} 10-3$ & M & BG & VISUAL SPCTR CHNGE INPX & CORR & HARK & 50008 & $\mathrm{~T}$ & $\mathrm{~L}$ \\
\hline 9.88 & E-1 M & PRO & PANOL-1 & 25 & 7.03 & $\mathrm{x} 10-3$ & M & $\mathrm{BG}$ & VISUAL SPCTR CHNGE INPX & CORR & HARK & 50008 & $\mathrm{~T}$ & $\mathrm{~L}$ \\
\hline 6.54 & E-1 M & PRO & PANOL-2 & 25 & 9.53 & $\mathrm{x} 10-3$ & M & BG & VISUAL SPCTR CHNGE INPX & CORR & HARK & 50008 & $\mathrm{~T}$ & $\mathrm{~L}$ \\
\hline 1.046 & $6 \mathrm{E} O \mathrm{M}$ & PRO & PANOL-2 & 25 & 7.62 & $\mathrm{x} 10-3$ & M & BG & VISUAL SPCTR CHNGE INPX & CORR & HARK & 50008 & $\mathrm{~T}$ & $\mathrm{~L}$ \\
\hline 8.00 & $\mathrm{E} O \mathrm{H}$ & PRO & PANOL-2 & 25 & 9.2 & $\mathrm{x} 10-3$ & M & $\mathrm{BB}$ & EQUIV CONDCTNCE GRAPH & BROW & GRIE & 49014 & $\mathrm{~T}$ & 3 \\
\hline 1.619 & $\vartheta \mathrm{E} 1 \mathrm{H}$ & PRO & PANOL-2 & 25 & 1.04 & $\mathrm{x} 10-2$ & M & $\mathrm{BB}$ & EQUIV CONDCTNCE GRAPH & BROW & GRIE & 49014 & $\mathrm{~T}$ & 3 \\
\hline 5. & E $2 Y$ & PRE & ESSURE & 25.0 & 1.39 & $x 10-2$ & M & CB & SPECFC CONDCTNCE GRAPH & OSUG & SATO & 65036 & G & $\mathrm{L}$ \\
\hline 1. & E $3 \mathrm{Y}$ & PRE & ISSURE & 25.0 & 1.41 & $\mathrm{x} 10-2$ & M & $\mathrm{CB}$ & SPECFC CONDCTNCE GRAPH & OSUG & SATO & 65036 & G & L \\
\hline 2. & E $3 Y$ & PRE & ISSURE & 25.0 & 1.42 & $\mathrm{x} 10-2$ & M & $\mathrm{CB}$ & SPECFC CONDCTNCE GRAPH & OSUG & SATO & 65036 & G & L \\
\hline 4.84 & E-5 M & UND & DECANOL-1 & 25 & 1.06 & $\mathrm{x} 10-2$ & M & BG & VISUAL SPCTR CHNGE INPX & CORR & HARK & 50008 & $\mathrm{~T}$ & L \\
\hline 6.39 & E-5 M & UND & DECANOL-1 & 25 & 9.43 & $\mathrm{x} 10-3$ & M & BG & VISUAL SPCTR CHNGE INPX & CORR & HARK & 50008 & $\mathrm{~T}$ & L \\
\hline 8.31 & $\mathrm{E}-5 \mathrm{M}$ & UND & DECANOL-1 & 25 & 9.19 & $\mathrm{x} 10-3$ & M & BG & VISUAL SPCTR CHNGE INPX & CORR & HARK & 50008 & $\mathrm{~T}$ & L \\
\hline 1.01 & $E-4 M$ & UND & DECANOL-1 & 25 & 7.47 & $\mathrm{x} 10-3$ & M & $\mathrm{BG}$ & VISUAL SPCTR CHNGE INPX & CORR & HARK & 50008 & $\mathrm{~T}$ & L \\
\hline 1.28 & $E-4 M$ & UND & DECANOL-1 & 25 & 7.07 & $x 10-3$ & M & $\mathrm{BG}$ & VISUAL SPCTR CHNGE INPX & CORR & HARK & 50008 & $\mathrm{~T}$ & $\mathrm{~L}$ \\
\hline 5.66 & $\mathrm{EOH}$ & TER & RTIARY BUTANOL & 25 & 7.9 & $\mathrm{x} 10-3$ & M & $\mathrm{BB}$ & EQUIV CONDCTNCE GRAPH & BROW & GRIE & 49014 & $\mathrm{~T}$ & 3 \\
\hline 7.88 & $\mathrm{E} O \mathrm{H}$ & TER & RTIARY BUTANOL & 25 & 7.4 & $x 10-3$ & M & $\mathrm{BB}$ & EQUIV CONDCTNCE GRAPH & BROW & GRIE & 49014 & $\mathrm{~T}$ & 3 \\
\hline 6.39 & $E-3 M$ & TRI & IETHYL CARBINOL & 25 & 1.18 & $x 10-2$ & $M$ & $\mathrm{BG}$ & VISUAL SPCTR CHNGE INPX & CORR & HARK & 50008 & $\mathrm{~T}$ & L \\
\hline 1.22 & $\mathrm{E}-2 \mathrm{M}$ & TRI & IETHYL CARBINOL & 25 & 1.11 & $\mathrm{x} 10-2$ & M & BG & VISUAL SPCTR CHNGE INPX & CORR & HARK & 50008 & $\mathrm{~T}$ & $\mathrm{~L}$ \\
\hline 2.22 & $E-2 M$ & TRI & IETHYL CARBINOL & 25 & 1.01 & $x 10-2$ & M & $\mathrm{BG}$ & VISUAL SPCTR CHNGE INPX & CORR & HARK & 50008 & $T$ & L \\
\hline 3.08 & $E-2 M$ & TRI & IETHYL CARBINOL & 25 & 9.32 & $x 10-3$ & M & BG & VISUAL SPCTR CHNGE INPX & CORR & HARK & 50008 & $T$ & $\mathrm{~L}$ \\
\hline 3.72 & $\mathrm{E}-2 \mathrm{M}$ & TRI & IETHYL CARBINOL & 25 & 8.44 & $\times 10-3$ & M & BG & VISUAL SPCTR CHNGE INPX & CORR & HARK & 50008 & $\mathrm{~T}$ & $\mathrm{~L}$ \\
\hline 4.27 & $E-2 M$ & TRI & IETHYL CARBINOL & 25 & 7.76 & $x 10-3$ & M & BG & VISUAL SPCTR CHNGE INPX & CORR & HARK & 50008 & $\mathrm{~T}$ & $\mathrm{~L}$ \\
\hline 4.80 & $E-2 M$ & TRI & IETHYL CARBINOL & 25 & 7.27 & $x_{10-3}$ & M & BG & VISUAL SPCTR CHNGE INPX & CORR & HARK & 50008 & $\mathrm{~T}$ & $\mathrm{~L}$ \\
\hline 2.5 & $\mathrm{E}-3 \mathrm{M}$ & 003 & & 30 & 2.5 & $x 10-3$ & M & $\mathrm{BE}$ & EQUIV CONDCTNCE GRAPH & RALS & EGGE & 48020 & $\mathrm{~K}$ & $\mathrm{~L}$ \\
\hline 1. & $\begin{array}{lll}E-1 & K \\
2.0 & \text { E } & 0\end{array}$ & $\mathrm{CL}-$ & $\begin{array}{l}\text { - ION } \\
\text { PH OF SOLUTION }\end{array}$ & UNK & 3.50 & $\times 10-3$ & M & $\mathrm{CB}$ & SURFACE TENSION LOG PLOT & ROE & BRAS & 54013 & $\mathrm{~T}$ & $\mathrm{~L}$ \\
\hline 2.52 & $\begin{array}{l}E-5 \mathrm{M} \\
6.00 \mathrm{E}-2\end{array}$ & ${ }_{M}^{\mathrm{DEC}}$ & $\begin{array}{r}\text { CANOL-1 } \\
\text { K CL }\end{array}$ & 25 & 4.43 & $x 10-3$ & $M$ & BG & VISUAL SPCTR CHNGE INPX & CORR & HARK & 50008 & $\mathrm{~T}$ & $\mathrm{~L}$ \\
\hline 4.53 & $\begin{array}{l}\mathrm{E}-5 \mathrm{M} \\
6.00 \mathrm{E}-2\end{array}$ & ${ }_{M}^{\mathrm{DEC}}$ & $\begin{array}{r}\text { KANOL-1 } \\
\text { K CL }\end{array}$ & 25 & 3.98 & $\mathrm{x} 10-3$ & $M$ & BG & VISUAL SPCTR CHNGE INPX & CORR & HARK & 50008 & $\mathrm{~T}$ & $\mathrm{~L}$ \\
\hline 4.96 & $\begin{array}{l}\mathrm{E}-5 \mathrm{M} \\
3.00 \mathrm{E}-2\end{array}$ & $\mathrm{M}^{\mathrm{DEC}}$ & $\begin{array}{r}\text { CANOL-1 } \\
\text { K CL }\end{array}$ & 25 & 6.00 & $x 10-3$ & $M$ & BG & VISUAL SPCTR CHNGE INPX & CORR & HARK & 50008 & $\mathrm{~T}$ & L \\
\hline 5.52 & $\begin{array}{l}\mathrm{E}-5 \mathrm{M} \\
1.50 \mathrm{E}-2\end{array}$ & ${ }_{M}^{\mathrm{DEC}}$ & $\begin{array}{r}\text { CANOL-1 } \\
\text { K CL }\end{array}$ & 25 & 6.73 & $\mathrm{x} 10-3$ & M & BG & VISUAL SPCTR CHNGE INPX & CORR & HARK & 50008 & $\mathrm{~T}$ & $\mathrm{~L}$ \\
\hline 6.32 & $\begin{array}{l}\mathrm{E}-5 \mathrm{M} \\
6.00 \mathrm{E}-2\end{array}$ & $\mathrm{MEC}$ & $\begin{array}{r}\text { CANOL-1 } \\
\text { K CL }\end{array}$ & 25 & 3.70 & $\mathrm{X} 10-3$ & M & BG & VISUAL SPCTR CHNGE INPX & CORR & HARK & 50008 & $\mathrm{~T}$ & $\mathrm{~L}$ \\
\hline 6.81 & $\begin{array}{ll}E-5 & M \\
7.5 & E-3\end{array}$ & ${ }_{M}$ DEC & $\begin{array}{r}\text { CANOL-1 } \\
\text { K CL }\end{array}$ & 25 & 8.63 & $\mathrm{x} 10-3$ & M & BG & VISUAL SPCTR CHNGE INPX & CORR & HARK & 50008 & $\mathrm{~T}$ & L \\
\hline 8.05 & $\begin{array}{l}E-5 \mathrm{M} \\
6.00 \mathrm{E}-2\end{array}$ & $M^{\mathrm{DEC}}$ & $\begin{array}{r}\text { CANOL-1 } \\
\text { K CL }\end{array}$ & 25 & 3.53 & $\mathrm{X} 10-3$ & M & BG & VISUAL SPCTR CHNGE INPX & CORR & HARK & 50008 & $\mathrm{~T}$ & L \\
\hline 8.23 & $\begin{array}{l}\mathrm{E}-5 \mathrm{M} \\
3.00 \mathrm{E}-2\end{array}$ & $\mathrm{M}^{\mathrm{DEC}}$ & $\begin{array}{l}\text { CANOL-1 } \\
\text { K CL }\end{array}$ & 25 & 4.98 & $\mathrm{X} 10-3$ & M & BG & VISUAL SPCTR CHNGE INPX & CORR & HARK & 50008 & $\mathrm{~T}$ & L \\
\hline 8.86 & $\begin{array}{l}E-5 \mathrm{M} \\
6.00 \mathrm{E}-2\end{array}$ & $M_{M}^{\mathrm{DEC}}$ & $\begin{array}{r}\text { CANOL-I } \\
\text { K CL }\end{array}$ & 25 & 3.11 & $\mathrm{x} 10-3$ & M & BG & VISUAL SPCTR CHNGE INPX & CORR & HARK & 50008 & $\mathrm{~T}$ & L \\
\hline 9.42 & $\begin{array}{l}\mathrm{E}-5 \mathrm{M} \\
1.50 \mathrm{E}-2\end{array}$ & $M^{\mathrm{DEC}}$ & $\begin{array}{r}\text { CANOL-1 } \\
\text { K CL }\end{array}$ & 25 & 5.74 & $\mathrm{X} 10-3$ & $M$ & BG & VISUAL SPCTR CHNGE INPX & CORR & HARK & 50008 & $\mathrm{~T}$ & L \\
\hline 1.01 & $\begin{array}{l}\mathrm{E}-4 \mathrm{M} \\
6.00 \mathrm{E}-2\end{array}$ & $M^{D E C}$ & $\begin{array}{r}\text { CANOL-1 } \\
\text { K CL }\end{array}$ & 25 & 2.95 & $\mathrm{x} 10-3$ & M & BG & VISUAL SPCTR CHNGE INPX & CORR & HARK & 50008 & $\mathrm{~T}$ & L \\
\hline 1.03 & $\begin{array}{l}\mathrm{E}-4 \mathrm{M} \\
3.00 \mathrm{E}-2\end{array}$ & $M_{M}^{D E C}$ & $\begin{array}{r}\text { CANOL-1 } \\
\text { K CL }\end{array}$ & 25 & 4.14 & $\mathrm{x} 10-3$ & M & BG & VISUAL SPCTR CHNGE INPX & CORR & HARK & 50008 & $\mathrm{~T}$ & L \\
\hline 1.10 & $\begin{array}{l}E-4 M \\
7.5 \quad E-3\end{array}$ & $M_{M}^{D E C}$ & $\begin{array}{r}\text { CANOL-1 } \\
\mathrm{K} \mathrm{CL}\end{array}$ & 25 & 6.95 & $\mathrm{x} 10-3$ & M & BG & VISUAL SPCTR CHNGE INPX & CORR & HARK & 50008 & $\mathrm{~T}$ & L \\
\hline 1.26 & $\begin{array}{l}\mathrm{E}-4 \mathrm{M} \\
3.00 \mathrm{E}-2\end{array}$ & DEC & CANOL-1 & 25 & 3.82 & $\mathrm{X} 10-3$ & M & BG & VISUAL SPCTR CHNGE INPX & CORR & HARK & 50008 & $\mathrm{~T}$ & $\mathrm{~L}$ \\
\hline
\end{tabular}

Concentration units: $\mathrm{A}-\operatorname{mol} \%$; $\mathrm{B}-\mathrm{vol} \%$ solvent: $\mathrm{C}-\mathrm{mol} \%$ surfactant mixture; 


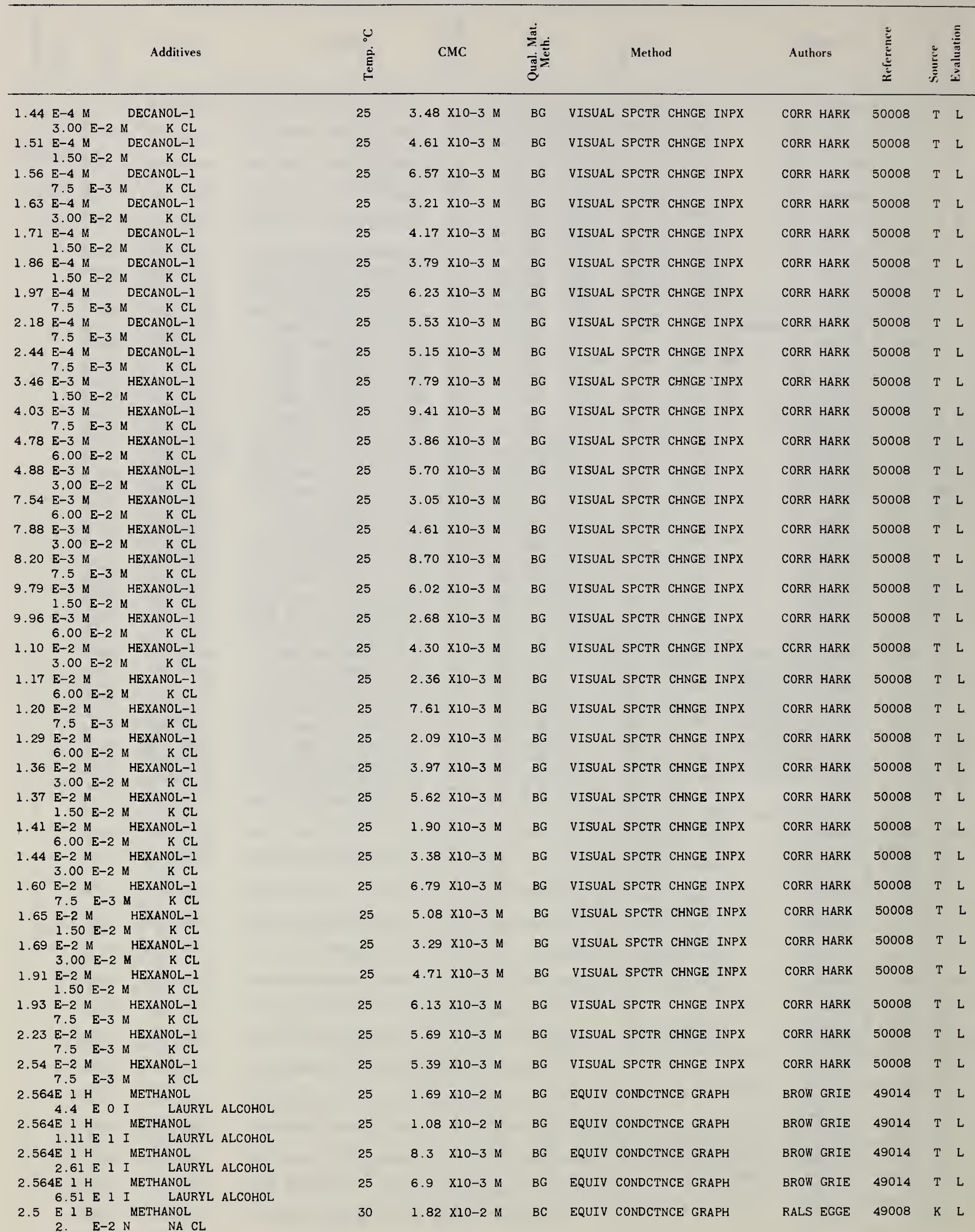

Concentration units: $\mathrm{A}-$ mol \%; $\mathrm{B}-$ vol \% solvent: $\mathrm{C}-$ mol \% surfactant mixture; D-wt/vol \%; $\mathrm{E}-\%$ saturation; $\mathrm{H}-$ wt $\%$ solvent; I-mol \% surfactant; $\mathrm{K}$-normality counterions; $\mathrm{M}$-molar; $\mathrm{N}$-normal; $\mathrm{P}-$ wt $\% ; \mathrm{Q}$ - wt \% surfactant: $\mathrm{R}$-varied; $\mathrm{S}-$ mol/ kg; $\mathrm{T}$ - wt \% surfactant mixture; $\mathrm{U}-\mathrm{mol} /(\mathrm{l}$ orkg); $\mathrm{W}$ - molal; $\mathrm{Y}-\mathrm{atm}$. Detailson page 222. 


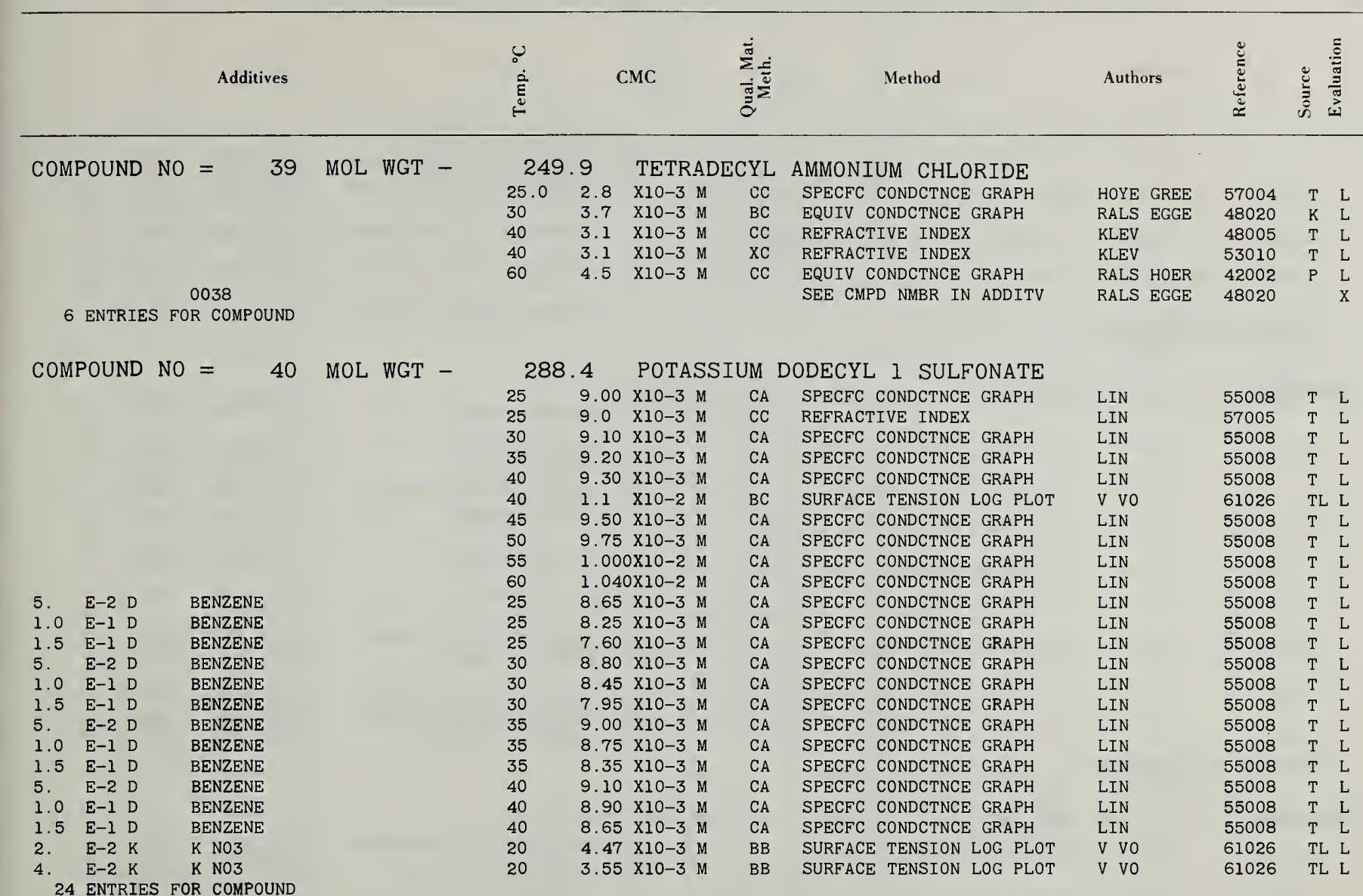

\section{COMPOUND NO $=41$ MOL WGT -}

1. $E-1 M \quad K C L$

2. $\mathrm{E}-2 \mathrm{M}$ NA CL

4. $E-2 M \quad N A C L$

1.0 E-1 M NA CL

5. E $2 Y$

1. E 3 Y

1.5 E 3 Y

2. E 3 Y

3. $\mathrm{E} 3 \mathrm{Y}$

4. $E 3 Y$

5. E 3 Y

$2.59 \mathrm{E} I \mathrm{I}$

$5.00 \mathrm{E} I \mathrm{C}$

$7.59 \mathrm{E} I \mathrm{C}$

$4.96 \mathrm{E}-1 \mathrm{~W}$

3.8 E-3 M NA BR
22 ENTRIES FOR COMPOUND

NA CL

PRESSURE

PRESSURE

PRESSURE

PRESSURE

PRESSURE

PRESSURE

0042

0042

0042

$\mathrm{NA}$ CL
263.9 DODECYL TRIMETHYL AMMONIUM CHLORIDE

$\begin{array}{llll}23 & 5.70 \times 10-1 & \mathrm{D} & \mathrm{CC} \\ & 2.159 \times 10-2 & \mathrm{M} & \\ 25 & 1.72 \times 10-2 & \mathrm{M} & \mathrm{BB} \\ 25 & 2.0 \times 10-2 & \mathrm{M} & \mathrm{BC} \\ 25.0 & 2.03 \times 10-2 & \mathrm{M} & \mathrm{CB} \\ 30 & 2.28 \times 10-2 & \mathrm{M} & \mathrm{BC} \\ 30 & 1.6 \times 10-2 & \mathrm{M} & \mathrm{XG} \\ \mathrm{UNK} & 2.28 \times 10-2 & \mathrm{M} & \mathrm{BC} \\ 25 & 7.5 \times 10-3 & \mathrm{M} & \mathrm{BC} \\ 23 & 4.20 \times 10-1 & \mathrm{D} & \mathrm{CC} \\ & 1.591 \times 10-2 & \mathrm{M} & \\ 23 & 3.10 \times 10-1 & \mathrm{D} & \mathrm{CC} \\ & 1.174 \times 10-2 & \mathrm{M} & \\ 23 & 1.90 \times 10-1 & \mathrm{D} & \mathrm{CC} \\ & 7.199 \times 10-3 & \mathrm{M} & \\ 25.0 & 2.09 \times 10-2 & \mathrm{M} & \mathrm{CB} \\ 25.0 & 2.11 \times 10-2 & \mathrm{M} & \mathrm{CB} \\ 25.0 & 2.04 \times 10-2 & \mathrm{M} & \mathrm{CB} \\ 25.0 & 1.98 \times 10-2 & \mathrm{M} & \mathrm{CB} \\ 25.0 & 1.87 \times 10-2 & \mathrm{M} & \mathrm{CB} \\ 25.0 & 1.83 \times 10-2 & \mathrm{M} & \mathrm{CB} \\ 25.0 & 1.81 \times 10-2 & \mathrm{M} & \mathrm{CB} \\ 25 & 1.14 \times 10-2 & \mathrm{M} & \mathrm{BB} \\ 25 & 7.47 \times 10-3 \mathrm{M} & \mathrm{BB} \\ 25 & 5.01 \times 10-3 \mathrm{M} & \mathrm{BB} \\ 31.5 & 3.8 \times 10-3 \mathrm{M} & \mathrm{BC} \\ & & & \end{array}$

C
B
C
C
C
C
C
B
$\mathrm{CB}$
$\mathrm{CB}$
$\mathrm{CB}$
$\mathrm{BB}$
$\mathrm{BB}$
$\mathrm{BB}$
$\mathrm{BC}$

TURBIDITY PLT LITE SCATR

UNSPECIFIED CONDUCTANCE

SURFACE TENSION LOG PLOT

SPECFC CONDCTNCE GRAPH

EQUIV CONDCTNCE GRAPH

VISUAL SPCTR CHNGE

UNSPECIFIED CONDUCTANCE

SURFACE TENSION LOG PLOT

TURBIDITY PLT LITE SCATR

TURBIDITY PLT LITE SCATR

TURBIDITY PLT LITE SCATR

SPECFC CONDCTNCE GRAPH

SPECFC CONDCTNCE GRAPH

SPECFC CONDCTNCE GRAPH

SPECFC CONDCTNCE GRAPH

SPECFC CONDCTNCE GRAPH

SPECFC CONDCTNCE GRAPH

SPECFC CONDCTNCE GRAPH

UNSPEC SOLUBLZTN SDN 4

UNSPEC SOLUBLZTN SDN 4

UNSPEC SOLUBLZTN SDN 4

DEBYE PLT LIGHT SCATTER
KUSH PARK

HOYE MARM WEIN ZOGR

OSUG SATO

RALS BROO

KLEV

CELL EGGE

WEIN ZOGR

KUSH PARK

KUSH PARK

KUSH PARK

OSUG SATO OSUG SATO OSUG SATO OSUG SATO OSUG SATO OSUG SATO OSUG SATO HOYE MARM HOYE MARM HOYE MARM ANAC GHOS

\begin{tabular}{|c|c|}
\hline 7006 & $\mathrm{~T}$ \\
\hline 61002 & $\begin{array}{l}M \\
T\end{array}$ \\
\hline 65026 & $\mathrm{~T}$ \\
\hline 65036 & $G$ \\
\hline 49013 & $\mathrm{~T}$ \\
\hline 53010 & $\mathrm{~T}$ \\
\hline 52001 & $\mathrm{~T}$ \\
\hline 65026 & $\mathrm{~T}$ \\
\hline 57006 & $\mathrm{~T}$ \\
\hline & \\
\hline & \\
\hline 57006 & $\mathrm{~T}$ \\
\hline 65036 & $\begin{array}{l}\mathrm{M} \\
\mathrm{G}\end{array}$ \\
\hline 65036 & G \\
\hline 65036 & G \\
\hline 65036 & G \\
\hline 65036 & G \\
\hline 65036 & G \\
\hline 65036 & G \\
\hline 61002 & $\mathrm{~T}$ \\
\hline 61002 & $\mathrm{~T}$ \\
\hline 61002 & $T$ \\
\hline
\end{tabular}

COMPOUND NO $=42$ MOL WGT -

292.0 TETRADECYL TRIMETHYL AMMONIUM CHLORIDE

\begin{tabular}{|c|c|c|c|c|c|c|}
\hline 23 & $\begin{array}{l}1.20 \times 10-1 \\
4.109 \times 10-3\end{array}$ & $\mathrm{CC}$ & TURBIDITY PLT LITE SCATR & KUSH PARK & 57006 & $\begin{array}{l}\mathrm{T} \\
\mathrm{M}\end{array}$ \\
\hline & $3.0 \times 10-3 \mathrm{~N}$ & $\mathrm{HE}$ & EQUIV CONDCTNCE GRAPH & BRAD MCBA & 48009 & $\mathrm{~T}$ \\
\hline & $4.47 \times 10-3 \mathrm{~N}$ & BB & UNSPECIFIED CONDUCTANCE & HOYE MARM & 61002 & $\mathrm{~T}$ \\
\hline & $3.6 \times 10-3 \mathrm{~N}$ & $\mathrm{XG}$ & VISUAL SPCTR CHNGE & KLEV & 53010 & $\mathrm{~T}$ \\
\hline
\end{tabular}

Concentration units: $\mathrm{A}-\operatorname{mol} \%$ : $\mathrm{B}-\mathrm{vol} \%$ solvent: $\mathrm{C}-$ mol $\%$ surfactant mixture; counterions; $\mathrm{M}-$ molar; $\mathrm{N}$-normal; $\mathrm{P}-$ wt \%; $\mathrm{Q}-$ wt $\%$ surfactant; $\mathrm{R}$ - varied; $\mathrm{S}-$ mol/ $D-w t / v o l ~ \% ; E-\%$ saturation; $\mathrm{H}-$ wt $\%$ solvent; $1-$ mol \% surfactant; $\mathrm{K}-$ normality $\mathrm{kg} ; \mathrm{T}-\mathrm{wt} \%$ surfactant mixture; $\mathrm{U}-\mathrm{mol} /(\mathrm{l}$ orkg): $\mathrm{W}-$ molal; $\mathrm{Y}-$ atm. Detailson page 222. 


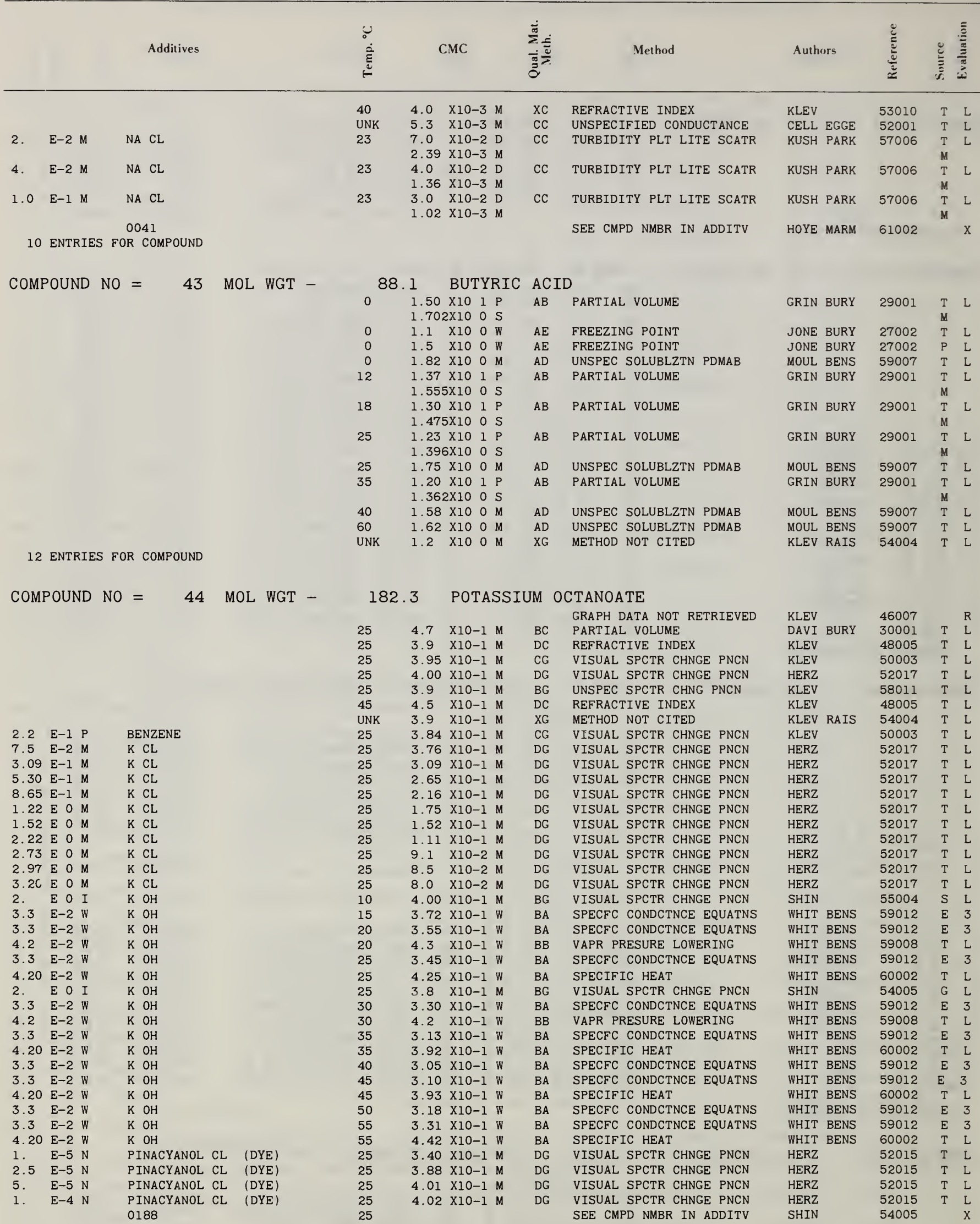

Concentration units: $\mathrm{A}-$ mol \%; $\mathrm{B}-$ vol \% solvent; $\mathrm{C}-$ mol \% surfactant mixture; $\mathrm{D}-\mathrm{wt} / \mathrm{vol} \%$; $\mathrm{E}-\%$ saturation; $\mathrm{H}-$ wt $\%$ solvent; $\mathrm{I}-$ mol \% surfactant; $\mathrm{K}-$ normality counterions; $\mathrm{M}$-molar; $\mathrm{N}$-normal; $\mathrm{P}-$ wt \%: $\mathrm{O}$-wt \% surfactant; $\mathrm{R}$-varied; $\mathrm{S}-$ mol $\mathrm{kg} ; \mathrm{T}-\mathrm{wt} \%$ surfactant mixture; $\mathrm{U}-\mathrm{mol} /(\mathrm{l}$ or $\mathrm{kg}) ; \mathrm{W}-$ molal; $\mathrm{Y}$ - atm. Detailson page 222. 


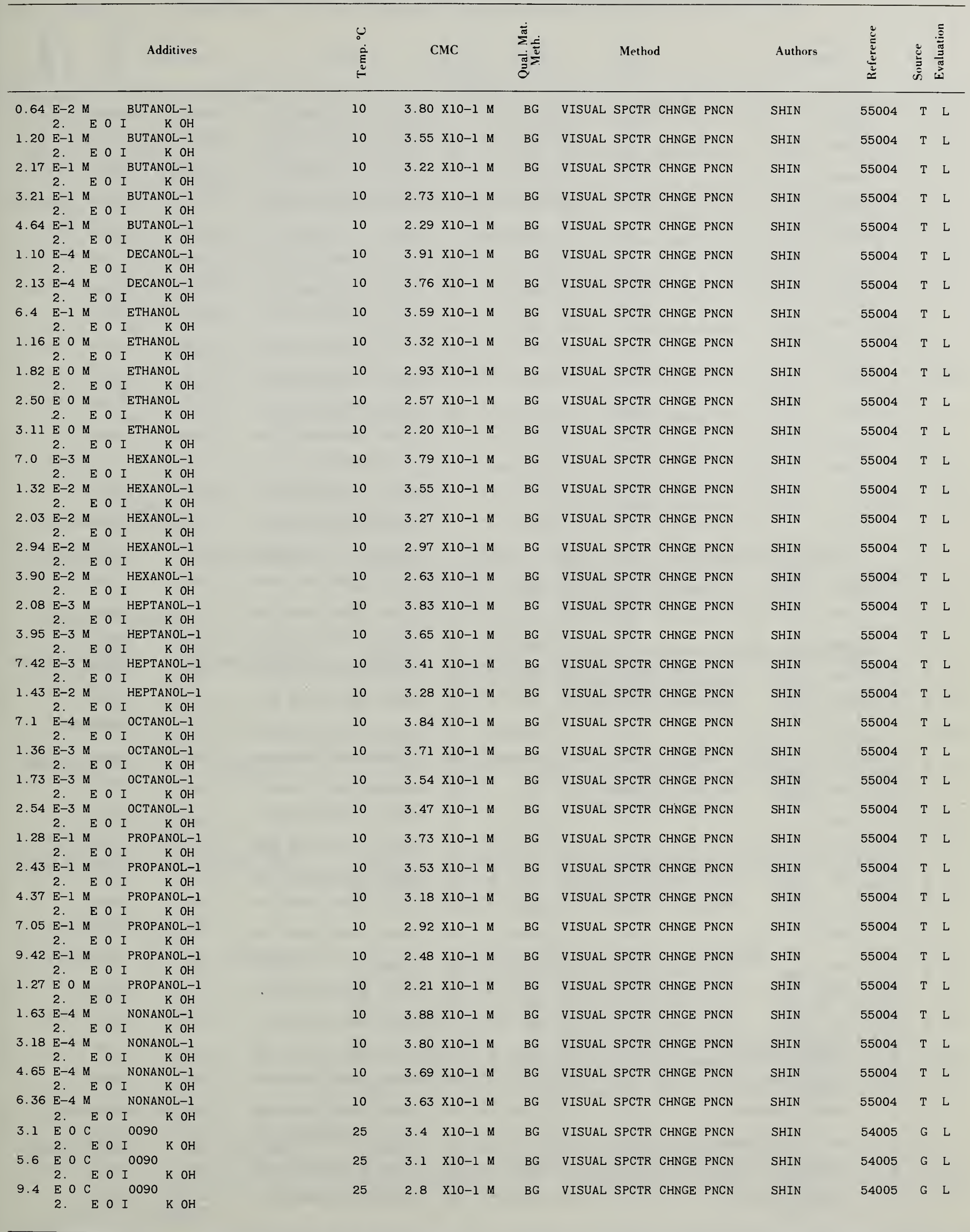

Concentration units: $\mathrm{A}-\mathrm{mol} \%$; $\mathrm{B}-$ vol \% solvent; $\mathrm{C}-$ mol \% surfactant mixture $\mathrm{D}-\mathrm{wt} / \mathrm{vol} \%$; E-\% saturation; $\mathrm{H}-\mathrm{wt} \%$ solvent; I-mol \% surfactant; $\mathrm{K}-$ normality counterions; $\mathrm{M}$-molar; $\mathrm{N}$-normal; $\mathrm{P}$ - wt \%; $\mathrm{Q}$ - wt \% surfactant; $\mathrm{R}$-varied; $\mathrm{S}$-mol/ $\mathrm{kg}$; $\mathrm{T}-$ wt \% surfactant mixture; $\mathrm{U}-\mathrm{mol} /(\mathrm{l}$ or $\mathrm{kg}) ; \mathrm{W}-$ molal; $\mathrm{Y}-\mathrm{atm}$. Details on page 222. 


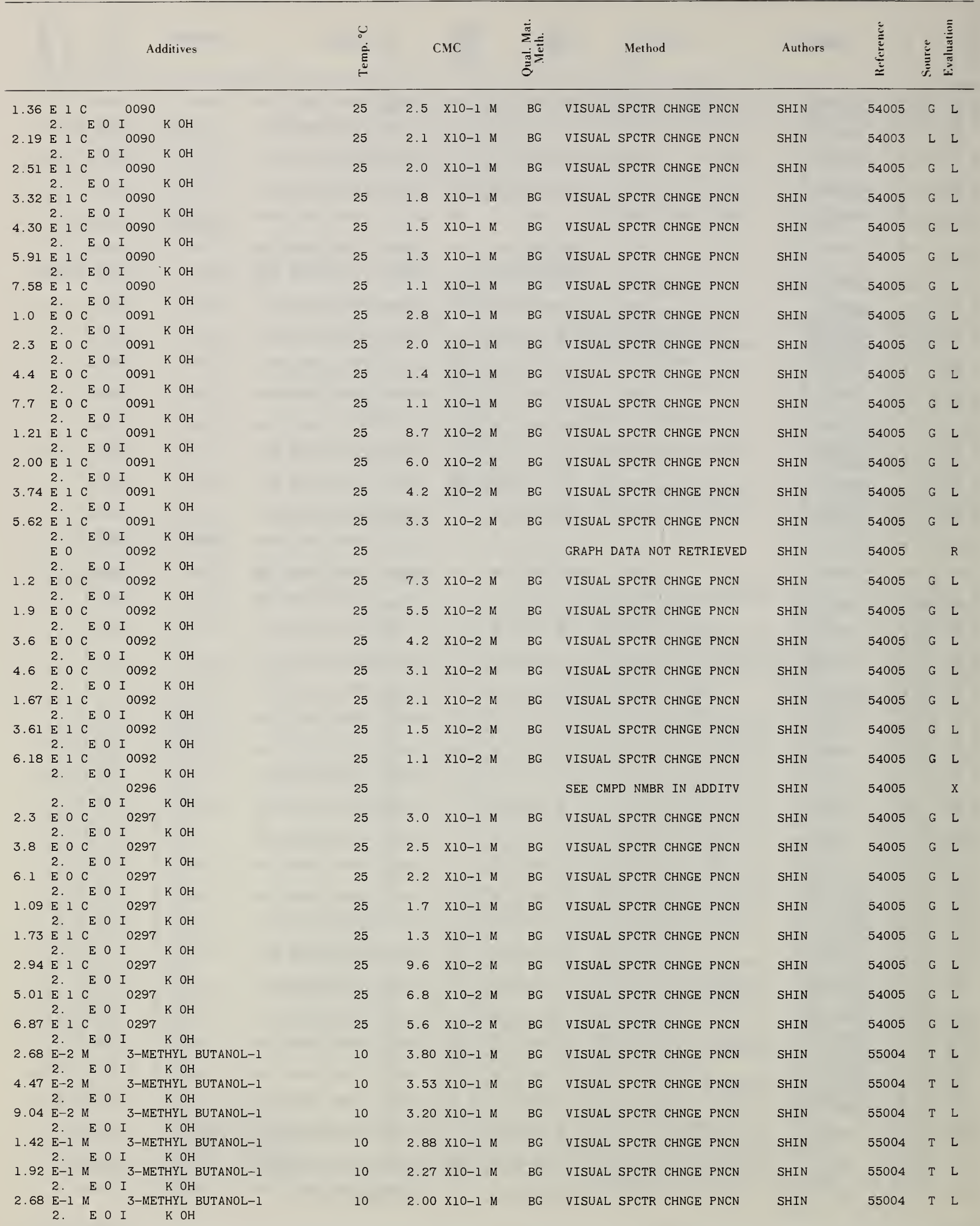

Concentration units: $\mathrm{A}-\mathrm{mol} \% ; \mathrm{B}-$ vol \% solvent; $\mathrm{C}-$ mol \% surfactant mixture; 


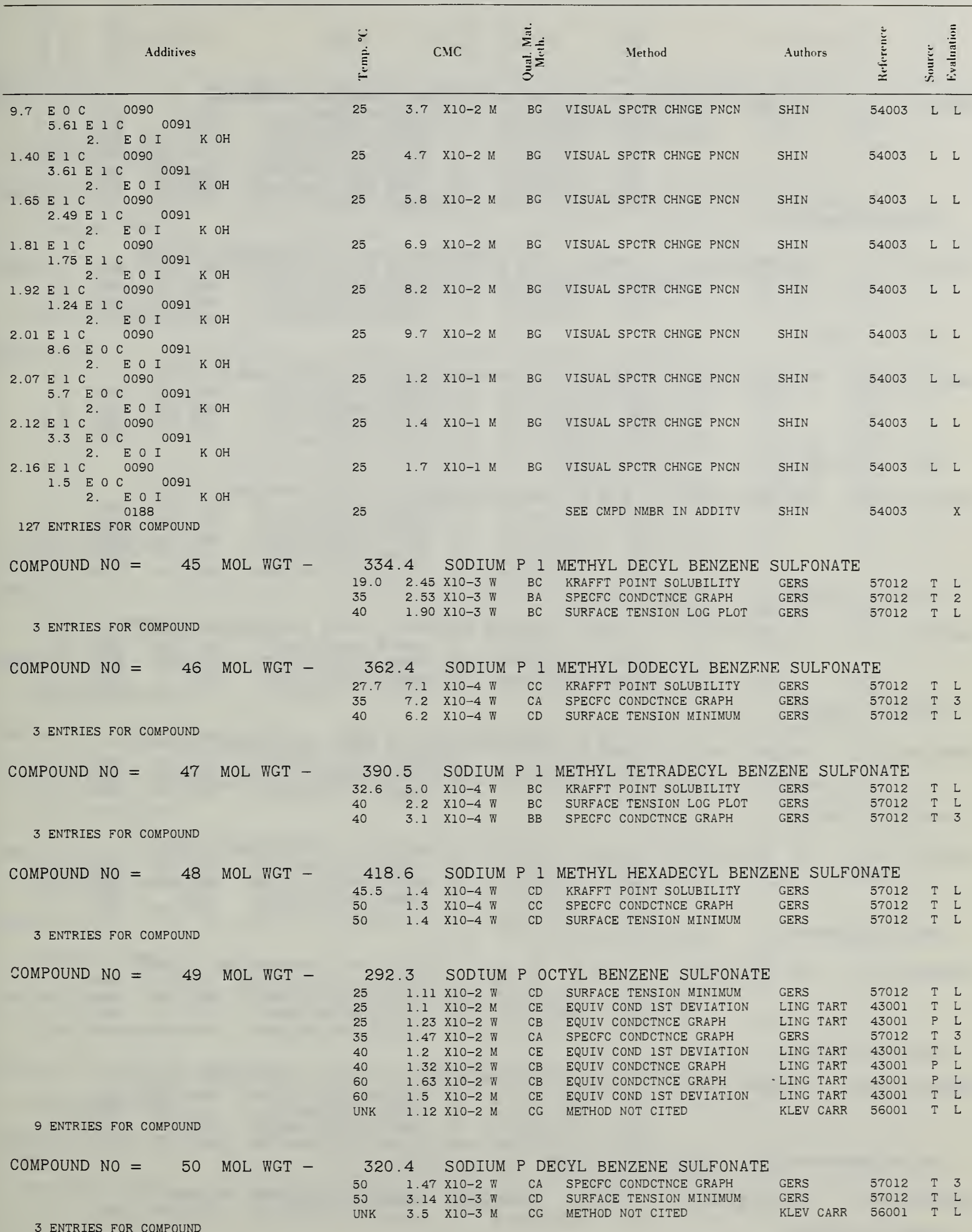

\footnotetext{
Concentration units: $\mathrm{A}-\mathrm{mol} \%$; $\mathrm{B}-\mathrm{vol} \%$ solvent; $\mathrm{C}-$ mol $\%$ surfactant mixture;
$\mathrm{D}-$ wt/vol \%; $\mathrm{E}-\%$ saturation: $\mathrm{H}-$ wt \% solvent; I-mol \% surfactant; K-normality
} counterions; $\mathrm{M}-$ molar; $\mathrm{N}-$ normal; $\mathrm{P}-\mathrm{wt} \% ; \mathrm{Q}-\mathrm{wt} \%$ surfactant; $\mathrm{R}-$ varied; $\mathrm{S}-$ mol/ 


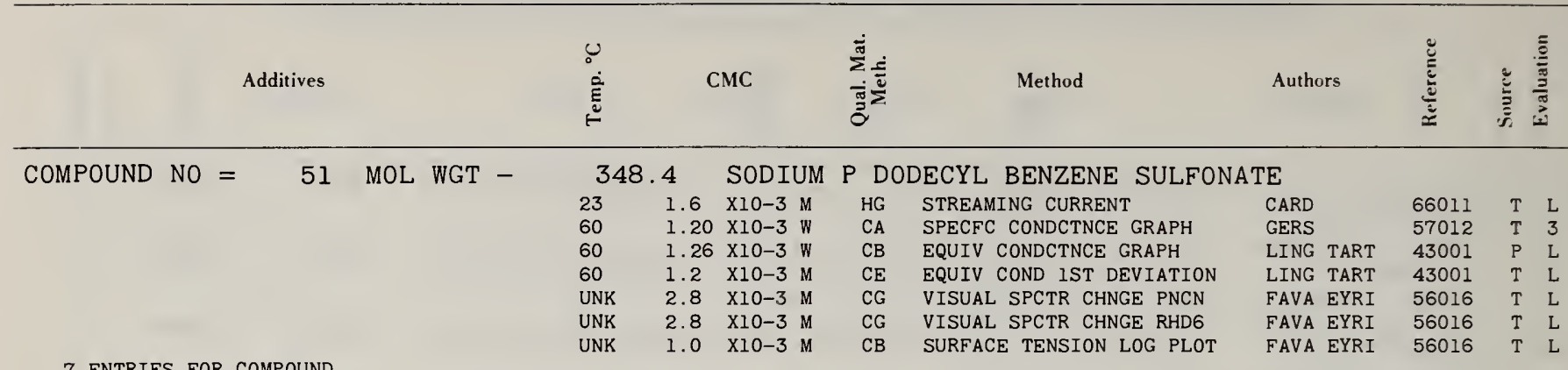

7 ENTRIES FOR COMPOUND

388.5 SODIUM HEXADECYL MONO OXYETHYLENE SULFATE

$\begin{array}{lllllllll}25 & 2.2 & \text { XI0-4 M } & \text { CG } & \text { VISUAL SPCTR CHNGE PNCN } & \text { WEIL BIST } & 58003 & \text { T } & \text { L } \\ 25 & 2.1 & \text { XI0-4 M } & \text { CC } & \text { SURFACE TENSION LOG PLOT } & \text { WEIL BIST } & 58003 & \text { T } & \text { L } \\ 25 & 2.34 & \text { XI0-4 M } & \text { CC } & \text { EQUIV CONDCTNCE GRAPH } & \text { WEIL BIST } & 58003 & \text { T } & \text { L } \\ 50 & 2.4 & \text { X10-4 M } & \text { CG } & \text { VISUAL SPCTR CHNGE PNCN } & \text { WEIL STIR } & 59004 & \text { T } & \text { L }\end{array}$

4 ENTRIES FOR COMPOUND
432.6 SODIUM HEXADECYL DI OXYETHYLENE SULFATE

$25 \quad 1.4 \quad$ X10-4 M CG VISUAL SPCTR CHNGE PNCN

$25 \quad 1.34 \times 10-4 \mathrm{M} \quad \mathrm{CC}$ EQUIV CONDCTNCE GRAPH

$50 \quad 1.4 \quad$ X10-4 M CG VISUAL SPCTR CHNGE PNCN
WEIL BIST WEIL BIST WEIL BIST WEIL STIR
58003

3 T $L$

$T$

4 ENTRIES FOR COMPOUND

5 ENTRIES FOR COMPOUND

476.6

\begin{tabular}{|c|c|c|}
\hline & & \\
\hline 25 & 1.0 & $\times 10-4$ \\
\hline & 7.0 & $\mathrm{X} 10-5 \mathrm{M}$ \\
\hline & 1.23 & $\times 10-4$ \\
\hline & 1.95 & $\times 10-4$ \\
\hline & 1.2 & $\mathrm{x} 10-4$ \\
\hline
\end{tabular}

CG

$\mathrm{CG}$

$\mathrm{CD}$
$\mathrm{CD}$

$\mathrm{CG}$
ECYL TRI OXYETHYLENE SULFATE

VISUAL SPCTR CHNGE PNCN SURFACE TENSION LOG PLOT EQUIV COND MAX BEGINING SURFACE TENSION UNSPEC

VISUAL SPCTR CHNGE PNCN
WEIL BIST WEIL BIST WEIL BIST GOTT

WEIL STIR
$58003 \quad T \quad L$ 58003 T $\quad$ L 58003 T $60018 \quad G \quad$ L

59004 T
COMPOUND NO $=55$ MOL WGT -

4 ENTRIES FOR COMPOUND

$\begin{array}{lll}520.7 & \text { SODIUM } \\ 25 & 8.0 & \times 10-5 \mathrm{M} \\ 25 & 1.0 & \times 10-4 \mathrm{M} \\ 50 & 1.01 & \mathrm{X10-4} \mathrm{M} \\ 50 & 1.2 & \mathrm{X10-4} \mathrm{M}\end{array}$

HEXADECYL TETRA OXYETHYLENE SULFATE CD SURFACE TENSION LOG PLOT CG VISUAL SPCTR CHNGE PNCN CD SURFACE TENSION UNSPEC

CG VISUAL SPCTR CHNGE PNCN
WEIL BIST

WEIL BIST

GOTT

WEIL STIR
$58003 \quad T \quad L$

$58003 \quad T \quad L$ 60018 G L

59004 T L
COMPOUND NO $=56$ MOL WGT -

3 ENTRIES FOR COMPOUND
416.6 SODIUM OCTADECYL MONO OXYETHYLENE SULFATE

$\begin{array}{llllll}25^{\circ} & 1.1 & \text { XIO-4 M } & \text { BG } & \text { VISUAL SPCTR CHNGE PNCN } & \text { WEIL BIST } \\ 25 & 1.9 & \text { X10-4 M } & \text { BD } & \text { SURFACE TENSION LOG PLOT } & \text { WEIL BIST } \\ 50 & 9.0 & \text { XI0-5 M } & \text { CG } & \text { VISUAL SPCTR CHNGE PNCN } & \text { WEIL STIR }\end{array}$

58003 T L 58003 T L 59004 T L
COMPOUND NO $=57$ MOL WGT -

4 ENTRIES FOR COMPOUND

\begin{tabular}{|c|c|c|c|c|}
\hline & & ODIUM & OCTAD & OXYET \\
\hline 5 & 7.0 & $\mathrm{X} 10-5 \mathrm{M}$ & VISUA & SPCTR CHNGE PNCN \\
\hline & 8.0 & $\mathrm{X} 10-5 \mathrm{M}$ & SURFAC & E TENSION LOG PLOT \\
\hline 50 & 1.00 & $\mathrm{X} 10-4 \mathrm{M}$ & SURFACI & E TENSION UNSPEC \\
\hline & 70 & $\mathrm{X} 10-5 \mathrm{M}$ & VISUAL & SPCTR CHNGE PNCN \\
\hline
\end{tabular}

$\mathrm{CC}$
SULFATE

WEIL BIST WEIL BIST GOTT

WEIL STIR
58003

58003

60018

59004
COMPOUND NO $=58$ MOL WGT -

4 ENTRIES FOR COMPOUND

COMPOUND NO $=59 \cdot$ MOL WGT -

4 ENTRIES FOR COMPOUND

COMPOUND NO $=60$ MOL WGT -

I ENTRIES FOR COMPOUND
504.7 SODIUM OCTADECYL TRI OXYETHYLENE SULFATE

$\begin{array}{lll}25 & 5.0 & \mathrm{X} 10-5 \mathrm{M} \\ 25 & 5.0 & \mathrm{XI0-5} \mathrm{M} \\ 50 & 6.98 & \mathrm{XI0-5} \mathrm{M} \\ 50 & 7.0 & \mathrm{X} 10-5 \mathrm{M}\end{array}$

CD SURFACE TENSION LOG PLOT

CG VISUAL SPCTR CHNGE PNCN

CD SURFACE TENSION UNSPEC

CG VISUAL SPCTR CHNGE PNCN
WEIL BIST WEIL BIST GOTT WEIL STIR
58003

58003

60018

59004
T $L$

T $\mathrm{L}$

G L

T L
548.7 SODIUM OCTADECYL TETRA OXYETHYLENE SULFATE

$\begin{array}{lllllllll}25 & 4.0 & \text { X10-5 M } & \text { CD } & \text { SURFACE TENSION LOG PLOT } & \text { WEIL BIST } & 58003 & \text { T } & \text { L } \\ 25 & 4.0 & \text { X10-5 M } & \text { CG } & \text { VISUAL SPCTR CHNGE PNCN } & \text { WEIL BIST } & 58003 & \text { T } & \text { L } \\ 50 & 3.97 & \text { X10-5 M } & \text { CD } & \text { SURFACE TENSION UNSPEC } & \text { GOTT } & 60018 & \text { G } & \text { L } \\ 50 & 7.0 & \text { X10-5 M } & \text { CG } & \text { VISUAL SPCTR CHNGE PNCN } & \text { WEIL STIR } & 59004 & \text { T } & \text { L }\end{array}$

Concentration units: $\mathrm{A}-\mathrm{mol} \% ; \mathrm{B}-$ vol \% solvent; $\mathrm{C}-$ mol \% surfactant mixture; $\mathrm{D}-\mathrm{wt} / \mathrm{vol} \% ; \mathrm{E}-\%$ saturation; $\mathrm{H}-\mathrm{wt} \%$ solvent; I-mol \% surfactant; $\mathrm{K}-$ normality counterions; $\mathrm{M}$ - molar; $\mathrm{N}$ - normal; $\mathrm{P}$-wi \%; $\mathrm{Q}$ - wt \% surfactant; $\mathrm{R}$-varied; $\mathrm{S}$-mol/ kg: T - wt \% surfactant mixture; $\mathrm{U}-$ mol/(l orkg); $\mathrm{W}-$ molal; $\mathrm{Y}$ - atm. Detailson page 222. 


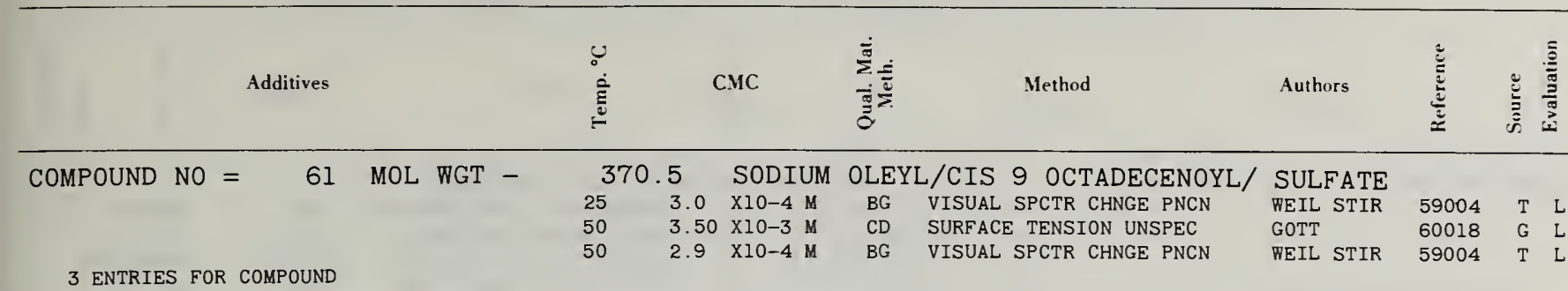

COMPOUND NO $=62$ MOL WGT -

1 ENTRIES FOR COMPOUND

COMPOUND NO $=63$ MOL WGT -

2 ENTRIES FOR COMPOUND

COMPOUND NO $=64$ MOL WGT -

E 00001

11 ENTRIES FOR COMPOUND

COMPOUND NO $=65$ MOL WGT -

1 ENTRIES FOR COMPOUND

COMPOUND NO $=66$

MOL WGT -

1 ENTRIES FOR COMPOUND

COMPOUND NO $=67$ MOL WGT -

2.22 E I Q N-C1O GLYCEROL ETHER

2 ENTRIES FOR COMPOUND

COMPOUND NO $=68$ MOL WGT -

1 ENTRIES FOR COMPOUND

COMPOUND NO $=69$ MOL WGT -

1 ENTRIES FOR COMPOUND

COMPOUND NO $=70$ MOL WGT -

1 ENTRIES FOR COMPOUND

$$
\text { COMPOUND NO }=71 \text { MOL WGT - }
$$

1 ENTRIES FOR COMPOUND

COMPOUND NO $=72$ MOL WGT -

2 ENTRIES FOR COMPOUND
370.5 SODIUM ELAIDYL/TRANS 9 OCTADECENOYL/SULFATE

$\begin{array}{lllllllll}50 & 1.8 & \text { X10-4 M CG VISUAL SPCTR CHNGE PNCN WEIL STIR } 59004 & \text { T } & \text { L }\end{array}$

\begin{tabular}{lllllllll}
\multicolumn{1}{c}{441.4} & SODIUM & 9 & 10 & DICHLORO OCTADECYL & SULFATE \\
25 & 3.1 & XIO-4 M & BG & VISUAL SPCTR CHNGE PNCN & WEIL STIR & 59004 & T & L \\
50 & 2.6 & XI0-4 M & BG & VISUAL SPCTR CHNGE PNCN & WEIL STIR & 59004 & T & L
\end{tabular}

372.5 SODIUM OCTADECYL 1 SULFATE

\begin{tabular}{|c|c|c|c|c|c|c|c|}
\hline & 53005 & & & VALUES FRM REF IN CMC & KLIN LANG & 57022 & \\
\hline 40.0 & 1.65 & $\mathrm{X} 10-4 \mathrm{M}$ & $\mathrm{BE}$ & SPECFC CONDCTNCE GRAPH & EVAN & 56006 & $\mathrm{~T}$ \\
\hline 40.0 & 3.0 & $\mathrm{X} 10-4 \mathrm{M}$ & $\mathrm{BD}$ & EQUIV CONDCTNCE GRAPH & EVAN & 56006 & $\mathrm{KL}$ \\
\hline 50 & 1.7 & $\mathrm{X} 10-4 \mathrm{M}$ & $\mathrm{CD}$ & EQUIV CONDCTNCE GRAPH & LANG & 53005 & 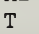 \\
\hline 50 & 2.31 & $\mathrm{X} 10-4 \mathrm{M}$ & $\mathrm{CD}$ & SURFACE TENSION UNSPEC & GOTT & 60 & G \\
\hline & 1.9 & $\mathrm{X} 10-4 \mathrm{M}$ & BB & INTERFACIAL TENSION LOGM & KLIN LANG & & \\
\hline 50 & 2.3 & $\mathrm{X} 10-4 \mathrm{M}$ & BB & SURFACE TENSION LOG PLOT & KLIN LANG & 57022 & 1 \\
\hline & 1.1 & $\mathrm{X} 10-4 \mathrm{M}$ & CG & VISUAL SPCTR CHNGE PNCN & WEIL STIR & & \\
\hline 50 & 1.1 & $\mathrm{X} 10-4 \mathrm{M}$ & BG & VISUAL SPCTR CHNGE PNCN & WEIL STIR & 63013 & $T$ \\
\hline VK & $\begin{array}{l}1.0 \\
2.68\end{array}$ & $\begin{array}{l}X 10-2 \text { D } \\
\times 10-4\end{array}$ & XG & VISUAL SPCTR CHNGE PNCN & DEMC & 1031 & \\
\hline & & & & GRAPH & LANG & 53005 & \\
\hline
\end{tabular}

499.7 TRIETHANOLAMMONIUM OCTADECYL 1 SULFATE

$\begin{array}{llllllll}50 & 7.0 & \text { X10-5 M CG VISUAL SPCTR CHNGE PNCN WEIL STIR } 59004 & \text { T } & \text { L }\end{array}$

232.2 SODIUM OCTYL 2 SULFATE

$\begin{array}{llllllll}40.0 & 1.80 & \times 10-1 & M & \text { BB SPECFC CONDCTNCE GRAPH } & \text { EVAN } & 56006 & \text { T } 3\end{array}$

288.3 SODIUM DODECYL 2 SULFATE

$55 \quad 2.82 \times 10-1$ D CG VISUAL SPCTR CHNGE PNCN

$55 \quad \begin{aligned} & 9.781 \times 10-3 \mathrm{M} \\ & 1.76 \times 10-1 \mathrm{D}\end{aligned}$

SCHI FOWK $57014 \quad$ T $\quad$ L

SCHI FOWK $57014 \stackrel{\text { M }}{\text { T }} \begin{aligned} & \text { L } \\ & \text { M }\end{aligned}$

302.3 SODIUM TRIDECYL 2 SULFATE

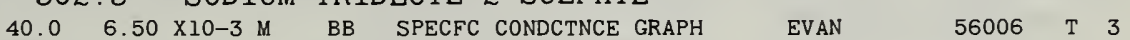

330.4 SODIUM PENTADECYL 2 SULFATE

$\begin{array}{llllllll}40.0 & 1.71 & \text { X10-3 M } & \text { BB SPECFC CONDCTNCE GRAPH } & \text { EVAN } & 56006 & \text { T } & L\end{array}$

358.5 SODIUM HEPTADECYL 2 SULFATE

$40.0 \quad 4.9 \quad \times 10-4 \mathrm{M} \quad \mathrm{BC}$ SPECFC CONDCTNCE GRAPH

EVAN

56006 T L'

372.5 SODIUM OCTADECYL 2 SULFATE

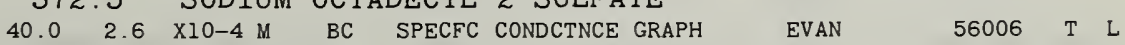

274.3 SODIUM UNDECYL 3 SULFATE

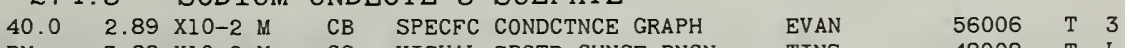

$\begin{array}{llllllllllllll}4 M & 3.88 & \times 10-2 & M & \text { CG } & \text { VISUAL SPCTR CHNGE PNCN } & \text { WINS } & 48008 & T & L\end{array}$
Concentration units: $\mathrm{A}-\mathrm{mol} \% ; \mathrm{B}-\mathrm{vol} \%$ solvent; $\mathrm{C}-$ mol \% surfactant mixture; D-wt/vol \%; E-\% saturation; $\mathrm{H}-$ wt \% solvent; I-mol \% surfactant; K-normality counterions; $\mathrm{M}$-molar; $\mathrm{N}$ - normal; $\mathrm{P}$ - wt \%; $\mathrm{Q}$-wt \% surfactant; $\mathrm{R}$-varied; $\mathrm{S}-$ mol/ kg; $T$ - wt \% surfactant mixture; $U-$ mol/( or kg); $\mathrm{W}$ - molal; $\mathrm{Y}$ - atm. Details on page 222. 


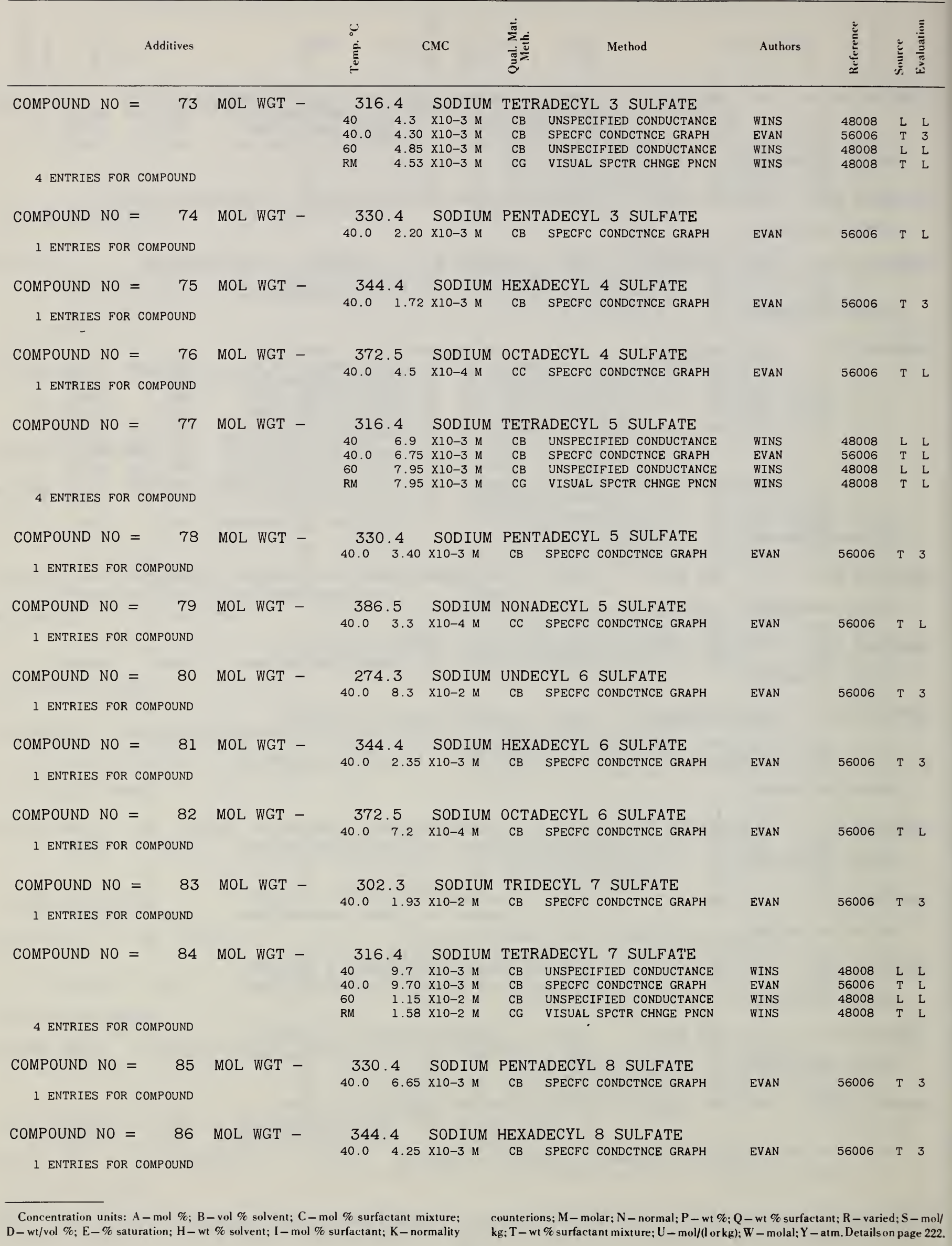




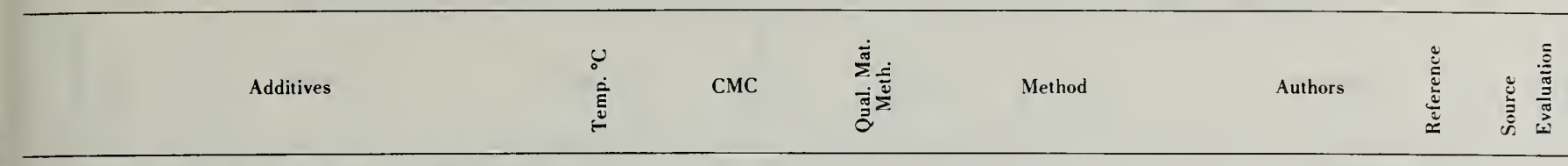

COMPOUND NO $=87$ MOL WGT -

1 ENTRIES FOR COMPOUND

COMPOUND NO $=88$ MOL WGT -

1 ENTRIES FOR COMPOUND

COMPOUND NO $=89$ MOL WGT -

1 ENTRIES FOR COMPOUND

COMPOUND NO $=90$ MOL WGT -

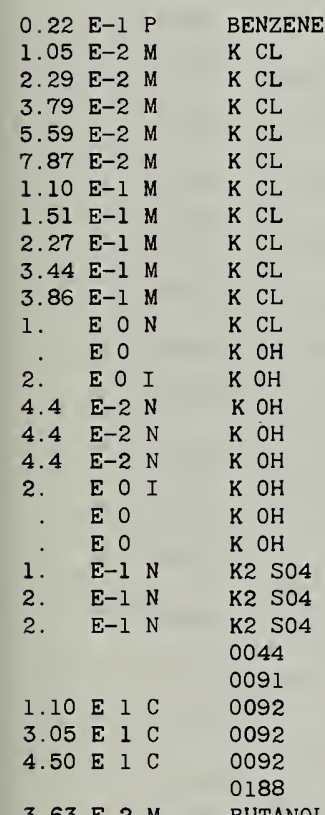

$3.63 \mathrm{E}-2 \mathrm{M}$ BUTANOL-1 2. E O I $\mathrm{K} \mathrm{OH}$

$6.74 \mathrm{E}-2 \mathrm{M}$ BUTANOL-1

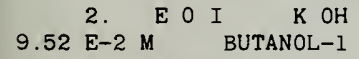
2. E O I K K OH

$1.44 \mathrm{E}-1 \mathrm{M}$ BUTANOL-1 2. $\mathrm{E} O \mathrm{I} \quad \mathrm{K} O \mathrm{H}$

$1.80 \mathrm{E}-1 \mathrm{M}$ BUTANOL-1 2. E O I $\mathrm{K} O \mathrm{H}$

$2.29 \mathrm{E}-1 \mathrm{M}$ BUTANOL-1 2. E O I $\mathrm{K} \mathrm{OH}$

$6.4 \mathrm{E}-5 \mathrm{M}$ DECANOL-1 2. E O I $\mathrm{K} \mathrm{OH}$

$1.20 \mathrm{E}-4 \mathrm{M}$ DECANOL-1 2. E O I $\mathrm{K} \mathrm{OH}$

$1.64 \mathrm{E}-4 \mathrm{M}$ DECANOL-1 2. E O I $\mathrm{K}$ OH

$5.44 \mathrm{E}-1 \mathrm{M}$ ETHANOL 2. $\mathrm{E} O \mathrm{I} \quad \mathrm{K}$ OH

358.5 SODIUM HEPTADECYL 9 SULFATE

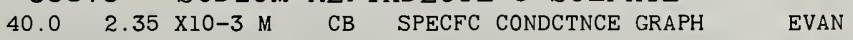

56006 T L

386.5 SODIUM 1 NONYL DECYL SULFATE

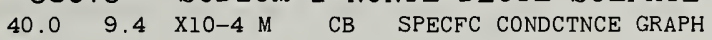

EVAN

56006 T $\quad$ L

526.8 SODIUM 1 TETRADECYL PENTADECYL SULFATE

40.0 8. X10-5 M CG EQUIV CONDCTNCE MAXIMUM EVAN

56006 T $\quad$ L

210.3 POTASSIUM DECANOATE

\begin{tabular}{|c|c|c|c|c|c|c|c|c|}
\hline & & & & GRAPH DATA NOT RETRIEVED & KLEV & & 46007 & \\
\hline 25 & 9.9 & $\mathrm{X} 10-2 \mathrm{M}$ & DB & REFRACTIVE INDEX & KLEV & & 48005 & $\mathrm{~T}$ \\
\hline 25 & 1.00 & $\mathrm{X} 10-1 \mathrm{M}$ & CG & VISUAL SPCTR CHNGE PNCN & KLEV & & 50003 & T \\
\hline 25 & 9.98 & $\mathrm{X} 10-2 \mathrm{M}$ & DG & VISUAL SPCTR CHNGE PNCN & HERZ & & 52015 & $\mathrm{~T}$ \\
\hline 25 & 9.5 & $\mathrm{x} 10-2 \mathrm{M}$ & BG & UNSPEC SPCTR CHNG PNCN & KLEV & & 58011 & $\mathrm{~T}$ \\
\hline 25 & 9.98 & $\mathrm{X} 10-2 \mathrm{M}$ & DG & VISUAL SPCTR CHNGE PNCN & HERZ & & 52017 & $\mathrm{~T}$ \\
\hline 30 & 1.06 & $\mathrm{X} 10-1 \mathrm{M}$ & DB & FOTOMTR SOLUBLZTN PDMAB & KOLT & STRI & 48016 & $\mathrm{~T}$ \\
\hline 45 & 9.8 & $\mathrm{X} 10-2 \mathrm{M}$ & DB & REFRACTIVE INDEX & KLEV & & 48005 & $\mathrm{~T}$ \\
\hline 45 & 1.18 & $\mathrm{X} 10-1 \mathrm{M}$ & DB & REFRACTIVE INDEX & KLEV & & 48005 & $\mathrm{~T}$ \\
\hline 50 & 1.05 & $\mathrm{X}_{10} \mathrm{O}-1 \mathrm{M}$ & DB & FOTOMTR SOLUBLZTN PDMAB & KOLT & STRI & 48016 & $T$ \\
\hline 50 & 48016 & & & VALUES FRM REF IN CMC & KOLT & STRI & 49005 & \\
\hline JNK & 9.5 & $\mathrm{X} 10-2 \mathrm{M}$ & DG & VISUAL SPCTR CHNGE PNCN & CORR & HARK & 46015 & $\mathrm{~T}$ \\
\hline 25 & 9.3 & $\mathrm{X} 10-2 \mathrm{M}$ & CG & VISUAL SPCTR CHNGE PNCN & KLEV & & 50003 & $\mathrm{~T}$ \\
\hline 25 & 9.49 & $\mathrm{X} 10-2 \mathrm{M}$ & DG & VISUAL SPCTR CHNGE PNCN & HERZ & & 52017 & $\mathrm{~T}$ \\
\hline 25 & 9.17 & $\mathrm{X} 10-2 \mathrm{M}$ & DG & VISUAL SPCTR CHNGE PNCN & HERZ & & 52017 & $\mathrm{~T}$ \\
\hline 25 & 8.85 & $\mathrm{X} 10-2 \mathrm{M}$ & DG & VISUAL SPCTR CHNGE PNCN & HERZ & & 52017 & $\mathrm{~T}$ \\
\hline 25 & 8.38 & $\mathrm{X} 10-2 \mathrm{M}$ & DG & VISUAL SPCTR CHNGE PNCN & HERZ & & 52017 & $\mathrm{~T}$ \\
\hline 25 & 7.87 & $\mathrm{X} 10-2 \mathrm{M}$ & DG & VISUAL SPCTR CHNGE PNCN & HERZ & & 52017 & $\mathrm{~T}$ \\
\hline 25 & 7.31 & $\mathrm{XlO}-2 \mathrm{M}$ & DG & VISUAL SPCTR CHNGE PNCN & HERZ & & 52017 & $\mathrm{~T}$ \\
\hline 25 & 6.45 & $\mathrm{X} 10-2 \mathrm{M}$ & DG & VISUAL SPCTR CHNGE PNCN & HERZ & & 52017 & $\mathrm{~T}$ \\
\hline 25 & 5.68 & $\mathrm{X} 10-2 \mathrm{M}$ & DG & VISUAL SPCTR CHNGE PNCN & HERZ & & 52017 & $\mathrm{~T}$ \\
\hline 25 & 4.69 & $\mathrm{X} 10-2 \mathrm{M}$ & DG & VISUAL SPCTR CHNGE PNCN & HERZ & & 52017 & $\mathrm{~T}$ \\
\hline 25 & 4.29 & $\mathrm{X} 10-2 \mathrm{M}$ & DG & VISUAL SPCTR CHNGE PNCN & HERZ & & 52017 & $\mathrm{~T}$ \\
\hline 50 & 2.2 & $\mathrm{X} 10-2 \mathrm{M}$ & $\mathrm{DC}$ & FOTOMTR SOLUBLZTN PDMAB & KOLT & STRI & 49005 & $\mathrm{~T}$ \\
\hline 0 & 1.68 & $\mathrm{X} 10-1 \mathrm{~W}$ & $\mathrm{CB}$ & VAPR PRESURE LOWERING & HUFF & MCBA & 51004 & $\mathrm{~T}$ \\
\hline 10 & 1.09 & $\mathrm{X} 10-1 \mathrm{M}$ & BG & VISUAL SPCTR CHNGE PNCN & SHIN & & 55004 & $\mathrm{~s}$ \\
\hline 20 & 7. & $\mathrm{X} 10-2 \mathrm{M}$ & $\mathrm{XG}$ & VISCOSITY MINIMUM & SATA & TYUZ & 53006 & $\mathrm{~T}$ \\
\hline 20 & 5.7 & $\mathrm{X} 10-2 \mathrm{M}$ & $\mathrm{CE}$ & DIFFUSION COEFFICIENT & TYUZ & & 61025 & $\mathrm{~T}$ \\
\hline 20 & 1.06 & $\mathrm{X} 10-1 \mathrm{M}$ & $\mathrm{CE}$ & DIFFUSION COEFFICIENT & TYUZ & & 61025 & $\mathrm{~T}$ \\
\hline 25 & 1.00 & $\mathrm{X} 10-1 \mathrm{M}$ & BG & VISUAL SPCTR CHNGE PNCN & SHIN & & 54005 & G \\
\hline 3 & 9.8 & $\mathrm{X} 10-2 \mathrm{~W}$ & $\mathrm{CB}$ & VAPR PRESURE LOWERING & HUFF & MCBA & 1004 & $\mathrm{~T}$ \\
\hline 50 & 1.15 & $\mathrm{X} 10-1 \mathrm{~W}$ & $\mathrm{CB}$ & VAPR PRESURE LOWERING & HUFF & MCBA & 51004 & $\mathrm{~T}$ \\
\hline 5 & 8.2 & $\mathrm{X} 10-2 \mathrm{M}$ & DB & FOTOMTR SOLUBLZTN PDMAB & KOLT & STRI & 49005 & $\mathrm{~T}$ \\
\hline 50 & 6.5 & $\mathrm{X} 10-2 \mathrm{M}$ & DB & FOTOMTR SOLUBLZTN PDMAB & KOLT & STRI & 49005 & $\mathrm{~T}$ \\
\hline 50 & 4.5 & $\mathrm{X} 10-2 \mathrm{M}$ & DC & FOTOMTR SOLUBLZTN PDMAB & KOLT & STRI & 49005 & $\mathrm{~T}$ \\
\hline 25 & & & & SEE CMPD NMBR IN ADDITV & SHIN & & 54005 & \\
\hline UNK & & & & SEE CMPD NMBR IN ADDITV & CORR & HARK & 46015 & \\
\hline 25 & 5.2 & $\mathrm{X} 10-2 \mathrm{M}$ & DC & REFRACTIVE INDEX & KLEV & & 48005 & $\mathrm{~T}$ \\
\hline 2 & 1.5 & $\mathrm{X} 10-2 \mathrm{M}$ & DD & REFRACTIVE INDEX & KLEV & & 48005 & $\mathrm{~T}$ \\
\hline 25 & 1.2 & $\mathrm{X} 10-2 \mathrm{M}$ & DD & REFRACTIVE INDEX & KLEV & & 48005 & $\mathrm{~T}$ \\
\hline 2 & & & & SEE CMPD NMBR IN ADDITV & SHIN & & 54005 & \\
\hline 10 & 1.01 & $\mathrm{X} 10-1 \mathrm{M}$ & $B G$ & VISUAL SPCTR CHNGE PNCN & SHIN & & 55004 & $\mathrm{~T}$ \\
\hline & 9.4 & $\mathrm{X} 10-2 \mathrm{M}$ & BG & VISUAL SPCTR CHNGE PNCN & SHIN & & 55004 & $\mathrm{~T}$ \\
\hline & 8.8 & $\mathrm{X} 10-2 \mathrm{M}$ & BG & VISUAL SPCTR CHNGE PNCN & SHIN & & 55004 & $\mathrm{~T}$ \\
\hline & 8.1 & $\mathrm{X} 10-2 \mathrm{M}$ & BG & VISUAL SPCTR CHNGE PNCN & SHIN & & 55004 & $\mathrm{~T}$ \\
\hline & 7.2 & $\mathrm{X} 10-2 \mathrm{M}$ & BG & VISUAL SPCTR CHNGE PNCN & SHIN & & 55004 & $\mathrm{~T}$ \\
\hline & 6.4 & $\mathrm{X} 10-2 \mathrm{M}$ & BG & VISUAL SPCTR CHNGE PNCN & SHIN & & 55004 & $\mathrm{~T}$ \\
\hline & 1.06 & $\mathrm{X} 10-1 \mathrm{M}$ & BG & VISUAL SPCTR CHNGE PNCN & SHIN & & 55004 & $\mathrm{~T}$ \\
\hline & 1.02 & $\mathrm{X} 10-1 \mathrm{M}$ & BG & VISUAL SPCTR CHNGE PNCN & SHIN & & 55004 & $\mathrm{~T}$ \\
\hline & 1.01 & $\mathrm{X} 10-1 \mathrm{M}$ & BG & VISUAL SPCTR CHNGE PNCN & SHIN & & 55004 & 1 \\
\hline & 9.7 & $\mathrm{X} 10-2 \mathrm{M}$ & BG & VISUAL SPCTR CHNGE PNCN & SHIN & & 55004 & $\mathrm{~T}$ \\
\hline
\end{tabular}

Concentration units: A-mol \%; B-vol \% solvent; $\mathrm{C}-$ mol \% surfactant mixture; $\quad$ counterions; $\mathrm{M}-$ molar; $\mathrm{N}-$ normal; $\mathrm{P}-\mathrm{wt} \% ; \mathrm{Q}-\mathrm{wt} \%$ surfactant; $\mathrm{R}-\mathrm{varied} ; \mathrm{S}-$ mol/ D-wt/vol \%; E-\% saturation; H-wt \% solvent; I-mol \% surfactant; K-normality $\quad$ kg; T-wt \% surfactant mixture; U-mol// or kg); W-molal; $Y-$ atm. Details on page 222. 


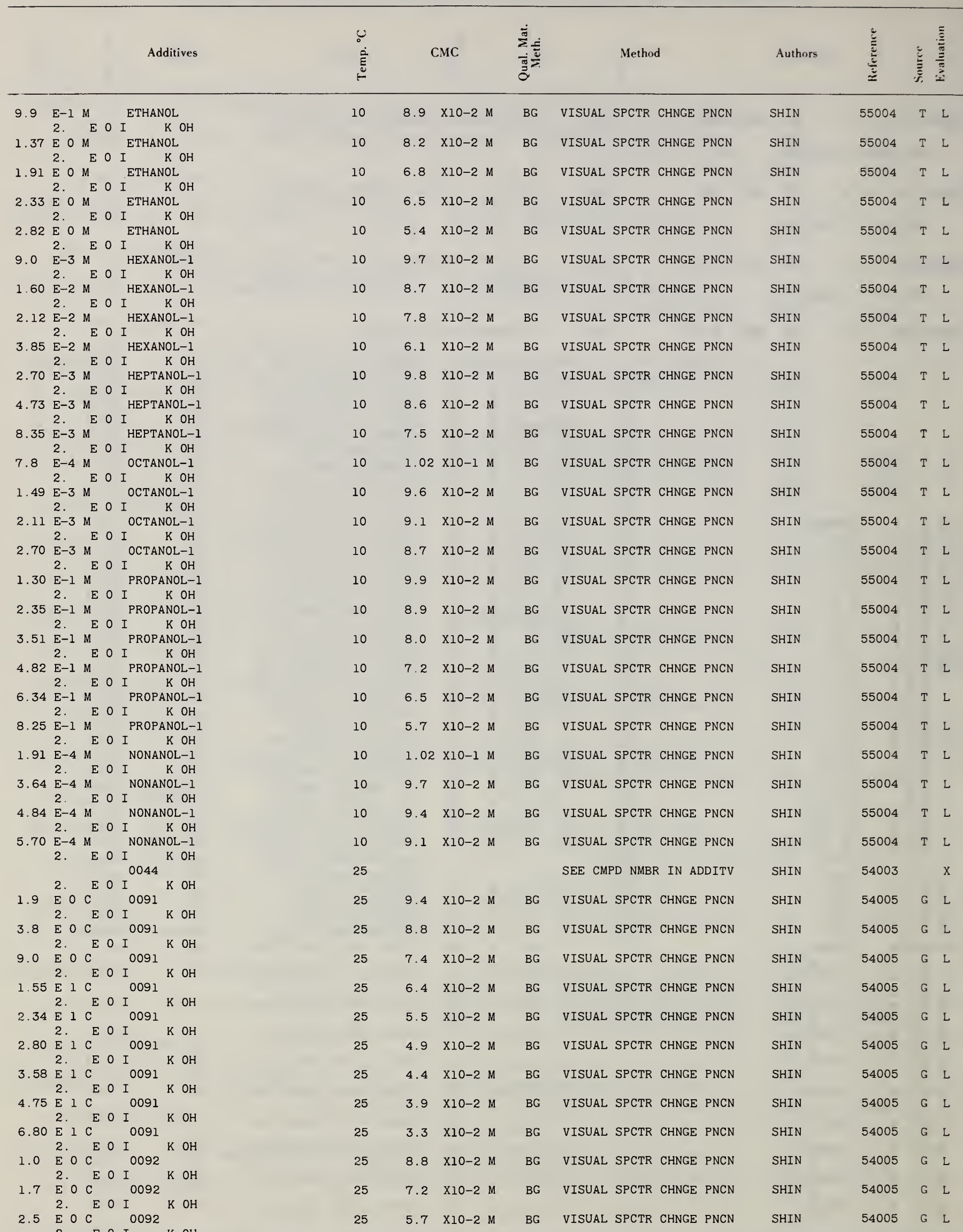

\footnotetext{
2. E $O$ I $K$ OH
} 


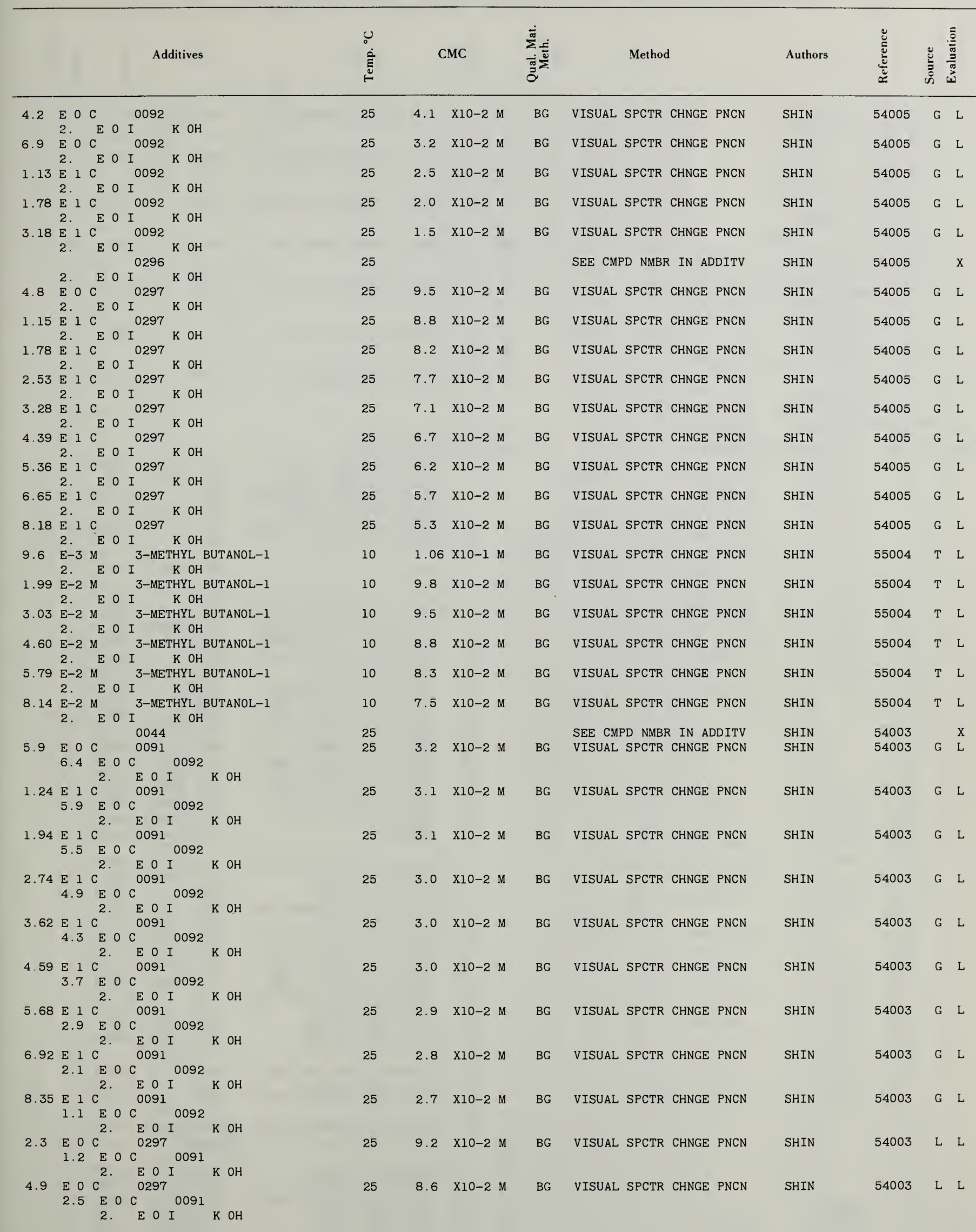

Concentration units: $\mathrm{A}-\mathrm{mol} \% ; \mathrm{B}-$ vol \% solvent; $\mathrm{C}-$ mol \% surfactant mixture;

counterions; $\mathrm{M}$-molar; $\mathrm{N}$-normal; $\mathrm{P}$ - wt \%; $\mathrm{Q}-$ wt \% surfactant; $\mathrm{R}$-varied; $\mathrm{S}-\mathrm{mol} /$ D-wt/vol \%; E-\% saturation; $\mathrm{H}-$ wt \% solvent; I-mol \% surfactant; $\mathrm{K}-$ normality 


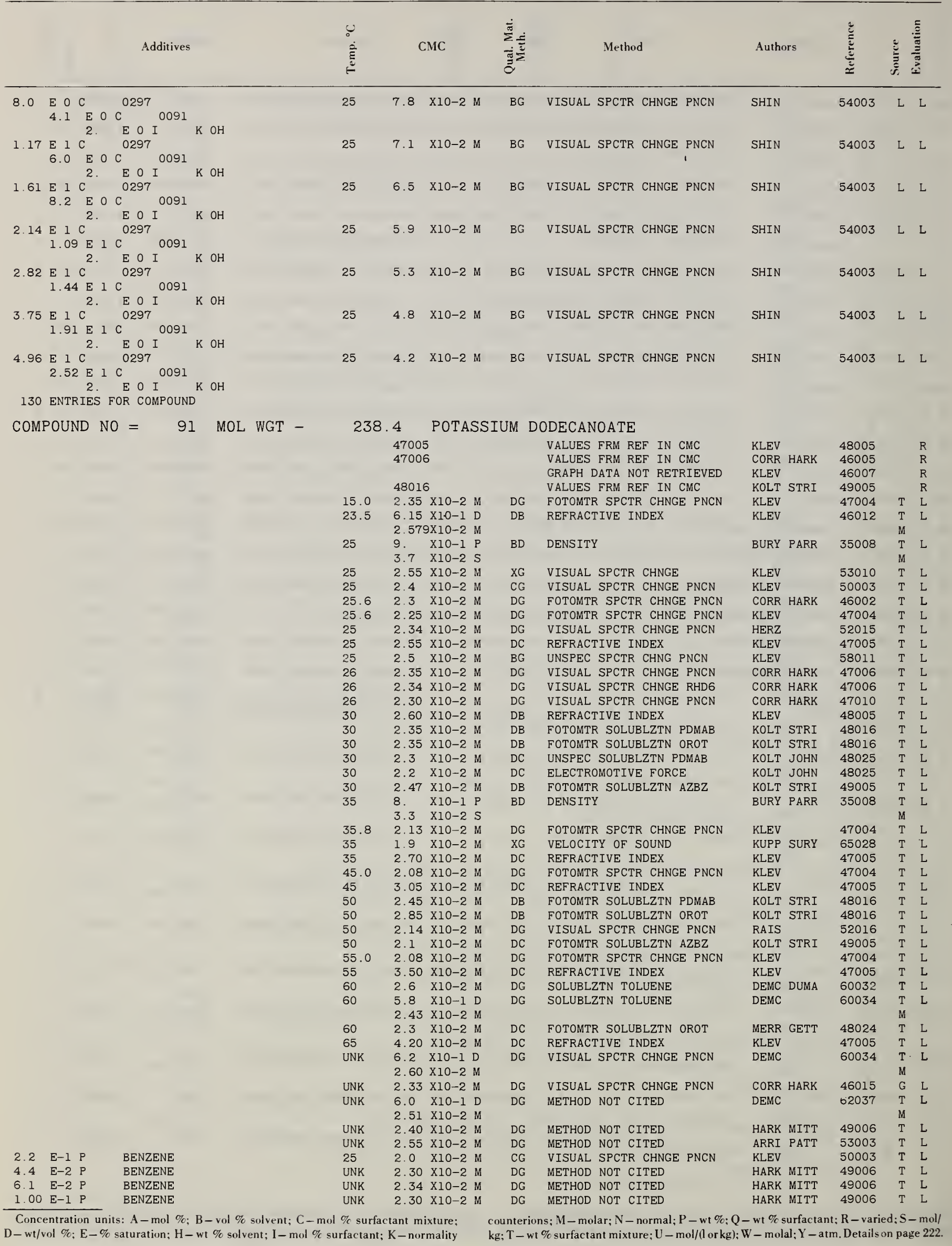




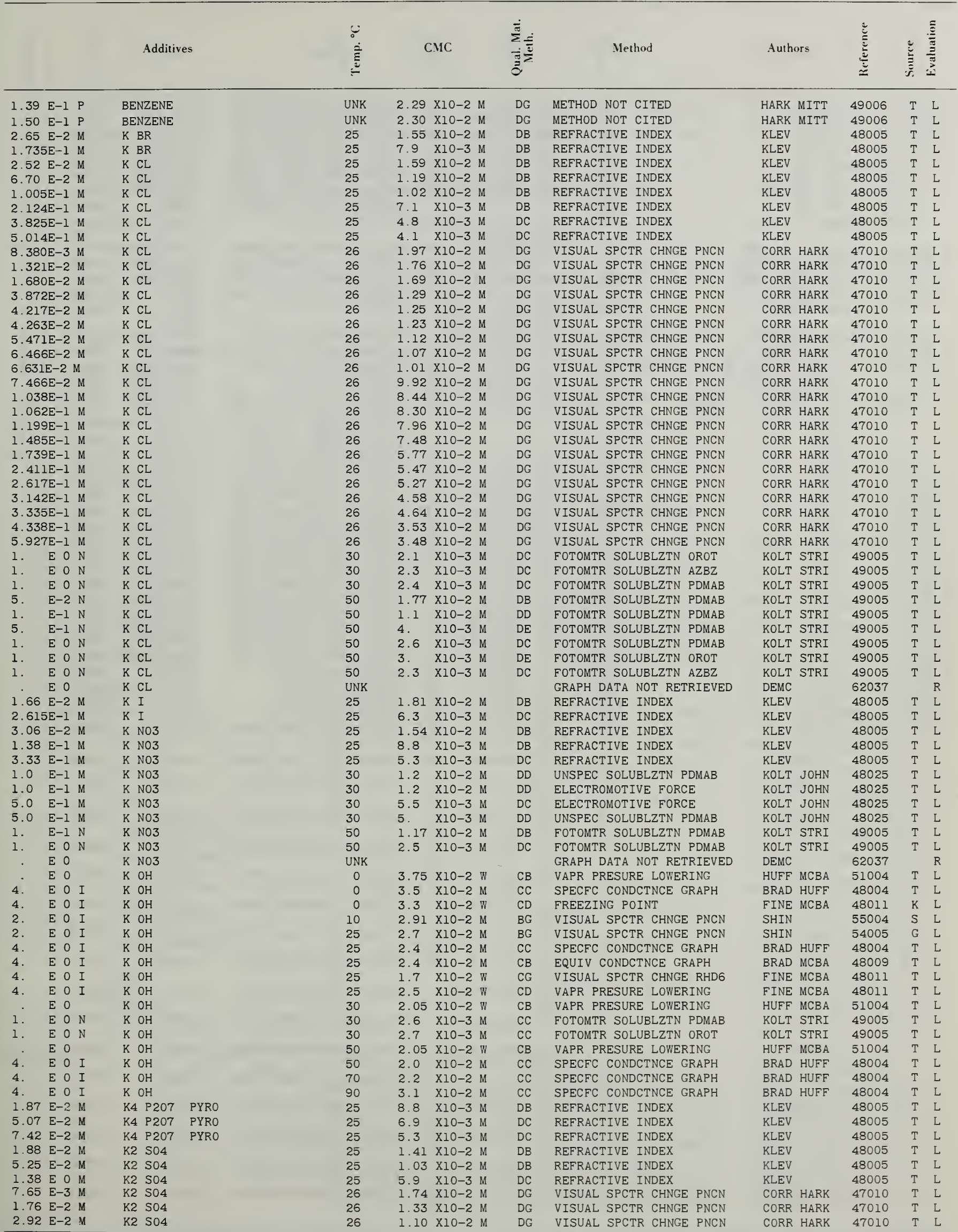

Concentration units: $\mathrm{A}-\operatorname{mol} \% ; \mathrm{B}-\mathrm{vol} \%$ solvent; $\mathrm{C}-$ mol $\%$ surfactant mixture;

$\mathrm{D}-\mathrm{wt} / \mathrm{vol} \% ; \mathrm{E}-\%$ saturation; $\mathrm{H}-$ wt \% solvent; I-mol \% surfactant; $\mathrm{K}$ - normality

counterions: $\mathrm{M}$-molar: $\mathrm{N}$-normal: $\mathrm{P}$ - wt $\%: \mathrm{Q}-\mathrm{wt} \%$ surfactant; $\mathrm{R}$-varied; $\mathrm{S}$-mol/ kg: T-wt \% surfactant mixture; $\mathrm{U}-\mathrm{mol} /(\mathrm{l}$ orkg); $\mathrm{W}-$ molal; $\mathrm{Y}-\mathrm{atm}$. Detailson page 222. 


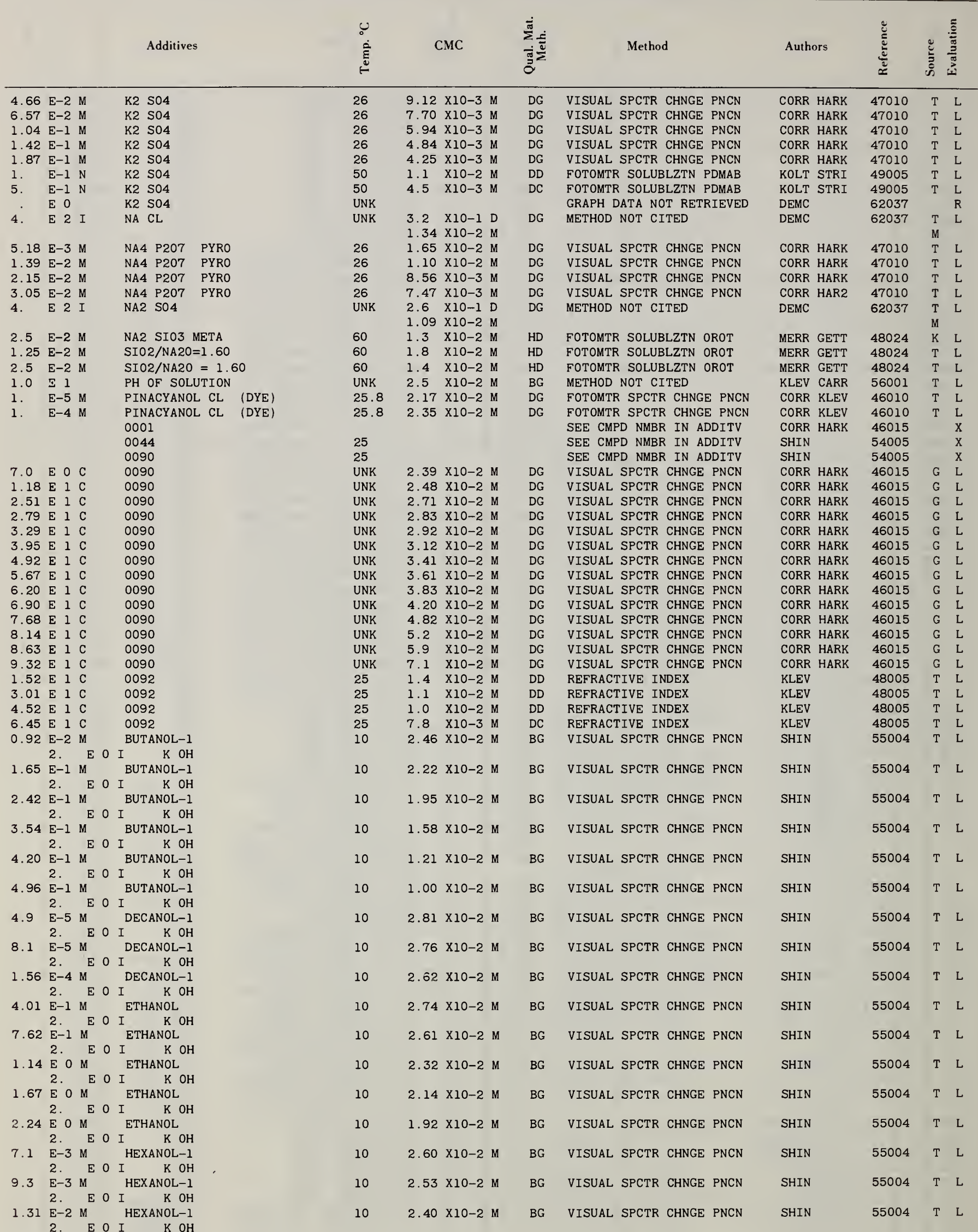

Concentration units: $\mathrm{A}-$ mol $\% ; \mathrm{B}-$ vol \% solvent; $\mathrm{C}-$ mol \% surfactant mixture; 
Additives

CMC

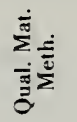

Method

Authors

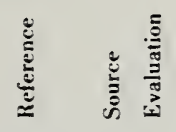

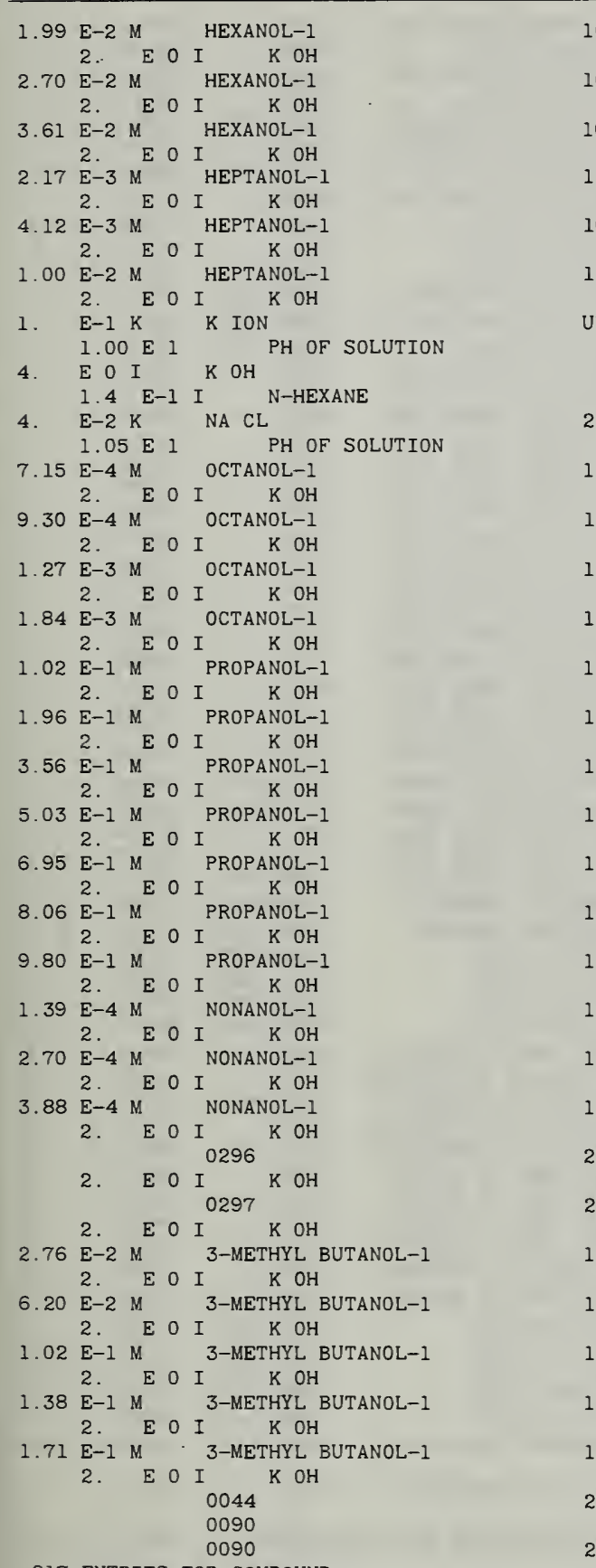

$102.18 \times 10-2 \mathrm{M}$ BG VISUAL SPCTR CHNGE PNCN

$101.85 \times 10-2 \mathrm{M}$ BG VISUAL SPCTR CHNGE PNCN

$10 \quad 1.58 \times 10-2 \mathrm{M} \quad \mathrm{BG}$

$10 \quad 2.73 \times 10-2 M \quad B G$

10

10

$2.58 \times 10-2 \mathrm{M}$

BG

$1.88 \times 10-2 \mathrm{M}$

UNK $1.37 \times 10-2 \mathrm{M} \quad \mathrm{CB}$

$0 \quad 2.8 \times 10-2 \mathrm{~W} \quad \mathrm{CD}$ FREEZING POINT

20

10

10

10

10

10

10

10

10

10

10

10

10

10

10

25

25

10

10

10

10

10

25

25
$1.7 \times 10-2 \mathrm{M} \quad \mathrm{BC}$ INTERFACIAL TENSION LOGM

$2.65 \times 10-2 \mathrm{M}$ BG VISUAL SPCTR CHNGE PNCN

$2.58 \times 10-2 \mathrm{M}$

$2.40 \times 10-2 \mathrm{M}$

$2.32 \times 10-2 \mathrm{M}$

$2.70 \times 10-2 \mathrm{M}$

$2.59 \times 10-2 \mathrm{M}$

$2.44 \times 10-2 \mathrm{M}$

$2.28 \times 10-2 \mathrm{M}$

$2.09 \times 10-2 \mathrm{M}$

$1.82 \times 10-2 \mathrm{M}$

$1.67 \times 10-2 \mathrm{M}$

$2.79 \times 10-2 \mathrm{M}$

$2.69 \times 10-2 \mathrm{M}$

$2.61 \times 10-2 \mathrm{M}$

$2.67 \times 10-2 \mathrm{M}$

$2.31 \times 10-2 \mathrm{M}$

$1.91 \times 10-2 \mathrm{M}$

$1.61 \times 10-2 \mathrm{M}$

1. $45 \times 10-2 \mathrm{M}$
BG

VISUAL SPCTR CHNGE PNCN

VISUAL SPCTR CHNGE PNCN

VISUAL SPCTR CHNGE PNCN

VISUAL SPCTR CHNGE PNCN

SURFACE TENSION LOG PLOT

VISUAL SPCTR CHNGE PNCN

VISUAL SPCTR CHNGE PNCN

VISUAL SPCTR CHNGE PNCN

VISUAL SPCTR CHNGE PNCN

VISUAL SPCTR CHNGE PNCN

VISUAL SPCTR CHNGE PNCN

VISUAL SPCTR CHNGE PNCN

BG

BG

BG

BG

BG

BG

VISUAL SPCTR CHNGE PNCN

SEE CMPD NMBR IN ADDITV

SEE CMPD NMBR IN ADDITV

BG

VISUAL SPCTR CHNGE PNCN

BG

BG

BG

BG

SEE CMPD NMBR IN ADDITV SEE CMPD NMBR IN ADDITV SEE CMPD NMBR IN ADDITV
SHIN

SHIN

SHIN

SHIN

SHIN

SHIN

ROE BRAS

FINE MCBA

$\mathrm{V}$ vo

SHIN

SHIN

SHIN

SHIN

SHIN

SHIN

SHIN

SHIN

SHIN

SHIN

SHIN

SHIN

SHIN

SHIN

SHIN

SHIN

SHIN

SHIN

SHIN

SHIN

SHIN

SHIN

SHIN

SHIN
$55004 \quad \mathrm{~T} \quad$ L

$55004 \quad \mathrm{~T} \quad \mathrm{~L}$

55004 T L

$55004 \quad \mathrm{~T} \quad \mathrm{~L}$

$55004 \quad \mathrm{~T} \quad \mathrm{~L}$

$55004 \quad \mathrm{~T} \quad \mathrm{~L}$

54013 T L

48011 T L

60025 T L

$55004 \quad \mathrm{~T} \quad \mathrm{~L}$

$55004 \quad \mathrm{~T} \quad \mathrm{~L}$

$55004 \quad \mathrm{~T} \quad \mathrm{~L}$

55004 T L

$55004 \quad \mathrm{~T} \quad \mathrm{~L}$

$55004 \quad \mathrm{~T} \quad \mathrm{~L}$

$55004 \quad \mathrm{~T} \quad \mathrm{~L}$

55004 T L

$55004 \quad \mathrm{~T} \quad \mathrm{~L}$

$55004 \quad \mathrm{~T} \quad \mathrm{~L}$

$55004 \quad \mathrm{~T} \quad$ L

$55004 \quad \mathrm{~T} \quad \mathrm{~L}$

$55004 \quad \mathrm{~T} \quad \mathrm{~L}$

55004 T L

$54005 \quad X$

$54003 x$

55004 T L

$55004 \quad \mathrm{~T} \quad \mathrm{~L}$

55004 T L

$55004 \quad \mathrm{~T} \quad \mathrm{~L}$

$55004 \quad \mathrm{~T} \quad \mathrm{~L}$

54003

54003

54003
COMPOUND NO $=92$ MOL WGT -

\begin{tabular}{llll}
266.5 & POTASS \\
\multicolumn{4}{c}{47005} \\
25 & 6.3 & $\times 10-3$ & $M$ \\
25.6 & 6.0 & $\times 10-3$ & $M$ \\
25.8 & 6.4 & $\times 10-3$ & $M$ \\
25.6 & 6.4 & $\times 10-3$ & $M$ \\
25 & 5.9 & $\times 10-3$ & $M$ \\
25 & 6.6 & $\times 10-3$ & $M$ \\
25 & 5.8 & $\times 10-3$ & $M$ \\
30 & 7.0 & $X 10-3$ & $M$ \\
35.8 & 5.7 & $\times 10-3$ & $M$
\end{tabular}

Concentration units: $\mathrm{A}-\mathrm{mol} \%$; $\mathrm{B}-$ vol $\%$ solvent; $\mathrm{C}-\mathrm{mol} \%$ surfactant mixture: D-wt/vol \%; E-\% saturation; $\mathrm{H}-$ wt $\%$ solvent; $\mathrm{I}-$ mol \% surfactant; $\mathrm{K}$ - normality counterions; $\mathrm{M}$-molar; $\mathrm{N}$-normal: $\mathrm{P}-$ wt $\% ; \mathrm{Q}$ - wt $\%$ surfactant; $\mathrm{R}$-varied; $\mathrm{S}-$ mol kg: T - wt \% surfactant mixture; $\mathrm{U}-\mathrm{mol} /(\mathrm{lorkg})$; $\mathrm{W}$ - molal; $\mathrm{Y}$-atm. Details on page 222. 


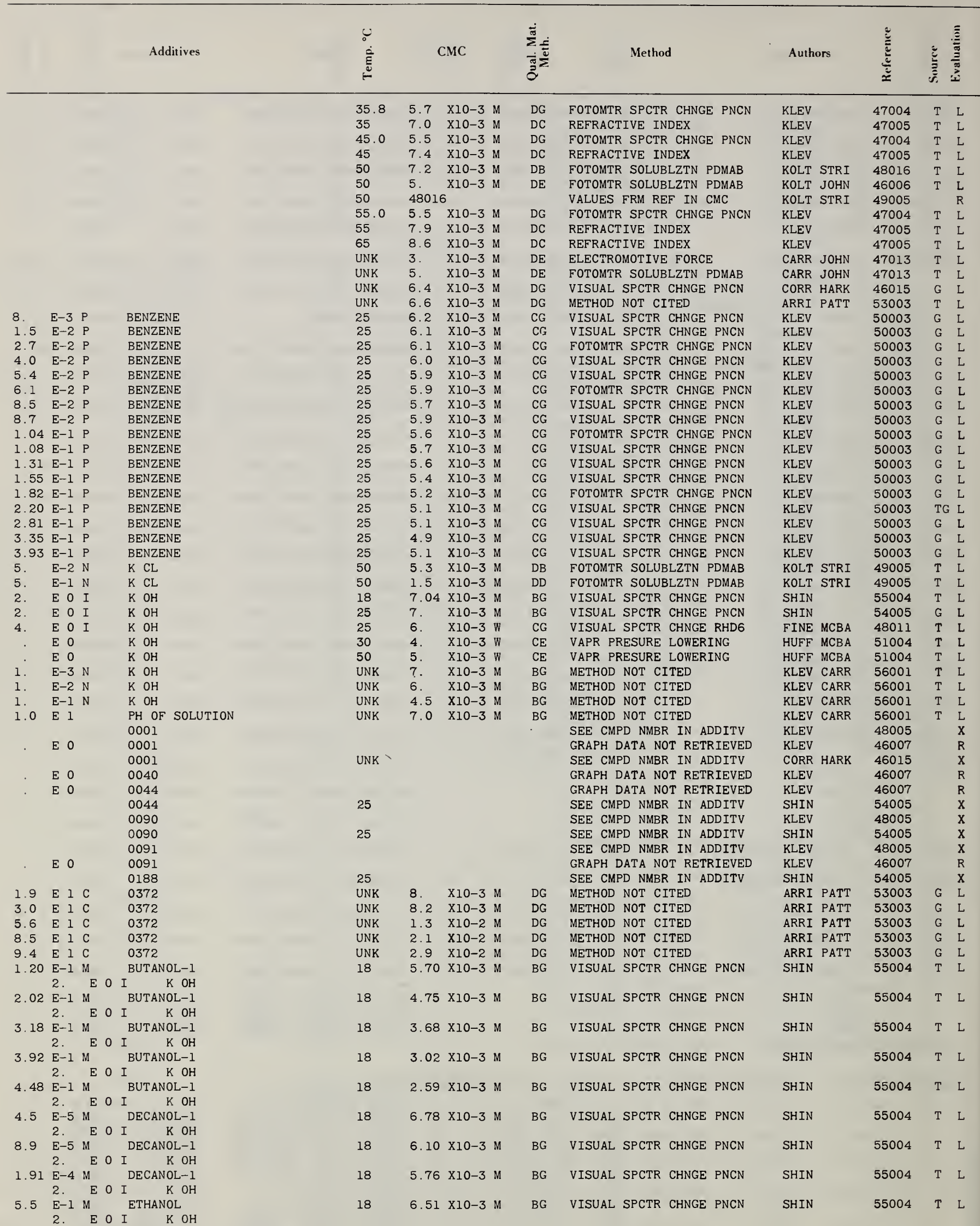

Concentration units: A-mol \%; B-vol \% solvent; $\mathrm{C}-$ mol \% surfactant mixture;

counterions; $\mathrm{M}$ - molar: $\mathrm{N}$ - normal; $\mathrm{P}$ - wt \%: $\mathrm{Q}$ - wt \% surfactant; $\mathrm{R}$ - varied; $\mathrm{S}$ - mol/ D-wt/vol \%; E-\% saturation; $\mathrm{H}-$ wt \% solvent; $\mathrm{I}-\mathrm{mol} \%$ surfactant; $\mathrm{K}-$ normality 


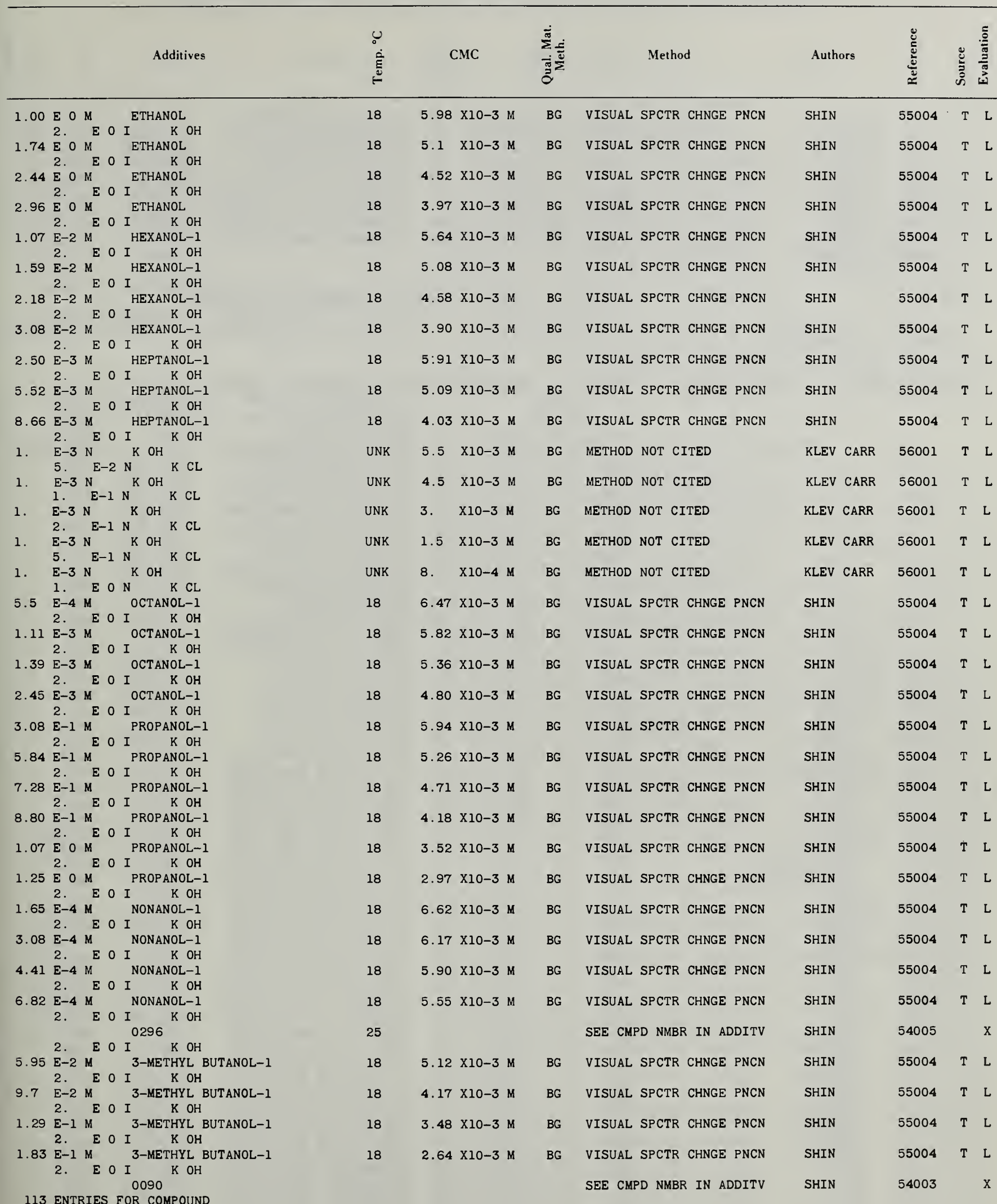

252.2 OCTYL TRIMETHYL AMMONIUM BROMIDE $20 \quad 2.82 \times 10-1 \mathrm{M}$ BB INTERFACIAL TENSION LOGM $25 \quad 1.4 \quad \times 10-1 M$
CD REFRACTIVE INDEX

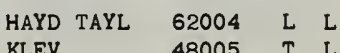

Concentration units: $\mathrm{A}-\mathrm{mol} \% ; \mathrm{B}-\mathrm{vol} \%$ solvent; $\mathrm{C}-$ mol $\%$ surfactant mixture; D-wt/vol \%: E-\% saturation; $\mathrm{H}-$ wt $\%$ solvent: I-mot \% surfactant; $\mathrm{K}$-normality counterions; $\mathrm{M}$ - molar: $\mathrm{N}$-normal; $\mathrm{P}-\mathrm{wt} \%$; $\mathrm{Q}-$ wt \% surfactant; $\mathrm{R}$ - varied; $\mathrm{S}$ - mol/ kg; T - wt \% surfactant mixture; $U-$ mol/( 1 or kg); W - molal; $Y$ - atm. Details on page 222. 


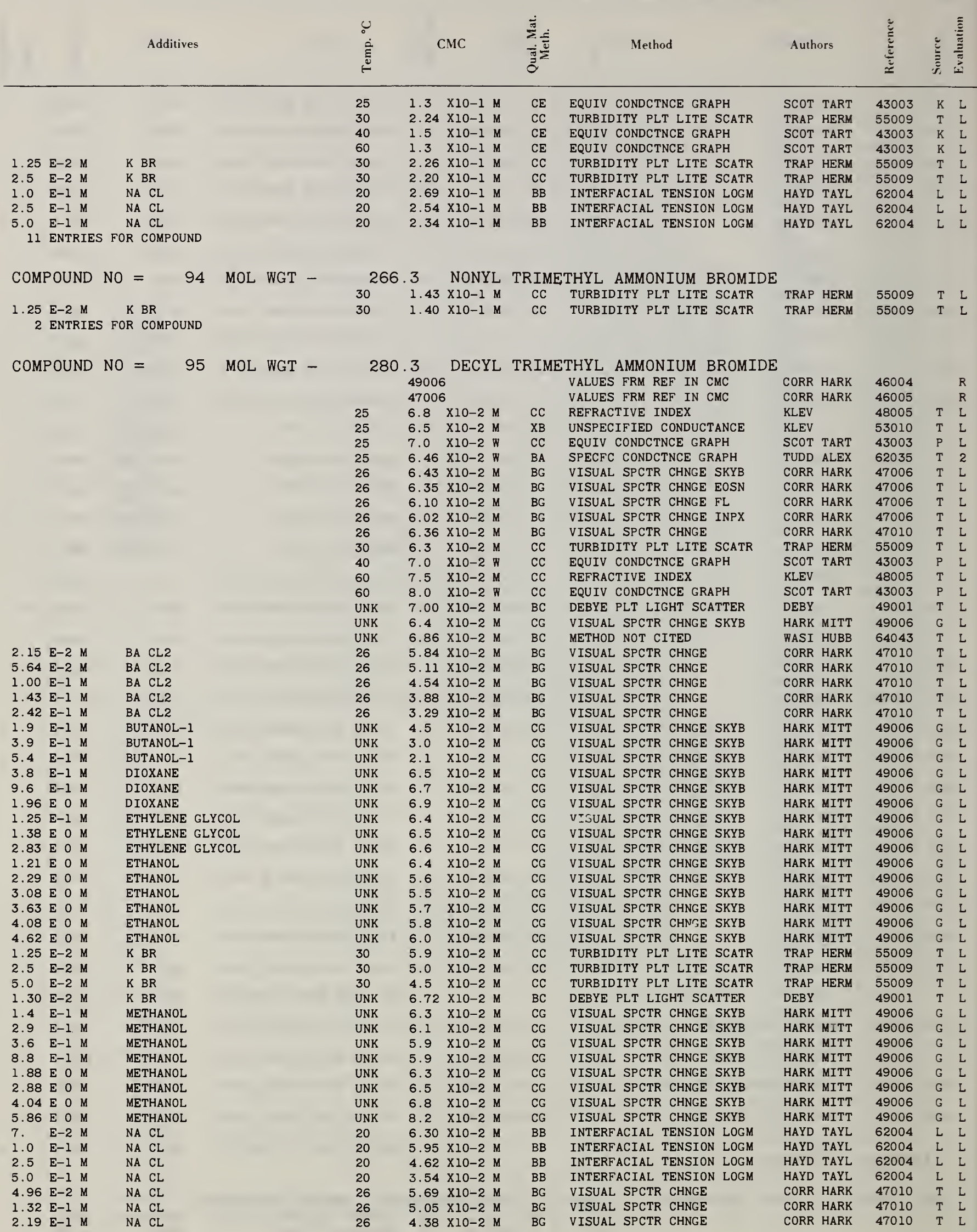

Concentration units: $\mathrm{A}-\mathrm{mol} \%: \mathrm{B}-\mathrm{vol} \%$ solvent: $\mathrm{C}-$ mol \% surfactant mixture; D-wt/vol \%: E- \% saturation: $\mathrm{H}-$ wt \% solvent: I-mol \% surfactant; K-normality 


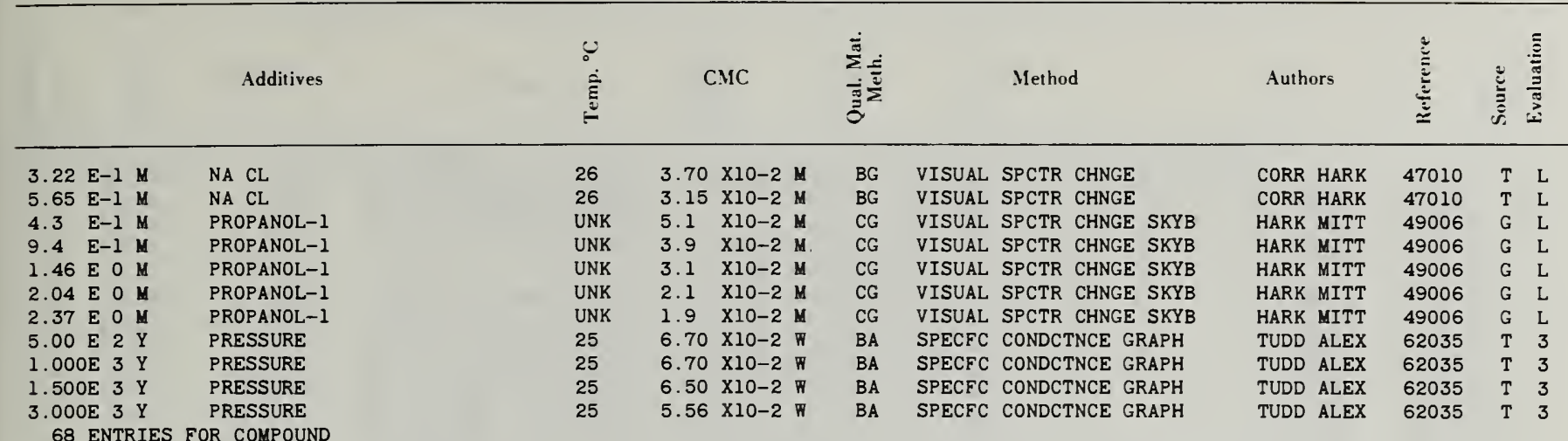

COMPOUND NO $=96$ MOL WGT -

$1.25 \mathrm{E}-2 \mathrm{M} \quad \mathrm{K}$ BR

$2.5 \mathrm{E}-2 \mathrm{M} \quad \mathrm{K}$ BR

3 ENTRIES FOR COMPOUND

\subsection{UNDECYL TRIMETHYL AMMONIUM BROMIDE}

\begin{tabular}{|c|c|c|}
\hline 30 & 3.6 & $X 10-2 N$ \\
\hline 30 & 3.1 & $\times 10-2$ \\
\hline 30 & 2.7 & $\times 10-2$ \\
\hline
\end{tabular}

\author{
$\mathrm{CC}$
}

TURBIDITY PLT LITE SCATR

CC TURBIDITY PLT LITE SCATR

TURBIDITY PLT LITE SCATR
TRAP HERM TRAP HERM TRAP HERM
55009 T $\quad$ L 55009 T $\quad$ L $\begin{array}{lll}55009 & \mathrm{~T} & \mathrm{~L}\end{array}$

\section{COMPOUND NO $=97$ MOL WGT -}

308.4 DODECYL TRIMETHYL AMMONIUM BROMIDE

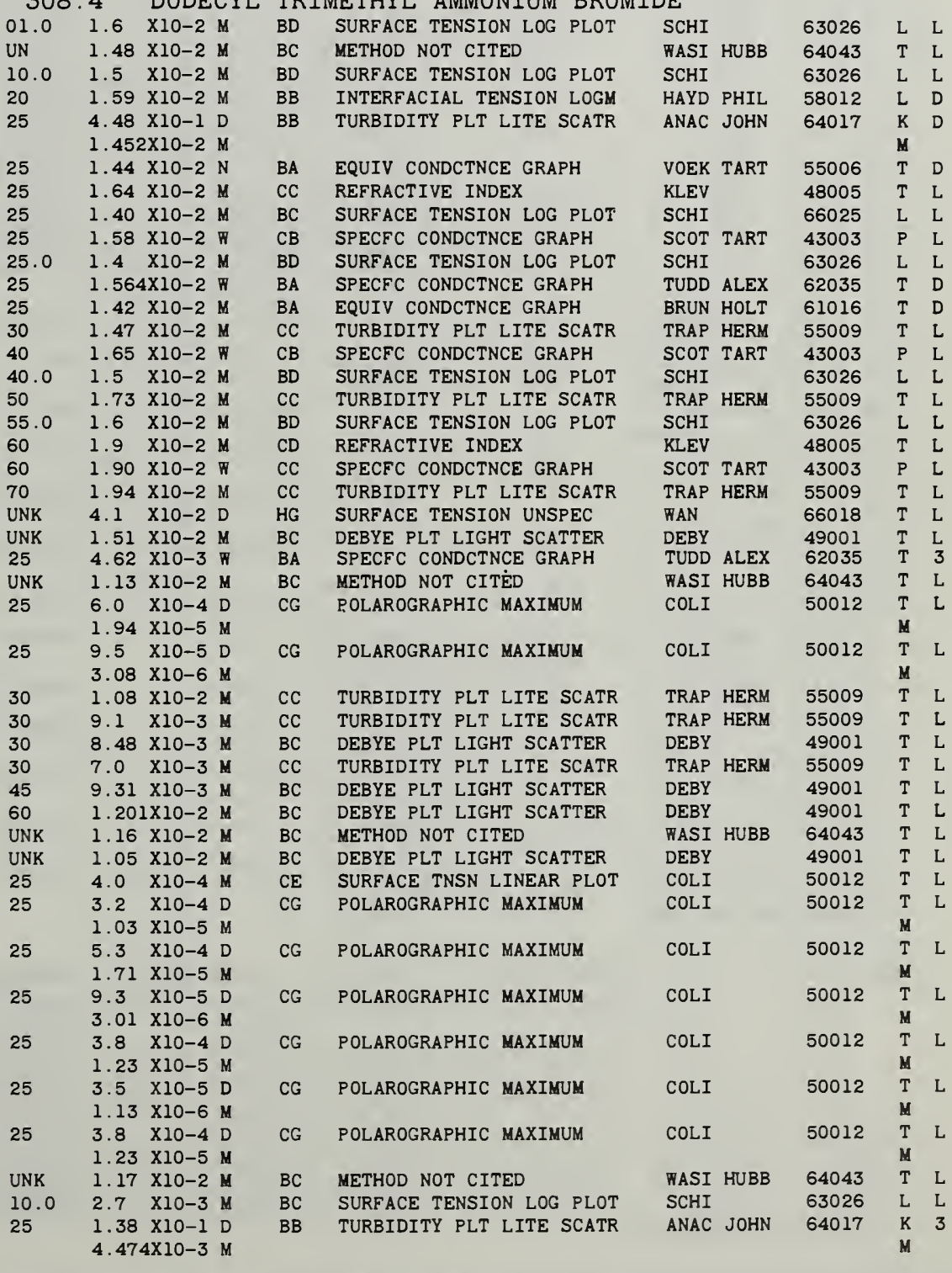

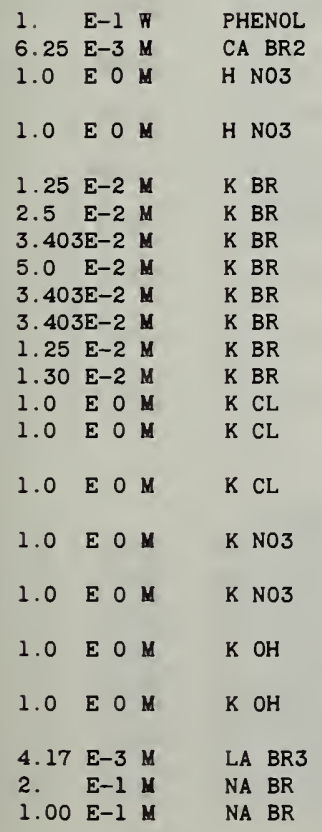

Concentration units: $\mathrm{A}-$ mol $\% ; \mathrm{B}-$ vol $\%$ solvent: $\mathrm{C}-$ mol \% surfactant mixture; D-witvol \%; E-\% saturation; $\mathrm{H}-\mathrm{wt} \%$ solvent; $\mathrm{I}-$ mol \% surfactant; $\mathrm{K}-$ normality counterions; $\mathrm{M}$-molar: $\mathrm{N}$ - normal; $\mathrm{P}-$ wt $\%: \mathrm{Q}-\mathrm{wt} \%$ surfactant; $\mathrm{R}$ - varied: $\mathrm{S}-$ mol/ kg: $\mathrm{T}-\mathrm{wt} \%$ surfactant mixture: $\mathrm{U}-\mathrm{mol} /(\mathrm{l}$ or kg); $\mathrm{W}-$ molal; $Y-$ atm. Details on page 222. 


\begin{tabular}{|c|c|c|c|c|c|c|c|c|c|c|c|c|c|c|}
\hline 2. & E-1 & M & NA BR & 25 & 2.0 & $x 10-3$ & M & $\mathrm{BD}$ & SURFACE TENSION LOG PLOT & SCHI & & 66025 & $\mathrm{~L}$ & L \\
\hline 2 . & $E-1$ & M & NA $B R$ & 25.0 & 2.5 & $x 10-3$ & M & $\mathrm{BC}$ & SURFACE TENSION LOG PLOT & SCHI & & 63026 & $\mathrm{~L}$ & L \\
\hline 4. & $\mathrm{E}-1$ & M & NA BR & 25 & 1.0 & $x 10-3$ & M & $\mathrm{BD}$ & SURFACE TENSION LOG PLOT & SCHI & & 66025 & $\bar{L}$ & $\mathrm{~L}$ \\
\hline 5.02 & $\mathrm{E}-1$ & M & NA BR & 25 & $\begin{array}{l}6.2 \\
2.01\end{array}$ & $\begin{array}{l}\times 10-2 \\
\times 10-3\end{array}$ & $\begin{array}{l}D \\
M\end{array}$ & BB & TURBIDITY PLT LITE SCATR & ANAC & JOHN & 64017 & $\begin{array}{l}K \\
M\end{array}$ & 3 \\
\hline 8. & $E-1$ & M & NA BR & 25 & 6.3 & $x 10-4$ & M & $\mathrm{BC}$ & SURFACE TENSION LOG PLOT & $\mathrm{SCHI}$ & & 66025 & L & $\mathrm{L}$ \\
\hline 5.02 & $E-1$ & W & NA BR & 31.5 & 1.9 & $x 10-3$ & M & $\mathrm{BD}$ & DEBYE PLT LIGHT SCATTER & ANAC & GHOS & 63016 & $\mathrm{~T}$ & $\mathrm{~L}$ \\
\hline 2. & E-1 & M & NA $B R$ & 40.0 & 3.0 & $x 10-3$ & M & $\mathrm{BC}$ & SURFACE TENSION LOG PLOT & $\mathrm{SCHI}$ & & 63026 & L & $\mathrm{L}$ \\
\hline 2 . & $E-1$ & M & NA BR & 55.0 & 3.2 & $\times 10-3$ & M & $\mathrm{BC}$ & SURFACE TENSION LOG PLOT & $\mathrm{SCHI}$ & & 63026 & $\mathrm{~L}$ & L \\
\hline 7 . & $E-2$ & M & NA CL & 20 & 8.7 & $x 10-3$ & M & BB & INTERFACIAL TENSION LOGM & HAYD & TAYL & 60011 & L & L \\
\hline 1.0 & E-1 & M & $\mathrm{NA} C L$ & 20 & 7.5 & $\times 10-3$ & M & $\mathrm{BB}$ & INTERFACIAL TENSION LOGM & HAYD & TAYL & 60011 & L & L \\
\hline 2.5 & $\mathrm{E}-1$ & M & NA CL & 20 & 4.25 & $x 10-3$ & M & BB & INTERFACIAL TENSION LOGM & HAYD & TAYL & 60011 & $\mathrm{~L}$ & $\mathrm{~L}$ \\
\hline 5.0 & $E-1$ & M & NA CL & 20 & 2.57 & $\times 10-3$ & M & BB & INTERFACIAL TENSION LOGM & HAYD & TAYL & 60011 & $\mathrm{~L}$ & $\mathrm{~L}$ \\
\hline 5.00 & E 2 & $\mathrm{Y}$ & PRESSURE & 25 & 1.61 & $\times 10-2$ & W & $\mathrm{BA}$ & SPECFC CONDCTNCE GRAPH & TUDD & ALEX & 62035 & $\mathrm{~T}$ & 3 \\
\hline 1.000 & $O E 3$ & $Y$ & PRESSURE & 25 & 1.616 & $6 \times 10-2$ & $W$ & $\mathrm{BA}$ & SPECFC CONDCTNCE GRAPH & TUDD & ALEX & 62035 & $\mathrm{~T}$ & 3 \\
\hline 1.500 & $\mathrm{OE} 3$ & $Y$ & PRESSURE & 25 & 1.56 & $\times 10-2$ & W & $\mathrm{BA}$ & SPECFC CONDCTNCE GRAPH & TUDD & ALEX & 62035 & $\mathrm{~T}$ & 3 \\
\hline 3.000 & $O E 3$ & $Y$ & PRESSURE & 25 & 1.272 & $2 \times 10-2$ & W & $\mathrm{BA}$ & SPECFC CONDCTNCE GRAPH & TUDD & ALEX & 62035 & $\mathrm{~T}$ & 3 \\
\hline 5. & E-1 & M & UREA & 25 & 1.56 & $x 10-2$ & $M$ & BA & EQUIV CONDCTNCE GRAPH & BRUN & HOLT & 61016 & $\mathrm{~T}$ & 3 \\
\hline 2.0 & E 0 & M & UREA & 25 & 2.04 & $\times 10-2$ & M & BA & EQUIV CONDCTNCE GRAPH & BRUN & HOLT & 61016 & $\mathrm{~T}$ & 3 \\
\hline 6.0 & E 0 & M & UREA & 25 & 4.54 & $x 10-2$ & M & BA & EQUIV CONDCTNCE GRAPH & BRUN & HOLT & 61016 & $\mathrm{~T}$ & 3 \\
\hline 1.0 & E 1 & $\mathrm{C}$ & 0115 & 25 & 6.3 & $\times 10-4$ & M & $\mathrm{BC}$ & SURFACE TENSION LOG PLOT & SCHI & & 66025 & $\mathrm{~L}$ & L \\
\hline 2.5 & E 1 & $\mathrm{C}$ & 0115 & 25 & 2.5 & $x 10-4$ & $M$ & $\mathrm{BC}$ & SURFACE TENSION LOG PLOT & SCHI & & 66025 & $\bar{L}$ & $\mathrm{~L}$ \\
\hline 5.0 & E 1 & $\mathrm{C}$ & 0115 & 25 & 1.25 & $\times 10-4$ & M & $\mathrm{BC}$ & SURFACE TENSION LOG PLOT & $\mathrm{SCHI}$ & & 66025 & $\mathrm{~L}$ & $\mathrm{~L}$ \\
\hline 1.0 & E 1 & $\mathrm{C}$ & 0116 & 25 & 6.3 & $\times 10-4$ & M & $\mathrm{BC}$ & SURFACE TENSION LOG PLO'T & $\mathrm{SCHI}$ & & 66025 & $\mathrm{~L}$ & $\mathrm{~L}$ \\
\hline 2.5 & E 1 & $\mathrm{C}$ & 0116 & 25 & 2.0 & $\times 10-4$ & $M$ & $\mathrm{BD}$ & SURFACE TENSION LOG PLOT & SCHI & & 66025 & $\mathrm{~L}$ & L \\
\hline 5.0 & E 1 & $\mathrm{C}$ & 0116 & 25 & 8.0 & $\times 10-5$ & M & $\mathrm{BC}$ & SURFACE TENSION LOG PLOT & SCHI & & 66025 & L & $\mathrm{L}$ \\
\hline 1.0 & E 1 & C & 0325 & 25 & 8.0 & $\mathrm{X} 10-4$ & M & $\mathrm{BC}$ & SURFACE TENSION LOG PLOT & $\mathrm{SCHI}$ & & 66025 & $\bar{L}$ & $\mathrm{~L}$ \\
\hline 2.5 & E 1 & $\mathrm{C}$ & 0325 & 25 & 6.0 & $\times 10-4$ & M & $\mathrm{BC}$ & SURFACE TENSION LOG PLOT & SCHI & & 66025 & $\bar{L}$ & $\mathrm{~L}$ \\
\hline 5.0 & E 1 & $\mathrm{C}$ & 0325 & 25 & 4.0 & $\times 10-4$ & M & $\mathrm{BC}$ & SURFACE TENSION LOG PLOT & $\mathrm{SCHI}$ & & 66025 & $\mathrm{~L}$ & $\mathrm{~L}$ \\
\hline 7.5 & E 1 & $\mathrm{C}$ & 0325 & 25 & 2.0 & $\times 10-4$ & M & $\mathrm{BD}$ & SURFACE TENSION LOG PLOT & SCHI & & 66025 & $\mathrm{~L}$ & $\mathrm{~L}$ \\
\hline 1.0 & E 1 & C & 0327 & 25 & 2.0 & $x 10-3$ & M & $\mathrm{BD}$ & SURFACE TENSION LOG PLOT & SCHI & & 66025 & $\mathrm{~L}$ & $\mathrm{~L}$ \\
\hline 2.5 & E 1 & $\mathrm{C}$ & 0327 & 25 & 2.5 & $\times 10-4$ & M & $\mathrm{BD}$ & SURFACE TENSION LOG PLOT & $\mathrm{SCHI}$ & & 66025 & $\mathrm{~L}$ & $\mathrm{~L}$ \\
\hline 5.0 & E 1 & C & 0327 & 25 & 1.25 & $\times 10-4$ & M & $\mathrm{BC}$ & SURFACE TENSION LOG PLOT & SCHI & & 66025 & $\mathrm{~L}$ & $\mathrm{~L}$ \\
\hline \multirow[t]{2}{*}{5.0} & E 0 & M & $\mathrm{NH} 3$ & 25 & 3.0 & $\times 10-4$ & $\mathrm{D}$ & CG & POLAROGRAPHIC MAXIMUM & COLI & & 50012 & $\mathrm{~T}$ & $\mathrm{~L}$ \\
\hline & 1. & E-1 & NH4 CL & & 9.72 & $\times 10-6$ & M & & & & & & $M$ & \\
\hline \multirow[t]{2}{*}{5.00} & E 2 & $\mathrm{Y}$ & PRESSURE & 25 & 5.00 & $x 10-3$ & $\mathrm{~W}$ & $\mathrm{BA}$ & SPECFC CONDCTNCE GRAPH & TUDD & ALEX & 62035 & $\mathrm{~T}$ & 3 \\
\hline & 1. & E-1 & W PHENOL & & & & & & & & & & & \\
\hline \multirow{2}{*}{$1.000 \mathrm{~B}$} & OE 3 & $\mathrm{Y}$ & PRESSURE & 25 & 5.03 & $x 10-3$ & W & BA & SPECFC CONDCTNCE GRAPH & TUDD & ALEX & 62035 & $\mathrm{~T}$ & 3 \\
\hline & 1 . & $E-1$ & PHENOL & & & & & & & & & & & \\
\hline \multirow{2}{*}{$1.500 \mathrm{~B}$} & OE 3 & $\mathrm{Y}$ & PRESSURE & 25 & 4.88 & $\times 10-3$ & $w$ & BA & SPECFC CONDCTNCE GRAPH & TUDD & ALEX & 62035 & $\mathrm{~T}$ & 3 \\
\hline & 1. & $\mathrm{E}-1$ & PHENOL & & & & & & & & & & & \\
\hline \multirow{2}{*}{$3.000 \mathrm{~B}$} & OE 3 & $\mathrm{Y}$ & PRESSURE & 25 & 3.88 & $\mathrm{x} 10-3$ & W & BA & SPECFC CONDCTNCE GRAPH & TUDD & ALEX & 62035 & $\mathrm{~T}$ & 3 \\
\hline & 1. & E-1 & W PHENOL & & & & & & & & & & & \\
\hline \multirow[t]{2}{*}{1.0} & E 1 & $\mathrm{C}$ & 0327 & 25 & 3.0 & $\mathrm{X} 10-4$ & M & $\mathrm{BD}$ & SURFACE TENSION LOG PLOT & SCHI & & 66025 & $\mathrm{~L}$ & $\mathrm{~L}$ \\
\hline & 2. & $E-1$ & NA BR & & & & & & & & & & & \\
\hline \multirow[t]{2}{*}{1.0} & E 1 & $\mathrm{C}$ & 0327 & 25 & 1.25 & $\mathrm{X} 10-4$ & M & $\mathrm{BC}$ & SURFACE TENSION LOG PLOT & $\mathrm{SCHI}$ & & 66025 & $\mathrm{~L}$ & $\mathrm{~L}$ \\
\hline & 4. & $E-1$ & $M \quad N A B R$ & & & & & & & & & & & \\
\hline \multirow[t]{2}{*}{1.0} & E 1 & $\mathrm{C}$ & 0327 & 25 & 1.0 & $\mathrm{X} 10-4$ & M & $\mathrm{BD}$ & SURFACE TENSION LOG PLOT & $\mathrm{SCHI}$ & & 66025 & $\mathrm{~L}$ & $\mathrm{~L}$ \\
\hline & 8. & E-1 & M NA BR & & & & & & & & & & & \\
\hline \multirow[t]{2}{*}{2.5} & E 1 & $\mathrm{C}$ & 0327 & 25 & 2.0 & $\times 10-4$ & M & $\mathrm{BD}$ & SURFACE TENSION LOG PLOT & $\mathrm{SCHI}$ & & 66025 & $\mathrm{~L}$ & $\mathrm{~L}$ \\
\hline & 2 . & $E-1$ & $M \quad N A B R$ & & & & & & & & & & & \\
\hline \multirow[t]{2}{*}{2.5} & E 1 & $\mathrm{C}$ & 0327 & 25 & 1.25 & $\times 10-4$ & M & $\mathrm{BC}$ & SURFACE TENSION LOG PLOT & $\mathrm{SCHI}$ & & 66025 & $\mathrm{~L}$ & L \\
\hline & 4. & E-1 & M NA BR & & & & & & & & & & & \\
\hline \multirow[t]{2}{*}{2.5} & E 1 & $\mathrm{C}$ & 0327 & 25 & 1.0 & $\mathrm{x} 10-4$ & M & $\mathrm{BD}$ & SURFACE TENSION LOG PLOT & $\mathrm{SCHI}$ & & 66025 & $\mathrm{~L}$ & $\mathrm{~L}$ \\
\hline & 8. & $E-1$ & $M \quad N A B R$ & & & & & & & & & & & \\
\hline \multirow[t]{2}{*}{5.0} & E 1 & $\mathrm{C}^{2}$ & 0327 & 25 & 1.25 & $\times 10-4$ & M & $\mathrm{BC}$ & SURFACE TENSION LOG PLOT & $\mathrm{SCHI}$ & & 66025 & $\mathrm{~L}$ & $\mathrm{~L}$ \\
\hline & 2. & $E-1$ & $M \quad N A B R$ & & & & & & & & & & & \\
\hline 5.0 & E 1 & $\mathrm{C}$ & 0327 & 25 & 1.25 & $\times 10-4$ & M & $\mathrm{BC}$ & SURFACE TENSION LOG PLOT & $\mathrm{SCHI}$ & & 66025 & $\mathrm{~L}$ & $\mathrm{~L}$ \\
\hline & 4. & $E-1$ & M NA BR & & & & & & & & & & & \\
\hline 5.0 & E 1 & $\mathrm{C}$ & 0327 & 25 & 1.0 & $\times 10-4$ & M & $\mathrm{BD}$ & SURFACE TENSION LOG PLOT & SCHI & & 66025 & $\mathrm{~L}$ & L \\
\hline
\end{tabular}

90 ENTRIES FOR COMPOUND

COMPOUND NO $=98$ MOL WGT -

$\begin{array}{llll}336.4 & \text { TETRADE } \\ 30 & 3.51 & \times 10-3 \mathrm{M} \\ 30 & 3.6 & \times 10-3 \mathrm{M} \\ 50 & 4.2 & \times 10-3 & \mathrm{M} \\ 70 & 4.2 & \times 10-3 & \mathrm{M} \\ \text { UNK } & 8.2 & \times 10-2 & \mathrm{D} \\ 30 & 2.1 & \times 10-3 & \mathrm{M} \\ 30 & 1.6 & \times 10-3 \mathrm{M} \\ 30 & 1.3 & \times 10-3 & \mathrm{M} \\ \text { UNK } & 1.76 & \times 10-3 & \mathrm{M} \\ 30 & 4.2 & \times 10-4 & \mathrm{M}\end{array}$

$$
\begin{aligned}
& \text { ECYL } \\
& \text { BB } \\
& C C \\
& C C \\
& \text { CC } \\
& \text { HG } \\
& C C \\
& C D \\
& C D \\
& B C \\
& B C
\end{aligned}
$$

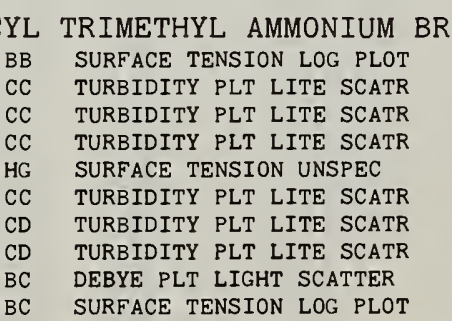

Authors

$\begin{array}{llll}1.25 & \mathrm{E}-2 & \mathrm{M} & \mathrm{K} \\ 2.5 & \mathrm{E}-2 & \mathrm{M} & \mathrm{K} \\ 5.0 & \mathrm{E}-2 & \mathrm{M} & \mathrm{KR} \\ 1.30 & \mathrm{E}-2 & \mathrm{M} & \mathrm{BR} \\ 5 . & \mathrm{K}-2 & \mathrm{BR} \\ 10 & \text { ENTRIES } & \text { FOR BR COMPOUND } \\ 10 & \end{array}$

Concentration units: $\mathrm{A}-\mathrm{mol} \%$; $\mathrm{B}-\mathrm{vol} \%$ solvent; $\mathrm{C}-$ mol \% surfactant mixture; counterions; $\mathrm{M}-$ molar; $\mathrm{N}-$ normal; $\mathrm{P}-\mathrm{wt} \% ; \mathrm{Q}-$ wt $\%$ surfactant; $\mathrm{R}$ - varied; $\mathrm{S}-$ mol/ D-wt/vol \%; E-\% saturation; $\mathrm{H}-\mathrm{wt} \%$ solvent: I-mol \% surfactant; $\mathrm{K}-$ normality 
Additives
$\mathrm{CMC}$

Method

Authors

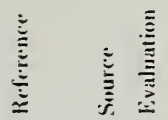

99 MOL WGT -

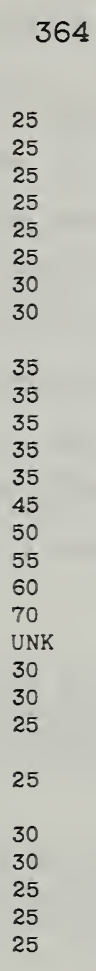

$1.0 \mathrm{EOM} \mathrm{KC}$

1.0 E O M $\quad \mathrm{K} \mathrm{NO3}$

1.0 E O M K NO3

1.0 E O M K NO3

1.0 E O M K NO3

$1.0 \mathrm{EOM} \quad \mathrm{K} \mathrm{OH}$

$1.0 \mathrm{E} \mathrm{OM} \mathrm{K} \mathrm{OH}$

$\mathrm{E} O \quad \mathrm{~K} \mathrm{OH}$

E $0 \quad$ (C4H9) $4 \mathrm{~N} \mathrm{BR} \mathrm{/NORMAL}$

E O (C2H5) $4 \mathrm{~N} \mathrm{BR}$

E $0 \quad$ (CH3) N BR

E 0 (C3H7) $4 \mathrm{~N}$ BR /NORMAL

1.0 E $1 \mathrm{C} \quad 0117$

2.5 E 1 C 0117

5.0 E I C 0117

E $0 \quad 0427$

1.0 E $1 \mathrm{C} \quad 0535$

2.5 E $1 C \quad 0535$

5.0 E I C $\quad 0535$

5.0 E O M NH3

5.0 E $O M^{E-1 ~ M ~ N H 4 ~ C L ~}$

$$
\text { 1. } \mathrm{E}-1 \mathrm{M} \mathrm{NH} 4 \mathrm{CL}
$$

$1.00 \mathrm{E} \mathrm{O}$ PH OF SOLUTION

$1.50 \mathrm{E}-5 \mathrm{M}$ BROMPHENOL BLUE

$1.00 \mathrm{E} \mathrm{O} \quad \mathrm{PH}$ OF SOLUTION

$1.50 \mathrm{E}-5 \mathrm{M}$ BROMPHENOL BLUE

$1.00 \mathrm{E} \mathrm{O} \quad$ PH OF SOLUTION

$3.00 \mathrm{E}-5 \mathrm{M}$ BROMPHENOL BLUE

$1.00 \mathrm{E} \mathrm{O} \quad \mathrm{PH}$ OF SOLUTION $3.00 \mathrm{E}-5 \mathrm{M}$ BROMPHENOL BLUE

$1.00 \mathrm{E} O \quad$ PH OF SOLUTION
364.5 HEXADECYL TRIMETHYLAMMONIUM BROMIDE

QUESTIONABLE CRITERION

GRAPH DATA NOT RETRIEVED

DEBY ANAC COHE VASS

SURFACE TENSION LOG PLOT

8. $0 \times 10-4 \mathrm{M}$

$9.8 \times 10-4 \mathrm{M}$

5. $\times 10-4 \mathrm{~W}$

$9.20 \times 10-4 \mathrm{~W}$

$3.3 \times 10-2 \mathrm{D}$

$3.3 \times 10-2 \mathrm{D}$

4. $\mathrm{X} 10-4 \mathrm{M}$

$3.0 \times 10-2 \mathrm{D}$

$8.23 \times 10-4 \mathrm{M}$

$9.5 \times 10-4 \mathrm{M}$

$9.8 \times 10-4 \mathrm{M}$

9. $\times 10-4 \mathrm{M}$

1. $020 \times 10-3 \mathrm{~W}$

$9.1 \times 10-4 \mathrm{M}$

1. $155 \times 10-3 \mathrm{~W}$

$1.5 \times 10-3 \mathrm{M}$

1. 320X10-3 $\mathrm{W}$

$1.0 \times 10-3 \mathrm{M}$

$1.5 \times 10-3 \mathrm{M}$

$5.1 \times 10-2 \mathrm{D}$

$7.0 \times 10-5 \mathrm{D}$

$1.92 \times 10-6 \mathrm{M}$

$3.8 \times 10-4 \mathrm{D}$

$1.04 \times 10-5 \mathrm{M}$

$8.2 \times 10-4 \mathrm{M}$

$6.0 \times 10-5 \mathrm{M}$

$2.0 \times 10-4 \mathrm{M}$

$7.4 \times 10-5 \mathrm{D}$

$2.03 \times 10-6 \mathrm{M}$

$3.5 \times 10-4 \mathrm{D}$

$9.60 \times 10-6 \mathrm{M}$

$7.4 \times 10-5 \mathrm{D}$

$6.5 \times 10-5 \mathrm{D}$

$1.78 \times 10-6 \mathrm{M}$

$2.03 \times 10-6 \mathrm{M}$

$4.2 \times 10-4 \mathrm{D}$

$2.3 \times 10-4 \mathrm{D}$

$6.31 \times 10-6 \mathrm{M}$

1. $15 \times 10-5 \mathrm{M}$

$2.1 \times 10-5 \mathrm{D}$

$5.76 \times 10-7 \mathrm{M}$

$1.3 \times 10-4 \mathrm{D}$

$3.56 \times 10-6$

SPECFC CONDCTNCE GRAPH

VISUAL SPCTR CHNGE

SPECFC CONDCTNCE GRAPH

METHOD NOT CITED

FOTOMTR SOLUBLZTN OROT

TURBIDITY PLT LITE SCATR

REFRACTIVE INDEX

EQUIV CONDCTNCE GRAPH

SPECFC CONDCTNCE GRAPH

EQUIV CONDCTNCE GRAPH

SPECFC CONDCTNCE GRAPH

VELOCITY OF SOUND

SPECFC CONDCTNCE GRAPH

TURBIDITY PLT LITE SCATR

SPECFC CONDCTNCE GRAPH

REFRACTIVE INDEX

TURBIDITY PLT LITE SCATR

SURFACE TENSION UNSPEC

QUESTIONABLE CRITERION

QUESTIONABLE CRITERION

CG POLAROGRAPHIC MAXIMUM

CG POLAROGRAPHIC MAXIMUM

TURBIDITY PLT LITE SCATR

QUESTIONABLE CRITERION

SURFACE TNSN LINEAR PLOT

SURFACE TENSION MINIMUM

CG POLAROGRAPHIC MAXIMUM

CG POLAROGRAPHIC MAXIMUM

CG POLAROGRAPHIC MAXIMUM

CG POLAROGRAPHIC MAXIMUM

SCHI

SCOT TART

FINE MCBA

GINN HARR

GINN KINN

TRAP HERM

STEI COHE

HART COLL

HART COLL

HART COLL

CZER

KUPP SURY

CZER

TRAP HERM

CZER

KLEV

TRAP HERM

STEI COHE

STEI COHE

COLI

COLI

TART

STEI COHE

COLI

COLI

COLI

COLI

COLI

COLI

CG POLAROGRAPHIC MAXIMUM

CG POLAROGRAPHIC MAXIMUM

COLI

COLI

CG POLAROGRAPHIC MAXIMUM

COLI

CG POLAROGRAPHIC MAXIMUM

COLI

QUESTIONABLE CRITERION QUESTIONABLE CRITERION QUESTIONABLE CRITERION QUESTIONABLE CRITERION QUESTIONABLE CRITERION

$8.0 \times 10-4 \mathrm{M}$ CC SURFACE TENSION LOG PLOT

$4.5 \times 10-5 \mathrm{M}$ CC SURFACE TENSION LOG PLOT

$1.4 \times 10-5 \mathrm{M}$

SURFACE TENSION LOG PLOT

GRAPH DATA NOT RETRIEVED

$8.0 \times 10-4 \mathrm{M}$ CC SURFACE TENSION LOG PLOT

$3.0 \times 10-5 \mathrm{M}$ CD SURFACE TENSION LOG PLOT

$1.3 \times 10-5 \mathrm{M}$

SURFACE TENSION LOG PLOT

POLAROGRAPHIC MAXIMUM

2. $19 \times 10-6 \mathrm{M}$

$1.5 \times 10-4 \mathrm{D}$

$4.11 \times 10-6 \mathrm{M}$

POLAROGRAPHIC MAXIMUM

FOTOMTR SPCTR CHNGE BRPB

STEI COHE

STEI COHE

STEI COHE

STEI COHE

STEI COHE

SCHI

SCHI

SCHI

LANG

SCHI

SCHI

SCHI

COLI

COLI

X10-5 M C

COLI

6. X10-5 M CG FOTOMTR SPCTR CHNGE BRPB

COLI

FOTOMTR SPCTR CHNGE BRPB

COLI

FOTOMTR SPCTR CHNGE BRPB

COLI

FOTOMTR SPCTR CHNGE BRPB
51001

61027

66025

43003

48011

6030

61014

61015

55009

65012

36001

36001

36001

66030

65028

66030

55009

48005

55009

66018

65012

65012
50012

50012

59010

65012

50012

50012

50012

50012

50012

50012

50012

50012

50012

65012

65012

65012

65012

65012

66025

66025

66025

53005

66025

66025

66025

50012

50012

51008

51008

51008

51008

51008

$R$
$R$

L L

P L

T

T

T L

T L

T L

$\begin{array}{ll}M & \\ P & D\end{array}$

$P$ D

T L

T D

T L

T L

T 3

T L

T L

T $R$

T $\quad \mathrm{L}$

M

T

M

$R$

T L

$\begin{array}{ll}\text { T } & \text { L } \\ \text { T } & \text { L }\end{array}$

T L

M

T 


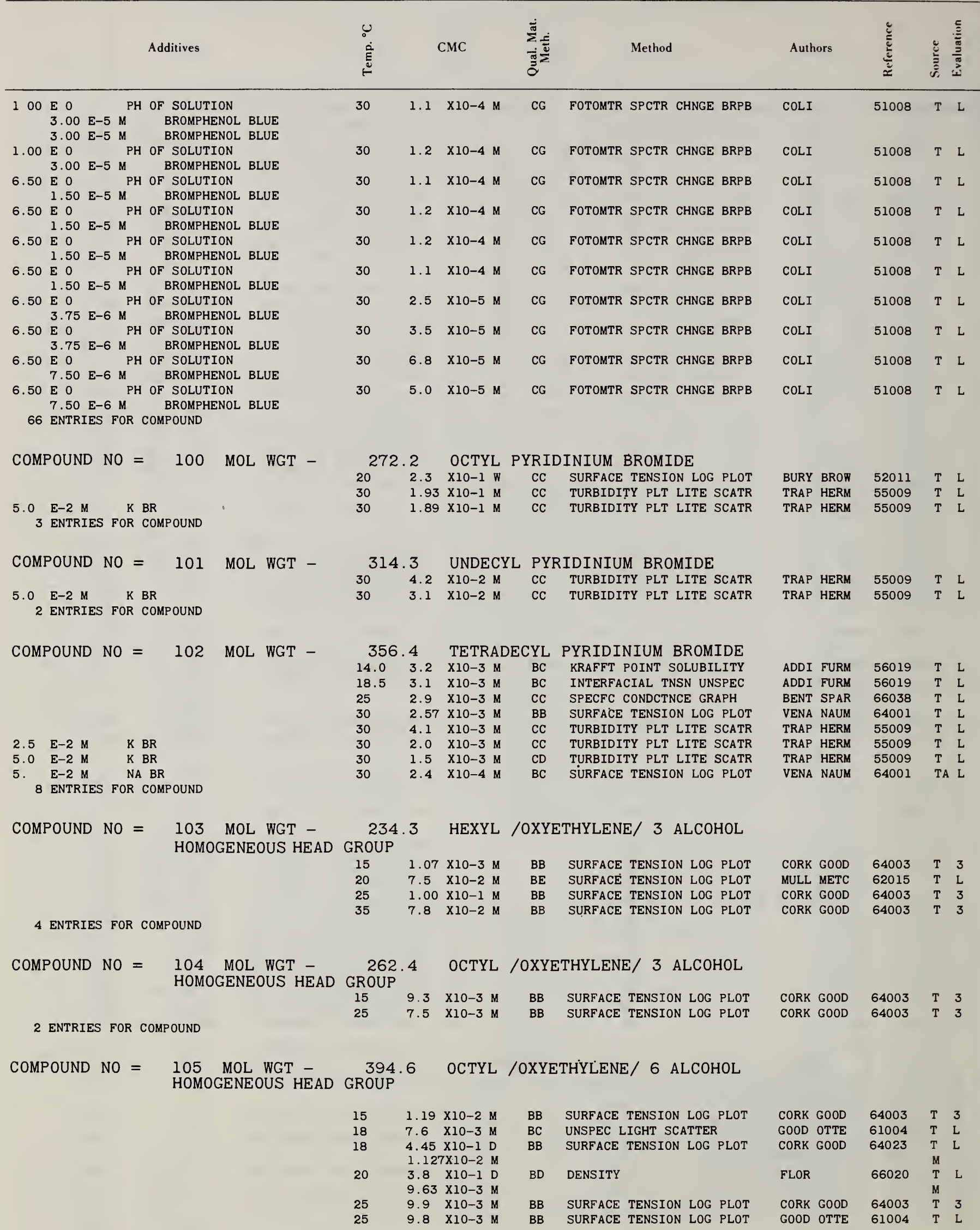

Concentration units: A-mol \%; $\mathrm{B}-\mathrm{vol} \%$ solvent: $\mathrm{C}-$ mol \% surfactant mixture; D-wt/vol \%: E-\% saturation; H-wt \% solvent; I-mol \% surfactant; K-normality counterions; $\mathrm{M}$-molar; $\mathrm{N}$ - normal; $\mathrm{P}-\mathrm{wt} \% ; \mathrm{Q}$ - wt \% surfactant; $\mathrm{R}$-varied; $\mathrm{S}-$ mol/ kg; $\mathrm{T}-$ wt \% surfactant mixture; $\mathrm{U}-\mathrm{mol} /(\mathrm{l}$ or kg); $\mathrm{W}-$ molal; $\mathrm{Y}-\mathrm{atm}$. Details on page 222. 
Complete Table of Critical Micelle Concentration Values - Continued

Additives

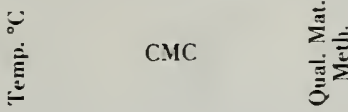

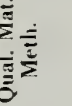

Method

Authors

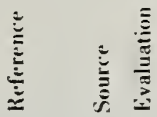

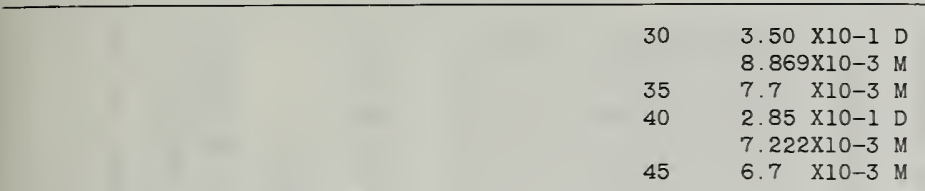

$\begin{array}{lll}\text { BB } & \text { SURFACE TENSION LOG PLOT } \\ \text { BB } & \text { SURFACE TENSION LOG PLOT } \\ \text { BB } & \text { SURFACE TENSION LOG PLOT } \\ \text { BB } & \text { SURFACE TENSION LOG PLOT }\end{array}$

CORK GOOD

64023

T L

CORK GOOD

64003

$\begin{array}{ll}M & \\ T & 3\end{array}$

10 ENTRIES FOR COMPOUND

$6.7 \times 10-3 \mathrm{M}$

CORK GOOD

CORK GOOD

64003

\section{COMPOUND NO $=106$ MOL WGT - HOMOGENEOUS HEAD 526.7
GROUP}

\begin{tabular}{|c|c|c|}
\hline 15 & 1.6 & $\mathrm{X} 10-2 \mathrm{M}$ \\
\hline 2 & 1.3 & $\mathrm{X} 10-2 \mathrm{M}$ \\
\hline 3 & 1.1 & $\mathrm{X} 10-2$ \\
\hline
\end{tabular}

3 ENTRIES FOR COMPOUND

1. 1 X10-2 M

\section{MOL WGT - 290.4
HOMOGENEOUS HEAD GROUP \\ 9 DECYL /OXYETHYLENE/ 3 ALCOHOL}

$15 \quad 7.3 \quad \mathrm{X} 10-4 \mathrm{M}$ $256.0 \times 10-4 \mathrm{M}$

3 ENTRIES FOR COMPOUND
BB SURFACE TENSION LOG PLOT BB SURFACE TENSION LOG PLOT BB SURFACE TENSION LOG PLOT
CORK GOOD CORK GOOD CORK GOOD
64003 64003 64003
T 3 $\begin{array}{ll}T & 3 \\ T & 3\end{array}$

\section{COMPOUND NO $=$ \\ 108 MOL WGT - HOMOGENEOUS HEAD}

\section{6 DECYL /OXYETHYLENE/ 6 ALCOHOL}

\section{GROUP}

$\begin{array}{llll}15 & 1.14 \times 10-3 & \mathrm{M} \\ 20 & 9.2 & \times 10-4 & \mathrm{M} \\ 20 & 9.6 & \times 10-4 & \mathrm{M} \\ 23.5 & 9.5 & \times 10-4 & \mathrm{M} \\ 25 & 9.0 \times 10-4 & \mathrm{M} \\ 25 & 3.80 \times 10-2 & \mathrm{D} \\ & 8.991 \times 10-4 & \mathrm{M} \\ 35 & 6.6 \mathrm{X} 10-4 & \mathrm{M} \\ 35 & 2.79 \times 10-2 & \mathrm{D} \\ & 6.601 \times 10-4 & \mathrm{M} \\ 45 & 6.4 \mathrm{X} 10-4 & \mathrm{M} \\ 45 & 1.83 \times 10-2 & \mathrm{D} \\ & 4.330 \times 10-4 & \mathrm{M}\end{array}$

BB SURFACE TENSION LOG PLOT BD SURFACE TENSION LOG PLOT BC REFRACTIVE INDEX

BG FOTOMTR SPCTR CHNG I2 BB SURFACE TENSION LOG PLOT

SURFACE TENSION LOG PLOT SURFACE TENSION LOG PLOT

SURFACE TENSION LOG PLOT: SURFACE TENSION LOG PLOT
$4.330 \times 10-4 \mathrm{M}$
CORK GOOD CARL CHAL DONB JAN

CARL CHAL

CORK GOOD

CORK GOOD

CORK GOOD

CORK GOOD

CORK GOOD CORK GOOD
64003 64009 63021

64009 64003 64023

64003 64023

64003 64023

10 ENTRIES FOR COMFOUND

554.8 DECYL/OXYETHYLENE/ 9 ALCOHOL

$\begin{array}{llr}\text { COMPOUND NO }= & 109 \text { MOL WGT }- & 554 \\ \text { HOMOGENEOUS HEAD GROUP }\end{array}$

$\begin{array}{llllll}\text { GROUF } & 15 & 1.4 & \text { X10-3 M } & \text { BB } & \text { SURFACE TENSION LOG PLOT } \\ 25 & 1.3 & \text { X10-3 M } & \text { BB } & \text { SURFACE TENSION LOG PLOT } \\ 35 & 1.1 & \times 10-3 & \text { M } & \text { BB } & \text { SURFACE TENSION LOG PLOT }\end{array}$

3 ENTRIES FOR COMPOUND
CORK GOOD CORK GOOD CORK GOOD
64003 $64003 \quad T \quad 3$ 64003 T 3

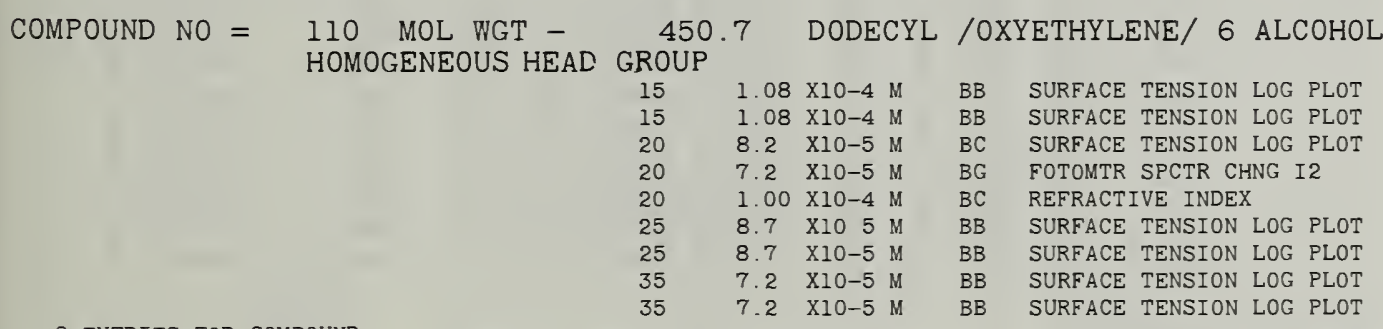

9 ENTRIES FOR COMPOUND

\subsection{LITHIUM DODECYL 1 SULFATE}

$\begin{array}{llll}10 & 7.0 & \times 10-3 & M \\ 25 & 7.9 & \times 10-3 & M \\ 25 & 8.77 & \times 10-3 & M \\ 25 & 8.93 & \times 10-3 & M \\ 40 & 1.05 & \times 10-2 & M \\ 45 & 8.0 & \times 10-3 & M \\ 50 & 6.9 & \times 10-3 & M \\ 40 & 2.5 & \times 10-3 & M \\ 40 & 9.7 & \times 10-3 & M \\ 40 & 9.6 & \times 10-3 & M\end{array}$

CORK GOOD GOOD OTTE CARL CHAL CARL CHAL DONB JAN

CORK GOOD GOOD OTTE CORK GOOD GOOD OTTE
64003 61004 64009 64009 63021 64003 61004 64003 61004

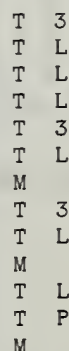

\begin{abstract}
COMPOUND NO $=111$ MOL WGT -
\end{abstract}

$\begin{array}{ll}\text { BB } & \text { SURFACE TENSION LOG PLOT } \\ \text { BB } & \text { SURFACE TENSION LOG PLOT } \\ \text { BA } & \text { EQUIV CONDCTNCE GRAPH } \\ \text { BA } & \text { SPECFC CONDCTNCE GRAPH } \\ \text { CB } & \text { SPECFC CONDCTNCE GRAPH } \\ \text { BB } & \text { SURFACE TENSION LOG PLOT } \\ \text { DG } & \text { VISUAL SPCTR CHNGE PNCN } \\ \text { CG } & \text { FOTOMTR SPCTR CHNGE RHD6 } \\ \text { CG } & \text { FOTOMTR SPCTR CHNGE RHD6 } \\ \text { CG } & \text { FOTOMTR SPCTR CHNGE RHD6 }\end{array}$

$\begin{array}{llll}\text { SCHI } & 64020 & \text { T } & \text { L } \\ \text { SCHI } & 64020 & \text { T } & \text { L } \\ \text { MYSE PRIN } & 59002 & \text { TL } & 2 \\ \text { MYSE PRIN } & 59002 & \text { TL } & 2 \\ \text { MEGU KOND } & 56020 & \text { T } & \text { L } \\ \text { SCHI } & 64020 & \text { T } & \text { L } \\ \text { RAIS } & 52016 & \text { T } & \text { L } \\ \text { MEGU KOND } & 56020 & \text { T } & \text { L } \\ \text { MEGU KOND } & 56020 & \text { T } & \text { L } \\ \text { MEGU KOND } & 56020 & \text { T } & \text { L }\end{array}$

Concentration units: $\mathrm{A}-\mathrm{mol} \%$; $\mathrm{B}-\mathrm{vol} \%$ solvent: $\mathrm{C}-\mathrm{mol} \%$ surfactant mixture: $\mathrm{D}-\mathrm{wt} / \mathrm{vol} \%: \mathrm{E}-\%$ saturation: $\mathrm{H}-\mathrm{wt} \%$ solvent; $\mathrm{I}-\mathrm{mol} \%$ surfactant: $\mathrm{K}-$ normality counterions; $\mathrm{M}$-molar; $\mathrm{N}$-normal; $\mathrm{P}-\mathrm{wt} \%$; $\mathrm{Q}-$ wt $\%$ surfactant; $\mathrm{R}$-varied; $\mathrm{S}-\mathrm{mol} /$ kg: T-wt \% surfactant mixture; $\mathrm{U}-\mathrm{mol} /(\mathrm{l}$ or kg): $\mathrm{W}-$ molal: $\mathrm{Y}-\mathrm{atm}$. Details on page 222. 


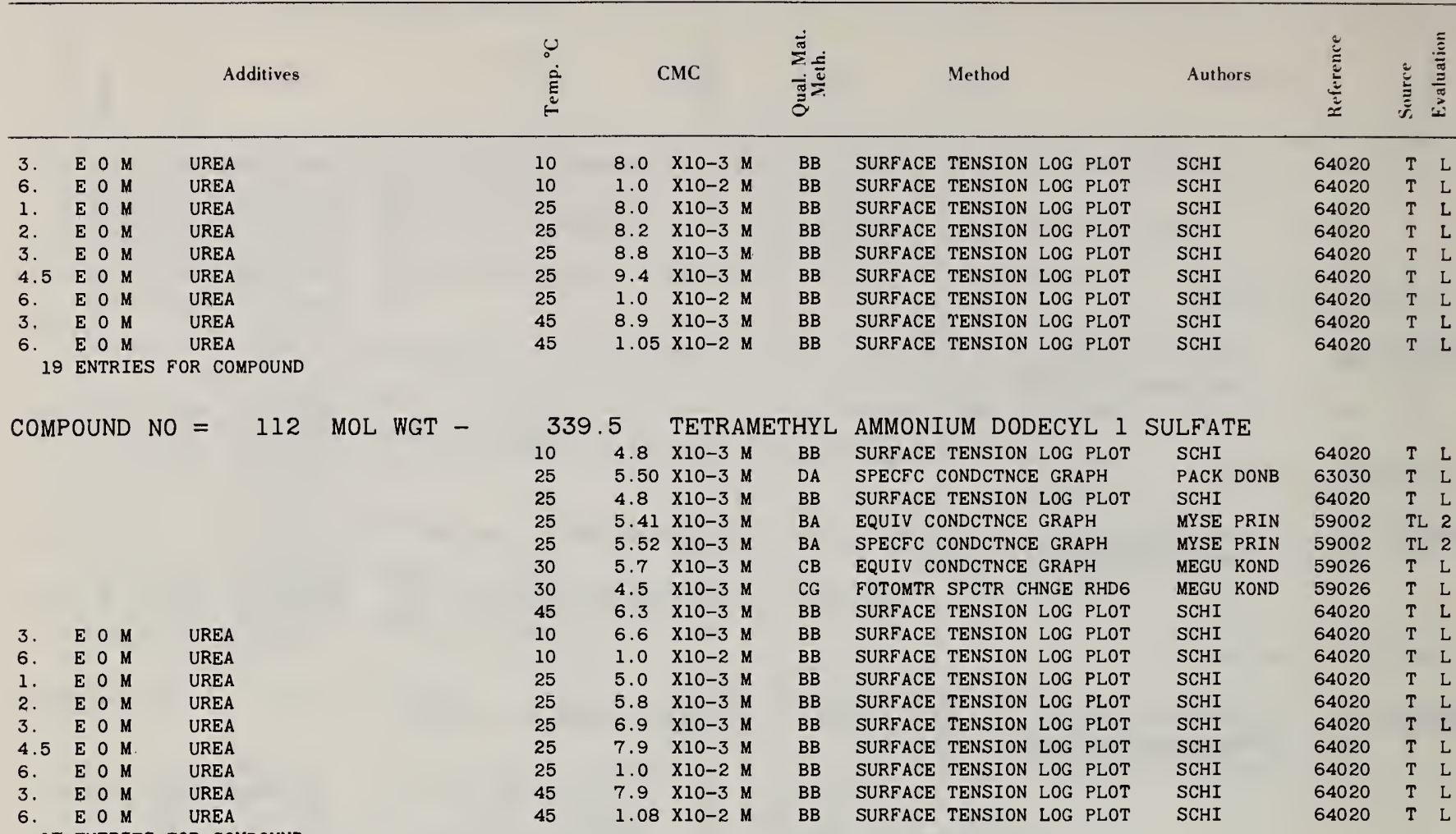

COMPOUND NO $=113$ MOL WGT -

$\begin{array}{lllll}\text { 3. } & \text { E } & 0 & M & \\ \text { 6. } & \text { E } & 0 & M & \text { UREA } \\ \text { 3. } & \text { U } & 0 & M & \text { UREA } \\ \text { 6. } & \text { E } & 0 & M & \text { UREA } \\ \text { 3. } & \text { E } & 0 & M & \text { UREA } \\ 6 . & \text { U } & 0 & M & \text { UREA } \\ \text { UREA }\end{array}$

12 ENTRIES FOR COMPOUND
420.5 SODIUM DODECYL TRI-OXYETHYLENE SULFATE

$\begin{array}{llllllllll}01.0 & 1.25 & \text { X10-4 M } & \text { CC } & \text { SURFACE TENSION LOG PLOT } & \text { SCHI } & 63026 & \mathrm{~L} & \mathrm{~L} \\ 10 & 1.1 & \text { X10-4 M } & \text { CC } & \text { SURFACE TENSION LOG PLOT } & \text { SCHI } & 64020 & \text { T } & \text { L } \\ 25 & 1.0 & \text { X10-4 M } & \text { CC } & \text { SURFACE TENSION LOG PLOT } & \text { SCHI } & 64020 & \text { T } & \text { L } \\ 45 & 1.2 & \text { X10-4 M } & \text { CC } & \text { SURFACE TENSION LOG PLOT } & \text { SCHI } & 64020 & \text { T } & \text { L } \\ 50 & 1.97 & \text { X10-3 M } & \text { CD } & \text { SURFACE TENSION UNSPEC } & \text { GOTT } & 60018 & \text { G } & \text { P } \\ 55.0 & 1.4 & \text { X10-4 M } & \text { CD } & \text { SURFACE TENSION LOG PLOT } & \text { SCHI } & 63026 & \text { L } & \text { L } \\ 10 & 1.55 & \text { X10-4 M } & \text { CC } & \text { SURFACE TENSION LOG PLOT } & \text { SCHI } & 64020 & \text { T } & \text { L } \\ 10 & 2.85 & \text { X10-4 M } & \text { CC } & \text { SURFACE TENSION LOG PLOT } & \text { SCHI } & 64020 & \text { T } & \text { T } \\ 25 & 1.5 & \text { X10-4 M } & \text { CC } & \text { SURFACE TENSION LOG PLOT } & \text { SCHI } & 64020 & \text { T } & \text { T } \\ 25 & 2.5 & \text { X10-4 M } & \text { CC } & \text { SURFACE TENSION LOG PLOT } & \text { SCHI } & 64020 & \text { T } & \text { L } \\ 45 & 1.93 & \text { X10-4 M M } & \text { CC } & \text { SURFACE TENSION LOG PLOT } & \text { SCHI } & 64020 & \text { T } & \text { L } \\ 45 & 2.70 & \text { X10-4 M } & \text { CC } & \text { SURFACE TENSION LOG PLOT } & \text { SCHI } & 64020 & \text { T } & \text { L }\end{array}$

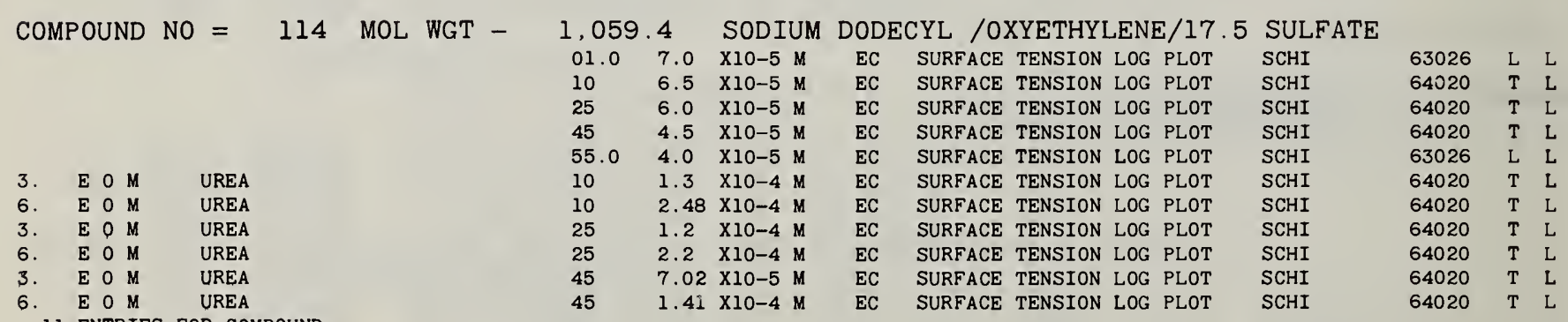

COMPOUND NO $=115$ MOL WGT - 494.7 DODECYL /OXYETHYLENE/7 ALCOHOL REDUCED POLYDISPERSION OF HEAD GROUPS

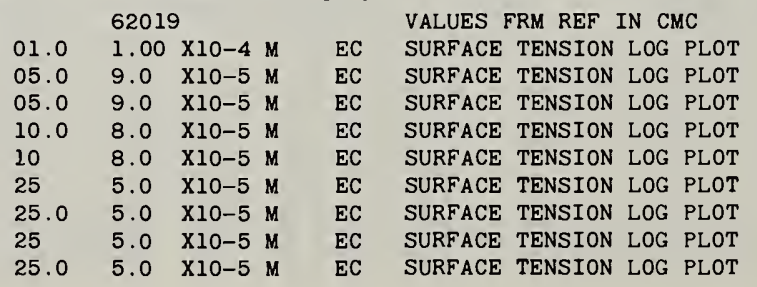

$\begin{array}{llll}\text { SCHI ATLA } & 62020 & & \text { R } \\ \text { SCHI } & 63026 & \text { L } & \text { L } \\ \text { SCHI } & 63026 & \text { L } & \text { L } \\ \text { SCHI MANN } & 66001 & \text { L } & \text { L } \\ \text { SCHI } & 63026 & \text { L } & \text { L } \\ \text { SCHI } & 64020 & \text { T } & \text { L } \\ \text { SCHI } & 66025 & \text { L } & \text { L } \\ \text { SCHI } & 62019 & \text { T } & \text { L } \\ \text { SCHI } & 64020 & \text { T } & \text { L } \\ \text { SCHI MANN } & 66001 & \text { L } & \text { L }\end{array}$

Concentration units: $\mathrm{A}-\mathrm{mol} \% ; \mathrm{B}-\mathrm{vol} \%$ solvent; $\mathrm{C}-$ mol \% surfactant mixture; counterions; $\mathrm{M}-$ molar; $\mathrm{N}-$ normal; $\mathrm{P}-\mathrm{wt} \% ; \mathrm{Q}-$ wt $\%$ surfactant; $\mathrm{R}$ - varied: $\mathrm{S}-$ mol/ D-wt/vol \%; E-\% saturation; $\mathrm{H}-$ wt \% solvent: I - mol \% surfactant; $\mathrm{K}-$ normality 


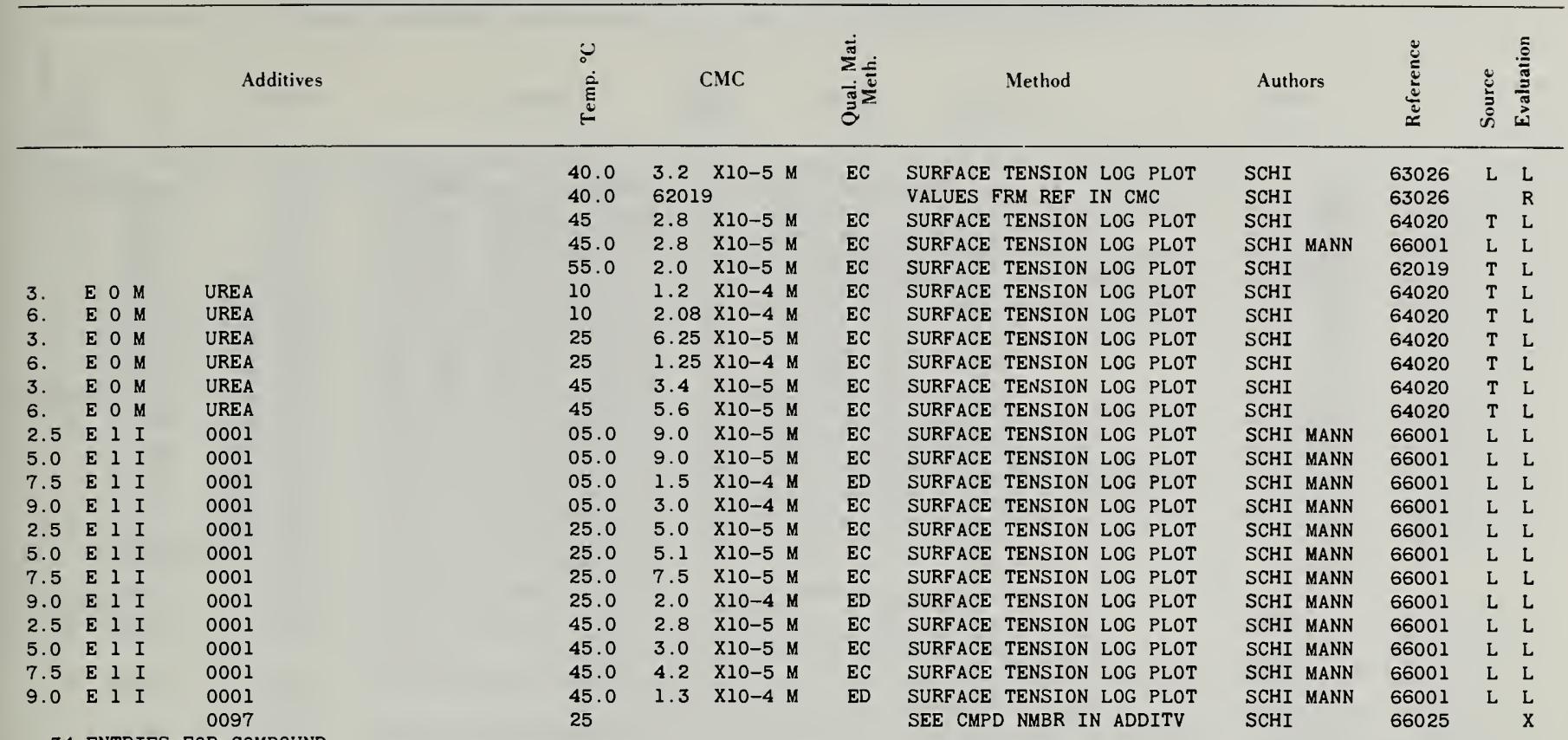

34 ENTRIES FOR COMPOUND

COMPOUND NO $=116$ MOL WGT $-1,508.1$ DODECYL /OXYETHYLENE/30 ALCOHOL REDUCED POLYDISPERSION OF HEAD GROUPS

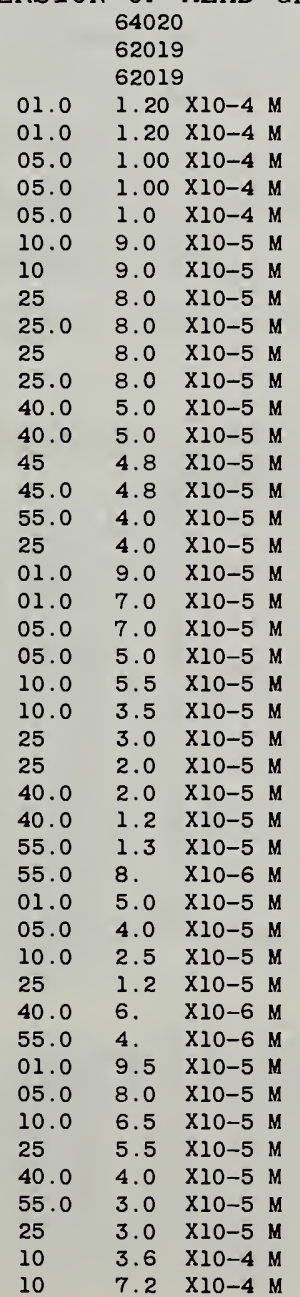

VALUES FRM REF IN CMC VALUES FRM REF IN CMC VALUES FRM REF IN CMC SURFACE TENSION LOG PLOT SURFACE TENSION LOG PLOT SURFACE TENSION LOG PLOT SURFACE TENSION LOG PLOT SURFACE TENSION LOG PLOT SURFACE TENSION LOG PLOT SURFACE TENSION LOG PLOT SURFACE TENSION LOG PLOT SURFACE TENSION LOG PLOT SURFACE TENSION LOG PLOT SURFACE TENSION LOG PLOT SURFACE TENSION LOG PLOT SURFACE TENSION LOG PLOT SURFACE TENSION LOG PLOT SURFACE TENSION LOG PLOT SURFACE TENSION LOG PLOT SURFACE TENSION LOG PLOT SURFACE TENSION LOG PLOT SURFACE TENSION LOG PLOT SURFACE TENSION LOG PLOT SURFACE TENSION LOG PLOT SURFACE TENSION LOG PLOT SURFACE TENSION LOG PLOT SURFACE TENSION LOG PLOT SURFACE TENSION LOG PLOT SURFACE TENSION LOG PLOT SURFACE TENSION LOG PLOT SURFACE TENSION LOG PLOT SURFACE TENSION LOG PLOT SURFACE TENSION LOG PLOT SURFACE TENSION LOG PLOT SURFACE TENSION LOG PLOT SURFACE TENSION LOG PLOT SURFACE TENSION LOG PLOT SURFACE TENSION LOG PLOT SURFACE TENSION LOG PLOT SURFACE TENSION LOG PLOT SURFACE TENSION LOG PLOT SURFACE TENSION LOG PLOT SURFACE TENSION LOG PLOT SURFACE TENSION LOG PLOT SURFACE TENSION LOG PLOT SURFACE TENSION LOG PLOT SURFACE TENSION LOG PLOT

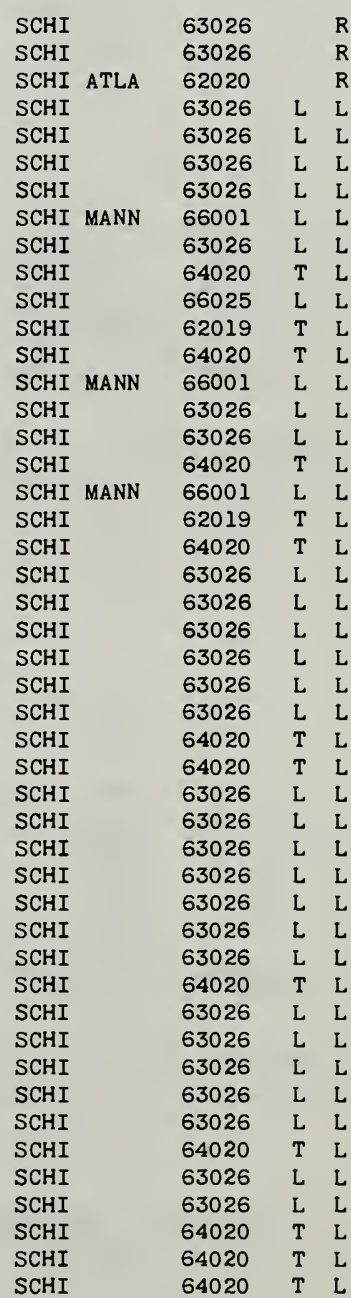

counterions; $\mathrm{M}$-molar; $\mathrm{N}$ - normal; $\mathrm{P}$ - wt \%; $\mathrm{Q}$ - wt \% surfactant; $\mathrm{R}$-varied; $\mathrm{S}$ - mol/ kg; T - wt \% surfactant mixture; $U-$ mol/ $(l \circ \mathrm{kg}) ; \mathrm{W}-$ molal; $\mathrm{Y}-\mathrm{atm}$. Details on page 222.
Concentration units: $\mathrm{A}-\operatorname{mol} \% ; \mathrm{B}-$ vol \% solvent: $\mathrm{C}-$ mol \% surfactant mixture D-wt/vol \%; E-\% saturation; $\mathrm{H}-$ wt \% solvent; $\mathrm{I}-\mathrm{mol} \%$ surfactant; $\mathrm{K}-$ normality 


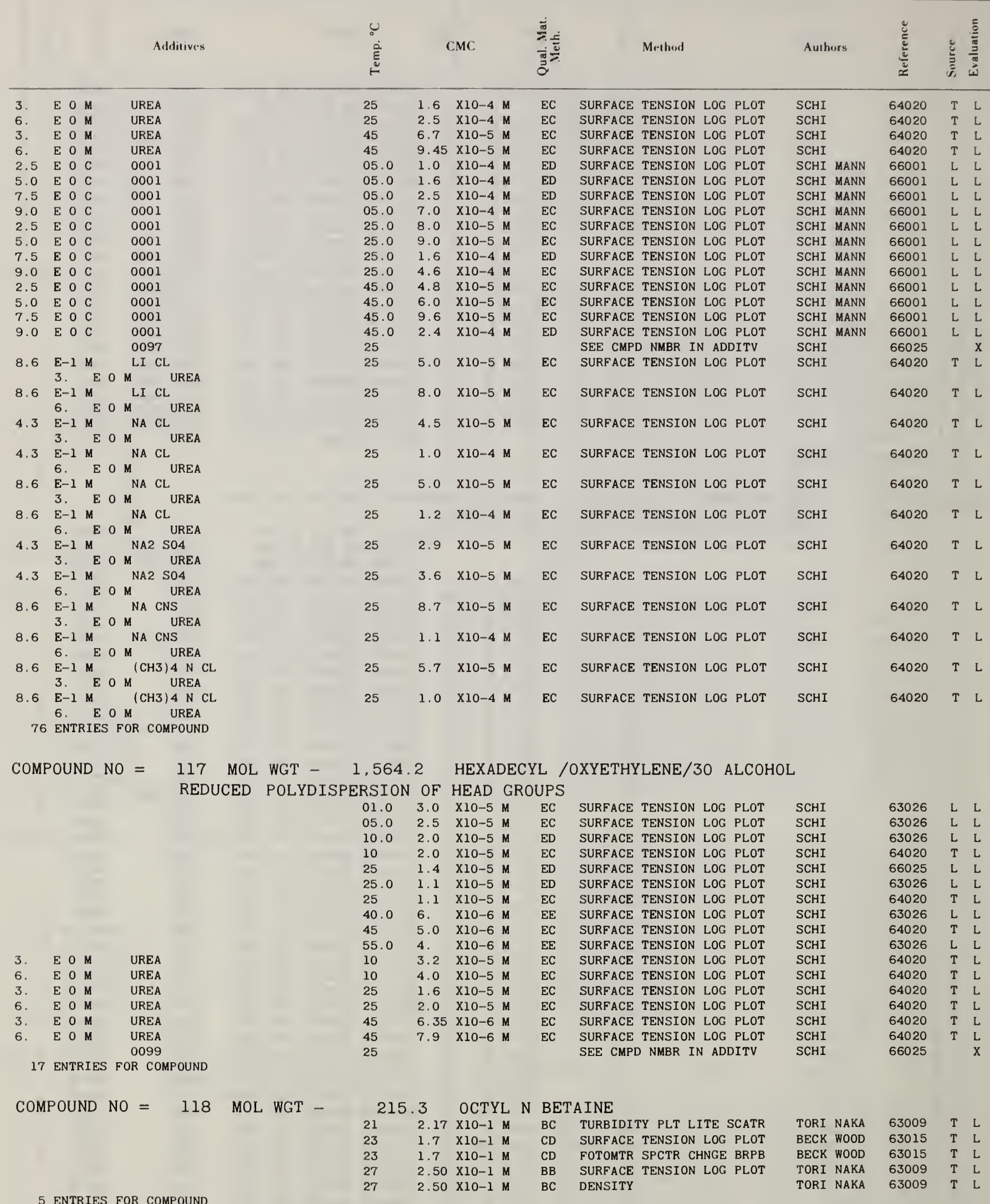

5 ENTRIES FOR COMPOUND

TORI NAKA BECK WOOD

TORI NAKA

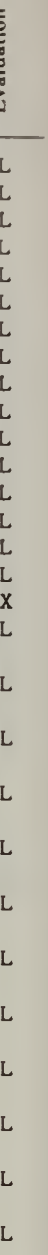




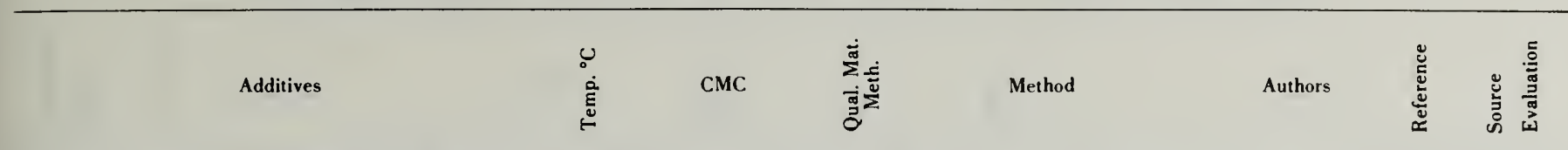

COMPOUND NO $=119$ MOL WGT -

5 ENTRIES FOR COMPOUND

COMPOUND NO $=120$ MOL WGT -

5 ENTRIES FOR COMPOUND

COMPOUND NO $=121$ MOL WGT -

13 ENTRIES FOR COMPOUND

COMPOUND NO $=122$ MOL WGT -

5 ENTRIES FOR COMPOUND

COMPOUND NO $=123$ MOL WGT -

5 ENTRIES FOR COMPOUND

COMPOUND NO $=124$ MOL WGT -

1 ENTRIES FOR COMPOUND

COMPOUND NO $=125$ MOL WGT -

1 ENTRIES FOR COMPOUND

COMPOUND NO $=126$ MOL WGT -

1 ENTRIES FOR COMPOUND

COMPOUND NO $=127$ MOL WGT $4.95 \mathrm{E}-1 \mathrm{~W}$ NA I03 5.1 E-3 M NA BR

1 ENTRIES FOR COMPOUND

COMPOUND NO $=128$ MOL WGT $4.94 \mathrm{E}-1 \mathrm{~W}$ NA HCO2 FORMATE $6.0 \mathrm{E}-3 \mathrm{M}$ NA BR

1 ENTRIES FOR COMPOUND

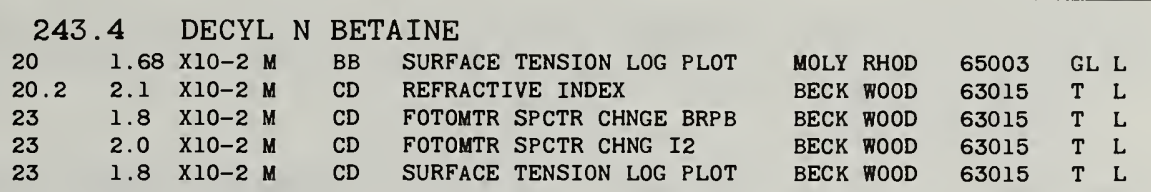

257.4 UNDECYL N BETAINE

$\begin{array}{lllllllll}20 & 7.1 & \text { XIO-3 M } & \text { BB } & \text { SURFACE TENSION LOG PLOT } & \text { MOLY RHOD } & 65003 & \text { GL L } \\ 20.2 & 6.4 & \text { X10-3 M } & \text { CC } & \text { REFRACTIVE INDEX } & \text { BECK WOOD } & 63015 & \text { T } & \text { L } \\ 23 & 6.0 & \text { X10-3 M } & \text { CC } & \text { FOTOMTR SPCTR CHNGE BRPB } & \text { BECK WOOD } & 63015 & \text { T } & \text { L } \\ 23 & 6.4 & \text { X10-3 M } & \text { CC } & \text { FOTOMTR SPCTR CHNG I2 } & \text { BECK WOOD } & 63015 & \text { T } & \text { L } \\ 23 & 6.6 & \text { X10-3 M } & \text { CC } & \text { SURFACE TENSION LOG PLOT } & \text { BECK WOOD } & 63015 & \text { T } & \text { L }\end{array}$

271.4 DODECYL N BETAINE

$10.2 \quad 2.0 \quad X 10-3 \mathrm{M}$ BC REFRACTIVE INDEX

20.2 2.1 X10-3 M CD REFRACTIVE INDEX

$20 \quad 2.00 \times 10-3 \mathrm{M}$ BB SURFACE TENSION LOG PLOT

$20.2 \quad 2.1 \quad \mathrm{X} 10-3 \mathrm{M}$ BC REFRACTIVE INDEX

$23 \quad 1.8$ X10-3 M CD SURFACE TENSION LOG PLOT

$23 \quad 1.6 \times 10-3 \mathrm{M}$ CD FOTOMTR SPCTR CHNGE BRPB

$23 \quad 1.8 \times 10-3 \mathrm{M}$ CD FOTOMTR SPCTR CHNG I2

$25.2 \quad 2.2 \times 10-3 \mathrm{M}$ BC REFRACTIVE INDEX

$30.0 \quad 2.3 \quad \mathrm{X} 10-3 \mathrm{M}$ BC REFRACTIVE INDEX

$35.7 \quad 2.4 \quad X 10-3 \mathrm{M}$ BC REFRACTIVE INDEX

$45.12 .6 \quad X 10-3 \mathrm{M}$ BC REFRACTIVE INDEX

$49.7 \quad 2.7 \quad X 10-3 \mathrm{M}$ BC REFRACTIVE INDEX

$57.0 \quad 2.8 \quad X 10-3 \mathrm{M} \quad \mathrm{BC}$ REFRACTIVE INDEX

MOLY RHOD

BECK WOOD

MOLY RHOD

MOLY RHOD

BECK WOOD

BECK MOOD

BECK WOOD

MOLY RHOD

MOLY RHOD

MOLY RHOD

MOLY RHOD

MOLY RHOD

MOLY RHOD

65003

63015

65003

65003

63015

63015

63015

65003

65003

65003

65003

65003

$65003 \quad \mathrm{GL}$
MOLY RHOD BECK WOOD BECK WOOD BECK WOOD BECK WOOD
65003 GL L

63015 T L

63015 T $\mathrm{L}$

$63015 \mathrm{~T} \mathrm{~L}$

$63015 \mathrm{~T} \mathrm{~L}$

\subsection{HEXADECYL N BETAINE}

$\begin{array}{lllll}20.2 & 1.6 & \text { XIO-5 M } & \text { CD } & \text { REFRACTIVE INDEX } \\ 20 & 2.52 & \times 10-5 \mathrm{M} & \text { BB } & \text { SURFACE TENSION LOG PLOT } \\ 23 & 2.0 & \times 10-5 \mathrm{M} & \mathrm{CD} & \text { SURFACE TENSION LOG PLOT } \\ 23 & 1.8 & \mathrm{X} 10-5 \mathrm{M} & \mathrm{CD} & \text { FOTOMTR SPCTR CHNGE BRPB } \\ 23 & 1.8 & \mathrm{X} 10-5 \mathrm{M} & \mathrm{CD} & \text { FOTOMTR SPCTR CHNG I2 }\end{array}$

BECK WOOD MOLY RHOD BECK WOOD BECK WOOD BECK WOOD
63015 T L 65003 GL L $63015 \mathrm{~T}$ 63015 T L 63015 T L
307.9 DODECYL N BETAINE HYDROCHLORIDE $23 \quad 1.98 \times 10-3 \mathrm{M}$ CC SURFACE TENSION LOG PLOT
BECK WOOD 63015 T L

Concentration units: $\mathrm{A}-\mathrm{mol} \% ; \mathrm{B}-$ vol \% solvent; $\mathrm{C}-$ mol \% surfactant mixture; 


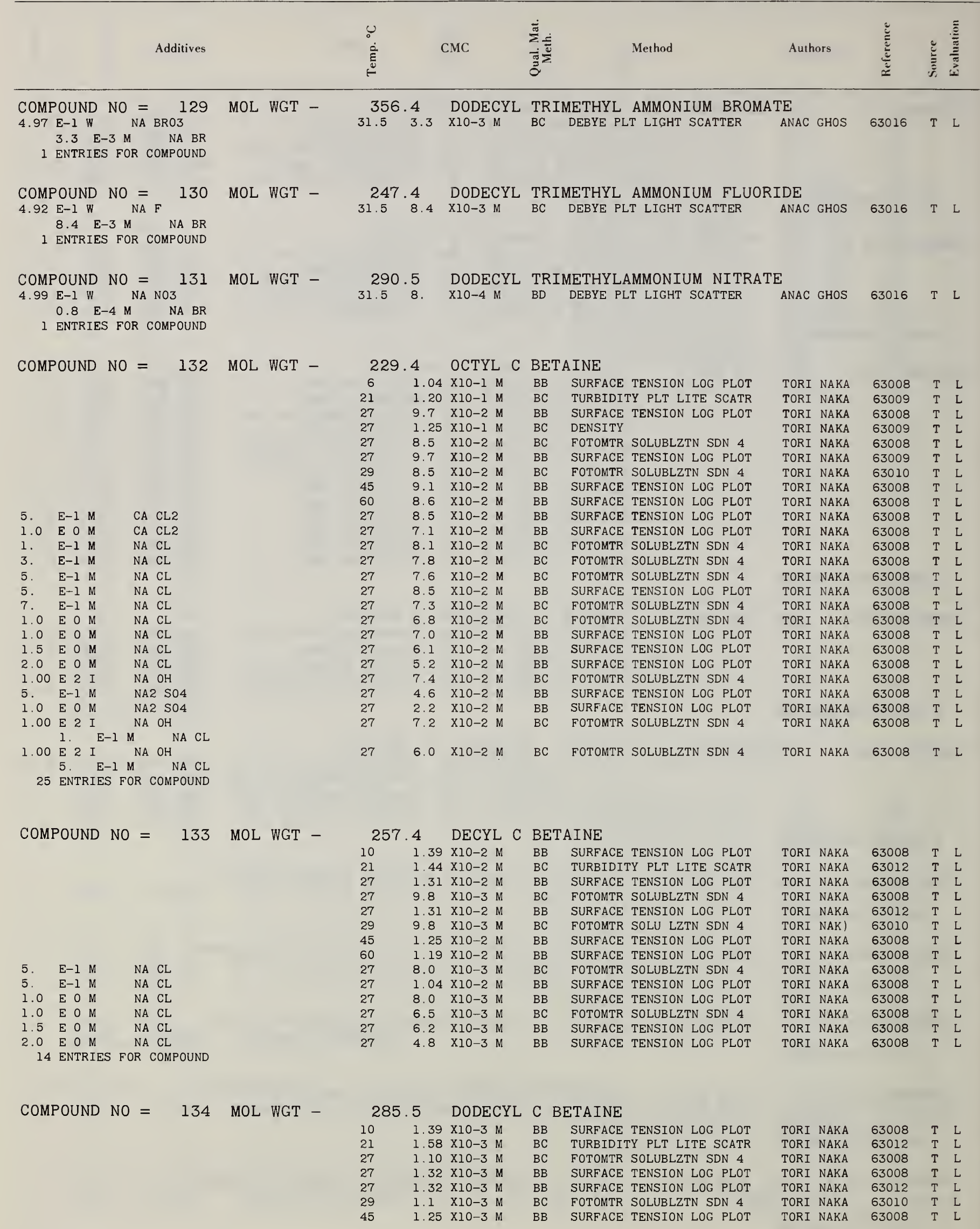

\footnotetext{
Concentration units: $\mathrm{A}-\operatorname{mol} \% ; \mathrm{B}-$ vol \% solvent; $\mathrm{C}-$ mol \% surfactant mixture; $\mathrm{D}-\mathrm{wt} / \mathrm{vol} \%$; $\mathrm{E}-\%$ saturation; $\mathrm{H}-$ wt $\%$ solvent; $\mathrm{I}-\mathrm{mol} \%$ surfactant; $\mathrm{K}-$ normality
}

counterions; $\mathrm{M}$ - molar; $\mathrm{N}$ - normal; $\mathrm{P}-\mathrm{wt} \% ; \mathrm{Q}-$ wt $\%$ surfactant; $\mathrm{R}$-varied; $\mathrm{S}-\mathrm{mol} /$ $\mathrm{k}(\mathrm{r} ; \mathrm{T}-\mathrm{wt} \%$ surfactant mixture; $\mathrm{U}-\mathrm{mol} /(\mathrm{l}$ orkg); $\mathrm{W}-$ molal; $\mathrm{Y}-\mathrm{atm}$. Details on page 222. 


\begin{tabular}{|c|c|c|c|c|c|c|c|c|c|c|c|c|}
\hline & & Additives & 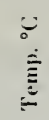 & & $\mathrm{CMC}$ & 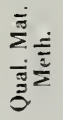 & & Method & Authors & 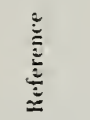 & 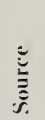 & 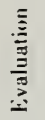 \\
\hline & & & 60 & 1.20 & $\mathrm{X} 10-3 \mathrm{M}$ & $\mathrm{BB}$ & SURFACE & TENSION LOG PLOT & TORI NAKA & 63008 & $\mathrm{~T}$ & L \\
\hline 5. & $E-1 \quad M$ & NA CL & 27 & 7.5 & $\mathrm{X} 10-4 \mathrm{M}$ & $\mathrm{BC}$ & FOTOMTR & SOLUBLZTN SDN 4 & TORI NAKA & 63008 & $\mathrm{~T}$ & L \\
\hline 5. . & $E-1 \quad M$ & NA CL & 7 & 9.6 & $\mathrm{X} 10-4 \mathrm{M}$ & $\mathrm{BB}$ & SURFACE & TENSION LOG PLOT & TORI NAKA & 63008 & $\mathrm{~T}$ & $\mathrm{~L}$ \\
\hline 1.0 & E O M & NA CL & 27 & 6.8 & $\mathrm{X} 10-4 \mathrm{M}$ & $\mathrm{BB}$ & SURFACE & TENSION LOG PLOT & TORI NAKA & 63008 & $\mathrm{~T}$ & L \\
\hline 1.0 & E $O M$ & NA CL & 27 & 5.3 & $\mathrm{X} 10-4 \mathrm{M}$ & $\mathrm{BC}$ & FOTOMTR & SOLUBLZTN SDN 4 & TORI NAKA & 63008 & $\mathrm{~T}$ & L \\
\hline 1.5 & $E O M$ & NA $C L$ & 27 & 4.8 & $\mathrm{X} 10-4 \mathrm{M}$ & $\mathrm{BB}$ & SURFACE & TENSION LOG PLOT & TORI NAKA & 63008 & $\mathrm{~T}$ & $\bar{L}$ \\
\hline 2.0 & E O M & NA $C L$ & 27 & 3.4 & $\mathrm{X} 10-4 \mathrm{M}$ & $\mathrm{BB}$ & SURFACE & TENSION LOG PLOT & TORI NAK) & 63008 & $\mathrm{~T}$ & $\mathrm{~L}$ \\
\hline
\end{tabular}

COMPOUND NO $=135$ MOL WGT -

1. $E-1 M$ NA CL 2 ENTRIES FOR COMPOUND \begin{tabular}{llllll}
\multicolumn{2}{c}{265.8} & OCTYL & C & BETAINE HYDROCHLORIDE \\
27 & 6.0 & X10-2 M & & BC & FOTOMTR SOLUBLZTN SDN 4 \\
27 & 4.2 & X10-2 M & M & BC & FOTOMTR SOLUBLZTN SDN 4
\end{tabular}
TORI NAKA TORI NAKA
63008 T $\quad$ L 63008 T $\quad$ L

COMPOUND NO $=136$ MOL WGT -

237.3 SODIUM ALPHA DIMETHYL AMINO CAPRATE

1. E-I M NA CL

2 ENTRIES FOR COMPOUND

$\begin{array}{llllllllll}27 & 9.6 & \text { XIO-2 M } & \text { BC } & \text { FOTOMTR SOLUBLZTN SDN } 4 & \text { TORI NAKA } & 63008 & \text { T } & \text { L } \\ 27 & 7.0 & \text { X10-2 M } & \text { BC } & \text { FOTOMTR SOLUBLZTN SDN } 4 & \text { TORI NAKA } & 63008 & \text { T } & \text { L }\end{array}$

COMPOUND NO $=137$ MOL WGT -

251.8 ALPHA DIMETHYLAMINO CAPRIC ACID HYDROCHLORIDE

1. E-1 M NA CL

2 ENTRIES FOR COMPOUND

$\begin{array}{lllllllllll}27 & 6.0 & \text { XIO-2 M } & \text { BC } & \text { FOTOMTR SOLUBLZTN SDN } & \text { TORI NAKA } 63008 & \text { T } & \text { L } \\ 27 & 4.2 & \text { X10-2 M } & \text { BC } & \text { FOTOMTR SOLUBLZTN SDN } 4 & \text { TORI NAKA } 63008 & \text { T L } & \end{array}$

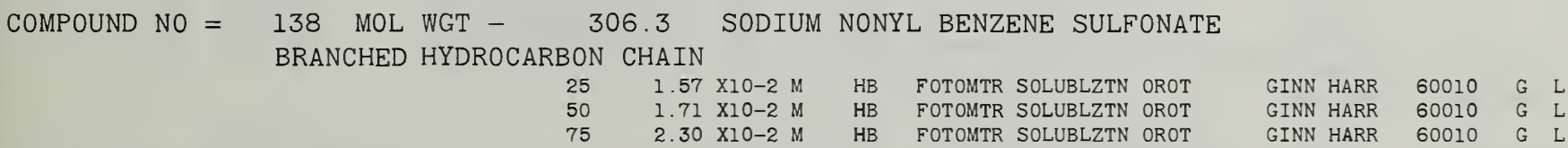

3 ENTRIES FOR COMPOUND

$2.30 \times 10-2 \mathrm{M}$ HB FOTOMTR SOLUBLZTN OROT

$\begin{array}{llll}\text { GINN HARR } & 60010 & \text { G } & \text { L } \\ \text { GINN HARR } & 60010 & \text { G } & \text { L }\end{array}$

COMPOUND NO $=139$ MOL WGT - 348.4 SODIUM DODECYL BENZENE SULFONATE BRANCHED HYDROCARBON CHAIN

C12 DIETHANOLAMIDE

C12 DIETHANOLAMIDE

CI2 DIETHANOLAMIDE

NA CL

$\mathrm{NA} \mathrm{CL}$

$\mathrm{NA} 2 \mathrm{CO3}$

$\mathrm{NA} 2 \mathrm{CO} 3$

$\mathrm{NA} \mathrm{OH}$

$\mathrm{NA} \mathrm{OH}$

NA4 P207 PYRO

NA4 P207 PYRO

NA P04

NA P04

NA14 P12037 POLY

NA14 P12037 POLY

NA53 P500154 POLY

NA2 SIO3 META

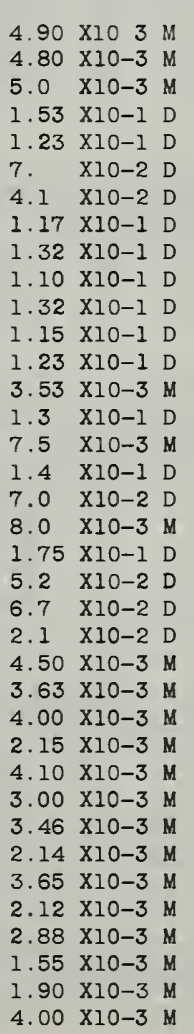

$4.90 \times 103 \mathrm{M}$

$\mathrm{HC}$

$\mathrm{HC}$

$\mathrm{HB}$

$\mathrm{HC}$

$\mathrm{HC}$

HG

HG

HE

$\mathrm{HG}$

HG

$\mathrm{HC}$

$\mathrm{HC}$

$\mathrm{HB}$

$\mathrm{HB}$

$\mathrm{HC}$

$\mathrm{HB}$

$\mathrm{HC}$

$\mathrm{HC}$

$\mathrm{HB}$

HE

HG

$\mathrm{HC}$

$\mathrm{HC}$

$\mathrm{HC}$

$\mathrm{HC}$

$\mathrm{HC}$

$\mathrm{HC}$

$\mathrm{HC}$

$\mathrm{HC}$

$\mathrm{HC}$

$\mathrm{HC}$

$\mathrm{HC}$
$\mathrm{HC}$

$\mathrm{HC}$

$\mathrm{HC}$

$\mathrm{HC}$
QUESTIONABLE CRITERION FOTOMTR SOLUBLZTN OROT FOTOMTR SOLUBLZTN OROT FOTOMTR SOLUBLZTN OROT SPECFC CONDCTNCE GRAPH SURFACE TENSION UNSPEC VISUAL SPCTR CHNGE PNCN FOTOMTR SPCTR CHNGE PNCN TETHOD NOT CITED

VISUAL SPCTR CHNGE PNCN FOTOMTR SPCTR CHNGE PNCN SPECFC CONDCTNCE GRAPH FUTOMTR SOLUBLZTN OROT SURFACE TENSION LOG PLOT SURFACE TENSION LOG PLOT FOTOMTR SOLUBLZTN OROT FOTOMTR SOLUBLZTN OROT FOTOMTR SOLUBLZTN OROT FOTOMTR SOLUBLZTN OROT FOTOMTR SOLUBLZTN OROT METHOD NOT CITED

FOTOMTR SPCTR CHNGE PNCN SPECFC CONDCTNCE GRAPH SURFACE TENSION UNSPEC FOTOMTR SOLUBLZTN OROT FOTOMTR SOLUBLZTN OROT FOTOMTR SOLUBLZTN OROT FOTOMTR SOLUBLZTN OROT FOTOMTR SOLUBLZTN OROT FOTOMTR SOLUBLZTN OROT FOTOMTR SOLUBLZTN OROT FOTOMTR SOLUBLZTN OROT FOTOMTR SOLUBLZTN OROT FOTOMTR SOLUBLZTN OROT FOTOMTR SOLUBLZTN OROT FOTOMTR SOLUBLZTN OROT FOTOMTR SOLUBLZTN OROT FOTOMTR SOLUBLZTN OROT
YANG FOST 53015 GINN KINN 59009 GINN HARR 60010 GINN HARR 58008 GINN HARR GINN HARR GINN HARR 58008 GINN HARR 61014 GINN HARR 58008 GINN HARR 58008 GINN HARR 58008 GINN KINN MANK

MANK

GINN KINN

GINN HARR GINN KINN GINN KINN GINN HARR GINN HARR GINN HARR GINN HARR GINN HARR GINN KINN GINN KINN GINN KINN GINN KINN GINN KINN GINN KINN GINN KINN GINN KINN GINN KINN GINN KINN GINN KINN GINN KINN GINN KINN GINN KINN 


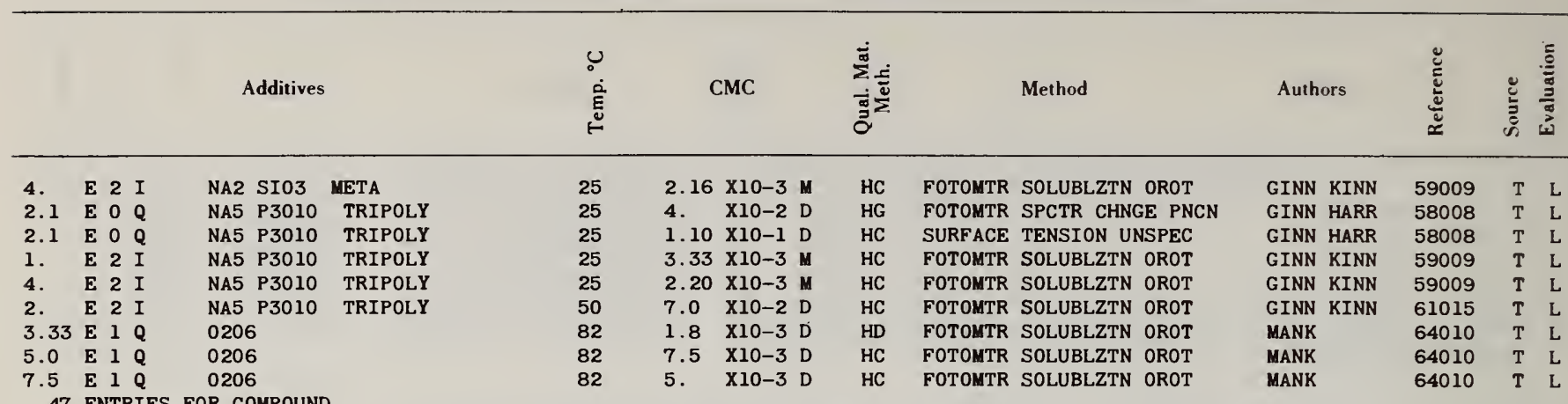
COMPOUND NO $=140$ MOL WGT - 320.4 SODIUM DECYL BENZENE SULFONATE BRANCHED HYDROCARBON CHAIN
$\begin{array}{llllll}25 & 4.1 & \mathrm{X} 10-3 \mathrm{M} & \text { HB } & \text { FOTOMTR SOLUBLZTN OROT } \\ 50 & 4.8 & \mathrm{X} 10-3 \mathrm{M} & \text { HB } & \text { FOTOMTR SOLUBLZTN OROT }\end{array}$

3 ENTRIES FOR COMPOUND
GINN HARR GINN HARR GINN HARR
60010

60010

60010
G L

G L

G L
GINN HARR GINN KINN GINN HARR GINN KINN GINN HARR GINN KINN GINN KINN
60010 G L 61015 T L 60010 G L 61015 T L 60010 G L 61015 T L 61015 T $\mathrm{L}$

2. E 2 I NA5 P3010 TRIPOLY

$3.1 \times 10-2 \mathrm{D}$

HC

FOTOMTR SOLUBLZTN OROT

7 ENTRIES FOR COMPOUND

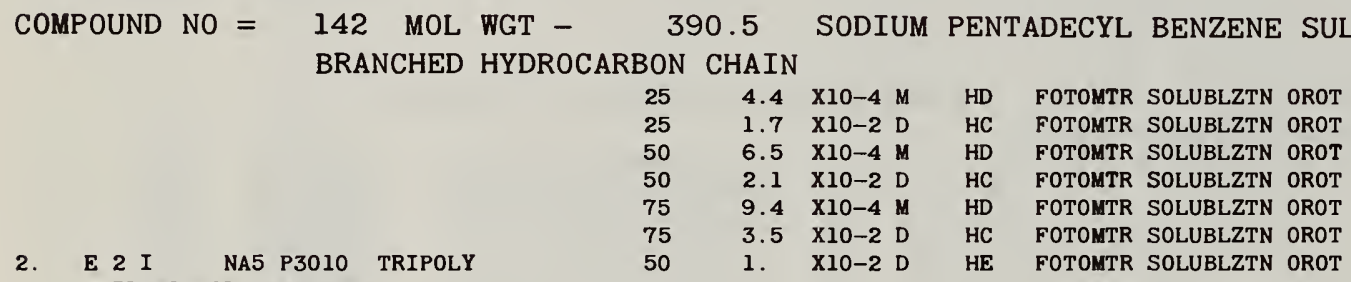

GINN HARR GINN KINN GINN HARR GINN KINN GINN HARR GINN KINN GINN KINN
60010 G L $61015 \mathrm{~T}$ 60010 G L 61015 T L 60010 G L 61015 T $\mathrm{L}$ 61015 T L

7 ENTRIES FOR COMPOUND

378.6 DECYL /OXYETHYLENE/ 5.0 ALCOHOL

COMPOUND NO $=$
143 MOL WGT -
BRANCHED CHAIN, NATURAL OE DISTRIBUTION

$\begin{array}{llllll}25 & 6.3 & \text { X10-2 } & \text { D } & \text { HB } & \text { FOTOMTR SOLUBLZTN OROT } \\ 50 & 6.0 & \text { X10-2 } & \text { D } & \text { HB } & \text { FOTOMTR SOLUBLZTN OROT }\end{array}$

$75 \quad 4.0 \quad$ X10-2 D HB FOTOMTR SOLUBLZTN OROT

GINN HARR GINN HARR GINN HARR
60010 60010

60010
3 ENTRIES FOR COMPOUND

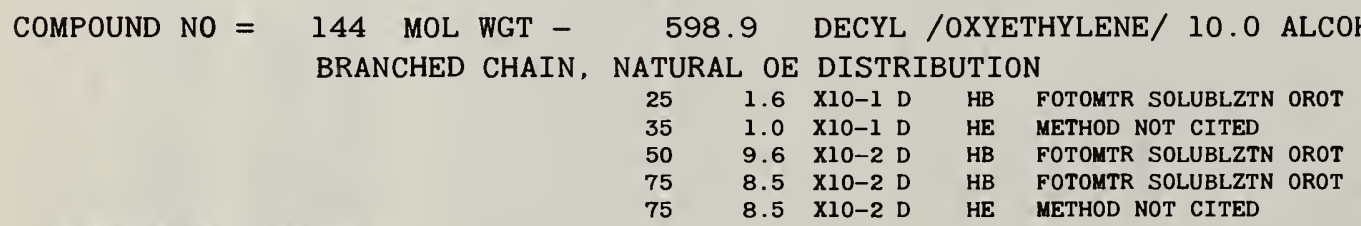

5 ENTRIES FOR COMPOUND

\section{COMPOUND NO $=145$ MOL WGT $-\quad 814.8$ DECYL /OXYETHYLENE/ 14.9 ALCOHOL BRANCHED CHAIN, NATURAL OE DISTRIBUTION}

$\begin{array}{lllllll}25 & 2.8 & \text { X10-1 } & \text { D } & \text { HB } & \text { FOTOMTR SOLUBLZTN OROT } \\ 50 & 1.44 & \text { X10-1 } & \text { D } & \text { HB } & \text { FOTOMTR SOLUBLZTN OROT } \\ 75 & 1.2 & \text { X10-1 } & \text { D } & \text { HB } & \text { FOTOMTR SOLUBLZTN OROT }\end{array}$

4 ENTRIES FOR COMPOUND
HB FOTOMTR SOLUBLZTN OROT

METHOD NOT CITED
GINN HARR GINN HARR GINN HARR GINN HARR
GINN HARR GINN HARR GINN HARR GINN HARR GINN HARR
60010 T L $61014 \mathrm{~T} \mathrm{~L}$ 60010 T L 60010 T L 61014 T L

\section{COMPOUND NO $=146$ MOL WGT $-1,039.5$ DECYL /OXYETHYLENE/ 20.0 ALCOHOL BRANCHED CHAIN, NATURAL OE DISTRIBUTION}

60010 T 60010 T $\mathrm{L}$ 60010 T $\mathrm{L}$ 61014 T L
Concentration units: $\mathrm{A}-$ mol \%; $\mathrm{B}-$ vol \% solvent; $\mathrm{C}-$ mol \% surfactant mixture; D-wt/vol \%; E-\% saturation; $\mathrm{H}-$ wt \% solvent; I-mol \% surfactant; $\mathrm{K}$ - normality counterions; $\mathrm{M}$ - molar; $\mathrm{N}$ - normal; $\mathrm{P}$ - wt \%; $\mathrm{Q}$ - wt \% surfactant; $\mathrm{R}$-varied; $\mathrm{S}$ - mol/

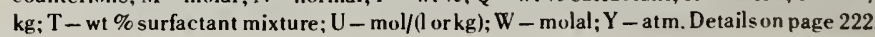




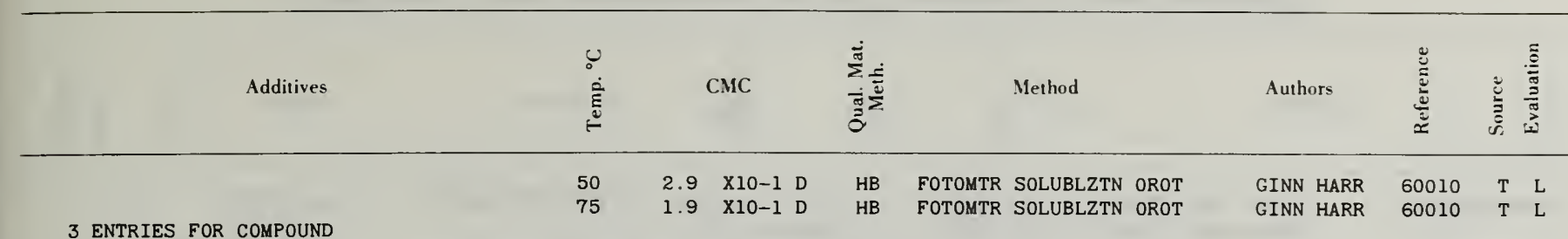

COMPOUND NO $=147$ MOL WGT $-1,453.6 \quad$ DECYL /OXYETHYLENE/ 29.4 ALCOHOL BRANCHED CHAIN, NATURAL OE DISTRIBUTION

3 ENTRIES FOR COMPOUND

$\begin{array}{llllllllll}25 & 8.3 & \text { X10-1 } & \text { D } & \text { HB } & \text { FOTOMTR SOLUBLZTN OROT } & \text { GINN HARR } & 60010 & \text { T } & \text { L } \\ 50 & 4.5 & \text { XIO-1 } & \text { D } & \text { HB } & \text { FOTOMTR SOLUBLZTN OROT } & \text { GINN HARR } & 60010 & \text { T } & \text { L } \\ 75 & 3.4 & \text { XIO-1 D } & \text { HB } & \text { FOTOMTR SOLUBLZTN OROT } & \text { GINN HARR } & 60010 & \text { T } & \text { L }\end{array}$

COMPOUND NO $=148$ MOL WGT -429.5 TRIDECYL /OXYETHYLENE/ 5.2 ALCOHOL BRANCHED CHAIN, NATURAL OE DISTRIBUTION

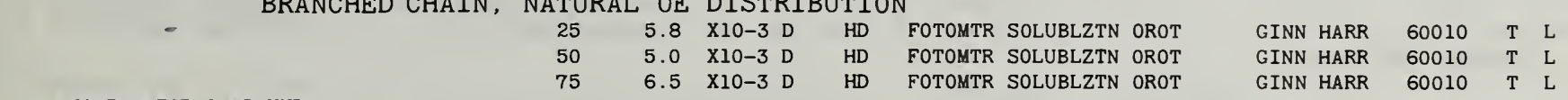

3 ENTRIES FOR COMPOUND $\begin{array}{lllll}5.0 & \times 10-3 & D & \text { HD } & \text { FOTOMTR SOLUBLZTN ORO } \\ 6.5 & \times 10-3 & \text { D } & \text { HD } & \text { FOTOMTR SOLUBLZTN OROT }\end{array}$ GINN HARR 60010 T L

\section{COMPOUND NO $=149$ MOL WGT - 645.4 TRIDECYL /OXYETHYLENE/ 10.1 ALCOHOL} BRANCHED CHAIN, NATURAL OE DISTRIBUTION

$\begin{array}{llllllllll}25 & 9 . & \text { X10-3 D } & \text { HD } & \text { FOTOMTR SOLUBLZTN OROT } & \text { GINN KINN } & 59009 & \text { T } & \text { L } \\ 25 & 1.0 & \text { X10-2 D } & \text { HC } & \text { FOTOMTR SOLUBLZTN OROT } & \text { GINN HARR } & 60010 & \text { T } & \text { L } \\ 25 & 9.2 & \text { X10-3 } & \text { D } & \text { HE } & \text { METHOD NOT CITED } & \text { GINN HARR } & 61014 & \text { T } & \text { L } \\ 25 & 9.2 & \text { X10-3 D } & \text { HC } & \text { FOTOMTR SOLUBLZTN OROT } & \text { GINN KINN } & 61015 & \text { T } & \text { L } \\ 50 & 7.8 & \text { X10-3 D } & \text { HC } & \text { FOTOMTR SOLUBLZTN OROT } & \text { GINN HARR } & 60010 & \text { T } & \text { L } \\ 50 & 7.8 & \text { X10-3 D } & \text { HC } & \text { FOTOMTR SOLUBLZTN OROT } & \text { GINN KINN } & 61015 & \text { T } & \text { L } \\ 75 & 7.6 & \text { X10-3 D } & \text { HC } & \text { FOTOMTR SOLUBLZTN OROT } & \text { GINN HARR } & 60010 & \text { T } & \text { L } \\ 75 & 7.6 & \text { X10-3 D } & \text { HE } & \text { METHOD NOT CITED } & \text { GINN HARR } & 61014 & \text { T } & \text { L } \\ 75 & 7.57 & \text { X10-3 D } & \text { HC } & \text { FOTOMTR SOLUBLZTN OROT } & \text { GINN KINN } & 61015 & \text { T } & \text { L } \\ 25 & 9 . & \text { X10-3 D } & \text { HD } & \text { FOTOMTR SOLUBLZTN OROT } & \text { GINN KINN } & 59009 & \text { T } & \text { L }\end{array}$

4. E 2 I NA4 P207 PYRO 10 ENTRIES FOR COMPOUND

COMPOUND NO $=150$ MOL WGT BRANCHED CHAIN, NATURAL OE DISTRIBUTION

870.1 TRIDECYL /OXYETHYLENE/ 15.2 ALCOHOL $\begin{array}{llllll}25 & 2.3 & \times 10-2 & \mathrm{D} & \text { HC } & \text { FOTOMTR SOLUBLZTN OROT } \\ 50 & 1.5 & \times 10-2 & \mathrm{D} & \text { HC } & \text { FOTOMTR SOLUBLZTN OROT }\end{array}$

3 ENTRIES FOR COMPOUND

$\begin{array}{lllll}1.5 & \times 10-2 & D & \text { HC } & \text { FOTOMTR SOLUBLZTN OROT } \\ 1.0 & \times 10-2 & D & \text { HC } & \text { FOTOMTR SOLUBLZTN OROT }\end{array}$

GINN HARR GINN HARR GINN HARR
60010 60010 60010
T $\quad$ L T L T L

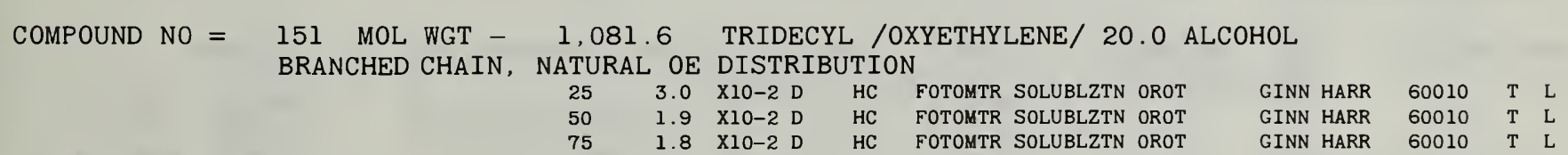

3 ENTRIES FOR COMPOUND

3 ENTRIES FOR COMPOUND

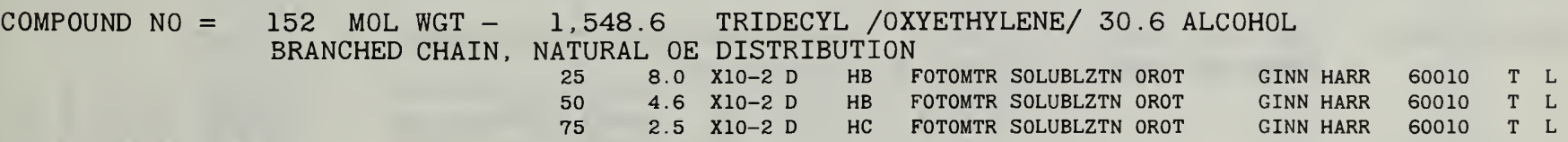

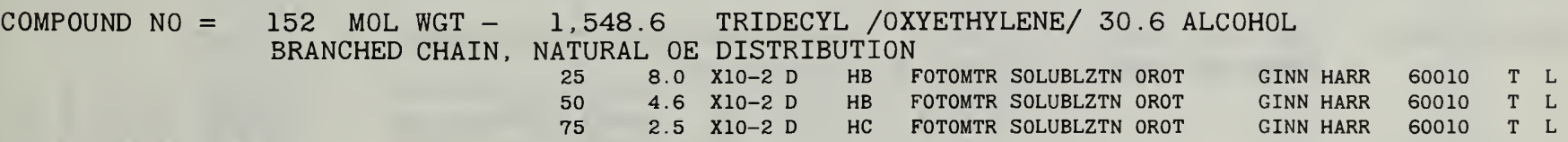

COMPOUND NO $=153$ MOL WGT - 440.6 NONYL BENZENE /OXYETHYLENE/ 5.0 ALCOHOL BRANCHED CHAIN, NATURAL OE DISTRIBUTION

$\begin{array}{llllllllll}25 & 2.5 & \text { X10-3 } & \text { D } & \text { HD } & \text { FOTOMTR SOLUBLZTN OROT } & \text { GINN HARR } & 60010 & \text { T } & \text { L } \\ 50 & 1.0 & \text { X10-3 } & \text { D } & \text { HD } & \text { FOTOMTR SOLUBLZTN OROT } & \text { GINN HARR } & 60010 & \text { T } & \text { L } \\ 75 & 1.9 & \text { X10-3 } & \text { D } & \text { HD } & \text { FOTOMTR SOLUBLZTN OROT } & \text { GINN HARR } & 60010 & \text { T } & \text { L }\end{array}$

3 ENTRIES FOR COMPOUND

$1.0 \times 10-3 \mathrm{D}$ HD FOTOMTR SOLUBLZTN OROT

$75 \quad 1.9 \times 10-3 \quad D$

652.1 NONYL BENZENE /OXYETHYLENE/ 9.8 ALCOHOL

COMPOUND NO $=154$ MOL WGT BRANCHED CHAIN, NATURAL OE DISTRIBUTION

$\begin{array}{llllllllll}25 & 4.0 & \text { X10-3 } & \text { D } & \text { HD } & \text { FOTOMTR SOLUBLZTN OROT } & \text { GINN HARR } & 60010 & \text { T } & \text { L } \\ 50 & 4.3 & \text { X10-3 } & \text { D } & \text { HD } & \text { FOTOMTR SOLUBLZTN OROT } & \text { GINN HARR } & 60010 & \text { T } & \text { L } \\ 75 & 4.2 & \text { X10-3 } & \text { D } & \text { HD } & \text { FOTOMTR SOLUBLZTN OROT } & \text { GINN HARR } & 60010 & \text { T } & \text { L }\end{array}$

3 ENTRIES FOR COMPOUND

GINN HARR

60010 T $L$

Concentration units: $\mathrm{A}-$ mol $\%$; $\mathrm{B}-\mathrm{vol} \%$ solvent; $\mathrm{C}-$ mol $\%$ surfactant mixture; $\mathrm{D}-$ wt $/$ vol \%; E-\% saturation; $\mathrm{H}-$ wt \% solvent; I - mol \% surfactant; $\mathrm{K}-$ normality counterions; $\mathrm{M}$-molar; N-normal; P-wt \%; Q-wt \% surfactant: $\mathrm{R}$-varied; $\mathrm{S}$ - mol/ kg: $\mathrm{T}-\mathrm{wt} \%$ surfactant mixture; $\mathrm{U}-\mathrm{mol} /(\mathrm{l}$ or kg); $\mathrm{W}$ - molal; $\mathrm{Y}$ - atm. Details on page 222. 


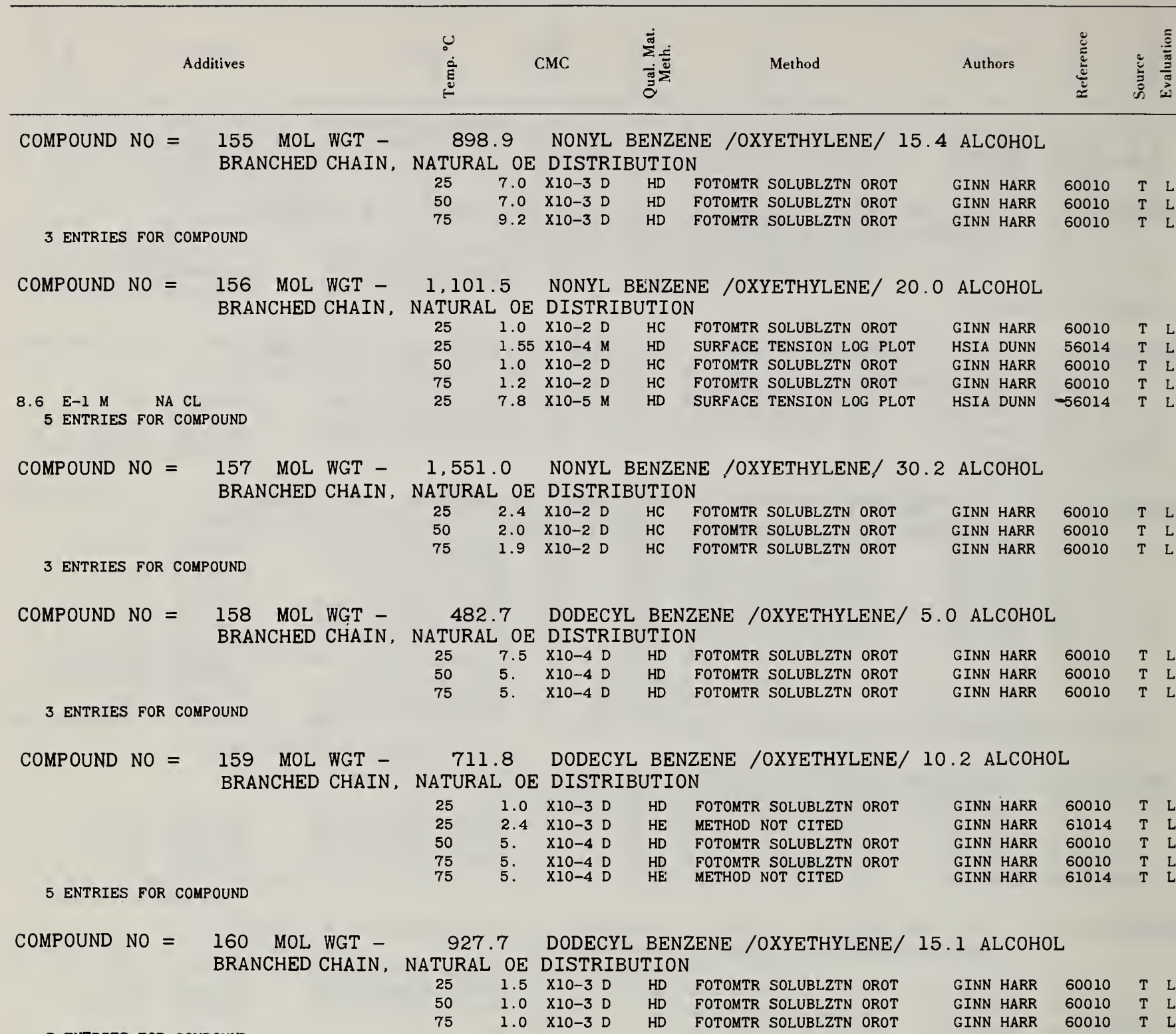

3 ENTRIES FOR COMPOUND

$$
\begin{aligned}
& 1.0 \times 10-2 \mathrm{D} \\
& 1.55 \times 10-4 \mathrm{M} \\
& 1.0 \times 10-2 \mathrm{D} \\
& 1.2 \times 10-2 D
\end{aligned}
$$

HD

$\mathrm{HC}$ FOTOMTR SOLUBLZTN OROT GINN HARR GINN HARR

5 ENTRIES FOR COMPOUND $\begin{array}{lr}157 \text { MOL WGT }- & 1,551 \\ \text { BRANCHED CHAIN, NATURAL }\end{array}$

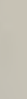

t




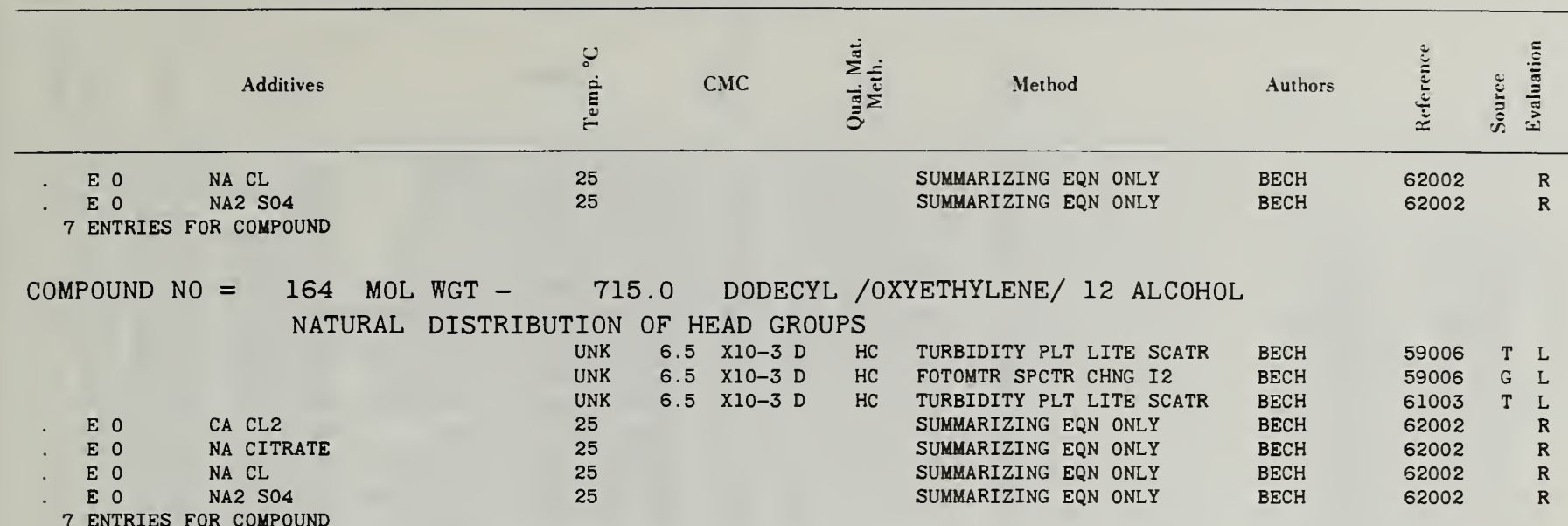

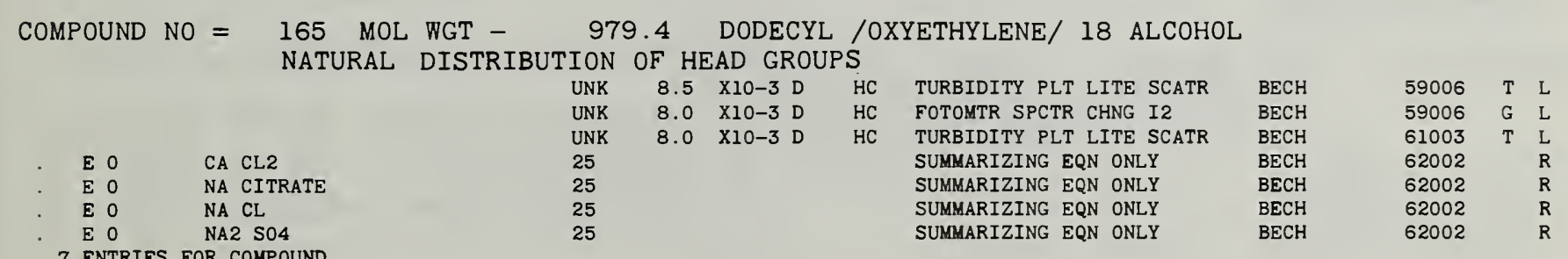

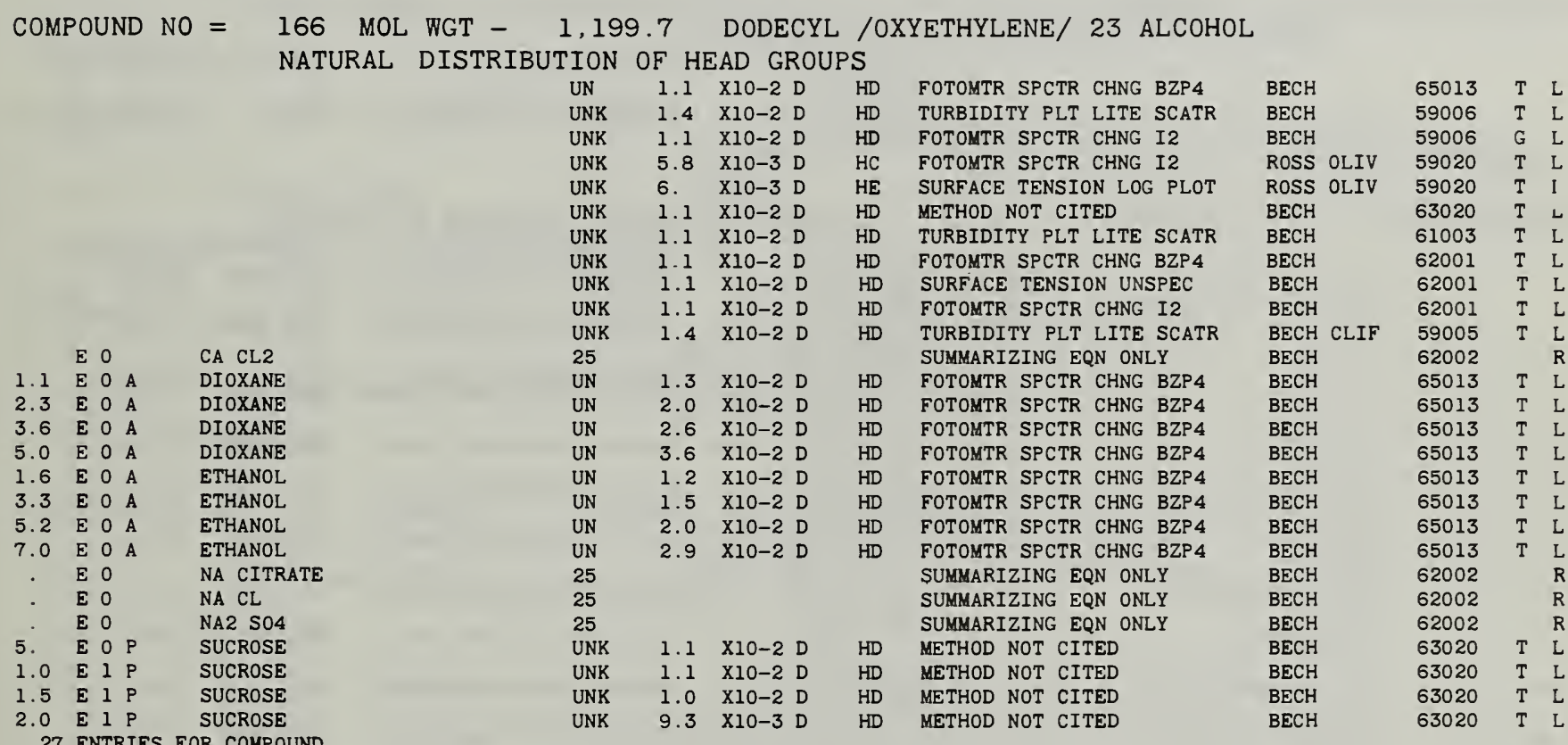

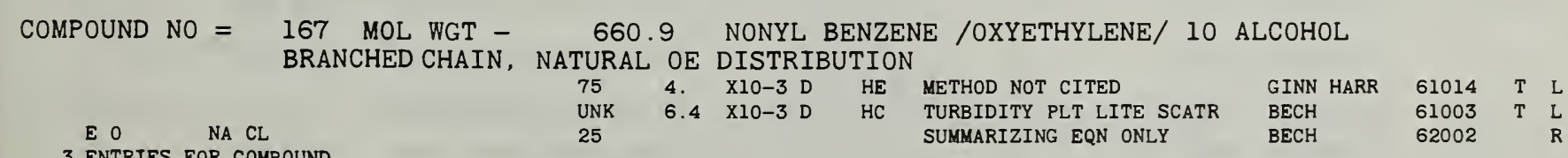

3 ENTRIES FOR COMPOUND

UNK $6.4 \times 10-3 \mathrm{D}$ HC
881.2 NONYL BENZENE /OXYETHYLENE/ 15 ALCOHOL

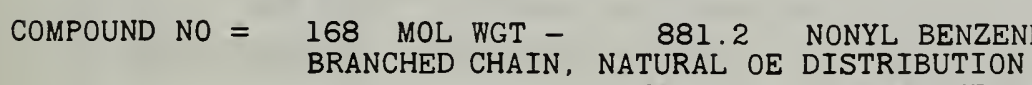

$\begin{array}{lllllllll}25 & 1.2 & \text { X10-4 M } & \text { HD } & \text { SURFACE TENSION LOG PLOT } & \text { HSIA DUNN } & 56014 & \text { T } & \text { L } \\ 25 & 8.3 & \text { X10-3 D } & \text { HC } & \text { TURBIDITY PLT LITE SCATR } & \text { BECH } & 62002 & \text { T } & \text { L } \\ 25 & 7.7 & \text { X10-3 D } & \text { HC } & \text { FOTOMTR SPCTR CHNG I2 } & \text { BECH } & 62002 & \text { T } & \text { L } \\ 25 & 8.1 & \text { X10-3 D } & \text { HC } & \text { SURFACE TENSION UNSPEC } & \text { BECH } & 62002 & \text { T } & \text { L } \\ \text { UNK } & 7.7 & \text { X10-3 D } & \text { HC } & \text { TURBIDITY PLT LITE SCATR } & \text { BECH } & 61003 & \text { T } & \text { L }\end{array}$

Concentration units: $\mathrm{A}-\mathrm{mol} \% ; \mathrm{B}-\mathrm{vol} \%$ solvent; $\mathrm{C}-\mathrm{mol} \%$ surfactant mixture; D-wt/vol \%; E-\% saturation; $\mathrm{H}-$ wt \% solvent; $\mathrm{I}-$ mol \% surfactant; $\mathrm{K}-$ normality counterions; $\mathrm{M}-$ molar; $\mathrm{N}$ - normal; $\mathrm{P}-$ wt \%; $\mathrm{Q}-$ wt \% surfactant; $\mathrm{R}$-varied; $\mathrm{S}-$ mol/ kg; $\mathrm{T}$ - wt \% surfactant mixture; $\mathrm{U}-\mathrm{mol} /(\mathrm{l}$ or $\mathrm{kg}) ; \mathrm{W}$ - molal; $\mathrm{Y}$ - atm. Details on page 222. 


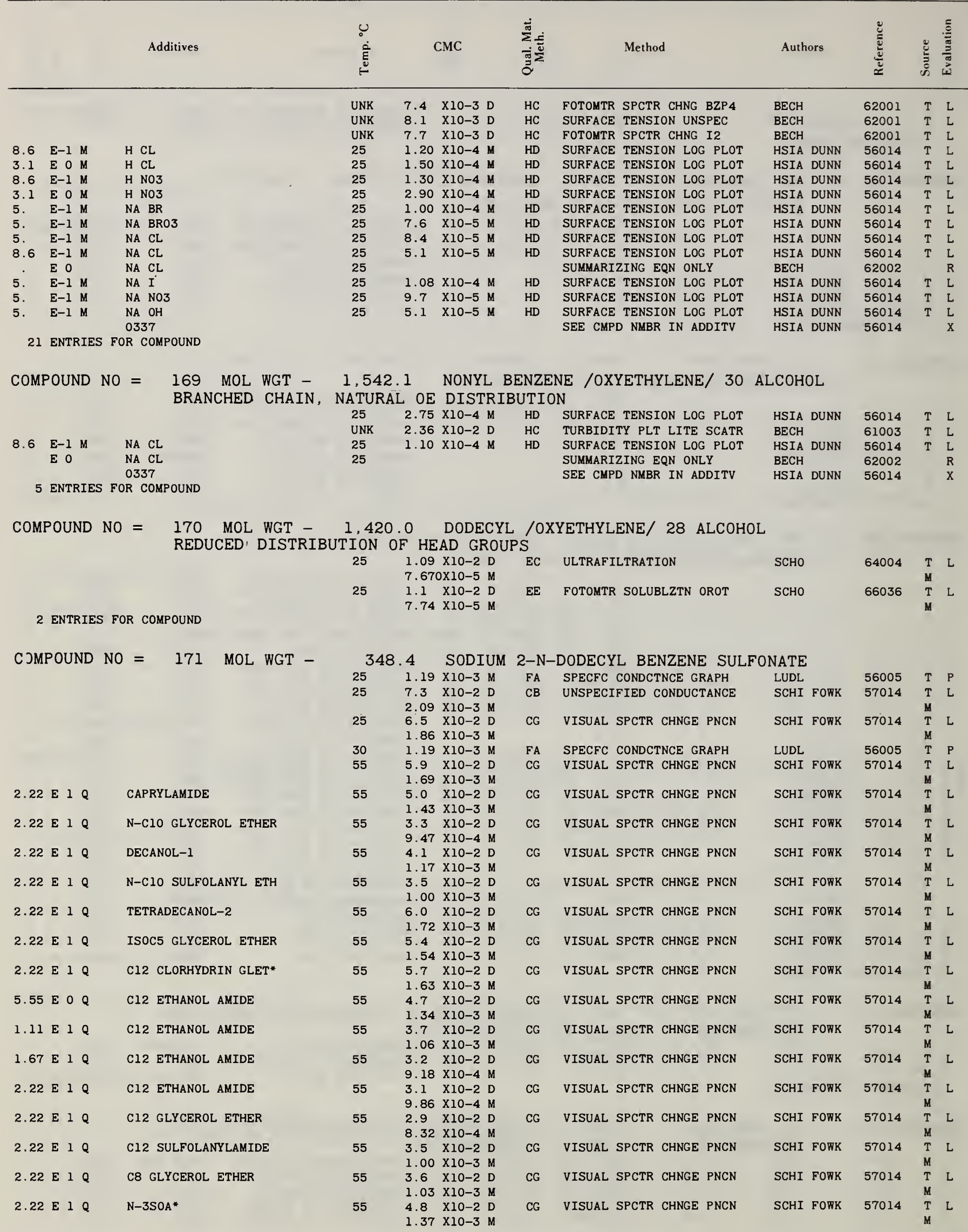

Concentration units: $\mathrm{A}-\mathrm{mol} \% ; \mathrm{B}-\mathrm{vol} \%$ solvent; $\mathrm{C}-$ mol \% surfactant mixture; D-wt/vol \%; E-\% saturation; $\mathrm{H}-$ wt $\%$ solvent; $\mathrm{I}-$ mol \% surfactant; $\mathrm{K}-$ normality counterions; $\mathrm{M}$-molar; $\mathrm{N}$-normal; $\mathrm{P}$ - wt \%; $\mathrm{Q}-$ wt \% surfactant: $\mathrm{R}$-varied: $\mathrm{S}$ - mol/ kg: T - wt \% surfactant mixture; $\mathrm{U}-\mathrm{mol} /(\mathrm{l}$ or kg); $\mathrm{W}-$ molal; $\mathrm{Y}-\mathrm{atm}$. Details on page 222. 


\section{Additives}

CMC

\begin{tabular}{|c|c|c|c|c|c|c|}
\hline 2.22 & E $1 Q$ & & TMCH & HCGLET & & \\
\hline 2.0 & $E \perp Q$ & & NA2 & S04 & & \\
\hline 2.0 & $\begin{array}{l}E 11 \\
5.1\end{array}$ & 0 & $Q^{\text {NA2 }}$ & $\begin{array}{l}\mathrm{SO4} \\
\mathrm{Cl} 2\end{array}$ & ETHANOL & AMIDE \\
\hline 2.0 & $\begin{array}{l}E 1 Q \\
8.8\end{array}$ & & $Q^{\text {NA2 }}$ & $\begin{array}{l}\mathrm{SO} 4 \\
\mathrm{C} 12\end{array}$ & ETHANOL & AMIDE \\
\hline 2.0 & $\begin{array}{l}E 1 Q \\
3.52 \mathrm{E}\end{array}$ & & $Q^{\text {NA2 }}$ & $\begin{array}{l}\mathrm{S} 04 \\
\mathrm{C} 12\end{array}$ & & AMIDE \\
\hline 8.0 & $\begin{array}{l}E 1 Q \\
4.4 \quad E\end{array}$ & 0 & $Q^{\text {NA2 }}$ & $\begin{array}{l}\mathrm{S} 04 \\
\mathrm{C} 12\end{array}$ & ETHANOL & AMIDE \\
\hline 8.0 & $\begin{array}{l}E 1 Q \\
8.8\end{array}$ & 0 & $Q^{\text {NA2 }}$ & $\begin{array}{l}\mathrm{SO} 4 \\
\mathrm{C} 12\end{array}$ & ETHANOL & AMIDE \\
\hline 8.0 & $\begin{array}{l}E 1 Q Q \\
1.76 \mathrm{E}\end{array}$ & 1 & $Q^{\text {NA2 }}$ & $\begin{array}{l}\mathrm{SO} 4 \\
\mathrm{C} 12\end{array}$ & ETHANOL & AMIDE \\
\hline 8.0 & $\begin{array}{l}E 1 Q Q \\
3.52 \mathrm{E}\end{array}$ & & $Q^{\text {NA2 }}$ & $\begin{array}{l}\mathrm{SO} 4 \\
\mathrm{Cl} 2\end{array}$ & ETHANOL & AMID \\
\hline
\end{tabular}

29 ENTRIES FOR COMPOUND
2.22 E 1 Q N-C10 GLYCEROL ETHER

2.22 E $1 Q$ C12 ETHANOL AMIDE

2. 22 E $1 Q$ C8 GLYCEROL ETHER

4 ENTRIES FOR COMPOUND

COMPOUND NO $=173$ MOL WGT -

2.22 E $1 Q$ C12 ETHANOL AMIDE

2 ENTRIES FOR COMPOUND

COMPOUND NO $=174$ MOL WGT -

2.22 E $1 Q$ C12 ETHANOL AMIDE

2 ENTRIES FOR COMPOUND

COMPOUND NO $=175$ MOL WGT -

$2.22 \dot{E} 1 Q$ N-C1O GLYCEROL ETHEER

2 ENTRIES FOR COMPOUND

COMPOUND NO $=176$ MOL WGT -

2.22 E $1 Q$ N-C1O GLYCEROL ETHER

2 ENTRIES FOR COMPOUND

COMPOUND NO $=177$ MOL WGT -

2.22 E $1 \mathrm{Q}$ N-C1O GLYCEROL ETHER

2 ENTRIES FOR COMPOUND

COMPOUND NO $=178$ MOL WGT -
Method

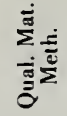

55

$14 \times 10-3 \mathrm{M}$

$3.6 \times 10-2 \mathrm{D}$

$1.14 \times 10-3 \mathrm{M}$

$2.8 \times 10-2 \mathrm{D}$

$8.03 \times 10-4 \mathrm{M}$

$3.4 \times 10-2 \mathrm{D}$

$9.75 \times 10-4 \mathrm{M}$

$3.1 \times 10-2 \mathrm{D}$

$8.89 \times 10-4 \mathrm{M}$

$2.8 \times 10-2 \mathrm{D}$

$8.03 \times 10-4 \mathrm{M}$

$2.7 \times 10-2 \mathrm{D}$

$7.74 \times 10-4 \mathrm{M}$
CG

CG

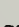

CG

CG

CG

CG

CG

CG
VISUAL SPCTR CHNGE PNCN

ISUAL SPCTR CHNGE PNCN

VISUAL SPCTR CHNGE PNCN

VISUAL SPCTR CHNGE PNCN

VISUAL SPCTR CHNGE PNCN

VISUAL SPCTR CHNGE PNCN

VISUAL SPCTR CHNGE PNCN

VISUAL SPCTR CHNGE PNCN

VISUAL SPCTR CHNGE PNCN
SCHI FOWK $57014 \quad \mathrm{~T}$ L

SCHI FOWK $57014 \stackrel{\text { T }}{\mathrm{T}}$

SCHI FOWK 57014 T L

SCHI FOWK 57014 T L

SCHI FOWK

SCHI FOWK

SCHI FOWK

SCHI FOWK
SCHI FOWK 57014 T L

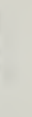

292.3 SODIUM 2-N-OCTYL BENZENE SULFONATE

\begin{tabular}{|c|c|c|c|c|c|c|c|c|}
\hline $\begin{array}{l}5.56 \times 10-1 \mathrm{D} \\
1.902 \times 10-2 \mathrm{M}\end{array}$ & CG & VISUAL & SPCTR & CHNGE & PNCN & $\mathrm{SCHI}$ & FOWK & 57014 \\
\hline $\begin{array}{l}3.47 \times 10-1 \mathrm{D} \\
1.187 \times 10-2 \mathrm{M}\end{array}$ & CG & VISUAL & SPCTR & CHNGE & PNCN & SCHI & FOTK & 57014 \\
\hline $\begin{array}{l}3.43 \times 10-1 \mathrm{D} \\
1.173 \times 10-2 \mathrm{M}\end{array}$ & CG & VISUAL & SPCTR & CHNGE & PNCN & $\mathrm{SCHI}$ & FOWK & 57014 \\
\hline $\begin{array}{l}3.33 \times 10-1 \mathrm{D} \\
1.139 \times 10-2 \mathrm{M}\end{array}$ & CG & VISUAL & SPCTR & CHNGE & PNCN & $\mathrm{SCHI}$ & FOWK & 57014 \\
\hline
\end{tabular}

320.4 SODIUM 2-N-DECYL BENZENE SULFONATE

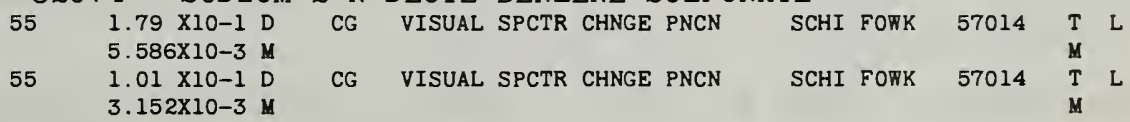

376.5 SODIUM 2-N-TETRADECYL BENZENE SULFONATE

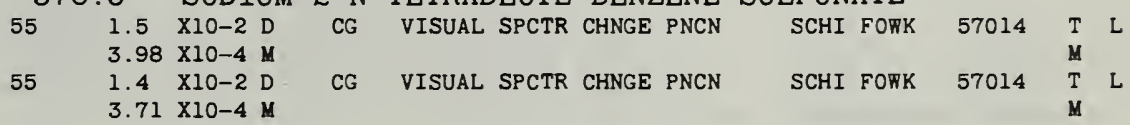

272.3 SODIUM DODECANE 2-SULFONATE

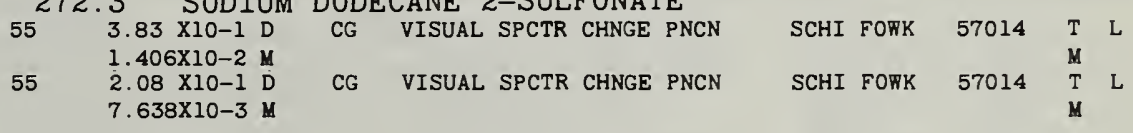

300.4 SODIUM TETRADECANE 2-SULFONATE

$\begin{array}{llllllllll}55 & 1.13 \times 10-1 & \text { D } & \text { CG } & \text { VISUAL SPCTR CHNGE PNCN } & \text { SCHI F.TKK } & 57014 & \text { T } & \text { L } \\ 55 & 3.761 \times 10-3 & \text { M } & & & & & & \text { M } & \\ 5 & 6.9 \times 10-2 & \text { D } \\ 5 & 2.29 \times 10-3 & \text { CG } & \text { VISUAL SPCTR CHNGE PNCN } & \text { SCHI } & \text { TK } & 57014 & \text { T } & \text { L }\end{array}$

328.4 SODIUM HEXADECANE 2-SULFONATE

$\begin{array}{llllll}55 & 3.5 & \text { X10-2 } & \text { D } & \text { CG } & \text { VISUAL SPCTR CHNGE PNCN } \\ 55 & 1.06 & \text { X10-3 } & \text { M } & & \\ 5 & 2.2 & \text { X10-2 } & \text { D } \\ & 6.69 & \text { X CG } & \text { CG } & \text { MISUAL SPCTR CHNGE PNCN }\end{array}$

$\begin{array}{llll}\text { SCHI FONK } & 57014 & \text { T } & \text { L } \\ & & \text { M } & \\ \text { SCHI FONK } & 57014 & \text { T } & \text { L } \\ & & \text { M } & \end{array}$

356.5 SODIUM OCTADECANE 2-SULFONATE

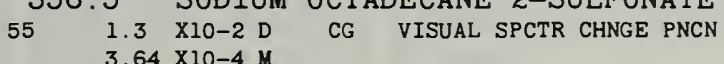

SCHI FOWK $57014 \quad$ T $\quad$ L
Concentration units: $\mathrm{A}-\mathrm{mol} \%$; $\mathrm{B}-$ vol \% solvent; $\mathrm{C}-$ mol \% surfactant mixture; D-wt/vol \%; E-\% saturation; $\mathrm{H}-$ wt $\%$ solvent; I-mol \% surfactant; $\mathrm{K}$ - normality counterions; $\mathrm{M}$-molar; $\mathrm{N}$-normal; $\mathrm{P}$ - wt \%; $\mathrm{Q}$ - wt \% surfactant; $\mathrm{R}$-varied; $\mathrm{S}-\mathrm{mol} /$ kg; T - wt \% surfactant mixture; $\mathrm{U}-\mathrm{mol} /(\mathrm{l}$ or kg); $\mathrm{W}$ - molal; $\mathrm{Y}-\mathrm{atm}$. Details on page 222. 


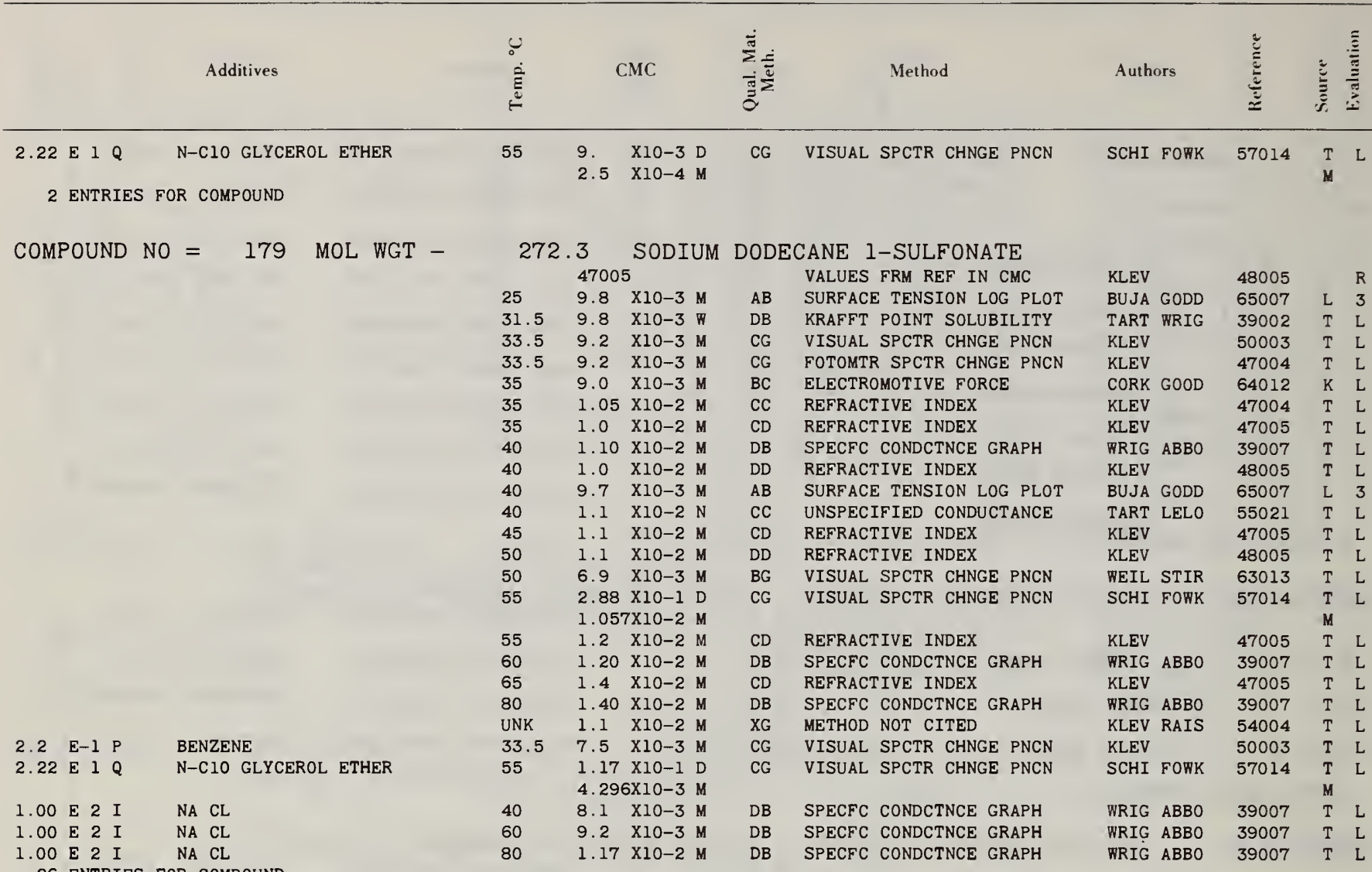

COMPOUND NO $=181$ MOL WGT -

7 ENTRIES FOR COMPOUND

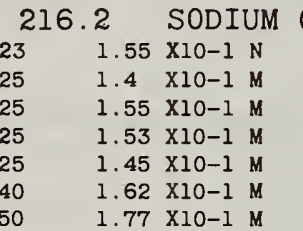

$\begin{array}{ll}\text { OCTYL } & \text { 1-SULFONATE } \\ \text { CB } & \text { UNSPECIFIED CONDUCTANCE } \\ \text { CG } & \text { VISUAL SPCTR CHNGE PNCN } \\ \text { CC } & \text { REFRACTIVE INDEX } \\ \text { CC } & \text { REFRACTIVE INDEX } \\ \text { CG } & \text { FOTOMTR SPCTR CHNGE PNCN } \\ \text { CC } & \text { REFRACTIVE INDEX } \\ \text { CC } & \text { REFRACTIVE INDEX }\end{array}$

TART LELO

LELO TART

KLEV

KLEV

KLEV

KLEV

KLEV

$\begin{array}{lll}55021 & \text { T } & 3 \\ 51003 & \text { L } & \text { L } \\ 48005 & \text { T } & 3 \\ 47004 & \text { T } & \text { L } \\ 47004 & \text { T } & \text { L } \\ 48005 & \text { T } & 3 \\ 48005 & \text { T } & \text { L }\end{array}$

244.3 SODIUM DECYL 1-SULFONATE

\begin{tabular}{|c|c|c|}
\hline & 47005 & \\
\hline 22.5 & 4.0 & $\mathrm{X} 10-2 \mathrm{~W}$ \\
\hline 25 & 3.8 & $\mathrm{X} 10-2 \mathrm{M}$ \\
\hline 25 & 4.2 & $\times 10-2 \mathrm{M}$ \\
\hline 25 & 4.1 & $\times 10-2 \mathrm{~N}$ \\
\hline 25 & 3.8 & $\times 10-2$ \\
\hline 26 & 4.00 & $\times 10-2 \mathrm{~N}$ \\
\hline 26 & 3.87 & $\times 10-2$ \\
\hline 26 & 4.00 & $\times 10-2 \mathrm{~K}$ \\
\hline 30 & 3.8 & $\mathrm{X} 10-2 \mathrm{~N}$ \\
\hline 30 & $\begin{array}{l}1.066 \\
4.363\end{array}$ & $\begin{array}{l}6 \times 100 \\
3 \times 10-2\end{array}$ \\
\hline 35 & 4.2 & $\mathrm{X} 10-2 \mathrm{~N}$ \\
\hline 40 & 4.0 & $\times 10-2$ \\
\hline 40 & 4.1 & $\times 10-2$ \\
\hline 45 & 4.5 & $\times 10-2$ \\
\hline 50 & 4.5 & $\mathrm{x} 10-2$ \\
\hline 55 & 4.9 & $\times 10-2$ \\
\hline 60 & 4.3 & $\mathrm{x} 10-2$ \\
\hline 65 & 5.5 & $\times 10-2$ \\
\hline 80 & 5.8 & $\mathrm{X} 10-2$ \\
\hline UNK & 4.00 & $x 10-2$ \\
\hline 25 & 3.4 & $\times 10-2$ \\
\hline UNK & 3.81 & $\times 10-2$ \\
\hline UNK & 3.86 & $x 10-2$ \\
\hline UNK & 3.89 & $\times 10-2$ \\
\hline
\end{tabular}

$\begin{array}{llll}\text { KLEV } & 48005 & & \text { R } \\ \text { CORR HARK } & 46005 & & \text { R } \\ \text { TART WRIG } & 39002 & \text { T } & \text { L } \\ \text { KLEV } & 50003 & \text { T } & \text { L } \\ \text { KLEV } & 47004 & \text { T } & \text { L } \\ \text { KLEV } & 47005 & \text { T } & \text { L } \\ \text { KLEV } & 47004 & \text { T } & \text { L } \\ \text { CORR HARK } & 47006 & \text { T } & \text { L } \\ \text { CORR HARK } & 47006 & \text { T } & \text { L } \\ \text { CORR HARK } & 47010 & \text { T } & \text { L } \\ \text { TART LELO } & 55021 & \text { T } & \text { L } \\ \text { SCHO } & 66036 & \text { T } & 2 \\ & & \text { M } & \\ \text { KLEV } & 47005 & \text { T } & \text { L } \\ \text { WRIG ABBO } & 39007 & \text { T } & \text { L } \\ \text { KLEV } & 48005 & \text { T } & \text { L } \\ \text { KLEV } & 47005 & \text { T } & \text { L } \\ \text { KLEV } & 48005 & \text { T } & \text { L } \\ \text { KLEV } & 47005 & \text { T } & \text { L } \\ \text { WRIG ABBO } & 39007 & \text { T } & \text { L } \\ \text { KLEV } & 47005 & \text { T } & \text { L } \\ \text { WRIG ABBO } & 39007 & \text { T } & \text { L } \\ \text { HARK MITT } & 49006 & \text { T } & \text { L } \\ \text { KLEV } & 50003 & \text { T } & \text { L } \\ \text { HARK MITT } & 49006 & \text { T } & \text { L } \\ \text { HARK MITT } & 49006 & \text { T } & \text { L } \\ \text { HARK MITT } & 49006 & \text { T } & \text { L }\end{array}$

$\begin{array}{llll}2.2 & E-1 & P & \text { BENZENE } \\ 3.40 & \text { E-2 } & P & \text { BENZENE } \\ 5.50 & \text { E-2 } & P & \text { BENZENE } \\ 8.9 & \text { E-2 } & P & \text { BENZENE }\end{array}$

82 MOL WGT -
Concentration units: $\mathrm{A}-\mathrm{mol} \%$; $\mathrm{B}-\mathrm{vol} \%$ solvent; $\mathrm{C}-$ mol \% surfactant mixture; D-wt/vol \%; E-\% saturation; $\mathrm{H}-$ wt \% solvent; I-mol \% surfactant; $\mathrm{K}-$ normality counterions; $\mathrm{M}$ - molar; $\mathrm{N}$ - normal; $\mathrm{P}$ - wt \%; Q-wt \% surfactant; R-varied; $\mathrm{S}-$ mol/ $\mathrm{kg} ; \mathrm{T}-\mathrm{wt} \%$ surfactant mixture; $\mathrm{U}-\mathrm{mol} /(\mathrm{l}$ or $\mathrm{kg}) ; \mathrm{W}-$ molal; $\mathrm{Y}-\mathrm{atm}$. Details on page 222. 


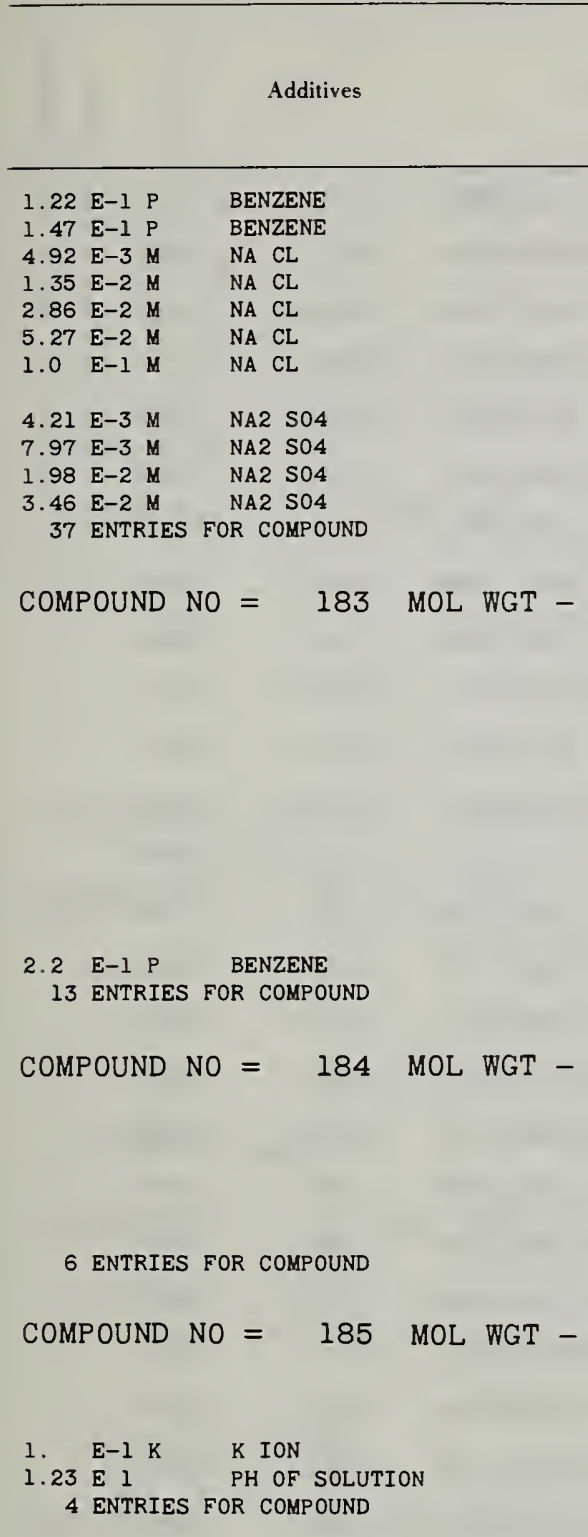

COMPOUND NO $=186$ MOL WGT -

4 ENTRIES FOR COMPOUND

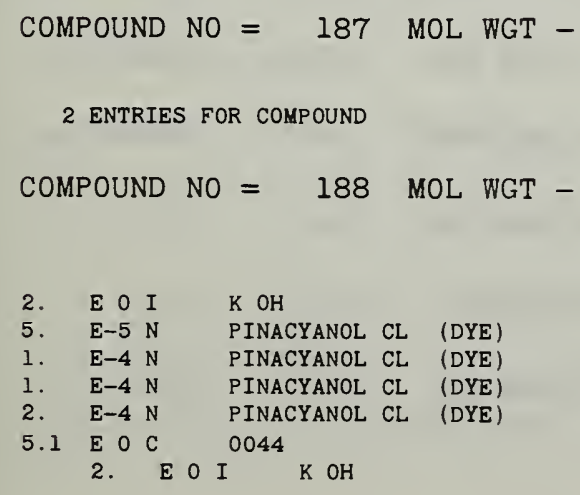

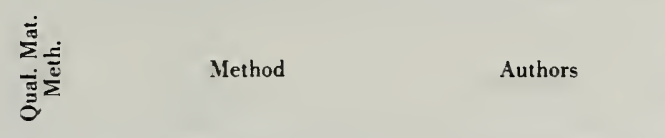

$\begin{array}{llll}\text { UNK } & 3.82 \times 10-2 \mathrm{M} & \text { CG } & \text { METHOD NOT CITED } \\ \text { UNK } & 3.85 \times 10-2 \mathrm{M} & \text { CG } & \text { METHOD NOT CITED } \\ 26 & 3.82 \times 10-2 \mathrm{M} & \text { CG } & \text { VISUAL SPCTR CHNGE PNCN } \\ 26 & 3.50 \times 10-2 \mathrm{M} & \text { CG } & \text { VISUAL SPCTR CHNGE PNCN } \\ 26 & 3.17 \times 10-2 \mathrm{M} & \text { CG } & \text { VISUAL SPCTR CHNGE PNCN } \\ 26 & 2.93 \times 10-2 \mathrm{M} & \text { CG } & \text { VISUAL SPCTR CHNGE PNCN } \\ 30 & 5.364 \times 10-1 \mathrm{D} & \mathrm{BA} & \text { FOTOMTR SOLUBLZTN OROT } \\ & 2.195 \times 10-2 \mathrm{M} & & \\ 26 & 3.72 \times 10-2 \mathrm{M} & \mathrm{CG} & \text { VISUAL SPCTR CHNGE PNCN } \\ 26 & 3.52 \times 10-2 \mathrm{M} & \text { CG } & \text { VISUAL SPCTR CHNGE PNCN } \\ 26 & 3.12 \times 10-2 \mathrm{M} & \text { CG } & \text { VISUAL SPCTR CHNGE PNCN } \\ 26 & 2.73 \times 10-2 \mathrm{M} & \text { CG } & \text { VISUAL SPCTR CHNGE PNCN }\end{array}$

HARK MITT HARK MITT

CORR HARK

CORR HARK

CORR HARK

CORR HARK

$\mathrm{SCHO}$

CORR HARK CORR HARK

CORR HARK

CORR HARK

$\begin{array}{lll}49006 & \text { T } & \text { L } \\ 49006 & \text { T } & \text { L } \\ 47010 & \text { T } & \text { L } \\ 47010 & \text { T } & \text { L } \\ 47010 & \text { T } & \text { L } \\ 47010 & \text { T } & \text { L } \\ 66036 & \text { T } & 2 \\ & \text { M } & \\ 47010 & \text { T } & \text { L } \\ 47010 & \text { T } & \text { L } \\ 47010 & \text { T } & \text { L } \\ 47010 & \text { T } & \text { L }\end{array}$

300.4 SODIUM TETRADECYL 1-SULFONATE $\begin{array}{llllll}39.5 & 2.7 & X 10-3 & \mathrm{~W} & \text { DB } & \text { KRAFFT POINT SOLUBILITY } \\ 40 & 2.5 & \times 10-3 \mathrm{M} & \text { DB } & \text { SPECFC CONDCTNCE GRAPH }\end{array}$

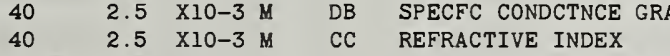

$42.52 .5 \quad$ X10-3 M CG VISUAL SPCTR CHNGE PNCN 42.52 .5 X10-3 M CG FOTOMTR SPCTR CHNGE PNCN

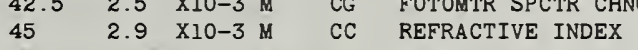

$453.15 \times 10-3 \mathrm{M}$ CC REFRACTIVE INDEX

$50 \quad 2.9 \times 10-3 \mathrm{M}$ CC REFRACTIVE INDEX

$502.03 \times 10-3 \mathrm{M}$ BG VISUAL SPCTR CHNGE PNCN

$60 \quad 3.3 \times 10-3 \mathrm{M} \quad$ DB SPECFC CONDCTNCE GRAPH

$60 \quad 3.3 \times 10-3 \mathrm{~N} \quad$ DC UNSPECIFIED CONDUCTANCE

$80 \quad 4.6 \quad \times 10-3 \mathrm{M} \quad$ DB SPECFC CONDCTNCE GRAPH

$42.5 \quad 1.8 \quad$ X10-3 M $\quad$ CG VISUAL SPCTR CHNGE PNCN

$\begin{array}{llll}\text { TART WRIG } & 39002 & \text { T } & \text { L } \\ \text { WRIG ABBO } & 39007 & \text { T } & \text { L } \\ \text { KLEV } & 48005 & \text { T } & \text { L } \\ \text { KLEV } & 50003 & \text { T } & \text { L } \\ \text { KLEV } & 47004 & \text { T } & \text { L } \\ \text { KLEV } & 48005 & \text { T } & \text { L } \\ \text { KLEV } & 47004 & \text { T } & \text { L } \\ \text { KLEV } & 48005 & \text { T } & \text { L } \\ \text { WEIL STIR } & 63013 & \text { T } & \text { L } \\ \text { WRIG ABBO } & 39007 & \text { T } & \text { L } \\ \text { TART LELO } & 55021 & \text { TA } & \text { L } \\ \text { WRIG ABBO } & 39007 & \text { T } & \text { L } \\ \text { KLEV } & 50003 & \text { T } & \text { L }\end{array}$

328.4 SODIUM HEXADECYL I-SULFONATE $47.5 \quad 1.05$ X10-3 $\mathrm{W}$ DB KRAFFT POINT SOLUBILITY $\begin{array}{llllll}50 & 7 . & \text { X10-4 M } & \text { CD } & \text { REFRACTIVE INDEX } \\ 50 & 8.0 & \text { X10-4 M } & \text { CG } & \text { FOTONTR SPCTR CHNGE PNCN }\end{array}$ $\begin{array}{llll}8.0 & \text { X10-4 M } & \text { CG } & \text { FOTONTR SPCTR CHNGE PNCN } \\ 4.5 & \text { X10-4 M } & \text { BG } & \text { VISUAL SPCTR CHNGE PNCN }\end{array}$ 9. X10-4 M CD REFRACTIVE INDEX $1.15 \times 10-3 \mathrm{M}$ CC REFRACTIVE INDEX

$\begin{array}{llll}\text { TART HRIG } & 39002 & \text { T } & \text { L } \\ \text { KLEV } & 48005 & \text { T } & \text { L } \\ \text { KLEV } & 47004 & \text { T } & \text { L } \\ \text { WEIL STIR } & 63013 & \text { T } & \text { L } \\ \text { KLEV } & 48005 & \text { T } & \text { L } \\ \text { KLEV } & 47004 & \text { T } & \text { L }\end{array}$

294.5 POTASSIUM HEXADECANOATE

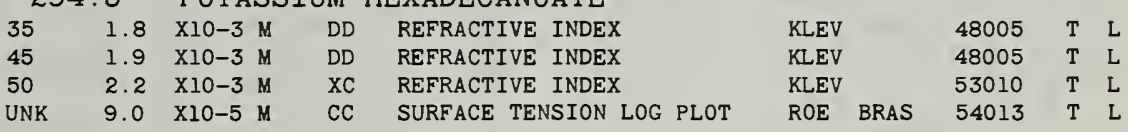

277.9 HEXADECYL AMMONIUM CHLORIDE

$\begin{array}{lllllllll}40 & 1.07 & \text { X10-3 M } & \text { CB } & \text { SPECFC CONDCTNCE GRAPH } & \text { SHIR TAMA } & 57016 & \text { P } & \text { L } \\ 50 & 8 . & \text { XIO-4 M } & \text { CD } & \text { REFRACTIVE INDEX } & \text { KLEV } & 48005 & \text { TA } & \text { L } \\ 55 & 8.5 & \text { XIO-4 M } & \text { XE } & \text { REFRACTIVE INDEX } & \text { KLEV } & 53010 & \text { T } & \text { L } \\ 60 & 9.9 & \text { XIO-4 M } & \text { CC } & \text { EQUIV CONDCTNCE GRAPH } & \text { RALS HOER } & 42002 & \text { P } & \text { L }\end{array}$

306.0 OCTADECYL AMMONIUM CHLORIDE 60 3. XIO-4 M CD EQUIV CONDCTNCE GRAPH $60 \quad 2.5 \quad \mathrm{X} 10-4 \mathrm{M} \quad \mathrm{CD}$ REFRACTIVE INDEX

RALS HOER 42002 T $\quad$ L KLEV 48005 T $\quad$ L

154.2 POTASSIUM HEXANOATE

$\begin{array}{lllllll}25 & 1.55 & \text { X10 } & 0 & \mathrm{M} & \text { DC } & \text { REFRACTIVE INDEX } \\ 25 & 1.68 & \text { X10 } & 0 & \mathrm{M} & \text { BG } & \text { UNSPEC SPCTR CHNG PNCN } \\ \text { UNK } & 1.5 & \text { X10 } & 0 & \mathrm{M} & \text { XG } & \text { METHOD NOT CITED } \\ 25 & 1.60 & \text { X10 } & 0 & \mathrm{M} & \text { BG } & \text { VISUAL SPCTR CHNGE PNCN } \\ 25 & 1.22 & \text { X10 } & 0 & \mathrm{M} & \text { DG } & \text { VISUAL SPCTR CHNGE PNCN } \\ 25 & 1.49 & \text { X10 } & 0 & \mathrm{M} & \text { DG } & \text { VISUAL SPCTR CHNGE PNCN } \\ 25 & 1.0 & \text { X10 } & 0 & \mathrm{M} & \text { DG } & \text { FOTOMTR SPCTR CHNGE PNCN } \\ 25 & 1.48 & \text { X10 } & 0 & \mathrm{M} & \text { DG } & \text { VISUAL SPCTR CHNGE PNCN } \\ 25 & 1.28 & \text { X10 } & 0 & \mathrm{M} & \text { BG } & \text { VISUAL SPCTR CHNGE PNCN }\end{array}$

$\begin{array}{llll}\text { KLEV } & 48005 & \text { T } & \text { L } \\ \text { KLEV } & 58011 & \text { T } & \text { L } \\ \text { KLEV RAIS } & 54004 & \text { T } & \text { L } \\ \text { SHIN } & 54005 & \text { G } & \text { L } \\ \text { HERZ } & 52015 & \text { T } & \text { L } \\ \text { HERZ } & 52015 & \text { T } & \text { L } \\ \text { HERZ } & 52015 & \text { T } & \text { L } \\ \text { HERZ } & 52015 & \text { T } & \text { L } \\ \text { SHIN } & 54005 & \text { G } & \text { L }\end{array}$

Concentration units: $\mathrm{A}-\mathrm{mol} \%$; $\mathrm{B}-$ vol $\%$ solvent; $\mathrm{C}-$ mol $\%$ surfactant mixture; $\mathrm{D}-\mathrm{wt} / \mathrm{vol} \%$; E-\% saturation; $\mathrm{H}-$ wt \% solvent; I-mol \% surfactant; $\mathrm{K}-$ normality counterions; $\mathrm{M}-$ molar; $\mathrm{N}$ - normal; $\mathrm{P}-\mathrm{wt} \% ; \mathrm{Q}-\mathrm{wt} \%$ surfactant; $\mathrm{R}$ - varied; $\mathrm{S}-\mathrm{mol} /$ $\mathrm{kg} ; \mathrm{T}-\mathrm{w} \mathrm{\%} \%$ surfactant mixtu re; $\mathrm{U}-\mathrm{mol} /(\mathrm{l}$ or $\mathrm{kg}) ; \mathrm{W}-$ molal; $\mathrm{Y}-\mathrm{atm}$. Details on page 222. 


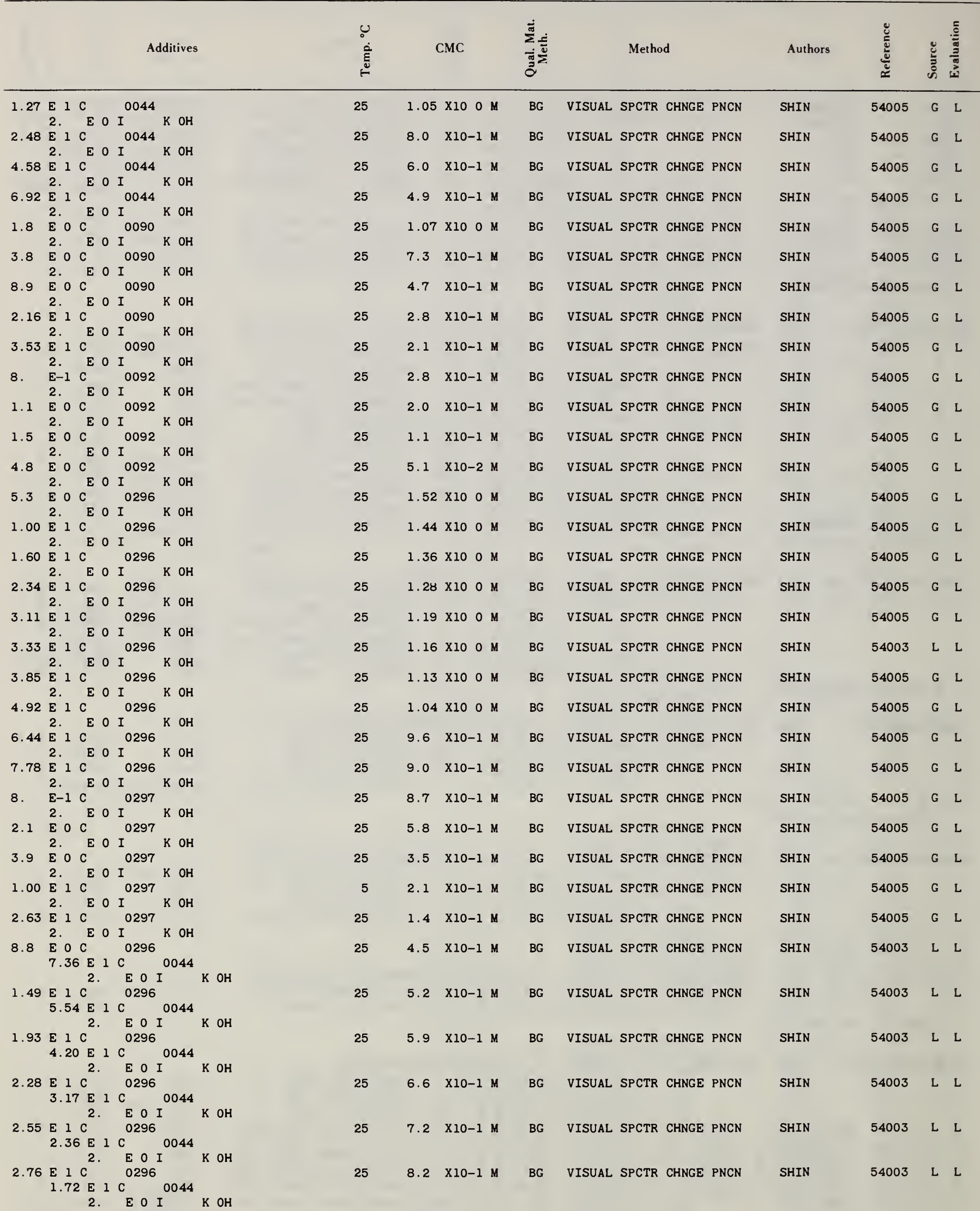

Concentration units: A-mol \%; B-vol \% solvent; $\mathrm{C}-$ mol \% surfactant mixture; D-wt/vol \%; E-\% saturation; $\mathrm{H}-$ wt \% solvent; I-mol \% surfactant; $\mathrm{K}-$ normality

counterions; $\mathrm{M}$-molar; $\mathrm{N}$-normal; $\mathrm{P}$-wt \%; $\mathrm{Q}$ - wt \% surfactant; $\mathrm{R}$-varied; $\mathrm{S}$-mol/ $\mathrm{kg} ; \mathrm{T}-\mathrm{wt} \%$ surfactant mixture; $\mathrm{U}-\mathrm{mol} /(\mathrm{lorkg}) ; \mathrm{W}-$ molal; $\mathrm{Y}-\mathrm{atm}$. Details on page 222 


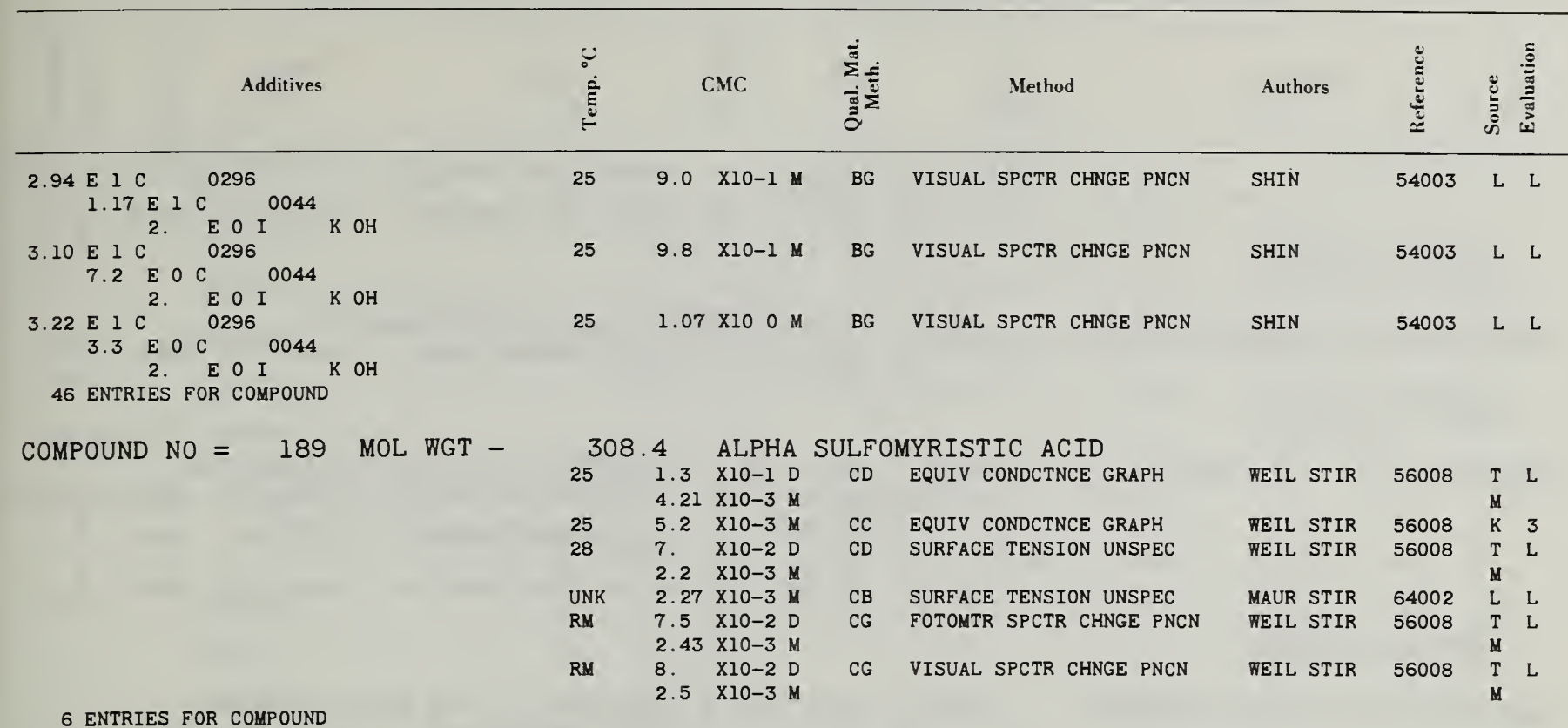

\begin{abstract}
COMPOUND NO $=190$ MOL WGT -
\end{abstract}
4 ENTRIES FOR COMPOUND

COMPOUND NO $=191$ MOL WGT -

4 ENTRIES FOR COMPOUND

COMPOUND NO $=192$ MOL WGT -

2 ENTRIES FOR COMPOUND

COMPOUND NO $=193$ MOL WGT -

1 ENTRIES FOR COMPOUND

COMPOUND NO $=194$ MOL WGT -

4 ENTRIES FOR COMPOUND

COMPOUND NO $=195$ MOL WGT -

\subsection{ALPHA SULFOPALMITIC ACID}

$\begin{array}{llll}28 & 2 . & \times 10-2 & D \\ & 5.9 & \times 10-4 & M \\ \text { UNK } & 6.0 & \times 10-4 & M \\ \text { RM } & 2.3 & \text { X10-2 } & \text { D } \\ & 6.83 & \times 10-4 & M \\ \text { RM } & 1.7 & \times 10-2 & D \\ & 5.05 & \times 10-4 & M\end{array}$

CE SURFACE TENSION UNSPEC

CB SURFACE TENSION UNSPEC

CG FOTOMTR SPCTR CHNGE PNCN

WEIL STIR

MAUR STIR

WEIL STIR

MEIL STIR

CG VISUAL SPCTR CHNGE PNCN

HEIL STIR

56008 T $\quad$ L

64002 L L

56008 T L

$56008 \quad \frac{M}{T} \quad$ L

364.5 ALPHA SULFOSTEARIC ACID

\begin{tabular}{|c|c|c|c|c|c|c|c|}
\hline 28 & 5 & $\mathrm{X} 10-3 \mathrm{D}$ & $\mathrm{CD}$ & SURFACE TENSION UNSPEC & FEIL STIR & 56008 & $\mathrm{~T}$ \\
\hline & 1.3 & $\mathrm{X} 10-4 \mathrm{M}$ & & & & & M \\
\hline UNK & 1.4 & $\mathrm{X} 10-4 \mathrm{M}$ & $C D$ & SURFACE TENSION UNSPEC & MAUR STIR & 64002 & L \\
\hline RM & $\begin{array}{l}4 . \\
1.0\end{array}$ & $\begin{array}{l}\times 10-3 D \\
\times 10-4\end{array}$ & CG & FOTOMTR SPCTR CHNGE PNCN & WEIL STIR & 56008 & $\begin{array}{l}T \\
M\end{array}$ \\
\hline aM & $\begin{array}{l}5 . \\
1.3\end{array}$ & $\begin{array}{l}\times 10-3 \mathrm{D} \\
\times 10-4 \mathrm{M}\end{array}$ & CG & TR CHNGE PNCN & WEIL STIR & 56008 & $\begin{array}{l}\mathrm{T} \\
\mathrm{M}\end{array}$ \\
\hline
\end{tabular}

386.5 SODIUM ETHYL ALPHA SULFOPALMITATE

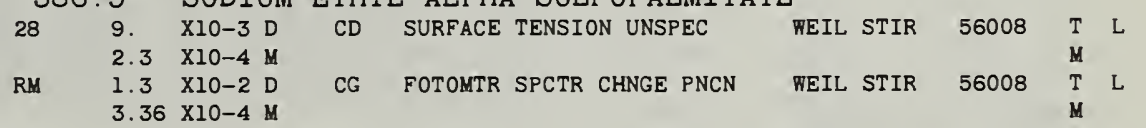

400.5 SODIUM PROPYL ALPHA SULFOPALMITATE

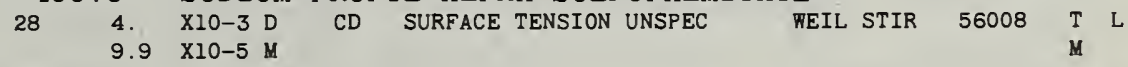

400.5 SODIUM METHYL ALPHA SULFOSTEARATE

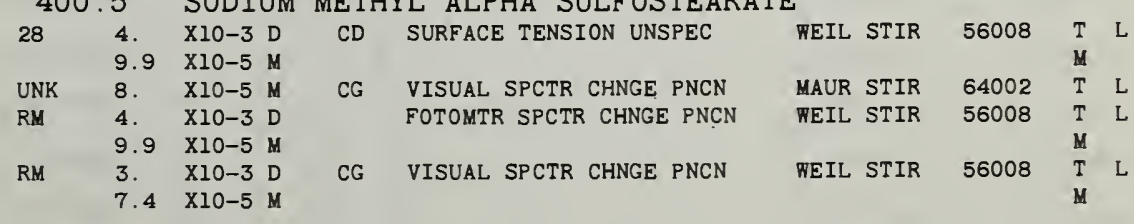

414.5 SODIUM ETHYL ALPHA SULFOSTEARATE

28 5. X10-3 D CD SURFACE TENSION UNSPEC TEIL STIR 56008 T $\mathrm{L}$
Concentration units: $\mathrm{A}-\mathrm{mol} \% ; \mathrm{B}-\mathrm{vol} \%$ solvent; $\mathrm{C}-\mathrm{mol} \%$ surfactant mixture; D-wt/vol \%; E-\% saturation; $\mathrm{H}-$ wt $\%$ solvent; I-mol \% surfactant; $\mathrm{K}-$ normality counterions; M-molar; N-normal; P-wt \%; Q-wt \% surfactant; R-varied; S-mol/ $\mathrm{kg} ; \mathrm{T}-\mathrm{wt} \%$ surfactant mixture; $\mathrm{U}-\mathrm{mol} /(\mathrm{lor} \mathrm{kg}) ; \mathrm{W}-$ molal; $\mathrm{Y}$ - atm. Details on page 222. 


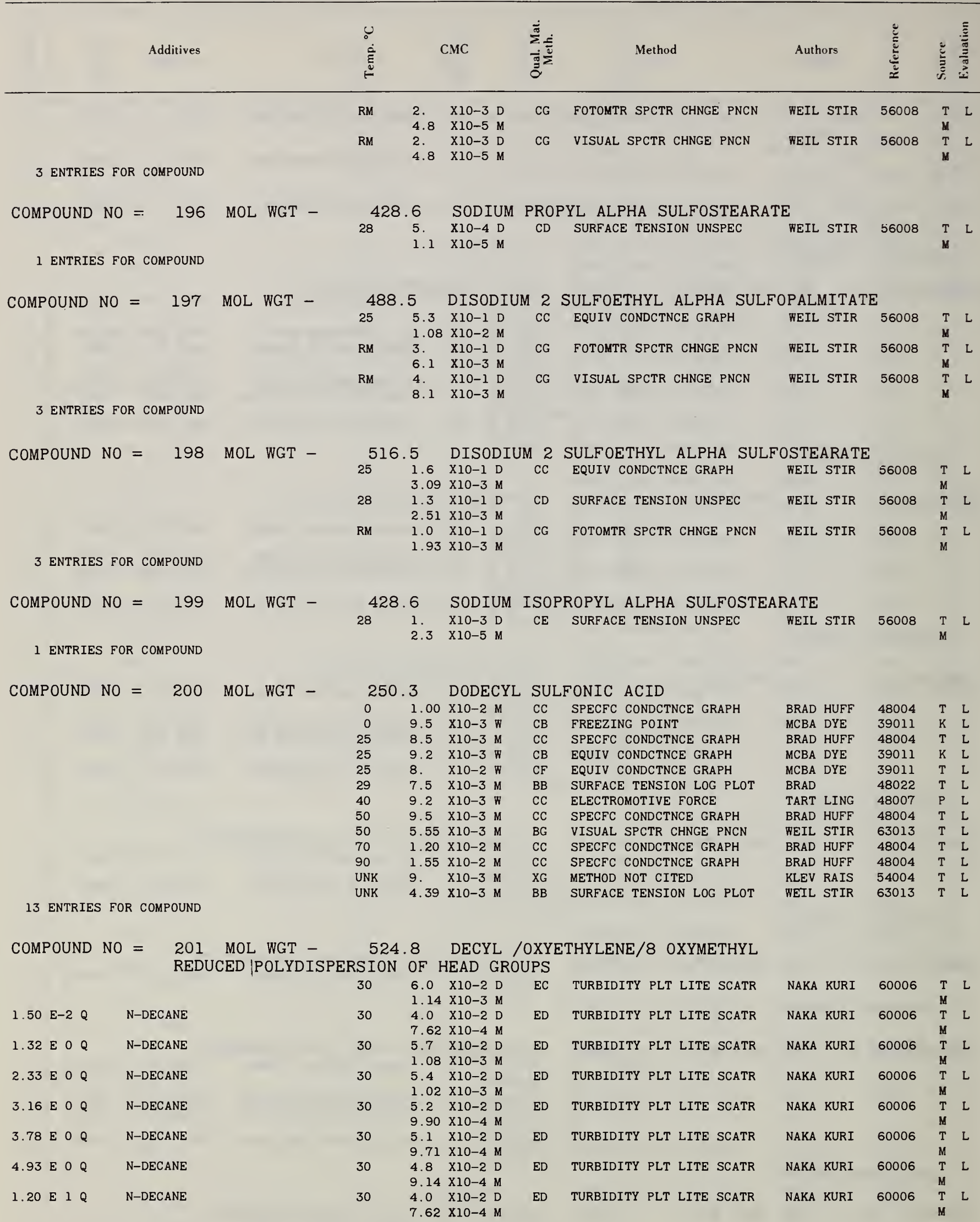

Concentration units: $\mathrm{A}-\mathrm{mol} \% ; \mathrm{B}-\mathrm{vol} \%$ solvent; $\mathrm{C}-$ mol \% surfactant mixture; D-wt/vol \%; E-\% saturation; $\mathrm{H}-$ wt \% solvent; I-mol \% surfactant; K-normality counterions; $\mathrm{M}$-molar; $\mathrm{N}$-normal; $\mathrm{P}$ - wt \%: $\mathrm{Q}$ - wt \% surfactant; $\mathrm{R}$-varied; $\mathrm{S}-\mathrm{mol} /$ kg; $\mathrm{T}-\mathrm{wt} \%$ surfactant mixture; $\mathrm{U}-\mathrm{mol} /(\mathrm{l}$ orkg); $\mathrm{W}-$ molal; $\mathrm{Y}$ - atm. Detailson page 222 


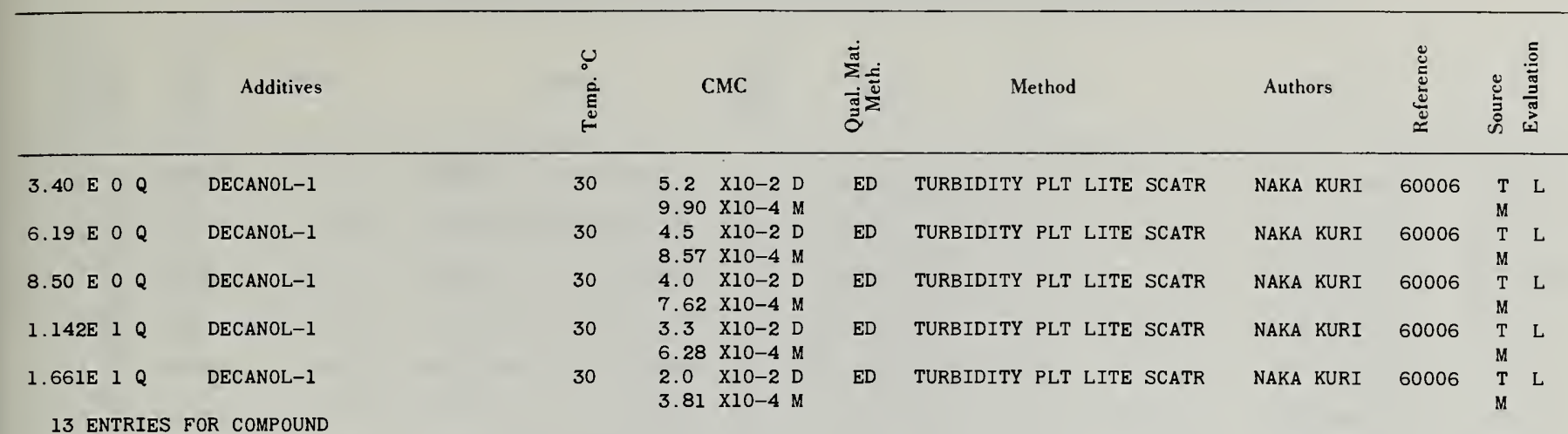

COMPOUND NO $=202$ MOL WGT - 657.0 DECYL /OXYETHYLENE/11 OXYMETHYL REDUCED POLYDISPERSION OF HEAD GROUPS

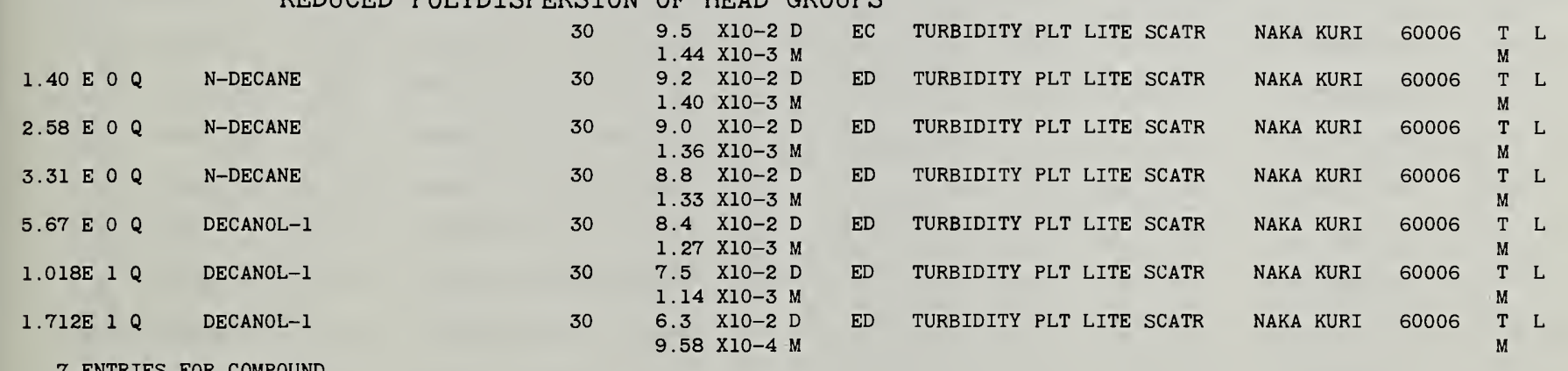

7 ENTRIES FOR COMPOUND

235.8 DECYL TRIMETHYL AMMONIUM CHLORIDE

$\begin{array}{lllllllll}25 & 6.5 & \text { X10-2 M } & \text { XG } & \text { VISUAL SPCTR CHNGE } & \text { KLEV } & 53010 & \text { T } & \text { L } \\ 25 & 6.11 & \text { X10-2 M } & \text { BB } & \text { UNSPECIFIED CONDUCTANCE } & \text { HOYE MARM } & 61002 & \text { T } & 3 \\ 25 & 1.96 & \text { X10-2 M } & \text { BB } & \text { UNSPEC SOLUBLZTN SDN 4 } & \text { HOYE MARM } & 61002 & \text { T } & 3\end{array}$

3.33 E 1 C 0041 3 ENTRIES FOR COMPOUND
MOL WGT -

COMPOUND NO $=204$ MOL WGT REDUCED POLYDISPERSION OF HEAD GROUPS

\begin{tabular}{|c|c|c|c|c|c|c|c|}
\hline 9.7 & $\begin{array}{l}1.70 \times 10-1 \\
2.425 \times 10-3\end{array}$ & $\begin{array}{l}\mathrm{D} \\
\mathrm{M}\end{array}$ & $\mathrm{EC}$ & TURBIDITY & PLT LITE & SCATR & KURI \\
\hline 29.0 & $\begin{array}{l}1.10 \times 10-1 \\
1.569 \times 10-3\end{array}$ & $\begin{array}{l}\mathrm{D} \\
\mathrm{M}\end{array}$ & EC & TURBIDITY & PLT LITE & SCATR & KURI \\
\hline 30 & $\begin{array}{l}1.05 \times 10-1 \\
1.497 \times 10-3\end{array}$ & $\begin{array}{l}\mathrm{D} \\
\mathrm{M}\end{array}$ & EC & TURBIDITY & PLT LITE & SCATR & KURI \\
\hline 45 & $\begin{array}{l}8.2 \times 10-2 \\
1.16 \times 10-3\end{array}$ & $\begin{array}{l}\mathrm{D} \\
\mathrm{M}\end{array}$ & EC & TURBIDITY & PLT LITE & SCATR & KURI \\
\hline 50.7 & $\begin{array}{ll}7.8 & \times 10-2 \\
1.11 & \times 10-3\end{array}$ & $\begin{array}{l}D \\
M\end{array}$ & ED & TURBIDITY & PLT LITE & SCATR & KURI \\
\hline 50 & $\begin{array}{ll}7.8 & \times 10-2 \\
1.11 & \times 10-3\end{array}$ & & EC & TURBIDITY & PLT LITE & SCATR & KURI \\
\hline 58.5 & $\begin{array}{l}7.0 \times 10-2 \\
9.98 \times 10-4\end{array}$ & & ED & TURBIDITY & PLT LITE & SCATR & KURI \\
\hline 69.7 & $\begin{array}{l}6.2 \times 10-2 \\
8.84 \times 10-4\end{array}$ & & ED & TURBIDITY & PLT LITE & SCATR & KURI \\
\hline 73.4 & $\begin{array}{l}6.0 \times 10-2 \\
8.55 \times 10-4\end{array}$ & & ED & TURBIDITY & PLT LITE & SCATR & KURI \\
\hline 75.0 & $\begin{array}{l}6.0 \times 10-2 \\
8.55 \times 10-4\end{array}$ & & ED & TURBIDITY & PLT LITE & SCATR & KURI \\
\hline 09.6 & $\begin{array}{l}1.65 \times 10-1 \\
2.353 \times 10-3\end{array}$ & & ED & TURBIDITY & PLT LITE & SCATR & KURI \\
\hline 10.0 & $\begin{array}{l}1.63 \times 10-1 \\
2.325 \times 10-3\end{array}$ & & ED & TURBIDITY & PLT LITE & SCATR & KURI \\
\hline 30.0 & $\begin{array}{l}1.05 \times 10-1 \\
1.497 \times 10-3\end{array}$ & & ED & TURBIDITY & PLT LITE & SCATR & KURI \\
\hline 30.0 & $\begin{array}{l}1.02 \times 10-1 \\
1.455 \times 10-3\end{array}$ & $\begin{array}{l}\text { D } \\
M\end{array}$ & ED & TURBIDITY & PLT LITE & SCATR & KURI \\
\hline 50.0 & $\begin{array}{l}7.8 \times 10-2 \\
1.11 \times 10-3\end{array}$ & $\begin{array}{l}D \\
M\end{array}$ & ED & TURBIDITY & PLT LITE & SCATR & KURI \\
\hline 50.0 & $\begin{array}{l}7.0 \times 10-2 \\
9.98 \times 10-4\end{array}$ & $\begin{array}{l}D \\
M\end{array}$ & ED & TURBIDITY & PLT LITI & SCATR & KURI \\
\hline 60.0 & $7.0 \times 10-2$ & R & ED & TURBIDITY & PLT LIT & SCATR & KURI \\
\hline
\end{tabular}

$\begin{array}{lll}62009 & \text { T } & \text { L } \\ & \text { M } & \\ 62009 & \text { T } & \text { L } \\ & \text { M } & \\ 62009 & \text { T } & \text { L } \\ & \text { M } & \\ 62009 & \text { T } & \text { L } \\ & \text { M } & \\ 62009 & \text { T } & \text { L } \\ & \text { M } & \\ 62009 & \text { T } & \text { L } \\ & \text { M } & \\ 62009 & \text { T } & \text { L } \\ & \text { M } & \\ 62009 & \text { T } & \text { L } \\ & \text { M } & \\ 62009 & \text { T } & \text { L } \\ & \text { M } & \\ 62009 & \text { T } & \text { L } \\ & \text { M } & \\ 62009 & \text { T } & \text { L } \\ & \text { M } & \\ 62009 & \text { T } & \text { L } \\ & \text { M } & \\ 62009 & \text { T } & \text { L } \\ & \text { M } & \\ 62009 & \text { T } & \text { L } \\ & \text { M } & \\ 62009 & \text { T } & \text { L } \\ & \text { M } & \\ 62009 & \text { T } & \text { L } \\ & \text { M } & \\ 62009 & \text { T } & \text { L } \\ & \text { M } & \\ & & \\ & \end{array}$

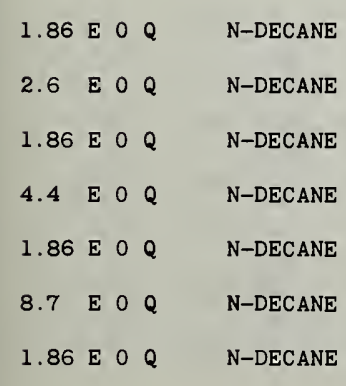

1.86 E $0 Q \quad$ N-DECANE

Concentration units: $\mathrm{A}-\mathrm{mol} \% ; \mathrm{B}-\mathrm{vol} \%$ solvent; $\mathrm{C}-$ mol \% surfactant mixture; D-wt/vol \%: E-\% saturation; $H-w t \%$ solvent; $I-$ mol \% surfactant; $K$ - normality counterions; $\mathrm{M}$ - molar; $\mathrm{N}$-normal; $\mathrm{P}-\mathrm{wt} \%$; $\mathrm{Q}$ - wt \% surfactant; $\mathrm{R}$ - varied; $\mathrm{S}-$ mol/ $\mathrm{kg} ; \mathrm{T}-\mathrm{wt} \%$ surfactant mixture; $\mathrm{U}-\mathrm{mol} /(\mathrm{l}$ or $\mathrm{kg}) ; \mathrm{W}-$ molal; $\mathrm{Y}-\mathrm{atm}$. Detailson page 222. 


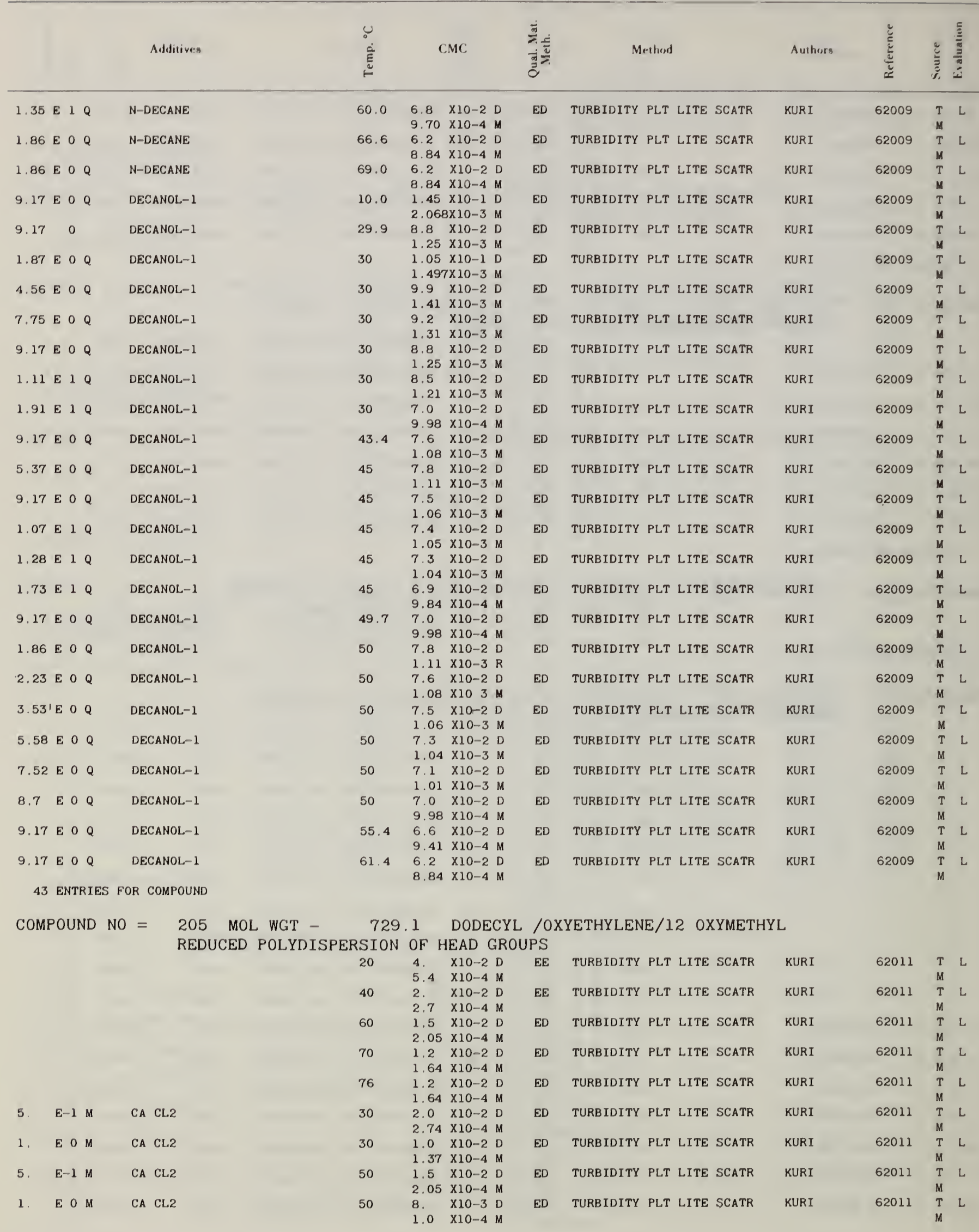

Concentration units: $\mathrm{A}-$ mol $\%$; $B-$ vol \% solvent; $\mathrm{C}-$ mol \% surfactant mixture; counterions; $\mathrm{M}$ - molar; $\mathrm{N}$-normal; $\mathbf{P}-$ wt \%; $\mathrm{Q}-$ wt \% surfactant; $\mathrm{R}$-varied; $\mathrm{S}-\mathrm{mol} /$ D-wt/vol \%; E-\% saturation; II-wt \% solvent; I-mol \% surfactant; K- mormality 


\begin{tabular}{|c|c|c|c|c|c|c|c|c|c|c|c|c|c|c|c|c|c|c|}
\hline \multirow[b]{2}{*}{1.} & \multirow[b]{2}{*}{ E 0} & & \multirow{2}{*}{\multicolumn{2}{|c|}{$\begin{array}{l}\text { Additives } \\
\text { CA CL2 }\end{array}$}} & \multirow{2}{*}{ 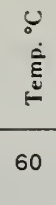 } & \multicolumn{3}{|c|}{ CMC } & \multirow{2}{*}{ 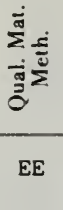 } & \multicolumn{4}{|c|}{ Method } & \multicolumn{2}{|c|}{ Authors } & \multirow{2}{*}{ 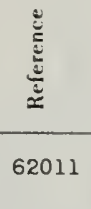 } & \multirow{2}{*}{ 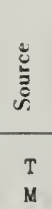 } & \multirow{2}{*}{ 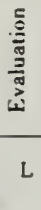 } \\
\hline & & & & & & $\begin{array}{l}5 . \\
6.8\end{array}$ & $\begin{array}{l}\times 10-3 \\
\times 10-5\end{array}$ & $\begin{array}{l}D \\
M\end{array}$ & & TURBIDITY & PLT & LITE & SCATR & KURI & & & & \\
\hline 5. & E-1 & M & $\mathrm{CA}$ & CL2 & 64 & $\begin{array}{l}1.0 \\
1.37\end{array}$ & $\begin{array}{l}\times 10-2 \\
\times 10-4\end{array}$ & $\begin{array}{l}D \\
M\end{array}$ & ED & TURBIDITY & PLT & LITE & SCATR & KURI & & 62011 & $\begin{array}{l}\mathrm{T} \\
\mathrm{M}\end{array}$ & $\mathrm{L}$ \\
\hline 2. & E-1 & $\mathbf{M}$ & NA & $\mathrm{CL}$ & 30 & $\begin{array}{l}2.3 \\
3.15\end{array}$ & $\begin{array}{l}\times 10-2 \\
\times 10-4\end{array}$ & $\begin{array}{l}D \\
M\end{array}$ & ED & TURBIDITY & PLT & LITE & SCATR & KURI & NAKA & 62010 & $\begin{array}{l}\mathrm{T} \\
\mathrm{M}\end{array}$ & $\mathrm{L}$ \\
\hline 2. & $E-1$ & M & NA & $\mathrm{CL}$ & 30 & $\begin{array}{l}2.3 \\
3.15\end{array}$ & $\begin{array}{l}\times 10-2 \\
\times 10-4\end{array}$ & $\begin{array}{l}\mathrm{D} \\
\mathrm{M}\end{array}$ & ED & TURBIDITY & PLT & LITE & SCATR & KURI & & 62011 & $\begin{array}{l}\mathrm{T} \\
\mathrm{M}\end{array}$ & L \\
\hline 1. & E 0 & $\mathbf{M}$ & NA & $\mathrm{CL}$ & 30 & $\begin{array}{l}2.0 \\
2.74\end{array}$ & $\begin{array}{l}\times 10-2 \\
\times 10-4\end{array}$ & $\begin{array}{l}D \\
M\end{array}$ & ED & TURBIDITY & PLT & LITE & SCATR & KURI & & 62011 & $\begin{array}{l}T \\
M\end{array}$ & $\mathrm{~L}$ \\
\hline 2. & $E-1$ & M & NA & $\mathrm{CL}$ & 50 & $\begin{array}{l}1.6 \\
2.19\end{array}$ & $\begin{array}{l}X 10-2 \\
\times 10-4\end{array}$ & $\begin{array}{l}D \\
M\end{array}$ & ED & TURBIDITY & PLT & LITE & SCATR & KURI & NAKA & 62010 & $\begin{array}{l}\mathrm{T} \\
\mathrm{M}\end{array}$ & $\mathrm{L}$ \\
\hline 2. & E-1 & M & NA & $\mathrm{CL}$ & 50 & $\begin{array}{l}1.6 \\
2.19\end{array}$ & $\begin{array}{l}\times 10-2 \\
\times 10-4\end{array}$ & $\begin{array}{l}D \\
M\end{array}$ & ED & TURBIDITY & PLT & LITE & SCATR & KURI & & 62011 & $\begin{array}{l}\mathrm{T} \\
\mathrm{M}\end{array}$ & L \\
\hline 1. & E 0 & M & NA & $\mathrm{CL}$ & 50 & $\begin{array}{l}1.5 \\
2.05\end{array}$ & $\begin{array}{l}\times 10-2 \\
\times 10-4\end{array}$ & $\begin{array}{l}D \\
M\end{array}$ & ED & TURBIDITY & PLT & LITE & SCATR & KURI & & 62011 & $\begin{array}{l}\mathrm{T} \\
\mathrm{M}\end{array}$ & $\mathrm{L}$ \\
\hline 1. & E 0 & M & NA & $\mathrm{CL}$ & 60 & $\begin{array}{l}1.2 \\
1.64\end{array}$ & $\begin{array}{l}X 10-2 \\
\times 10-4\end{array}$ & $\begin{array}{l}D \\
M\end{array}$ & ED & TURBIDITY & PLT & LITE & SCATR & KURI & & 62011 & $\begin{array}{l}\mathrm{T} \\
\mathrm{M}\end{array}$ & L \\
\hline 2. & $E-1$ & M & NA & $\mathrm{CL}$ & 70 & $\begin{array}{l}1.2 \\
1.64\end{array}$ & $\begin{array}{l}\times 10-2 \\
\times 10-4\end{array}$ & $\begin{array}{l}D \\
M\end{array}$ & ED & TURBIDITY & PLT & LITE & SCATR & KURI & NAKA & 62010 & $\begin{array}{l}\mathrm{T} \\
\mathrm{M}\end{array}$ & L \\
\hline 2. & $E-1$ & M & NA & $\mathrm{CL}$ & 70 & $\begin{array}{l}1.2 \\
1.64\end{array}$ & $\begin{array}{l}\times 10-2 \\
\times 10-4\end{array}$ & $\begin{array}{l}D \\
M\end{array}$ & ED & TURBIDITY & PLT & LITE & SCATR & KURI & & 62011 & $\begin{array}{l}\mathrm{T} \\
\mathrm{M}\end{array}$ & $\mathrm{L}$ \\
\hline 2. & $\begin{array}{l}E-1 \\
5\end{array}$ & ${ }^{\mathrm{M}} \mathrm{E}$ & $\mathrm{T}^{\mathrm{NA}}$ & $\begin{array}{l}\mathrm{CL} \\
0001\end{array}$ & 30 & $\begin{array}{l}2.3 \\
3.15\end{array}$ & $\begin{array}{l}\times 10-2 \\
\times 10-4\end{array}$ & $\begin{array}{l}D \\
M\end{array}$ & ED & TURBIDITY & PLT & LITE & SCATR & KURI & NAKA & 62010 & $\begin{array}{l}\mathrm{T} \\
\mathrm{M}\end{array}$ & L \\
\hline 2. & $\begin{array}{l}E-1 \\
2.0\end{array}$ & ${ }^{\mathrm{M}} 1$ & $T^{N A}$ & ${ }_{0001}^{C L}$ & 30 & $\begin{array}{l}2.5 \\
3.42\end{array}$ & $\begin{array}{l}\times 10-2 \\
\times 10-4\end{array}$ & $\begin{array}{l}D \\
M\end{array}$ & ED & TURBIDITY & PLT & LITE & SCATR & KURI & NAKA & 62010 & $\begin{array}{l}\mathrm{T} \\
\mathrm{M}\end{array}$ & $\mathrm{L}$ \\
\hline 2. & $\begin{array}{l}E-1 \\
4.0\end{array}$ & M 1 . & $\mathrm{T}^{\mathrm{NA}}$ & ${ }_{0001}^{\mathrm{CL}}$ & 30 & $\begin{array}{l}2.8 \\
3.84\end{array}$ & $\begin{array}{l}\times 10-2 \\
\times 10-4\end{array}$ & $\begin{array}{l}D \\
M\end{array}$ & ED & TURBIDITY & PLT & LITE & SCATR & KURI & NAKA & 62010 & $\begin{array}{l}\mathrm{T} \\
\mathrm{M}\end{array}$ & $\mathrm{L}$ \\
\hline 2. & $\begin{array}{l}E-1 \\
6.0\end{array}$ & M 1 . & $\mathrm{T}^{\mathrm{NA}}$ & $\begin{array}{l}\text { CL } \\
0001\end{array}$ & 30 & $\begin{array}{l}2.8 \\
3.84\end{array}$ & $\begin{array}{l}\times 10-2 \\
\times 10-4\end{array}$ & $\begin{array}{l}\mathrm{D} \\
\mathrm{M}\end{array}$ & ED & TURBIDITY & PLT & LITE & SCATR & KURI & NAKA & 62010 & $\begin{array}{l}\mathrm{T} \\
\mathrm{M}\end{array}$ & $\mathrm{L}$ \\
\hline 2. & $\begin{array}{l}E-1 \\
8.0\end{array}$ & ${ }^{\mathrm{M}} \mathrm{I}$ & $T^{N A}$ & $\begin{array}{l}\mathrm{CL} \\
\quad 0001\end{array}$ & 30 & $\begin{array}{l}2.9 \\
3.97\end{array}$ & $\begin{array}{l}\times 10-2 \\
\times 10-4\end{array}$ & $\begin{array}{l}\mathrm{D} \\
\mathrm{M}\end{array}$ & ED & TURBIDITY & PLT & LITE & SCATR & KURI & NAKA & 62010 & $\begin{array}{l}\mathrm{T} \\
\mathrm{M}\end{array}$ & $\mathrm{L}$ \\
\hline 2. & $\begin{array}{l}E-1 \\
5\end{array}$ & ${ }^{\mathrm{M}} 0$ & $T^{N A}$ & $\begin{array}{l}\mathrm{CL} \\
0001\end{array}$ & 50 & $\begin{array}{l}2.2 \\
3.01\end{array}$ & $\begin{array}{l}\times 10-2 \\
\times 10-4\end{array}$ & $\begin{array}{l}D \\
M\end{array}$ & ED & TURBIDITY & PLT & LITE & SCATR & KURI & NAKA & 62010 & $\begin{array}{l}\mathrm{T} \\
\mathrm{M}\end{array}$ & L \\
\hline 2. & $\begin{array}{l}E-1 \\
2.0\end{array}$ & ${ }^{\mathrm{M}} 1$ & $T^{N A}$ & ${ }_{0001} L^{C L}$ & 50 & $\begin{array}{l}2.5 \\
3.42\end{array}$ & $\begin{array}{l}\times 10-2 \\
\times 10-4\end{array}$ & $\begin{array}{l}D \\
M\end{array}$ & ED & TURBIDITY & PLT & LITE & SCATR & KURI & NAKA & 62010 & $\begin{array}{l}\mathrm{T} \\
\mathrm{M}\end{array}$ & L \\
\hline 2. & $\begin{array}{l}E-1 \\
4.0\end{array}$ & E 1 & $T^{N A}$ & ${ }_{0001}^{C L}$ & 50 & $\begin{array}{l}3.0 \\
4.11\end{array}$ & $\begin{array}{l}\times 10-2 \\
\times 10-4\end{array}$ & $\begin{array}{l}\mathrm{D} \\
\mathrm{M}\end{array}$ & $E D$ & TURBIDITY & PLT & LITE & SCATR & KURI & NAKA & 62010 & M & $\mathrm{L}$ \\
\hline 2. & $\begin{array}{l}\text { E-1 } \\
6.0\end{array}$ & ${ }^{\mathrm{M}} 1$ & $T^{N A}$ & $\begin{array}{l}\mathrm{CL} \\
\quad 0001\end{array}$ & 50 & $\begin{array}{l}3.3 \\
4.52\end{array}$ & $\begin{array}{l}\times 10-2 \\
\times 10-4\end{array}$ & $\begin{array}{l}D \\
M\end{array}$ & ED & TURBIDITY & PLT & LITE & SCATR & KURI & NAKA & 62010 & $\begin{array}{l}\mathrm{T} \\
\mathrm{M}\end{array}$ & L \\
\hline 2. & $\begin{array}{l}E-1 \\
8.0\end{array}$ & M 1 & $T^{N A}$ & $\begin{array}{l}\mathrm{CL} \\
\quad 0001\end{array}$ & 50 & $\begin{array}{l}3.6 \\
4.93\end{array}$ & $\begin{array}{l}\times 10-2 \\
\times 10-4\end{array}$ & $\begin{array}{l}D \\
M\end{array}$ & ED & TURBIDITY & PLT & LITE & SCATR & KURI & NAKA & 62010 & $\begin{array}{l}\mathrm{T} \\
\mathrm{M}\end{array}$ & $\mathrm{L}$ \\
\hline 2. & $\begin{array}{l}E-1 \\
5\end{array}$ & $\begin{array}{l}\mathrm{M} \\
\mathrm{E}\end{array}$ & $T^{N A}$ & 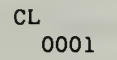 & 70 & $\begin{array}{l}2.0 \\
2.74\end{array}$ & $\begin{array}{l}\times 10-2 \\
\times 10-4\end{array}$ & $\begin{array}{l}D \\
M\end{array}$ & $\mathrm{ED}$ & TURBIDITY & PLT & LITE & SCATR & KURI & NAKA & 62010 & $\begin{array}{l}\mathrm{T} \\
\mathrm{M}\end{array}$ & L \\
\hline 2. & $\begin{array}{l}E-1 \\
2.0\end{array}$ & ${ }^{M}$ E 1 & $T^{N A}$ & $\begin{array}{l}\mathrm{CL} \\
\quad 0001\end{array}$ & 70 & $\begin{array}{l}2.6 \\
3.56\end{array}$ & $\begin{array}{l}\times 10-2 \\
\times 10-4\end{array}$ & $\begin{array}{l}D \\
M\end{array}$ & ED & TURBIDITY & PLT & LITE & SCATR & KURI & NAKA & 62010 & $\begin{array}{l}\mathrm{T} \\
\mathrm{M}\end{array}$ & $\mathrm{L}$ \\
\hline 2. & $\begin{array}{l}E-1 \\
4.0\end{array}$ & E 1 & $T^{N A}$ & $\begin{array}{l}\mathrm{CL} \\
\quad 0001\end{array}$ & 70 & $\begin{array}{l}3.4 \\
4.66\end{array}$ & $\begin{array}{l}\times 10-2 \\
\times 10-4\end{array}$ & $\begin{array}{l}\mathrm{D} \\
\mathrm{M}\end{array}$ & $E D$ & TURBIDITY & PLT & LITE & SCATR & KURI & NAKA & 62010 & $\begin{array}{l}\mathrm{T} \\
\mathrm{M}\end{array}$ & $\mathrm{L}$ \\
\hline 2. & $\begin{array}{l}\text { E-1 } \\
6.0 \\
\text { E-1 } \\
8.0\end{array}$ & $\begin{array}{ccc}\mathrm{M}^{\mathrm{E}} & 1 \\
\mathrm{E} & 1 & \\
& 1\end{array}$ & $T_{T}^{N A}$ & $\begin{array}{l}\mathrm{CL} \\
\mathrm{O} 001 \\
\mathrm{CL} \\
0001\end{array}$ & $\begin{array}{l}70 \\
70\end{array}$ & $\begin{array}{l}4.0 \\
5.48 \\
5.0 \\
6.85\end{array}$ & $\begin{array}{l}\times 10-2 \\
\times 10-4 \\
\times 10-2 \\
\times 10-4\end{array}$ & $\begin{array}{l}D \\
M \\
D \\
M\end{array}$ & $\begin{array}{l}E D \\
E D\end{array}$ & $\begin{array}{l}\text { TURBIDITY } \\
\text { TURBIDITY }\end{array}$ & $\begin{array}{l}\text { PLT } \\
\text { PLT }\end{array}$ & $\begin{array}{l}\text { LITE } \\
\text { LITE }\end{array}$ & $\begin{array}{l}\text { SCATR } \\
\text { SCATR }\end{array}$ & $\begin{array}{l}\text { KURI } \\
\text { KURI }\end{array}$ & $\begin{array}{l}\text { NAKA } \\
\text { NAKA }\end{array}$ & $\begin{array}{l}62010 \\
62010\end{array}$ & $\begin{array}{c}\mathrm{T} \\
\mathrm{M} \\
\mathrm{T} \\
\mathrm{M}\end{array}$ & $\begin{array}{l}\text { L } \\
\text { L }\end{array}$ \\
\hline
\end{tabular}

6249 TRITON X-100(P-T-OCTYL BENZENE/OXYETHYLENE/9 206 MOL WGT - 6249 TRITON X
NATURAL DISTKIBU'TION UF HEAD GROUPS

$\begin{array}{llll}0 & 9 . & \times 10-4 & W \\ 26 & 1.5 & \times 10-2 & D \\ 30 & 5 . & \times 10-2 & D \\ \text { UNK } & 1.6 & \times 10-2 & D \\ 30 & 4 . & \times 10-2 & D \\ 25 & 1.8 & \times 10-3 & D \\ 25 & 2.2 & \times 10-3 & D \\ 25 & 1.6 & \times 10-3 & D \\ 25 & 3.7 & \times 10-3 & D \\ 25 & 2.0 & \times 10-3 & D \\ 25 & 2.2 & \times 10-3 & D \\ 25 & 1.5 & \times 10-3 & D \\ 25 & 2.5 & \times 10-3 & D \\ 25 & 2.2 & \times 10-3 & D \\ 25 & 2.0 & \times 10-3 & D \\ 25 & 2.0 & \times 10-3 & D\end{array}$

HD
HD
HD
HE
HG
HG
HG
HG
HG
HE
HG
HG
HE
HG
HG

FREEZING POINT

SURFACE TENSION LOG PLOT

TURBIDITY PLT LITE SCATR

FOTOMTR SPCTR CHNG I2

TURBIDITY PLT LITE SCATR

POLAROGRAPHIC MAXIMUM

POLAROGRAPHIC MAXIMUM

POLAROGRAPHIC MAXIMUM

POLAROGRAPHIC MAXIMUM

POLAROGRAPHIC MAXIMUM

SURFACE TNSN LINEAR PLOT

POLAROGRAPHIC MAXIMUM

POLAROGRAPHIC MAXIMUM

SURFACE TNSN LINEAR PLOT

POLAROGRAPHIC MAXIMUM
POLAROGRAPHIC MAXIMUM

$\begin{array}{llll}\text { GONI MCBA } & 47007 & \text { T } & \text { L } \\ \text { MANK } & 64010 & \text { T } & \text { L } \\ \text { KURI } & 62011 & \text { T } & \text { L } \\ \text { ROSS OLIV } & 59020 & \text { T } & \text { L } \\ \text { KURI } & 62011 & \text { T } & \text { L } \\ \text { COLI } & 50012 & \text { T } & \text { L } \\ \text { COLI } & 50012 & \text { T } & \text { L } \\ \text { COLI } & 50012 & \text { T } & \text { L } \\ \text { COLI } & 50012 & \text { T } & \text { L } \\ \text { COLI } & 50012 & \text { T } & \text { L } \\ \text { COLI } & 50012 & \text { T } & \text { L } \\ \text { COLI } & 50012 & \text { T } & \text { L } \\ \text { COLI } & 50012 & \text { T } & \text { L } \\ \text { COLI } & 50012 & \text { T } & \text { L } \\ \text { COLI } & 50012 & \text { T } & \text { L } \\ \text { COLI } & 50012 & \text { T } & \text { L }\end{array}$

Concentration units: $\mathrm{A}-\mathrm{mol} \%$; $\mathrm{B}-$ vol \% solvent; $\mathrm{C}-$ mol \% surfactant mixture; D-wt/vol \%; E-\% saturation; $\mathrm{H}-$ wt \% solvent; I-mol \% surfactant; $\mathrm{K}$ - normality counterions; $\mathrm{M}$-molar; $\mathrm{N}$ - normal; $\mathrm{P}-\mathrm{wt} \% ; \mathrm{Q}-\mathrm{wt} \%$ surfactant; $\mathrm{R}$-varied: $\mathrm{S}-$ mol/ kg; $\Upsilon$ - wt \% surfactant mixture; $U-$ mol/(l orkg); $W-$ molal; $Y$ - atm. Details on page 222. 


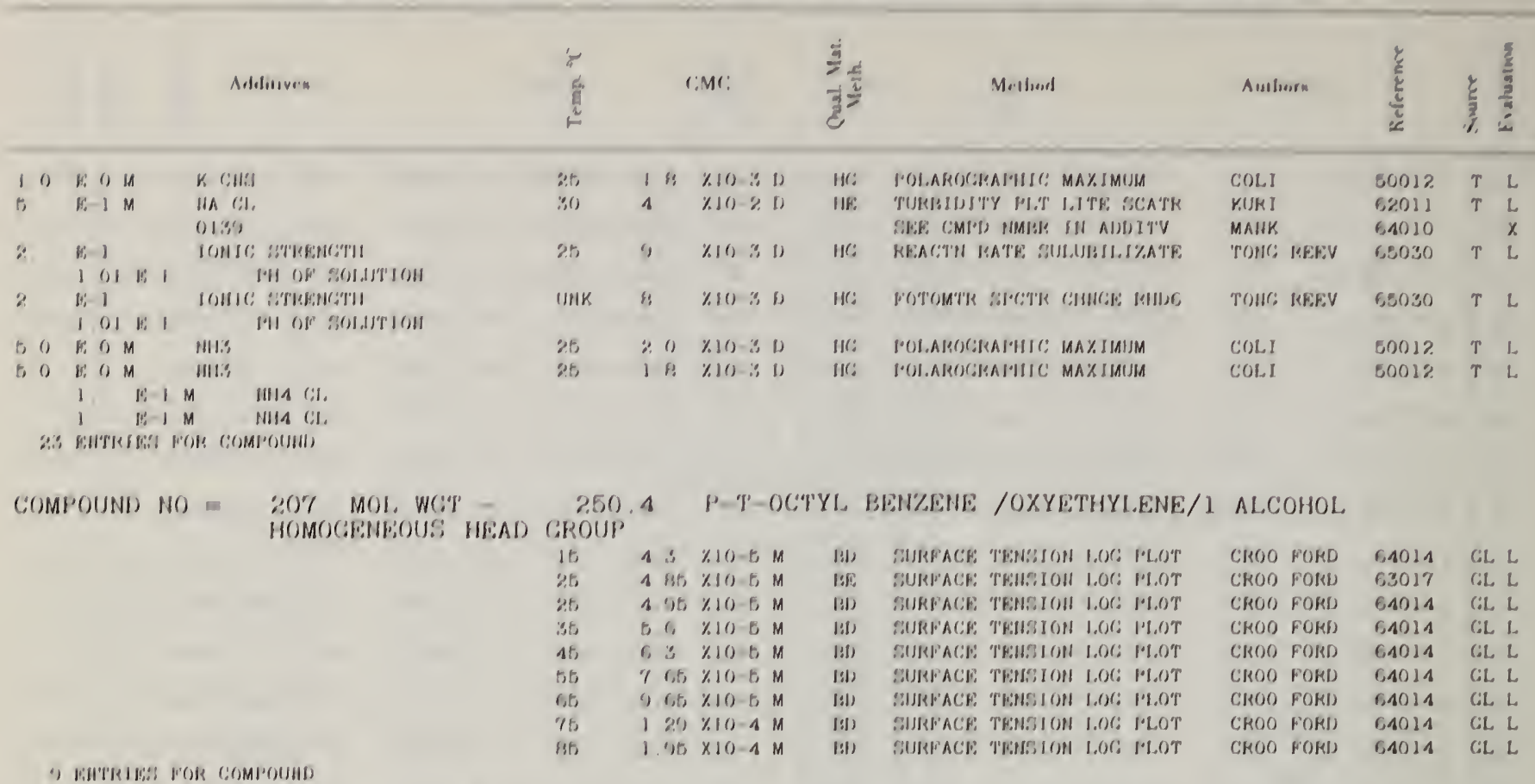

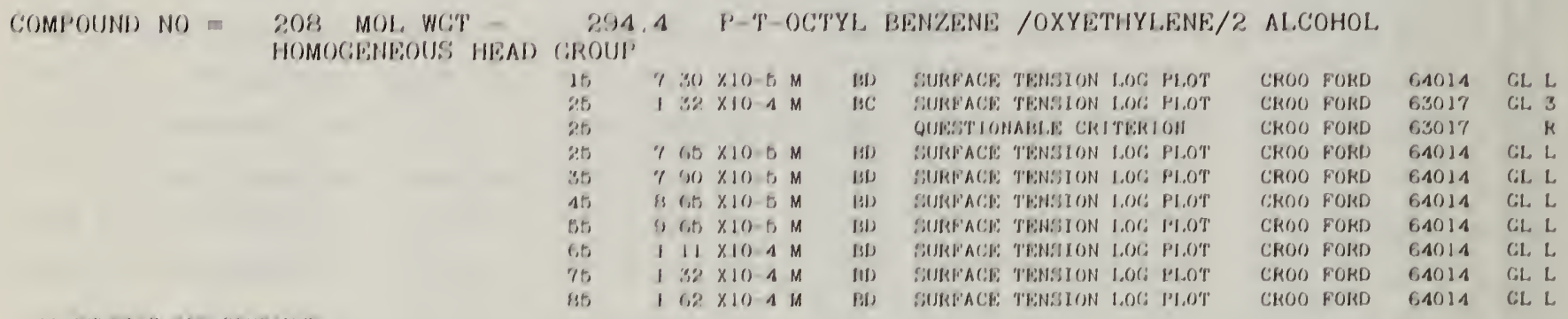

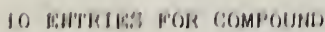

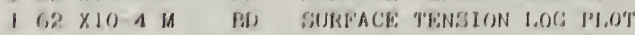

CKOO FOKD

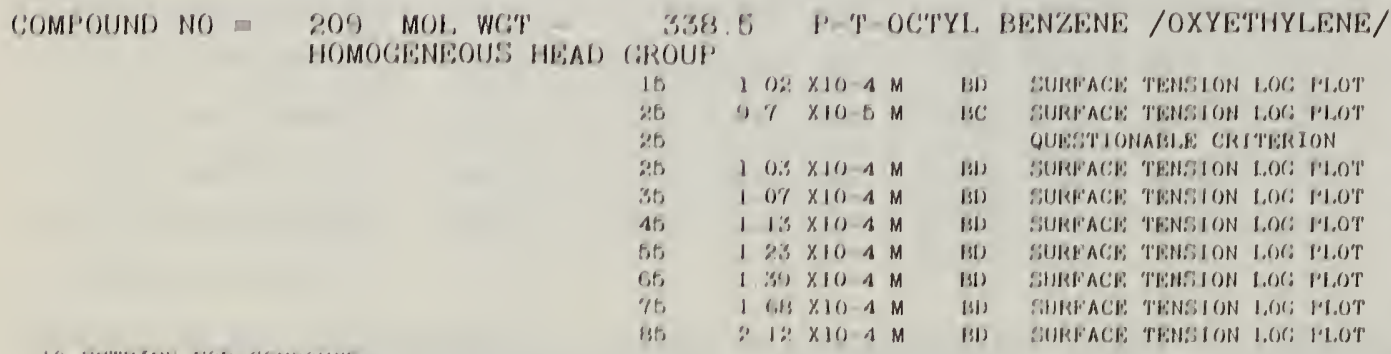

ALCOHOL

CKOO FORII CKOO FOKD CHOO FORD CROO FOFD CKOO FOKD CROO FOKD CKOO FOKD C.ROO FOKD CHOO FOKI) (KOO FOKS)

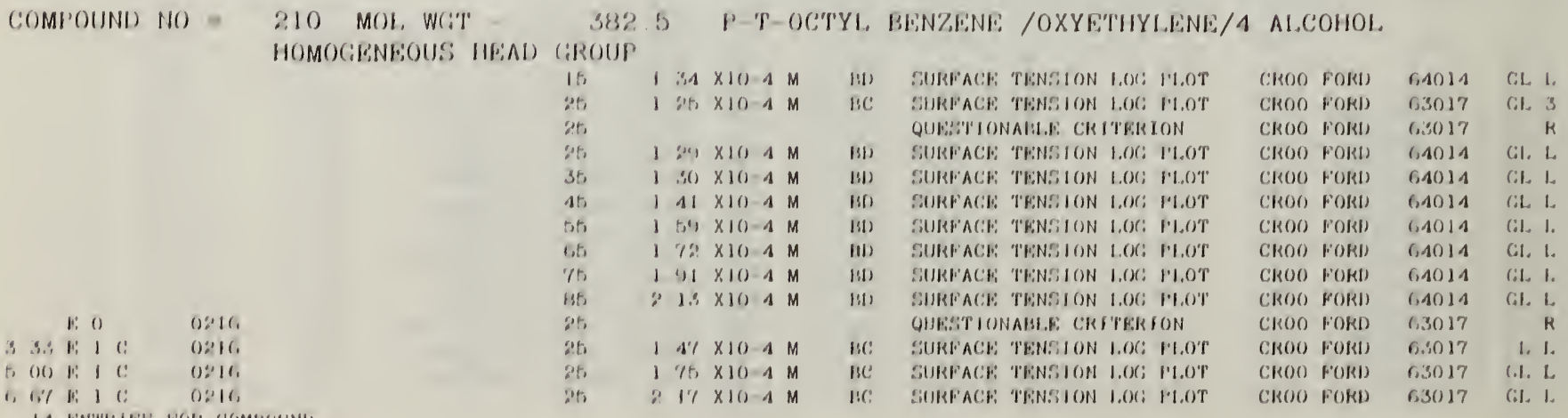

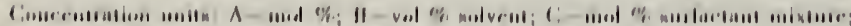

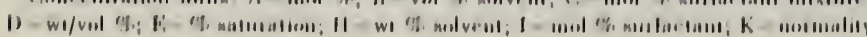

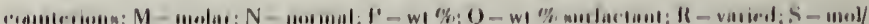

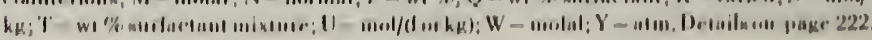




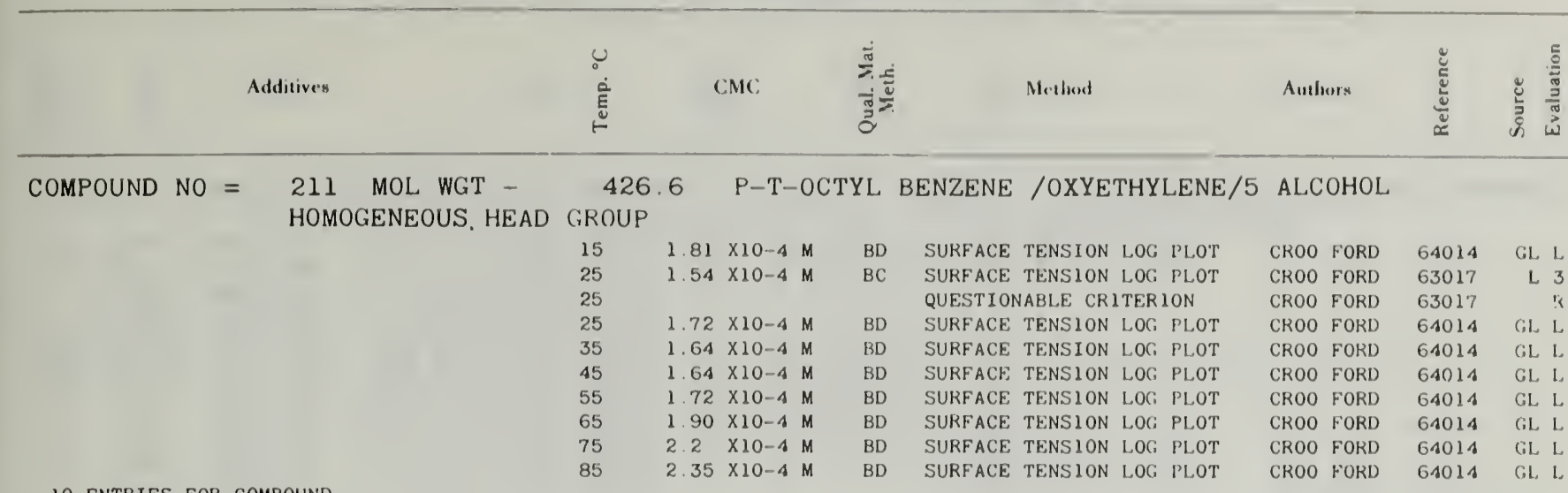

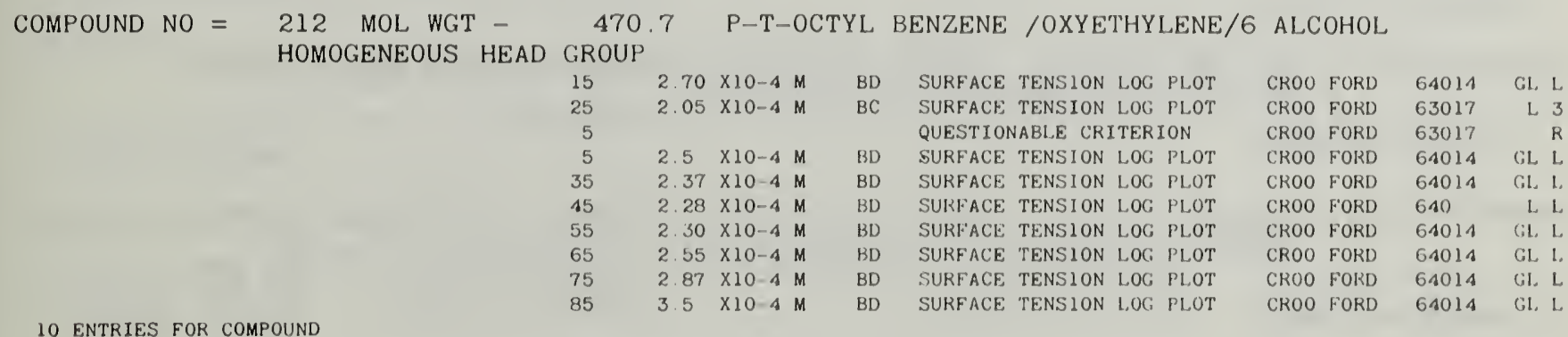

10 ENTRIES FOR COMPOUND

514.7 P-T-OCTYL BENZENE /OXYETHYLENE/7 ALCOHOL

COMPOUND NO $=\begin{aligned} & 213 \text { MOL WG' }-514 \\ & \text { iHOMOGENEOUS'HEAD GROUP }\end{aligned}$

$\begin{array}{ll}15 & 2.91 \times 10-4 \mathrm{M} \\ 25 & 2.46 \times 10-4 \mathrm{M} \\ 25 & \\ 25 & 2.68 \times 10-4 \mathrm{M} \\ 35 & 2.44 \times 10-4 \mathrm{M} \\ 45 & 2.43 \times 10-4 \mathrm{M} \\ 55 & 2.41 \times 104 \mathrm{M} \\ 65 & 2.50 \times 10-4 \mathrm{M} \\ 75 & 2.68 \times 10-4 \mathrm{M} \\ 85 & 2.90 \times 10-4 \mathrm{M}\end{array}$

10 ENTRIES FOR COMPOUND
BD SURFACE, TENSION LOG PLOT BC SURFACE TENSION LOG PLOT QULSTIONABLE CRITERION BI) SURFACE TENSION LOG PLOT $B D$ BD $B D$ $B D$ $\mathrm{BD}$
$\mathrm{BD}$ SURFACE TENSION LOG PLOT SURFACE TENSION LOG PLOT SURFACE TENSION LOG PLOT SURFACE TENSION LOC PLOT SURFACE TENSION LOG PLOT SURFACE TENSION LOG PIOT

CROO FORD CROO FORD CROO FORD CROO FORD CROO FORD CROO FORD CROO FORD CROO FORD CROO FORD CROO FORD
64014 GL, 1 63017 L. 3 $63017 \quad \mathrm{~K}$ $64014 \mathrm{GL}, \mathrm{L}$ 61011 Gi. L 64011 (iL L 64014 (IL L 64014 (:L L 64014 (iL L $64014 \quad$ GL L

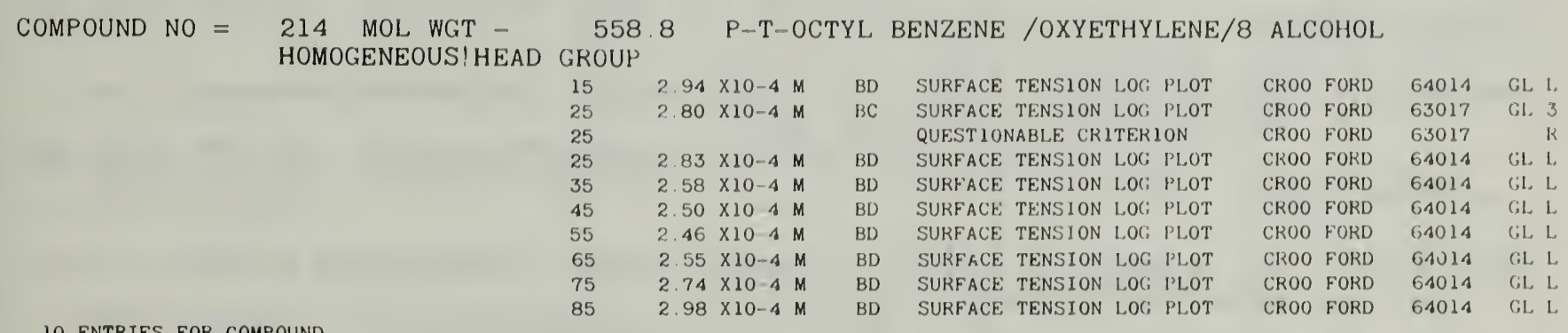

10 ENTRIES FOR COMPOUND

602.8 P-T-OCTYL BENZENE /OXYETHYLENE/9 ALCOHOL

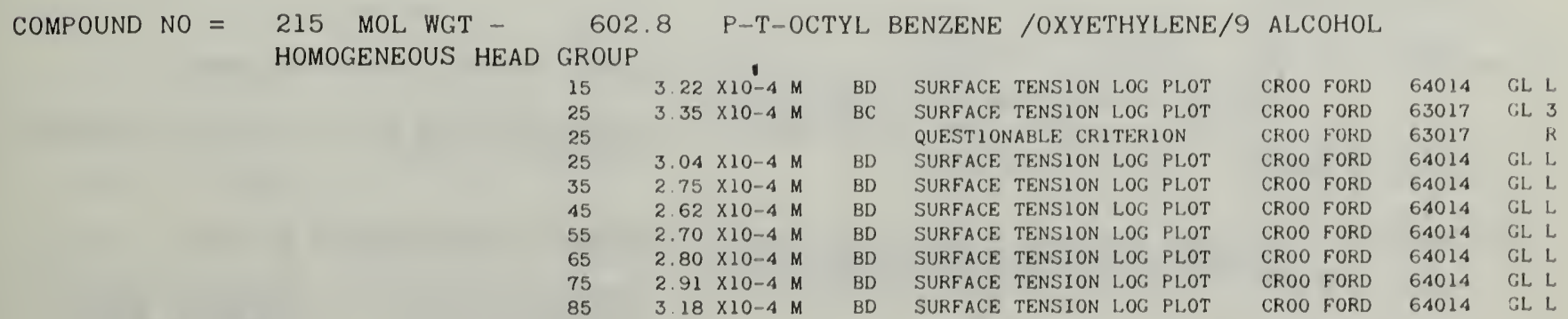

10 ENTRIES FOR COMPOUND

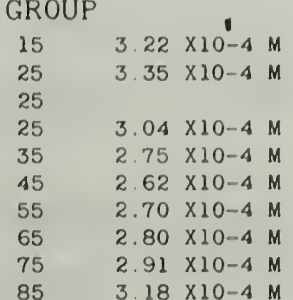

Concentration units: $\mathrm{A}-\operatorname{mol} \% ; \mathrm{B}-\mathrm{vol} \%$ solvent: $\mathrm{C}-\mathrm{mol} \%$ surfactant mixture; D - wt/vol \%; $\mathbf{E}-\%$ saturation; H-wt \% solvent; I - mol \% surfuctant; $\mathrm{K}$ - normality connerions; M - molar; N - normal; $P$ - wt \%; $Q$ - wt \% surfactant; $R$ - varied; $S$ - mool/

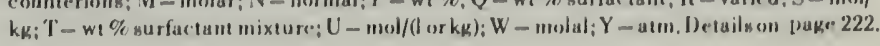




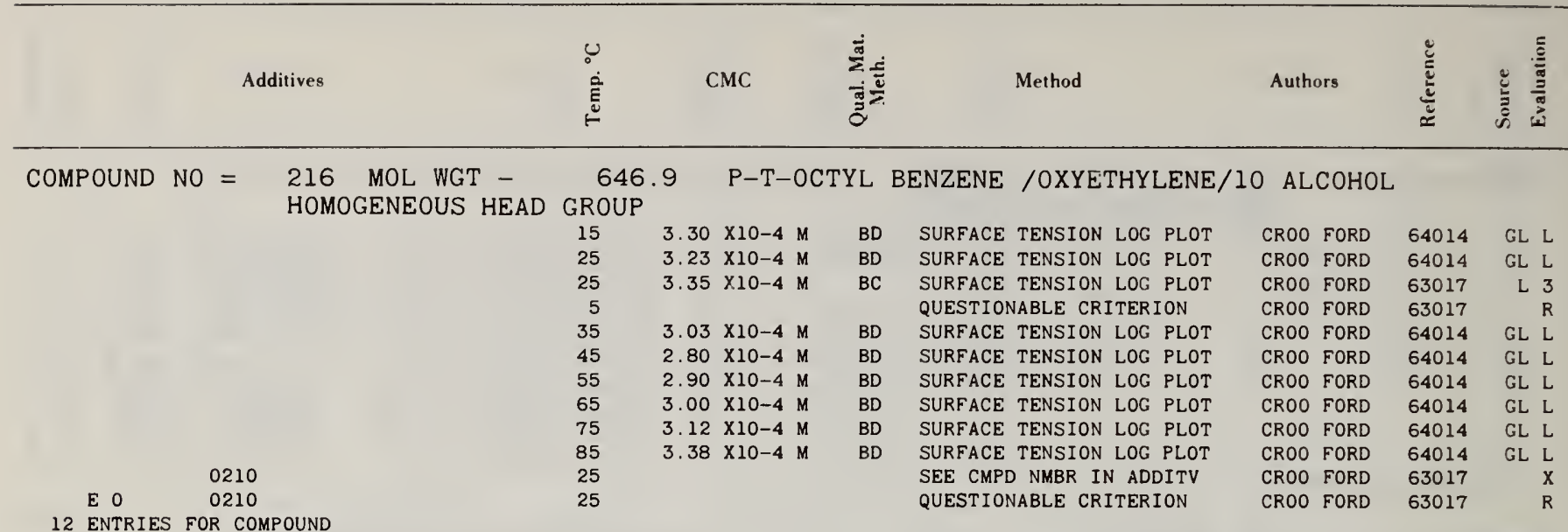

\section{COMPOUND NO $=217$ MOL WGT - 250.4 P-T-OCTYL BENZENE /OXYETHYLENE/I ALCOHOL} NATURAL DISTRIBUTION OF HEAD GROUPS

1 ENTRIES FOR COMPOUND

$254.85 \times 10-5 \mathrm{M}$ EE SURFACE TENSION LOG PLOT CROO FORD

63017

GL L

\section{COMPOUND NO $=218$ MOL WGT - NATURAL DISTRIBUTION OF HEAD GROUPS}

2 ENTRIES FOR COMPOUND
SURFACE TENSION LOG PLOT QUESTIONABLE CRITERION
CROO FORD CROO FORD
63017 63017
GL L R

\section{COMPOUND NO $=219$ MOL WGT - NATURAL DISTRIB$$
P
$$ \\ ON OF HEAD GROUPS

\begin{abstract}
$1.14 \times 10-4 \times$
\end{abstract} 25

2 ENTRIES FOR COMPOUND

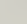

SURFACE TENSION LOG PLOT CROO FORD QUESTIONABLE CRITERION

CROO FORD

63017

63017

GL L

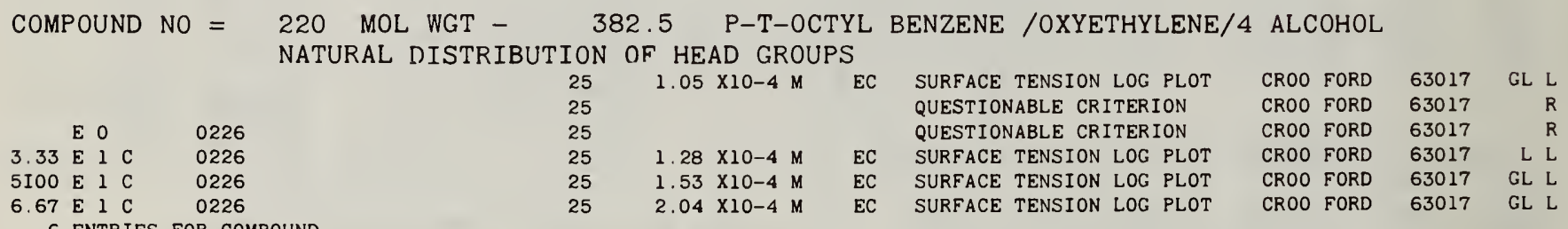

426.6 P-T-OCTYL BENZENE /OXYETHYLENE/5 ALCOHOL 221 MOL WGT - 426.6 P-T-OCTY
NATURAL DISTRIBUTION OF HEAD GROUPS

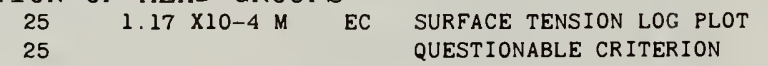

CROO FORD CROO FORD

63017 63017

GL L

2 ENTRIES FOR COMPOUND

470.7 P-T-OSTYL BENZENE /OXYETHYLENE/6 ALCOHOL $\begin{aligned} \text { COMPOUND NO }= & 222 \text { MOL WGT } \\ & \text { NATURAL DISTRIBUTION OF HEAD GROUPS }\end{aligned}$

2 ENTRIES FOR COMPOUND

$25 \quad 1.80 \times 10-4 \mathrm{M}$ EC SURFACE TENSION LOG PLOT CROO FORD
QUESTIONABLE CRITERION CROO FORD

63017 63017

$L$
$R$

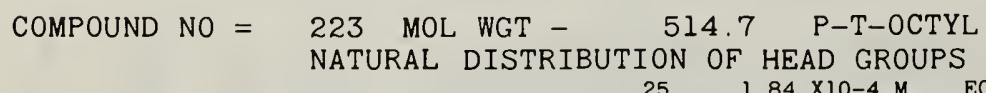

2 ENTRIES FOR COMPOUND $25 \quad 1.84 \times 10-4 \mathrm{M}$ EC SURFACE TENSION LOG PLOT CROO FORD 25 QUESTIONABLE CRITERION CROO FORD

COMPOUND NO $=\quad 224$ MOL WGT $-558.8 \quad$ P-T-OCTYL
NATURAL DISTRIBUTION OF HEAD GROUPS

2 ENTRIES FOR COMPOUND
25

$2.47 \times 10-4$

\section{EC}

SURFACE TENSION LOG PLOT QUESTIONABLE CRITERION
ALCOHOL

CROO FORD CROO FORD
63017 63017
GL L
Concentration units: $\mathrm{A}-$ mol \%; $\mathrm{B}-\mathrm{vol} \%$ solvent; $\mathrm{C}-$ mol \% surfactant mixture; D-wt/vol \%; E- \% saturation; $\mathrm{H}-$ wt $\%$ solvent; 1 - mol \% surfactant; $\mathrm{K}$-normality counterions; $\mathrm{M}$ - molar; $\mathrm{N}$ - normal: $\mathrm{P}-$ wt \%; $\mathrm{Q}$ - wt \% surfactant; $\mathrm{R}$-varied; $\mathrm{S}-$ mol/ kg; $\mathrm{T}-\mathrm{wt} \%$ surfactant mixture; $\mathrm{U}-\mathrm{mol} /(\mathrm{l}$ or kg); $\mathrm{W}-$ molal; $\mathrm{Y}$ - atm. Details on page 222. 


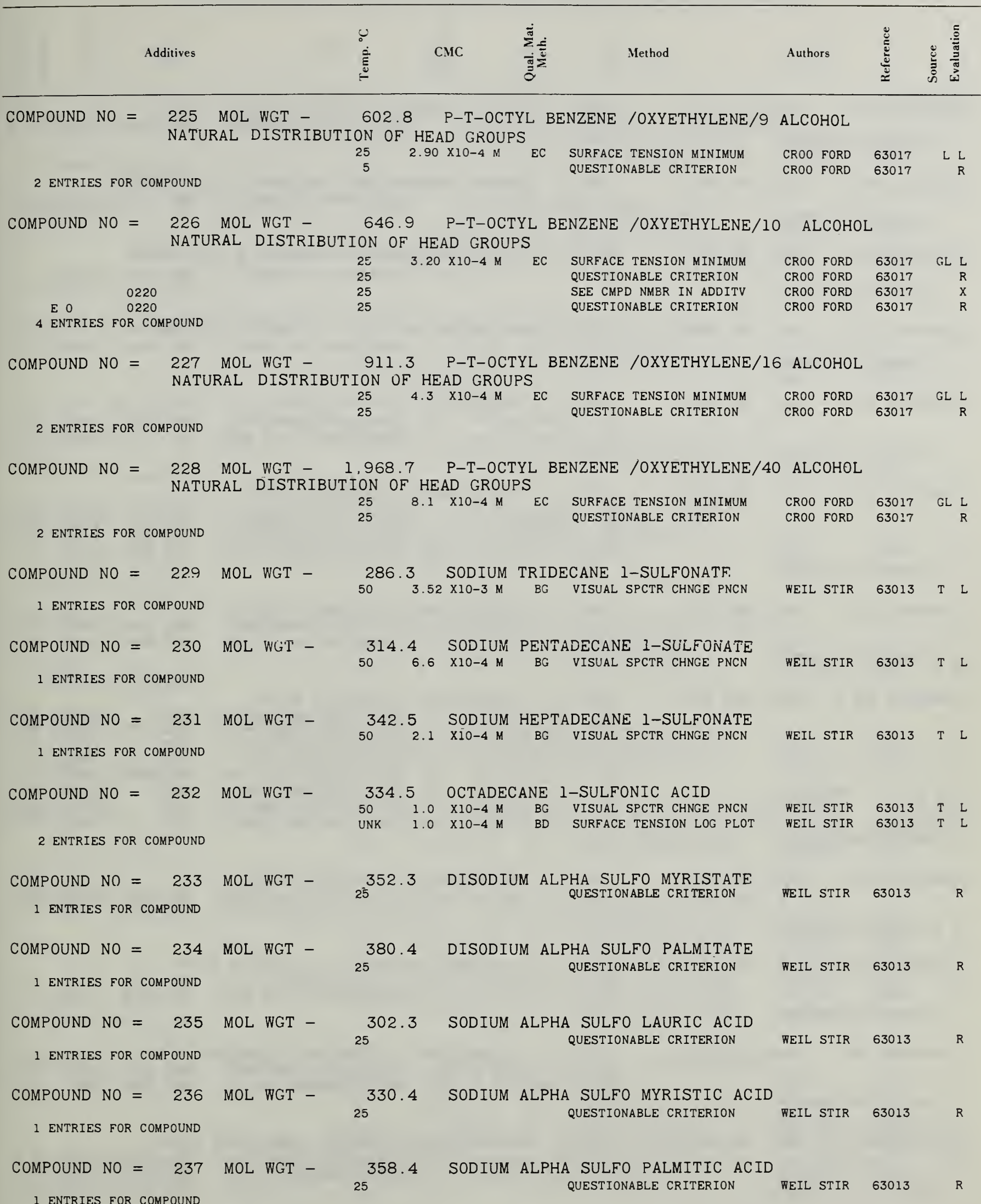

Concentration units: $\mathrm{A}-\mathrm{mol} \% ; \mathrm{B}-\mathrm{vol} \%$ solvent; $\mathrm{C}-$ mol \% surfactant mixture; 


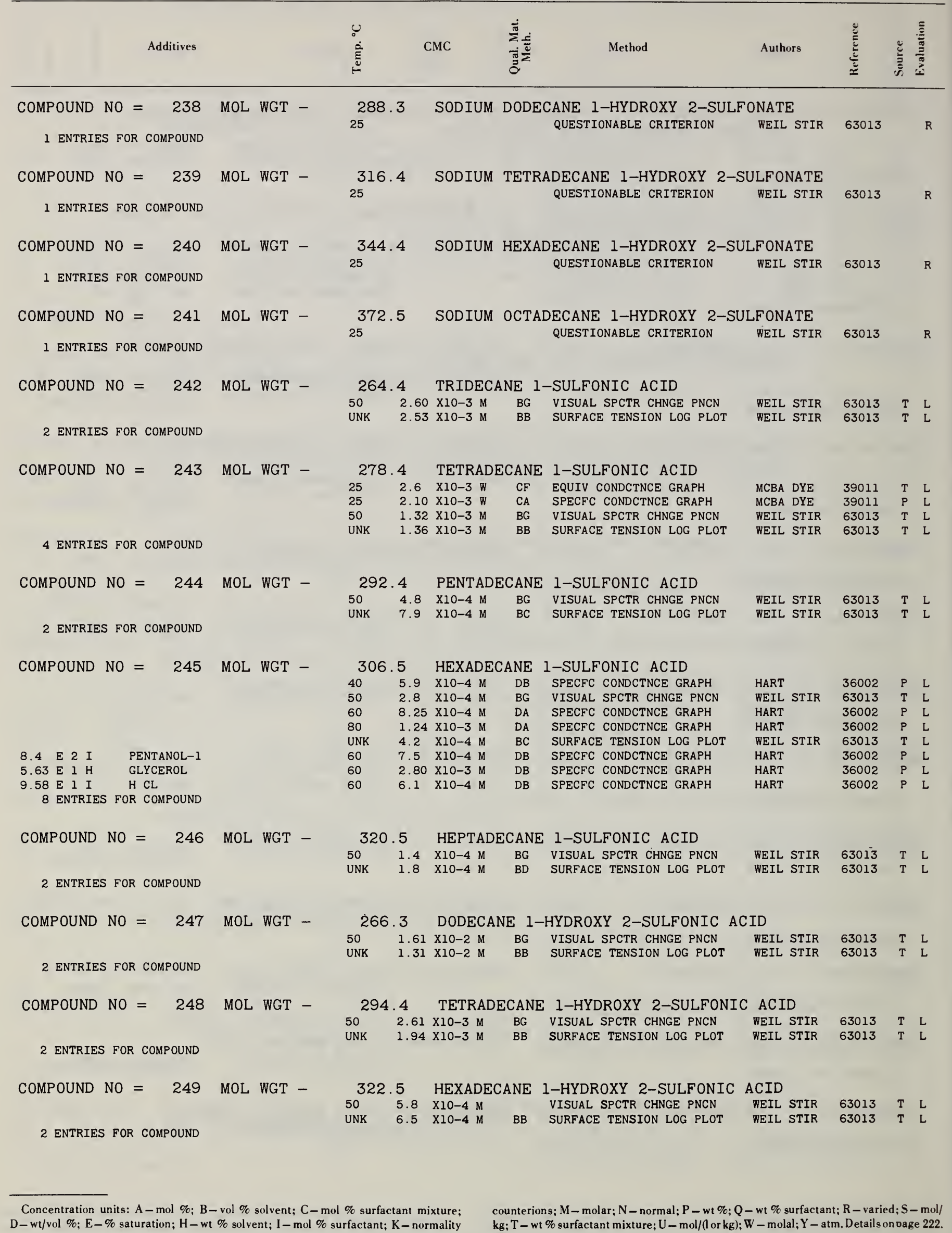




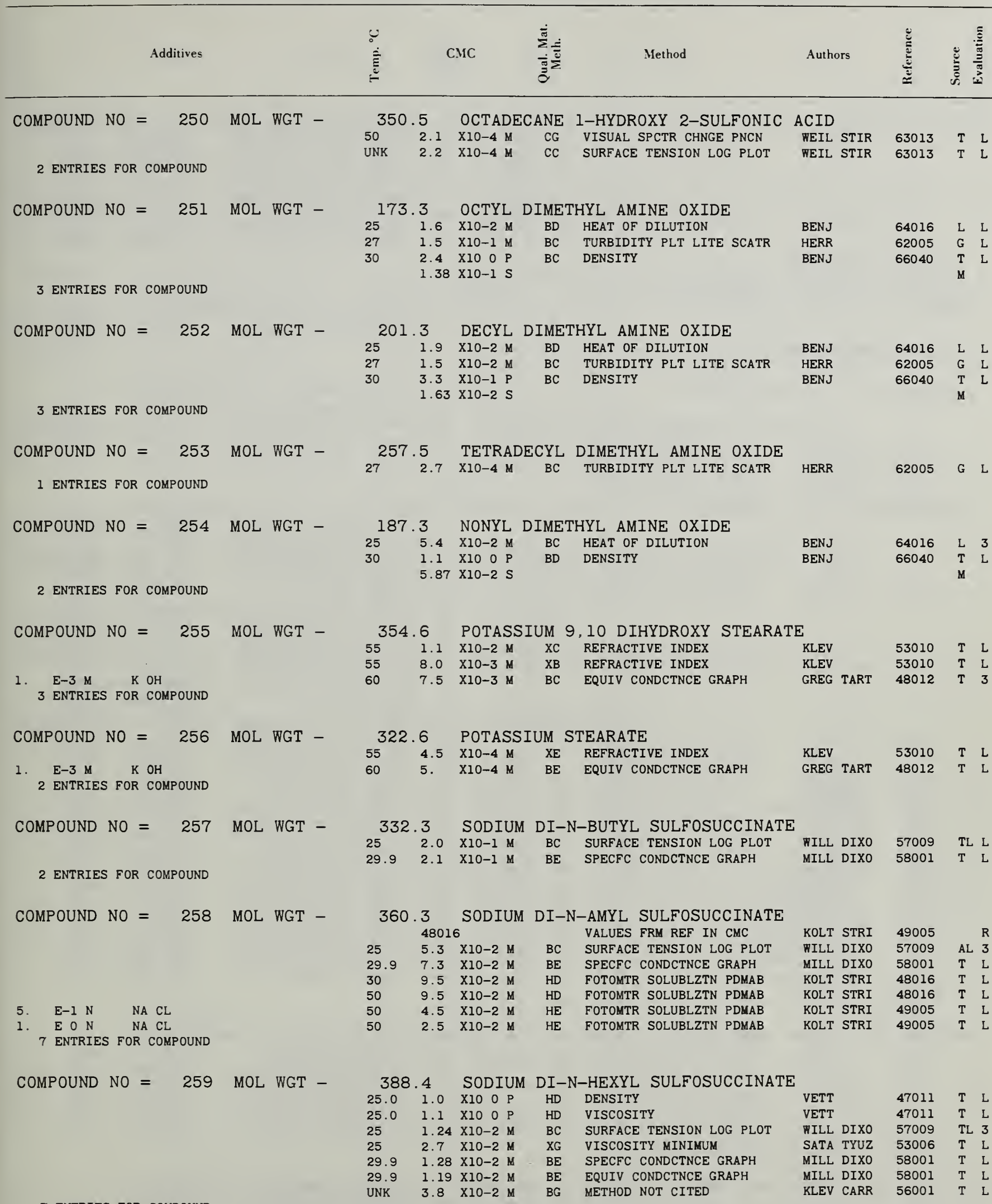

7 ENTRIES FOR COMPOUND

444.5 SODIUM DI-N-OCTYL SULFOSUCCINATE

$20 \quad 4.5$ X10-3 M HG FOTOMTR SPCTR CHNGE PNCN TAUB KONS 60033 T L

COMPOUND NO $=260$ MOL WGT - 


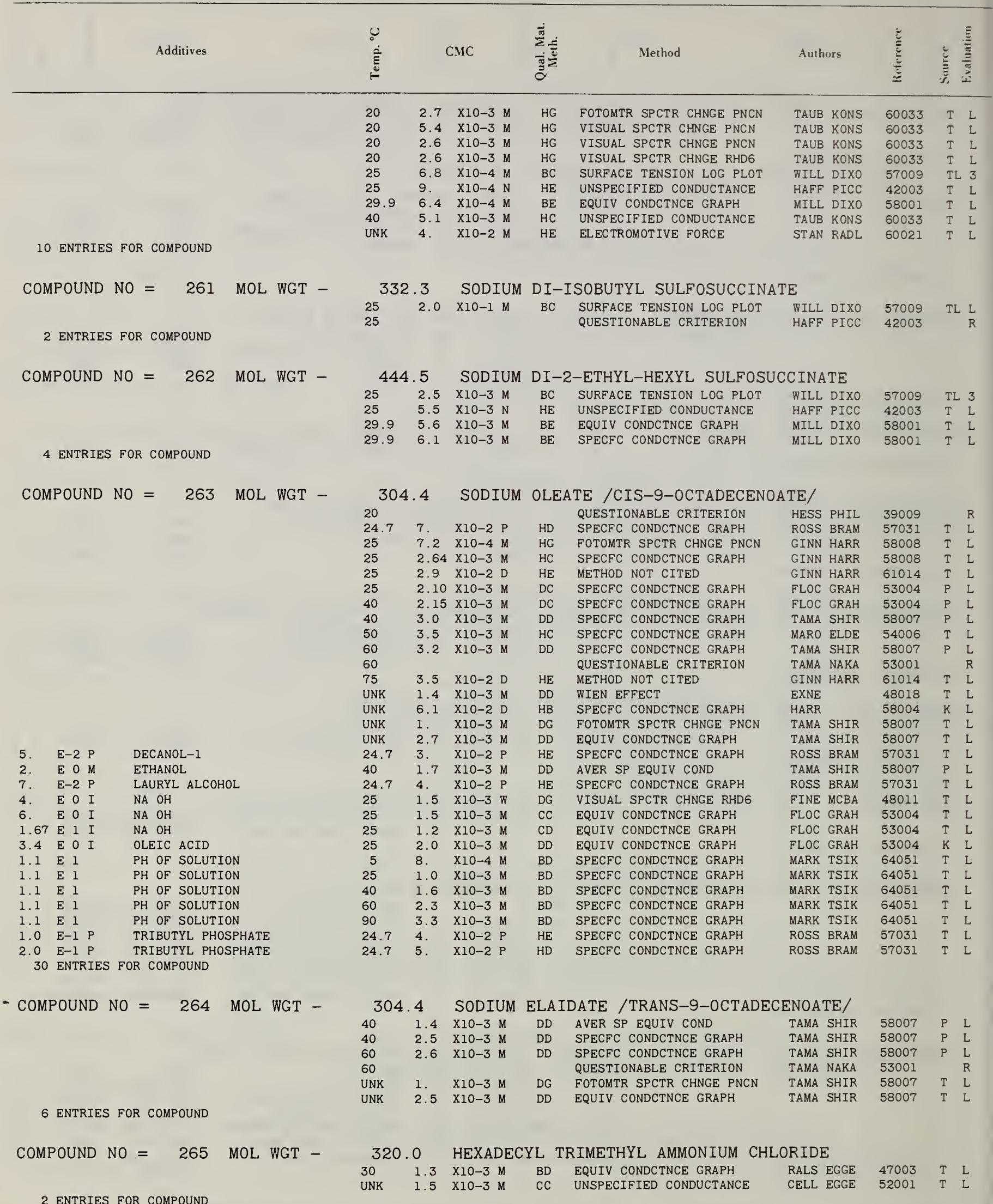

2 ENTRIES FOR COMPOUND 


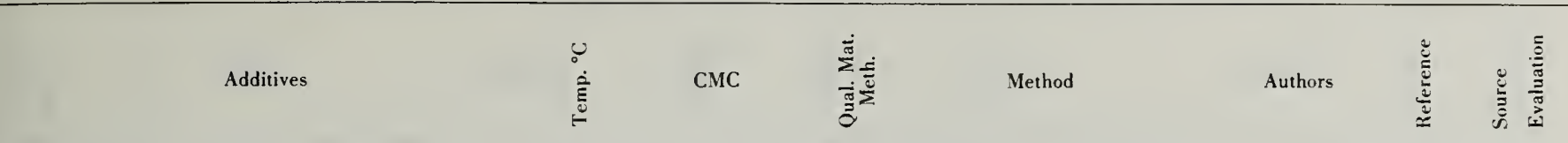

COMPOUND NO $=266$ MOL WGT -

1 ENTRIES FOR COMPOUND

COMPOUND NO $=267$ MOL WGT -

1 ENTRIES FOR COMPOUND

COMPOUND NO $=268$ MOL WGT -

1 ENTRIES FOR COMPOUND

COMPOUND NO $=269$ MOL WGT -

1 ENTRIES FOR COMPOUND

COMPOUND NO $=270$ MOL WGT -

4.74 E O H METHANOL

5 ENTRIES FOR COMPOUND

COMPOUND NO $=271$ MOL WGT -

2 ENTRIES FOR COMPOUND

COMPOUND NO $=272$ MOL WGT -

1 ENTRIES FOR COMPOUND

COMPOUND NO $=273$ MOL WGT -
350.0 HEXADECYL DIMETHYL 2-HYDROXYETHYL AMMONIUM CHLORIDE

$30 \quad 1.2 \quad \times 10-3 \mathrm{M} \quad$ CC EQUIV CONDCTNCE GRAPH RALS EGGE 47003 T $\mathrm{L}$

380.1 HEXADECYL DI-/2-HYDROXYETHYL/METHYL AMMONIUM CHLORIDE

$30 \quad 1.0 \quad \mathrm{X} 10-3 \mathrm{M} \quad \mathrm{CC}$ EQUIV CONDCTNCE GRAPH RALS EGGE 47003 T L

410.1 HEXADECYL TRI-/2-HYDROXYETHYL/ AMMONIUM CHLORIDE $30 \quad 1.0 \quad \times 10-3 \mathrm{M} \quad$ CC EQUIV CONDCTNCE GRAPH $\quad$ RALS EGGE $47003 \quad \mathrm{~T}$ L

380.1 HEXADECYLDIMETHYL2, 3-DIHYDROXYPROPYLAMMONIUM CHLORIDE $30 \quad 1.6 \quad \mathrm{X} 10-3 \mathrm{M} \quad$ CC $\quad$ EQUIV CONDCTNCE GRAPH $\quad$ RALS EGGE $47003 \quad \mathrm{~T}$ L

348.1 OCTADECYL TRIMETHYLAMMONIUM CHLORIDE

$\begin{array}{lllllllll}25 & 3.4 & \text { X10-4 M } & \text { BD } & \text { EQUIV CONDCTNCE GRAPH } & \text { GRIE KRAU } & 48010 & \text { T } & \text { L } \\ 30 & 4 . & \text { X10-4 M } & \text { CE } & \text { EQUIV CONDCTNCE GRAPH } & \text { RALS EGGE } & 47003 & \text { T } & \text { L } \\ 30 & 3 . & \text { X10-4 M } & \text { CD } & \text { EQUIV CONDCTNCE GRAPH } & \text { RALS EGGE } & 47003 & \text { K } & \text { L } \\ \text { UNK } & 3.46 & \text { X10-4 M } & \text { CC } & \text { UNSPECIFIED CONDUCTANCE } & \text { CELL EGGE } & 52001 & \text { T } & \text { L } \\ 25 & 4.00 & \text { X10-4 M } & \text { BB } & \text { EQUIV CONDCTNCE GRAPH } & \text { GRIE KRAU } & 48010 & \text { P } & 3\end{array}$

306.3 SODIUM TRI-ISO-PROPYL BENZENE SULFONATE

$\begin{array}{lllllllll}31 & 6.5 & X 10-2 M & \text { FC } & \text { KRARFT POINT SOLUBILITY } & \text { SHUC LING } & 49004 & \mathrm{~K} & \mathrm{~L}\end{array}$

284.3 TRI-ISOPROPYL BENZENE SULFONIC ACID

$\begin{array}{lllllllll}50 & 5.5 & \times 10-2 \mathrm{M} & \text { FB EQUIV CONDCTNCE GRAPH } & \text { SHUC LING } & 49004 & \text { T } & 3\end{array}$
Concentration units: $\mathrm{A}-\mathrm{mol} \% ; \mathrm{B}-\mathrm{vol} \%$ solvent: $\mathrm{C}-$ mol \% surfactant mixture;

$\mathrm{D}-$ wt $/ \mathrm{vol} \%$; E- \% saturation; H-wt \% solvent; I-mol \% surfactant; K-normality

\subsection{SODIUM DODECANOATE}

42004

$2.7 \times 10-2 \mathrm{M} \quad \mathrm{DC}$

$2.84 \times 10-2 \mathrm{M}$

2. $60 \times 10-2 \mathrm{M}$

$2.64 \times 10-2 \mathrm{M}$

$2.6 \times 10-2 \mathrm{M}$

$2.77 \times 10-2 \mathrm{M}$

$2.37 \times 10-2 \mathrm{M}$

$2.44 \times 10-2 \mathrm{M}$

2. $30 \times 10-2 \mathrm{M}$

$2.72 \times 10-2 \mathrm{M}$

$2.55 \times 10-2 \mathrm{M}$

$2.53 \times 10-2 \mathrm{M}$

$2.66 \times 10-2 \mathrm{M}$

$2.50 \times 10-2 \mathrm{M}$

$2.64 \times 10-2 \mathrm{M}$

$2.5 \times 10-2 \mathrm{M}$

$2.69 \times 10-2 \mathrm{M}$

$2.72 \times 10-2 \mathrm{M}$

$2.55 \times 10-2 \mathrm{M}$

$2.3 \times 10-2 \mathrm{M}$

$2.80 \times 10-2 \mathrm{M}$

$1.91 \times 10-2 \mathrm{M}$

48016

$2.15 \times 10-2 \mathrm{M}$

$2.6 \times 10-2 \mathrm{M}$

$2.6 \times 10-2 \mathrm{M}$

$2.51 \times 10-2 \mathrm{M}$

$2.4 \times 10-2 \mathrm{M}$

$2.7 \times 10-2 \mathrm{M}$

\section{VALUES FR}

QUESTIONABLE CRITERION

EQUIV CONDCTNCE GRAPH

FOTOMTR SOLUBLZTN PDMAB

FOTOMTR SOLUBLZTN OROT

VISUAL SPCTR CHNGE

EQUIV CONDCTNCE GRAPH

VISUAL SPCTR CHNGE PNCN

SPECFC CONDCTNCE GRAPH

SURFACE TENSION MINIMUM

EQUIV CONDCTNCE GRAPH

FOTOMTR SOLUBLZTN PDMAB

FOTOMTR SOLUBLZTN OROT

EQUIV CONDCTNCE GRAPH

SPECFC CONDCTNCE GRAPH

EQUIV CONDCTNCE GRAPH

FOTOMTR SOLUBLZTN PDMAB

EQUIV CONDCTNCE GRAPH

FQUIV CONDCTNCE GRAPH

FOTOMTR SOLUBLZTN PDMAB

SURFACE TENSION MINIMUM

VISUAL SPCTR CHNGE PNCN

VALUES FRM REF IN CMC

SPECFC CONDCTNCE GRAPH

PH OR HYDROLYSIS

SOLUBLZTN TOLUENE

SOLUBLZTN TOLUENE

FOTOMTR SOLUBLZTN OROT

ULTRAFILTRATION
FOTOMTR SOLUBLZTN PDMAB

$\begin{array}{llll}\text { BOTR CRES } & 60024 & & \text { R } \\ \text { EKWA } & 40003 & & \text { R } \\ \text { EKWA LIND } & 41004 & & \text { R } \\ \text { EKWA } & 27001 & \text { L } & \text { L } \\ \text { SATA TYUZ } & 53006 & \text { T } & \text { L } \\ \text { HESS PHIL } & 39009 & & \text { R } \\ \text { EKWA } & 42004 & \text { P } & \text { L } \\ \text { GINN KINN } & 59009 & \text { T } & \text { L } \\ \text { GINN KINN } & 59009 & \text { T } & \text { L } \\ \text { KLEV } & 53010 & \text { T } & \text { L } \\ \text { EKWA } & 42004 & \text { P } & \text { L } \\ \text { MERR GETT } & 48024 & \text { T } & \text { L } \\ \text { CAMP LAKS } & 65024 & \text { T } & 3 \\ \text { CAMP LAKS } & 65024 & \text { T } & \text { L } \\ \text { EKWA } & 42004 & \text { P } & \text { L } \\ \text { KOLT STRI } & 48016 & \text { T } & \text { L } \\ \text { KOLT STRI } & 48016 & \text { T } & \text { L } \\ \text { EKWA } & 42004 & \text { P } & \text { L } \\ \text { CAMP LAKS } & 65024 & \text { T } & \text { L } \\ \text { EKWA } & 42004 & \text { P } & \text { L } \\ \text { KOLT JOHN } & 46006 & \text { T } & \text { L } \\ \text { EKWA } & 42004 & \text { P } & \text { L } \\ \text { EKWA } & 42004 & \text { P } & \text { L } \\ \text { KOLT STRI } & 48016 & \text { T } & \text { L } \\ \text { KOLT JOHN } & 46006 & \text { T } & \text { L } \\ \text { CAMP LAKS } & 65024 & \text { T } & \text { L } \\ \text { RAIS } & 52016 & \text { T } & \text { L } \\ \text { KOLT STRI } & 49005 & & \text { R } \\ \text { MARO ELDE } & 54006 & \text { T } & \text { L } \\ \text { STAU } & 39006 & \text { G } & \text { L } \\ \text { DEMC DUMA } & 60032 & \text { T } & \text { L } \\ \text { DEMC } & 60034 & \text { T } & \text { L } \\ & & \text { M } & \\ \text { MERR GETT } & 48024 & \text { T } & \text { L } \\ \text { EKWA } & 27001 & \text { L } & \text { L } \\ & & & \end{array}$

counterions; M-molar; $\mathrm{N}$ - normal; $\mathrm{P}$ - wt \%; $\mathrm{Q}$ - wt \% surfactant; $\mathrm{R}$-varied; $\mathrm{S}-\mathrm{mol} /$ kg; T - wt \% surfactant mixture; $\mathrm{U}-\mathrm{mol} /(\mathrm{l}$ or kg); $\mathrm{W}-$ molal; $\mathrm{Y}-\mathrm{atm}$. Details on page 222. 


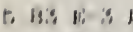

I $11 \mathrm{NO}: \mathrm{M}$

$\therefore$ III $11: 19: 14$

2 ist 11; $\because 14$

s) 60 11: $: 1 \mathrm{M}$

1 Uf: $11 ;: M$ Ii: 1

: IIS $11: \because \mathrm{M}$

$\therefore$ () $11: \therefore \mathrm{M}$

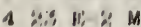

6. 1 II: $\because \mathrm{M}$

Vi: - I II

Ii - I II

V. 0 II

Ii) 0

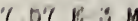

$10: 16: M$

I $: 14$ Vif : M

$\therefore 6,11 \%: M$

$\therefore 6:$ II: $\because M$

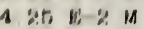

1 f.6, Ii: : $M$

P B6 $\mathrm{n}_{\mathrm{i}}: \mathrm{M}$

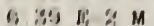

II: (I)

(i) $(0$

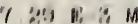

1. 07 11: $\because \mathrm{M}$

$\therefore 17 \mathrm{H} \div \mathrm{M}$

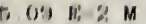

6. An $\mathrm{Bi}: \mathrm{M}$

7 13A N1: $: M$

in: 0 i

ii: 0

H: I II

Hil 1 II

in: 0

1. 131 Hi: $: \mathrm{M}$

$\therefore 15 \mathrm{H}: \mathrm{M}$

$4 \therefore H: M$

1. 1 II: $\therefore \mathrm{M}$

$\therefore 0111: \because M$

$\because, 0, \quad 11: M M$

$\therefore$ ID $10: M$

(1) \& 1 is $\mathrm{M}$

15,4 II: : M

$\because$ i) $l: \because M$

$\therefore 41 \mathrm{II}: \mathrm{M}$

40 Hi: $\mathrm{M}$

Ii, ()

Iit ()

(1) I) $11:$ : $\mathrm{M}$

1 cif li: $\because \mathrm{M}$

$\because 1 / 18$ lli: $\because \mathrm{M}$

$\because 11 \%$ 11: $: \mathrm{M}$

a $4011: \because: M$ II:

6) 151 HI: $\therefore$

$7,698 \mathrm{NI} M$

$4: 2 \mathrm{VI} \therefore \mathrm{M}$

I 114 11: $\because \mathrm{M}$

1 : 11 : $M$

atrit bi: $M$

(19 II: $\mathrm{M}$

b) it if: $: \mathrm{M}$

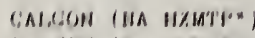

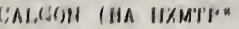

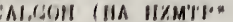

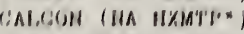

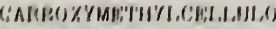

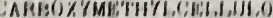

IIA II l:C):

IIA $1 \%$.

IIA $(\%$,

IA (i)

in lis.

IIA CI.

ia Ci

$\mathrm{IA} \mathrm{Cl}$

IIA CIL.

IIA: (U):

11A: $(\%):$

IIA: CO):

1A: C:

1A: $(: 0)$

IA: $60 \%$

$11 \mathrm{~A}: 60 \%$

A: $8: 0$ :

11A: (:0):

HA:

IIA IIOS:

IIA GII

HA OHI

IIA (NI

HA OII

IIA OII

IIA OII

IIA (BII

IIA OII

IIA OHI

IIA OHI

IIA OII

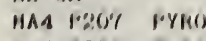

llaA loo' rYHO

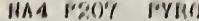

lina listi? P'YMO)

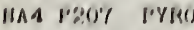

llaA l'so't PYRO

HAS räIT P'YRO

HA HOIA

IIA POCA

IIA rolas

IIA HoA

HA HOCA

HA l'()A

HA: :104

IIA:: BAO'l

HA: HAC)'

HA: HAOT

HA: $1: 40$ )?

11A2 BAOI'

IIA:? HAOT

HA: BHOS MEITA

HA: :IBO: MR:I'A

HA? IIIO), MHII'A

WA: BSOS: MH'I'A

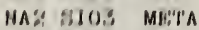

MA: HII): MH'TA

HA: HIO:S META

HA: HIOS MR:'A

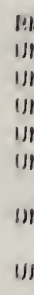

IIM $\because 0 \quad \% 10 \div 4$

IIIIK $2 \therefore \% 10 \div 19$

IIIIK $\therefore 2 \% 10 \geqslant 10$

IIIK $\therefore$.

lins to ', alo-1 ll

$\because 6,5 \times 10: 14$

1) 5 \% $10-1$ i

$\therefore, 6, \% 10: 14$

5. : $\%(10$ 1 11

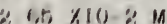

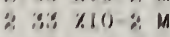

$\because$ af $\% 10: M$

औ1: $210 \div \mathrm{M}$

$21 \% 710: 14$

$1,08 \% 10 \% \mathrm{M}$

I $14: \% 10: M$

untr.

it

its

3

50

fic)

20

$\therefore$

$\therefore b$

to

st.

at,

IIIIV.

IIIIK

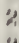

it

: its

(1)

()

IIIK

ats

$\therefore t$

$\therefore 1$,

:

:if

$\because 15$

$\therefore 6$

IIIKK

נII

$\therefore t$

IIIIK

:ti $\because$ ot $x i 0: \mathrm{M}$

1. $10 \times 10 \div: M$

I (B) $\mathrm{X}(0): \mathrm{M}$

I. fia $X 10: \because M$

1. $58: \times 10: \because M$

$1 \because 1 \times 10 \div \mathrm{M}$

$101 \times 10=: M$

if 6 X $10-3 \mathrm{M}$

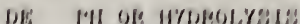

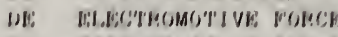

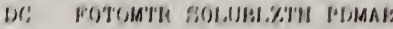

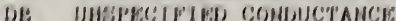

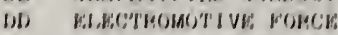

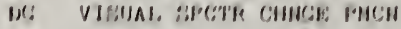

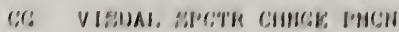

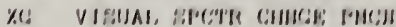

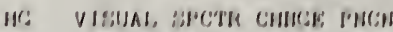

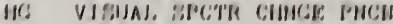

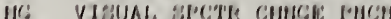

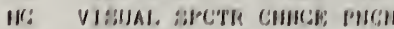

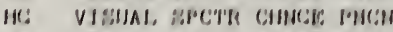

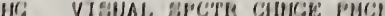

EKAIII BAT'A HO'T BH:TRIEVB:H

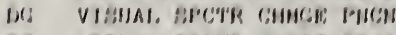

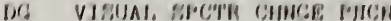

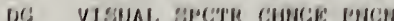

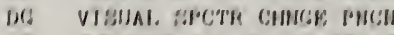

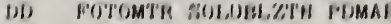

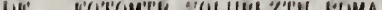

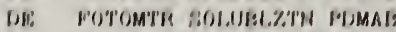

CRAPII DAT'A HO'T RE'TRIEVEIS

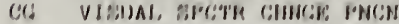

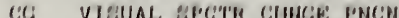

(e) VISHAL, BIC'TR CHISE: I'NCN

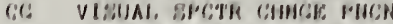

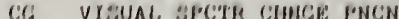

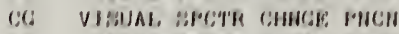

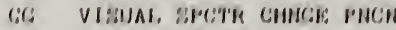

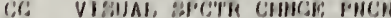

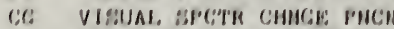

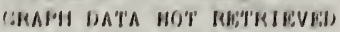

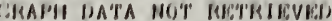

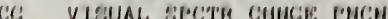

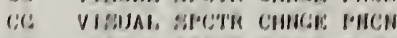

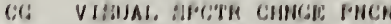

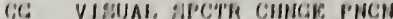

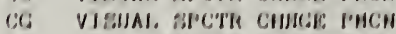

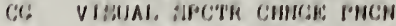

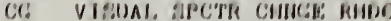

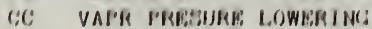

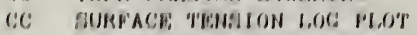

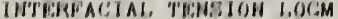

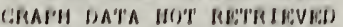

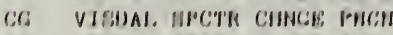

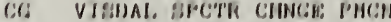

CU VIEIJAL, BOC'T'K CIINGE PIICN

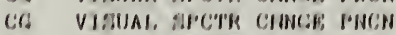

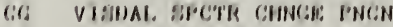

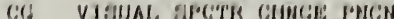

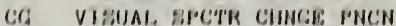

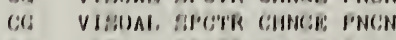

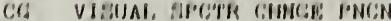

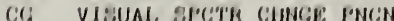

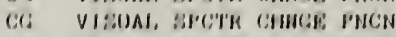

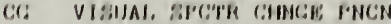

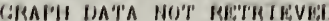

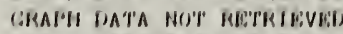

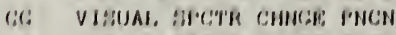

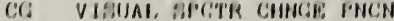

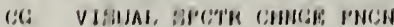

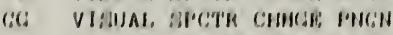

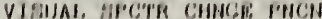

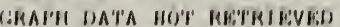

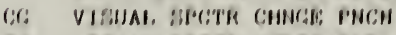

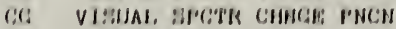

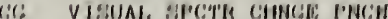

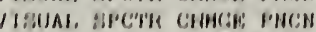

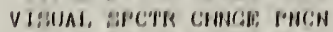

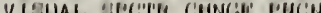

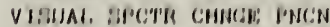

VISIIAAL. BFC'TR CHNCH: I'NC:N

EYWA

CARKI Jomi:

cinell Jormi

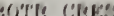

bothe lifelens

DKME:

DFME: 
Additives

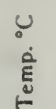

CMC

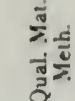

Merliod

Authors

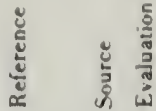

$8.9 E-3 M$

$1.52 \mathrm{E}-2 \mathrm{M}$

$1.57 \mathrm{E}-2 \mathrm{M}$

$2.05 \mathrm{E}-2 \mathrm{M}$

$2.47 \mathrm{E}-2 \mathrm{M}$

$3.34 \mathrm{E}-2 \mathrm{M}$

1.25 E-2 M

$2.5 \mathrm{E}-2 \mathrm{M}$

$9.8 \mathrm{E}-3 \mathrm{M}$

$2.09 \mathrm{E}-2 \mathrm{M}$

$2.84 \mathrm{E}-2 \mathrm{M}$

$3.46 \mathrm{E}-2 \mathrm{M}$

$4.35 \mathrm{E}-2 \mathrm{M}$

$5.82 \mathrm{E}-2 \mathrm{M}$

$2.5 \quad \mathrm{E}-2 \mathrm{M}$

$4.3 \mathrm{E}-3 \mathrm{M}$

$8.3 \mathrm{E}-3 \mathrm{M}$

1. $20 \mathrm{E}-2 \mathrm{M}$

$2.5 \mathrm{E}-2 \mathrm{M}$

1.1 E 1

1.1 E 1

1.1 E 1

1.1 E I

1.1 E I

1.1 E 1

1.1 E 1

3. $2 \mathrm{E}-4 \mathrm{M}$

1.1 E 1

$7.0 \quad \mathrm{E}-4 \mathrm{M}$

$1.1 \mathrm{E} 1$

$8.9 \mathrm{E}-4 \mathrm{M}$ 1.1 E 1

1. $20 \mathrm{E}-3 \mathrm{M}$ $1.1 \mathrm{E} 1$

$\begin{array}{rl}1.51 \mathrm{E}-3 \mathrm{M} & \\ 1.1 \mathrm{E} & 1\end{array}$ $1.1 \mathrm{E}$

1. 1 E 1

$3.2 \mathrm{E}-4 \mathrm{M}$ $1.1 \mathrm{E}$

$1.02 \mathrm{E}-3 \mathrm{M}$ $\begin{array}{ll}1.1 & \mathrm{E} \\ \mathrm{E}-3 \mathrm{M} & \end{array}$ 1.1 E 1

4. $10 \mathrm{E}-3 \mathrm{M}$ 1.1 E 1

4. 6 E-5 M 1. 1 E 1

$8.5 \mathrm{E}-5 \mathrm{M}$ 1.1 E 1

$1.05 \mathrm{E}-4 \mathrm{M}$ 1.1 E 1

1. $46 \mathrm{E}-4 \mathrm{M}$ 1.1 E 1

$1.61 \mathrm{E}-4 \mathrm{M}$ 1.1 E 1

$1.99 \mathrm{E}-4 \mathrm{M}$ 1.1 E 1

$7.7 \quad \mathrm{E}-3 \mathrm{M}$ 1.1 E 1

$1.12 \mathrm{E}-2 \mathrm{M}$ 1.1 E 1

$1.58 \mathrm{E}-2 \mathrm{M}$ 1.1 E 1

2. $20 \mathrm{E}-2 \mathrm{M}$ 1.1 E 1

2.92 E-2 M 1.1 E 1

3. $10 \mathrm{E}-2 \mathrm{M}$ 1.1 E 1

$4.02 \mathrm{E}-2 \mathrm{M}$ 1.1 E 1

$4.11 \mathrm{E}-2 \mathrm{M}$ 1.1 E 1

$4.97 \mathrm{E}-2 \mathrm{M}$ 1. 1 E 1
SI02/NA20 $=1.60$

SI02/NA2O $=1.60$

SI02/NA20 $=1.60$

SI02/NA20 $=1.60$

SI02/NA2O $=1.60$

SI02/NA20 $=1.60$

SI02/NA2O $=1,60$

SI02/NA2O $=1.60$

SI02/NA20 $=2.46$

$5102 /$ NA20 $=2.46$

SI02/NA20 $=2.46$

SI02/NA2O $=2.46$

SI02/NA20 $=2.46$

SI02/NA2O $=2.46$

SI02/NA2O $=2.46$

SIO2/NA2O $=3.93$

SI02/NA2O $=3.93$

SI02/NA2O $=3.93$

SI02/NA2O $=3.93$

PH OF SOLUTION

PH OF SOLUTION

PH OF SOLUTION

PH OF SOLUTION

PH OF SOLUTION

PH OF SOLUTION

PH OF SOLUTION

1.2 DECANE DIOL

PH OF SOLUTION

1,2 DECANE DIOL

PH OF SOLUTION

1,2 DECANE DIOL PH OF SOLUTION

1.2 DECANE DIOL

PH OF SOLUTION

1,2 DECANE DIOL

PH OF SOLUTION

1,2 DECANE DIOL

PH OF SOLUTION

1. 10 DECANE DIOL

PH OF SOLUTION

1,10 DECANE DIOL

PH OF SOLUTION

1. 10 DECANE DIOL

PH OF SOLUTION

1,10 DECANE DIOL PH OF SOLUTION

DECANOL-1

PH OF SOLUTION

DECANOL-1

PH OF SOLUTION

DECANOL-1

PH OF SOLUTION

DECANOL-1

PH OF SOLUTION

DECANOL-1

PH OF SOLUTION

DECANOL-1

PH OF SOLUTION

HEXANOL-1

PH OF SOLUTION

HEXANOL- 1

PH OF SOLUTION

HEXANOL-1

PH OF SOLUTION

HEXANOL-1

PH OF SOLUTION

HEXANOL-1

PH OF SOLUTION

HEXANOL-1

PH OF SOLUTION

HEXANOL-1

PH OF SOLUTION

HEXANOL-1

PH OF SOLUTION

HEXANOL-1

PH OF SOLUTION

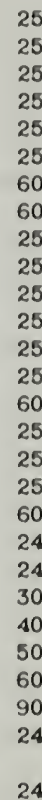

1. $47 \times 10-2 \mathrm{M}$

$1.51 \times 10-2 \mathrm{M}$

1. $32 \times 10-2 M$

1. $19 \times 10-2 \mathrm{M}$

$1.08 \times 10-2 \mathrm{M}$

$1.8 \times 10-2 \mathrm{M}$

$1.4 \times 10-2 M$

$1.96 \times 10-2 \mathrm{M}$

1. $56 \times 10-2 \mathrm{M}$

$1.42 \times 10-2 \mathrm{M}$

1. $30 \times 10-2 \mathrm{M}$

$1.09 \times 10-2 \mathrm{M}$

$9.75 \times 10-3 \mathrm{M}$

$1.4 \times 10-2$

$2.18 \times 10-2 \mathrm{M}$

$2.08 \times 10-2 \mathrm{M}$

$2.00 \times 10-2 \mathrm{M}$

$1.4 \times 10-2 \mathrm{M}$

$2.3 \times 10-2 \mathrm{M}$

$2.3 \times 10-2 M$

$2.25 \times 10-2$

$2.25 \times 10-2 \mathrm{M}$

$2.25 \times 10-2 \mathrm{M}$

$2.25 \times 10-2 M$

$2.25 \times 10-2 \mathrm{M}$

$2.11 \times 10-2 \mathrm{M}$

$1.86 \times 10-2 \mathrm{M}$

$1.80 \times 10-2 \mathrm{M}$

BG

24

24

24

24

24

24

24

24

24

24

24

24

24

24

24

24

24

24

24

24

24

$9.5 \times 10-3 \mathrm{M}$

$8.8 \times 10-3 \mathrm{M}$
$1.71 \times 10-2 \mathrm{M}$

HG VISUAL SPCTR CHNGE PNCN HG VISUAL SPCTR CHNGE PNCN HG VISUAL SPCTR CHNGE PNCN

HG VISUAL SPCTR CHNGE PNCN

HG VISUAL SPCTR CHNGE PNCN

HG VISUAL SPCTR CHNGE PNCN FOTOMTR SOLUBLZTN OROT FOTOMTR SOLUBLZTN OROT VISUAL SPCTR CHNGE PNCN VISUAL SPCTR CHNGE PNCN VISUAL SPCTR CHNGE PNCN VISUAL SPCTR CHNGE PNCN VISUAL SPCTR CHNGE PNCN VISUAL SPCTR CHNCE PNCN FOTOMTR SOLUBLZTN OROT VISUAL SPCTR CHNGE PNCN VISUAL SPCTR CHNGE PNCN VISUAL SPCTR CHNGE PNCN FOTOMTR SOLUBLZTN OROT FOTOMTR SPCTR CHNGE PNCN SURFACE TENSION LOG PLOT SPECFC CONDCTNCE GRAPH SPECFC CONDCTNCE GRAPH SPECFC CONDCTNCE GRAPH SPECFC CONDCTNCE GRAPH SPECFC CONDCTNCE GRAPH FOTOMTR SPCTR CHNGE PNCN

FOTOMTR SPCTR CHNGE PNCN

FOTOMTR SPCTR CHNGE PNCN

FOTOMTR SPCTR CHNGE PNCN

FOTOMTR SPCTR CHNGE PNCN

FOTOMTR SPCTR CHNGE PNCN

FOTOMTR SPCTR CHNGE PNCN

FOTOMTR SPCTR CHNGE PNCN

FOTOMTR SPCTR CHNGE PNCN

FOTOMTR SPCTR CHNGE PNCN

FOTOMTR SPCTR CHNGE PNCN

FOTOMTR SPCTR CHNGE PNCN

FOTOMTR SPCTR CHNGE PNCN

FOTOMTR SPCTR CHNGE PNCN

FOTOMTR SPCTR CHNGE PNCN

FOTOMTR SPCTR CHNGE PNCN

FOTOMTR SPCTR CHNGE PNCN

FOTOMTR SPCTR CHNGE PNCN

HARV

FOTOMTR SPCTR CHNGE PNCN

HARV

FOTOMTR SPCTR CHNGE PNCN

HARV

FOTOMTR SPCTR CHNGE PNCN

HARV

FOTOMTR SPCTR CHNGE PNCN

HARV

FOTOMTR SPCTR CHNGE PNCN

HARV

FOTOMTR SPCTR CHNGE PNCN

MERR GETT

MERR CETT

MERR GETT

MERR GETT

MERR GETT

MERR GETT

MERR GETT

MERR GETT

MERR GETT

MERR GETT

MERR GETT

MERR GET

MERR GETT

ERR GETT

MERR GET 


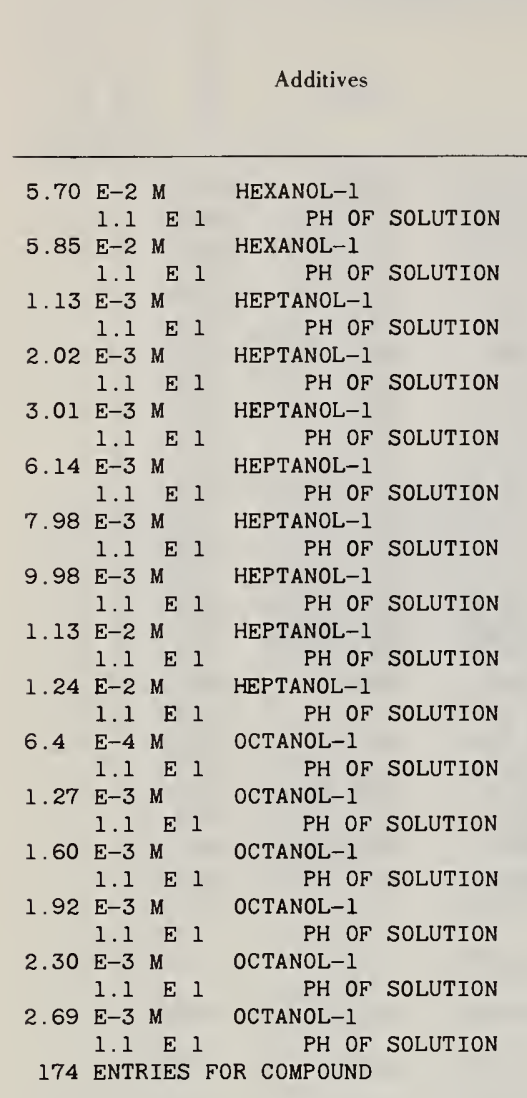

\section{COMPOUND NO $=274$ MOL WGT -}

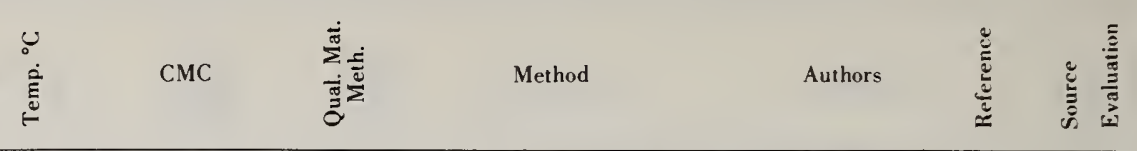

$\begin{array}{lllllllll}24 & 7.1 \times 10-3 \mathrm{M} & \text { BG } & \text { FOTOMTR SPCTR CHNGE PNCN } & \text { HARV } & 56018 & \text { G } & \text { L } \\ 24 & 7.5 \times 10-3 \mathrm{M} & \text { BG } & \text { FOTOMTR SPCTR CHNGE PNCN } & \text { HARV } & 56018 & \text { G } & \text { L } \\ 24 & 2.13 \times 10-2 \mathrm{M} & \text { BG } & \text { FOTOMTR SPCTR CHNGE PNCN } & \text { HARV } & 56018 & \text { G } & \text { L } \\ 24 & 2.06 \times 10-2 \mathrm{M} & \text { BG } & \text { FOTOMTR SPCTR CHNGE PNCN } & \text { HARV } & 56018 & \text { G } & \text { L } \\ 24 & 2.00 \times 10-2 \mathrm{M} & \text { BG } & \text { FOTOMTR SPCTR CHNGE PNCN } & \text { HARV } & 56018 & \text { G } & \text { L } \\ 24 & 1.68 \times 10-2 \mathrm{M} & \text { BG } & \text { FOTOMTR SPCTR CHNGE PNCN } & \text { HARV } & 56018 & \text { G } & \text { L } \\ 24 & 1.50 \times 10-2 \mathrm{M} & \text { BG } & \text { FOTOMTR SPCTR CHNGE PNCN } & \text { HARV } & 56018 & \text { G } & \text { L } \\ 24 & 1.40 \times 10-2 \mathrm{M} & \text { BG } & \text { FOTOMTR SPCTR CHNGE PNCN } & \text { HARV } & 56018 & \text { G } & \text { L } \\ 24 & 1.45 \times 10-2 \mathrm{M} & \text { BG } & \text { FOTOMTR SPCTR CHNGE PNCN } & \text { HARV } & 56018 & \text { G } & \text { L } \\ 24 & 1.55 \times 10-2 \mathrm{M} & \text { BG } & \text { FOTOMTR SPCTR CHNGE PNCN } & \text { HARV } & 56018 & \text { G } & \text { L } \\ 24 & 2.10 \times 10-2 \mathrm{M} & \text { BG } & \text { FOTOMTR SPCTR CHNGE PNCN } & \text { HARV } & 56018 & \text { G } & \text { L } \\ 24 & 1.90 \times 10-2 \mathrm{M} & \text { BG } & \text { FOTOMTR SPCTR CHNGE PNCN } & \text { HARV } & 56018 & \text { G } & \text { L } \\ 24 & 1.93 \times 10-2 \mathrm{M} & \text { BG } & \text { FOTOMTR SPCTR CHNGE PNCN } & \text { HARV } & 56018 & \text { G } & \text { L } \\ 24 & 1.90 \times 10-2 \mathrm{M} & \text { BG } & \text { FOTOMTR SPCTR CHNGE PNCN } & \text { HARV } & 56018 & \text { G } & \text { L } \\ 24 & 1.95 \times 10-2 \mathrm{M} & \text { BG } & \text { FOTOMTR SPCTR CHNGE PNCN } & \text { HARV } & 56018 & \text { G } & \text { L } \\ 24 & 1.95 \times 10-2 \mathrm{M} & \text { BG } & \text { FOTOMTR SPCTR CHNGE PNCN } & \text { HARV } & 56018 & \text { G } & \text { L }\end{array}$

340.0 HEXADECYL PYRIDINIUM CHLORIDE $\begin{array}{lllll}13.0 & 8.4 & \times 10-4 \mathrm{M} & \mathrm{BC} & \text { KRAFFT POINT SOLUBILITY }\end{array}$ $18.5 \quad 8.5 \times 10-4 \mathrm{M} \quad \mathrm{BC}$ INTERFACIAL TNSN UNSPEC $23.5 .0 \times 10-4 \mathrm{M}$ HG STREAMING CURRENT $\begin{array}{lllll}23 & 5.0 & \times 10-4 \mathrm{M} & \text { HG } & \text { STREAMING CURRENT } \\ 25 & 9 . & \mathrm{X} 10-4 \mathrm{M} & \mathrm{BD} & \text { EQUIV CONDCTNCE GRAPH }\end{array}$ $259.0 \quad \times 10-4 \mathrm{M} \quad$ BB FOTOMTR SOLUBLZTN AZBZ $25 \quad 6.7 \quad X 10-4 \mathrm{M} \quad$ BG EQUIV COND MAX BEGINING $253 . \quad \mathrm{X} 10-5 \mathrm{~W}$ MG VISUAL SPCTR CHNGE $2.2 \times 10-3 \mathrm{M}$ $2.36 \times 10-3 \mathrm{M}$ $4.0 \times 10-4 \mathrm{M}$ $1.8 \times 10-4 \mathrm{M}$ 8. $\times 10-5 \mathrm{M}$ $1.6 \times 10-4 \mathrm{M}$ $1.2 \times 10-4 \mathrm{M}$ $8.3 \times 10-5 \mathrm{M}$ $3.2 \times 10-5 \mathrm{M}$ $6.9 \times 10-5 \mathrm{M}$ $3.3 \times 10-5 \mathrm{M}$ $9.6 \times 10-6 \mathrm{M}$ $5.8 \times 10-4 \mathrm{M}$

\section{VISUAL SPCTR CHNGE}

EQUIV COND IST DEVIATION SPECFC CONDCTNCE GRAPH FOTOMTR SOLUBLZTN AZBZ FOTOMTR SOLUBLZTN AZBZ FOTOMTR SOLUBLZTN AZBZ TURBIDITY PLT LITE SCATR TURBIDITY PLT LITE SCATR TURBIDITY PLT LITE SCATR TURBIDITY PLT LITE SCATR TURBIDITY PLT LITE SCATR TURBIDITY PLT LITE SCATR TURBIDITY PLT LITE SCATR EQUIV CONDCTNCE GRAPH

$\begin{array}{llll}\text { ADDI FURM } & 56019 & \text { T } & \text { L } \\ \text { ADDI FURM } & 56019 & \text { T } & \text { L } \\ \text { CARD } & 66011 & \text { T } & \text { L } \\ \text { MALS HART } & 34001 & \text { K } & \text { L } \\ \text { HART } & 38001 & \text { P } & 3 \\ \text { GRIE KRAU } & 49018 & \text { T } & \text { L } \\ \text { FINE MCBA } & 48011 & \text { T } & \text { L } \\ \text { HART } & 36002 & \text { T } & \text { L } \\ \text { HART } & 36002 & \text { P } & 3 \\ \text { HART } & 38001 & \text { P } & \text { L } \\ \text { HART } & 38001 & \text { P } & \text { L } \\ \text { HART } & 38001 & \text { P } & \text { L } \\ \text { ANAC } & 58009 & \text { G } & \text { L } \\ \text { ANAC } & 58009 & \text { G } & \text { L } \\ \text { ANAC } & 58009 & \text { G } & \text { L } \\ \text { ANAC } & 58009 & \text { G } & \text { L } \\ \text { 4NAC } & 58009 & \text { G } & \text { L } \\ \text { ANAC } & 58009 & \text { G } & \text { L } \\ \text { ANAC } & 58009 & \text { G } & \text { L } \\ \text { GRIE KRAU } & 49018 & \text { T } & 3\end{array}$

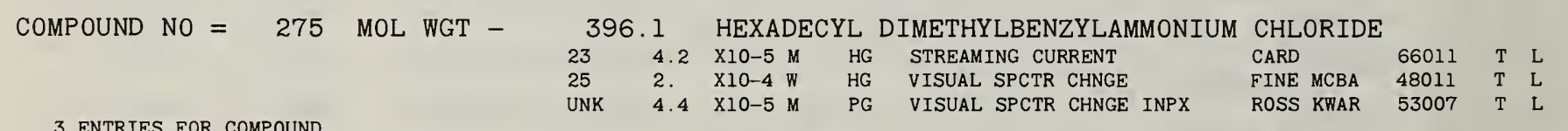

3 ENTRIES FOR COMPOUND

\section{COMPOUND NO $=276$ MOL WGT - 596.8 NONAETHYLENE GLYCOL MONODODECANOATE NATURAL DISTRIBUTION OF HEAD GROUPS}

1 ENTRIES FOR COMPOUND

$0 \quad 6.3 \quad \times 10-4 \mathrm{~W} \quad$ HD FREEZING POINT

GONI MCBA $47007 \quad$ T $\quad$ L

\section{COMPOUND NO $=277$ MOL WGT - 4. EOI C6H5 ( $\mathrm{CH} 3) 3 \mathrm{~N} \mathrm{OH}$ 1 ENTRIES FOR COMPOUND}

349.6 BENZYL TRIMETHYL AMMONIUM DODECANOATE

$25 \quad 1 \quad$ X10-2 M HE EQUIV CONDCTNCE GRAPH $\quad$ BRAD MCBA 48009 T $L$

Concentration units: $\mathrm{A}-\operatorname{mol} \% ; \mathrm{B}-$ vol $\%$ solvent; $\mathrm{C}-$ mol $\%$ surfactant mixture; $\mathrm{D}-$ wt/vol \%; $\mathrm{E}-\%$ saturation; $\mathrm{H}-$ wt $\%$ solvent; I-mol \% surfactant; $\mathrm{K}$ - normality counterions; $\mathrm{M}$-molar; $\mathrm{N}$ - normal; $\mathrm{P}-$ wt \%; $\mathrm{Q}$ - wt \% surfactant: $\mathrm{R}$ - varied; $\mathrm{S}-$ mol/ kg; $\mathrm{T}-\mathrm{wt} \%$ surfactant mixture; $\mathrm{U}-\mathrm{mol} /(\mathrm{lor} \mathrm{kg}) ; \mathrm{W}$ - molal: $\mathrm{Y}$-atm. Details on page 222. 
Additives

$\cup$
$\dot{\mathrm{E}}$

Method

Authors

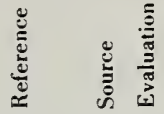

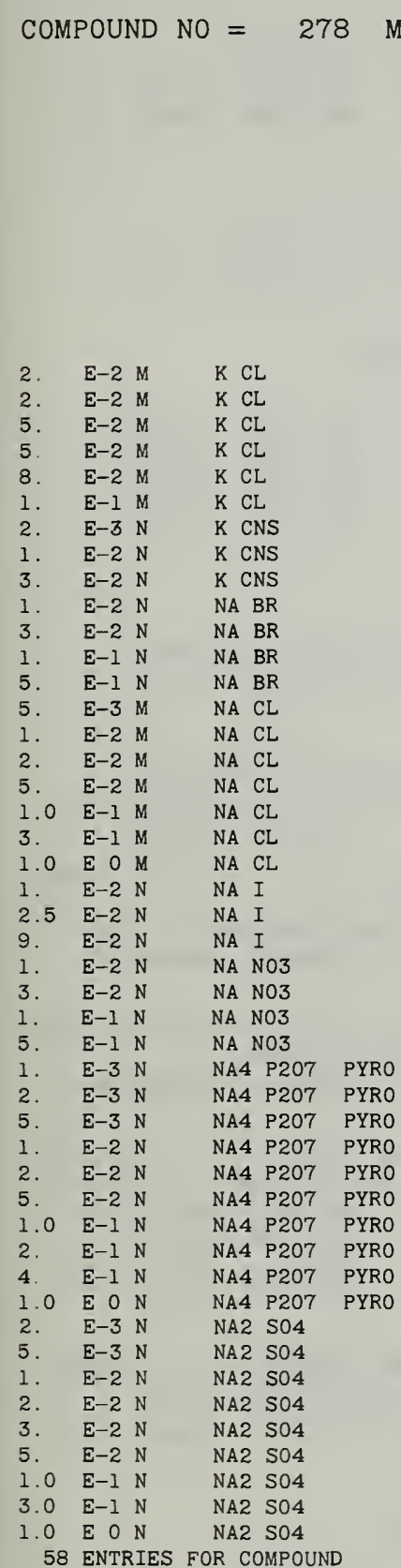

\subsection{DODECYL PYRIDINIUM CHLORIDE}

\section{$25 \quad 1.5 \quad \mathrm{X} 10-2 \mathrm{M}$}

$2.8 \times 10-3 M$

$1.47 \times 10-2 \mathrm{M}$

$1.46 \times 10-2 \mathrm{M}$

$1.4 \times 10-2 \mathrm{M}$

$2.0 \times 10-2 \mathrm{~W}$

$1.74 \times 10-2 \mathrm{M}$

$1.71 \times 10-2 \mathrm{M}$

$1.5 \times 10-2 \mathrm{M}$

$2.0 \times 10-2 \mathrm{~W}$

$1.51 \times 10-2 \mathrm{M}$

$1.13 \times 10-2 \mathrm{M}$

$1.22 \times 10-2 \mathrm{M}$

$8.46 \times 10-3 \mathrm{M}$

$8.60 \times 10-3 \mathrm{M}$

$6.88 \times 10-3 \mathrm{M}$

$2.3 \times 10-3 \mathrm{M}$

$7.5 \times 10-3 \mathrm{M}$

$3.5 \times 10-3 \mathrm{M}$

$2.4 \times 10-3 \mathrm{M}$

9.6. $\times 10-3 \mathrm{M}$

$6.5 \times 10-3 \mathrm{M}$

$2.8 \times 10-3 \mathrm{M}$

$1.5 \times 10-3 \mathrm{M}$

$1.33 \times 10-2 \mathrm{M}$

$1.16 \times 10-2 \mathrm{M}$

$9.3 \times 10-3 \mathrm{M}$

$6.5 \times 10-3 \mathrm{M}$

$4.9 \times 10-3 \mathrm{M}$

$2.9 \times 10-3 \mathrm{M}$

$1.4 \times 10-3 \mathrm{M}$

$6.5 \times 10-3 \mathrm{M}$

$4.0 \times 10-3 \mathrm{M}$

$2.5 \times 10-3 \mathrm{M}$

$9.6 \times 10-3 \mathrm{M}$

$5.6 \times 10-3 \mathrm{M}$

$3.4 \times 10-3 \mathrm{M}$

$1.9 \times 10-3 \mathrm{M}$

$1.01 \times 10-2 \mathrm{M}$

$7.5 \times 10-3 \mathrm{M}$

$6.0 \times 10-3 \mathrm{M}$

$4.7 \times 10-3 \mathrm{M}$

$4.6 \times 10-3 \mathrm{M}$

$4.8 \times 10-3 \mathrm{M}$

$4.6 \times 10-3 \mathrm{M}$

$4.0 \times 10-3 \mathrm{M}$

$3.3 \times 10-3 \mathrm{M}$

$2.2 \times 10-3 \mathrm{M}$

$1.11 \times 10-2 \mathrm{M}$

$8.6 \times 10-3 \mathrm{M}$

$7.8 \times 10-3 \mathrm{M}$

$7.1 \times 10-3 \mathrm{M}$

$6.7 \times 10-3 \mathrm{M}$

$5.9 \times 10-3 \mathrm{M}$

$5.4 \times 10-3 \mathrm{M}$

$3.8 \times 10-3 \mathrm{M}$

$1.8 \times 10-3 \mathrm{M}$
XG QUESTIONABLE CRITERI

HE EQUIV CONDCTNCE GRAPH

CC TURBIDITY PLT LITE SCATR

CB SURFACE TENSION UNSPEC

BC SURFACE TENSION LOG PLOT

CC VAPR PRESURE LOWERING

CB SPECFC CONDCTNCE GRAPH

CG FOTOMTR SPCTR CHNGE EOSN

XC REFRACTIVE INDEX

CC VAPR PRESURE LOWERING

CG VISUAL SPCTR CHNGE EOSN

CC TURBIDITY PLT LITE SCATR

CB SURFACE TENSION UNSPEC

CC TURBIDITY PLT LITE SCATR

CB SURFACE TENSION UNSPEC

CC TURBIDITY PLT LITE SCATR

BC SURFACE TENSION LOG PLOT

CG VISUAL SPCTR CHNGE EOSN

CG VISUAL SPCTR CHNGE EOSN

CG VISUAL SPCTR CHNGE EOSN

CG VISUAL SPCTR CHNGE EOSN

CG VISUAL SPCTR CHNGE EOSN

CG VISUAL SPCTR CHNGE EOSN

CG VISUAL SPCTR CHNGE EOSN

CG VISUAL SPCTR CHNGE EOSN

CG VISUAL SPCTR CHNGE EOSN

CG VISUAL SPCTR CHNGE EOSN

CG VISUAL SPCTR CHNGE EOSN

CG VISUAL SPCTR CHNGE EOSN

CG VISUAL SPCTR CHNGE EOSN

CG VISUAL SPCTR CHNGE EOSN

CG VISUAL SPCTR CHNGE EOSN

CG VISUAL SPCTR CHNGE EOSN

CG VISUAL SPCTR CHNGE EOSN

CG VISUAL SPCTR CHNGE EOSN

CG VISUAL SPCTR CHNGE EOSN

CG VISUAL SPCTR CHNGE EOSN

CG VISUAL SPCTR CHNGE EOSN

CG VISUAL SPCTR CHNGE EOSN

CG VISUAL SPCTR CHNGE EOSN

CG VISUAL SPCTR CHNGE EOSN

CG VISUAL SPCTR CHNGE EOSN

CG VISUAL SPCTR CHNGE EOSN

CG VISUAL SPCTR CHNGE EOSN

CG VISUAL SPCTR CHNGE EOSN

CG VISUAL SPCTR CHNGE EOSN

CG VISUAL SPCTR CHNGE EOSN

CG VISUAL SPCTR CHNGE EOSN

¿G VISUAL SPCTR CHNGE EOSN

CG VISUAL SPCTR CHNGE EOSN

CG VISUAL SPCTR CHNGE EOSN

CG VISUAL SPCTR CHNGE EOSN

CG VISUAL SPCTR CHNGE EOSN

CG VISUAL SPCTR CHNGE EOSN

CG VISUAL SPCTR CHNGE EOSN

CG VISUAL SPCTR CHNGE EOSN

CG
KLEV

BRAD MCBA

FORD OTTE

FORD OTTE

WEIN ZOGR

HUFF MCBA

MEGU KOND

MEGU KOND

KLEV

HUFF MCBA

LANG

FORD OTTE

FORD OTTE

FORD OTTE

FORD OTTE

FORD OTTE

WEIN ZOGR

LANG

LANG

LANG

LANG

LANG

LANG

LANG

LANG

LANG

LANG

LANG

LANG

LANG

LANG

LANG

LANG

LANG

LANG

LANG

LANG

LANG

LANG

LANG

LANG

LANG

LANG

LANG

LANG

LANG

LANG

LANG

LANG

LANG

LANG

LANG

LANG

LANG
52013

53010

$48009 \quad T$

66028 T 3

66028 T $\mathrm{L}$

65026 T L

51004 T L

59024 T $\quad$ L

59024 T L

53010 T L

51004 T L

51005 G L

66028 T L

66028 T $L$

66028 T L

6628 T $\quad$ L

$65026 \mathrm{~T} \cdot \mathrm{L}$

51005 G L

51005 G L

51005 G L

51005 G L

51005 G L

51005 G L

51005 G L

51005 G L

51005 G L

51005 G L

51005 G L

51005 G L

51005 G L

51005 G L

51005 G L

51005 G L

51005 G L

51005 G L

51005 G L

51005 G L

51005 G L

51005 G L

51005 G L

51005 G L

51005 G L

51005 G L

51005 G L

51005 G L

51005 G L

51005 G L

51005 G L

51005 G L

51005 G L

51005 G L

51005 G L

51005 G L

$51005 \quad G \quad L$

51005 G L

51005 G L

51005 G L

COMPOUND NO $=279$ MOL WGT -

340.0 DODECYL BENZYL DIMETHYLAMMONIUM CHLORIDE

OUESTIONABLE CRITERTON

YANG FOST

$25 \quad 2.3 \quad \mathrm{X} 10-3 \mathrm{M} \quad \mathrm{HC}$ EQUIV CONDCTNCE GRAPH

BRAD MCBA

OSUG SATO

$25.0 \quad 7.8 \quad \times 10-3 \mathrm{M} \quad \mathrm{CB}$ SPECFC CONDCTNCE GRAPH

UNK $2.8 \quad X 10-3 \mathrm{M}$ PG VISUAL SPCTR CHNGE INPX

UNK $8.1 \quad \mathrm{X} 10-3 \mathrm{M}$ CC UNSPECIFIED CONDUCTANCE

$25.0 \quad 8.25 \times 10-3 \mathrm{M} \quad \mathrm{CB}$ SPECFC CONDCTNCE GRAPH

ROSS KWAR

CELL EGGE

OSUG SATO

$25.0 \quad 8.5 \times 10-3 \mathrm{M} \quad \mathrm{CB}$ SPECFC CONDCTNCE GRAPH

$25.0 \quad 8.2 \quad \mathrm{X} 10-3 \mathrm{M} \quad \mathrm{CB}$ SPECFC CONDCTNCE GRAPH

$25.0 \quad 7.5 \quad \mathrm{X} 10-3 \mathrm{M} \quad \mathrm{CB}$ SPECFC CONDCTNCE GRAPH

OSUG SATO

OSUG SATO

OSUG SATO

OSUG SATO

$\begin{array}{lllll}25.0 & 7.1 & X 10-3 \mathrm{M} & \mathrm{CB} & \text { SPECFC CONDCTNCE GRAPH } \\ 25.0 & 6.8 & \mathrm{X} 10-3 \mathrm{M} & \mathrm{CB} & \text { SPECFC CONDCTNCE GRAPH }\end{array}$

OSUG SATO

53015

48009

65036 G 3

53007 T $\quad$ L

52001 T $L$

65036 G 3

65036 G 3

65036 G 3

$65036 \quad G \quad 3$

$65036 \quad G \quad 3$

65036 G 3

11 ENTRIES FOR COMPOUND

Concentration units: $\mathrm{A}-\mathrm{mol} \%: \mathrm{B}-\mathrm{vol} \%$ solvent: $\mathrm{C}-\mathrm{mol} \%$ surfactant mixture: 


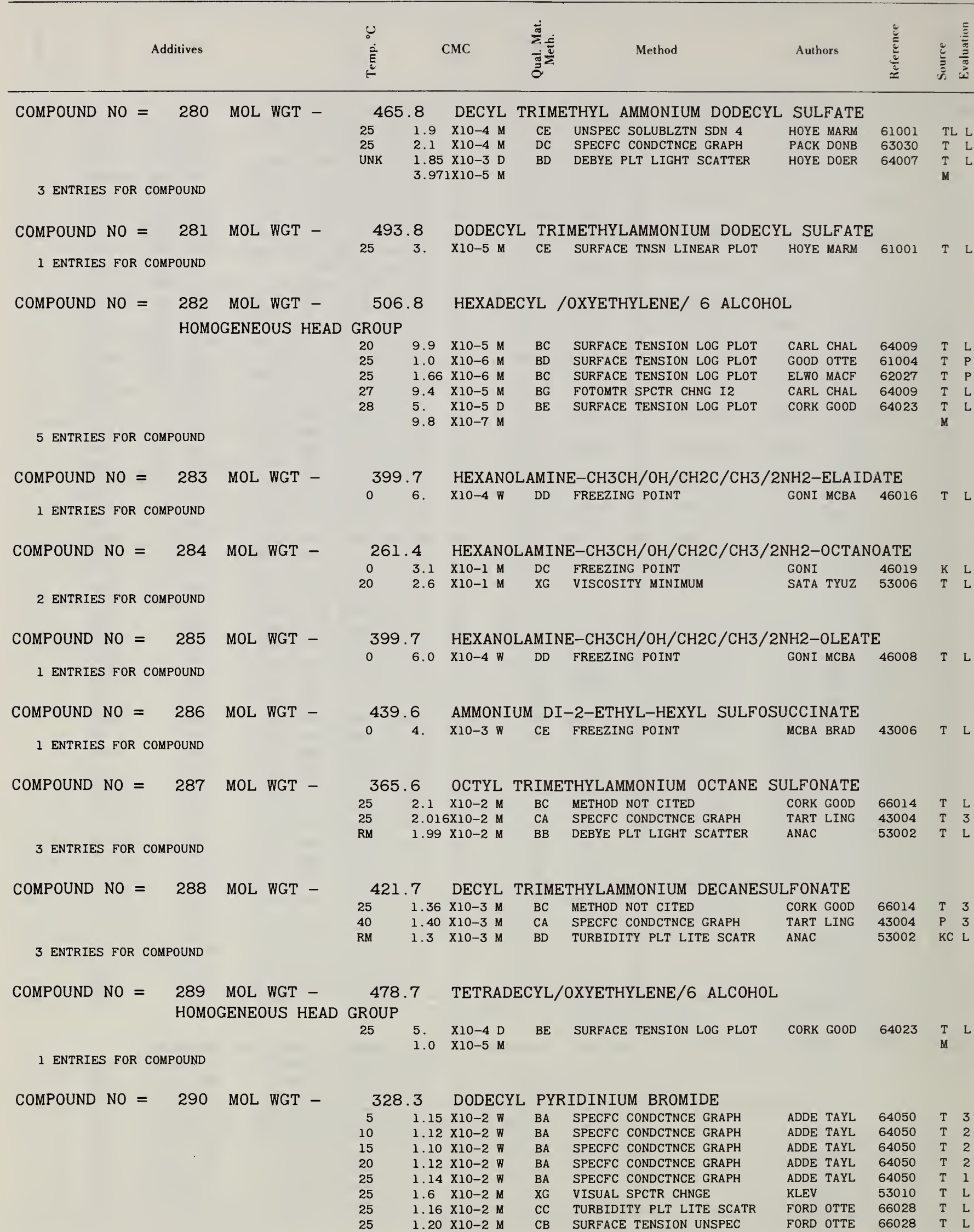

Concentration units: $\mathrm{A}-\mathrm{mol} \% ; \mathrm{B}-\mathrm{vol} \%$ solvent; $\mathrm{C}-$ mol \% surfactant mixture $\mathrm{D}-\mathrm{wt} / \mathrm{vol} \%$; E-\% saturation; $\mathrm{H}-$ wt \% solvent; I-mol \% surfactant; $\mathrm{K}-$ normality counterions; $\mathrm{M}$ - molar; $\mathrm{N}$ - normal; $\mathrm{P}$ - wt \%; $\mathrm{Q}$ - wt \% surfactant; $\mathrm{R}$-varied; $\mathrm{S}$ - mol/ kg; T-wt \% surfactant mixture; $U$ - mol/( orkg); W-molal; $Y$ - atm. Details on page 222. 


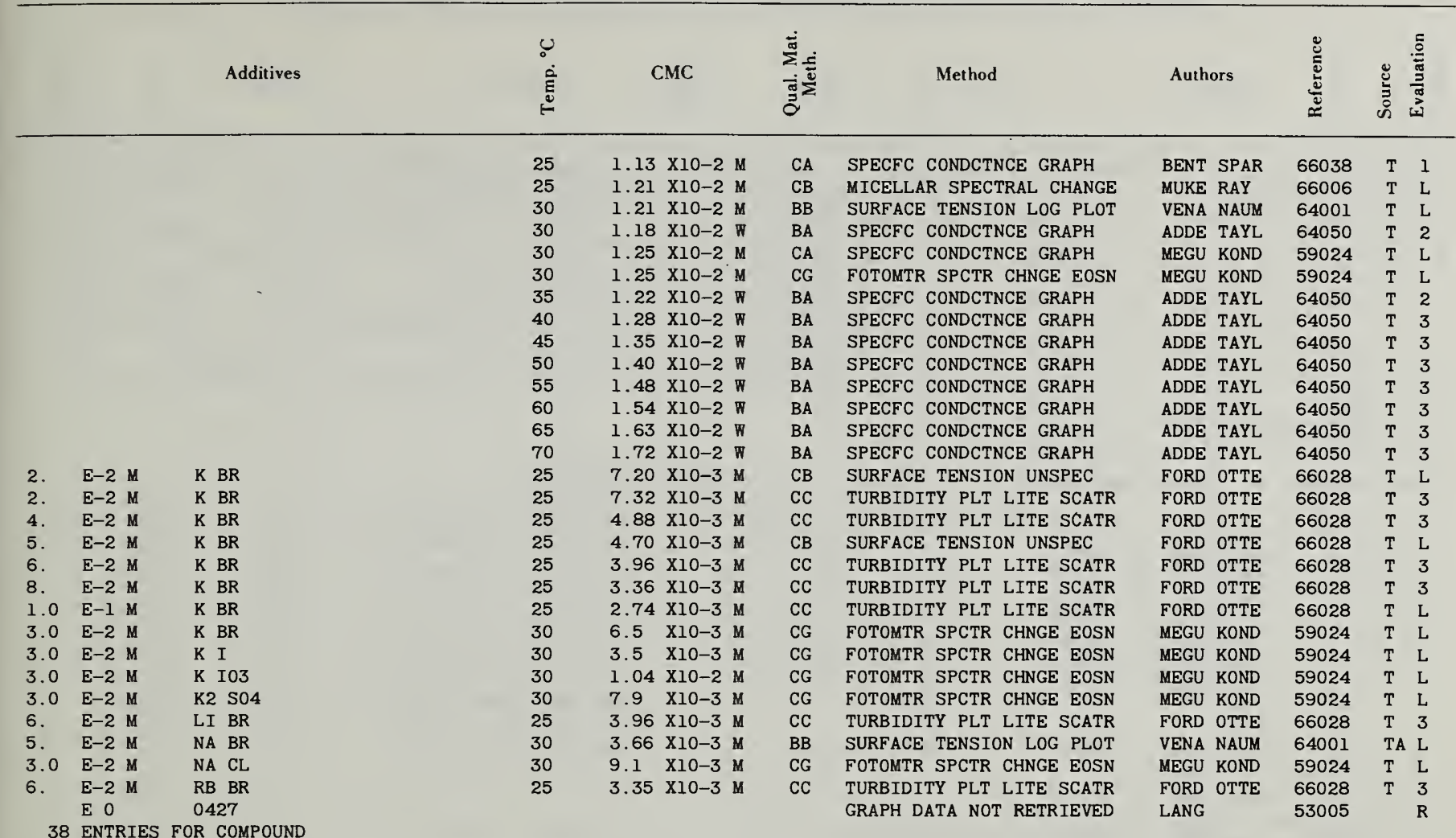

COMPOUND NO $=291$ MOL WGT -

5. E-2 M NA BR

2 ENTRIES FOR COMPOUND

COMPOUND NO $=292$ MOL WGT -

1 ENTRIES FOR COMPOUND

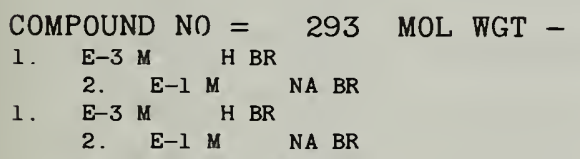

2 ENTRIES FOR COMPOUND \begin{tabular}{llllllllll}
420.6 & \multicolumn{1}{l}{ TETRADECYL } & TRIPROPYLAMMONIUM BROMIDE \\
30 & 2.05 & XIO-3 & M & BB & SURFACE TENSION LOG PLOT & VENA NAUM & 64001 & T & L \\
30 & 2.76 & XIO-4 M & BB & SURFACE TENSION LOG PLOT & VENA NAUM & 64001 & TA & L
\end{tabular}

448.7 HEXADECYL TRIPROPYLAMMONIUM BROMIDE

$30 \quad 5.7 \quad$ XIO-4 M $\quad$ BC SURFACE TENSION LOG PLOT VENA NAUM 6400I T L

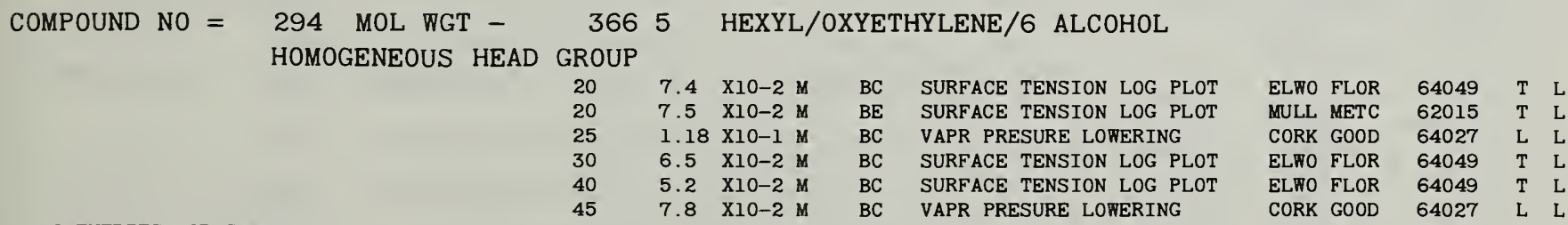

6 ENTRIES FOR COMPOUND

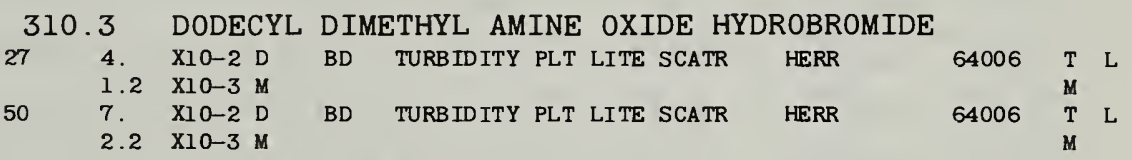

COMPOUND NO $=295$ MOL WGT -

246.2 SODIUM NONYL 1-SULFATE

\begin{tabular}{|c|c|c|c|c|c|c|c|c|}
\hline 20 & $6.5 \times 10-2$ & $\mathrm{M}$ & $\mathrm{BB}$ & INTERFACIAL TENSION LOGM & v vo & & 60025 & $\mathrm{~T}$ \\
\hline 21 & $1.66 \times 100$ & $\mathrm{D}$ & $\mathrm{BD}$ & REFRACTIVE INDEX & HUIS & & 64047 & $\mathrm{~T}$ \\
\hline 21 & $\begin{array}{l}1.59 \times 100 \\
6.458 \times 10-2\end{array}$ & $\mathrm{D}$ & BB & TURBIDITY PLT LITE SCATR & HUIS & & 64047 & $\mathrm{~T}$ \\
\hline 2 & $\begin{array}{l}1.35 \times 100 \\
5.483 \times 10-2\end{array}$ & $\begin{array}{l}\mathrm{D} \\
\mathrm{M}\end{array}$ & DB & REFRACTIVE INDEX & PR IN & HERM & 56011 & $\begin{array}{l}\mathrm{T} \\
\mathrm{M}\end{array}$ \\
\hline & $\begin{array}{l}1.45 \times 100 \\
5.889 \times 10-2\end{array}$ & $\begin{array}{l}\mathrm{D} \\
\mathrm{M}\end{array}$ & DB & TURBIDITY PLT LITE SCATR & PRIN & HERM & 56011 & $\begin{array}{l}\mathrm{T} \\
\mathrm{M}\end{array}$ \\
\hline
\end{tabular}

Concentration units: $\mathrm{A}-\mathrm{mol} \% ; \mathrm{B}-\mathrm{vol} \%$ solvent; $\mathrm{C}-$ mol \% surfactant mixture; D-wt/vol \%; E-\% saturation; $\mathrm{H}-$ wt \% solvent; I-mol \% surfactant; K-normality counterions; $\mathrm{M}-$ molar; $\mathrm{N}$ - normal; $\mathrm{P}$-wt \%; $\mathrm{Q}-$ wt \% surfactant; $\mathrm{R}$-varied; $\mathrm{S}$-mol/ kg; T - wt \% surfactant mixture; U-mol/(l or kg); W-molal; Y - atm. Detailson page 222. 


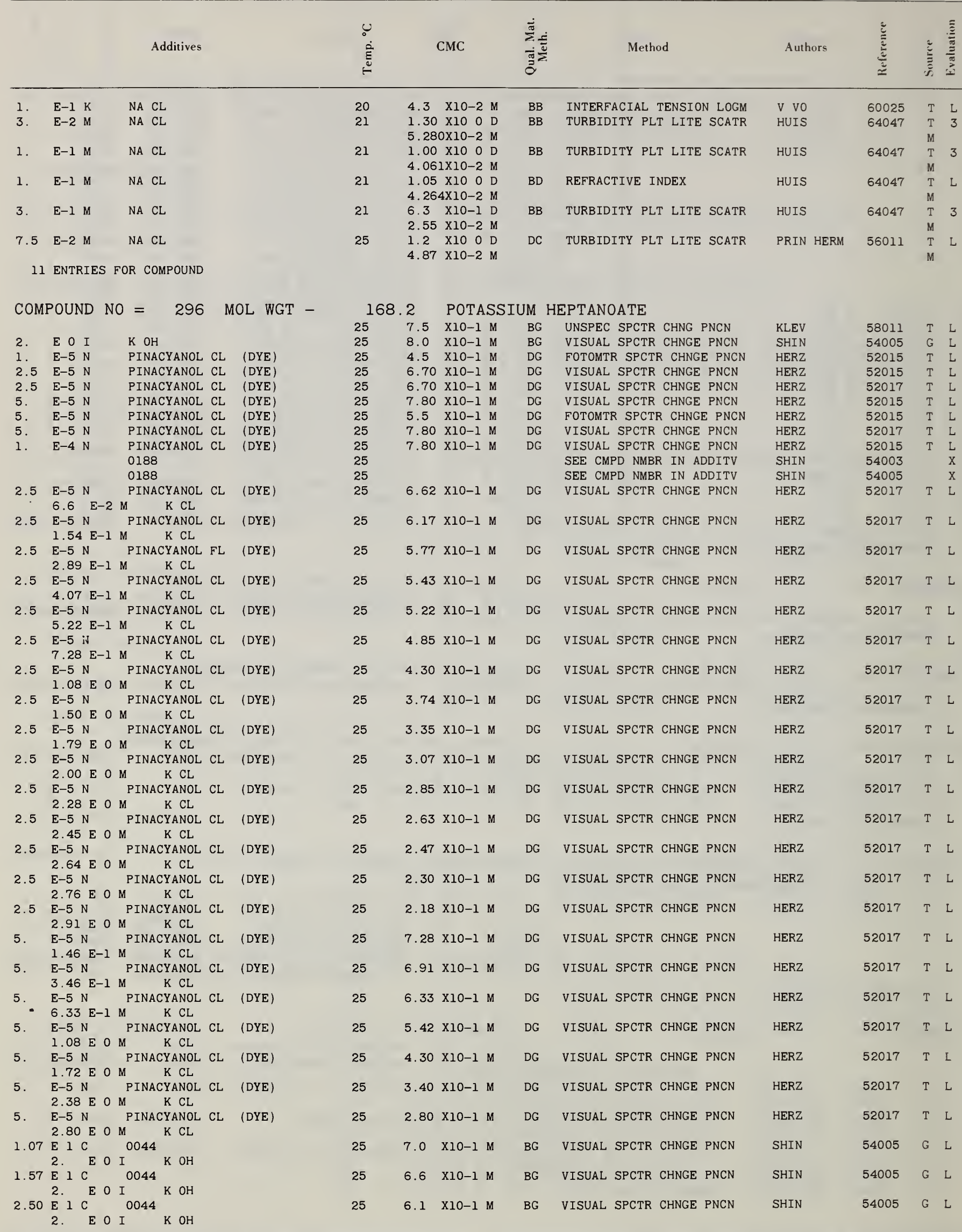

Concẹntration units: $\mathrm{A}-$ mol \%; $\mathrm{B}-$ vol \% solvent; $\mathrm{C}-$ mol \% surfactant mixture; D-wt/vol \%; E-\% saturation; H-wt \% solvent; I-mol \% surfactant; $\mathrm{K}-$ normality

counterions; $\mathrm{M}-$ molar; $\mathrm{N}$-normal; $\mathrm{P}-\mathrm{wt} \%$; $\mathrm{Q}-$ wt \% surfactant; $\mathrm{R}$-varied; $\mathrm{S}-$ mol/ $\mathrm{kg} ; \mathrm{T}-\mathrm{wt} \%$ surfactant mixture; $\mathrm{U}-\mathrm{mol} /(\mathrm{l}$ or $\mathrm{kg}) ; \mathrm{W}-$ molal; $\mathrm{Y}-$ atm. Details on page 222. 


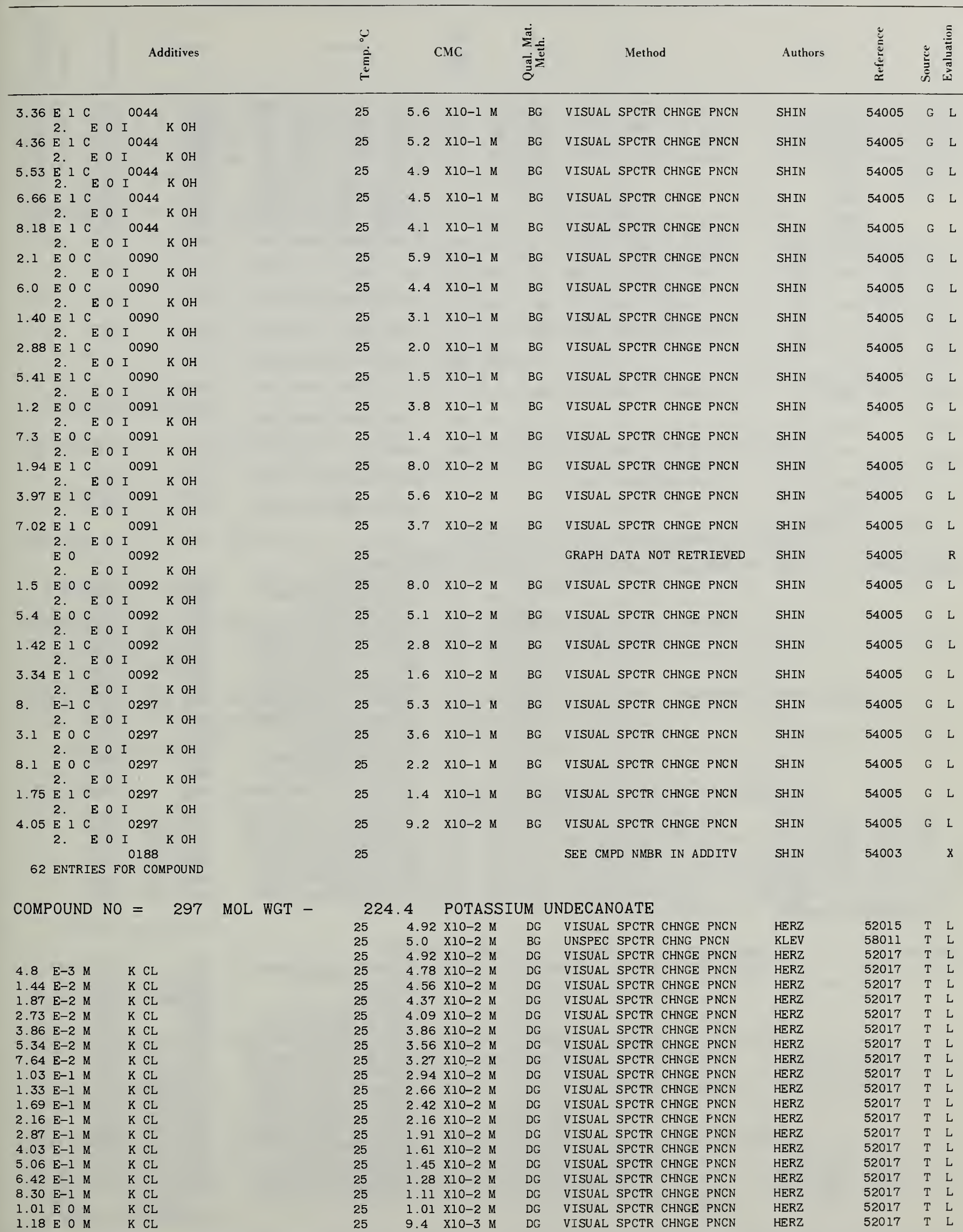

Concentration units: A-mol \%; B-vol \% solvent; $\mathrm{C}-$ mol \% surfactant mixture; 


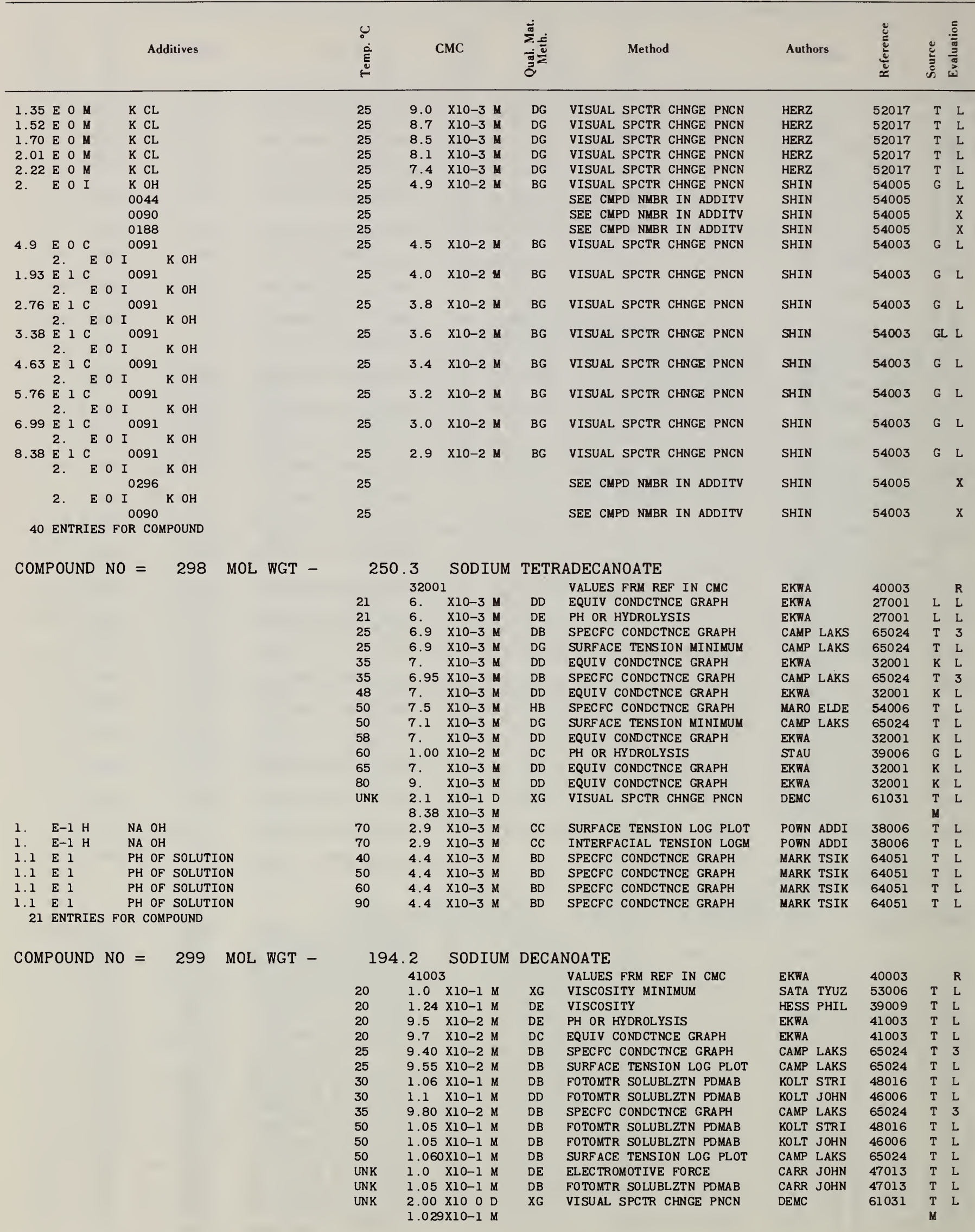

Concentration units: $\mathrm{A}-\mathrm{mol} \% ; \mathrm{B}-$ vol \% solvent; $\mathrm{C}-$ mol \% surfactant mixture;

counterions; $\mathrm{M}-$ molar; $\mathrm{N}$ - normal; $\mathrm{P}-$ wt \%; Q-wt \% surfactant; $\mathrm{R}$-varied; $\mathrm{S}-$ mol/ $\mathrm{D}-$ wt/vol \%; E-\% saturation; $\mathrm{H}$-wt \% solvent; I-mol \% surfactant; $\mathrm{K}$-normality 


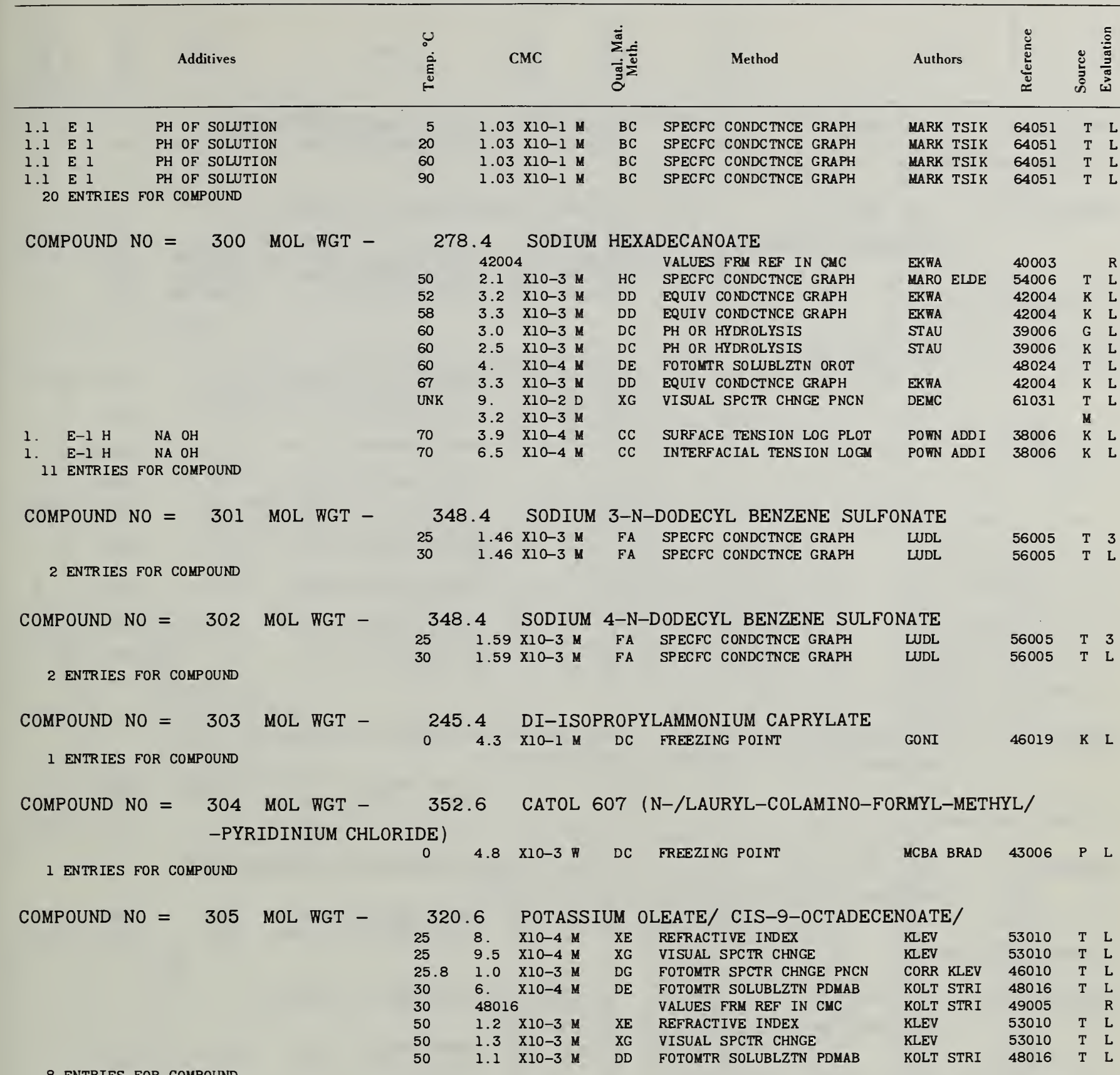

COMPOUND NO $=306$ MOL WGT -

1 ENTRIES FOR COMPOUND

COMPOUND NO $=307$ MOL WGT -

E $0 \quad \mathrm{NA2} \mathrm{S} 04$

3 ENTRIES FOR COMPOUND
496.9 DECYL TRIMETHYLAMMONIUM SULFATE UNK $5.03 \times 10-2 \mathrm{M}$ BC METHOD NOT CITED
WASI HUBB $64043 \quad \mathrm{~T} \quad \mathrm{~L}$

$$
\text { COMPOUND NO }=308 \text { MOL WGT - }
$$

$6.7 \quad$ E-3 M LA2 (S04) 3

2. E-2 M MG SO4

E $0 \quad \mathrm{NA2} \mathrm{S} 04$

4 ENTRIES FOR COMPOUND
553.0 DODECYL TRIMETHYLAMMONIUM SULFATE

$\begin{array}{lllllllll}25 & 1.64 & \text { XIO-2 N } & \text { BA } & \text { EQUIV CONDCTNCE GRAPH } & \text { VOEK TART } & 55006 & \text { T } & \text { L } \\ \text { UNK } & 9.3 & \text { XIO-3 M } & \text { BC } & \text { METHOD NOT CITED } & \text { WASI HUBB } & 64043 & \text { T } & \text { L } \\ \text { UNK } & & & & \text { QUESTIONABLE CRITERION } & \text { TASI HUBB } & 64043 & & \text { R }\end{array}$

609.1 TETRADECYL TRIMETHYLAMMONIUM SULFATE

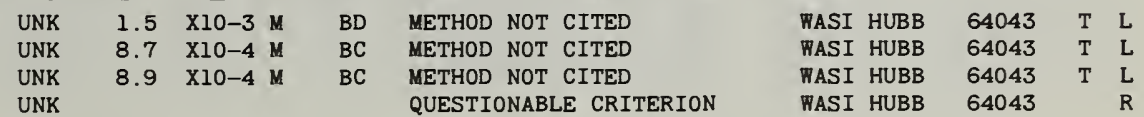

Concentration units: $\mathrm{A}-\mathrm{mol} \%$; $\mathrm{B}-$ vol \% solvent; $\mathrm{C}-\mathrm{mol} \%$ surfactant mixture; $\mathrm{D}-\mathrm{wt} / \mathrm{vol} \%$; E-\% saturation; $\mathrm{H}-\mathrm{wt} \%$ solvent; $\mathrm{I}-\mathrm{mol} \%$ surfactant; $\mathrm{K}$ - normality counterions; $\mathrm{M}$ - molar; $\mathrm{N}$ - normal; $\mathrm{P}$ - wt \%; Q-wt \% surfactant; $\mathrm{R}$ - varied; $\mathrm{S}-\mathrm{mol} /$ $\mathrm{kg} ; \mathrm{T}$ - wt \% surfactant mixture; $\mathrm{U}-\mathrm{mol} /(\mathrm{l}$ or $\mathrm{kg}) ; \mathrm{W}$ - molal; $\mathrm{Y}$ - atm. Details on page 222 


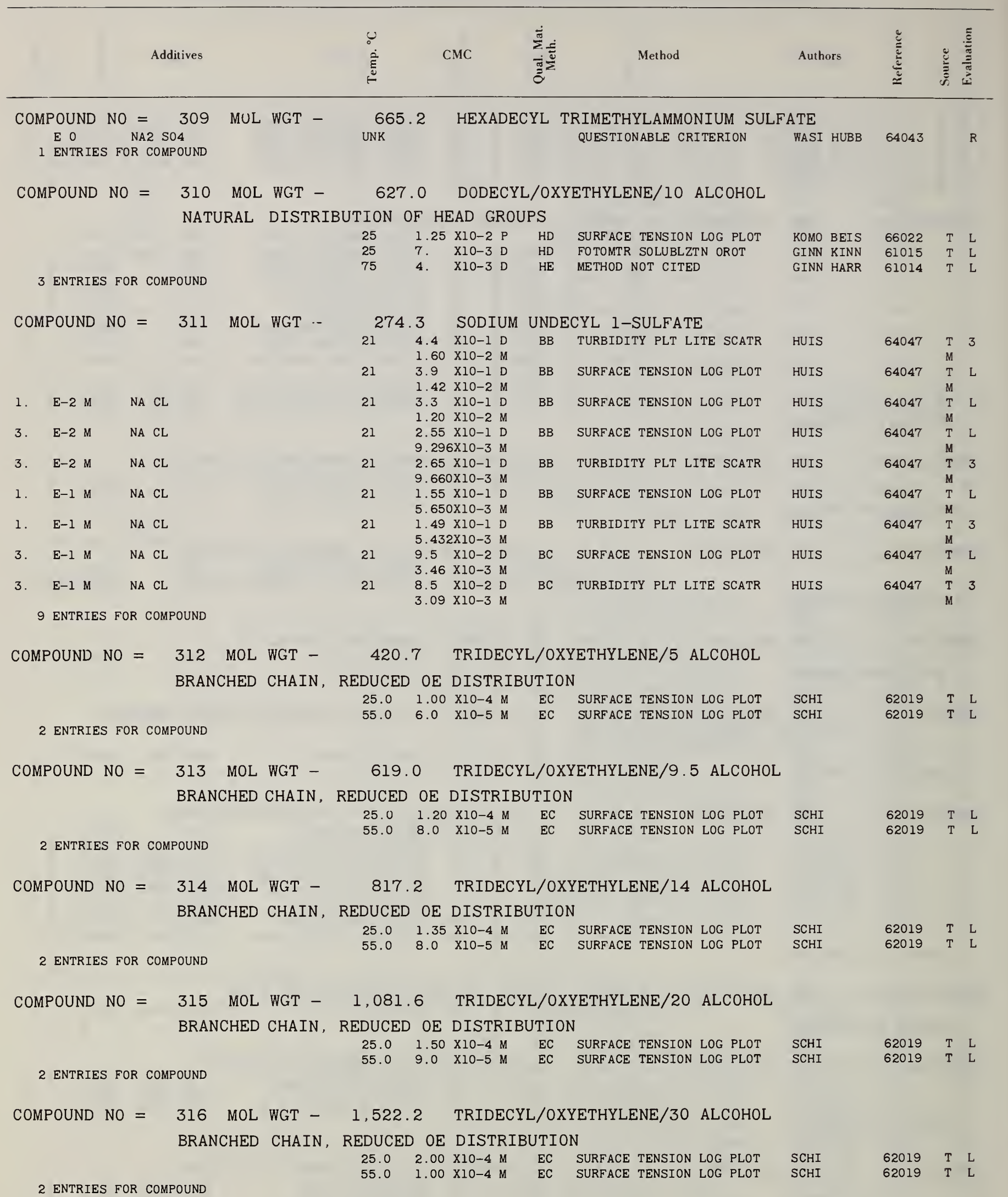




Additives Authors CMC M

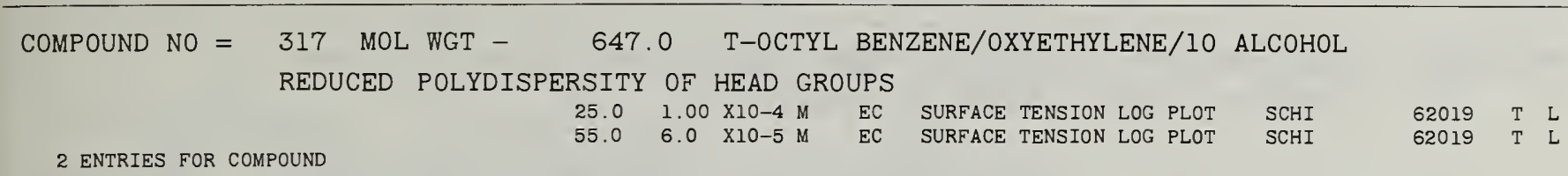

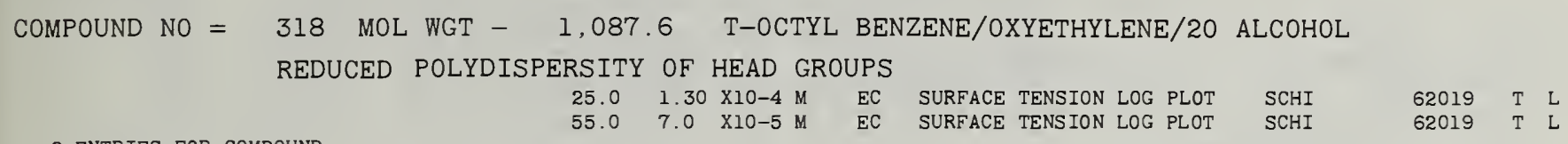

2 ENTRIES FOR COMPOUND

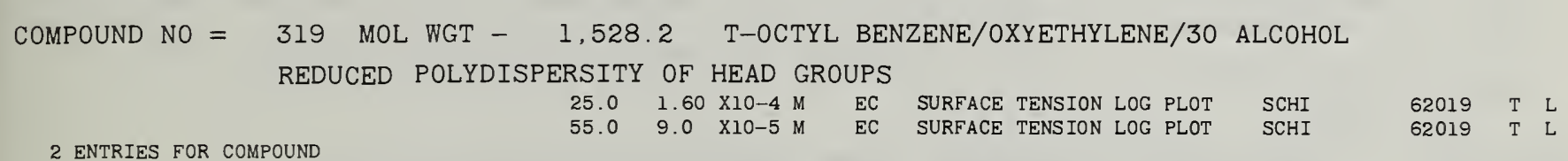

COMPOUND NO $=320$ MOL WGT - 661.0 NONYL BENZENE/OXYETHYLENE/1O ALCOHOL BRANCHED CHAIN, REDUCED OE DISTRIBUTION

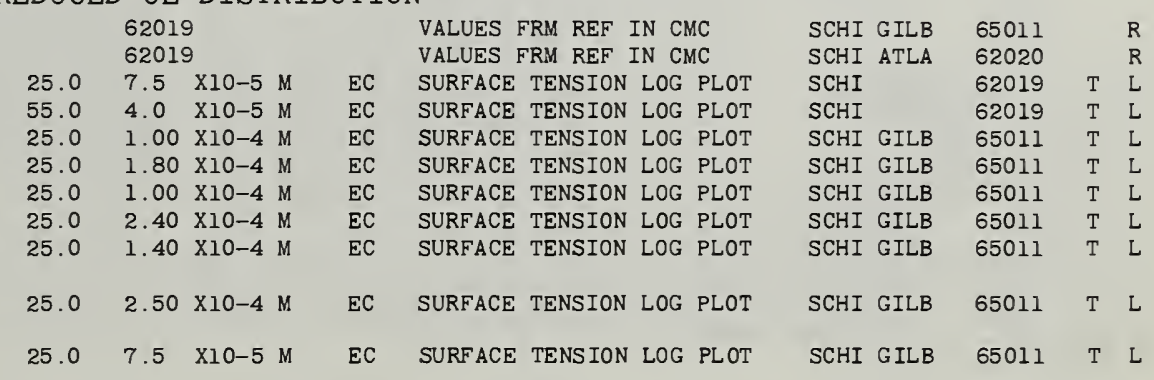

1.5 E O M DIOXANE

3.0 E O M DIOXANE

3. EOM UREA

6. E O M UREA

3. E $O M$ GUANIDINIUM CL

4.6 E $P$ PH OF SOLUTION

5. $\mathrm{E} O \mathrm{M}$ GUANIDINIUM $\mathrm{CL}$

$4.6 \div 0 \quad$ PH OF SOLUT ION

$\begin{array}{llll}\text { E O } & & \text { H CL } & \text { PH OF SOLUTION }\end{array}$

11 ENTRIES FOR COMPOUND
TSITY OF HEAD GROUPS

$9.0 \times 10-5 \mathrm{M}$ EC SURFACE TENSION LOG PLOT
62019 T $\quad$ L

\section{COMPOUND NO $=321$ MOL WGT $-\quad 881.3$ NONYL BENZENE/OXYETHYLENE/15 ALCOHOL} BRANCHED CHAIN, REDUCED OE DISTRIBUTION

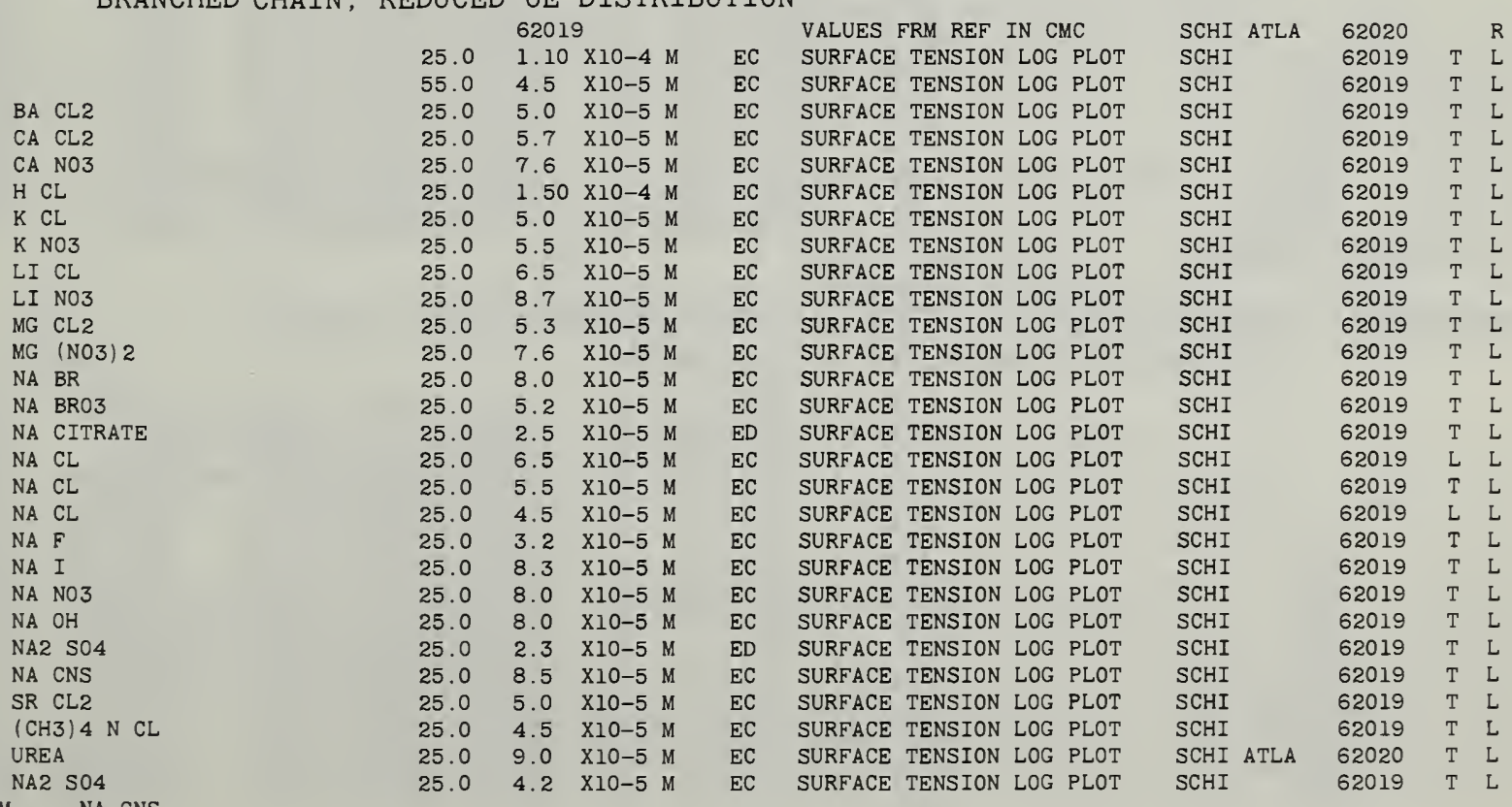

\begin{tabular}{|c|c|c|c|}
\hline 8.6 & $E-1$ & $\mathrm{~N}$ & $\mathrm{BA}$ CL2 \\
\hline 8.6 & $\mathrm{E}-1$ & $\mathrm{~N}$ & $\mathrm{CA} \quad \mathrm{CL} 2$ \\
\hline 8.6 & E-1 & $\mathrm{N}$ & $\mathrm{CA} \mathrm{NO3}$ \\
\hline 8.6 & $\mathrm{E}-1$ & M & $\mathrm{H} \mathrm{CL}$ \\
\hline 8.6 & $E-1$ & M & $\mathrm{K}$ CL \\
\hline 8.6 & $E-1$ & M & $\mathrm{K} \mathrm{NO3}$ \\
\hline 8.6 & $\mathrm{E}-1$ & M & LI CL \\
\hline 8.6 & E-1 & M & LI NO3 \\
\hline 8.6 & $E-1$ & $\mathrm{~N}$ & MG CL2 \\
\hline 8.6 & E-1 & $\mathrm{N}$ & MG $(\mathrm{NO3}) 2$ \\
\hline 8.6 & $\mathrm{E}-1$ & M & NA BR \\
\hline 8.6 & E-1 & M & NA BR03 \\
\hline 8.6 & E-1 & $\mathrm{N}$ & NA CITRATE \\
\hline 4.3 & E-1 & M & NA CL \\
\hline 8.6 & E-1 & M & NA CL \\
\hline 1.29 & EO & M & NA CL \\
\hline 8.6 & $E-1$ & M & NA F \\
\hline 8.6 & $E-1$ & M & NA I \\
\hline 8.6 & E-1 & M & NA NO3 \\
\hline 8.6 & E-1 & M & $\mathrm{NA} \mathrm{OH}$ \\
\hline 8.6 & $E-1$ & $\mathrm{~N}$ & NA2 SO4 \\
\hline 8.6 & $E-1$ & M & NA CNS \\
\hline 8.6 & $E-1$ & $\mathrm{~N}$ & SR CL2 \\
\hline 8.6 & E-1 & M & $(\mathrm{CH} 3) 4 \mathrm{~N} \mathrm{CL}$ \\
\hline 5. & $E-1$ & M & UREA \\
\hline \multirow[t]{2}{*}{8.6} & $E-1$ & $\mathrm{~N}$ & $\mathrm{NA2} \mathrm{SO}_{4}$ \\
\hline & 4.3 & $E-1$ & NA CNS \\
\hline
\end{tabular}

2.9 ENTRIES FOR COMPOUND
Concentration units: $\mathrm{A}-\mathrm{mol} \% ; \mathrm{B}-\mathrm{vol} \%$ solvent: $\mathrm{C}-$ mol $\%$ surfactant mixture; $\mathrm{D}-\mathrm{wt} / \mathrm{vol} \% ; \mathrm{E}-\%$ saturation; $\mathrm{H}-\mathrm{wt} \%$ solvent; $\mathrm{I}-\mathrm{mol} \%$ surfactant; $\mathrm{K}-$ normality counterions; $\mathrm{M}$ - molar; $\mathrm{N}$-normal; $\mathrm{P}-$ wt $\% ; \mathrm{Q}-$ wt $\%$ surfactant; $\mathrm{R}$-varied: $\mathrm{S}-$ mol/ $\mathrm{kg} ; \mathrm{T}-\mathrm{wt} \%$ surfactant mixture; $\mathrm{U}-\mathrm{mol} /(\mathrm{lor} \mathrm{kg}): \mathrm{W}$ - molal; $\mathrm{Y}$ - atm. Details on page 222. 


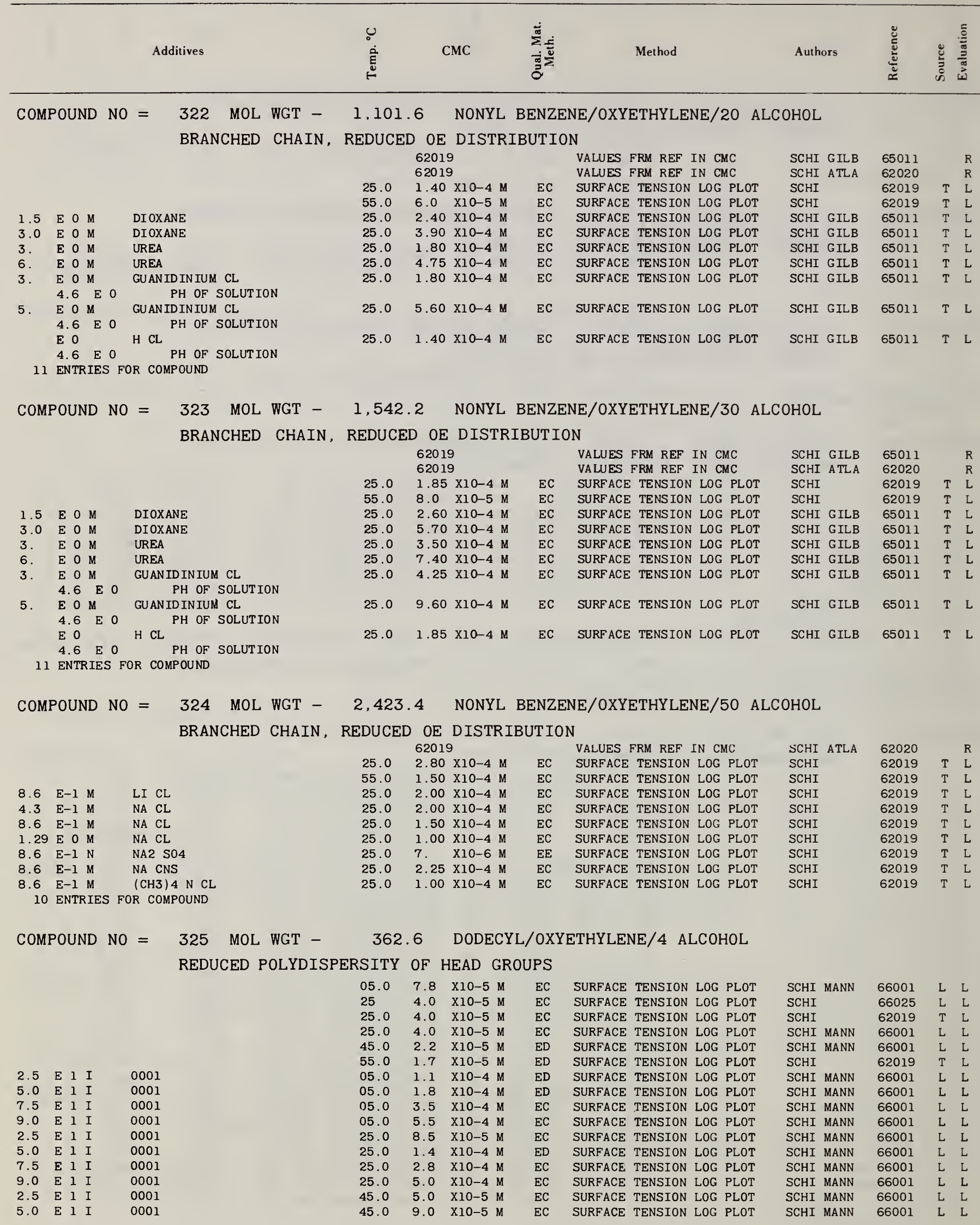

Concentration units: $\mathrm{A}-$ mol \%; $\mathrm{B}-$ vol \% solvent; $\mathrm{C}-$ mol \% surfactant mixture; 


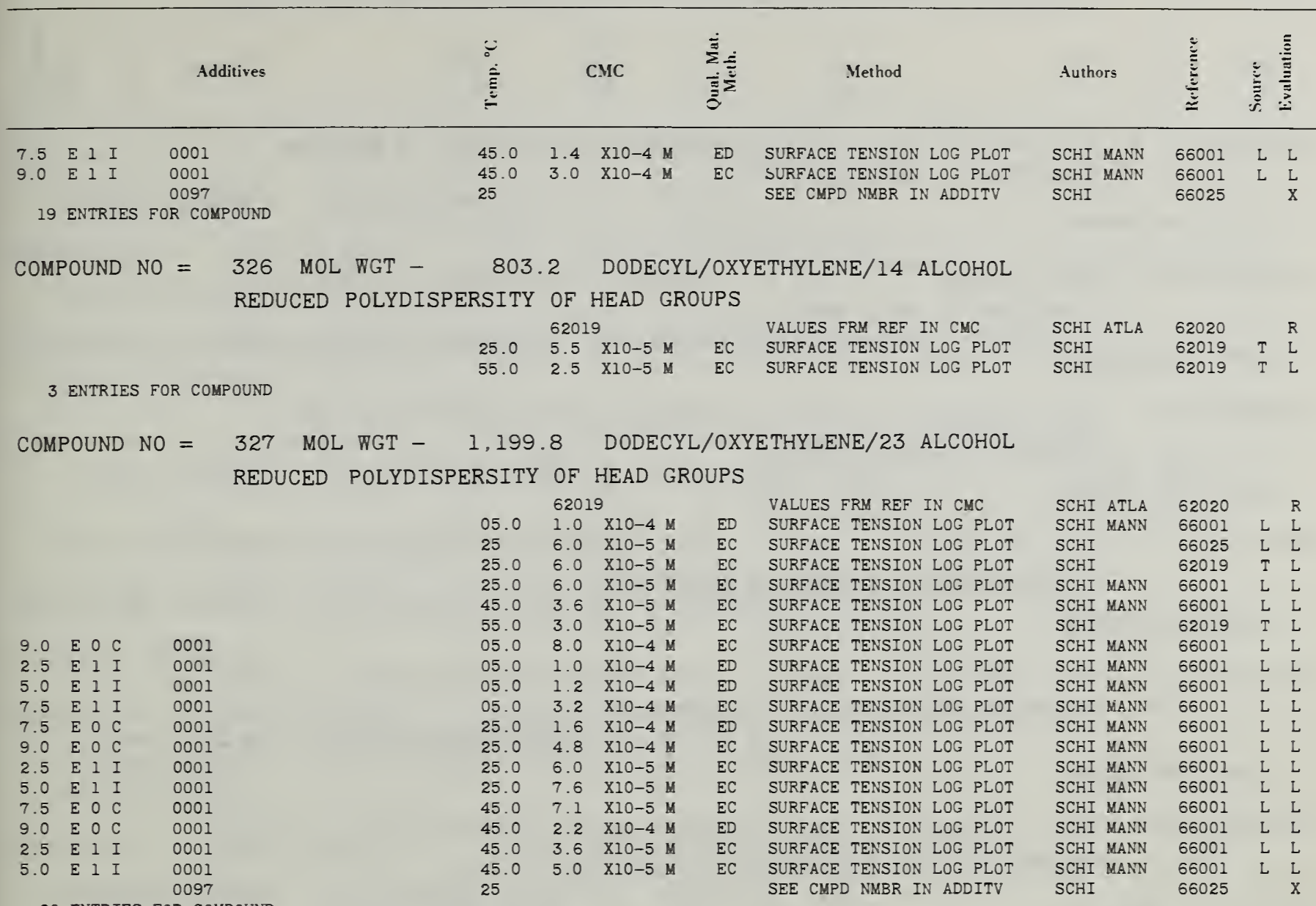

20 ENTRIES FOR COMPOUND

\section{COMPOUND NO $=328$ MOL WGT -887.4 OCTADECYL/OXYETHYLENE/14 ALCOHOL REDUCED POLYDISPERSITY OF HEAD GROUPS}

$\begin{array}{llllllllll} & & 62019 & & \text { VALUES FRN REF IN CMC } & \text { SCHI ATLA } & 62020 & R \\ 3 \text { ENTRIES FOR COMPOUND } & 25.0 & 6.0 & \text { X10-5 M } & \text { EC } & \text { SURFACE TENSION LOG PLOT } & \text { SCHI } & 62019 & \text { T } & \text { S } \\ & 55.0 & 2.0 & \text { X10-5 M } & \text { ED } & \text { SURFACE TENSION LOG PLOT } & \text { SCHI } & 62019 & \text { T } & \text { L }\end{array}$

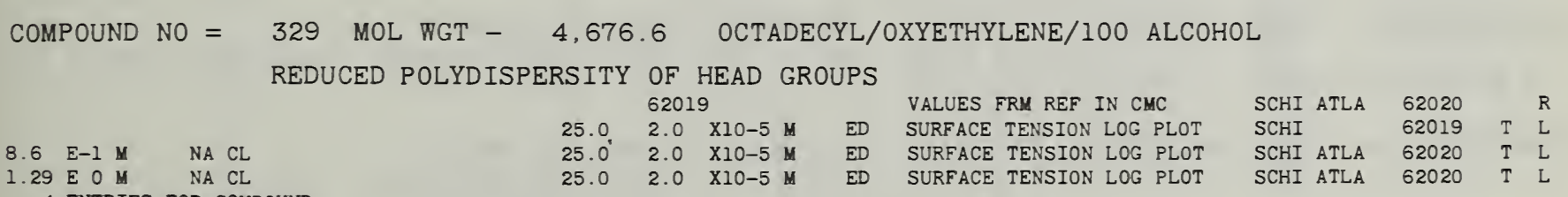

\section{COMPOUND NO $=330$ MOL WGT -550.9 HEXADECYL/OXYETHYLENE $/ 7$ ALCOHOL HOMOGENEOUS HEAD GROUP}

1 ENTRIES FOR COMPOUND $25 \quad 1.74$ X10-6 M BC SURFACE TENSION LOG PLOT ELTO MACF 62027 T 3

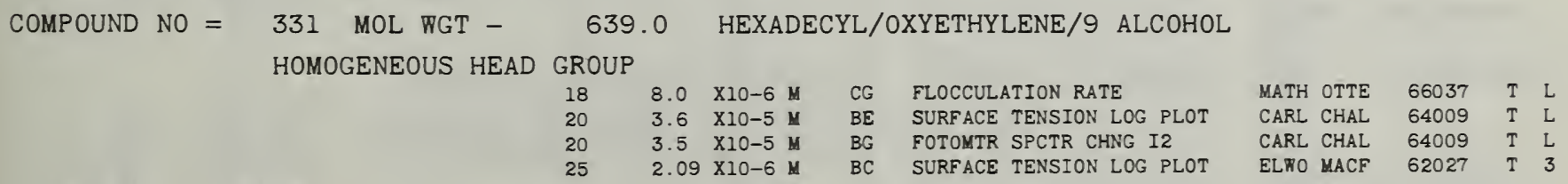

4 ENTRIES FOR COMPOUND 


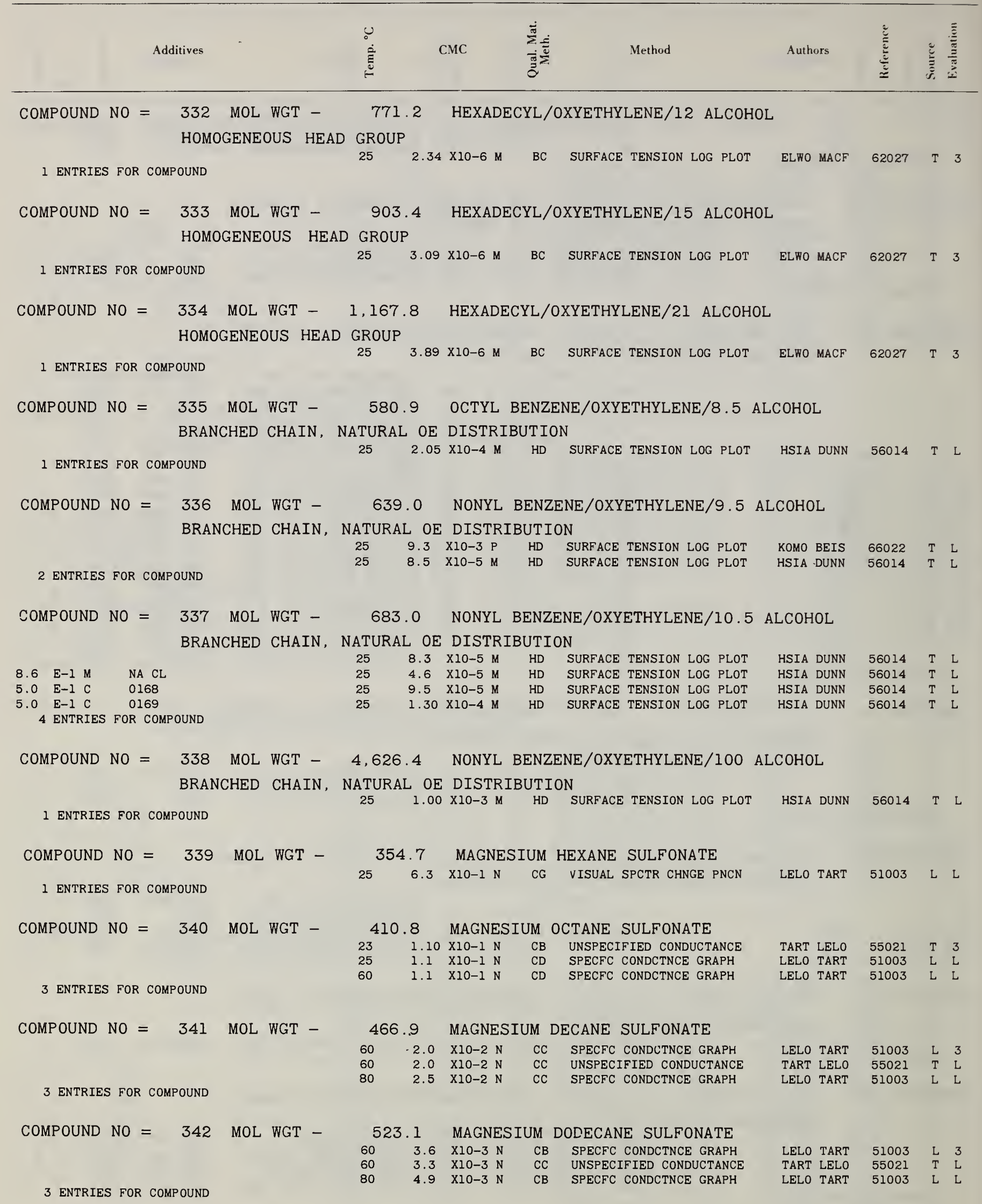

Concentration units: A-mol \%; B-vol \% solvent; $\mathrm{C}-$ mol \% surfactant mixture; counterions; $\mathrm{M}-$ molar; $\mathrm{N}-$ normal; $\mathrm{P}-$ wt $\% ; \mathrm{Q}-$ wt $\%$ surfactant; $\mathrm{R}$-varied; $\mathrm{S}-$ mol/ $\mathrm{D}-\mathrm{wt} / \mathrm{vol} \%$; E-\% saturation; $\mathrm{H}-$ wt \% solvent; $\mathrm{I}-$ mol \% surfactant; $\mathrm{K}$-normality kg; T-wt \% surfactant mixture; $\mathrm{U}-\mathrm{mol} /(\mathrm{l}$ or kg); $\mathrm{W}-$ molal; $\mathrm{Y}$ - atm. Details on page 222. 


Additives $\quad$ CMC

COMPOUND NO $=343$ MOL WGT -

1 ENTRIES FOR COMPOUND

COMPOUND NO $=344$ MOL WGT -

1 ENTRIES FOR COMPOUND

COMPOUND NO $=345$ MOL WGT -

3. $E-3 N$ NA CL

1 ENTRIES FOR COMPOUND

COMPOUND NO $=346$ MOL WGT -

$1.00 \mathrm{E} 2 \mathrm{I} \quad \mathrm{NA} \mathrm{BR}$

4 ENTRIES FOR COMPOUND

COMPOUND NO $=347$ MOL WGT -

$1.00 \mathrm{E} 2 \mathrm{I} \quad \mathrm{NA} B R$

2 ENTRIES FOR COMPOUND

174.2 SODIUM PENTANE SULFONA'I'E

$25 \quad 9.9 \times 10-1 \mathrm{M} \quad \mathrm{CD}$ METHOD NOT CITED

188.2 SODIUM HEXANE SULFONATE

$\begin{array}{llllllllll}25 & 4.6 & \mathrm{X} 10-1 & \mathrm{M} & \mathrm{CG} & \text { VISUAL SPCTR CHNGE PNCN } & \text { LELO TART } & 51003 & \mathrm{~L} & \mathrm{~L}\end{array}$

418.3 DIDODECYL DIMETHYLAMMONIUM CHLORIDE

1. X10-4 N CE UNSPECIFIED CONDUCTANCE TART LELO 55021 T $L$

437.8 DECYL TRIMETHYLAMMONIUM DECYL SULFATE

$\begin{array}{lllllllll} & 63014 & & \text { VALUES FRM REF IN CMC } & \text { CORK GOOD } & 65005 & & \text { R } \\ 25 & 4.6 & \text { X10-4 M } & \text { BB } & \text { SURFACE TENSION LOG PLOT } & \text { CORK GOOD } & 63014 & \text { L } & 3 \\ 25 & 4.5 & \text { X10-4 M } & \text { BC } & \text { METHOD NOT CITED } & \text { CORK GOOD } & 66014 & \text { T } & \text { L } \\ 25 & 4.6 & \text { X10-4 M } & \text { BB } & \text { SURFACE TENSION LOG PLOT } & \text { CORK GOOD } & 63014 & \text { L } & 3\end{array}$

381.6 OCTYL TRIMETHYLAMMOMIUM OCTYL SULFATE

$\begin{array}{lllllllll}25 & 7: 5 & \text { X10-3 M } & \text { BC } & \text { METHOD NOT CITED } & \text { CORK GOOD } & 66014 & \text { T } & \text { L } \\ 25 & 7.5 & \text { X10-3 M } & \text { BB } & \text { SURFACE TENSION LOG PLOT } & \text { CORK GOOD } & 65005 & \text { T } & 3\end{array}$

COMPOUND NO $=348$ MOL WGT - 443.2 NN-DIMETHYL 1-1-DIHYDROPENTADECAFLUORO OCTYL

AMINE N-OXIDE /C7F15CH2N/CH3/20/

1 ENTRIES FOR COMPOUND

$25 \quad 4.7 \quad \times 10-4 \mathrm{M}$

BB SURFACE TENSION LOG PLOT

CORK GOOD 65005 T 3

COMPOUND NO $=349$ MOL WGT -

1 ENTRIES FOR COMPOUND

COMPOUND NO $=350$ MOL WGT -

$3.7 \quad \mathrm{E}-2 \mathrm{M} \quad \mathrm{K} \mathrm{CL}$

8.7 E-2 M K CL

$1.59 \mathrm{E}-1 \mathrm{M} \quad \mathrm{K} \mathrm{CL}$

$2.78 \mathrm{E}-1 \mathrm{M} \quad \mathrm{K} \mathrm{CL}$

$4.61 \mathrm{E}-1 \mathrm{M} \quad \mathrm{K} \mathrm{CL}$

$6.77 \mathrm{E}-1 \mathrm{M} \quad \mathrm{K} \mathrm{CL}$

$8.55 \mathrm{E}-1 \mathrm{M} \quad \mathrm{K} \mathrm{CL}$

$1.29 \mathrm{E} \mathrm{O} \mathrm{M} \mathrm{K} \mathrm{CL}$

$1.63 \mathrm{E} \mathrm{OM} \quad \mathrm{K} \mathrm{CL}$

$1.92 \mathrm{E} O \mathrm{M} \quad \mathrm{K} \mathrm{CL}$

$2.11 \mathrm{E} O \mathrm{M} \quad \mathrm{K} C L$

$2.39 \mathrm{E} O \mathrm{M} \quad \mathrm{K} \mathrm{CL}$

$2.64 \mathrm{E} O \mathrm{M} \quad \mathrm{K} \mathrm{CL}$

17 ENTRIES FOR COMPOUND
356.5 SODIUM OCTADECANE 1-SULFONATE

$57.0 \quad 7.5 \quad \mathrm{X} 10-4 \mathrm{~W} \quad$ DB KRAFFT POINT SOLUBILITY
TART WRIG $39002 \quad \mathrm{~T} \quad \mathrm{~L}$

196.4 POTASSIUM NONANOATE

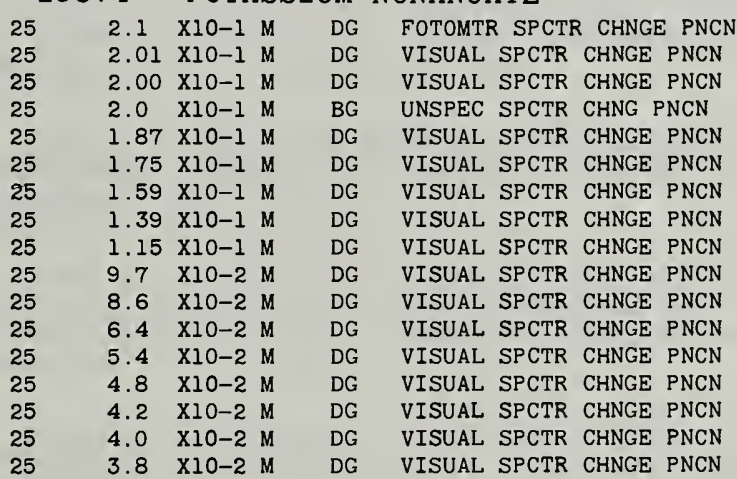

HERZ
HERZ
HERZ
KLEV
HERZ
HERZ
HERZ
HERZ
HERZ
HERZ
HERZ
HERZ
HERZ
HERZ
HERZ
HERZ

52015 T L

52015 T L

52017 T $\mathrm{L}$

58011 T $\quad$ L

$52017 \quad \mathrm{~T} \quad \mathrm{~L}$

52017 T $\quad$ L

52017 T $\quad$ L

52017 T $\quad$ L

52017 T $\quad$ L

52017 T $\mathrm{L}$

$52017 \quad \mathrm{~T} \quad \mathrm{~L}$

$52017 \quad \mathrm{~T} \quad \mathrm{~L}$

52017 T L

52017 TA L

52017 T L

52017 T $\quad$ L

52017 T $\quad$ L
-COMPOUND NO $=351$ MOL WGT -

\begin{tabular}{|c|c|c|}
\hline 1.34 & E-3 & \\
\hline 2.91 & $E-3$ & M \\
\hline 4.78 & E-3 & M \\
\hline 7.06 & $E-3$ & M \\
\hline 9.29 & E-3 & M \\
\hline 1.34 & $E-2$ & M \\
\hline 1.56 & $E-2$ & M \\
\hline 1.87 & $E-2$ & $M$ \\
\hline 2.51 & $E-2$ & M \\
\hline 2.76 & $E-2$ & M \\
\hline & $\pi$ & \\
\hline
\end{tabular}

252.5 POTASSIUM TRIDECANOATE

$\begin{array}{lll}25 & 1.26 \times 10-2 & M \\ 25 & 1.2 \times 10-2 & M \\ 25 & 1.26 \times 10-2 & M \\ 25 & 1.21 \times 10-2 & M \\ 25 & 1.16 \times 10-2 & M \\ 25 & 1.12 \times 10-2 & M \\ 25 & 1.06 \times 10-2 & M \\ 25 & 9.29 \times 10-3 & M \\ 25 & 8.92 \times 10-3 & M \\ 25 & 8.42 \times 10-3 & M \\ 25 & 8.00 \times 10-3 & M \\ 25 & 7.17 \times 10-3 & M \\ 25 & 6.90 \times 10-3 M \\ 25 & 6.54 \times 10-3 \mathrm{M}\end{array}$

$\begin{array}{lll} & \text { VISUAL SPCTR CHNGE PNCN } \\ \text { BG } & \text { UNSPEC SPCTR CHNG PNCN } \\ \text { DG } & \text { VISUAL SPCTR CHNGE PNCN } \\ \text { DG } & \text { VISUAL SPCTR CHNGE PNCN } \\ \text { DG } & \text { VISUAL SPCTR CHNGE PNCN } \\ \text { DG } & \text { VISUAL SPCTR CHNGE PNCN } \\ \text { DG } & \text { VISUAL SPCTR CHNGE PNCN } \\ \text { DG } & \text { VISUAL SPCTR CHNGE PNCN } \\ \text { DG } & \text { VISUAL SPCTR CHNGE PNCN } \\ \text { DG } & \text { VISUAL SPCTR CHNGE PNCN } \\ \text { DG } & \text { VISUAL SPCTR CHNGE PNCN } \\ \text { DG } & \text { VISUAL SPCTR CHNGE PNCN } \\ \text { DG } & \text { VISUAL SPCTR CHNGE PNCN } \\ \text { DG } & \text { VISUAL SPCTR CHNGE PNCN }\end{array}$

HERZ

KLEV

HERZ

HERZ

HERZ

HERZ

HERZ

HERZ

HERZ

HERZ

HERZ

HERZ

HERZ

HERZ

$\begin{array}{lll}52015 & \text { T } & \text { L } \\ 58011 & \text { T } & \text { L } \\ 52017 & \text { T } & \text { L } \\ 52017 & \text { T } & \text { L } \\ 52017 & \text { T } & \text { L } \\ 52017 & \text { T } & \text { L } \\ 52017 & \text { T } & \text { L } \\ 52017 & \text { T } & \text { L } \\ 52017 & \text { T } & \text { L } \\ 52017 & \text { T } & \text { L } \\ 52017 & \text { T } & \text { L } \\ 52017 & \text { T } & \text { L } \\ 52017 & \text { T } & \text { L } \\ 52017 & \text { T } & \text { L }\end{array}$

Concentration units: $\mathrm{A}-\mathrm{mol} \%$; $\mathrm{B}-\mathrm{vol} \%$ solvent; $\mathrm{C}-$ mol \% surfactant mixture; D-wt/vol \%; E-\% saturation; $\mathrm{H}-$ wt $\%$ solvent; I-mol \% surfactant; K-normality counterions; $\mathrm{M}$-molar; $\mathrm{N}$-normal; $\mathrm{P}$ - wt \%: $\mathrm{Q}-$ wt $\%$ surfactant; $\mathrm{R}$-varied; $\mathrm{S}-\mathrm{mol} /$ $\mathrm{kg} ; \mathrm{T}-$ wt \% surfactant mixture; $\mathrm{U}-\mathrm{mol} /(\mathrm{lor} \mathrm{kg})$; $\mathrm{W}$ - molal; $\mathrm{Y}$ - atm. Details on page 222 


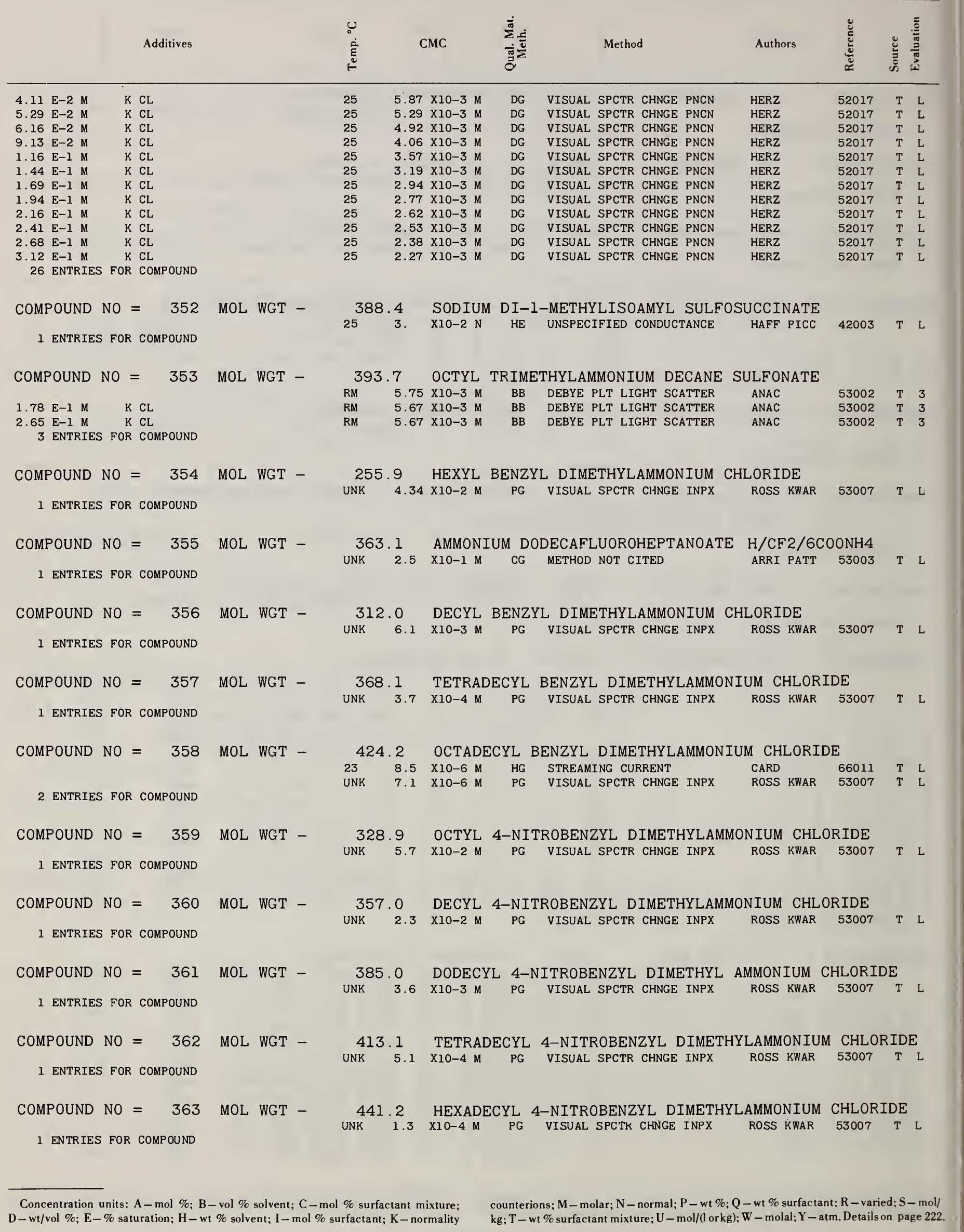


Additives

C. CMC

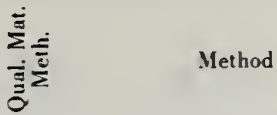

Method

Authors

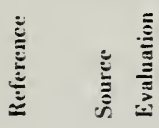

COMPOUND NO $=364$ MOL WGT -

I ENTRIES FOR COMPOUND

COMPOUND NO $=365$ MOL WGT -

1 ENTRIES FOR COMPOUND

COMPOUND NO $=366$ MOL WGT -

1 ENTRIES FOR COMPOUND

COMPOUND NO $=367$ MOL WGT -

1 ENTRIES FOR COMPOUND

COMPOUND NO $=368$ MOL WGT -

1 ENTRIES FOR COMPOUND

COMPOUND NO $=369$ MOL WGT -

1 ENTRIES FOR COMPOUND

COMPOUND NO $=370$ MOL WGT -

1 ENTRIES FOR COMPOUND

COMPOUND NO $=371$ MOL WGT -

1 ENTRIES FOR COMPOUND

COMPOUND NO $=372$ MOL WGT -

0092
2 ENTRIES FOR COMPOUND

COMPOUND NO $=373$ MOL WGT -

1 ENTRIES FOR COMPOUND

COMPOUND NO $=374$ MOL WGT -

2 ENTRIES FOR COMPOUND

COMPOUND NO $=375$ MOL WGT -

2 ENTRIES FOR COMPOUND

COMPOUND NO $=376$ MOL WGT -
469.2 OCTADECYL 4-NITROBENZYL DIMETHYLAMMONIUM CHLORIDE $\begin{array}{lllllllll}\text { UNK } & 2.9 & X 10-5 & M & \text { PG VISUAL SPCTR CHNGE INPX ROSS KWAR } 53007 & T & \text { L }\end{array}$

374.5 DODECYL 2-CHLOROBENZYL DIMETHYLAMMONIUM CHLORIDE $\begin{array}{llllllllll}\text { UNK } & 2.8 & \text { XIO-4 M } & \text { PG } & \text { VISUAL SPCTR CHNGE INPX } & \text { ROSS KWAR } & 53007 & \text { T } & \text { L }\end{array}$

408.9 DODECYL 2-4-DICHLOROBENZYL DIMETHYLAMMONIUMCHLORIDE $\begin{array}{lllllllll}\text { UNK } & 3.7 & \text { XIO-4 M } & \text { PG VISUAL SPCTR CHNGE INPX ROSS KWAR } 53007 \text { T L }\end{array}$

374.5 DODECYL 4-CHLOROBENZYL DIMETHYLAMMONIUM CHLORIDE UNK 4.2 XIO-4 M PG VISUAL SPCTR CHNGE INPX ROSS KWAR 53007 T L

401.0 DODECYL 2-HYDROXY-5-NITROBENZYL DIMETHYLAMMONIUM CHLORIDE UNK $6.9 \quad$ XIO-4 M PG VISUAL SPCTR CHNGE INPX ROSS KTAR 53007 T L

408.9 DODECYL 3-4-DICHLOROBENZYL DIMETHYLAMMONIUMCHLORIDE UNK $\quad 1.1 \quad$ XIO-3 M PG VISUAL SPCTR CHNGE INPX ROSS KTAR 53007 T L

384. 1 DODECYL 3-4-METHYLENEDIOXYBENZYL DIMETHYLAMMONIUM CHLORIDE UNK $3.8 \quad$ XIO-3 M $\quad$ PG VISUAL SPCTR CHNGE INPX ROSS KWAR 53007 T L

400.1 DODECYL 3-4-DIMETHOXYBENZYL DIMETHYLAMMONIUMCHLORIDE UNK $3.9 \quad$ XIO-3 M PG VISUAL SPCTR CHNGE INPX ROSS KFAR 53007 T L

463.1 AMMONIUM HEXADECAFLUORONONANOATE H/CF2/8 COO NH4 UNK 3.8 XIO-2 M CG METHOD NOT CITED ARRI PATT 53003 T L SEE CMPD NMBR IN ADDITV ARRI PATT $53003 \quad \mathrm{X}$

563.2 AMMONIUM EICOSAFLUOROUNDECANOATE H/CF2/10 COO NH4 UNK 9. XIO-3 M CG METHOD NOT CITED ARRI PATT 53003 T L

346.1 DODECAFLUOROHEPTANOIC ACID H/CF $2 / 6 \mathrm{COOH}$

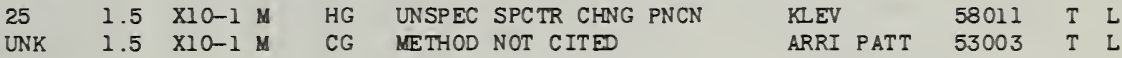
446.1 HEXADECAFLUORONONANOIC ACID H/CF $2 / 8 \mathrm{COOH}$ $\begin{array}{lllllllll}25 & 3 . & \text { XIO-2 M } & \text { HG } & \text { UNSPEC SPCTR CHNG PNCN } & \text { KLEV } & 58011 & \text { T } & \text { L } \\ \text { UNK } & 3 . & \text { XIO-2 } & \text { CG } & \text { METHOD NOT CITED } & \text { ARRI PATT } & 53003 & \text { T } & \text { L }\end{array}$

375.4 DODECYL PYRIDINIUM IODIDE

\begin{tabular}{|c|c|c|c|c|c|c|c|c|}
\hline & 60015 & & & VALUES FRM REF IN CMC & FORD & OTTE & 66028 & \\
\hline 8.0 & 4.1 & $\mathrm{x} 10-3 \mathrm{M}$ & $\mathrm{BC}$ & KRAFFT POINT SOLUBILITY & ADDI & FURM & 56019 & T \\
\hline .5 & 4.3 & $\mathrm{X} 10-3 \mathrm{M}$ & $\mathrm{BC}$ & INTERFACIAL TNSN UNSPEC & ADDI & FURM & 56019 & $\mathrm{~T}$ \\
\hline .3 & 5.10 & $\mathrm{X} 10-3 \mathrm{M}$ & $\mathrm{CB}$ & MICELLAR SPECTRAL CHANGE & MUKE & RAY & 66006 & \\
\hline .9 & 5.26 & $\mathrm{XI} 0-3 \mathrm{M}$ & $\mathrm{CB}$ & MICELLAR SPECTRAL CHANGE & MUKE & RAY & 66006 & \\
\hline & 5.60 & $\mathrm{X} 10-3 \mathrm{M}$ & DC & TURBIDITY PLT LITE SCATR & PARR & & 60015 & \\
\hline & 5.70 & $\mathrm{x} 10-3 \mathrm{M}$ & $\mathrm{CB}$ & SURFACE TENSION UNSPEC & FORD & OTTE & 66028 & \\
\hline & 5.26 & $\mathrm{X} 10-3 \mathrm{~m}$ & $D B$ & MICELLAR SPECTRAL CHANGE & MUKE & RAY & 3032 & \\
\hline & 5.0 & $x 10-3 M$ & CG & FOTOMTR SPCTR CHNGE EOSN & MEGU & KOND & 9024 & \\
\hline & 4.5 & $\mathrm{X} 10-3 \mathrm{~m}$ & $\mathrm{CA}$ & SPECFC CONDCTNCE GRAPH & MEGU & KOND & 59024 & \\
\hline & 5.60 & $\mathrm{X} 10-3 \mathrm{M}$ & $\mathrm{CB}$ & MICELLAR SPECTRAL CHANGE & TUEE & & 006 & \\
\hline & 5.85 & $x 10-3 M$ & $\mathrm{CB}$ & MICELLAR SPECTRAL CHANGE & MUKE & RAY & 66006 & \\
\hline & 6.30 & $\mathrm{X} 10-3 \mathrm{M}$ & CB & MICELLAR SPECTRAL CHANGE & MUKE & RAY & 66006 & \\
\hline
\end{tabular}

Concentration units: $\mathrm{A}-\mathrm{mol} \% ; \mathrm{B}-\mathrm{vol} \%$ solvent; $\mathrm{C}-\mathrm{mol} \%$ surfactant mixture; $\mathrm{D}-\mathrm{wt} / \mathrm{vol} \%$; E-\% saturation; $\mathrm{H}-\mathrm{wt} \%$ solvent; $\mathrm{I}-$ mol \% surfactant; $\mathrm{K}$ - normality

counterions; $\mathrm{M}$-molar; $\mathrm{N}$-normal; $\mathrm{P}$ - wt \%; Q - wt \% surfactant; $\mathrm{R}$-varied; $\mathrm{S}-$ mol/ kg; T-wt \% surfactant mixture; $U$ - mol/( orkg); $\mathbf{W}$-molal; $Y$-atm. Details on page 222. 


\begin{tabular}{|c|c|c|c|c|c|}
\hline 2.5 & $E-3$ & $M$ & K I & & \\
\hline 5.0 & $E-3$ & M & K I & & \\
\hline 1.00 & $E-2$ & M & K I & & \\
\hline 2. & $\mathrm{E}-2$ & M & K I & & \\
\hline 2.02 & E-2 & M & K I & & \\
\hline 5.01 & $E-2$ & M & K I & & \\
\hline 1.002 & $2 E-1$ & $M$ & K I & & \\
\hline 1. & $E-4$ & $M$ & NA2 & S203 & THIOSULF \\
\hline 1. & $E-3$ & M & NA2 & S203 & THIOSULF \\
\hline 1. & $E-3$ & M & NA2 & S203 & THIOSULF \\
\hline 3.4 & E 0 & $M$ & UREA & & \\
\hline 5.9 & E 0 & M & UREA & & \\
\hline 3.4 & E 0 & $M$ & UREA & & \\
\hline 5.9 & E 0 & $M$ & UREA & & \\
\hline 8.0 & E 0 & $M$ & UREA & & \\
\hline \multirow[t]{2}{*}{1.} & $\mathrm{E}-4$ & M & NA2 2 & S203 & THIOSULF \\
\hline & 3.4 & E 0 & M & UREA & \\
\hline \multirow[t]{2}{*}{1.} & $E-4$ & $\mathrm{M}$ & NA2 & S203 & THIOSULF \\
\hline & 5.9 & E 0 & M & UREA & \\
\hline \multirow[t]{2}{*}{1.} & $E-3$ & M & NA2 & S203 & THI OSULF \\
\hline & 9.6 & E-1 & M & UREA & \\
\hline \multirow[t]{2}{*}{1} & $E-3$ & M & NA2 & S203 & THIOSULF \\
\hline & 3.4 & E 0 & M & UREA & \\
\hline \multirow[t]{2}{*}{1.} & $E-3$ & M & NA 2 & $\mathrm{~S} 203$ & THIOSULF \\
\hline & 5.9 & E 0 & M & UREA & \\
\hline \multirow[t]{2}{*}{1.} & $E-3$ & M & NA 2 & S203 & THIOSULF \\
\hline & 9.6 & E-1 & M & UREA & \\
\hline \multirow[t]{2}{*}{1} & $E-3$ & $M$ & NA2 & $\mathrm{S} 203$ & THI OSULF \\
\hline & 3.4 & E 0 & M & UREA & \\
\hline 1 . & $E-3$ & M & NA 2 & S203 & THI OSULF \\
\hline & 5.9 & E 0 & & UREA & \\
\hline 41 & ENTR & IES F & POR CO & OMPOUND & \\
\hline
\end{tabular}

$\begin{array}{lllll}44.9 & 6.70 \times 10-3 \mathrm{M} & \text { CB } & \text { MICELLAR SPECTRAL CHANGE } \\ 45 & 6.70 \times 10-3 \mathrm{M} & \text { DB } & \text { MICELLAR SPECTRAL CHANGE } \\ \text { RM } & 5.0 \times 10-3 \mathrm{M} & \text { CC } & \text { MICELLAR SPECTRAL CHANGE } \\ \text { RM } & 5.3 \times 10-3 \mathrm{M} & \text { CG } & \text { VISUAL SPCTR CHNG SKYB } \\ \text { RM } & 5.6 \times 10-3 \mathrm{M} & \text { CC } & \text { FOTOMTR SOLUBLZTN OROT } \\ 25 & 4.53 \times 10-3 \mathrm{M} & \text { DC } & \text { TURBIDITY PLT LITE SCATR } \\ 25 & 3.87 \times 10-3 \mathrm{M} & \text { DC } & \text { TURB IDITY PLT LITE SCATR } \\ 25 & 2.94 \times 10-3 \mathrm{M} & \text { DC } & \text { TURBIDITY PLT LITE SCATR } \\ 25 & 1.80 \times 10-3 \mathrm{M} & \text { CB } & \text { SURFACE TENSION UNSPEC } \\ 30 & 1.94 \times 10-3 \mathrm{M} & \text { CB } & \text { MICELLAR SPECTRAL CHANGE } \\ 30 & 1.12 \times 10-3 \mathrm{M} & \text { CB } & \text { MICELLAR SPECTRAL CHANGE } \\ 30 & 6.5 \times 10-4 \mathrm{M} & \text { CB } & \text { MICELLAR SPECTRAL CHANGE } \\ 25 & 5.15 \times 10-3 \mathrm{M} & \text { DB } & \text { MICELLAR SPECTRAL CHANGE } \\ 25 & 4.75 \times 10-3 \mathrm{M} & \text { DB } & \text { MICELLAR SPECTRAL CHANGE } \\ 45 & 5.63 \times 10-3 \mathrm{M} & \text { DB } & \text { MICELLAR SPECTRAL CHANGE } \\ 25 & 9.34 \times 10-3 \mathrm{M} & \text { DB } & \text { MICELLAR SPECTRAL CHANGE } \\ 25 & 1.36 \times 10-2 \mathrm{M} & \text { DB } & \text { MICELLAR SPECTRAL CHANGE } \\ 45 & 1.18 \times 10-2 \mathrm{M} & \text { DB } & \text { MICELLAR SPECTRAL CHANGE } \\ 45 & 1.71 \times 10-2 \mathrm{M} & \text { DB } & \text { MICELLAR SPECTRAL CHANGE } \\ 45 & 2.13 \times 10-2 \mathrm{M} & \text { DB } & \text { MICELLAR SPECTRAL CHANGE } \\ 25 & 9.30 \times 10-3 \mathrm{M} & \text { DB } & \text { MICELLAR SPECTRAL CHANGE } \\ & & & \end{array}$

MUKE RAY MUKE RAY HARK DRIZ HARK DRIZ HARK DRIZ

PARR

PARR

PARR

FORD OTTE

MUKE RAY

MUKE RAY

MUKE RAY

MUKE RAY

MUKE RAY

MUKE RAY

MUKE RAY

MUKE RAY

MUKE RAY

MUKE RAY

MUKE RAY

MUKE RAY

MUKE RAY

MUKE RAY

MUKE RAY

MUKE RAY

MUKE RAY

MUKE RAY

MUKE RAY

63032 T L

\section{COMPOUND NO $=377$ MOL WGT - HOMOGENEOUS HEAD}

4 ENTRIES FOR COMPOUNL

406.7 DODECYL/OXYETHYLENE/ 5 ALCOHOL GROUP

$\begin{array}{llll}20 & 4.0 & \mathrm{X} 10-5 & \mathrm{M} \\ 20 & 3.5 & \mathrm{X} 10-5 & \mathrm{M} \\ 20 & 5.00 & \mathrm{X} 10-5 & \mathrm{M} \\ 23 & 5.7 & \mathrm{X} 10-5 & \mathrm{M}\end{array}$

BD

BD
SURFACE TENSION LOG PLOT FOTOMTR SPCTR CHNG I2 REFRACTIVE INDEX

SURFACE TENSION LOG PLOT
CARL CHAL CARL CHAL DONB JAN LANG
64009

64009 63021 60012 T

\begin{tabular}{|c|c|c|c|c|c|c|c|}
\hline \multirow[t]{4}{*}{ COMPOUND NO $=$} & \multirow[t]{4}{*}{$\begin{array}{l}378 \text { MOL WGT - } \\
\text { HOMOGENEOUS HEAD }\end{array}$} & \multicolumn{2}{|c|}{$\begin{array}{l}378.6 \\
\text { GROUP }\end{array}$} & \multicolumn{3}{|c|}{ DECYL/OXYETHYLENE/5 } & ALCOHOL \\
\hline & & 20 & 8.6 & $4 \mathrm{~N}$ & & SURI & \\
\hline & & 20 & 1.0 & $x 10-3$ & BG & FOTOMTR S & CTR CHNG I2 \\
\hline & & 20 & 7.8 & $\mathrm{xl0}-4 \mathrm{~N}$ & $\mathrm{BC}$ & REFRACTIVI & INDEX \\
\hline
\end{tabular}

3 ENTRIES FOR COMPOUND
2 ENTRIES FOR COMPOUND

$$
\begin{aligned}
& 379 \text { MOL WGT - } \\
& \text { HOMOGENEOUS HEAD }
\end{aligned}
$$

\section{GROUP} 20

\section{6}

4.2
6.4
DECYL/OXYETHYLENE/4 ALCOHOL

$\mathrm{Xl0-4} \mathrm{M}$ $\mathrm{X} 10-4 \mathrm{M} \quad \mathrm{BC}$
FOTOMTR SPCTR CHNG I2

SURFACE TENSION LOG PLOT
CARL CHAL 64009 T $\quad$ L CARL CHAL 64009 T L

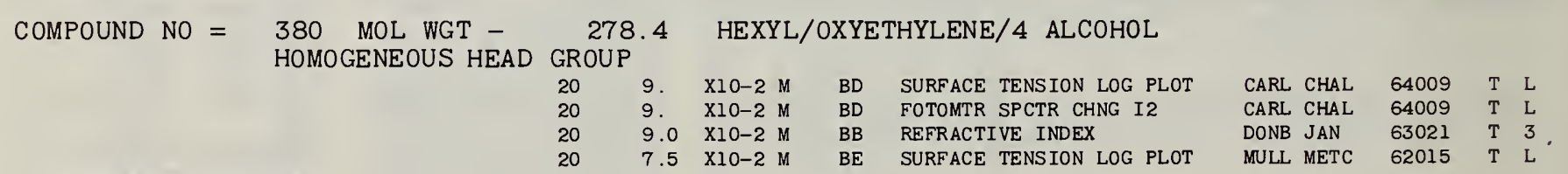

4 ENTR IES FOR COMPOUND

$\begin{array}{llll} & & & \\ \text { CARL CHAL } & 64009 & \text { T } & \text { L } \\ & 64009 & \text { T } & \text { L }\end{array}$ DONB JAN 63021 T $\quad$ L
Concentration units: $\mathrm{A}-\mathrm{mol} \% ; \mathrm{B}-\mathrm{vol} \%$ solvent; $\mathrm{C}-$ mol \% surfactant mixture; $\mathrm{D}-\mathrm{wt} / \mathrm{vol} \%$; E-\% saturation; $\mathrm{H}-$ wt $\%$ solvent; $\mathrm{I}-$ mol \% surfactant; $\mathrm{K}$ - normality counterions; $\mathrm{M}$ - molar; $\mathrm{N}-$ normal; $\mathrm{P}-$ wt $\% ; \mathrm{Q}-$ wt $\%$ surfactant; $\mathrm{R}$-varied; $\dot{\mathrm{S}}-\mathrm{mol} /$ kg; T - wt \% surfactant mixture; U-mol/(l orkg); W - molal; Y - atm. Details on page 222 


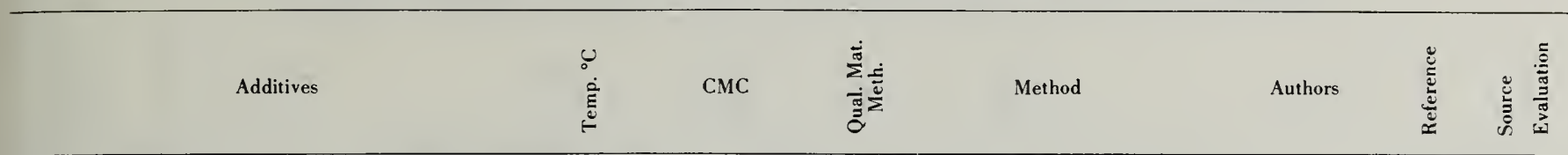

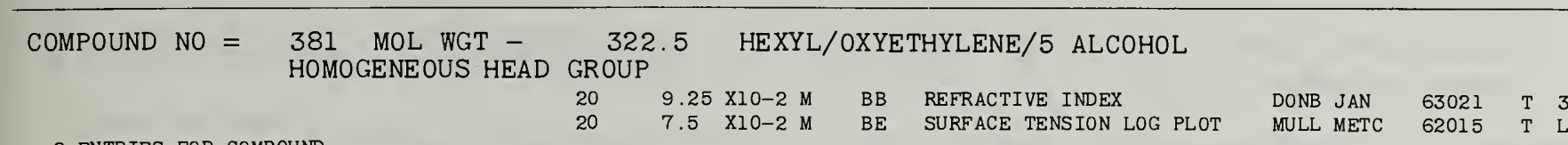

2 ENTRIES FOR COMPOUND

COMPOUND NO $=382$ MOL WGT -

1 ENTRIES FOR COMPOUND

COMPOUND NO $=383$ MOL WGT -

1 ENTRIES FOR COMPOUND

COMPOUND NO $=384$ MOL WGT -

1 ENTRIES FOR COMPOUND

COMPOUND NO $=385$ MOL WGT -

2 ENTRIES FOR COMPOUND

COMPOUND NO $=386$ MOL WGT -

3 ENTRIES FOR COMPOUND

COMPOUND NO $=387$ MOL WGT -

1 ENTRIES FOR COMPOUND

COMPOUND NO $=388$ MOL WGT -

1 ENTRIES FOR COMPOUND

COMPOUND NO $=389$ MOL WGT -

1 ENTRIES FOR COMPOUND

COMPOUND NO $=390$ MOL WGT -

1 ENTRIES FOR COMPOUND

COMPOUND NO $=391$ MOL WGT -

1 ENTRIES FOR COMPOUND

COMPOUND NO $=392$ MOL WGT -

1 ENTRIES FOR COMPOUND

COMPOUND NO $=393$ MOL WGT HOMOGENEOUS HEA

3 ENTRIES FOR COMPOUND
353.6 ETHYL TRIMETHYLAMMONIUM DODECYL SULFATE

254.30 XI0-3 M DA SPECFC CONDCTNCE GRAPH PACK DONB 63030 T $\quad$ L

381.6 BUTYL TRIMETHYLAMMONIUM DODECYL SULFATE $25 \quad 2.38 \times 10-3 \mathrm{M}$ DA SPECFC CONDCTNCE GRAPH PACK DONB

63030 T L

409.7 HEXYL TR IMETHYLAMMONIUM DODECYL SULFATE $25 \quad 1.25 \times 10-3 \mathrm{M}$ DB SPECFC CONDCTNCE GRAPH PACK DONB

63030 T L

$\begin{array}{rrrrrr}437.8 & \text { OCTYL } & \text { TRIMETHYLAMMONIUM DODECYL } & \text { SULFATE } \\ 25 & 4.3 & \text { XIO-4 M } & \text { BC } & \text { METHOD NOT CITED } & \text { CORK GOOD } \\ 25 & 4.0 & \text { X10-4 M } & \text { DB } & \text { SPECFC CONDCTNCE GRAPH } & \text { PACK DONB }\end{array}$

$66014 \quad$ T 3 63030 T $\quad$ L

\begin{tabular}{lllllllll}
\multicolumn{2}{c}{283.4} & AMMONIUM DODECYL SULFATE & & & & & \\
25 & 6.16 & X10-3 M & DA & SPECFC CONDCTNCE GRAPH & PACK DONB & 63030 & T & L \\
30 & 7.2 & X10-3 M & CB & EQUIV CONDCTNCE GRAPH & MEGU KOND & 59026 & T & L \\
30 & 6.6 & X10-3 M & CG & FOTOMTR SPCTR CHNGE RHD6 & MEGU KOND & 59026 & T & L
\end{tabular}

297.5 METHYLAMMONIUM DODECYL SULFATE $25 \quad 5.70 \times 10-3 \mathrm{M}$ DA SPECFC CONDCTNCE GRAPH PACK DONB 63030 T

311.5 ETHYLAMMONIUM DODECYL SULFATE $25 \quad 5.00 \times 10-3 \mathrm{M} \quad$ DA SPECFC CONDCTNCE GRAPH $\quad$ PACK DONB 63030 T

339.5 BUTYLAMMONIUM DODECYL SULFATE $252.92 \times 10-3 \mathrm{M} \quad$ DA SPECFC CONDCTNCE GRAPH PACK DONB $63030 \mathrm{~T} \quad \mathrm{~L}$

367.6 HEXYLAMMONIUM DODECYL SULFATE $25 \quad 1.12 \times 10-3 \mathrm{M} \quad$ DB SPECFC CONDCTNCE GRAPH

PACK DONB $63030 \quad$ T $\quad$ L

395.7 OCTYLAMMONIUM DODECYL SULFATE $25 \quad 2.8 \times 10-4 \mathrm{M} \quad$ DC SPECFC CONDCTNCE GRAPH

PACK DONB $63030 \quad$ T $\quad$ L

165.7 OCTYLAMMONIUM CHLORIDE

$25 \quad 1.75 \times 10-1 \mathrm{M} \quad \mathrm{XC}$ REFRACTIVE INDEX

KLEV $\quad 53010 \quad$ T $\quad$ L

\subsection{BUTYL/OXYETHYLENE/ 6 ALCOHOL}

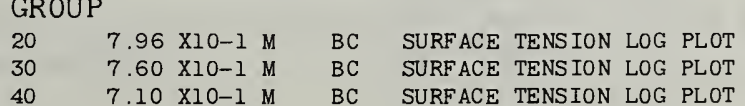

ELTO FLOR

ELWO FLOR

ELWO FLOR
64049 T 3

64049 T 3

64049 T $\quad$ L

\footnotetext{
$\begin{array}{ll}\text { COMPOUND NO }= & 394 \text { MOL WGT }- \\ \text { HOMOGENEOUS HEAD GROUP }\end{array}$

$\begin{aligned} & \text { COMPOUND NO }= 394 \text { MOL WGT }- \\ & \text { HOMOGENEOUS HEAD GROUP }\end{aligned}$
}

Concentration units: $\mathrm{A}-\mathrm{mol} \%$; $\mathrm{B}-\mathrm{vol} \%$ solvent; $\mathrm{C}-$ mol \% surfactant mixture;

counterions; $\mathrm{M}-$ molar; $\mathrm{N}-$ normal; $\mathrm{P}-$ wt \%; $\mathrm{Q}-$ wt $\%$ surfactant; $\mathrm{R}-$ varied; $\mathrm{S}-$ mol

$\mathrm{D}-\mathrm{wt} / \mathrm{vol} \% ; \mathrm{E}-\%$ saturation; $\mathrm{H}-\mathrm{wt} \%$ solvent; $\mathrm{I}-\mathrm{mol} \%$ surfactant; $\mathrm{K}$ - normality $\mathrm{kg} ; \mathrm{T}-\mathrm{wt} \%$ surfactant mixture; $\mathrm{U}-\mathrm{mol} /(\mathrm{l}$ or $\mathrm{kg})$; $\mathrm{W}-$ molal; $\mathrm{Y}-$ atm. Details on page 222. 


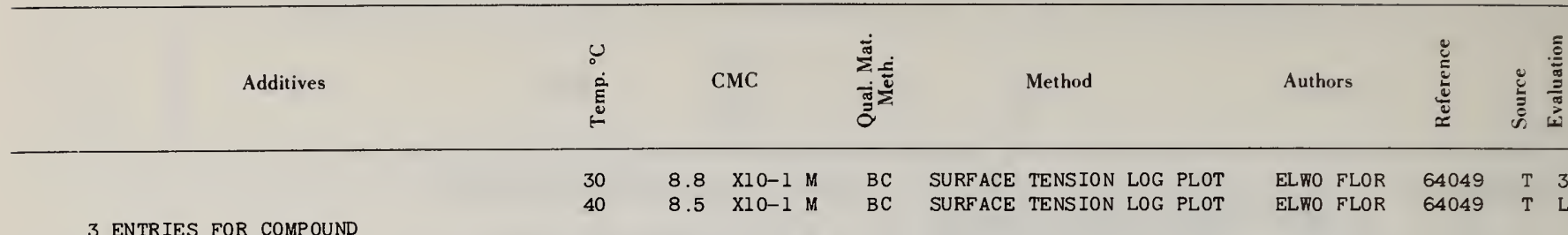

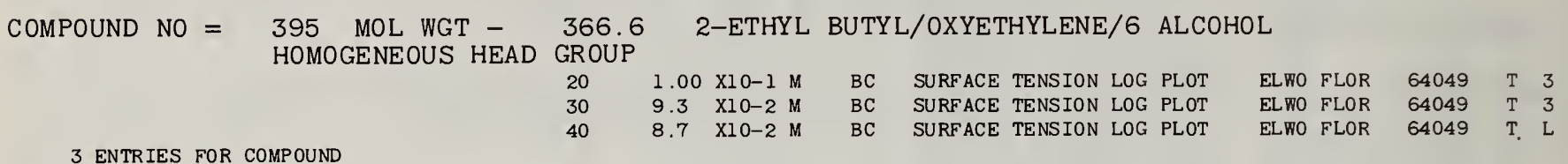

3 ENTRIES FOR COMPOUND

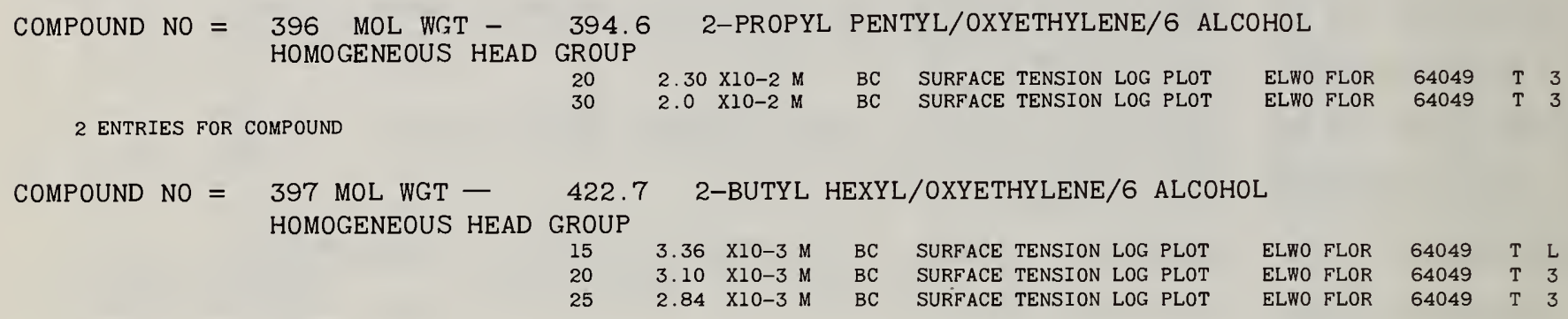

3 ENTRIES FOR COMPOUND

COMPOUND NO $=398$ MOL WGT - 554.9 2-BUTYL HEXYL/OXYETHYLENE/9 ALCOHOL HOMOGENEOUS HEAD GROUP

3 ENTRIES FOR COMPOUND

$\begin{array}{llll}20 & 3.20 \times 10-3 \mathrm{M} & \text { BC } & \text { SURFACE TENSION LOG PLOT } \\ 30 & 2.79 \times 10-3 \mathrm{M} & \text { BC } & \text { SURFACE TENSION LOG PLOT } \\ 40 & 2.43 \times 10-3 \mathrm{M} & \text { BC } & \text { SURFACE TENSION LOG PLOT }\end{array}$

ELWO FLOR ELWO FLOR ELWO FLOR

64049 T 3 64049 T 3 64049 T L

278.0 DODECYL DIMETHYL ETHYLAMMONIUM CHLORIDE

$\begin{array}{lllllllll}25 & 1.9 & \text { XIO-2 M } & \text { BC } & \text { SURFACE TENSION LOG PLOT } & \text { WEIN ZOGR } & 65026 & \text { T } & \text { L } \\ \text { UNK } & 2.13 & \text { X10-2 M } & \text { CC } & \text { UNSPECIFIED CONDUCTANCE } & \text { CELL EGGE } & 52001 & \text { T } & \text { L } \\ 25 & 7.0 & \text { X10-3 M } & \text { BC } & \text { SURFACE TENSION LOG PLOT } & \text { WEIN ZOGR } & 65026 & \text { T } & \text { L }\end{array}$

1. E-1 K CL

3 ENTRIES FOR COMPOUND

COMPOUND NO $=400$ MOL WGT -

1 ENTRIES FOR COMPOUND

COMPOUND NO $=401$ MOL WGT -

I ENTRIES FOR COMPOUND

COMPOUND NO $=402$ MOL WGT -

1 ENTRIES FOR COMPOUND

COMPOUND NO $=403$ MOL WGT -

1 ENTRIES FOR COMPOUND

$\begin{aligned} \text { COMPOUND NO }= & 404 \mathrm{MOL} \text { WGT }- \\ & 354.1 \\ & \text { } 6 \mathrm{H} 5 \mathrm{CH} 2 \mathrm{CH} 2 / \mathrm{N} / \mathrm{CH} 3 / 2 / \mathrm{Cl} 2 \mathrm{H} 25\end{aligned}$

1 ENTRIES FOR COMPOUND

$\begin{array}{lllllllll}\text { UNK } & 4.1 & \mathrm{X} 10-3 \mathrm{M} & \mathrm{CC} & \text { UNSPECIFIED CONDUCTANCE } & \text { CELL EGGE } & 52001 & \mathrm{~T} & \mathrm{~L}\end{array}$

COMPOUND NO $=405$ MOL WGT -

1 ENTIRES FOR COMPOUND

354.1 DODECYL METHYLETHYLBENZYLAMMONIUM CHLORIDE

$\begin{array}{lllllllll}\text { UNK } & 7.7 & \text { X10-3 M } & \text { CC UNSPECIFIED CONDUCTANCE } & \text { CELL EGGE } & 52001 & T & \text { L }\end{array}$

\section{COMPOUND NO $=406$ MOL WGT - \\ CHLORIDE}

$\mathrm{CF} 3 \mathrm{C} 6 \mathrm{H} 4 \mathrm{CH} 2 / \mathrm{N} / \mathrm{CH} 3 / 2 / \mathrm{C} 12 \mathrm{H} 25$

1 ENTRIES FOR COMPOUND

UNK $\quad 3.2 \times 10-3 \mathrm{M} \quad \mathrm{CC}$ UNSPECIFIED CONDUCTANCE $\quad$ CELL EGGE $52001 \quad \mathrm{~T} \quad \mathrm{~L}$

Concentration units: $\mathrm{A}-\mathrm{mol} \%$; $\mathrm{B}-$ vol $\%$ solvent; $\mathrm{C}-$ mol \% surfactant mixture; $\mathrm{D}-\mathrm{wt} / \mathrm{vol} \%$; E-\% saturation; $\mathrm{H}-$ wt $\%$ solvent; $\mathrm{I}-$ mol \% surfactant; $\mathrm{K}-$ normality counterions; $\mathrm{M}-$ molar; $\mathrm{N}-$ normal; $\mathrm{P}-$ wt $\% ; \mathrm{Q}-$ wt $\%$ surfactant; $\mathrm{R}$ - varied; $\mathrm{S}-\mathrm{mol} /$ $\mathrm{kg} ; \mathrm{T}-\mathrm{wt} \%$ surfactant mixture; $\mathrm{U}-\mathrm{mol} /(\mathrm{lorkg}) ; \mathrm{W}$-molal: $\mathrm{Y}$-atm. Detailson page 222. 


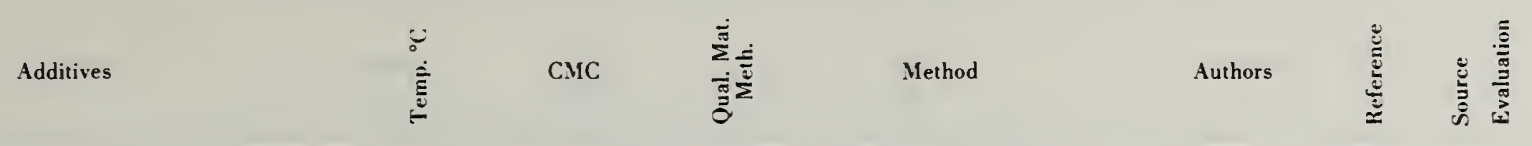

$\begin{array}{ll}\text { COMPOUND NO }= & 407 \text { MOL WGT }- \\ \text { C6H5CH2CH2/N/CH3/2/C } 12 \mathrm{H} 25 & \text { DODECYL DIMETHYL 3-PHENYLPROPYLAMMONIUM CHLORIDE }\end{array}$

1 ENTRIES FOR COMPOUND

COMPOUND NO $=408$ MOL WGT -

1 ENTRIES FOR COMPOUND

COMPOUND NO $=409$ MOL WGT -

3 ENTRIES FOR COMPOUND

COMPOUND NO $=410$ MOL WGT -

3 ENTRIES FOR COMPOUND

COMPOUND NO $=411$ MOL WGT -

1 ENTRIES FOR COMPOUND

COMPOUND NO $=412$ MOL WGT -

1 ENTRIES FOR COMPOUND

COMPOUND NO $=413$ MOL WGT -

1 ENTRIES FOR COMPOUND

COMPOUND NO $=414$ MOL WGT -

1 ENTRIES FOR COMPOUND

COMPOUND NO $=415$ MOL WGT -

1 ENTRIES FOR COMPOUND

COMPOUND NO $=416$ MOL WGT -

E $0 \quad$ PH OF SOLUTION

10 ENTRIES FOR COMPOUND

COMPOUND NO $=417$ MOL WGT -

$2.5 \quad \mathrm{E}-2 \mathrm{M} \quad \mathrm{HCL}$

5. $\mathrm{E}-2 \mathrm{M} \quad \mathrm{H} \mathrm{CL}$
344.6 POTASSIUM HEXADECANE 1-SULFONATE

$80 \quad 1.80 \times 10-3 \mathrm{~W} \quad \mathrm{CD}$ EQUIV CONDCTNCE GRAPH $\quad$ MURR HART 35001 K $\mathrm{L}$

415.6 TRIETHANOLAMMONIUM DODECYL SULFATE

$\begin{array}{lllllllll} & 59017 & & \text { VALUES FRM REF IN CMC } & \text { KASH } & 58021 & & \text { R } \\ 43 & 4 . & \text { X10-3 M } & \text { BE } & \text { SURFACE TNSN LINEAR PLOT } & \text { KASH EZAK } & 59017 & \text { T } & \text { L } \\ 40 & 4 . & \text { X10-3 M } & \text { BE } & \text { FOAMING PONER } & \text { KASH EZAK } & 59017 & \text { T } & \text { L }\end{array}$

353.5 MORPHOLINIUM DODECYL SULFATE

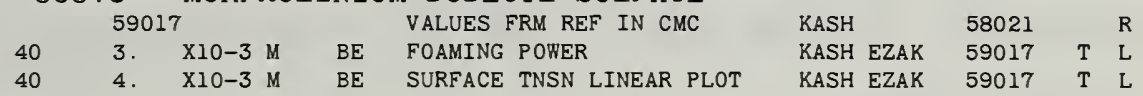

217.4 DECYLAMMONIUM ACETATE

UNK 4. X10-2 M CE METHOD NOT CITED SOMA HEAL 64035 T L

245.5 DODECYLAMMONIUM ACETATE

UNK $\quad 1.3 \quad \times 10-2 \mathrm{M} \quad \mathrm{CD}$ METHOD NOT CITED

SOMA HEAL 64035 T $\mathrm{L}$

273.5 TETRADECYLAMMONIUM ACETATE

UNK 4. X10-3 M CE METHOD NOT CITED

SOMA HEAL 64035 T L

301.6 HEXADECYLAMMONIUM ACETATE

UNK 8. X10-4 M CE METHOD NOT CITED

329.6 OCTADECYLAMMONIUM ACETATE

UNK 3 X10-4 M CE METHOD NOT CITED

\subsection{PERFLUORO HEXANOIC ACID}

QUESTIONABLE CRITERION QUESTIONABLE CRITERION

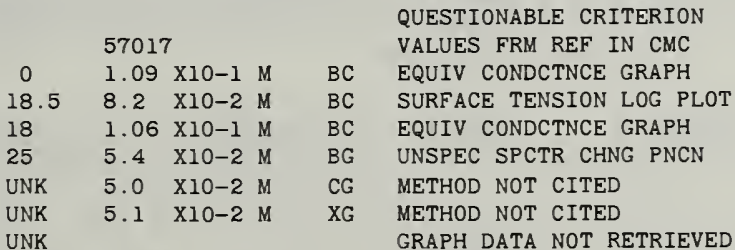

KLEV RAIS

KLEV VERG

KLEV VERG

KLEV VERG

KLEV RAIS

KLEV VERG

KLEV

KLEV CARR

KLEV RAIS

KLEV RAIS

54010

57017

56010

57017

54010

57017

58011

56001

54004

54004

$\begin{array}{ll} & R \\ & R \\ & R \\ P & 3 \\ T & L \\ P & 3 \\ T & L \\ T & L \\ T & L \\ & R\end{array}$

414.1 PERFLUORO OCTANOIC ACID

QUESTIONABLE CRITERION QUESTIONABLE CRITERION 57017 VALUES FRM REF IN CMC

\begin{tabular}{|c|c|c|c|c|}
\hline \multicolumn{4}{|c|}{57017} & \multirow{2}{*}{$\begin{array}{l}\text { VALUES FRM REF IN CMC } \\
\text { SURFACE TENSION LOG PLOT }\end{array}$} \\
\hline 18.5 & 9.0 & $\times 10-3$ & $B C$ & \\
\hline & 9.8 & $\mathrm{x} 10-3 \mathrm{~N}$ & BB & EQUIV CONDCTNCE GRAPH \\
\hline & 5.6 & $x 10-3$ & BG & UNSPEC SPCTR CHNG PNCN \\
\hline & 8.7 & $\times 10-3$ & $\mathrm{BC}$ & SURFACE TENSION LOG PLOT \\
\hline & 9.3 & $\mathrm{x} 10-3$ & BB & EQUIV CONDCTNCE GRAPH \\
\hline & 1.02 & $x 10-2$ & BB & EQUIV CONDCTNCE GRAPH \\
\hline & 5.1 & $\mathrm{x} 10-3$ & CG & METHOD NOT CITED \\
\hline & 3.2 & $x_{10}-3$ & $\mathrm{BC}$ & SURFACE TENSION LOG \\
\hline & & $\mathrm{x} 10-3 \mathrm{~N}$ & & SURFACE TENSION LOG PLOT \\
\hline
\end{tabular}

KLEV RATS KLEV VERG KLEV VERG

KLEV RAIS

KLEV VERG

KLEV

KLEV RAIS

KLEV VERG

KLEV VERG

KLEV CARR

KLEV RAIS

KLEV RAIS

54010

57017

56010

54010

$57017 \quad \mathrm{P} \quad 3$

58011 T $\quad$ L

54010 T $\quad$ L

$57017 \quad P \quad 3$

$57017 \quad P \quad 3$

56001 T L

54010 T $\quad$ L

54010 T $L$

Concentration units: $\mathrm{A}-\mathrm{mol} \%$; $\mathrm{B}-\mathrm{vol} \%$ solvent; $\mathrm{C}-\mathrm{mol} \%$ surfactant mixture;

counterions; $\mathrm{M}$-molar; $\mathrm{N}$-normal; $\mathrm{P}$-wt \%; $\mathrm{Q}-$ wt \% surfactant; $\mathrm{R}$ - varied; $\mathrm{S}-$ mol/ $\mathrm{D}-\mathrm{wt} / \mathrm{vol} \%$; E-\% saturation; $\mathrm{H}-\mathrm{wt} \%$ solvent: I - mol \% surfactant; K-normality 


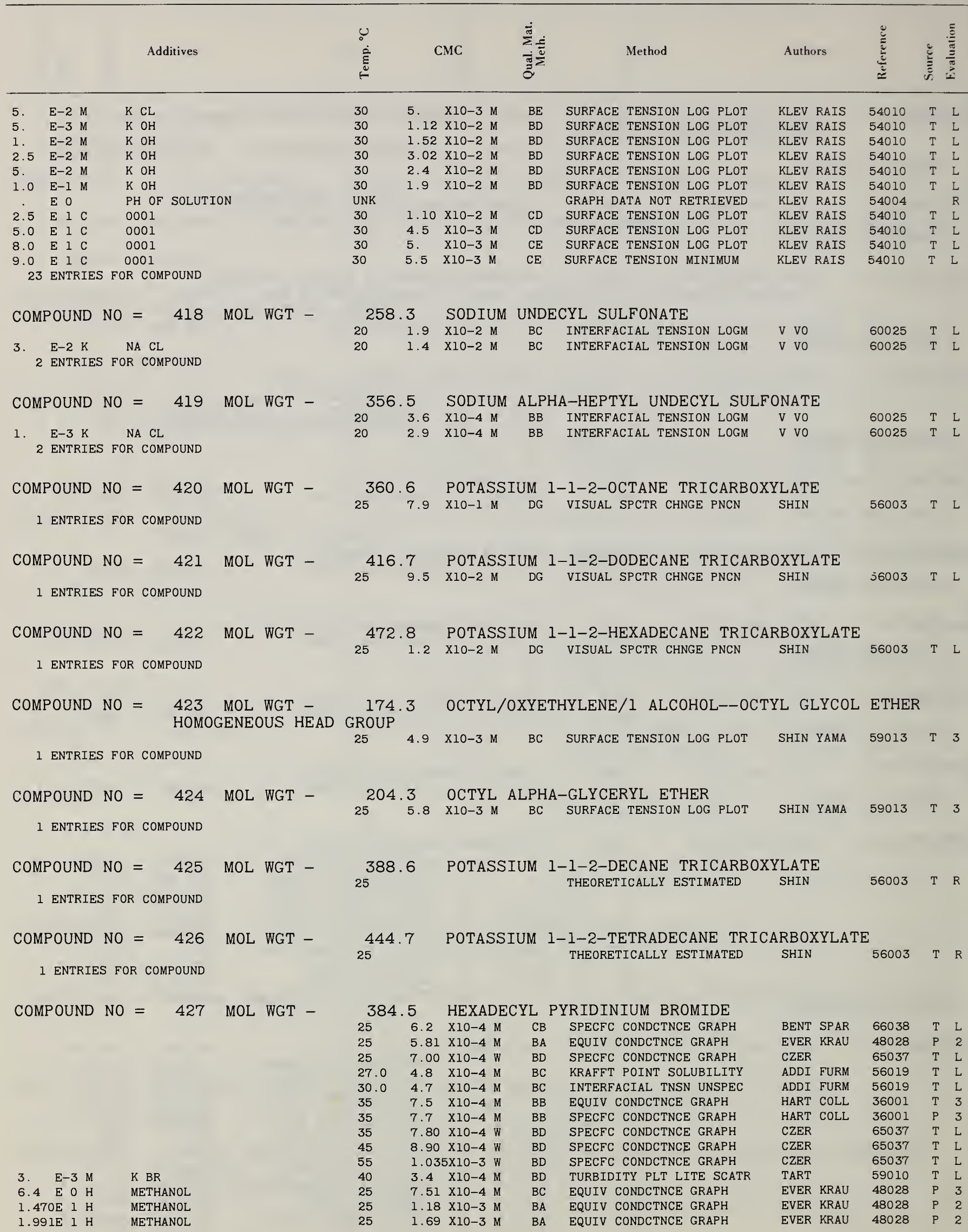

Concentration units: $\mathrm{A}-\mathrm{mol} \%$; $\mathrm{B}-$ vol \% solvent; $\mathrm{C}-$ mol \% surfactant mixture; 


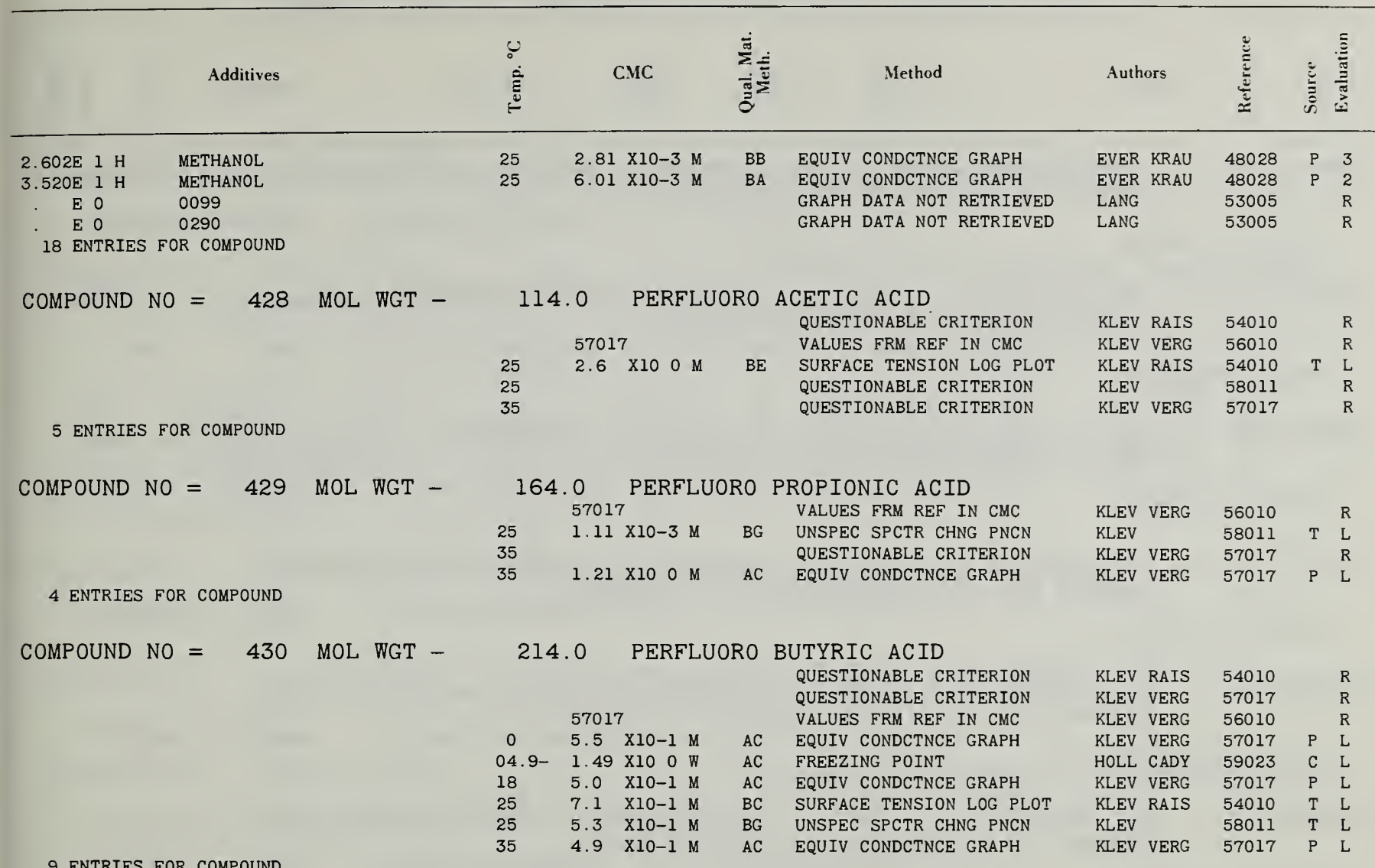

COMPOUND NO $=$ 431 MOL WGT -
BRANCHED CHAIN

1 ENTRIES FOR COMPOUND

574.9 TRIDECYL/OXO-PROCESS/ /OXYETHYLENE/8.5 ALCOHOL NATURAL OE DISTRIBUTION

$\begin{array}{lllllllllll}\text { UNK } & 8.8 & \text { X10-3 D HC FOTOMTR SPCTR CHNG I2 } & \text { BECH } & 59006 & G & \text { L }\end{array}$

\section{COMPOUND NO $=432$ MOL WGT - BRANCHED CHAIN}

E $O \quad N A C L$

2 ENTRIES FOR COMPOUND
641.0 TRIDECYL/OXO-PROCESS/ /OXYETHYLENE/10 ALCOHOL NATURAL OE DISTRIBUTION $\begin{array}{llllllllll}\text { UNK } & 8.1 & \text { X10-3 D } & \text { HC } & \begin{array}{l}\text { FOTOMTR SPCTR CHNG I2 } \\ \text { SUMMARIZING EQN ONLY }\end{array} & \text { BECH } & 59006 & \text { BECH } & \text { L } \\ 25 & & & & & 62002 & & \text { R }\end{array}$

861.3 TRIDECYL/OXO-PROCESS/ /OXYETHYLENE/15 ALCOHOL NATURAL OE DISTRIBUTION

$\begin{array}{lllllllll}\text { UNK } & 1.3 & \text { X10-2 D } & \text { HD } & \text { FOTOMTR SPCTR CHNG I2 } & \text { BECH } & 59006 & \text { G } & \text { L } \\ 25 & & & & \text { SUMMARIZING EQN ONLY } & \text { BECH } & 62002 & & \text { R }\end{array}$

E $0 \quad$ NA CL

433 MOL WGT BRANCHED CHAIN, $\begin{array}{lllll}\text { UNK } & 1.3 & \text { X10-2 D } & \text { HD } & \text { FOTOMTR SPCTR CHNG I2 } \\ 25 & & & & \text { SUMMARIZING EQN ONLY }\end{array}$ $\mathrm{BECH}$ 62002

2 ENTRIES FOR COMPOUND

COMPOUND NO $=434$ MOL WGT $-1,169.7$ TRIDECYL/OXO-PROCESS/ /OXYETHYLENE/22 ALCOHOL BRANCHED CHAIN, NATURAL OE DISTRIBUTION

$\begin{array}{llllll} & & & \\ \text { UNK } & 2.8 & \text { XIO-2 D } & \text { HD FOTOMTR SPCTR CHNG I2 } & \text { BECH } & 59006\end{array}$
$\begin{array}{lllllll}\text { UNK } & 2.8 & \text { X10-2 D } & \text { HD } & \text { FOTOMTR SPCTR CHNG I2 } & \text { BECH } & 59006 \\ 25 & & & & \text { SUMMARIZING EQN ONLY } & \text { BECH } & 62002\end{array}$ $62002 \quad R$

E $O \quad N A C L$

2 ENTRIES FOR COMPOUND

711.2 OCTADECYL/OXYETHYLENE/10 ALCOHOL QUESTIONABLE CRITERION VALUES FRM REF IN CMC BECH

62002 59006 $R$ 2 ENTRIES FOR COMPOUND 62002

\section{COMPOUND NO $=436$ MOL WGT $-1,151.8$ OCTADECYL/OXYETHYLENE/2O ALCOHOL} NATURAL DISTRIBUTION OF HEAD GROUPS

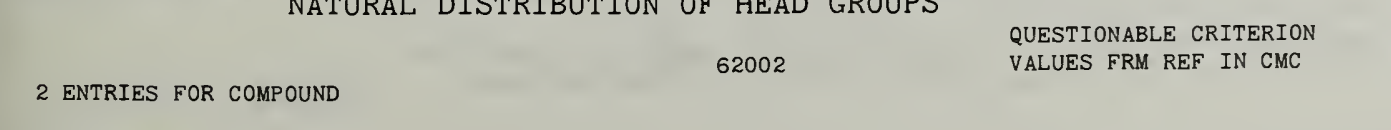




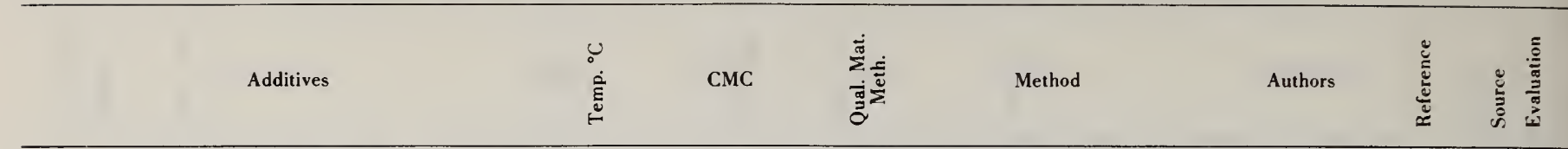

$\begin{aligned} \text { COMPOUND NO }= & 437 \text { MOL WGT }- \\ \text { NATURAL DISTRIBUTION OF } & 797.3 \text { OLEYL/CIS GROUPS } \\ \text { UNK } & 3.3 \text { X10-2 D }\end{aligned}$

1 ENTRIES FOR COMPOUND

UNK $\quad 3.3 \quad$ X10-2 D HD FOTOMTR SPCTR CHNG I2 2 BECH 59006 G L
COMPOUND NO $=438$ MOL WGT -973.5 OLEYL/CIS-9-OCTADECENOYL/ /OXYETHYLENE/16 ALCOHOL NATURAL DISTRIBUTION OF HEAD GROUPS
UNK $3.2 \times 10-2$ D HD FOTOMTR SPCTR CHNG I2 BECH 59006 G L

1 ENTRIES FOR COMPOUND

COMPOUND NO $=439$ MOL WGT $-1,149.7$ OLEYL/CIS-9-OCTADECENOYL/ /OXYETHYLENE/2O ALCOHOL NATURAL DISTRIBUTION OF HEAD GROUPS

1 ENTRIES FOR COMPOUND

UNK $2.9 \times 10-2$ D HD FOTOMTR SPCTR CHNG I2 BECH 59006 G L $\begin{array}{ll}\text { COMPOUND NO }= & 440 \text { MOL WGT } \\ \text { ISOMERS AND DISTRIBUTED MULTIPLE OE CHAINS }\end{array}$

1 ENTRIES FOR COMPOUND

UNK $\quad 1.3 \quad$ XIO-2 D HD FOTOMTR SPCTR CHNG I2 BECH

59006 G L

$\begin{aligned} & \text { COMPOUND NO }= 441 \text { MOL WGT - 699.0 POXETHYLENE/8 SORBITAN MONOLAURATE } \\ & \text { ISOMERS AND DISTRIBUTED MULTIPLE OE CHAINS } \\ & \text { UNK } 1.2 \text { X10-2 D HD FOTOMTR SPCTR CHNG I2 }\end{aligned}$

1 ENTRIES FOR COMPOUND

59006 G L

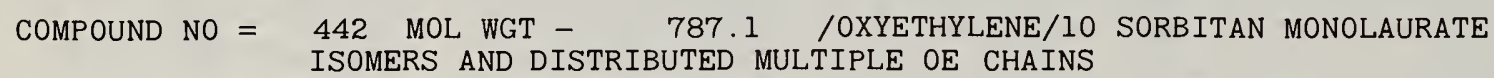

1 ENTRIES FOR COMPOUND

UNK $1.2 \times 10-2 D$ HD FOTOMTR SPCTR CHNG I2 BECH

$59006 \quad$ G L

$\begin{aligned} \text { COMPOUND NO }= & 443 \text { MOL WGT }- \text { 1,227.7 /OXYETHYLENE/2O SORBITAN MONOLAURATE } \\ & \text { ISOMERS AND DISTRIBUTED MULTIPLE OE CHAINS }\end{aligned}$

1 ENTRIES FOR COMPOUND

UNK $\quad 1.4 \times 10-2$ D $\quad$ HD FOTOMTR SPCTR CHNG I2

$59006 \quad$ G L

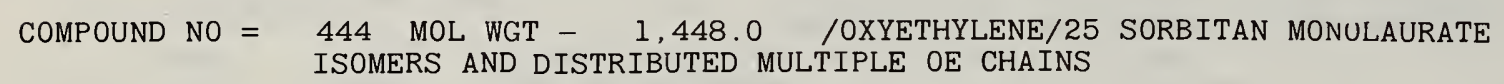

1 ENTRIES FOR COMPOUND

UNK $1.4 \quad \times 10-2 \mathrm{D} \quad$ HD FOTOMTR SPCTR CHNG I2

COMPOUND NO $=445$ MOL WGT -

292.3 SODIUM PARA-BIS-/N-BUTYL/ BENZENE SULFONATE

1 ENTRIES FOR COMPOUND

60 QUESTIONABLE CRITERION KOBE KLAM 60017

$\mathrm{R}$

COMPOUND NO $=446$ MOL WGT -

1 ENTRIES FOR COMPOUND

COMPOUND NO $=447$ MOL WGT -

1 ENTRIES FOR COMPOUND

COMPOUND NO $=448$ MOL WGT -

1. E-1 H NA OH

4 ENTRIES FOR COMPOUND

COMPOUND NO $=449$ MOL WGT -

1 ENTRIES FOR COMPOUND 348.5 SODIUM PARA-BIS-/N-HEXYL/ BENZENE SULFONATE
60
QUESTIONABLE CRITERION

$\mathrm{R}$

404.6 SODIUM PARA-BIS-/N-OCTYL/ BENZENE SULFONATE 60 QUESTIONABLE CRITERION KOBE KLAM 60017 R

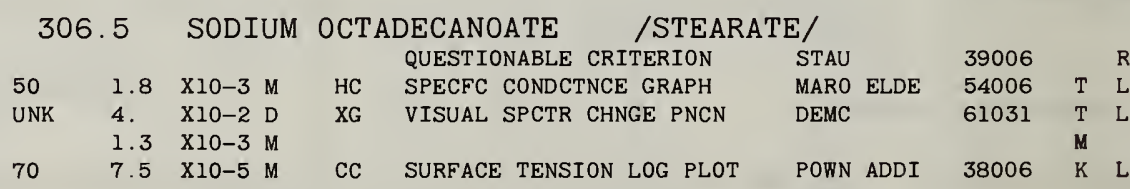

235.9 DODECYLMETHYL AMMONIUM CHLORIDE

$30 \quad 1.46 \times 10-2 \mathrm{M} \quad \mathrm{BC}$ EQUIV CONDCTNCE GRAPH $\quad$ RALS BRO0 $49013 \quad$ T 3
Concentration units: $\mathrm{A}-\mathrm{mol} \%$; $\mathrm{B}-$ vol \% solvent; $\mathrm{C}-$ mol \% surfactant mixture; $\mathrm{D}-\mathrm{wt} / \mathrm{vol} \%$; E-\% saturation; $\mathrm{H}-\mathrm{wt} \%$ solvent; I-mol \% surfactant; $\mathrm{K}$-normality counterions; $\mathrm{M}$ - molar; $\mathrm{N}$ - normal; $\mathrm{P}$ - wt \%; $\mathrm{Q}$ - wt \% surfactant; $\mathrm{R}$ - varied; $\mathrm{S}-$ mol/ $\mathrm{kg} ; \mathrm{T}-\mathrm{wt} \%$ surfactant mixtu re; $\mathrm{U}-\mathrm{mol} / \mathrm{l}$ or $\mathrm{kg}) ; \mathrm{W}-$ molal; $\mathrm{Y}-\mathrm{atm}$. Details on page 222. 


Additives $\quad$ CMC $\quad$ Method $\quad$ Authors

COMPOUND NO $=450$ MOL WGT -

1 ENTRIES FOR COMPOUND

COMPOUND NO $=451$ MOL WGT AMMONIUM CHLORIDE

249.9 DODECYLDIMETHYL AMMONIUM CHLORIDE

$\begin{array}{llllllll}30 & 1.61 \times 10-2 \mathrm{M} & \mathrm{BC} & \text { EQUIV CONDCTNCE GRAPH } & \text { RALS BRO0 } & 49013 & \mathrm{~T} & 3\end{array}$

448.1 PARA DI-ISOBUTYLPHENOXYETHOXYETHYL DIMETHYLBENZYL /HYAMINE $1622 /$

$\begin{array}{llllll}24.7 & 1.6 & \text { X10-1 } & \text { HC } & \text { GRAPH DATA NOT RETRIEVED } \\ 24.7 & 1.7 & \text { X } 10-1 & P & \text { HC } & \text { SPECFC CONDCTNCE GRAPH } \\ 24.7 & 9 . & \text { X10-2 } & \text { P } & \text { HD } & \text { SPECFC CONDCTNCE GRAPH } \\ 24.7 & 1.1 & \text { X10-1 } & \text { P } & \text { HD } & \text { SPECFC CONDCTNCE GRAPH } \\ 24.7 & 1.5 & \text { X10-1 P } & \text { HC } & \text { SPECFC CONDCTNCE GRAPH } \\ 24.7 & 1.4 & \text { X10-1 P } & \text { HC } & \text { SPECFC CONDCTNCE GRAPH } \\ 24.7 & 1.3 & \text { X10-1 P } & \text { HC } & \text { SPECFC CONDCTNCE GRAPH }\end{array}$

ROSS HUDS

COHE VASS

ROSS BRAM

ROSS BRAM

ROSS BRAM

ROSS BRAM

ROSS BRAM

ROSS BRAM

ROSS BRAM

57010

61027

$57031-T$

57031 T L

57031 T L

57031 T $L$

57031 T I

TRIBUTYL PHOSPHATE

2-ETHYL HEXANOL

9 ENTRIES FOR COMPOUND

COMPOUND NO $=452$ MOL WGT -

1 ENTRIES FOR COMPOUND

COMPOUND NO $=453$ MOL WGT -

1 ENTRIES FOR COMPOUND

COMPOUND NO $=454$ MOL WGT -

I ENTRIES FOR COMPOUND

COMPOUND NO $=455$ MOL WGT -

3 ENTRIES FOR COMPOUND
246.9 3-4-DICHLOROPERFLUORO BUTYRIC/KEL-F/ACID

$\begin{array}{lllllllll}25 & 7.0 & \text { X10-1 M } & \text { HG UNSPEC SPCTR CHNG PNCN } & \text { KLEV } & 58011 & T & \text { L }\end{array}$

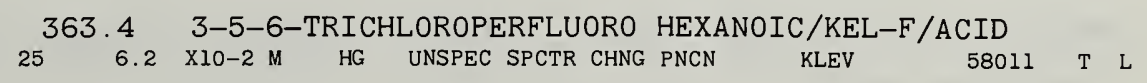

479.9 3-5-7-8-TETRACHLOROPERFLUORO OCTANOIC/KEL-F/ACID

$\begin{array}{llllllll}25 & 9.1 & \text { X10-3 M HG UNSPEC SPCTR CHNG PNCN } & \text { KLEV } & & & & \end{array}$
COMPOUND NO $=456$ MOL WGT -

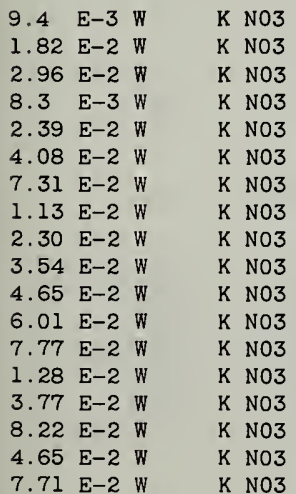

$\begin{array}{lll}76 & \text { ENTRIES FOR COMPOUND }\end{array}$
514.1 PERFLUORO DECANOIC ACID

\begin{tabular}{|c|c|c|c|c|}
\hline 25 & 4.8 & $\mathrm{X} 10-4 \mathrm{M}$ & BG & UNSPEC SPCTR CHNG PNCN \\
\hline & 8. & $\mathrm{X} 10-4 \mathrm{M}$ & $\mathrm{BC}$ & ACE TENSION LOG PLOT \\
\hline $\mathrm{N}$ & 8. & $\mathrm{X} 10-4 \mathrm{M}$ & $\mathrm{XG}$ & METHOD NOT CITED \\
\hline
\end{tabular}

KLEV

KLEV RAIS KLEV RAIS
58011 T $\mathrm{L}$

54010 T 54004 T L
COMPOUND NO $=457$ MOL WGT -

3 ENTRIES FOR COMPOUND
452.2 POTASSIUM PERFLUORO OCTANOATE

$\begin{array}{lll}25 & 2.88 \times 10-2 \mathrm{~W} \\ 30 & 2.74 \times 10-2 \mathrm{~W} \\ 30 & 2.63 \times 10-2 \mathrm{M} \\ 40 & 2.65 \times 10-2 \mathrm{~W} \\ 55 & 2.76 \times 10-2 \mathrm{~W} \\ 70 & 3.07 \times 10-2 \mathrm{~W} \\ 85 & 3.54 \times 10-2 \mathrm{~W} \\ \text { UNK } & 2.7 \times 10-2 \mathrm{M} \\ 30 & 2.43 \times 10-2 \mathrm{~W} \\ 30 & 2.22 \times 10-2 \mathrm{~W} \\ 30 & 2.01 \times 10-2 \mathrm{~W} \\ 40 & 2.40 \times 10-2 \mathrm{~W} \\ 40 & 2.07 \times 10-2 \mathrm{~W} \\ 40 & 1.79 \times 10-2 \mathrm{~W} \\ 40 & 1.46 \times 10-2 \mathrm{~W} \\ 55 & 2.42 \times 10-2 \mathrm{~W} \\ 55 & 2.17 \times 10-2 \mathrm{~W} \\ 55 & 1.93 \times 10-2 \mathrm{~W} \\ 55 & 1.82 \times 10-2 \mathrm{~W} \\ 55 & 1.65 \times 10-2 \mathrm{~W} \\ 55 & 1.49 \times 10-2 \mathrm{~W} \\ 70 & 2.75 \times 10-2 \mathrm{~W} \\ 70 & 2.22 \times 10-2 \mathrm{~W} \\ 70 & 1.82 \times 10-2 \mathrm{~W} \\ 85 & 2.59 \times 10-2 \mathrm{~W} \\ 85 & 2.27 \times 10-2 \mathrm{~W} \\ & \end{array}$

SHIN KATS SHIN KATS

KLEV RAIS

SHIN KATS

SHIN KATS

SHIN KATS

SHIN KATS

KLEV RAIS

SHIN KATS

SHIN KATS

SHIN KATS

SHIN KATS

SHIN KATS

SHIN KATS

SHIN KATS

SHIN KATS

SHIN KATS

SHIN KATS

SHIN KATS

SHIN KATS

SHIN KATS

SHIN KATS

SHIN KATS

SHIN KATS

SHIN KATS

SHIN KATS
64011 T 3

64011 T 3

$54010 \quad T \quad$ L

64011 T 3

64011 T 3

64011 T 3

64011 T 3

$54004 \quad T \quad L$

64011 L 3

64011 L 3

64011 L 3

64011 L 3

64011 L 3

64011 L 3

64011 L 3

64011 L 3

64011 L 3

64011 L 3

64011 L 3

64011 L 3

64011 L 3

64011 L 3

64011 L 3

64011 L 3

64011 L 3

64011 L 3

\subsection{1-4-HEXANEDIOL}

\begin{tabular}{lllllllll}
\multicolumn{1}{l}{118.2} & \multicolumn{1}{l}{$1-4-\mathrm{MEX}$} \\
24 & 1.9 & X10-1 M & BC & SURFACE TENSION LOG PLOT & KATO & 63037 & T & L \\
UNK & 2.0 & XIO-1 M & BC & FOTOMTR SOLUBLZTN SDN 4 & KATO & 63037 & T & L \\
UNK & 1.9 & XIO-1 M & BC & REFRACTIVE INDEX & KATO & 63037 & T & L
\end{tabular}

Concentration units: $\mathrm{A}-\mathrm{mol} \% ; \mathrm{B}-\mathrm{vol} \%$ solvent; $\mathrm{C}-$ mol \% surfactant mixture; $\mathrm{D}-$ wt/vol \%; E-\% saturation; $\mathrm{H}-$ wt \% solvent; I-mol \% surfactant; $\mathrm{K}-$ normality counterions; $\mathrm{M}-$ molar; $\mathrm{N}$ - normal; $\mathrm{P}$ - wt \%; $\mathrm{Q}-$ wt $\%$ surfactant; $\mathrm{R}$-varied; $\mathrm{S}-$ mol/ $\mathrm{kg} ; \mathrm{T}-$ wt \% surfactant mixture; $\mathrm{U}-\mathrm{mol} /(\mathrm{l}$ orkg); $\mathrm{W}-$ molal; $\mathrm{Y}-$ atm. Details on page 222. 


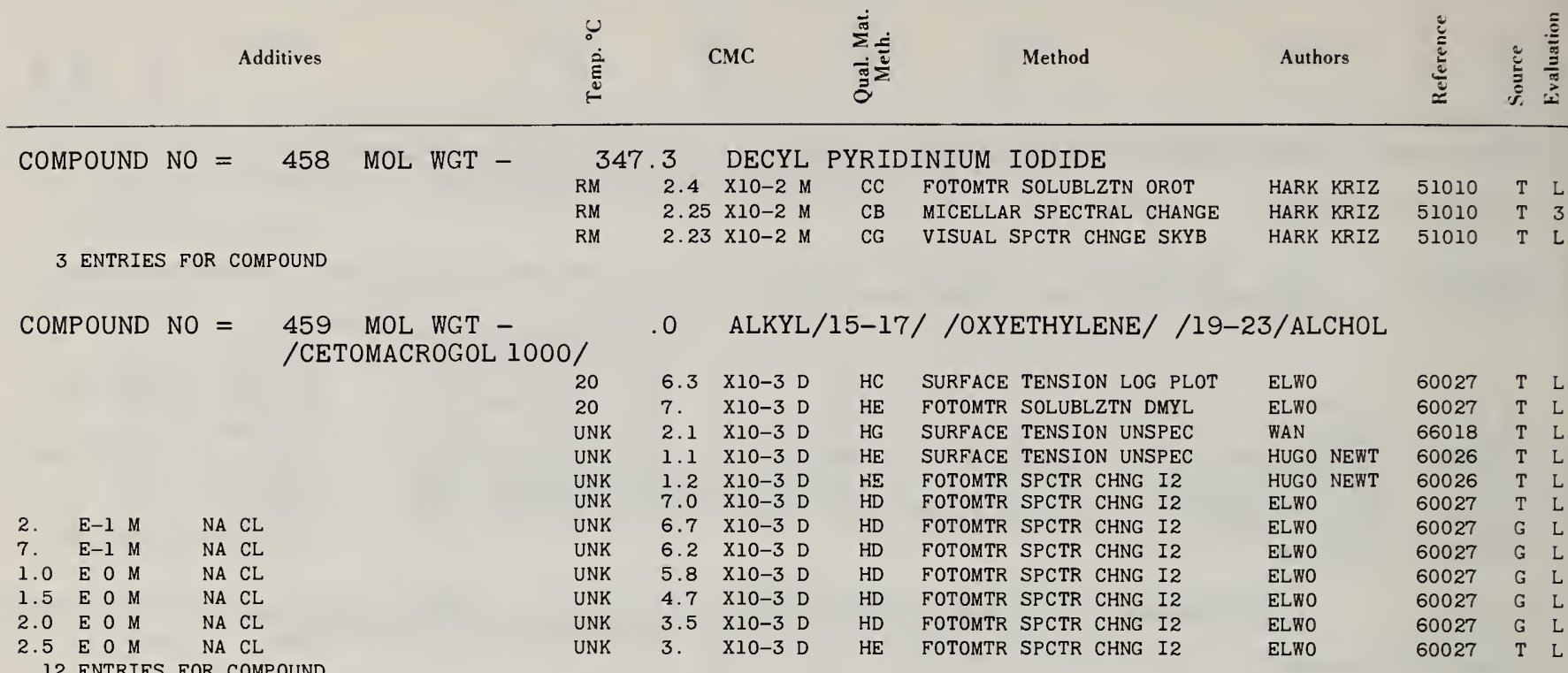

356.5 DODECYL TROPYLIUM MONOPHOSPHATE

$25 \quad 1.0 \quad \begin{array}{lll}\text { X10-3 M } & \text { BD SURFACE TENSION LOG PLOT ABUH MYSE } 65019 \text { T } & \text { L }\end{array}$

\section{COMPOUND NO $=460$ MOL WGT -

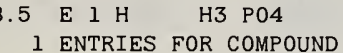

\section{COMPOUND NO $=461$ MCL WGT -} HOMOGENEOUS HEAD GROUP

2 ENTRIES FOR COMPOUND
462 MOL WGT -

$\begin{array}{ll}\text { NA } & C L \\ \text { NA } & C L \\ \text { NA } & C L \\ \text { NA } & C L \\ \text { NA } & C L \\ \text { NA } & C L \\ \text { NA } & C L \\ \text { NA } & C L \\ \text { NA } & C L\end{array}$

NA CL

11 ENTRIES FOR COMPOUND
118.2 BUTYL/OXYETHYLENE/I ALCOHOL--BUTYL GLYCOL ETHER $25 \quad 9.8 \quad \times 10-1 \mathrm{M} \quad \mathrm{CC}$ REFRACTIVE INDEX UNK 8.8 X10-1 M CC SURFACE TENSION LOG PLOT

DONB JAC DONB JACO

66019 T $L$ 66019 T L

$\begin{array}{llll}\text { BISW MUKH } & 60028 & \text { T } & \text { L } \\ \text { BISW MUKH } & 60028 & \text { T } & \text { L } \\ \text { BISW MUKH } & 60028 & \text { T } & \text { L } \\ \text { BISW MUKH } & 60028 & \text { T } & \text { L } \\ \text { BISW MUKH } & 60028 & \text { T } & \text { L } \\ \text { BISW MUKH } & 60028 & \text { T } & \text { L } \\ \text { BISW MUKH } & 60028 & \text { T } & \text { L } \\ \text { BISW MUKH } & 60028 & \text { T } & \text { L } \\ \text { BISW MUKH } & 60028 & \text { T } & \text { L } \\ \text { BISW MUKH } & 60028 & \text { T } & \text { L } \\ \text { BISW MUKH } & 60028 & \text { T } & \text { L }\end{array}$

\subsection{SODIUM MONOLAURIN SULFATE}

$25 \quad 5.2 \times 10-3 \mathrm{M} \quad$ CC SPECFC CONDCTNCE GRAPH

UNK $2.6 \quad \mathrm{X10-3} \mathrm{M} \quad \mathrm{CG}$ VISUAL SPCTR CHNGE RHD6

$25 \quad 3.4 \times 10-3 \mathrm{M} \quad$ CC SPECFC CONDCTNCE GRAPH

$25 \quad 1.6 \times 10-3 \mathrm{M} \quad \mathrm{CC}$ SPECFC CONDCTNCE GRAPH

$25 \quad 1.03 \times 10-3 \mathrm{M} \quad$ CD SPECFC CONDCTNCE GRAPH

$256.025 \times 10-\dot{M}$ CE SPECFC CONDCTNCE GRAPH

UNK $1.75 \times 10-3 \mathrm{M}$ CG VISUAL SPCTR CHNGE RHD6

UNK $\quad 1.3 \times 10-3 \mathrm{M} \quad$ CG VISUAL SPCTR CHNGE RHDG

UNK 9. X10-4 M CG VISUAL SPCTR CHNGE RHD6

UNK $\quad 6.3 \quad$ X10-4 M CG VISUAL SPCTR CHNGE RHD6

UNK $\quad 4.5 \quad \mathrm{X} 10-4 \mathrm{M} \quad \mathrm{CG} \quad$ VISUAL SPCTR CHNGE RHD6
BISW MUKH NATURAL DISTRIBUTION OF HEAD GROUPS
$28 \quad 2.47 \times 10-5 \mathrm{M}$

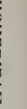

\section{COMPOUND NO $=463$ MOL WGT -705.0 NONYL BENZENE/OXYETHYLENE/11 ALCOHOL}

1 ENTRIES FOR COMPOUND

COMPOUND NO $=464$ MOL WGT - 881.3 NONYL BENZENE/OXYETHYLENE/15 ALCOHOL NATURAL DISTRIBUTION OF HEAD GROUPS

$\begin{array}{lllllll}21.2 & 3.10 \times 10-5 \mathrm{M} & \text { HD } & \text { SURFACE TENSION LOG PLOT } & \text { MANK } \\ 25 & 2.85 \times 10-5 \mathrm{M} & \text { HD } & \text { SURFACE TENSION LOG PLOT } & \text { MANK } \\ 28 & 2.71 \times 10-5 \mathrm{M} & \text { HD } & \text { SURFACE TENSION LOG PLOT } & \text { MANK } \\ 33.5 & 2.69 & \text { X10-5 } \mathrm{M} & \text { HD } & \text { SURFACE TENSION LOG PLOT } & \text { MANK } \\ 41.5 & 2.36 \times 10-5 \mathrm{M} & \text { HD } & \text { SURFACE TENSION LOG PLOT } & \text { MANK } \\ 45 & 2.27 \times 10-5 \mathrm{M} & \text { HD } & \text { SURFACE TENSION LOG PLOT } & \text { MANK }\end{array}$

66021 T $\quad$ L

66021 T $\quad$ L

66021 T $\quad$ L

66021 T $L$

66021 T L
6 ENTRIES FOR COMPOUND

MANK

MANK

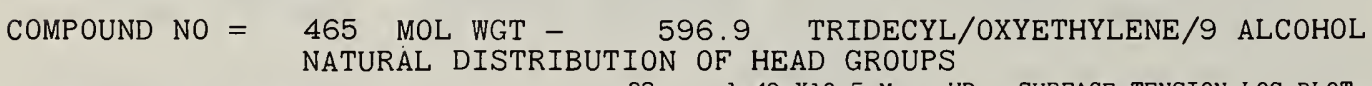
1 ENTRIES FOR COMPOUND

\section{NATURAL DISTRIBUTION OF HEAD GROUPS}

\author{
$28 \quad 1.49 \times 10-5 \mathrm{M}$
}

\section{SURFACE TENSION LOG PLOT}

Concentration units: $\mathrm{A}-\mathrm{mol} \%$; $\mathrm{B}-$ vol \% solvent; $\mathrm{C}-$ mol \% surfactant mixture; $\mathrm{D}-\mathrm{wt} / \mathrm{vol} \%$; E-\% saturation; $\mathrm{H}-$ wt \% solvent; $\mathrm{I}-$ mol \% surfactant; $\mathrm{K}-$ normality counterions; $\mathrm{M}-$ molar; $\mathrm{N}-$ normal; $\mathrm{P}-\mathrm{wt} \% ; \mathrm{Q}-$ wt \% surfactant; $\mathrm{R}$-varied; $\mathrm{S}-\mathrm{mol} /$ kg; $\mathrm{T}$ - wt \% surfactant mixture; $\mathrm{U}-\mathrm{mol} /(\mathrm{lor} \mathrm{kg}) ; \mathrm{W}$ - molal; $\mathrm{Y}$ - atm. Details on page 222. 


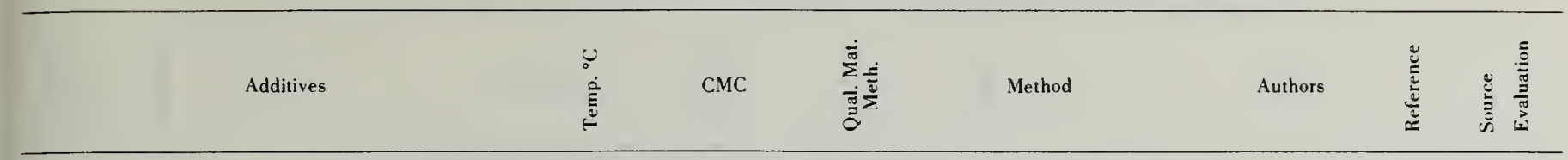

466 MOL WGT $-596.9 \quad$ TRIDECYL/SECONDARY/ /OXYETHYLENE/9 ALCOHOL
NATURAL DISTRIBUTION OF HEAD GROUPS

1 ENTRIES FOR COMPOUND

COMPOUND NO $=467$ MOL WGT - 729.1 TRIDECYL/SECONDARY/ /OXYETHYLENE/I2 ALCOHOL NATURAL DISTRIBUTION OF HEAD GROUPS

1 ENTRIES FOR COMPOUND

$28 \quad 8.67 \times 10-5 \mathrm{M}$

COMPOUND NO $=468$ MOL WGT - 459.6 DODECYL/OXYETHYLENE/6.2 ALCOHOL NATURAL DISTRIBUTION OF HEAD GROUPS

1 ENTRIES FOR COMPOUND

$30 \quad 8 \quad$ X10-5 M $\quad$ ED FOTOMTR SOLUBLZTN YLOB $\quad$ TOKI $\quad 64024$ T $\quad$ L

COMPOUND NO $=469$ MOL WGT - 521.2 DODECYL/OXYETHYLENE/7.6 ALCOHOL NATURAL DISTRIBUTION OF HEAD GROUPS

2 ENTRIES FOR COMPOUND

$\begin{array}{lllllllll}30 & 9 . & \text { X10-5 M } & \text { ED } & \text { FOTOMTR SOLUBLZTN YLOB } & \text { TOKI } & 64024 & \text { T } & \text { L } \\ 30 & 2.6 & \text { X10-4 M } & \text { EE } & \text { SURFACE TENSION LOG PLOT } & \text { TOKI } & 64024 & \text { T } & \text { L }\end{array}$

COMPOUND NO $=470$ MOL WGT $-\quad 653.4$ DODECYL/OXYETHYLENE/10.6 ALCOHOL NATURAL DISTRIBUTION OF HEAD GROUPS

1 ENTRIES FOR COMPOUND

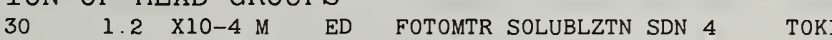

64024 T L

COMPOUND NO $=471$ MOL WGT $-\quad 882.5$ DODECYL/OXYETHYLENE/15.8 ALCOHOL NATURAL DISTRIBUTION OF HEAD GROUPS

2 ENTRIES FOR COMPOUND

$\begin{array}{lllllllll}30 & 1.8 & \text { X10-4 M } & \text { ED } & \text { FOTOMTR SOLUBLZTN YLOB } & \text { TOKI } & 64024 & \text { T } & \text { L } \\ 30 & 2.8 & \text { X10-4 M } & \text { EE } & \text { SURFACE TENSION LOG PLOT } & \text { TOKI } & 64024 & \text { T } & \text { L }\end{array}$

COMPOUND NO $=472$ MOL WGT $-1,085.2$ DODECYL/OXYETHYLENE/20.4 ALCOHOL NATURAL DISTRIBUTION OF HEAD GROUPS

1 ENTRIES FOR COMPOUND

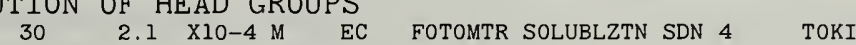

$64024 \quad T \quad L$

COMPOUND NO $=473$ MOL WGT $-1,380.4$ DODECYL/OXYETHYLENE/27.1 ALCOHOL NATURAL DISTRIBUTION OF HEAD GROUPS

2 ENTRIES FOR COMPOUND $30 \quad 2.5 \quad \times 10-4 \mathrm{M} \quad \mathrm{EC}$

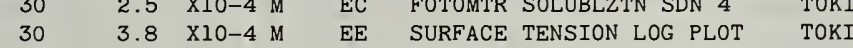

$64024 \quad \mathrm{~T} \quad \mathrm{~L}$ $64024 \quad T \quad L$

COMPOUND NO $=474$ MOL WGT $-1,887.1$ DODECYL/OXYETHYLENE/38.6 ALCOHOL NATURAL DISTRIBUTION OF HEAD GROUPS

2 ENTRIES FOR COMPOUND

$30 \quad 3.6 \quad$ X10-4 M EC FOTOMTR SOLUBLZTN SDN 4 TOKI
$30 \quad 5.0 \quad$ X10-4 M EE SURFACE TENSION LOG PLOT TOKI

COMPOUND NO $=475$ MOL WGT $-3,151.6$ DODECYL/OXYETHYLENE/67.3 ALCOHOL NATURAL DISTRIBUTION OF HEAD GROUPS

1 ENTRIES FOR COMPOUND $30 \quad 5.7 \quad \mathrm{X} 10-4 \mathrm{M} \quad \mathrm{EC}$

COMPOUND NO $=476$ MOL WGT -

6 ENTRIES FOR COMPOUND

\begin{tabular}{ccccll} 
& 166.2 & \multicolumn{2}{c}{ SODIUM } & \multicolumn{2}{l}{ OCTANOATE } \\
20 & 7. & X10-2 & M & XG & VISCOSITY MINIMUM \\
20 & 3.6 & X10-1 & M & DE & VISCOSITY \\
25 & 3.40 & X10-1 & M & DB & SPECFC CONDCTNCE GRAPH \\
25 & 3.51 & X10-1 & M & DB & SURFACE TENSION LOG PLOT \\
35 & 3.60 & X10-1 & M & DB & SPECFC CONDCTNCE GRAPH \\
50 & 3.85 & X10-1 & M & DB & SURFACE TENSION LOG PLOT
\end{tabular}

50
SATA TYUZ CAMP LAKS CAMP LAKS CAMP LAKS CAMP LAKS HESS PHIL
$64024 \quad$ T $\quad$ L 64024 T $\quad$ L

64024 T L
Concentration units: $\mathrm{A}-\operatorname{mol} \% ; \mathrm{B}-$ vol \% solvent: $\mathrm{C}-$ mol \% surfactant mixture; $\mathrm{D}-\mathrm{wt} / \mathrm{vol} \% ; \mathrm{E}-\%$ saturation; $\mathrm{H}-\mathrm{wt} \%$ solvent; $\mathrm{I}-$ mol \% surfactant; $\mathrm{K}-$ normality counterions; $\mathrm{M}-$ molar; $\mathrm{N}-$ normal; $\mathrm{P}-\mathrm{wt} \% ; \mathrm{Q}-\mathrm{wt} \%$ surfactant: $\mathrm{R}$ - varied; $\mathrm{S}-\mathrm{mol} /$ $\mathrm{kg}$; $\mathrm{T}-\mathrm{wt} \%$ surfactant mixture; $\mathrm{U}-\mathrm{mol} /(\mathrm{l}$ or $\mathrm{kg})$; $\mathrm{W}-$ molal; $\mathrm{Y}$ - atm. Details on page 222. 


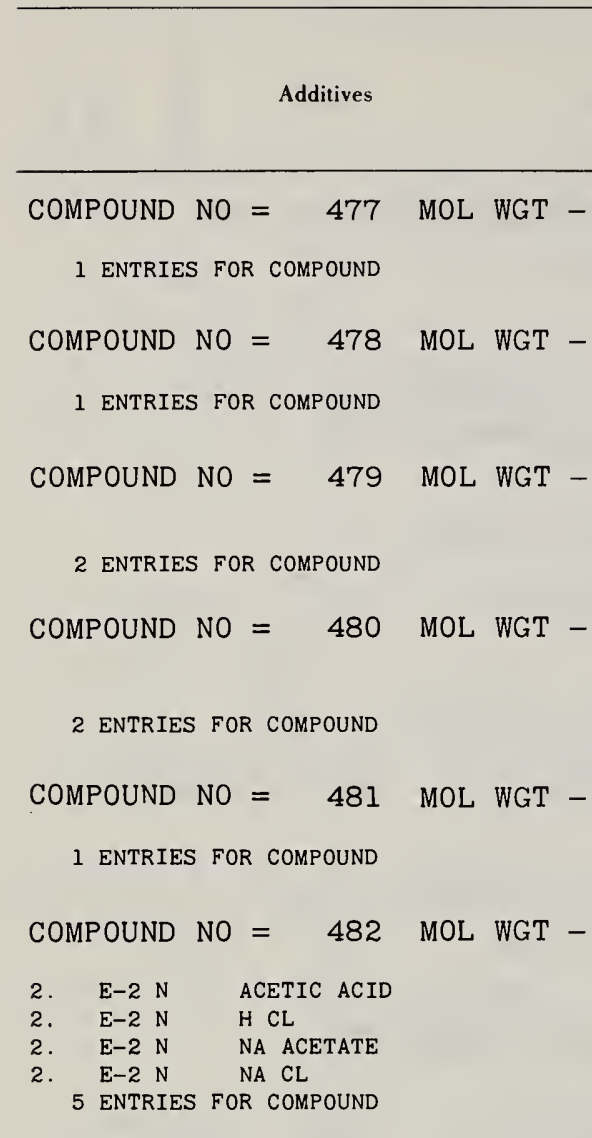

COMPOUND NO $=483$ MOL WGT -

1 ENTRIES FOR COMPOUND

COMPOUND NO $=484$ MOL WGT -

1 ENTRIES FOR COMPOUND

COMPOUND NO $=485$ MOL WGT -

1.1 E $1 \quad$ PH OF SOLUTION

1.1 E 1 PH OF SOLUTION

1.1 E 1 PH OF SOLUTION

1.1 E 1 PH OF SOLUTION

5 ENTRIES FOR COMPOUND

COMPOUND NO $=486$ MOL WGT -

1 ENTRIES FOR COMPOUND

COMPOUND NO $=487$ MOL WGT -

1.1 E 1 PH OF SOLUTION

1.1 E 1 PH OF SOLUTION

1.1 E 1 PH OF SOLUTION

5 ENTRIES FOR COMPOUND

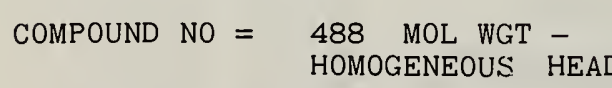

2 ENTRIES FOR COMPOUND

CMC $\quad$ Method $\quad$ Authors

392.6 OCTADECYL TRIMETHYLAMMONIUM BROMIDE

$23 \quad 3.0 \quad \times 10-4 \mathrm{M}$ HG STREAMING CURRENT

66011 T L

378.6 HEXADECYL DIMETHYLETHYLAMMONIUM BROMIDE $\begin{array}{llllllllll}23 & 4.8 & \text { X10-3 M GH STREAMING CURRENT } & \text { CARD } & 66011 & \text { T } & \text { L }\end{array}$

403.4 TETRADECYL PYRIDINIUM IODIDE $28.0 \quad 7.9 \times 10-4 \mathrm{M}$ BC KRAFFT POINT SOLUB $\perp L I T Y$ $40.0 \quad 1.2 \quad \times 10-3 \mathrm{M} \quad$ BD INTERFACIAL TNSN UNSPEC

431.5 HEXADECYL PYRIDINIUM IODIDE

$\begin{array}{lllll}35.0 & 3.1 & \times 10-4 \mathrm{M} & \mathrm{BC} & \text { KRAFFT POINT SOLUBILITY }\end{array}$

$50.0 \quad 4.4 \quad \mathrm{X} 10-4 \mathrm{M} \quad \mathrm{BC}$ INTERFACIAL TNSN UNSPEC

459.6 OCTADECYL PYRIDINIUM IODIDE

$\begin{array}{llll}45.5 & 1.3 & \times 10-4 \mathrm{M} & \text { BD }\end{array}$

$\begin{array}{llllll}248.4 & \text { DODECYL } & \text { AMMONIUM NITRATE } \\ 30 & 9.9 & \text { X10-3 M } & \text { BC } & \text { EQUIV CONDCTNCE GRAPH } \\ 30 & 9.9 & \text { X10-3 M } & \text { BC } & \text { EQUIV CONDCTNCE GRAPH } \\ 30 & 7.1 & \text { X10-3 M } & \text { BC } & \text { EQUIV CONDCTNCE GRAPH } \\ 30 & 6.9 & \text { X10-3 M } & \text { BC } & \text { EQUIV CONDCTNCE GRAPH } \\ 30 & 6.9 & \text { X10-3 M } & \text { BC } & \text { EQUIV CONDCTNCE GRAPH }\end{array}$

ADDI FURM ADDI FURM

$56019 \quad T \quad$ L 56019 T $\quad$ L

306.0 DIOCTYL DIMETHYL AMMONIUM CHLORIDE

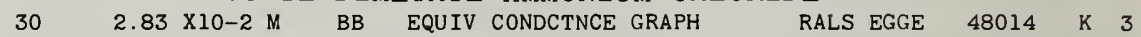

124.1 SODIUM PENTANOATE/VALERATE/

138.2 SODIUM HEXANOATE/CAPROATE/

$\begin{array}{rllll}20 & 1.57 \times 100 \mathrm{M} & \text { DE } & \text { VISCOSITY } \\ 5 & 6.60 \times 10-1 & \mathrm{M} & \text { BC } & \text { SPECFC CONDCTNCE GRAPH } \\ 20 & 7.30 \times 10-1 & \mathrm{M} & \text { BC } & \text { SPECFC CONDCTNCE GRAPH } \\ 60 & 8.90 \times 10-1 \mathrm{M} & \text { BC } & \text { SPECFC CONDCTNCE GRAPH } \\ 90 & 1.11 \times 100 \mathrm{M} & \text { BC } & \text { SPECFC CONDCTNCE GRAPH }\end{array}$

152.2 SODIUM HEPTANOATE

$20 \quad 9.5 \quad \mathrm{X} 10-1 \mathrm{M} \quad \mathrm{DE}$ VISCOSITY

\begin{tabular}{llllll}
180.2 & \multicolumn{2}{c}{ SODIUM } & NONANOATE \\
20 & 2.4 & $\times 10-1$ & $M$ & XG & VISCOSITY MINIMUM \\
20 & 2.2 & X10-1 M & DE & VISCOSITY \\
20 & 1.59 & $\times 10-1$ & $M$ & BC & SPECFC CONDCTNCE GRAPH \\
60 & 1.82 & X10-1 M & BC & SPECFC CONDCTNCE GRAPH \\
90 & 2.04 & X10-1 M & BC & SPECFC CONDCTNCE GRAPH
\end{tabular}
$20 \quad 2.35 \times 100 \mathrm{M}$ DD X-RAY DIFFRACTION
RALS EGGE

RALS EGGE

RALS EGGE

RALS EGGE

RALS EGGE

HESS PHIL MARK TSIK MARK TSIK MARK TSIK MARK TSIK

$49008 \quad \mathrm{~K} \quad 3$ $49008 \quad K \quad L$ $49008 \quad K \quad L$ $49008 \quad K \quad L$
39009 T $\quad$ L

$64051 \mathrm{~T} \quad \mathrm{~L}$

64051 T $\mathrm{L}$

64051 T L

64051 T L 49008 K L

494.8 DODECYL/OXYETHYLENE/7 ALCOHOL GROUP $\begin{array}{llllll}23 & 7.1 & \text { X10-5 M } & \text { PE } & \text { FOTOMTR SPCTR CHNG I2 } \\ 23 & 8.0 & \text { X10-5 M } & \text { PB } & \text { SURFACE TENSION LOG PLOT }\end{array}$ $\begin{array}{lllll}\text { HESS PHIL } & 39009 & \text { T } & \text { L } \\ & & & & \\ & & & & \\ \text { SATA TYUZ } & 53006 & \text { T } & \text { L } \\ \text { HESS PHIL } & 39009 & \text { T } & \text { L } \\ \text { MARK TSIK } & 64051 & \text { T } & \text { L } \\ \text { MARK TSIK } & 64051 & \text { T } & \text { L } \\ \text { MARK TSIK } & 64051 & \text { T } & \text { L }\end{array}$
Concentration units: $\mathrm{A}-\mathrm{mol} \%$; $\mathrm{B}-\mathrm{vol} \%$ solvent: $\mathrm{C}-$ mol \% surfactant mixture; D-wt/vol \%; E-\% saturation; $\mathrm{H}-$ wt \% solvent; I-moi \% surfactant; $\mathrm{K}$ - normality counterions; $\mathrm{M}$ - molar; $\mathrm{N}$ - normal; $\mathrm{P}$ - wt $\% ; \mathrm{Q}$ - wt \% surfactant: $\mathrm{R}$ - varied; $\mathrm{S}-\mathrm{mol} /$ $\mathrm{kg} ; \mathrm{T}-\mathrm{wt} \%$ surfactant mixture; $\mathrm{U}-$ mol/(0 or $\mathrm{kg}) ; \mathrm{W}-$ molal; $\mathrm{Y}$-atm. Details on page 222. 


\begin{tabular}{|c|c|c|c|c|c|c|c|}
\hline Additives & 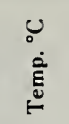 & СмС & 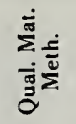 & Method & Authors & $\frac{\mathscr{E}}{\mathscr{E}}$ & 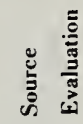 \\
\hline
\end{tabular}

\section{COMPOUND NO $=489$ MOL WGT -582.9 DODECYL/OXYETHYLENE/9 ALCOHOL HOMOGENEOUS HEAD GROUP}

2 ENTRIES FOR COMPOUND

$\begin{array}{llllllllll}23 & 8.3 & \text { X10-5 M } & \text { PE } & \text { FO'SOMTR SPCTR CHNG I2 } & \text { LANG } & 60012 & \text { T } & \text { L } \\ 23 & 1.0 & \text { X10-4 M } & \text { PC } & \text { SURFACE TENSION LOG PLOT } & \text { LANG } & 60012 & \text { T } & \text { L }\end{array}$

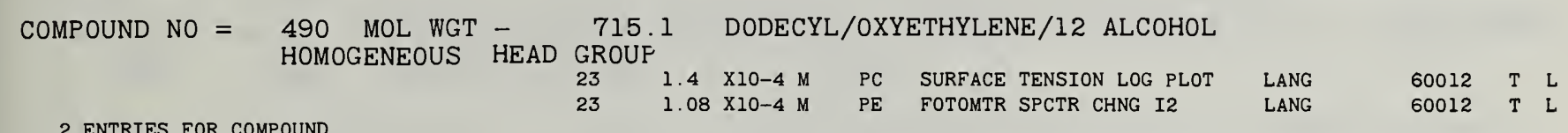

2 ENTRIES FOR COMPOUND

$\begin{array}{llll}\text { GROUF } & & & \\ 23 & 1.4 & \times 10-4 \mathrm{M} & \text { PC SURFACE TENSION LOG PLOT }\end{array}$

COMPOUND NO $=491$ MOL WGT -

6.0 E 1 H H2 504

7.8 E 1 H H2 SO4

9.6 E I H H2 $\mathrm{HO4}$

9.6 E 1 H H2 S04

9. E-1 M NA2 S04

4 ENTRIES FOR COMPOUND

$\begin{array}{llllllllll}356.5 & \text { DODECYL } & \text { TROPYLIUM } & \text { BISULFATE } & & & & & \\ 25 & 1.0 & \text { X10-3 M } & \text { BD } & \text { SURFACE TENSION LOG PLOT } & \text { ABUH MYSE } & 65019 & \text { T } & \text { L } \\ 25 & 2.2 & \text { X10-3 M } & \text { BC } & \text { SURFACE TENSION LOG PLOT } & \text { ABUH MYSE } & 65019 & \text { T } & 3 \\ 25 & 1.25 & \text { X10-2 M } & \text { BC } & \text { SURFACE TENSION LOG PLOT } & \text { ABUH MYSE } & 65019 & \text { T } & 3 \\ 25 & 5.4 & \text { X10-3 M } & \text { BC } & \text { SURFACE TENSION LOG PLOT } & \text { ABUH MYSE } & 65019 & \text { T } & 3\end{array}$

COMPOUND NO $=492$ MOL WGT -

348.5 SODIUM TETRAPROPYLENE/1-3-5-7-TETRAMETHYL-OCTYL/

2. E-1 M NA CL BENZENE SULFONATE

2 ENTRIES FOR COMPOUND

$75 \quad 3.14 \times 10-3 \mathrm{M} \quad$ BG VISUAL SPCTR CHNGE RHD6

$203.0 \quad 310-4 \mathrm{M} \quad$ CC SURFACE TENSION LOG PLOT

GRIE

$55028 \quad T \quad L$

COMPOUND NO $=493$ MOL WGT -

306.4 SODIUM P-NONYL BENZENE SULFONATE

1 ENTRIES FOR COMPOUND

$20 \quad 4.6 \quad$ XIO-3 M BB SURFACE TENSION LOG PLOT

HARR

59001 L 3

COMPOUND NO $=494$ MOL WGT -

2 ENTRIES FOR COMPOUND

COMPOUND NO $=495$ MOL WGT -

4 ENTRIES FOR COMPOUND

COMPOUND NO $=496$ MOL WGT -

1 ENTRIES FOR COMPOUND

COMPOUND NO $=497$ MOL WGT -

1 ENTRIES FOR COMPOUND

COMPOUND NO $=498$ MOL WGT -

1 ENTRIES FOR COMPOUND

COMPOUND NO $=499$ MOL WGT -

1 ENTRIES FOR COMPOUND

COMPOUND NO $=500$ MOL WGT -

1 ENTRIES FOR COMPOUND \begin{tabular}{lllll} 
& 0 & \multicolumn{2}{l}{ POTASSIUM } & DILINOLEATE \\
25.8 & 2.5 & XIO-4 M & HG & FOTOMTR SPCTR CHNGE PNCN \\
25.8 & 3.0 & X10-4 M & HC & SPECFC CONDCTNCE GRAPH
\end{tabular}

524.7 SUCROSE MONOLAURATE

$\begin{array}{lllll}20 & 1.85 & \text { XIO-4 M } & \text { BE } & \text { INTERFACIAL TENSION LOGM } \\ 20 & 1.3 & \text { X10-4 M } & \text { BE } & \text { SURFACE TENSION LOG PLOT } \\ 27.1 & 6.4 & \text { XIO-6 M } & \text { CD } & \text { SURFACE TENSION LOG PLOT } \\ 27.1 & 3.4 & \text { X10-4 M } & \text { CD } & \text { SURFACE TENSION LOG PLOT }\end{array}$

608.9 SUCROSE MONOSTEARATE

$50 \quad 1.1$ X10-5 M BE SURFACE TENSION LOG PLOT WACH HAYA 62023 TL L

552.7 SUCROSE MONOMYRISTATE

$20 \quad 2.58$ X10-5 M BE INTERFACIAL TENSION LOGM WACH HAYA 62023 T L

580.8 SUCROSE MONOPALMITATE

$50 \quad 5.5$ X10-5 M BE SURFACE TENSION LOG PLOT WACH HAYA 62023 TL L

819.3 SUCROSE DI-PALMITATE

QUESTIONABLE CRITERION WACH HAYA $62023 \quad R$

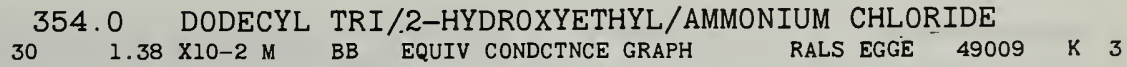

Concentration units: $\mathrm{A}-\mathrm{mol} \%$; $\mathrm{B}-\mathrm{vol} \%$ solvent: $\mathrm{C}-\mathrm{mol} \%$ surfactant mixture;

Concentration units: A-mol \%; $\mathrm{B}-\mathrm{vol} \%$ solvent: $\mathrm{C}-\mathrm{mol} \%$ surfactant mixture;
$\mathrm{D}-\mathrm{wt} / \mathrm{vol} \%$; $\mathrm{E}-\%$ saturation; $\mathrm{H}-\mathrm{wt} \%$ solvent; $\mathrm{I}-\mathrm{mol} \%$ surfactant; $\mathrm{K}-$ normality 


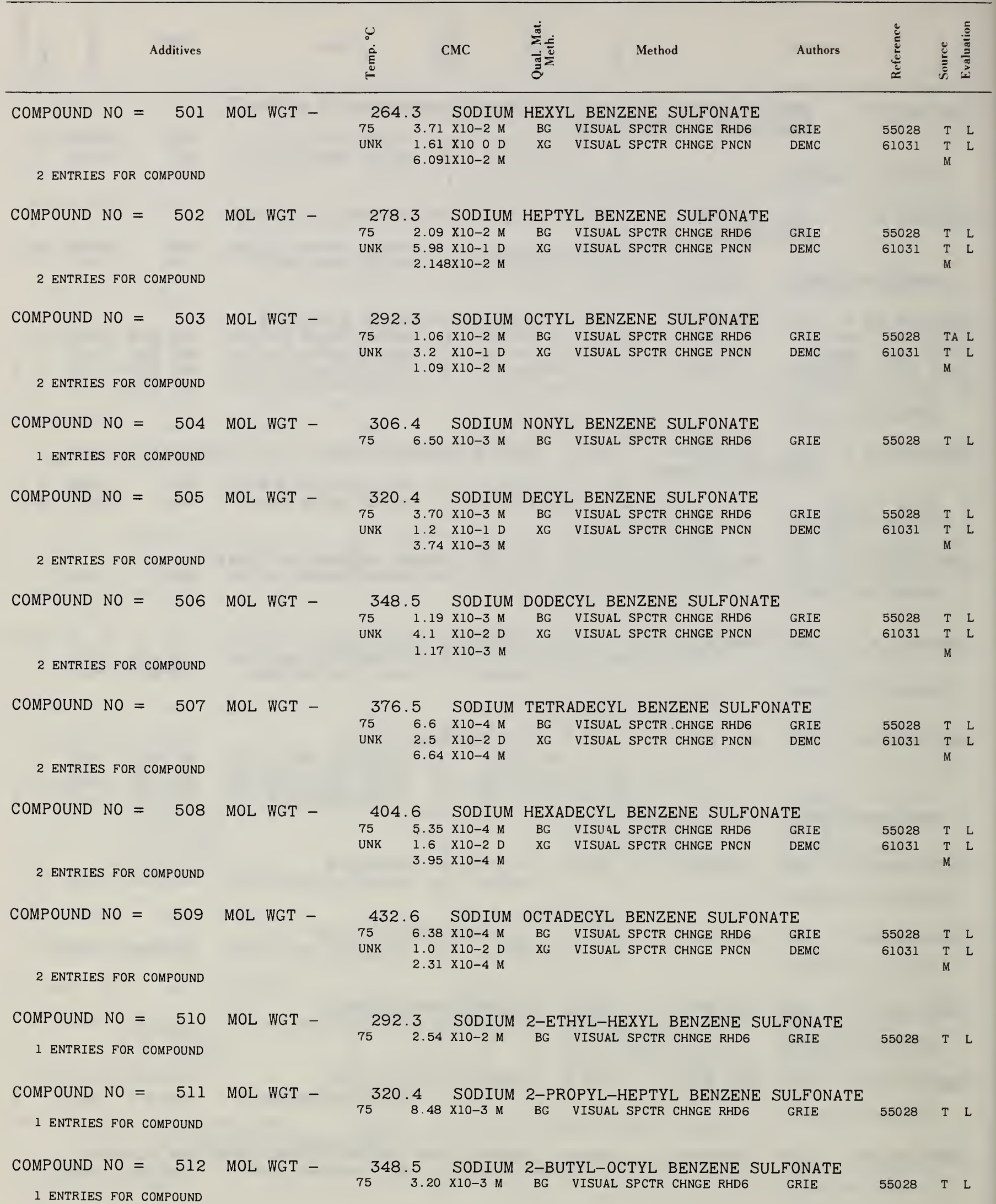

1 ENTRIES FOR COMPOUND

Concentration units: $\mathrm{A}-\mathrm{mol} \% ; \mathrm{B}-\mathrm{vol} \%$ solvent; $\mathrm{C}-$ mol \% surfactant mixture; counterions; $\mathrm{M}$ - molar; $\mathrm{N}$-normal; $\mathrm{P}$ - wt \%; $\mathrm{Q}-$ wt $\%$ surfactant; $\mathrm{R}$-varied; $\mathrm{S}-$ mol/ $\mathrm{D}-\mathrm{wt} / \mathrm{vol} \%$; $\mathrm{E}-\%$ saturation; $\mathrm{H}-$ wt \% solvent; $\mathrm{I}-$ mol \% surfactant; $\mathrm{K}$-normality 
Additives

\begin{tabular}{|c|c|c|c|c|}
\hline CMC & 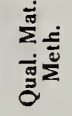 & Method & Authors & 巳ू \\
\hline
\end{tabular}

COMPOUND NO $=513$ MOL WGT -

1 ENTRIES FOR COMPOUND
376.5 SODIUM 2-AMYL-NONYL BENZENE SULFONATE

$753.32 \times 10-3 \mathrm{M}$ BG VISUAL SPCTR CHNGE RHD6 GRIE
$55028 \quad T \quad$ L

348.5 SODIUM 6-N-DODECYL BENZENE SULFONATE

$75 \quad 3.12 \times 10-3 \mathrm{M}$ BG VISUAL SPCTR CHNGE RHD6 GRIE

$55028 \quad$ T $\quad$ L

1 ENTRIES FOR COMPOUND

MOL WGT -

306.5 ISO-OCTYL/OXYETHYLENE/4 ALCOHOL

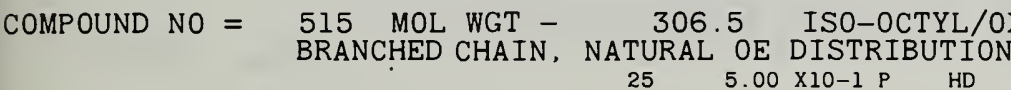

1 ENTRIES FOR COMPOUND

$255.00 \times 10-1 \mathrm{P}$ HD SURFACE TENSION LOG PLOT KOMO BEIS 66022 T L

COMPOUND NO $=516$ MOL WGT -320.5 NONYL/OXYETHYLENE/4 ALCOHOL

BRANCHED CHAIN, NATURAL OE DISTRIBUTION

1 ENTRIES FOR COMPOUND

KOMO BEIS 66022 T L

COMPOUND NO $=517$ MOL WGT - 598.9 DECYL/OXO-PROCESS/ /OXYETHYLENE/1O ALCOHOL BRANCHED CHAIN, NATURAL OE DISTRIBUTION

1 ENTRIES FOR COMPOUND

$251.30 \times 10-1 \mathrm{P}$ HD SURFACE TENSION LOG PLOT KOMO BEIS 66022 T $\mathrm{L}$

COMPOUND NO $=518$ MOL WGT $-\quad 450.7$ TRIMETHYLNONYL/OXYETHYLENE/6 ALCOHOL

BRANCHED CHAIN, NATURAL OE DISTRIBUTION

1 ENTRIES FOR COMPOUND

$\begin{array}{lllllllll}25 & 8.4 & \text { X10-2 P HD SURFACE TENSION LOG PLOT KOMO BEIS } 66022 & \text { T } & \text { L }\end{array}$

COMPOUND NO $=519$ MOL WGT - 334.6 DECYL/OXO-PROCESS/ /OXYETHYLENE/4 ALCOHOL

BRANCHED CHAIN, NATURAL OE DISTRIBUTION

1 ENTRIES FOR COMPOUND

256.6 X10-2 P HD SURFACE TENSION LOG PLOT KOMO BEIS 66022 T L

COMPOUND NO $=520$ MOL WGT -524.8 UNDECYL/OXYETHYLENE/8 ALCOHOL NATURAL DISTRIBUTION OF HEAD GROUPS

1 ENTRIES FOR COMPOUND

$25 \quad 2.3 \times 10-2 \mathrm{P} \quad$ HD SURFACE TENSION LOG PLOT KOMO BEIS 66022 T $\mathrm{L}$

COMPOUND NO $=521$ MOL WGT -641.0 TRIDECYL/OXYETHYLENE/IO ALCOHOL

BRANCHED CHAIN, NATURAL OE DISTRIBUTION

1 ENTRIES FOR COMPOUND

$25 \quad 9.4$ X10-3 P HD SURFACE TENSION LOG PLOT KOMO BEIS 66022 T L

COMPOUND NO $=522$ MOL WGT -727.9 DECYL BENZENE ORTHO/OXYETHYLENE/11.2 ALCOHOL NATURAL DISTRIBUTION OF HEAD GROUPS

1 ENTRIES FOR COMPOUND

$252.15 \times 10-3 \mathrm{P}$ HD SURFACE TENSION LOG PLOT KOMO BEIS 66022 T L

COMPOUND NO $=523$ MOL WGT -719.1 DECYL BENZENE PARA/OXYETHYLENE/11.0 ALCOHOL NATURAL DISTRIBUTION OF HEAD GROUPS

1 ENTRIES FOR COMPOUND

$25 \quad 1.4 \times 10-3 \mathrm{P}$ HD SURFACE TENSION LOG PLOT KOMO BEIS 66022 T L

COMPOUND NO $=524$ MOL WGT $-\quad 859.3$ HEXADECYL/OXYETHYLENE/14 ALCOHOL

BRANCHED CHAIN, NATURAL OE DISTRIBUTION

1 ENTRIES FOR COMPOUND

$25 \quad 1.3$ XIO-3 P HD SURFACE TENSION LOG PLOT KOMO BEIS 66022 T L

Concentration units: $\mathrm{A}-\mathrm{mol} \%$; $\mathrm{B}-$ vol \% solvent; $\mathrm{C}-$ mol \% surfactant mixture;

counterions; $\mathrm{M}-$ molar; $\mathrm{N}-$ normal; $\mathrm{P}-$ wt \%; Q-wt \% surfactant; $\mathrm{R}$-varied; $\mathrm{S}-$ mol/

D-wt/vol \%; E-\% saturation; H-wt \% solvent; I-mol \% surfactant; $\mathrm{K}-$ normality

$\mathrm{kg} ; \mathrm{T}$ - wt \% surfactant mixture; $\mathrm{U}-\mathrm{mol} /(\mathrm{l}$ or kg); $\mathrm{W}$ - molal; $\mathrm{Y}-\mathrm{atm}$. Detailson page 222. 


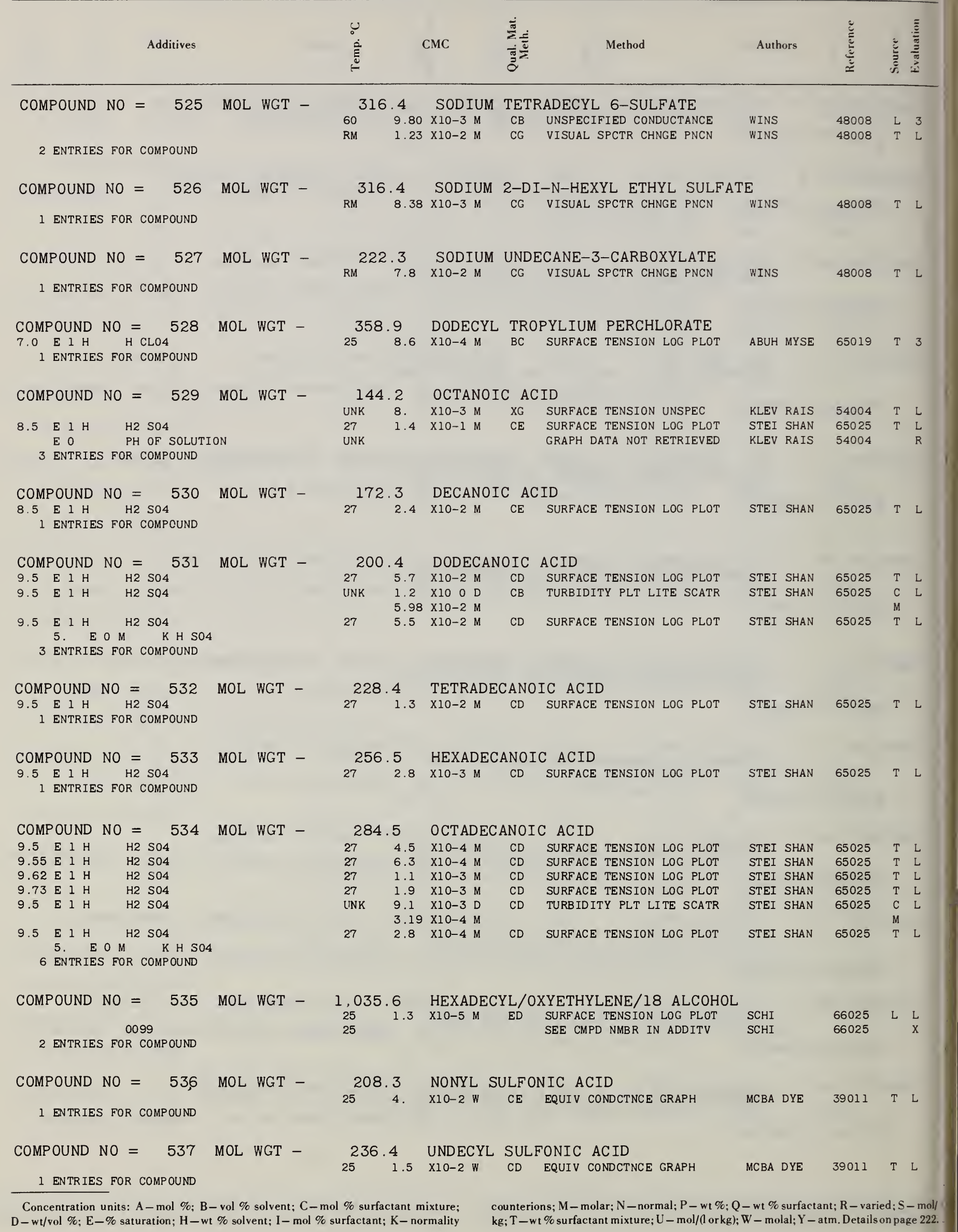




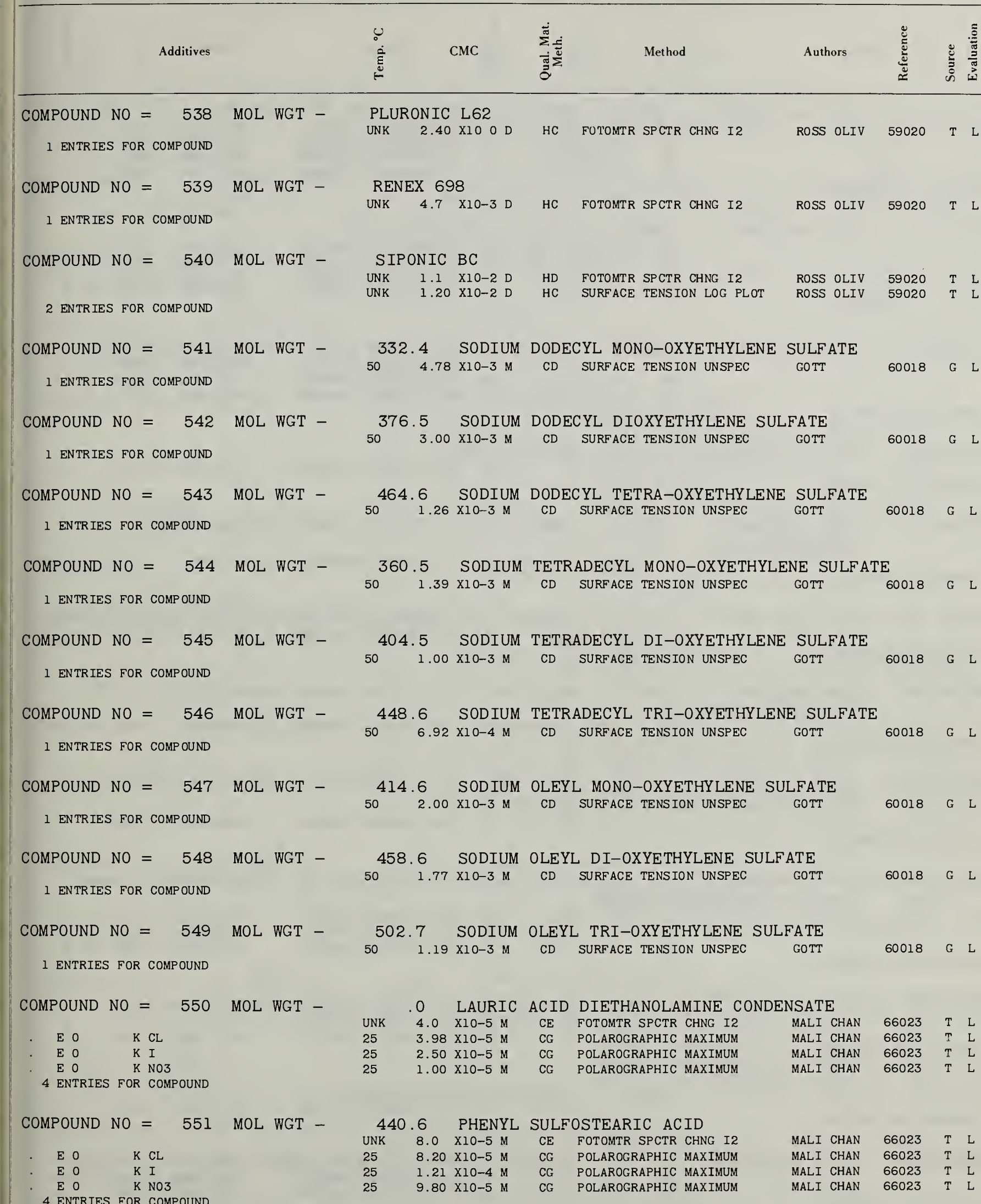

Concentration units: $\mathrm{A}-$ mol $\% ; \mathrm{B}-$ vol \% solvent; $\mathrm{C}-$ mol \% surfactant mixture; $\mathrm{D}-\mathrm{wt} / \mathrm{vol} \% ; \mathrm{E}-\%$ saturation; $\mathrm{H}-\mathrm{wt} \%$ solvent; $\mathrm{I}-\mathrm{mol} \%$ surfactant; $\mathrm{K}$-normality counterions; $\mathrm{M}$-molar; $\mathrm{N}$ - normal; $\mathrm{P}$ - wt \%; $\mathrm{Q}$ - wt \% surfactant; $\mathrm{R}$ - varied; $\mathrm{S}-\mathrm{mol} /$ $\mathrm{kg} ; \mathrm{T}$ - wt \% surfactant mixture; $\mathrm{U}-\mathrm{mol} /(\mathrm{lor} \mathrm{kg}) ; \mathrm{W}-$ molal; $\mathrm{Y}$ - atm. Details on page 222. 


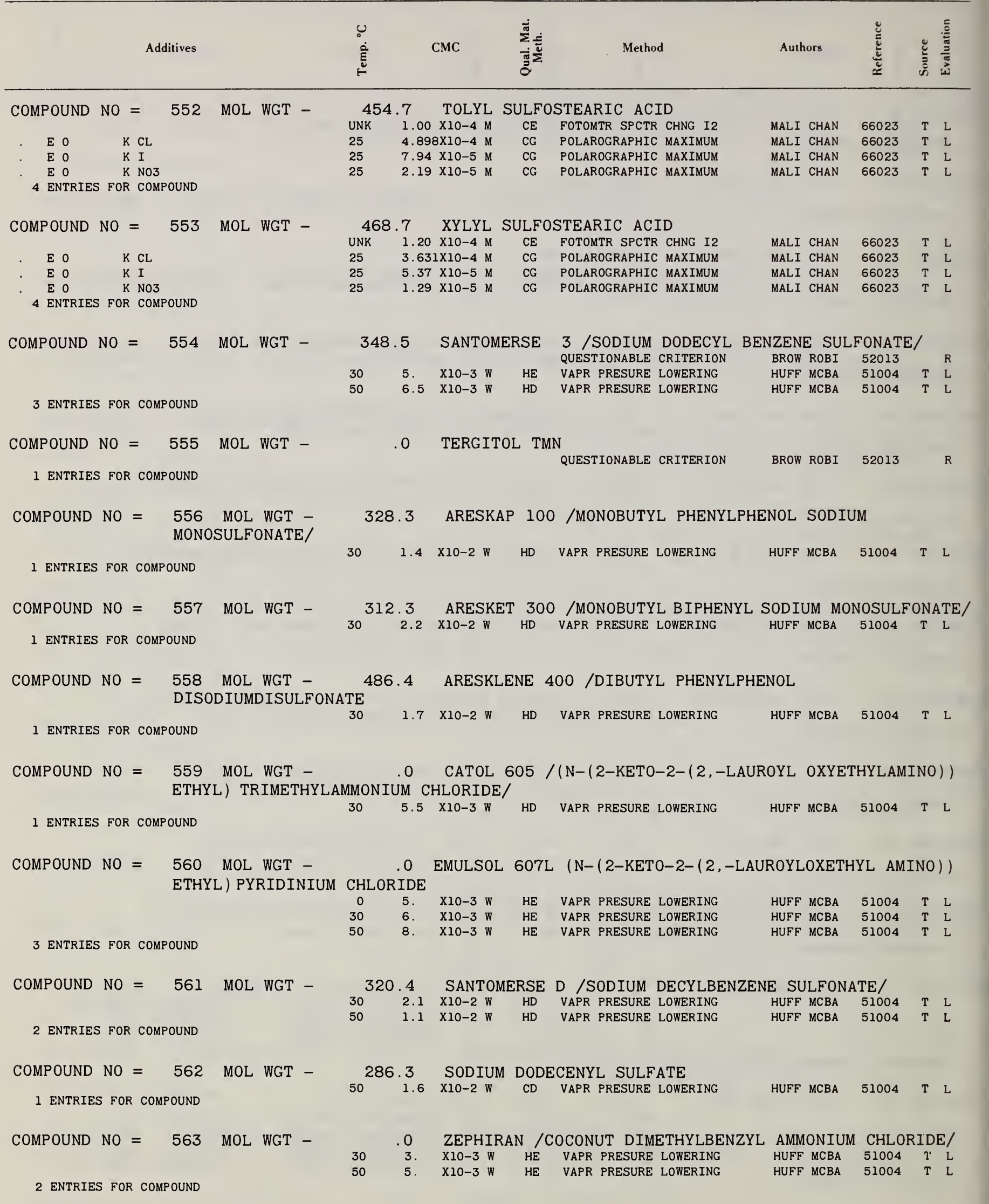

2 ENTRIES FOR COMPOUND

\footnotetext{
Concentration units: $\mathrm{A}-\mathrm{mol} \%$; $\mathrm{B}-\mathrm{vol} \%$ solvent; $\mathrm{C}-$ mol \% surfactant mixture; $\mathrm{D}-\mathrm{wt} / \mathrm{vol} \%$; E-\% saturation; $\mathrm{H}-$ wt \% solvent; I-mol \% surfactant; $\mathrm{K}$ - normality
} 


Additives $\quad$ CMC Nethod

\section{COMPOUND NO $=564$ \\ 1 ENTRIES FOR COMPOUND}

COMPOUND NO $=565$ MOL WGT -

1 ENTRIES FOR COMPOUND

COMPOUND NO $=566$ MOL WGT -

I ENTRIES FOR COMPOUND

COMPOUND NO $=567$ MOL WGT -

1 ENTRIES FOR COMPOUND

COMPOUND NO $=568$ MOL WGT -

1. E-1 M MG S04

7 ENTRIES FOR COMPOUND

COMPOUND NO $=569$ MOL WGT -

1 ENTRIES FOR COMPOUND

COMPOUND NO $=570$ MOL WGT -

2 ENTRIES FOR COMPOUND

COMPOUND NO $=571$ MOL WGT -

5 ENTRIES FOR COMPOUND

COMPOUND NO $=572$ MOL WGT -

1. $\mathrm{E}-1 \mathrm{M} \quad \mathrm{CO} \mathrm{SO} 4$

4 ENTRIES FOR COMPOUND

COMPOUND NO $=573$ MOL WGT -

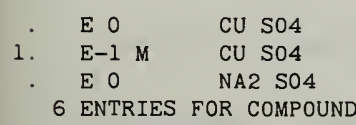

COMPOUND NO $=574$ MOL WGT -

2 ENTRIES FOR COMPOUND

COMPOUND NO $=575$ MOL WGT -

$.0 \quad$ NA OSR

$50 \quad 2.3 \quad \mathrm{X} 10-3 \mathrm{M}$ HC SPECFC CONDCTNCE GRAPH

MARO ELDE 54006 T L

.0 DAXAD 11

$50 \quad 8.0$ X10-2 D HC SPECFC CONDCTNCE GRAPH MARO ELDE 54006 T L

.0 AQUAREX D

$50 \quad 3.0$ X10-1 D HC SPECFC CONDCTNCE GRAPH MARO ELDE 54006 T $\mathrm{L}$

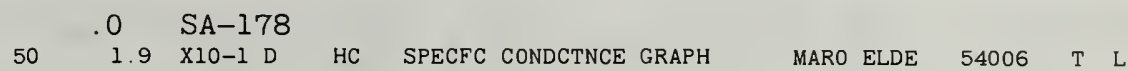

\begin{tabular}{llllllllll}
555.1 & \multicolumn{1}{l}{ MAGNESIUM DODECYL SULFATE } \\
25 & 8.8 & X10-4 M & BB & SURFACE TENSION LOG PLOT & MIYA & 60029 & T & L \\
30 & 1.25 & X10-3 M & BB & UNSPECIFIED CONDUCTANCE & SATA IWAM & 63034 & T & 3 \\
40 & 1.1 & X10-3 M & BC & SURFACE TENSION LOG PLOT & MIYA & 60029 & T & L \\
40 & 1.0 & X10-3 M & BG & VISUAL SPCTR CHNGE PNCN & MIYA & 60029 & T & L \\
40 & 1.1 & X10-3 M & BG & VISUAL SPCTR CHNGE RHD6 & MIYA & 60029 & T & L \\
54 & 1.1 & X10-3 M & BC & SURFACE TENSION LOG PLOT & MIYA & 60029 & T & L \\
30 & 3. & X10-4 M & BE & SURFACE TENSION LOG PLOT & SATA IWAM & 63034 & T & L
\end{tabular}

618.4 STRONTIUM DODECYL SULFATE

$67 \quad 1.1 \quad$ X10-3 M BC SURFACE TENSION LOG PLOT MIYA 60029 T

737.9 LEAD DODECYL SULFATE

$54 \quad 1.0 \quad$ X10-3 M $\quad$ BC SURFACE TENSION LOG PLOT MIYA $60029 \quad$ T $\quad$ L

$\begin{array}{llllllllll}67 & 9.8 & \text { XIO-4 M } & \text { BB } & \text { SURFACE TENSION LOG PLOT MIYA } & 60029 & \text { T } & \text { L }\end{array}$

585.7 MANGANESE DODECYL SULFATE

$25-1] \times 10-3 M \quad B C$ SURFACE TENSION

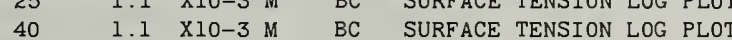

$40 \quad 1.1 \times 10-3 \mathrm{M}$ BG VISUAL SPCTR CHNGE RHDG

$40 \quad 1.2 \times 10-3 \mathrm{M} \quad \mathrm{BG}$ VISUAL SPCTR CHNGE PNCN

$54 \quad 1.1$ X10-3 M $\quad$ BC SURFACE TENSION LOG PLOT

MIYA

MIYA

MIYA

MIYA

MIYA

589.7 COBALTOUS DODECYL SULFATE

$30 \quad 1.23 \times 10-3 \mathrm{M} \quad \mathrm{BB}$ UNSPECIFIED CONDUCTANCE

$40 \quad 1.3 \times 10-3 \mathrm{M} \quad$ BG VISUAL SPCTR CHNGE RHD6

$40 \quad 1.0 \quad$ XIO-3 M $\quad$ BG VISUAL SPCTR CHNGE PNCN

30 3. X10-4 M BE SURFACE TENSION LOG PLOT

SATA IWAM

MIYA

MIYA

SATA IWAM

$63034 \quad \mathrm{~T} \quad 3$

60029 T L

60029 T $\quad$ L

$63034 \mathrm{~T}$ L

594.3 CUPRIC DODECYL SULFATE

\begin{tabular}{|c|c|c|c|c|c|c|c|c|}
\hline 30 & 1.20 & $\mathrm{X} 10-3 \mathrm{M}$ & BB & UNSPECIFIED CONDUCTANCE & SATA & IWAM & 63034 & $\mathrm{~T}$ \\
\hline 40 & 1.3 & $\mathrm{X} 10-3 \mathrm{M}$ & BG & VISUAL SPCTR CHNGE RHD6 & MIYA & & 60029 & $\mathrm{~T}$ \\
\hline 40 & 1.2 & $\mathrm{X} 10-3 \mathrm{M}$ & BG & $\begin{array}{l}\text { VISUAL SPCTR CHNGE PNCN } \\
\text { GRAPH DATA NOT RETRIEVED }\end{array}$ & $\begin{array}{l}\text { MIYA } \\
\text { SATA }\end{array}$ & IWAM & $\begin{array}{l}60029 \\
63034\end{array}$ & $\mathrm{~T}$ \\
\hline 30 & 3. & $\mathrm{X} 10-4 \mathrm{M}$ & $\mathrm{BE}$ & $\begin{array}{l}\text { SURFACE TENSION LOG PLOT } \\
\text { GRAPH DATA NOT RETRIEVED }\end{array}$ & $\begin{array}{l}\text { SATA } \\
\text { SATA }\end{array}$ & $\begin{array}{l}\text { IWAM } \\
\text { IWAM }\end{array}$ & $\begin{array}{l}63034 \\
63034\end{array}$ & $\mathrm{~T}$ \\
\hline
\end{tabular}

596.1 ZINC DODECYL SULFATE

$\begin{array}{llllllll}40 & 1.1 & \text { X10-3 M } & \text { BG VISUAL SPCTR CHNGE RHD6 } & \text { MIYA } & 60029 & \text { T } & \text { L }\end{array}$ $40 \quad 1.1 \quad \mathrm{X} 10-3 \mathrm{M} \quad \mathrm{BG}$ VISUAL SPCTR CHNGE PNCN MIYA 60029 T

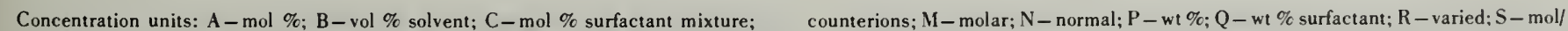

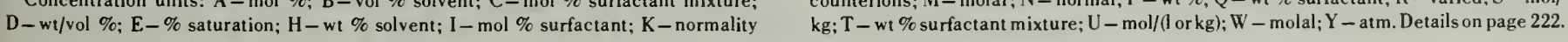




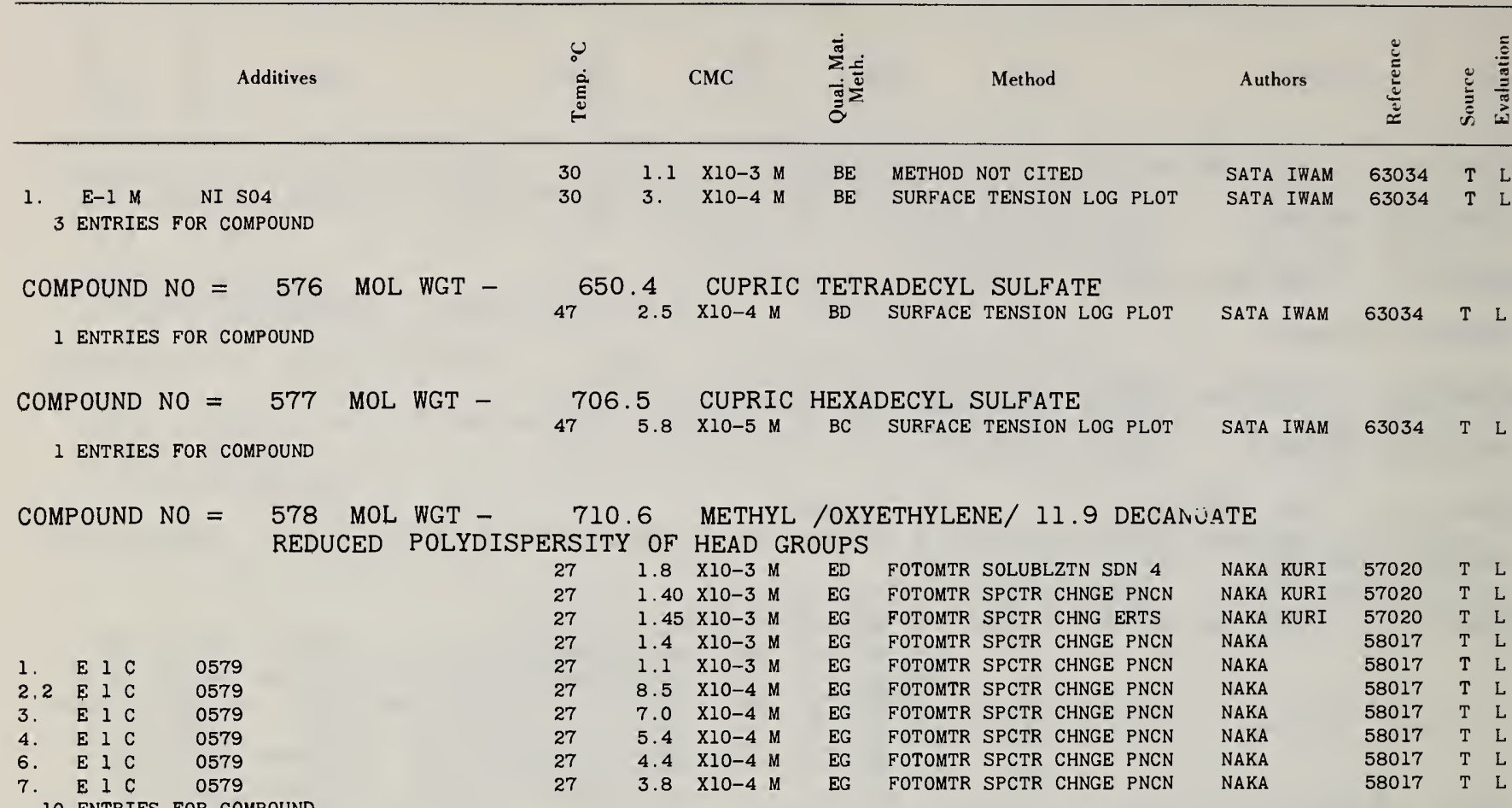

COMPOUND NO $=579$ MOL WGT - 765.1 METHYL /OXYETHYLENE/ 12.5 DODECANOATE REDUCED POLYDISPERSITY OF HEAD GROUPS

\begin{tabular}{|c|c|c|c|c|c|c|c|}
\hline 27 & 3.5 & $\mathrm{X} 10-4 \mathrm{M}$ & EG & FOTOMTR SPCTR CHNGE PNCN & NAKA KURI & 57020 & $\mathrm{~T}$ \\
\hline 27 & 3.4 & $\mathrm{X} 10-4 \mathrm{M}$ & EG & FOTOMTR SPCTR CHNG ERTS & NAKA KURI & 57020 & $\mathrm{~T}$ \\
\hline 27 & 2.8 & $\mathrm{X} 10-4 \cdot \mathrm{M}$ & ED & FOTOMTR SOLUBLZTN SDN 4 & NAKA KURI & 57020 & $\mathrm{~T}$ \\
\hline 2 & 3.4 & $\mathrm{X} 10-4 \mathrm{M}$ & EG & $\begin{array}{l}\text { FOTOMTR SPCTR CHNGE PNCN } \\
\text { SEE CMPD NMBR IN ADDITV }\end{array}$ & $\begin{array}{l}\text { NAKA } \\
\text { NAKA }\end{array}$ & $\begin{array}{l}58017 \\
58017\end{array}$ & $\mathrm{~T}$ \\
\hline
\end{tabular}

5 ENTRIES FOR COMPOUND SEE CMPD NMBR IN ADDITV

494.7 METHYL /OXYETHYLENE/ 7.0 DECANOATE

$\begin{aligned} \text { COMPOUND NO }= & 580 \text { MOL WGT - 494.7 METHYL /OXY } \\ & \text { REDUCED POLYDISPERSITY OF HEAD GROUPS }\end{aligned}$

REDUCED POLYDISPERSITY OF HEAD GROUPS

$\begin{array}{lllll}27 & 1.0 & \text { X10-3 M } & \text { ED } & \text { FOTOMTR SOLUBLZTN SDN } 4 \\ 27 & 8.0 & \text { X10-4 M } & \text { EG } & \text { FOTOMTR SPCTR CHNGE PNCN }\end{array}$

NAKA KURI

$9.5 \times 10-4 \mathrm{M}$

FOTOMTR SPCTR CHNG ERTS

NAKA KURI

$57020 \quad$ T $\quad$ L

57020 T $\quad$ L

3 ENTRIES FOR COMPOUND

640.1 METHYL /OXYETHYLENE/ 10.3 DECANOATE

581 MOL WGT -
REDUCED POLYDISPERSITY OF

$\begin{array}{lllll}27 & 1.4 & \text { X10-3 M } & \text { ED } & \text { FOTOMTR SOLUBLZTN SDN } 4 \\ 27 & 1.05 & \text { X10-3 M } & \text { EG } & \text { FOTOMTR SPCTR CHNGE PNCN } \\ 27 & 1.15 & \text { X10-3 M } & \text { EG } & \text { FOTOMTR SPCTR CHNG ERTS }\end{array}$

NAKA KURI

NAKA KURI

NAKA KURI

57020 T $\quad$ L

57020 T L

3 ENTRIES FOR COMPOUND

$1.15 \times 10-3 \mathrm{M}$ EG FOTOMTR SPCTR CHNG ERTS

57020 T $\quad$ L

COMPOUND NO $=582$ MOL WGT -891.3 METHYL /OXYETHYLENE/ 16.0 DECANOATE NATURAL DISTRIBUTION OF HEAD GROUPS

1 ENTRIES FOR COMPOUND

$27 \quad 1.6 \quad$ X10-3 M GG FOTOMTR SPCTR CHNGE PNCN

NAKA KURI $57020 \quad$ T $\quad$ L

COMPOUND NO $=583$ MOL WGT -478.7 METHYL /OXYETHYLENE/ 6.0 DODECANOATE REDUCED POLYDISPERSITY OF HEAD GROUPS

$\begin{array}{llllllllll}27 & 1.6 & \text { X10-4 M } & \text { ED } & \text { FOTOMTR SOLUBLZTN SDN 4 } & \text { NAKA KURI } & 57020 & \text { T } & \text { L } \\ 27 & 1.5 & \text { X10-4 M } & \text { EG } & \text { FOTOMTR SPCTR CHNGE PNCN } & \text { NAKA KURI } & 57020 & \text { T } & \text { L } \\ 27 & 2.0 & \text { X10-4 M } & \text { EG } & \text { FOTOMTR SPCTR CHNG ERTS } & \text { NAKA KURI } & 57020 & \text { T } & \text { L }\end{array}$

3 ENTRIES FOR COMPOUND

$1.5 \times 10-4 \mathrm{M} \quad$ EG FOTOMTR SPCTR CHNGE PNCN

NAKA KURI

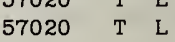

\section{COMPOUND NO $=584$ MOL WGT -584.5 METHYL /OXYETHYLENE/ 8.4 DODECANOATE REDUCED POLYDISPERSITY OF HEAD GROUPS}

$\begin{array}{lllllllll}27 & 2.0 & \text { X10-4 M } & \text { ED } & \text { FOTOMTR SOLUBLZTN SDN } 4 & \text { NAKA KURI } & 57020 & \text { T } & \text { L }\end{array}$

Concentration units: $\mathrm{A}-\mathrm{mol} \% ; \mathrm{B}-\mathrm{vol} \%$ solvent: $\mathrm{C}-\mathrm{mol} \%$ surfactant mixture $\mathrm{D}-\mathrm{wt} / \mathrm{vol} \%$; E-\% saturation; $\mathrm{H}-$ wt $\%$ solvent; I-mol \% surfactant; $\mathrm{K}$-normality counterions; $\mathrm{M}$-molar; $\mathrm{N}$-normal; $\mathrm{P}-$ wt \%; $\mathrm{Q}$ - wt \% surfactant; $\mathrm{R}$-varied; $\mathrm{S}-\mathrm{mol} /$ $\mathrm{kg} ; \mathrm{T}-$ wt \% surfactant mixture; $\mathrm{U}-\mathrm{mol} /(\mathrm{l}$ orkg); $\mathrm{W}-$ molal; $\mathrm{Y}-$ atm. Details on page 222. 


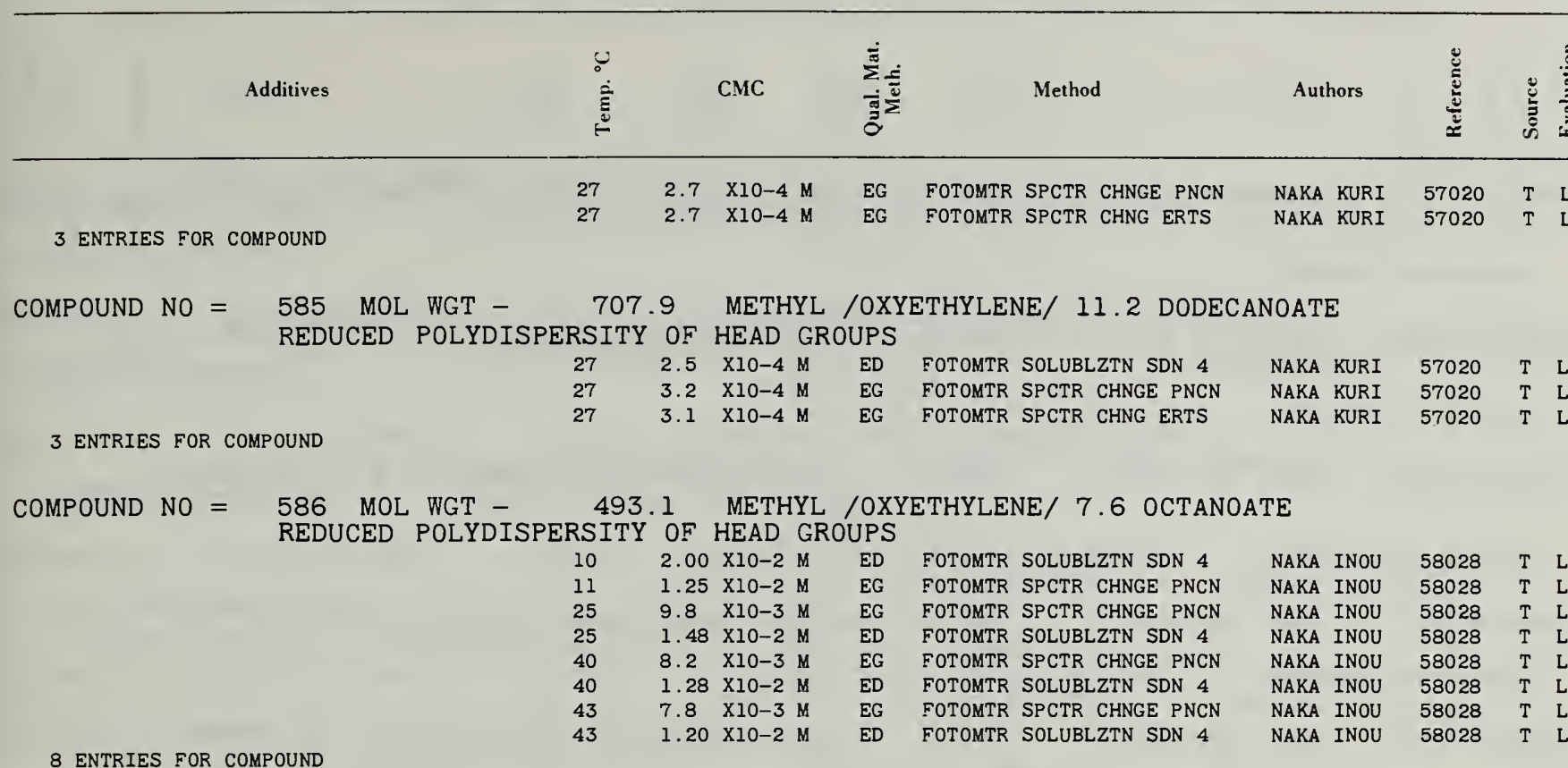

\section{COMPOUND NO $=587$ MOL WGT -}

2 ENTRIES FOR COMPOUND

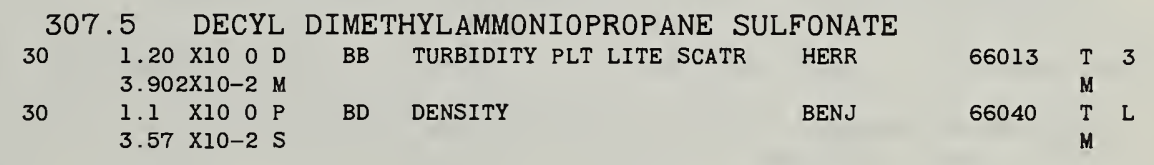

COMPOUND NO $=588$ MOL WGT -

2. $E-1 M \quad N A C L$

1. EO M NA CL

4 ENTRIES FOR COMPOUND

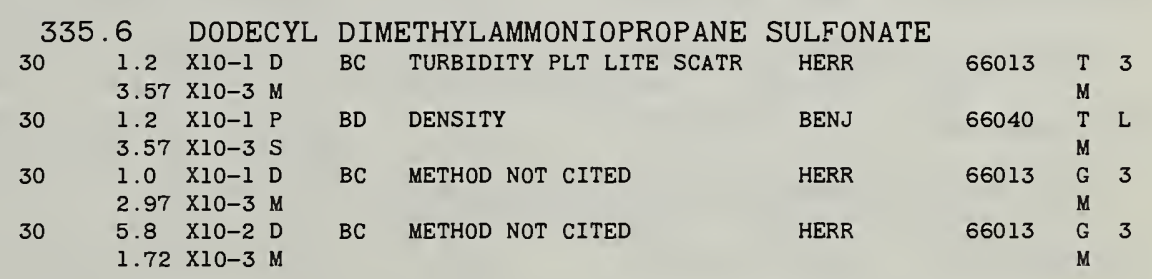

COMPOUND NO $=589$ MOL WGT -

2 ENTRIES FOR COMPOUND

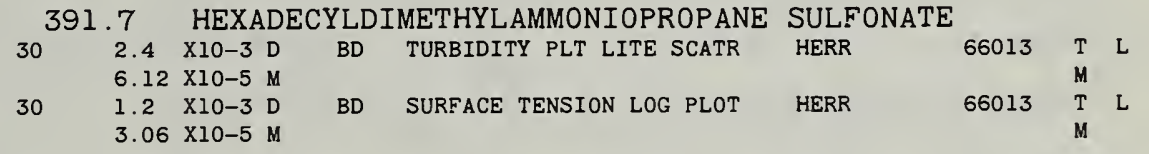

COMPOUND NO $=590$ MOL WGT -

299.6 DODECYL DIMETHYLAMMONIOPROPANE CARBOXYLATE $\begin{array}{lllllllll}30 & \begin{array}{llll}1.6 & \text { X10-1 } & \text { D } \\ 5.34 & \times 10-3 & \text { M }\end{array} & \text { BC } & \text { TURBIDITY PLT LITE SCATR } & \text { HERR } & 66013 & \text { T } & 3 \\ & & & & & & & \end{array}$

1 ENTRIES FOR COMPOUND

COMPOUND NO $=591$ MOL WGT -

352.5 DODECYL DIMETHYLPHOSPHONIOPROPANE SULFONATE

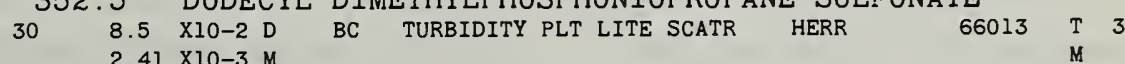

1 ENTRIES FOR COMPOUND

COMPOUND NO $=592$ MOL WGT -

299.6 DODECYL N-DIETHYL N-BETAINE

$30 \quad 8.4 \quad \times 10-2 \quad D \quad X C$ METHOD NOT CITED $2.80 \times 10-3 \mathrm{M}$

HERR $\quad 66013 \quad$ T L

1 ENTRIES FOR COMPOUND

COMPOUND NO $=593$ MOL WGT -

351.6 DIMETHYL DODECYLAMMONIOPROPANE HYDROXY SULFONATE

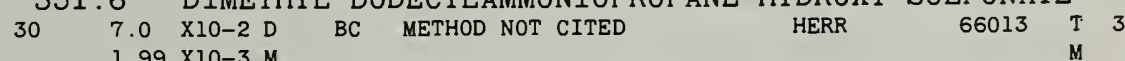

1 ENTRIES FOR COMPOUND

Concentration units: $\mathrm{A}-\mathrm{mol} \% ; \mathrm{B}-$ vol \% solvent; $\mathrm{C}-\mathrm{mol} \%$ surfactant mixture; D-wt/vol \%; E-\% saturation; $\mathrm{H}$ - wt \% solvent; I- mol \% surfactant; K-normality counterions; $\mathrm{M}$-molar; $\mathrm{N}$ - normal; $\mathrm{P}$ - wt \%; $\mathrm{Q}$ - wt \% surfactant: $\mathrm{R}$ - varied; $\mathrm{S}-\mathrm{mol} /$ kg; $\mathrm{T}-$ wt $\%$ surfactant mixture; $\mathrm{U}-\mathrm{mol} /(\mathrm{l}$ orkg); $\mathrm{W}$ - molal; $\mathrm{Y}$ - atm. Details on page 222. 


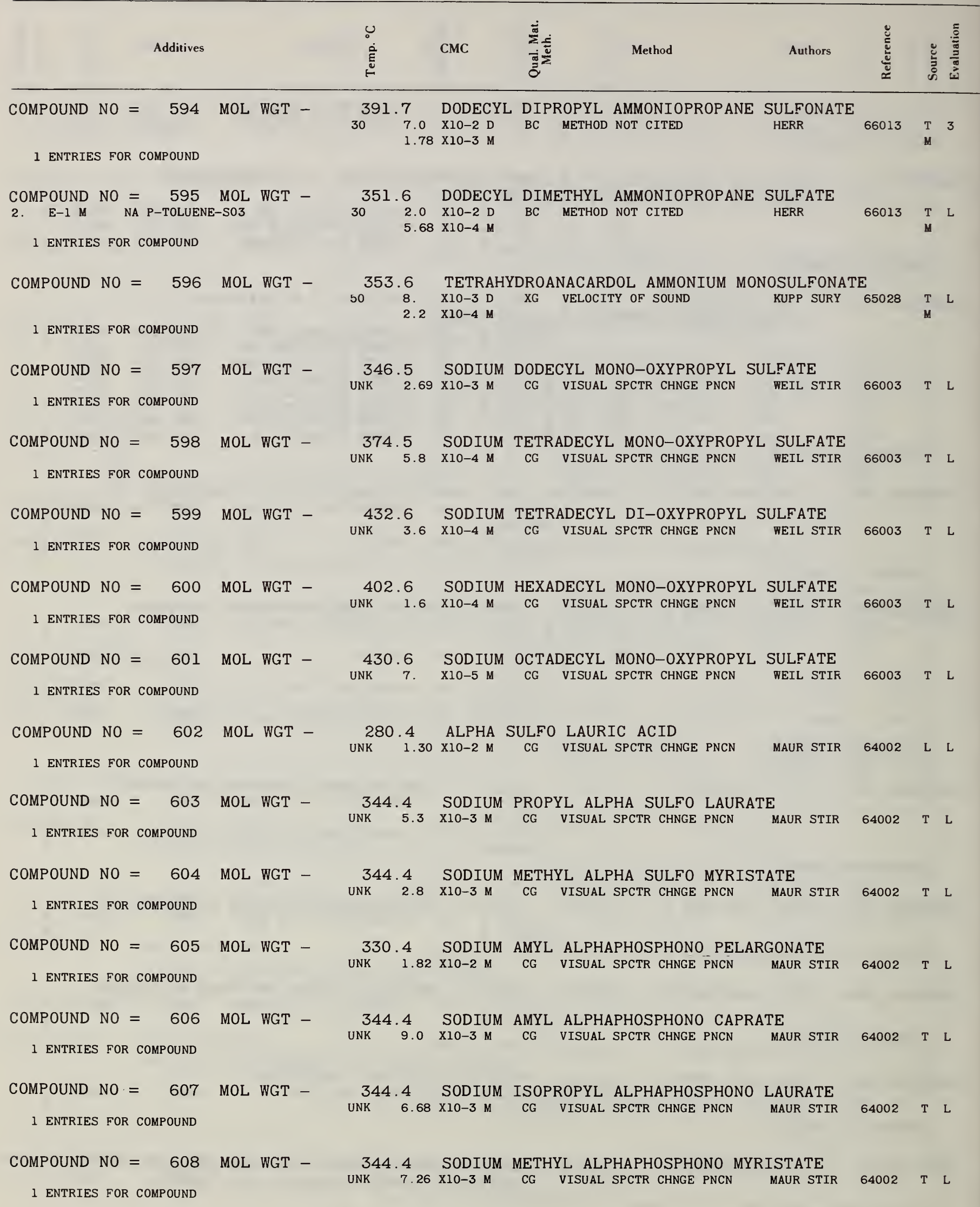

Concentration units: $\mathrm{A}-\operatorname{mol} \% ; \mathrm{B}-$ vol \% solvent; $\mathrm{C}-$ mol \% surfactant mixture;

counterions; $\mathrm{M}$ - molar; $\mathrm{N}$ - normal; $\mathrm{P}$ - wt \%; $\mathrm{Q}$ - wt \% surfactant; $\mathrm{R}$-varied; $\mathrm{S}-$ mol/ $\mathrm{D}-\mathrm{wt} / \mathrm{vol} \% ; \mathrm{E}-\%$ saturation; $\mathrm{H}-\mathrm{wt} \%$ solvent; $\mathrm{I}-$ mol \% surfactant; $\mathrm{K}$-normality 


\begin{tabular}{|c|c|c|c|c|c|c|c|}
\hline Additives & $\begin{array}{l}u \\
\dot{\delta} \\
\dot{\varepsilon}\end{array}$ & СмС & 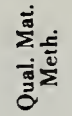 & Method & Authors & שֶّ & 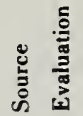 \\
\hline
\end{tabular}

COMPOUND NO $=609$ MOL WGT -

1 ENTRIES FOR COMPOUND

COMPOUND NO $=610$ MOL WGT -

I ENTRIES FOR COMPOUND

COMPOUND NO $=611$ MOL WGT -

1 ENTRIES FOR COMPOUND

COMPOUND NO $=612$ MOL WGT -

1 ENTRIES FOR COMPOUND

COMPOUND NO $=613$ MOL WGT -

I ENTRIES FOR COMPOUND

COMPOUND NO $=614$ MOL WGT -

1 ENTRIES FOR COMPOUND

COMPOUND NO $=615$ MOL WGT -

1 ENTRIES FOR COMPOUND

COMPOUND NO $=616$ MOL WGT -

1 ENTRIES FOR COMPOUND

COMPOUND NO $=617$ MOL WGT -

1 ENTRIES FOR COMPOUND

COMPOUND NO $=618$ MOL WGT -

1 ENTRIES FOR COMPOUND

COMPOUND NO $=619$ MOL WGT -

1 ENTRIES FOR COMPOUND

COMPOUND NO $=620$ MOL WGT -

1 ENTRIES FOR COMPOUND

COMPOUND NO $=621$ MOL WGT -

1 ENTRIES FOR COMPOUND

COMPOUND NO $=622$ MOL WGT -

I ENTRIES FOR COMPOUND

COMPOUND NO $=623$ MOL WGT -

1 ENTRIES FOR COMPOUND

COMPOUND NO $=624$ MOL WGT -

1 ENTRIES FOR COMPOUND
400.5 SODIUM ISOPROPYL ALPHAPHOSPHONO PALMITATE

UNK 3.2 X10-4 M CG VISUAL SPCTR CHNGE PNCN MAUR STIR 64002 T L

400.5 SODIUM METHYL ALPHAPHOSPHONO STEARATE

$\begin{array}{llllllll}\text { UNK } & 2.9 & \text { X10-4 M CG VISUAL SPCTR CHNGE PNCN } & \text { MAUR STIR } & 64002 & T & \text { L }\end{array}$

238.2 ALPHAPHOSPHONO PELARGONIC ACID

UNK $3.02 \times 10-2 \mathrm{M}$ CB SURFACE TENSION UNSPEC MAUR STIR 64002 LT L

252.3 ALPHAPHOSPHONO DECANOIC ACID

UNK 1.35 X10-2 M CB SURFACE TENSION UNSPEC MAUR STIR 64002 LT L

280.3 ALPHAPHOSPHONO DODECANOIC ACID

UNK $2.5 \quad \mathrm{X} 10-3 \mathrm{M} \quad$ CC SURFACE TENSION UNSPEC MAUR STIR 64002 LT L

308.4 ALPHAPHOSPHONO TETRADECANOIC ACID

$\begin{array}{lllllll}\text { UNK } & 5.8 & \text { X10-4 M } & \text { CB SURFACE TENSION UNSPEC } & \text { MAUR STIR } 64002 & \text { LT L }\end{array}$

336.5 ALPHAPHOSPHONO HEXADECANOIC ACID

UNK $8.9 \quad$ XIO-5 M $\quad$ CB SURFACE TENSION UNSPEC MAUR STIR 64002 LT L

274.3 MONOSODIUM ALPHAPHOSPHONO DECANOATE

UNK $4.89 \times 10-2 \mathrm{M}$ CG VISUAL SPCTR CHNGE PNCN MAUR STIR 64002 L L

302.3 MONOSODIUM ALPHAPHOSPHONO DODECANOATE

UNK $1.10 \quad \times 10-2 M \quad$ CG VISUAL SPCTR CHNGE PNCN MAUR STIR 64002 L L

330.4 MONOSODIUM ALPHAPHOSPHONO TETRADECANOATE

UNK $2.50 \times 10-3 \mathrm{M}$ CG VISUAL SPCTR CHNGE PNCN MAUR STIR 64002 L L

358.4 MONOSODIUM ALPHAPHOSPHONO HEXADECANOATE

UNK $5.2 \quad$ XI0-4 M CG VISUAL SPCTR CHNGE PNCN MAUR STIR 64002 L L

324.3 DISODIUM ALPHAPHOSPHONO DODECANOATE

UNK $3.06 \times 10-2 \mathrm{M}$ CG VISUAL SPCTR CHNGE PNCN MAUR STIR 64002 L L

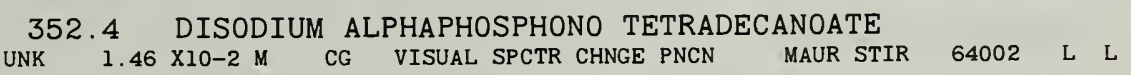

380.4 DISODIUM ALPHAPHOSPHONO HEXADECANOATE

$\begin{array}{lllllll}\text { UNK } & 6.7 & \text { XIO-3 M } & \text { CG VISUAL SPCTR CHNGE PNCN MAUR STIR } & 64002 & \text { L L }\end{array}$

408.5 DISODIUM ALPHAPHOSPHONO OCTADECANOATE

$\begin{array}{lllllll} & \text { UNK } & 2.9 & \text { XIO-3 M CG VISUAL SPCTR CHNGE PNCN MAUR STIR } 64002 & \text { L L }\end{array}$

374.3 TRISODIUM ALPHAPHOSPHONO TETRADECANOATE

UNK $3.02 \times 10-2 \mathrm{M}$ CG VISUAL SPCTR CHNGE PNCN MAUR STIR 64002 L L
Concentration units: $\mathrm{A}-\mathrm{mol} \% ; \mathrm{B}-\mathrm{vol} \%$ solvent; $\mathrm{C}-\mathrm{mol} \%$ surfactant mixture; D-wt/vol \%; E-\% saturation; $\mathrm{H}-$ wt \% solvent; I-mol \% surfactant; $\mathrm{K}$-normality counterions; $\mathrm{M}$-molar; $\mathrm{N}$-normal; $\mathrm{P}$ - wt \%; $\mathrm{Q}$ - wt \% surfactant; $\mathrm{R}$-varied; $\mathrm{S}$-mol/ kg; $\mathrm{T}$ - wt \% surfactant mixture; $\mathrm{U}-\mathrm{mol} /(\mathrm{l}$ or $\mathrm{kg}) ; \mathrm{W}-$ molal; $\mathrm{Y}$ - atm. Detailson page 222. 


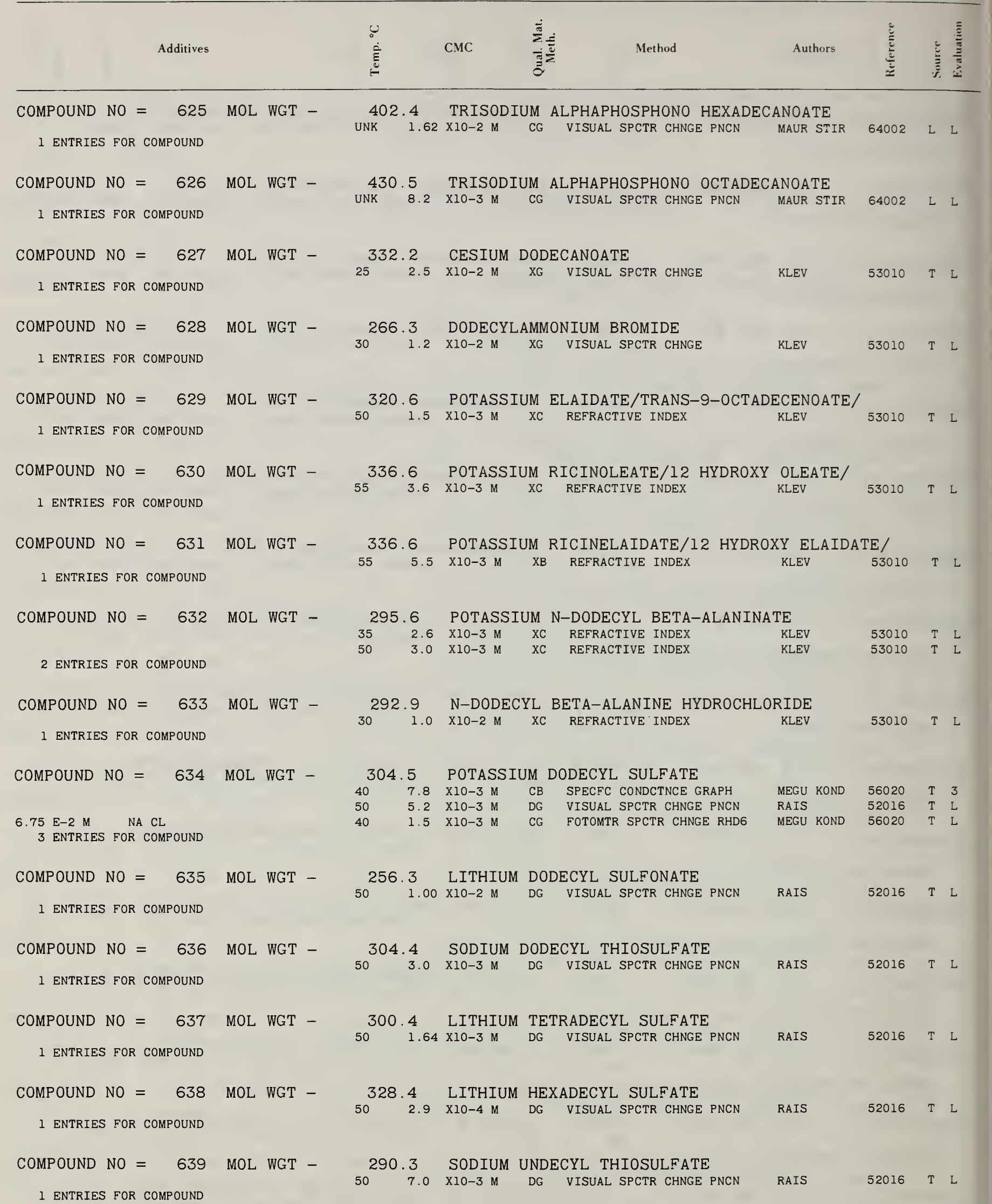

Concentration units: $\mathrm{A}-\mathrm{mol} \% ; \mathrm{B}-$ vol \% solvent; $\mathrm{C}-$ mol \% surfactant mixture; $\mathrm{D}-\mathrm{wt} / \mathrm{vol} \%$; E- \% saturation; $\mathrm{H}-$ wt $\%$ solvent; $\mathrm{I}-$ mol \% surfactant; $\mathrm{K}$-normality 


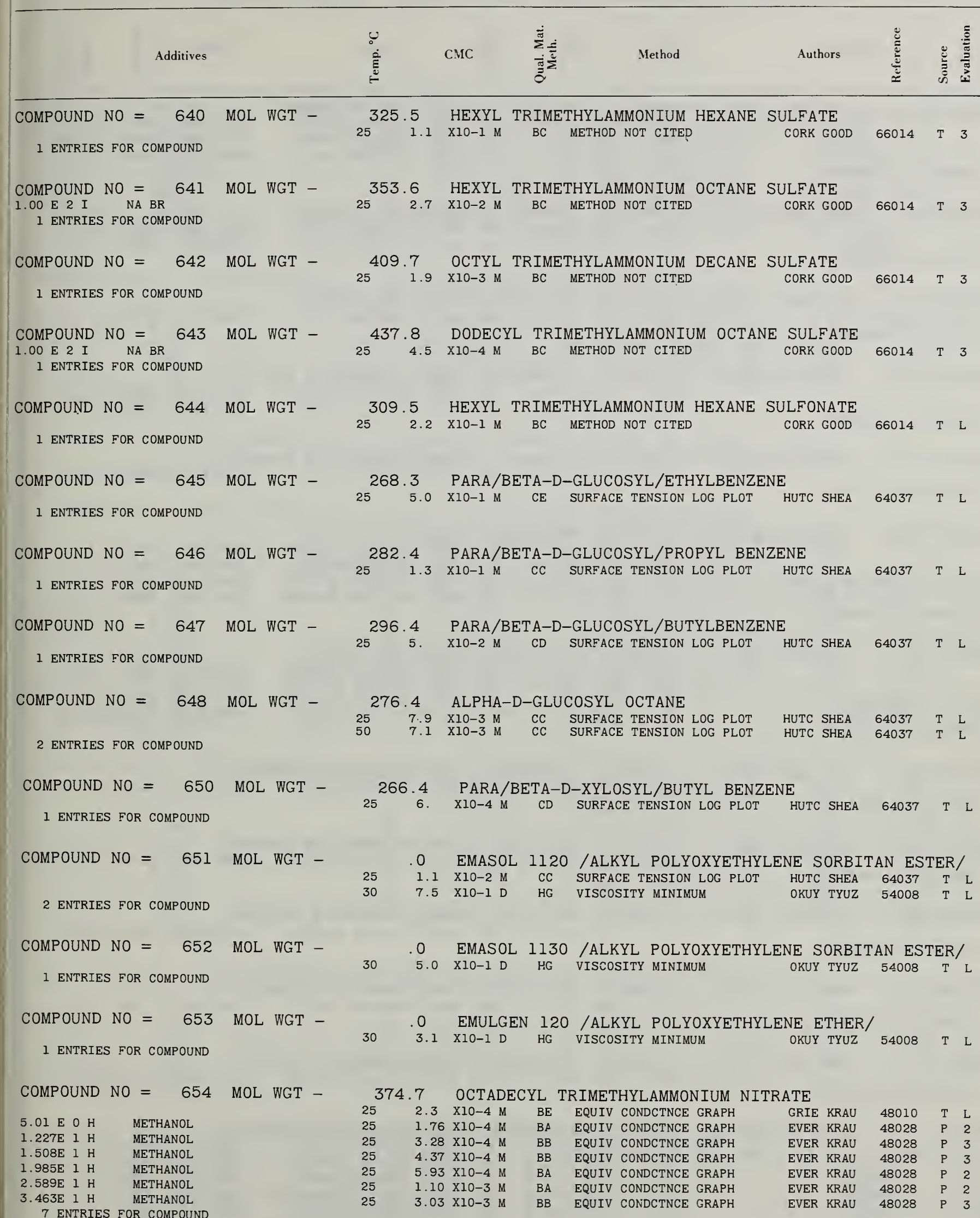

Concentration units: $\mathrm{A}-\mathrm{mol} \%$; $\mathrm{B}-\mathrm{vol} \%$ solvent; $\mathrm{C}-\mathrm{mol} \%$ surfactant mixture; $\mathrm{D}-$ wt/vol \%; E-\% saturation; $\mathrm{H}-$ wt \% solvent; I-mol \% surfactant; $\mathrm{K}$ - normality counterions; M-molar; N-normal; P-wt \%; Q - wt \% surfactant; $\mathrm{R}$-varied; $\mathrm{S}$ - mol/ kg; T - wt \% surfactant mixture; $U-$ mol/(l or kg); W - molal; $Y$ - atm. Details on page 222. 
$\frac{\text { Additives }}{\text { COMPOUND NO }=655 \text { MOL WGT - }}$

3 ENTRIES FOR COMPOUND

\begin{tabular}{|c|c|c|c|c|}
\hline 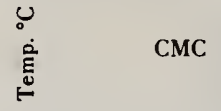 & 离 & Method & Authors & ֻัّँّ \\
\hline
\end{tabular}

\begin{tabular}{lllll}
368.1 & \multicolumn{3}{c}{ OCTADECYL } & PYRIDINIUM CHLORIDE \\
25 & 2.4 & X10-4 M & BB & EQUIV CONDCTNCE GRAPH \\
25 & $2.40 \times 10-4 \mathrm{M}$ & BB & EQUIV CONDCTNCE GRAPH \\
25 & 2.54 & X10-4 M & BC & EQUIV CONDCTNCE GRAPH
\end{tabular}

GRIE KRAU

$25 \quad 2.54 \times 10-4 \mathrm{M} \quad \mathrm{BC}$ EQUIV CONDCTNCE GRAPH

$\begin{array}{llll}48028 & P & 3 \\ & & \end{array}$

COMPOUND NO $=656$ MOL WGT -

2.0 E 1 H METHANOL

2 ENTRIES FOR COMPOUND

$\begin{array}{lll}394.7 & \text { OCTADECYL PYRIDINIUM NITRATE } \\ 25 & 1.28 \times 10-4 \mathrm{M} & \text { BB }\end{array}$

$\begin{array}{llll}25 & 1.28 \times 10-4 \mathrm{M} & \text { BB } & \text { EQUIV CONDCTNCE GRAPH } \\ 25 & 5.76 \times 10-4 \mathrm{M} & \text { BB } & \text { EQUIV CONDCTNCE GRAPH }\end{array}$

EVER KRAU

EVER KRAU

$48028 \quad$ T 3

COMPOUND NO $=657$ MOL WGT 2.0 E 1 H METHANOL

1 ENTRIES FOR COMPOUND

\subsection{OCTADECYL PYRIDINIUM BROMIDE}

$256.10 \times 10-4 \mathrm{M}$ BB EQUIV CONDCTNCE GRAPH $\quad$ EVER KRAU 48028 P 3

COMPOUND NO $=658$ MOL WGT -

2 ENTRIES FOR COMPOUND

COMPOUND NO $=659$ MOL WGT -

1 ENTRIES FOR COMPOUND

COMPOUND NO $=660$ MOL WGT -

7.9 E $0 \mathrm{H}$ TERTIARY BUTANOL

4 ENTRIES FOR COMPOUND

COMPOUND NO $=661$ MOL WGT -

$1.62 \mathrm{E} 1 \mathrm{H}$ METHANOL

$1.62 \mathrm{E} I \mathrm{H}$ METHANOL

4 ENTRIES FOR COMPOUND

1 ENTRIES FOR COMPOUND

COMPOUND NO $=663$ MOL WGT -

1 ENTRIES FOR COMPOUND

COMPOUND NO $=664$ MOL WGT -

1 ENTRIES FOR COMPOUND

COMPOUND NO $=665$ MOL WGT -

1 ENTRIES FOR COMPOUND

COMPOUND NO $=666$ MOL WGT -

1 ENTRIES FOR COMPOUND

COMPOUND NO $=667$ MOL WGT -

$\begin{array}{lllll}9.91 & \text { E } & 0 & H & \text { ACETONE } \\ 9.91 & \text { E } & 0 & H & \text { ACETONE } \\ 1.39 & \text { E } & 1 & H & \text { ACETONE } \\ 1.39 & \text { E } & 1 & H & \text { ACETONE } \\ 2.07 & \text { E } & 1 & H & \text { ACETONE }\end{array}$

7.9 E O H TERTIARY BUTANOL

COMPOUND NO $=662$ MOL WGT -

\begin{tabular}{lllllllll}
\multicolumn{1}{c}{440.6} & \multicolumn{1}{l}{ OCTADECYL } & TRIMETHYLAMMONIUM BROMATE \\
25 & 3.1 & X10-4 M & BG & EQUIV COND IST DEVIATION & GRIE KRAU & 48010 & T & L \\
25 & 3.31 & X10-4 M M & BB & EQUIV CONDCTNCE GRAPH & GRIE KRAU & 48010 & P & 3
\end{tabular}

357.7 OCTADECYL TRIMETHYLAMMONIUM FORMATE

$\begin{array}{llllllllll}25 & 4.4 & \times 10-4 & \mathrm{M} & \text { BC } & \text { EQUIV CONDCTNCE GRAPH } & \text { GRIE KRAU } & 48010 & \text { T } & 3\end{array}$

\begin{tabular}{lllllllll}
\multicolumn{2}{c}{479.5} & \multicolumn{2}{l}{ HEXADECYL } & PYRIDINIUM IODATE \\
25 & 1.6 & X10-3 M & BG & EQUIV CONDCTNCE MAXIMUM & BROW GRIE & 49014 & T & L \\
25 & 9.9 & X10-4 M & BG & EQUIV COND MAX BEGINING & BROW GRIE & 49014 & T & L \\
25 & 1.35 & X10-4 M & BG & EQUIV COND MAX BEGINING & BROW GRIE & 49014 & T & L \\
25 & 9. & X10-4 M & BG & EQUIV CONDCTNCE MAXIMUM & BROW GRIE & 49014 & T & L
\end{tabular}

\begin{tabular}{lllllllll}
\multicolumn{2}{c}{507.6} & \multicolumn{2}{l}{ OCTADECYL } & PYRIDINIUM IODATE & & & & \\
25 & 5. & X10-4 M & BG & EQUIV CONDCTNCE MAXIMUM & BROW GRIE & 49014 & T & L \\
25 & 9. & X10-5 M & BG & EQUIV COND MAX BEGINING & BROW GRIE & 49014 & T & L \\
25 & 8. & X10-4 M & BG & EQUIV CONDCTNCE MAXIMUM & BROW GRIE & 49014 & T & L \\
25 & 2.9 & X10-4 M & BG & EQUIV COND MAX BEGINING & BROW GRIE & 49014 & T & L
\end{tabular}

482.7 OCTADECYL TRIETHYLAMMONIUM BROMATE

$\begin{array}{lllllllll}25 & 2.5 & \text { X10-4 M } & \text { BC } & \text { EQUIV CONDCTNCE GRAPH } & \text { MCDO KRAU } & 51009 & \text { T } & 3\end{array}$

524.8 OCTADECYL TRIPROPYLAMMONIUM BROMATE

$25 \quad 1.25 \times 10-4 \mathrm{M}$ BG EQUIV COND MAX BEGINING $\quad$ MCDO KRAU 51009 T $\mathrm{L}$

566.9 OCTADECYL TRIBUTYLAMMONIUM BROMATE

$25 \quad 5.3 \quad \mathrm{X} 10-5 \mathrm{M}$ BG EQUIV COND MAX BEGINING MCDO KRAU 51009 TK L

608.9 OCTADECYL TRIAMYLAMMONIUM BROMATE

$25 \quad 1.6 \quad$ X10-5 M BG EQUIV COND MAX BEGINING MCDO KRAU 51009 TK L

538.8 HEXADECYL TRIBUTYLAMMONIUM BROMATE

$25 \quad 3.3$ X10-4 M BG EQUIV COND MAX BEGINING MCDO KRAU 51009 TK L

713.4 OCTADECYL TRIMETHYLAMMONIUM OXALATE

$\begin{array}{lllllllll}25 & 6.4 & \text { X10-5 M } & \text { BE } & \text { EQUIV CONDCTNCE GRAPH } & \text { YOUN GRIE } & 49017 & \text { T } & \text { L } \\ 25 & 1.44 & \text { X10-4 M } & \text { BG } & \text { EQUIV COND 1ST DEVIATION } & \text { YOUN GRIE } & 49017 & \text { T } & \text { L } \\ 25 & 1.61 & \text { X10-4 M } & \text { BB } & \text { EQUIV CONDCTNCE GRAPH } & \text { YOUN GRIE } & 49017 & \text { P } & 3 \\ 25 & 2.56 & \text { X10-4 M } & \text { BG } & \text { EQUIV COND 1ST DEVIATION } & \text { YOUN GRIE } & 49017 & \text { T } & \text { L } \\ 25 & 2.56 & \text { X10-4 M } & \text { BB } & \text { EQUIV CONDCTNCE GRAPH } & \text { YOUN GRIE } & 49017 & \text { P } & 3 \\ 25 & 5.29 & \text { X10-4 M } & \text { BG } & \text { EQUIV COND 1ST DEVIATION } & \text { YOUN GRIE } & 49017 & \text { T } & \text { L }\end{array}$

Concentration units: $\mathrm{A}-\mathrm{mol} \% ; \mathrm{B}-\mathrm{vol} \%$ solvent; $\mathrm{C}-$ mol \% surfactant mixture; D-wt/vol \%; E-\% saturation; $\mathrm{H}-$ wt $\%$ solvent; I- mol \% surfactant; $\mathrm{K}-$ normality

counterions; $\mathrm{M}-$ molar; $\mathrm{N}$ - normal; $\mathrm{P}-\mathrm{wt} \%$; $\mathrm{Q}-\mathrm{wt} \%$ surfactant; $\mathrm{R}$ - varied; $\mathrm{S}-\mathrm{mol} /$ $\mathrm{kg} ; \mathrm{T}-\mathrm{wt} \%$ surfactant mixture; $\mathrm{U}-\mathrm{mol} /(\mathrm{l}$ orkg); $\mathrm{W}-$ molal; $\mathrm{Y}-\mathrm{atm}$. Details on page 222. 


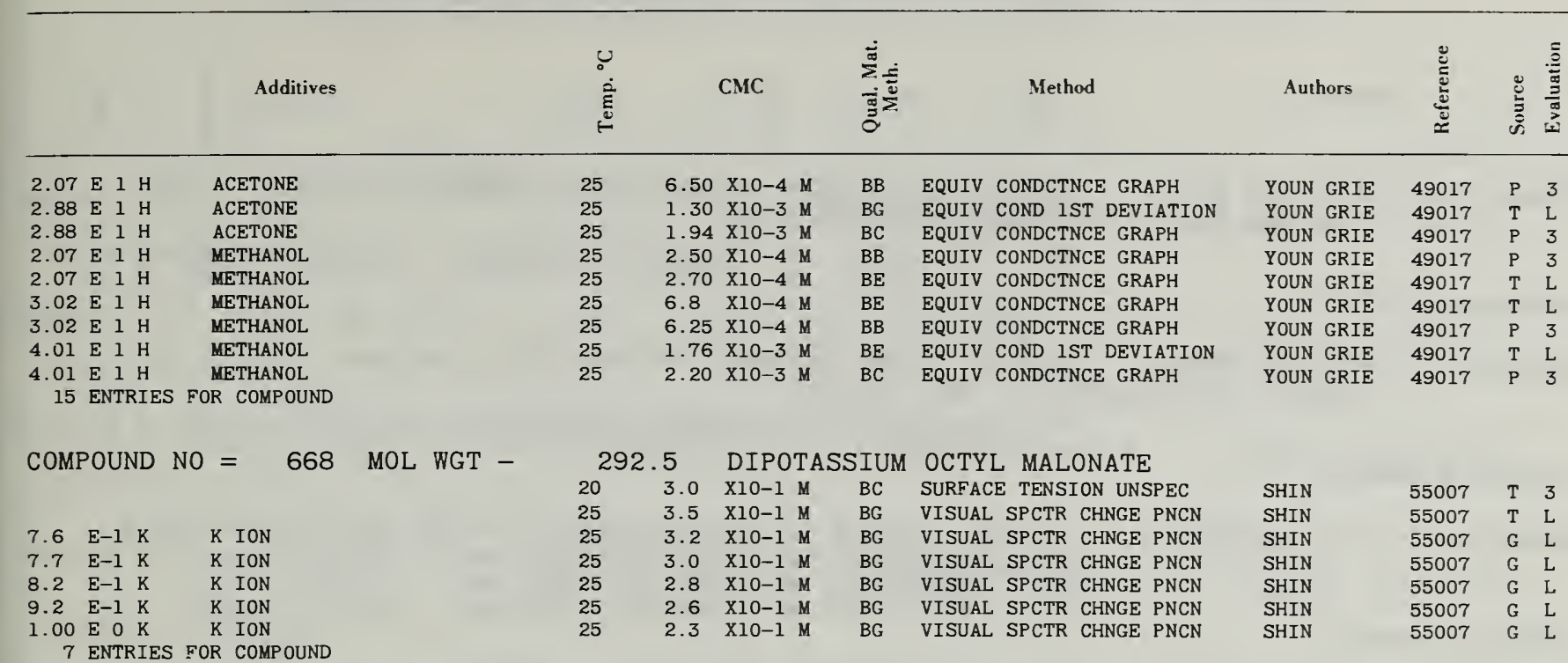

\section{COMPOUND NO $=669$ MOL WGT - \\ 1 ENTRIES FOR COMPOUND}

\subsection{DIPOTASSIUM DECYL MALONATE 25 \\ BG THEORETICALLY ESTIMATED}

348.6 DIPOTASSIUM DODECYL MALONATE $4.8 \times 10-2 \mathrm{M}$ BC SURFACE TENSION UNSPEC

$4.8 \times 10-2 M \quad$ BG FOTOMTR SPCTR CHNGE PNCN

$4.8 \times 10-2 \mathrm{M}$ BG VISUAL SPCTR CHNGE PNCN

$5.0 \times 10-2 \mathrm{M}$ BB FOTOMTR SOLUBLZTN 2NPA

$4.2 \times 10-2 \mathrm{M}$ BG VISUAL SPCTR CHNGE PNCN

$3.9 \times 10-2 M$ BG VISUAL SPCTR CHNGE PNCN

3.4 X10-2 M BG VISUAL SPCTR CHNGE PNCN

$2.6 \times 10-2 M \quad B G$ VISUAL SPCTR CHNGE PNCN

$2.1 \times 10-2 M \quad B G$ VISUAL SPCTR CHNGE PNCN

$1.9 \times 10-2 \mathrm{M}$ BG VISUAL SPCTR CHNGE PNCN

$1.6 \times 10-2 \mathrm{M}$ BG VISUAL SPCTR CHNGE PNCN

$1.3 \times 10-2 M \quad B G$ VISUAL SPCTR CHNGE PNCN

1.1 X10-2 M BG VISUAL SPCTR CHNGE PNCN
SHIN

SHIN

SHIN

SHIN

SHIN

SHIN

SHIN

SHIN

SHIN

SHIN

SHIN

SHIN

SHIN
COMPOUND NO $=671$ MOL WGT -

$\begin{array}{llll}3.7 & E-2 & K & \text { K ION } \\ 4.0 & \text { E-2 } & \text { K } & \text { K ION } \\ 4.5 & \text { E-2 } & \text { K } & \text { K ION } \\ 5.4 & \text { E-2 } & \text { K } & \text { K ION } \\ 7.0 & \text { E-2 } & \text { K } & \text { K ION } \\ 9.7 & \text { E-2 } & \text { K } & \text { K ION } \\ 1.23 & \text { E-1 } & \text { K } & \text { K ION }\end{array}$

10 ENTRIES FOR COMPOUND
376.7 DIPOTASSIUM TETRADECYL MALONATE

$\begin{array}{lllll}20 & 1.9 & \text { X10-2 } & \text { M } & \text { BC } \\ 25 & 1.7 & \text { X10-2 } & \text { M } & \text { BG } \\ 25 & 1.8 & \text { X10-2 } & \text { M } & \text { BC } \\ 25 & 1.5 & \text { X10-2 } & \text { M } & \text { BG } \\ 25 & 1.4 & \text { X10-2 } & \text { M } & \text { BG } \\ 25 & 1.2 & \text { X10-2 } & \text { M } & \text { BG } \\ 25 & 9.6 & \text { X10-3 } & \text { M } & \text { BG } \\ 25 & 7.1 & \text { X10-3 } & \text { M } & \text { BG } \\ 25 & 5.4 & \text { X10-3 } & \text { M } & \text { BG } \\ 25 & 4.4 & \text { X } 10-3 & M & \text { BG }\end{array}$

TETRADECYL MALONATE TENSION UNSPEC

VISUAL SPCTR CHNGE PNCN FOTOMTR SOLUBLZTN 2NPA VISUAL SPCTR CHNGE PNCN VISUAL SPCTR CHNGE PNCN VISUAL SPCTR CHNGE PNCN VISUAL SPCTR CHNGE PNCN VISUAL SPCTR CHNGE PNCN VISUAL SPCTR CHNGE PNCN VISUAL SPCTR CHNGE PNCN
SHIN

SHIN

SHIN

SHIN

SHIN

SHIN

SHIN

SHIN

SHIN

SHIN

$\begin{array}{lll}55007 & \text { T } & \text { L } \\ 55007 & \text { T } & \text { L } \\ 55007 & \text { T } & 3 \\ 55007 & \text { G } & \text { L } \\ 55007 & \text { G } & \text { L } \\ 55007 & \text { G } & \text { L } \\ 55007 & \text { G } & \text { L } \\ 55007 & \text { G } & \text { L } \\ 55007 & \text { G } & \text { L } \\ 55007 & \text { G } & \text { L }\end{array}$

404.7 DIPOTASSIUM HEXADECYL MALONATE

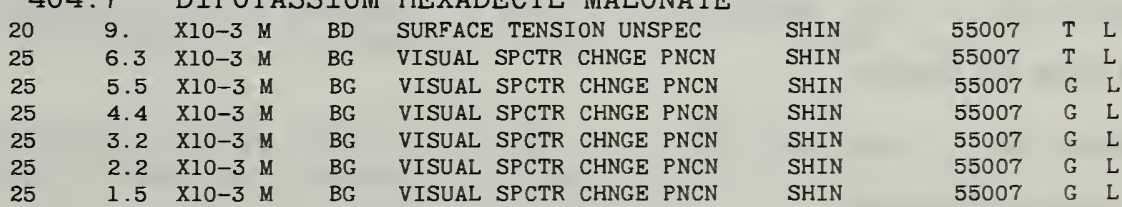

432.8 DIPOTASSIUM OCTADECYL MALONATE $25 \quad 2.3 \quad \mathrm{X} 10-3 \mathrm{M}$ BG VISUAL SPCTR CHNGE PNCN
SHIN

$55007 \quad T \quad$ L

1 ENTRIES FOR COMPOUND

\footnotetext{
Concentration units: $\mathrm{A}-\mathrm{mol} \% ; \mathrm{B}-\mathrm{vol} \%$ solvent; $\mathrm{C}-$ mol $\%$ surfactant mixture; $\mathrm{D}-\mathrm{wt} / \mathrm{vol} \% ; \mathrm{E}-\%$ saturation; $\mathrm{H}-\mathrm{wt} \%$ solvent: $\mathrm{I}-\mathrm{mol} \%$ surfactant; $\mathrm{K}$ - normality
} counterions; $\mathrm{M}-$ molar; $\mathrm{N}-$ normal; $\mathrm{P}-\mathrm{wt} \%$; Q - we \% surfactant; $\mathrm{R}$ - varied: $\mathrm{S}-\mathrm{mol} /$ 


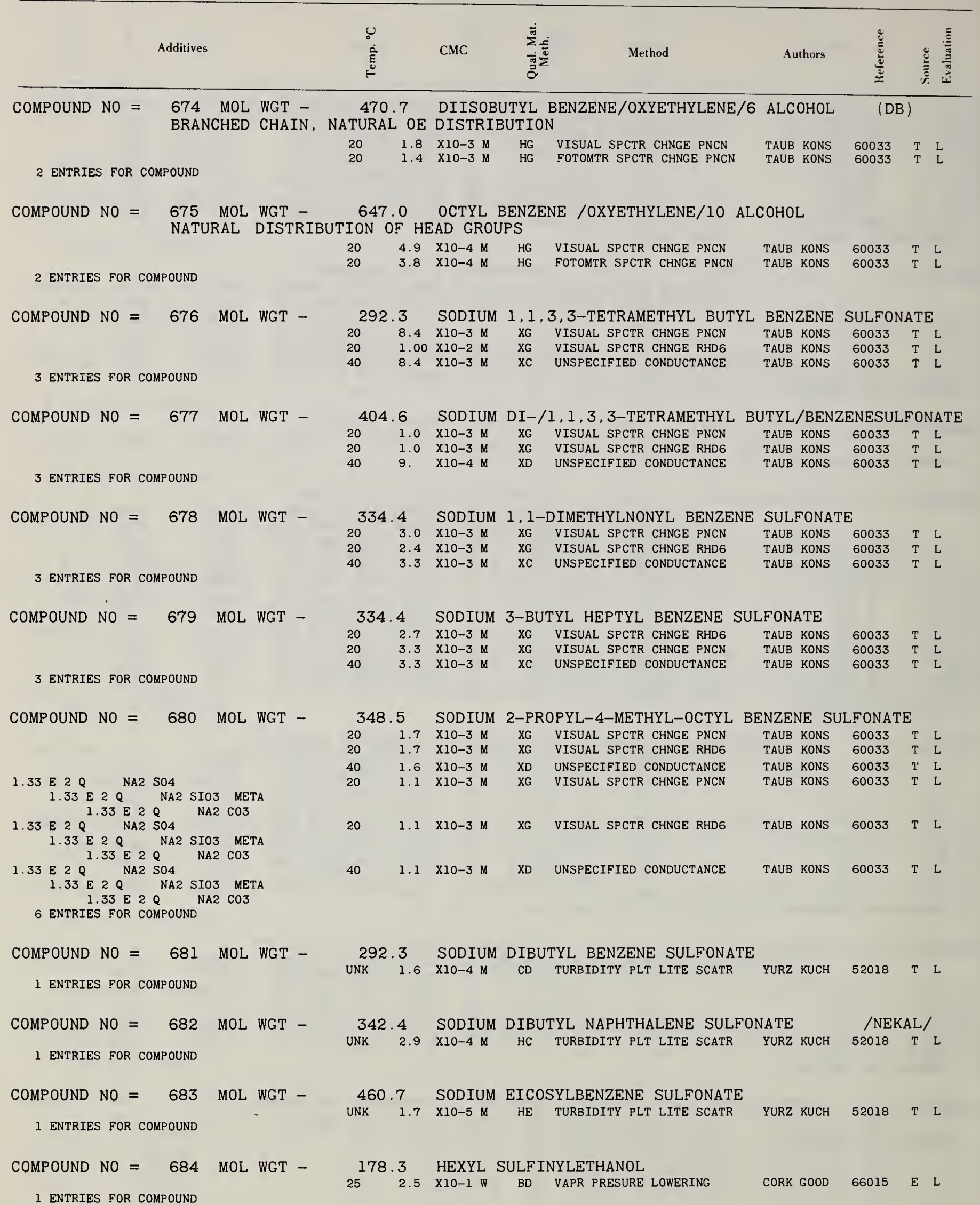

1 ENTRIES FOR COMPOUND 


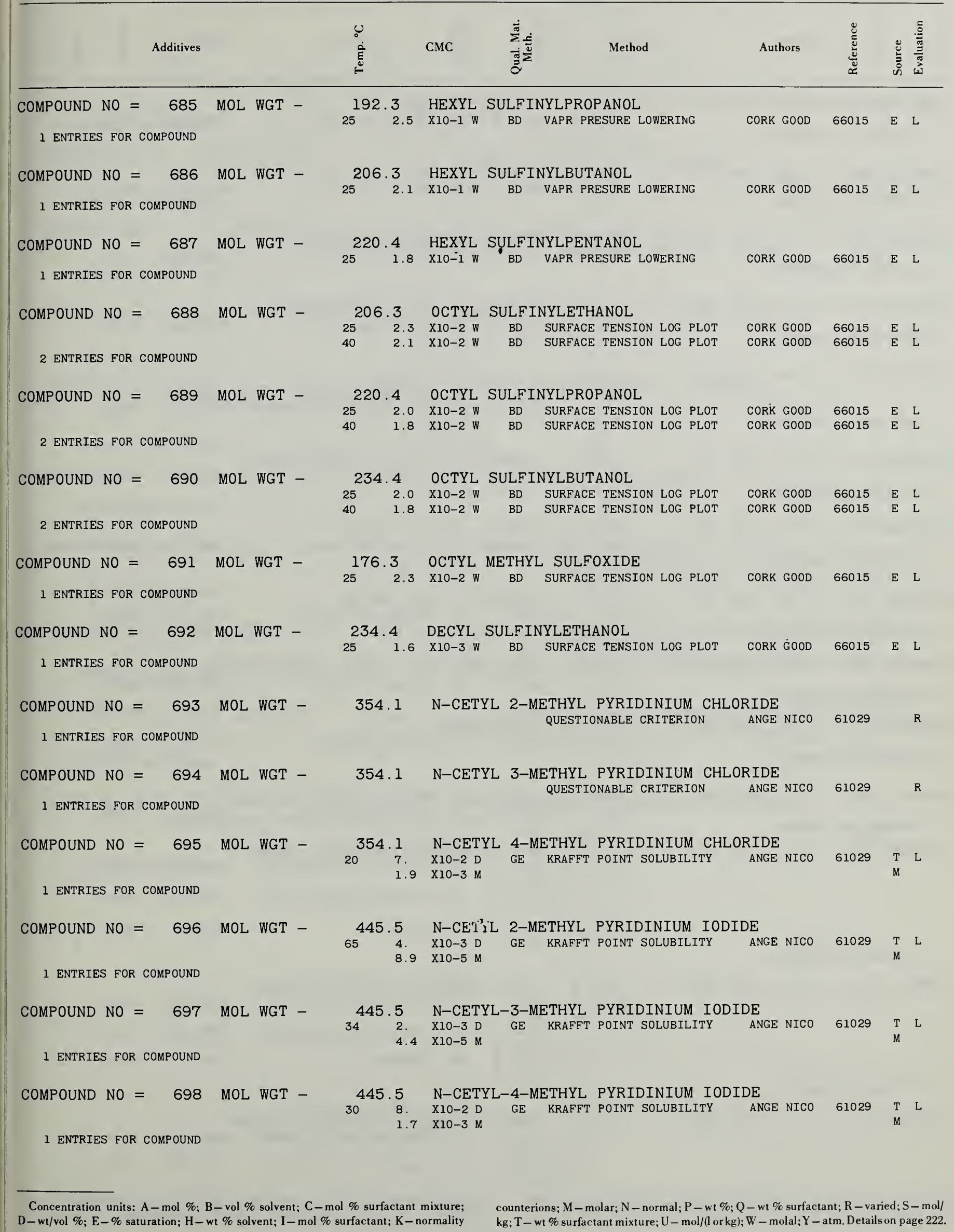




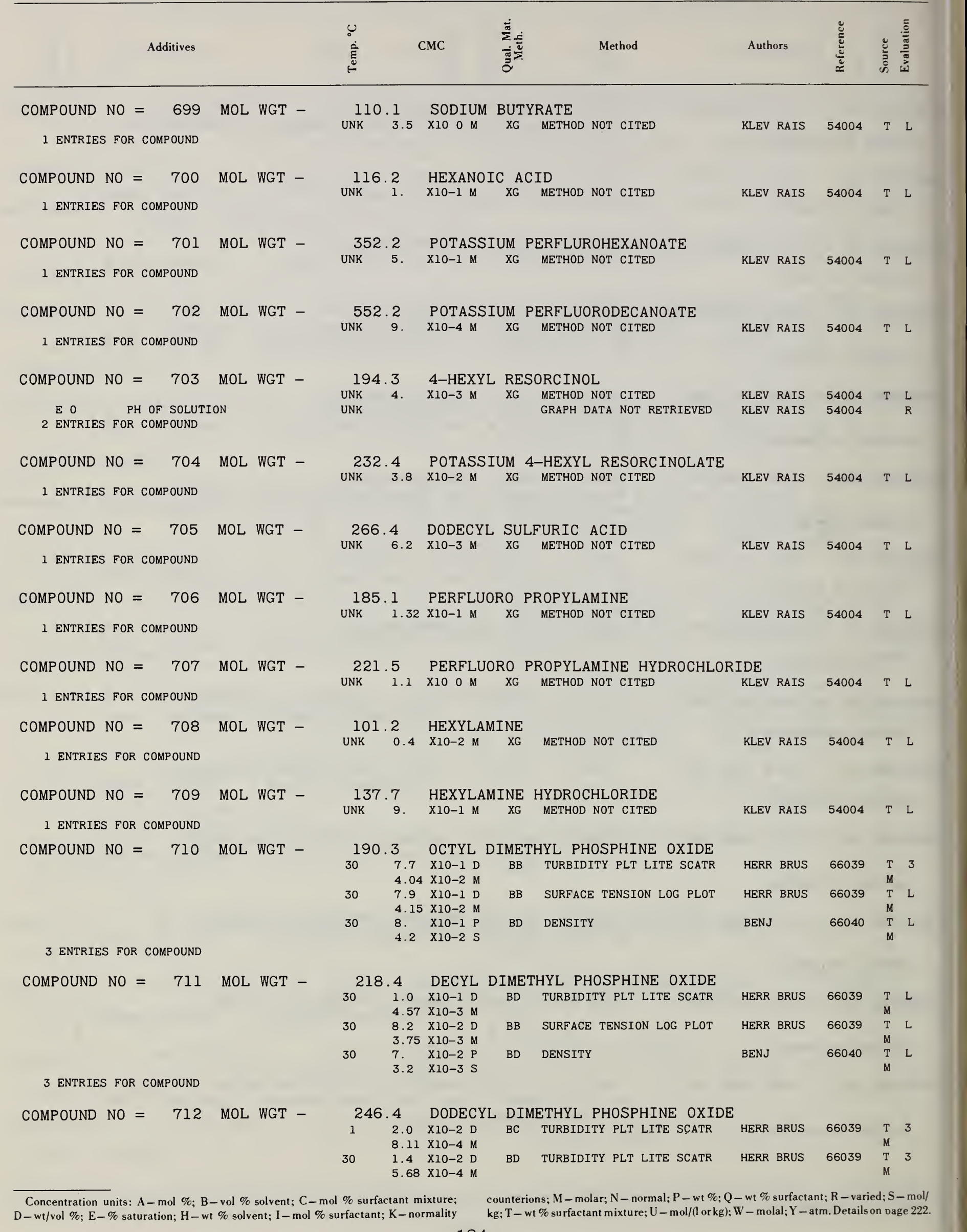


Complete Table of Critical Micelle Concentration Values-Continued

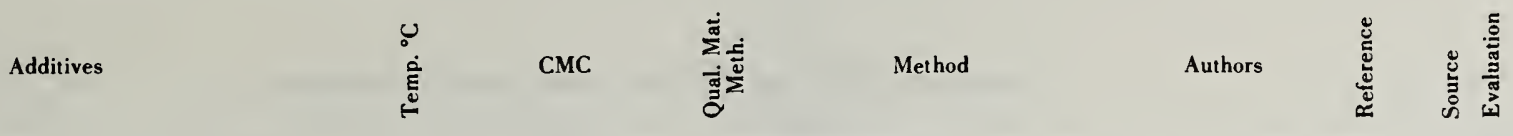

30 8. XIO-3 D BD SURFACE TENSION LOG PLOT HERR BRUS $66039 \quad T \quad$ L

3 ENTRIES FOR COMPOUND

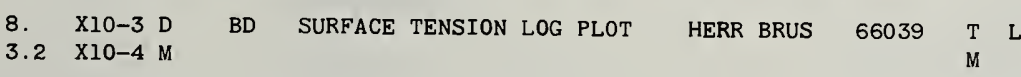

COMPOUND NO $=713$ MOL WGT -

$\begin{array}{lllll}215.4 & \text { UNDECYL } & \text { DIMETHYL AMINE OXIDE } \\ 30 & 1.3 & \text { X10-1 P } & \text { BD DENSITY } \\ 6.03 & \text { X10-3 } & \mathrm{S}\end{array}$

1 ENTRIES FOR COMPOUND $1.3 \times 10-1 \mathrm{P}$
$6.03 \times 10-3 \mathrm{~S}$

BENJ

$66040 \quad$ T $\quad$ L

COMPOUND NO $=714$ MOL WGT -

145.3 HEXYL DIMETHYL AMINE OXIDE

UNK $3.0 \quad \mathrm{X} 10 \quad \mathrm{P} P$ BE METHOD NOT CITED $2.06 \times 100$

BENJ

$66040 \quad$ T $\quad$ L

1 ENTRIES FOR COMPOUND

COMPOUND NO $=715$ MOL WGT -

159.3 HEPTYL DIMETHYL AMINE OXIDE UNK $1.0 \quad \times 101 \mathrm{P}$ BE METHOD NOT CITED $6.27 \times 10-1 \mathrm{~S}$

BEN

$66040 \underset{M}{T} \quad$ L

1 ENTRIES FOR COMPOUND

COMPOUND NO $=716$ MOL WGT - 891.3 DODECYL /OXYETHYLENE/16 ALCOHOL REDUCED POLYDISPERSITY OF HEAD GROUPS

$252.205 \times 10-2$ D EE FOTOMTR SOLUBLZTN OROT

$2.473 \times 10-4 \mathrm{M}$

$66036 \quad \mathrm{~T}$

1 ENTRIES FOR COMPOUND

COMPOUND NO $=717$ MOL WGT -

378.4 DODECYLQUINOLINIUM BROMIDE

1 ENTRIES FOR COMPOUND

$25 \quad 4.80 \times 10-3 \mathrm{M}$ BB SURFACE TENSION LOG PLOT

FEW GILB 58031 T 3

COMPOUND NO $=718$ MOL WGT -

2 ENTRIES FOR COMPOUND

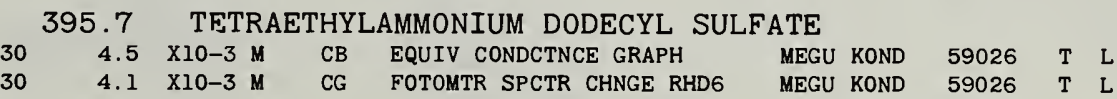

COMPOUND NO $=719$ MOL WGT -

\begin{tabular}{ccccccccc}
\multicolumn{7}{c}{507.9} & \multicolumn{2}{c}{ TETRABUTYLAMMONIUM DODECYL SULFATE } \\
30 & 1.3 & XI0-3 M & CC & EQUIV CONDCTNCE GRAPH & MEGU KOND & 59026 & T & L \\
30 & 1.0 & X10-3 M & CG & FOTOMTR SPECTR CHNGE RHD6 & MEGU KOND & 59026 & T & L
\end{tabular}

2 ENTRIES FOR COMPOUND

COMPOUND NO $=720$ MOL WGT -

733.2 1-6-DITRIMETHYLAMMONIUM-HEXANE/DODECYL SULFATE/2

$\begin{array}{lllllllllll}30 & 9.6 & \mathrm{XIO}-4 \mathrm{M} & \mathrm{CB} & \text { EQUIV CONDCTNCE GRAPH } & \text { MEGU KOND } & 59026 & \mathrm{~T} & 3\end{array}$

1 ENTRIES FOR COMPOUND

COMPOUND NO $=721$ MOL WGT $-1,542.1$ NONYL BENZENE /OXYETHYLENE/30 ALCOHOL

BRANCHED CHAIN REDUCED OE DISTRIBUTION

1 ENTRIES FOR COMPOUND

$25 \quad 4.22 \times 10-2 \mathrm{D} \quad \mathrm{EC}$

2.736 X10-4 M

ULTRAFILTRATION

SCHO

$64004 \quad T \quad$ L

Concentration units: $\mathrm{A}-\mathrm{mol} \% ; \mathrm{B}-\mathrm{vol} \%$ solvent; $\mathrm{C}-$ mol $\%$ surfactant mixture; D-wt/vol \%; E-\% saturation; $\mathrm{H}-$ wt \% solvent; I-mol \% surfactant; $\mathrm{K}-$ normality counterions; M-molar; N-normal; P - wt \%; Q-wt \% surfactant; $\mathrm{R}$-varied; $\mathrm{S}-$ mol/

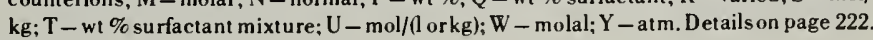




\section{Index of Additives Giving Compound Numbers}

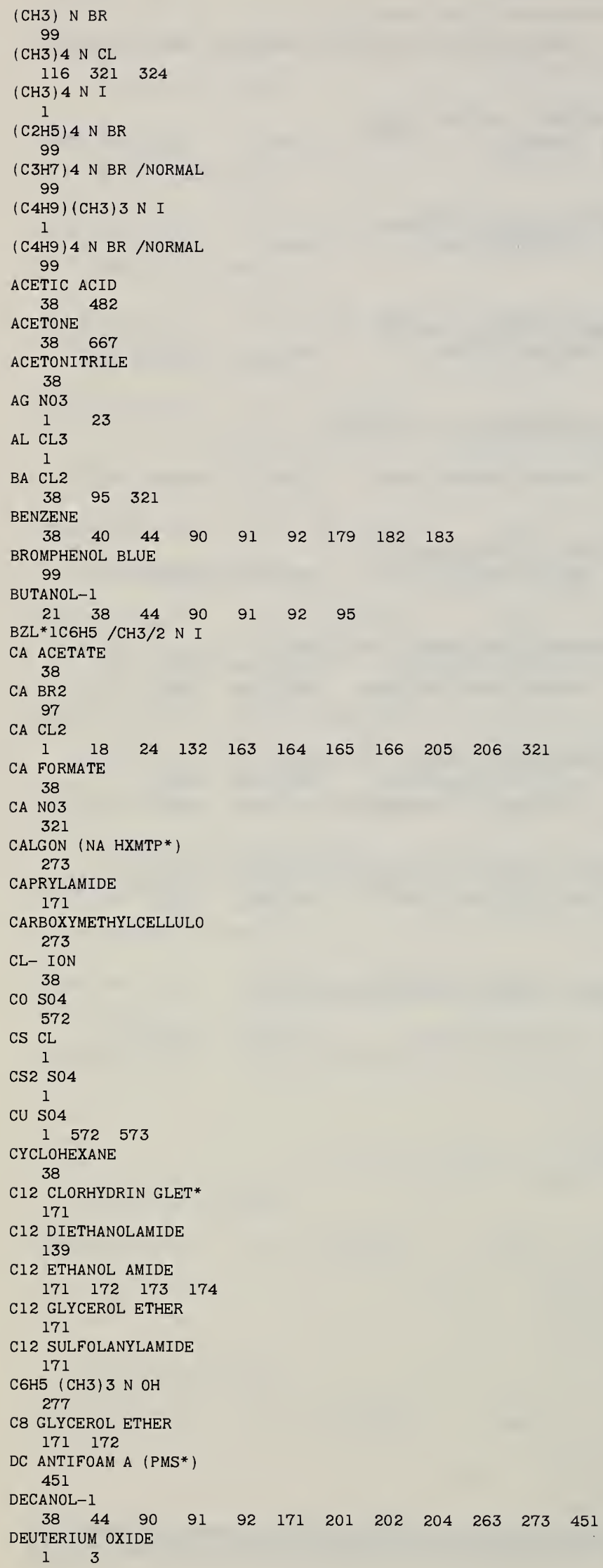


Index of Additives Giving Compound Numbers-Continued

MG S04

308568

N-C10 GLYCEROL ETHER

$\begin{array}{llllllll}1 & 171 & 172 & 175 & 176 & 177 & 178 & 179\end{array}$

N-C10 SULFOLANYL ETH

171

N-DECANE

$201202 \quad 204$

N-HEPTANE

1

N-HEXANE

$$
3891
$$

$\mathrm{N}-3 \mathrm{SOA}$ *

NA ACETATE

$\begin{array}{cc}38 & 482 \\ \text { NA } & \text { BR }\end{array}$

$\begin{array}{llllllll}1 & 41 & 97 & 98 & 102 & 127 & 128 & 129\end{array}$

$\begin{array}{llllllll}130 & 131 & 168 & 278 & 290 & 291 & 293 & 321\end{array}$

$\begin{array}{lll}346 & 347 & 643\end{array}$

NA BR03

$\begin{array}{lll}129 & 168 & 321\end{array}$

NA CITRATE

$\begin{array}{lllll}163 & 164 & 165 & 166 & 321\end{array}$

NA CL

$\begin{array}{llllllll}1 & 2 & 3 & 4 & 5 & 18 & 21 & 22\end{array}$

$\begin{array}{llllllll}24 & 38 & 41 & 42 & 91 & 93 & 95 & 97\end{array}$

$\begin{array}{llllllll}111 & 116 & 132 & 133 & 134 & 135 & 136 & 137\end{array}$

$\begin{array}{lllllllll}139 & 156 & 163 & 164 & 165 & 166 & 167 & 168\end{array}$

$\begin{array}{llllllll}169 & 179 & 182 & 205 & 206 & 258 & 273 & 274\end{array}$

$\begin{array}{lllllllll}278 & 290 & 295 & 311 & 321 & 324 & 329 & 337\end{array}$

$\begin{array}{lllllllll}345 & 418 & 419 & 432 & 433 & 434 & 459 & 462\end{array}$

NA CLO4

$\begin{array}{lll}492 & 588 & 634\end{array}$

3

NA CNS

$\begin{array}{lll}116 & 321 & 324\end{array}$

NA $F$

$1 \quad 130 \quad 321$

NA HCO2 FORMATE

$38 \quad 128$

NA $\mathrm{HCO} 3$

273

NA I

$\begin{array}{llll}1 & 168 & 278 & 321\end{array}$

NA 103

$\begin{array}{lll}127 & 168 & 32\end{array}$

NA NO3

$\begin{array}{cccccccc}1 & 23 & 24 & 38 & 131 & 273 & 278 & \\ \text { NA } & \text { OH } & & & & & & \\ 132 & 139 & 168 & 263 & 273 & 298 & 300 & 321\end{array}$

NA P-TOLUENE-SO3 295

NA PO4

$139 \quad 273$

NA SUCCINATE

38

$\mathrm{NA}+\mathrm{ION}$

1

NA14 P12037 POLY

$$
139
$$

NA2 $B 407$

273

$\mathrm{NA} 2 \mathrm{CO} 3$

$\begin{array}{lll}139 & 273 \quad 680\end{array}$

NA2 SI03 META $\begin{array}{llll}91 & 139 & 273 & 680\end{array}$

NA2 $\mathrm{SO} 4$

$\begin{array}{llllllll}1 & 18 & 91 & 116 & 132 & 163 & 164 & 165\end{array}$

$\begin{array}{llllllll}166 & 171 & 182 & 273 & 278 & 307 & 308 & 309\end{array}$

$\begin{array}{llll}321 & 324 & 573 & 680\end{array}$

NA2 S203 THIOSULF

$$
376
$$

NA4 P207 PYRO

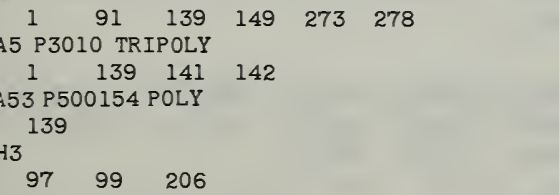

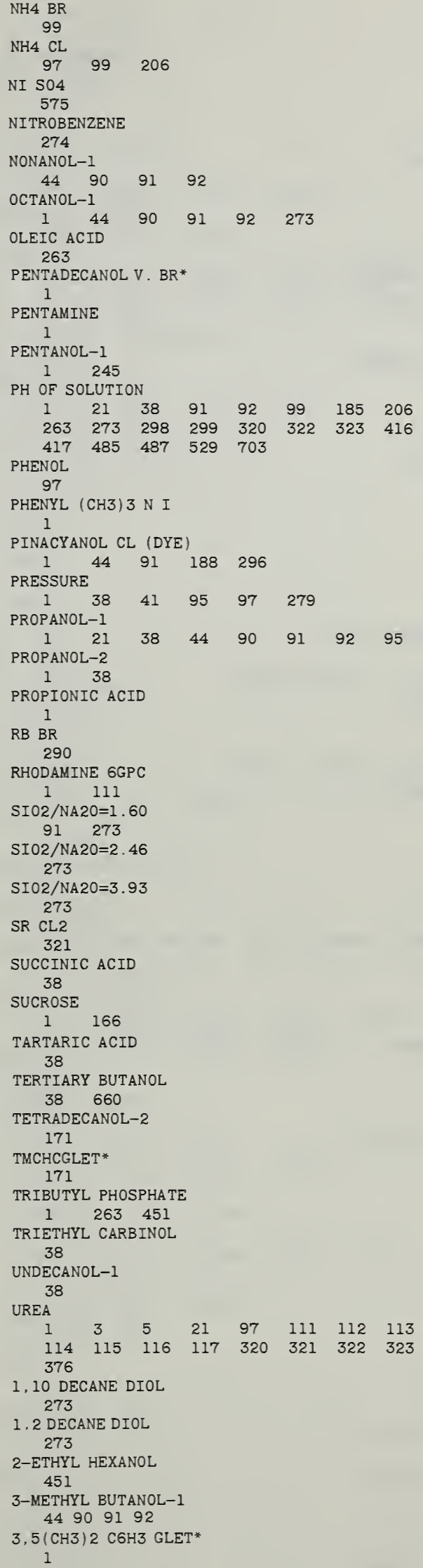


Index to References by Additives

(CH3) $4 \mathrm{~N} \mathrm{BR}$ 65012

(CH3) $4 \mathrm{~N} \mathrm{CL}$ 6201964020

(CH3) $4 \mathrm{~N} \mathrm{I}$ 53012

(C2H5) $4 \mathrm{~N} \mathrm{BR}$ 65012

(C3H7) $4 \mathrm{~N}$ BR /NORMAL 65012

(C4H9) $\mathrm{CH} 33 \mathrm{~N} \mathrm{I}$ 53012

(C4H9) $4 \mathrm{~N} \mathrm{BR} \mathrm{/NORMAL}$ 65012

ACETIC ACID 49008

ACETONE $48023 \quad 49017$

ACETONITRILE 48023

AG NO3 62006

AL CL3 51005

BA CL2 $47010 \quad 49008 \quad 62019$

BENZENE $\begin{array}{llll}48021 & 49006 & 50003 & 55008\end{array}$

BROMPHENOL BLUE 51008

BUTANOL-1 $49006 \quad 50008 \quad 55004 \quad 66012$

BZL* $\mathrm{C} 6 \mathrm{H} 5 / \mathrm{CH} 3 / 2 \mathrm{~N} \mathrm{I}$ 53012

CA ACETATE 49008

CA BR2 64043

CA CL2 $\begin{array}{llllll}51005 & 61008 & 62002 & 62011 & 62019 & 63008\end{array}$

CA FORMATE 49008

CA NO3 62019

CALGON (NA HXMTP*) 48024

CAPRYLAMIDE 57014

CARBOXYMETHYLCELLULO 48024

CL- ION 54013

Co 504 63034

CS CL 53012

CS2 504 53012

CU 504 63034

CYCLOHEXANE 48021

C12 CLORHYDRIN GLET* 57014

C12 DIETHANOLAMIDE 58008

Cl2 ETHANOL AMIDE 57014

C12 GLYCEROL ETHER 57014

C12 SULFOLANYLAMIDE 57014
C6H5 (CH3) $3 \mathrm{~N} \mathrm{OH}$ 48009

C8 GLYCEROL ETHER 57014

DC ANTIFOAM A (PMS*) 57031

DECANOL-1 $\begin{array}{lllllll}50008 & 55004 & 56018 & 57014 & 57031 & 60006 & 62009\end{array}$

DEUTERIUM OXIDE 66002

DIOXANE

$\begin{array}{lllll}46004 & 49006 & 65011 & 65013 & 65020\end{array}$

ETHANOL

$\begin{array}{lllllll}40004 & 46001 & 48023 & 49006 & 50008 & 55004 & 57013\end{array}$ $58007 \quad 65013 \quad 66012$

ETHYLENE GLYCOL $46004 \quad 49006$

FORMIC ACID 49008

GLYCEROL 3600246004

GUANIDINIUM CL 6501166012

GUANIDINIUM $\mathrm{CO}$ 66012

$\mathrm{H}$ BR

$64006 \quad 65012$

$\mathrm{H} \mathrm{CL}$

$\begin{array}{lllllll}36002 & 49008 & 54010 & 56014 & 57021 & 62005 & 62019\end{array}$ $64016 \quad 65011$

$\mathrm{H} \mathrm{CLO4}$

5001265019

$\mathrm{H} \mathrm{N03}$

$49008 \quad 50012 \quad 56014$

HEPTANOL-1 $5000855004 \quad 56018$

HEXANOL-1 $\begin{array}{llll}50008 & 55004 \quad 56018 \quad 59018\end{array}$

HEXYL AMMONIUM CL 48020

H2 504

6501965025

IONIC STRENGTH

65030

ISOC5 GLYCEROL ETHER 57014

$\mathrm{K} B R$

$\begin{array}{lllllll}48005 & 49001 & 55009 & 59010 & 59024 & 64043 & 66028\end{array}$

K CL

$\begin{array}{lllllll}47010 & 48005 & 49005 & 50008 & 50012 & 51005 & 52017\end{array}$

$\begin{array}{llllllll}53002 & 54010 & 56001 & 56002 & 62019 & 62037 & 65026\end{array}$

$\mathrm{K}$ CNS

66028

5001251005

K H SO4

K I 65025

$\begin{array}{llllll}48005 & 59024 & 60015 & 66006 & 66023 & 66028\end{array}$

$\mathrm{K} \mathrm{I03}$ 59024

$\mathrm{K} \mathrm{N03}$

$\begin{array}{lllllll}48005 & 48025 & 49005 & 50012 & 61026 & 62019 & 62037\end{array}$ 6401166023

$\mathrm{K} \mathrm{OH}$

$\begin{array}{lllllll}48004 & 48009 & 48011 & 48012 & 49005 & 50012 & 51004\end{array}$

$\begin{array}{llllllll}53006 & 54003 & 54005 & 54010 & 55004 & 56001 & 59008\end{array}$

$5901260002 \quad 61025 \quad 65012$

$\mathrm{K}+\mathrm{ION}$

$54013 \quad 55007$

K2 $\mathrm{S} 04$

$\begin{array}{llllll}47010 & 48005 & 49005 & 53012 & 59024 & 62037\end{array}$ 
Index to References by Additives-Continued

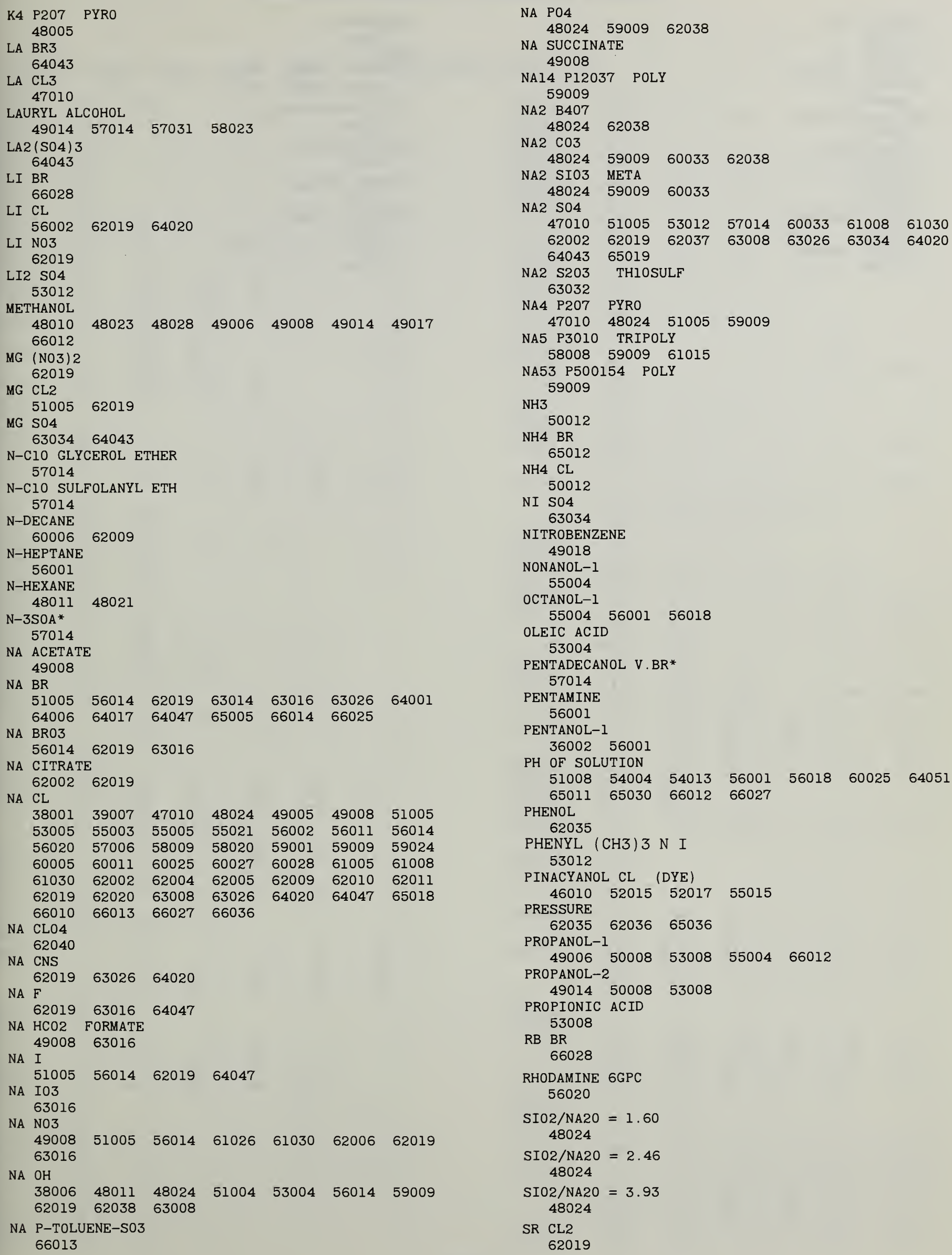

K4 P207 PYRO 48005

LA BR3 64043

LA CL3 47010

LAURYL ALCOHOL $\begin{array}{llll}49014 & 57014 & 57031 & 58023\end{array}$ 
Index to References by Additives-Continued

SUCCINIC ACID 49008

SUCROSE $63020 \quad 64034$

TARTARIC ACID 49008

TERTIARY BUTANOL 49014

TETRADECANOL-2 57014

TMCHCGLET* 57014

TRIBUTYL PHOSPHATE 57031

TRIETHYL CARBINOL 50008
UNDECANOL-1

50008

UREA

$47010 \quad 61016 \quad 62020$ $47010 \quad 48011 \quad 53010$

63032

64020

65011

1,10 DECANE DIOL 56018

1,2 DECANE DIOL 56018

2-ETHYL HEXANOL 57031

3-METHYL BUTANOL-1 55004

3,5 (CH3) 2 C6H3 GLET* 57014 
Index to Compounds by References

\begin{tabular}{|c|c|c|c|c|c|c|c|c|c|c|c|c|c|}
\hline 27001 & 273 & 298 & & & & & 48023 & 38 & & & & & \\
\hline 27002 & 43 & & & & & & 48024 & 91 & 273 & 300 & & & \\
\hline 29001 & 43 & & & & & & 48025 & 91 & & & & & \\
\hline 30001 & 44 & & & & & & 48027 & 38 & & & & & \\
\hline 32001 & 298 & & & & & & 48028 & 427 & 654 & 655 & 656 & 657 & \\
\hline 34001 & 274 & & & & & & 49001 & 95 & 97 & 98 & & & \\
\hline 35001 & 408 & & & & & & 49004 & 271 & 272 & & & & \\
\hline 35008 & 91 & & & & & & 49005 & 38 & 90 & 91 & 92 & 258 & 273 \\
\hline 36001 & 99 & 427 & & & & & & 305 & & & & & \\
\hline 36002 & 245 & 274 & & & & & 49006 & 91 & 95 & 182 & & & \\
\hline 38001 & 274 & & & & & & 49008 & 38 & 482 & & & & \\
\hline 38006 & 273 & 298 & 300 & 448 & & & 49009 & 500 & & & & & \\
\hline 39002 & 179 & 182 & 183 & 184 & 349 & & 49013 & 38 & 41 & 449 & 450 & & \\
\hline 39006 & 273 & 298 & 300 & 448 & & & 49014 & 38 & 660 & 661 & & & \\
\hline 39007 & 179 & 182 & 183 & & & & 49017 & 667 & & & & & \\
\hline \multirow[t]{2}{*}{39009} & 1 & 263 & 273 & 299 & 476 & 484 & 49018 & 274 & & & & & \\
\hline & 485 & 486 & 487 & & & & 50003 & 44 & 90 & 91 & 92 & 179 & 182 \\
\hline 39011 & 200 & 243 & 536 & 537 & & & & 183 & & & & & \\
\hline 40003 & 273 & 298 & 299 & 300 & & & 50008 & 38 & & & & & \\
\hline 40004 & 1 & & & & & & 50012 & 97 & 99 & 206 & & & \\
\hline 41003 & 299 & & & & & & 51001 & 99 & & & & & \\
\hline 41004 & 273 & & & & & 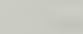 & 51003 & 181 & 339 & 340 & 341 & 342 & 343 \\
\hline 42002 & 37 & 38 & 39 & 186 & 187 & & & 344 & & & & & \\
\hline \multirow[t]{2}{*}{42003} & 1 & 2 & 3 & 260 & 261 & 262 & 51004 & 1 & 38 & 90 & 91 & 92 & 273 \\
\hline & 352 & & & & & & & 278 & 554 & 556 & 557 & 558 & 559 \\
\hline 42004 & 273 & 300 & & & & & & 560 & 561 & 562 & 563 & & \\
\hline 43001 & 49 & 51 & & & & & 51005 & 1 & 24 & 278 & & & \\
\hline 43003 & 93 & 95 & 97 & 99 & & & 51006 & 38 & & & & & \\
\hline 43004 & 287 & 288 & & & & & 51008 & 99 & & & & & \\
\hline 43006 & 286 & 304 & & & & & 51009 & 662 & 663 & 664 & 665 & 666 & \\
\hline 46001 & 38 & & & & & & 51010 & 376 & 458 & & & & \\
\hline 46002 & 91 & 92 & . & & & & 52001 & 41 & 42 & 265 & 270 & 279 & 399 \\
\hline 46004 & 38 & 95 & & & & & & 400 & 401 & 402 & 403 & 404 & 405 \\
\hline 46005 & 1 & 38 & 91 & 95 & 182 & & & 406 & 407 & & & & \\
\hline 46006 & 92 & 273 & 299 & & & & 52011 & 18 & 100 & & & & \\
\hline 46007 & 1 & 44 & 90 & 91 & 92 & & 52013 & 278 & 554 & 555 & & & \\
\hline 46008 & 285 & & & & & & 52015 & 44 & 90 & 91 & 92 & 188 & 296 \\
\hline 46010 & 5 & 91 & 92 & 305 & 494 & & & 297 & 350 & 351 & & & \\
\hline 46012 & 38 & 91 & & & & & 52016 & 1 & 91 & 111 & 273 & 634 & 635 \\
\hline 46015 & 1 & 90 & 91 & 92 & & & & 636 & 637 & 638 & 639 & & \\
\hline 46016 & 283 & & & & & & 52017 & 44 & 90 & 296 & 297 & 350 & 351 \\
\hline 46019 & 284 & 303 & & & & & 52018 & 681 & 682 & 683 & & & \\
\hline 47003 & 265 & 266 & 267 & 268 & 269 & 270 & 53001 & 263 & 264 & & & & \\
\hline \multirow[t]{2}{*}{47004} & 1 & 38 & 91 & 92 & 179 & 181 & 53002 & 287 & 288 & 353 & & & \\
\hline & 182 & 183 & 184 & & & & 53003 & 91 & 92 & 355 & 372 & 373 & 374 \\
\hline 47005 & 91 & 92 & 179 & 182 & & & & 375 & & & & & \\
\hline 47006 & 1 & 38 & 91 & 95 & 182 & & 53004 & 263 & & & & & \\
\hline 47007 & 206 & 276 & & & & & 53005 & 1 & 4 & 5 & 64 & 99 & 290 \\
\hline 47010 & 1 & 38 & 91 & 95 & 182 & & & 427 & & & & & \\
\hline 47011 & 259 & & & & & & 53006 & 1 & 38 & 90 & 259 & 273 & 284 \\
\hline 47013 & 92 & 273 & 299 & & & & & 299 & 476 & 487 & & & \\
\hline 48004 & 91 & 200 & & & & & 53007 & 275 & 279 & 354 & 356 & 357 & 358 \\
\hline \multirow[t]{3}{*}{48005} & $\begin{array}{r}1 \\
91\end{array}$ & $\begin{array}{l}37 \\
92\end{array}$ & $\begin{array}{l}38 \\
93\end{array}$ & $\begin{array}{l}39 \\
95\end{array}$ & 44 & $\begin{array}{l}90 \\
99\end{array}$ & & 359 & 360 & 361 & 362 & 363 & 364 \\
\hline & $\begin{array}{r}179 \\
179\end{array}$ & $\begin{array}{r}92 \\
181\end{array}$ & $\begin{array}{r}93 \\
182\end{array}$ & $\begin{array}{r}95 \\
183\end{array}$ & $\begin{array}{r}97 \\
184\end{array}$ & $\begin{array}{r}99 \\
185\end{array}$ & & 365 & 366 & 367 & 368 & 369 & 370 \\
\hline & 186 & 187 & 188 & 100 & 184 & 185 & & 371 & & & & & \\
\hline 48007 & 200 & & & & & & 53008 & 1 & 4 & & & & \\
\hline \multirow[t]{2}{*}{48008} & 4 & 16 & 17 & 72 & 73 & 77 & 53010 & 37 & 38 & 39 & 41 & 42 & 91 \\
\hline & 84 & 525 & 526 & 527 & . & & & 95 & 185 & 186 & 203 & 255 & 256 \\
\hline 48009 & 42 & 91 & 277 & 278 & 279 & & & 273 & 278 & 290 & 305 & 392 & 627 \\
\hline 48010 & 270 & 654 & 655 & 658 & 659 & & & 628 & 629 & 630 & 631 & 632 & 633 \\
\hline \multirow[t]{2}{*}{48011} & 91 & 92 & 99 & 263 & 273 & 274 & 53012 & 1 & & & & & \\
\hline & 275 & & & & & & 53015 & 139 & 279 & & & & \\
\hline 48012 & 255 & 256 & & & & & 54003 & 44 & 90 & 91 & 92 & 188 & 296 \\
\hline 48014 & 483 & & & & & & & 297 & & & & & \\
\hline \multirow[t]{2}{*}{48016} & 38 & 90 & 91 & 92 & 258 & 273 & 54004 & 1 & 43 & 44 & 179 & 188 & 200 \\
\hline & 299 & 305 & & & & & & 416 & 417 & 455 & 456 & 529 & 699 \\
\hline 48018 & 263 & & & & & & & 700 & 701 & 702 & 703 & 704 & 705 \\
\hline 48020 & 38 & 39 & & & & & & 706 & 707 & 708 & 709 & & \\
\hline 48021 & 38 & & & & & & 54005 & 44 & 90 & 91 & 92 & 188 & 296 \\
\hline 48022 & 1 & 200 & & & & & & 297 & & & & & \\
\hline
\end{tabular}


Index to Compounds by References-Continued

\begin{tabular}{|c|c|c|c|c|c|c|c|c|c|c|c|c|c|}
\hline 54006 & $\begin{array}{r}38 \\
564\end{array}$ & $\begin{array}{l}263 \\
565\end{array}$ & $\begin{array}{l}273 \\
566\end{array}$ & $\begin{array}{l}298 \\
567\end{array}$ & .300 & 448 & $\begin{array}{l}58017 \\
58020\end{array}$ & $\begin{array}{r}578 \\
1\end{array}$ & 579 & & & & \\
\hline 54008 & 651 & 652 & 653 & & & & 58021 & 409 & 410 & & & & \\
\hline 54010 & 1 & 416 & 417 & 428 & 430 & 455 & 58023 & 1 & & & & & \\
\hline 54013 & $\begin{array}{r}456 \\
1\end{array}$ & & & & & & 58028 & 586 & & & & & \\
\hline 55003 & 1 & 50 & 91 & 185 & & & 58031 & 717 & 492 & 493 & & & \\
\hline 55004 & 44 & 90 & 91 & 92 & & & $\begin{array}{l}59001 \\
59002\end{array}$ & $\begin{array}{r}1 \\
111\end{array}$ & 112 & & & & \\
\hline 55005 & 1 & & & & & & 59004 & 1 & 5 & 52 & 53 & 54 & 55 \\
\hline 55006 & 97 & 307 & & & & & & 56 & 57 & 58 & 59 & 60 & 61 \\
\hline 55007 & 668 & 669 & 670 & 671 & 672 & 673 & & 62 & 63 & 64 & 65 & & \\
\hline 55008 & 40 & & & & & & 59005 & 166 & & & & & \\
\hline \multirow[t]{2}{*}{55009} & 93 & 94 & 95 & 96 & 97 & 98 & 59006 & 163 & 164 & 165 & 166 & 431 & 432 \\
\hline & 99 & 100 & 101 & 102 & & & & 433 & 434 & 435 & 436 & 437 & 438 \\
\hline 55015 & 1 & & & & & & & 439 & 440 & 441 & 442 & 443 & 444 \\
\hline 55018 & 1 & & & & & & 59007 & 43 & & & & & \\
\hline \multirow[t]{2}{*}{55021} & 1 & 3 & 4 & 179 & 181 & 182 & 59008 & 44 & & & & 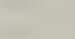 & \\
\hline & 183 & 340 & 341 & 342 & 345 & & 59009 & 139 & 149 & 273 & & & \\
\hline \multirow[t]{3}{*}{55028} & 492 & 501 & 502 & 503 & 504 & 505 & 59010 & 99 & 427 & & & & \\
\hline & 506 & 507 & 508 & 509 & 510 & 511 & 59012 & 44 & & & & & \\
\hline & 512 & 513 & 514 & & & & 59013 & 18 & 423 & 424 & & & \\
\hline \multirow[t]{2}{*}{56001} & 1 & 38 & 49 & 50 & 91 & 92 & 59015 & 1 & & & & & \\
\hline & 259 & 416 & 417 & & & & 59016 & 1 & 38 & 273 & & & \\
\hline 56002 & 1 & & & & & & 59017 & 409 & 410 & & & & \\
\hline 56003 & 420 & 421 & 422 & 425 & 426 & & 59018 & 1 & 3 & & & & \\
\hline 56005 & 171 & 301 & 302 & & & & 59020 & 166 & 206 & 538 & 539 & 540 & \\
\hline \multirow[t]{6}{*}{56006} & 1 & 2 & 4 & 5 & 15 & 16 & 59023 & 430 & & & & & \\
\hline & 17 & 64 & 66 & 68 & 69 & 70 & 59024 & 278 & 290 & 376 & 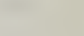 & - & \\
\hline & 71 & 72 & 73 & 74 & 75 & 76 & 59026 & 112 & 386 & 718 & 719 & 720 & \\
\hline & 77 & 78 & 79 & 80 & 81 & 82 & 60001 & 37 & 38 & & & & \\
\hline & 83 & 84 & 85 & 86 & 87 & 88 & 60002 & 44 & & & & & \\
\hline & $\begin{array}{l}89 \\
36\end{array}$ & & & & & & 60004 & 1 & & & & & \\
\hline 56008 & $\begin{array}{r}36 \\
194\end{array}$ & 189 & 190 & 191 & 192 & 193 & 60005 & 38 & & & & & \\
\hline 56010 & $\begin{array}{l}194 \\
416\end{array}$ & 195 & 196 & 197 & 198 & 199 & 60006 & 201 & 202 & & & & \\
\hline 56011 & $\begin{array}{r}416 \\
1\end{array}$ & 417 & 428 & 429 & 430 & & 60008 & 6 & 7 & 8 & 9 & 10 & 11 \\
\hline & & 2 & 3 & 4 & 5 & 295 & & 12 & 13 & 14 & & & \\
\hline 56014 & 156 & 168 & 169 & 335 & 336 & 337 & 60010 & 1 & 138 & 139 & 140 & 141 & 142 \\
\hline & 338 & & & & & ( & & 143 & 144 & 145 & 146 & 147 & 148 \\
\hline 56016 & 51 & & & & & & & 149 & 150 & 151 & 152 & 153 & 154 \\
\hline 56018 & 273 & & & & & & & 155 & 156 & 157 & 158 & 159 & 160 \\
\hline 56019 & 102 & 274 & 376 & 427 & 479 & 480 & & 161 & 162 & & & & \\
\hline & 481 & & & & & & 60011 & 1 & 97 & & & & \\
\hline 56020 & 1 & 111 & 634 & & & & 60012 & 377 & 488 & 489 & 490 & & \\
\hline 57004 & 37 & 38 & 39 & & & & 60015 & 376 & & & & & \\
\hline 57005 & 1 & 40 & & & & & 60017 & 445 & 446 & 447 & & & \\
\hline 57006 & 38 & 41 & 42 & & & & 60018 & 1 & 4 & 5 & 54 & 55 & 57 \\
\hline 57009 & 257 & 258 & 259 & 260 & 261 & 262 & & 58 & 59 & 61 & 64 & 113 & 541 \\
\hline 57010 & 451 & & & & 20 & W & & 542 & 543 & 544 & 545 & 546 & 547 \\
\hline 57011 & 1 & 2 & 3 & & & & & 548 & 549 & & & & \\
\hline 57012 & 45 & 46 & 47 & 48 & 49 & 50 & 60020 & 1 & & & & & \\
\hline & 51 & & & & & & 60021 & 1 & 260 & & 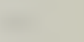 & & \\
\hline 57013 & 1 & & & & & & 60024 & 1 & 38 & 273 & & 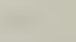 & \\
\hline 57014 & 1 & 67 & 171 & 172 & 173 & 174 & 60025 & 1 & 91 & 295 & 418 & 419 & \\
\hline & 175 & 176 & 177 & 178 & 179 & & 60026 & 459 & & & & & \\
\hline 57016 & 186 & & & & & & 60027 & 459 & & & & 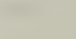 & \\
\hline 57017 & 416 & 417 & 428 & 429 & 430 & & 60028 & 462 & & & & & \\
\hline 57020 & $\begin{array}{l}578 \\
584\end{array}$ & $\begin{array}{l}579 \\
585\end{array}$ & 580 & 581 & 582 & 583 & 60029 & 1 & 24 & 568 & 569 & 570 & 571 \\
\hline 57021 & 38 & 000 & & & & & & 572 & 573 & 574 & & & \\
\hline 57022 & 1 & 2 & 3 & 4 & 5 & 64 & 60032 & 91 & 273 & & & & \\
\hline 57024 & 495 & & & & & & 60033 & 260 & 674 & 675 & 676 & 677 & 678 \\
\hline 57025 & 1 & & & & & & & 679 & 680 & & & & \\
\hline 57031 & 1 & 263 & 451 & & & & 60034 & 91 & 273 & & & & \\
\hline 58001 & 257 & 258 & 259 & 260 & 262 & & 61001 & 280 & 281 & & & & \\
\hline 58003 & 5 & 52 & 53 & 54 & 55 & 56 & 61002 & 41 & 42 & 203 & & & \\
\hline & 57 & 58 & 59 & & & & 61003 & 163 & 164 & 165 & 166 & 167 & $16 \varepsilon$ \\
\hline 58004 & 263 & & & & & & & 169 & & & & & \\
\hline 58007 & 263 & 264 & & & & & 61004 & 105 & 110 & 282 & & & \\
\hline 58008 & 1 & 139 & 263 & & & & 61005 & 3 & & & & & \\
\hline 58009 & 274 & & & & & & 61007 & 1 & 3 & 4 & 15 & 16 & 17 \\
\hline 58011 & 44 & 90 & 91 & 92 & 188 & 296 & 61008 & 18 & 19 & 20 & & & \\
\hline & 297 & 350 & 351 & 374 & 375 & 416 & 61014 & 1 & 99 & 139 & 144 & 145 & 149 \\
\hline & 417 & 428 & 429 & 430 & 452 & 453 & & 159 & 167 & 263 & 310 & 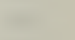 & \\
\hline & 454 & 455 & & & & & 61015 & 1 & $9 \varsigma$ & 139 & 141 & 142 & 149 \\
\hline 58012 & 1 & 97 & & & & & & 310 & & & & & \\
\hline
\end{tabular}


Index to Compounds by References-Continued

\begin{tabular}{|c|c|c|c|c|c|c|c|c|c|c|c|c|c|}
\hline 61016 & 97 & & & & & & 64004 & 170 & 721 & & & & \\
\hline 61017 & 1 & 3 & & & & & 64006 & 21 & 22 & 293 & & & \\
\hline 61025 & 90 & & & & & & 64007 & 280 & & & & & \\
\hline 61026 & 40 & 1 & & & & & 64009 & 108 & 110 & 282 & 331 & 377 & 378 \\
\hline 61027 & 99 & 451 & & & & & & 379 & 380 & & & & \\
\hline 61029 & 693 & 694 & 695 & 696 & 697 & 698 & 64010 & 139 & 206 & & & & \\
\hline 61030 & 273 & & & & & & 64011 & 456 & & & & & \\
\hline \multirow[t]{4}{*}{61031} & 1 & 2 & 3 & 4 & 5 & 64 & 64012 & 179 & & & & & \\
\hline & $27 \overline{3}$ & 298 & 299 & 300 & 448 & 501 & 64014 & 207 & 208 & 209 & 210 & 211 & 212 \\
\hline & 502 & 503 & 505 & 506 & 507 & 508 & & 213 & 214 & 215 & 216 & & \\
\hline & 509 & & & & & & 64016 & 21 & 22 & 251 & 252 & 254 & \\
\hline 62001 & 166 & 168 & & & & & 64017 & 1 & 97 & & & & \\
\hline \multirow[t]{2}{*}{62002} & 163 & 164 & 165 & 166 & 167 & 168 & 64020 & 1 & 3 & 5 & 111 & 112 & 113 \\
\hline & 169 & 432 & 433 & 434 & 435 & 436 & & 114 & 115 & 116 & 117 & & \\
\hline 62004 & 2 & 3 & 93 & 95 & & & 64023 & 105 & 108 & 282 & 289 & & \\
\hline 62005 & 21 & 22 & 251 & 252 & 253 & & 64024 & 468 & 469 & 470 & 471 & 472 & 473 \\
\hline 62006 & 1 & 23 & 24 & & & & & 474 & 475 & & & & \\
\hline \multirow[t]{3}{*}{62008} & 7 & 25 & 26 & 27 & 28 & 29 & 64025 & 1 & & & & & \\
\hline & 30 & 31 & 32 & 33 & 34 & 35 & 64027 & 294 & & & & & \\
\hline & 36 & & & & & & 64032 & 1 & & & & & \\
\hline 62009 & 1 & 204 & & & & & 64034 & 1 & & & & & \\
\hline 62010 & 1 & 205 & & & & & 64035 & 411 & 412 & 413 & 414 & 415 & \\
\hline 62011 & 205 & 206 & & & & & 64037 & 645 & 646 & 647 & 648 & 650 & 651 \\
\hline 62015 & 103 & 294 & 380 & 381 & & & 64043 & 95 & 97 & 306 & 307 & 308 & 309 \\
\hline \multirow[t]{4}{*}{62019} & 115 & 116 & 312 & 313 & 314 & 315 & 64047 & 1 & 2 & 3 & 295 & 311 & \\
\hline & 316 & 317 & 318 & 319 & 320 & 321 & 64049 & 294 & 393 & 394 & 395 & 396 & 397 \\
\hline & 322 & 323 & 324 & 325 & 326 & 327 & & 398 & & & & & \\
\hline & 328 & 329 & & & & & 64050 & 290 & & & & & \\
\hline \multirow{2}{*}{62020} & 115 & 116 & 320 & 321 & 322 & 323 & 64051 & 1 & 5 & 263 & 273 & 298 & 299 \\
\hline & 324 & 326 & 327 & 328 & 329 & & & 485 & 487 & & & & \\
\hline 62023 & 495 & 496 & 497 & 498 & 499 & & 65003 & 119 & 120 & 121 & 122 & 123 & \\
\hline 62027 & 282 & 330 & 331 & 332 & 333 & 334 & 65005 & 346 & 347 & 348 & & & \\
\hline 62035 & 95 & 97 & & & & & 65007 & 179 & & & & & \\
\hline 62036 & 1 & & & & & & 65011 & 320 & 322 & 323 & & & \\
\hline 62037 & 91 & & & & & & 65012 & 99 & & & & & \\
\hline 62038 & 273 & & & & & & 65013 & 166 & & & & & \\
\hline 62040 & 3 & & & & & & 65018 & 1 & 3 & & & & \\
\hline 63001 & 1 & 3 & 4 & & & & 65019 & 460 & 491 & 528 & & & \\
\hline 63008 & 132 & 133 & 134 & 135 & 136 & 137 & 65020 & 1 & 4 & 5 & & & \\
\hline 63009 & 118 & 132 & & & & & 65022 & 1 & 2 & 4 & & & \\
\hline 63010 & 132 & 133 & 134 & & & & 65024 & 273 & 298 & 299 & 476 & & \\
\hline 63012 & 133 & 134 & & & & & 65025 & 529 & 530 & 531 & 532 & 533 & 534 \\
\hline \multirow[t]{6}{*}{63013} & 1 & 4 & 5 & 9 & 10 & 64 & 65026 & 41 & 278 & 399 & & & \\
\hline & 179 & 183 & 184 & 200 & 229 & 230 & 65028 & 1 & 91 & 99 & 596 & & \\
\hline & 231 & 232 & 233 & 234 & 235 & 236 & 65030 & 1 & 206 & & & & \\
\hline & 237 & 238 & 239 & 240 & 241 & 242 & 65031 & 38 & & & & & \\
\hline & 243 & 244 & 245 & 246 & 247 & 248 & 65036 & 38 & 41 & 279 & & & \\
\hline & 249 & 250 & & & & & 65037 & 427 & & & & & \\
\hline 63014 & 346 & & & & & & 66001 & 1 & 115 & 116 & 325 & 327 & \\
\hline 63015 & 118 & 119 & 120 & 121 & 122 & 123 & 66002 & 1 & 3 & & & & \\
\hline & 124 & 125 & 126 & & & & 66003 & $59 \overline{7}$ & 598 & 599 & 600 & 601 & \\
\hline 63016 & 41 & 97 & 127 & 128 & 129 & 130 & 66006 & 290 & 376 & & & & \\
\hline & 131 & & & & & & 66007 & 1 & & & & & \\
\hline 63017 & 207 & 208 & 209 & 210 & 211 & 212 & 66010 & 1 & & & & & \\
\hline & 213 & 214 & 215 & 216 & 217 & 218 & 66011 & 51 & 274 & 275 & 358 & 477 & 478 \\
\hline & $\begin{array}{l}219 \\
225\end{array}$ & $\begin{array}{l}220 \\
226\end{array}$ & $\begin{array}{l}221 \\
227\end{array}$ & $\begin{array}{l}222 \\
228\end{array}$ & 223 & 224 & 66012 & 21 & & & & & \\
\hline 63020 & 166 & & & & & & 66013 & 587 & 588 & 589 & 590 & 591 & 592 \\
\hline 63021 & 108 & 110 & 377 & 378 & 380 & 381 & & 593 & 594 & 595 & & & \\
\hline 63026 & 1 & 97 & 113 & 114 & 115 & 116 & 66014 & 287 & 288 & 346 & 347 & 385 & 640 \\
\hline & 117 & & & & & & & 641 & 642 & 643 & 644 & & \\
\hline 63030 & 112 & 280 & 382 & 383 & 384 & 385 & 66015 & 684 & 685 & 686 & 687 & 688 & 689 \\
\hline & 386 & 387 & 388 & 389 & 390 & 391 & & 690 & 691 & 692 & & & \\
\hline 63032 & 376 & & & & & & 66018 & 1 & 97 & 98 & 99 & 459 & \\
\hline 63034 & 1 & 568 & 572 & 573 & 575 & 576 & 66019 & 461 & & & & & \\
\hline 63037 & $\begin{array}{l}577 \\
457\end{array}$ & & & & & & 66020 & 105 & & & & & \\
\hline 64001 & 98 & 102 & 290 & 291 & 292 & & 66021 & 139 & 463 & 464 & 465 & 466 & 467 \\
\hline 64002 & 26 & 189 & 190 & 191 & 194 & 602 & 66022 & 310 & 336 & 515 & 516 & 517 & 518 \\
\hline & 603 & 604 & 605 & 606 & 607 & 608 & & 519 & 520 & 521 & 522 & 523 & 524 \\
\hline & 609 & 610 & 611 & 612 & 613 & 614 & 66023 & 550 & 551 & 552 & 553 & & \\
\hline & $\begin{array}{l}615 \\
621\end{array}$ & $\begin{array}{l}616 \\
622\end{array}$ & $\begin{array}{l}617 \\
623\end{array}$ & 618 & $\begin{array}{l}619 \\
625\end{array}$ & $\begin{array}{l}620 \\
626\end{array}$ & 66025 & 97 & 99 & 115 & 116 & 117 & 325 \\
\hline 64003 & 103 & 104 & 105 & $\begin{array}{l}624 \\
106\end{array}$ & $\begin{array}{l}625 \\
107\end{array}$ & $\begin{array}{l}020 \\
108\end{array}$ & & 327 & 535 & & & & \\
\hline & 109 & 110 & & & & & 66027 & 21 & & & & & \\
\hline
\end{tabular}


Index to Compounds by References - Continued

716

66038
66039
66040 710

331
66040
4
711
251

21251

$710 \quad 711$
712

252

713
102

254

714
290

587

715 


\section{Index to References by Methods}

AVER COND BEGINING MAXIM 53008

AVER SP EQUIV COND $53008 \quad 58007 \quad 61007$

DEBYE PLT LIGHT SCATTER $\begin{array}{lllll}49001 & 53002 & 55005 & 63016 & 64007\end{array}$

DENSITY

$\begin{array}{llllll}35008 & 47011 & 60005 & 63009 & 66020 & 66040\end{array}$

DIFFUSION COEFFICIENT 61025

ELECTROMOTIVE FORCE

$\begin{array}{lllllll}47013 & 48007 & 48025 & 57021 & 59016 & 60021 & 63001\end{array}$ 6401264032

EMF ALONG CONC GRADIENT 59015

EQUIV COND MAX BEGINING $49014 \quad 49018 \quad 51009 \quad 58003$

EQUIV COND IST DEVIATION $\begin{array}{lllllll}36002 & 40004 & 43001 & 48010 & 49014 & 49017 & 53005\end{array}$ 55018

EQUIV CONDCTNCE GRAPH

$\begin{array}{lllllll}27001 & 32001 & 34001 & 35001 & 36001 & 39011 & 40004\end{array}$ $\begin{array}{lllllll}41003 & 42002 & 42004 & 43001 & 43003 & 47003 & 48009\end{array}$ $\begin{array}{llllllll}48010 & 48012 & 48014 & 48020 & 48021 & 48023 & 48028\end{array}$ $\begin{array}{llllllll}49004 & 49008 & 49009 & 49013 & 49014 & 49017 & 49018\end{array}$ $\begin{array}{llllllll}51009 & 53004 & 53005 & 53008 & 55005 & 55006 & 55018\end{array}$ $\begin{array}{llllllll}56006 & 56008 & 57013 & 57017 & 57025 & 58001 & 58003\end{array}$ $\begin{array}{lllllll}58007 & 59002 & 59026 & 61005 & 61016 & 61017 & 62040\end{array}$ 6403465020

EQUIV CONDCTNCE MAXIMUM 4901456006

FLOCCULATION RATE 66037

FOAMING POWER 59017

FOTOMTR SOLUBLZTN AZBZ $38001 \quad 49005$

FOTOMTR SOLUBLZTN DMYL 60027

FOTOMTR SOLUBLZTN OROT $\begin{array}{lllllll}48016 & 48024 & 49005 & 51010 & 55005 & 59009 & 60010\end{array}$ $61015 \quad 64010 \quad 66036$

FOTOMTR SOLUBLZTN PDMAB $\begin{array}{lllll}46006 & 47013 \quad 48016 \quad 49005 \quad 59009\end{array}$

FOTOMTR SOLUBLZTN SDN 4 $\begin{array}{llllll}57020 & 58028 & 63008 & 63010 & 63037 & 64024\end{array}$

FOTOMTR SOLUBLZTN YLOB 6402466027

FOTOMTR SOLUBLZTN 2NPA 55007

FOTOMTR SPCTR CHNG BZP4 6200165013

FOTOMTR SPCTR CHNG ERTS 57020

FOTOMTR SPCTR CHNG I2 $\begin{array}{lllllll}59006 & 59020 & 60012 & 60026 & 60027 & 62001 & 62002\end{array}$ 630156400966023

FOTOMTR SPCTR CHNGE BRPB $51008 \quad 63015$

FOTOMTR SPCTR CHNंGE EOSN 59024

FOTOMTR SPCTR CHNGE INPX 47004

FOTOMTR SPCTR CHNGE PNCN $\begin{array}{lllllll}46002 & 46010 & 47004 & 50003 & 52015 & 53012 & 55007\end{array}$ $\begin{array}{llllllll}55015 & 56008 & 56018 & 57020 & 57025 & 58007 & 58008\end{array}$ $58017 \quad 58028 \quad 60033$

FOTOMTR SPCTR CHNGE RHD6 $56020 \quad 59026 \quad 65030$

FREEZING POINT $\begin{array}{lllllll}27002 & 39011 & 43006 & 46008 & 46016 & 46019 & 47007\end{array}$ $48011-59023$
GRAPH DATA NOT RETRIEVED $\begin{array}{lllllll}46007 & 53005 & 54004 & 54005 & 61027 & 61030 & 62037\end{array}$ $62038 \quad 63034$

HEAT OF DILUTION $64016 \quad 66012$

INTERFACIAL TENSION LOGMI $\begin{array}{lllllll}38006 & 57022 & 58012 & 60011 & 60025 & 62004 & 62023\end{array}$ INTERFACIAL TNSN UNSPEC 56019

KRAFFT POINT SOLUBILITY $\begin{array}{llll}39002 & 49004 & 56019 & 57012 \quad 61029\end{array}$

METHOD NOT CITED

$\begin{array}{lllllll}49006 & 51003 & 53003 & 54004 & 56001 & 61014 & 62037\end{array}$ $\begin{array}{lllllll}63020 & 63034 & 64035 & 64043 & 66013 & 66014 & 66040\end{array}$

MICELLAR SPECTRAL CHANGE $51010 \quad 63032 \quad 66006$

PARTIAL VOLUME 2900130001

PH OR HYDROLYSIS $27001 \quad 39006 \quad 41003 \quad 60001$

POLAROGRAPHIC MAXIMUM 5001266023

QUESTIONABLE CRITERION $\begin{array}{llllllll}39006 & 39009 & 41004 & 42003 & 51001 & 52013 & 53001\end{array}$ $\begin{array}{llllllll}53015 & 54010 & 57010 & 57017 & 58011 & 60008 & 60017\end{array}$ $\begin{array}{lllllll}61029 & 62002 & 62023 & 63013 & 63015 & 63017 & 64043\end{array}$ 65012

REACTN RATE SULUBILIZATE 65030

REFRACTIVE INDEX

$\begin{array}{llllllll}46012 & 47004 & 47005 & 48005 & 53010 & 56011 & 57005\end{array}$ $\begin{array}{lllllll}63015 & 63021 & 63037 & 64047 & 65003 & 65012 & 66019\end{array}$

SEE CMPD NMBR IN ADDITV

$\begin{array}{lllllll}46015 & 48005 & 48020 & 51005 & 53003 & 53005 & 54003\end{array}$ $\begin{array}{llllllll}54005 & 54010 & 56014 & 58017 & 61002 & 61007 & 61008\end{array}$ $\begin{array}{llllllll}61017 & 62010 & 63001 & 63017 & 64010 & 66001 & 66025\end{array}$

SOLUBLZTN TOLUENE 6003260034

SPECFC CONDCTNCE EQUATNS $5701159012 \quad 66002$

SPECFC CONDCTNCE GRAPH

$\begin{array}{llllllll}36001 & 36002 & 39007 & 39011 & 42003 & 43003 & 43004\end{array}$ $\begin{array}{lllllll}46001 & 46010 & 48004 & 48027 & 51003 & 51006 & 53004\end{array}$ $\begin{array}{llllllll}54006 & 55005 & 55008 & 56005 & 56006 & 56020 & 57004\end{array}$ $\begin{array}{llllllll}57012 & 57016 & 57025 & 57031 & 58001 & 58004 & 58007\end{array}$ $\begin{array}{lllllll}58008 & 59002 & 59024 & 60020 & 60028 & 61005 & 61017\end{array}$ $\begin{array}{lllllll}62006 & 62035 & 62036 & 63030 & 64050 & 64051 & 65018\end{array}$ $\begin{array}{lllllll}65024 & 65031 & 65036 & 65037 & 66007 & 66030 & 66038\end{array}$

SPECIFIC HEAT 60002

STREAMING CURRENT 66011

SUMMARIZING EQN ONLY 62002

SURFACE TENSION LOG PLOT $\begin{array}{lllllll}38006 & 48022 & 52011 & 54010 & 54013 & 56014 & 56016\end{array}$ $\begin{array}{llllllll}56018 & 57009 & 57012 & 57022 & 57024 & 58003 & 58031\end{array}$ $\begin{array}{llllllll}59001 & 59013 & 59020 & 60004 & 60012 & 60027 & 60029\end{array}$ $\begin{array}{lllllll}61004 & 61008 & 61026 & 62015 & 62019 & 62020 & 62023\end{array}$ $\begin{array}{lllllll}62027 & 63008 & 63009 & 63012 & 63013 & 63014 & 63015\end{array}$ $\begin{array}{lllllll}63017 & 63026 & 63034 & 63037 & 64001 & 64003 & 64009\end{array}$ $\begin{array}{lllllll}64010 & 64014 & 64020 & 64023 & 64024 & 64037 & 64047\end{array}$ $\begin{array}{lllllll}64049 & 65003 & 65005 & 65007 & 65011 & 65019 & 65024\end{array}$ $\begin{array}{llllllll}65025 & 65026 & 66001 & 66007 & 66013 & 66015 & 66019\end{array}$ $66021 \quad 66022 \quad 66025 \quad 66039$ SURFACE TENSION MINIMUM $50012 \quad 54010 \quad 57012 \quad 63017 \quad 65024$ SURFACE TENSION UNSPEC $\begin{array}{lllllll}54004 & 55007 & 56008 & 58008 & 60018 & 60026 & 62001\end{array}$ $62002 \quad 64002 \quad 66018 \quad 66028$

SURFACE TNSN LINEAR PLOT $\begin{array}{llllll}50012 & 57025 & 58020 & 59017 & 61001 & 66007\end{array}$ 
Index to References by Methods-Continued

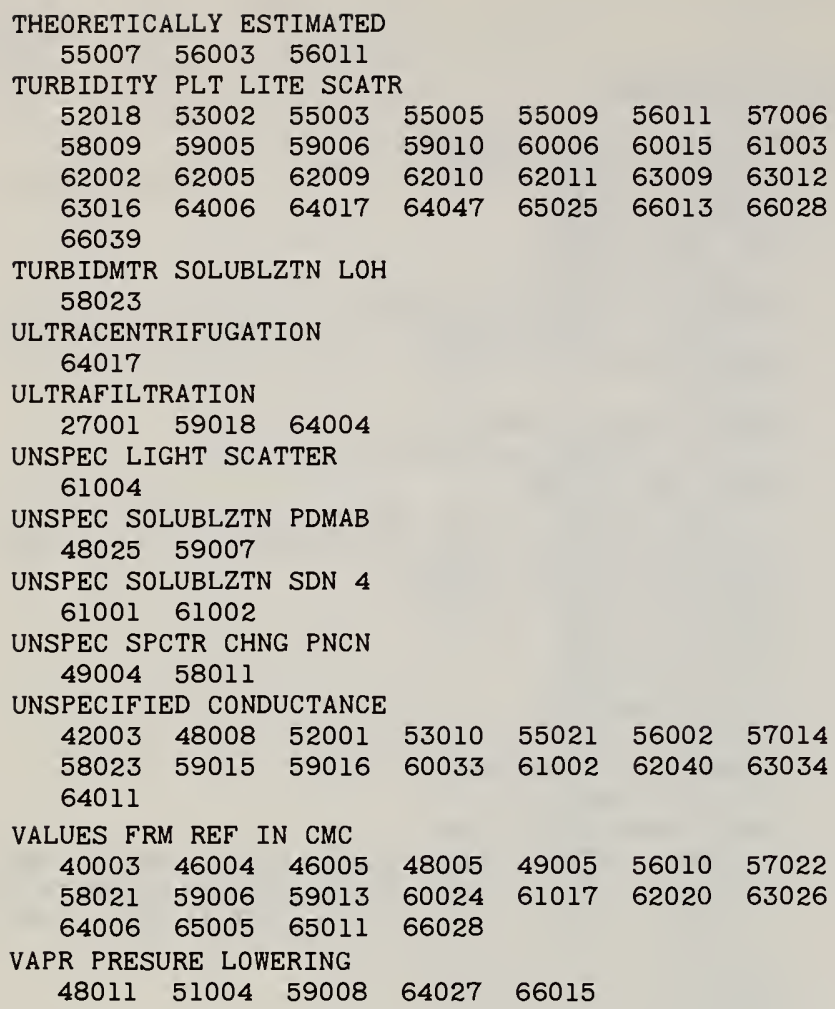

VELOCITY OF SOUND $6502265028 \quad 66010$

VISCOSITY

$\begin{array}{llll}39009 & 47011 & 57025 & 64025\end{array}$

VISCOSITY MINIMUM

$53006 \quad 54008$

VISUAL FLUOR CHNGE RHDG 56002

VISUAL SPCTR CHNGE

VISUAL SPCTR CHNGE DCFL 46004

VISUAL SPCTR CHNGE EOSN $47006 \quad 51005$

VISUAL SPCTR CHNGE FL 47006

VISUAL SPCTR CHNGE INPX $\begin{array}{llll}47006 & 47010 & 50008 & 53007\end{array}$

VISUAL SPCTR CHNGE PNCN $\begin{array}{lllllll}46015 & 47006 & 47010 & 48008 & 48024 & 50003 & 51003\end{array}$ $\begin{array}{llllllll}51005 & 52015 & 52016 & 52017 & 54003 & 54005 & 55004\end{array}$ $\begin{array}{llllllll}55007 & 56003 & 56008 & 56016 & 57014 & 58003 & 58008\end{array}$ $\begin{array}{lllllll}59004 & 60008 & 60029 & 60033 & 60034 & 61030 & 61031\end{array}$ $62008 \quad 63013 \quad 64002 \quad 66003$

VISUAL SPCTR CHNGE RHD6 $\begin{array}{lllllll}47006 & 48011 & 55028 & 56016 & 60021 & 60028 & 60029\end{array}$ 60033

VISUAL SPCTR CHNGE SKYB $47006 \quad 47010 \quad 49006 \quad 51010$

WIEN EFFECT 48018

X-RAY DIFFRACTION 39009 
Author Index

\begin{tabular}{|c|c|c|c|c|c|}
\hline Author & Journal & Vol. & Pg. & Year & $\begin{array}{c}\text { Reter- } \\
\text { ence } \\
\text { No. }\end{array}$ \\
\hline \multirow[t]{2}{*}{ ABBOTT AD } & J AM CHEM SOC & 61 & 549 & 1939 & 39007 \\
\hline & J PHYS CHEM & 59 & 1193 & 1955 & 55012 \\
\hline ABU-HAMDIYY .M & J PHYS CHEM & 69 & 1466 & 1965 & 65019 \\
\hline \multirow[t]{3}{*}{ ADAM NK } & TRANS FARADAY SOC & 32 & 653 & 1936 & 36004 \\
\hline & TRANS FARADAY SOC & 34 & 758 & 1938 & 38007 \\
\hline & TRANS FARADAY SOC & 42 & 523 & 1946 & 46021 \\
\hline ADCOCK WA & J AM CHEM SOC & 71 & 2835 & 1949 & 49015 \\
\hline ADDERSON JE & $\mathrm{J}$ COLLOID SCI & 19 & 495 & 1964 & 64050 \\
\hline \multirow[t]{3}{*}{ ADDISON CC } & TRANS FARADAY SOC & 33 & 851 & 1937 & 37003 \\
\hline & TRANS FARADAY SOC & 34 & 372 & 1938 & 38006 \\
\hline & $\mathrm{J}$ CHEM SOC & & 03229 & 1956 & 56019 \\
\hline ALEXANDER AE & J PHYS CHEM & 66 & 1839 & 1962 & 62035 \\
\hline \multirow[t]{8}{*}{ ANACKER EW } & J PHYS COLLOID CHEM & 55 & 644 & 1951 & 51001 \\
\hline & J COLLOID SCI & 8 & 402 & 1953 & 53002 \\
\hline & $J$ PHYS CHEM & 62 & 41 & 1958 & 58009 \\
\hline & $\mathrm{J}$ PHYS CHEM & 63 & 1022 & 1959 & 59011 \\
\hline & $\mathrm{J}$ CHEM EDUC & 37 & 36 & 1960 & 60005 \\
\hline & J PHYS CHEM & 67 & 1713 & 1963 & 63016 \\
\hline & $J$ PHYS CHEM & 68 & 81 & 1964 & 64017 \\
\hline & J PHYS CHEM & 68 & 3490 & 1964 & 64018 \\
\hline ANDRUS RW & $\mathrm{J}$ PHYS CHEM & 69 & 2357 & 1965 & 65030 \\
\hline ANGELESCU E. & REV CHIM AC.REP POP.ROUM. & 6 & 309 & 1961 & 61029 \\
\hline ARAI $H$ & KOGYO KAGAKU ZASSHI & 68 & 1090 & 1965 & 65017 \\
\hline ARISHI $S$ & J SCI HIROSHIMA UNIV, SER A-II & 22 & 57 & 1958 & 58023 \\
\hline \multirow[t]{3}{*}{ ARKIN L } & $\mathrm{J}$ AM CHEM SOC & 70 & 3965 & 1948 & 48017 \\
\hline & J COLLOID SCI & 4 & 537 & 1949 & 49012 \\
\hline & $\mathrm{J}$ AM CHEM SOC & 73 & 4574 & 1951 & 51007 \\
\hline ARRINGTON CH & J PHYS CHEM & 57 & 247 & 1953 & 53003 \\
\hline \multirow[t]{5}{*}{ ASCOLI F } & NATURE & 184 & 1482 & 1959 & 59014 \\
\hline & J MOL BIOL & 3 & 202 & 1961 & 61023 \\
\hline & J PHYS CHEM & 65 & 1991 & 1961 & 61024 \\
\hline & PONTIF ACAD SCIENTIAR. SCRIPTA VAR. & 22 & $/ 12 / 1$ & 1962 & 62021 \\
\hline & BIOPOLYMERS & 1 & 353 & 1963 & 63025 \\
\hline ATLAS SM & J PHYS CHEM & 66 & 1326 & 1962 & 62020 \\
\hline \multirow[t]{3}{*}{ AULT WC } & J AM OIL CHEMISTS SOC & 37 & 679 & 1960 & 60008 \\
\hline & J AM OIL CHEMISTS SOC & 39 & 55 & 1962 & 62008 \\
\hline & J AM OIL CHEMISTS SOC & 41 & 205 & 1964 & 64002 \\
\hline \multirow{2}{*}{$\begin{array}{l}\text { BAILEY JH } \\
\text { BAIR EJ }\end{array}$} & J COLLOID SCI & 8 & 385 & 1953 & 53007 \\
\hline & J AM CHEM SOC & 73 & 799 & 1951 & 51013 \\
\hline & J AM CHEM SOC & 73 & 1129 & 1951 & 51014 \\
\hline BALMBRA RR & $\begin{array}{l}\text { TRANS FARADAY SOC } \\
\text { TRANS FARADAY SOC }\end{array}$ & 58 & $\begin{array}{r}1661 \\
979\end{array}$ & 1962 & 62013 \\
\hline BARBULESCU EM & $\begin{array}{l}\text { TRANS FARADAY SOC } \\
\text { REV CHIM AC.REP POP.ROUM. }\end{array}$ & 60 & 979 & 1964 & 64023 \\
\hline BARR EA & $\begin{array}{l}\text { REV CHIM AC.REP POP.ROUM. } \\
\text { J AM OIL CHEMISTS SOC }\end{array}$ & $\begin{array}{r}6 \\
43\end{array}$ & 309 & 1961 & 61029 \\
\hline \multirow[t]{2}{*}{ BASCOM WD } & J COLLOID SCI & $\begin{array}{l}43 \\
13\end{array}$ & 569 & $\begin{array}{l}1966 \\
1958\end{array}$ & $\begin{array}{l}66003 \\
58024\end{array}$ \\
\hline & WORLD PETROL CONGR, PROC & v 6 & 18 & 1959 & 59021 \\
\hline BAYLEY CH & CAN J CHEM ENGR & F28 & 213 & 1950 & 50010 \\
\hline BECHER P & J COLLOID SCI & 14 & 519 & 1959 & 59005 \\
\hline & $J$ PHYS CHEM & 63 & 1675 & 1959 & 59006 \\
\hline 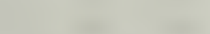 & $\mathrm{J}$ PHYS CHEM & 64 & 1221 & 1960 & 60003 \\
\hline & J COLLOID SCI & 16 & 49 & 1961 & 61003 \\
\hline - & J PHYS CHEM & 66 & 374 & 1962 & 62001 \\
\hline 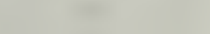 & J COLLOID SCI & 17 & 325 & 1962 & 62002 \\
\hline & J COLLOID SCI & 18 & 196 & 1963 & 63020 \\
\hline & $\mathrm{J}$ COLLOID SCI & 18 & 665 & 1963 & 63022 \\
\hline & PROC INTERN CONGR SURFACE ACTIVITY & $4 \mathrm{TH}$ & & 1964 & 64005 \\
\hline & $J$ PHYS CHEM & 68 & 3511 & 1964 & 64008 \\
\hline & $\mathrm{J}$ COLLOID SCI & 20 & 728 & 1965 & 65013 \\
\hline BECKETT AH & J PHARM PHARMACOL & 15 & 422 & 1963 & 63015 \\
\hline BEISWANGE. JPG & J AM OIL CHEMISTS SOC & 43 & 435 & 1966 & 66022 \\
\hline BENJAMIN L & J PHYS CHEM & 68 & 3575 & 1964 & 64016 \\
\hline & $\mathrm{J}$ COLLOID INTERFACE SCI & 22 & 386 & 1966 & 66012 \\
\hline & $J$ PHYS CHEM & 70 & 3790 & 1966 & 66040 \\
\hline BENSON GC & $\mathrm{J}$ PHYS CHEM & 61 & 593 & 1957 & 57002 \\
\hline & CAN J CHEM & 35 & 986 & 1957 & 57011 \\
\hline & J COLLOID SCI & 13 & 584 & 1958 & 58005 \\
\hline & TRANS FARADAY SOC & 54 & 1638 & 1958 & 58006 \\
\hline & CAN J CHEM & 37 & 2083 & 1959 & 59007 \\
\hline & CAN J CHEM & 37 & 2086 & 1959 & 59008 \\
\hline & TRANS FARADAY SOC & 55 & 1025 & 1959 & 59012 \\
\hline & $\mathrm{J}$ PHYS CHEM & 64 & 599 & 1960 & 60002 \\
\hline BENTON D & TRANS FARADAY SOC & 62 & 3244 & 1966 & 66038 \\
\hline BERRY RWH & $\mathrm{J}$ CHEM SOC & & 2264 & 1964 & 64028 \\
\hline
\end{tabular}




\begin{tabular}{|c|c|c|c|c|c|}
\hline Author & Journal & Vol. & Pg. & Year & $\begin{array}{c}\text { Refer- } \\
\text { ence } \\
\text { No. }\end{array}$ \\
\hline \multirow[t]{4}{*}{ BESPYATOV MP } & MASLOB ZHIR PROM & 26 & 24 & 1960 & 60030 \\
\hline & MASLOB ZHIR PROM & 26 & 26 & 1960 & 60031 \\
\hline & MASLOB ZHIR PROM & 28 & 20 & 1962 & 62039 \\
\hline & MASLOB ZHIR PROM & 29 & 19 & 1963 & 63038 \\
\hline BETZ MD & $\mathrm{J}$ AM CHEM SOC & 57 & 1905 & 1935 & 35007 \\
\hline \multirow{5}{*}{ BISTLINE RG } & $J$ PHYS CHEM & 62 & 1083 & 1958 & 58003 \\
\hline & $J$ AM OIL CHEMISTS SOC & 36 & 241 & 1959 & 59004 \\
\hline & $J$ AM OIL CHEMISTS SOC & 37 & 679 & 1960 & 60008 \\
\hline & $\mathrm{J}$ AM OIL CHEMISTS SOC & 39 & 55 & 1962 & 62008 \\
\hline & $J$ AM OIL CHEMISTS SOC & 40 & 538 & 1963 & 63013 \\
\hline BISWAS AK & $\mathrm{J}$ PHYS CHEM & 64 & 1 & 1960 & 60028 \\
\hline BJAASTAD SG & $J$ PHARM SCI & 54 & 1529 & 1965 & 65027 \\
\hline BOLAM TR & NATURE & 167 & 195 & 1951 & 51012 \\
\hline BOLDUAN OEA & $J$ PHYS CHEM & 47 & 94 & 1943 & 43008 \\
\hline \multirow[t]{14}{*}{ BOTRE C } & NATURE & 184 & 1482 & 1959 & 59014 \\
\hline & TRANS FARADAY SOC & 55 & 1975 & 1959 & 59015 \\
\hline & $J$ PHYS CHEM & 63 & 650 & 1959 & 59016 \\
\hline & PROC INTERN CONGR SURFACE ACTIVITY & 3RD & 302 & 1960 & 60024 \\
\hline & J MOL BIOL & 3 & 202 & 1961 & 61023 \\
\hline & $J$ PHYS CHEM & 65 & 1991 & 1961 & 61024 \\
\hline & ANN CHIM (ROME) & 52 & 1199 & 1962 & 62017 \\
\hline & PONTIF ACAD SCIENTIAR. SCRIPTA VAR. & 22 & $/ 12 / 1$ & 1962 & 62021 \\
\hline & J PHARM SCI & 52 & 1011 & 1963 & 63024 \\
\hline & BIOPOLYMERS & 1 & 353 & 1963 & 63025 \\
\hline & BIOCHEM BIOPHYS ACTA & 88 & 415 & 1964 & 64031 \\
\hline & $J$ PHYS CHEM & 68 & 3624 & 1964 & 64032 \\
\hline & RIC SCI & 6 & 71 & 1964 & 64033 \\
\hline & J PHARM SCI & 54 & 919 & 1965 & 65010 \\
\hline \multirow[t]{2}{*}{ BOYD GE } & $J$ AM CHEM SOC & 64 & 2067 & 1942 & 42008 \\
\hline & $J$ AM CHEM SOC & 64 & 498 & 1942 & 42009 \\
\hline \multirow[t]{5}{*}{ BRADY AP } & $J$ AM CHEM SOC & 65 & 2072 & 1943 & 43006 \\
\hline & $\mathrm{J}$ COLLOID SCI & 3 & 511 & 1948 & 48004 \\
\hline & J COLLOID SCI & 3 & 425 & 1948 & 48009 \\
\hline & J PHYS COLLOID CHEM & 3 & 57 & 1948 & 48022 \\
\hline & J PHYS COLLOID CHEM & 55 & 311 & 1951 & 51004 \\
\hline BRAMFITT TH & $J$ PHYS CHEM & 61 & 1261 & 1957 & 57031 \\
\hline BRASS PD & $J$ AM CHEM SOC & 76 & 4703 & 1954 & 54013 \\
\hline BROCKLEHURST $P$ & $\mathrm{~J}$ CHEM SOC & & 2264 & 1964 & 64028 \\
\hline BROOME FK & $\mathrm{J}$ AM CHEM SOC & 71 & 2145 & 1949 & 49008 \\
\hline & J AM CHEM SOC & 71 & 671 & 1949 & 49013 \\
\hline BROWN AS & J PHYS CHEM & 56 & 701 & 1952 & 52013 \\
\hline BROWN EL & J AM OIL CHEMISTS SOC & 38 & 361 & 1961 & 61019 \\
\hline BROWN GL & J AM CHEM SOC & 69 & 1835 & 1947 & 47001 \\
\hline & J AM CHEM SOC & 71 & 95 & 1949 & 49014 \\
\hline BROWNING J & TRANS FARADAY SOC & 48 & 209 & 1952 & 52011 \\
\hline BRUFANI M & J PHARM SCI & 54 & 919 & 1965 & 65010 \\
\hline BRUNING W & J AM CHEM SOC & 83 & 4865 & 1961 & 61016 \\
\hline BRUSHMILLER $\mathrm{J}$ & $J$ PHYS CHEM & 70 & 2909 & 1966 & 66039 \\
\hline BUJAKE JE & TRANS FARADAY SOC & 61 & 190 & 1965 & 65007 \\
\hline BUNBURY HM & TRANS FARADAY SOC & 31 & 208 & 1935 & 35003 \\
\hline BURY CR & PHIL MAG & 4 & 841 & 1927 & 27002 \\
\hline & $\mathrm{J}$ CHEM SOC & & 679 & 1929 & 29001 \\
\hline & $\mathrm{J}$ CHEM SOC & 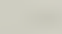 & 2263 & 1930 & 30001 \\
\hline & $\mathrm{J}$ CHEM SOC & & 626 & 1935 & 35008 \\
\hline & TRANS FARADAY SOC & 48 & 209 & 1952 & 52011 \\
\hline CADLE RD & $\mathrm{J}$ AM CHEM SOC & 73 & 5411 & 1951 & 51003 \\
\hline CADY $\mathrm{GH}$ & $\mathrm{J}$ PHYS CHEM & 63 & 757 & 1959 & 59023 \\
\hline CAMPBELL AN & CAN J CHEM & 40 & 839 & 1962 & 62034 \\
\hline & CAN J CHEM & 43 & 1004 & 1964 & 64026 \\
\hline & CAN J CHEM & 43 & 1729 & 1965 & 65024 \\
\hline CARDWELL PH & J COLLOID INTERFACE SCI & 22 & 430 & 1966 & 66011 \\
\hline & & 19 & 201 & 1964 & 64009 \\
\hline CARR CW & J PHYS COLLOID CHEM & 51 & 636 & 1947 & 47013 \\
\hline & J POLYMER SCI & 5 & 201 & 1950 & 50002 \\
\hline & J POLYMER SCI & 5 & 191 & 1950 & 50005 \\
\hline & $J$ PHYS CHEM & 60 & 1245 & 1956 & 56001 \\
\hline CARRINGTON RAG & $\mathrm{J}$ CHEM SOC & & 1701 & 1957 & 57007 \\
\hline CARTAN $F$ & $J$ CHEM EDUC & 37 & 36 & 1960 & 60005 \\
\hline CELLA JA & $\mathrm{J}$ AM CHEM SOC & 74 & 2061 & 1952 & 52001 \\
\hline CHALLIS RA & $\mathrm{J}$ COLLOID SCI & 19 & 201 & 1964 & 64009 \\
\hline CHAND $\mathrm{P}$ & J AM OIL CHEMISTS SOC & 43 & 446 & 1966 & 66023 \\
\hline CLIFTON NK & J COLLOID SCI & 14 & 519 & 1959 & 59005 \\
\hline CLUNIE JS & TRANS FARADAY SOC & 58 & 1661 & 1962 & 62013 \\
\hline & TRANS FARADAY SOC & 60 & 979 & 1964 & 64023 \\
\hline COHEN I & J COLLOID SCI & 9 & 243 & 1954 & 54011 \\
\hline & $J$ PHYS CHEM & 65 & 1781 & 1961 & 61027 \\
\hline & $J$ PHYS CHEM & 65 & 1774 & 1961 & 61028 \\
\hline & J COLLOID SCI & 20 & 732 & 1965 & 65012 \\
\hline COLE RH & J AM CHEM SOC & 71 & 2835 & 1949 & 49015 \\
\hline COLICHMAN EL & J AM CHEM SOC & 72 & 4036 & 1950 & 50012 \\
\hline & J AM CHEM SOC & 73 & 3385 & 1951 & 51008 \\
\hline
\end{tabular}




\begin{tabular}{|c|c|c|c|c|c|}
\hline Author & Jesurnal & Vol. & $P_{q}$. & Year & $\begin{array}{l}\text { Refer- } \\
\text { ence } \\
\text { No. }\end{array}$ \\
\hline COLL $\mathrm{H}$ & $\mathrm{J}$ COLLOID SCI & 17 & 220 & 1962 & 62003 \\
\hline COLLIE B & TRANS FARADAY SOC & 32 & 795 & 1936 & 36001 \\
\hline CONSOLE L & ANALES ASOC QUIM ARGENT & 53 & 39 & 1965 & 65034 \\
\hline COON RI & J PHYS CHEM & 57 & 923 & 1953 & 53014 \\
\hline \multirow[t]{15}{*}{ CORKILL JM } & TRANS FARADAY SOC & 57 & 1627 & 1961 & 61004 \\
\hline & TRANS FARADAY SOC & 58 & 206 & 1962 & 62006 \\
\hline & TRANS FARADAY SOC & 58 & 1661 & 1962 & 62013 \\
\hline & PROC ROY SOC & 273 & 84 & 1963 & 63014 \\
\hline & J PHYS CHEM & 67 & 935 & 1963 & 63018 \\
\hline & J COLLOID SCI & 18 & 401 & 1963 & 63019 \\
\hline & TRANS FARADAY SOC & 60 & 202 & 1964 & 64003 \\
\hline & TRANS FARADAY SOC & 60 & 986 & 1964 & 64012 \\
\hline & TRANS FARADAY SOC & 60 & 979 & 1964 & 64023 \\
\hline & TRANS FARADAY SOC & 60 & 996 & 1964 & 64027 \\
\hline & TRANS FARADAY SOC & 61 & 589 & 1965 & 65004 \\
\hline & TRANS FARADAY SOC & 61 & 583 & 1965 & 65005 \\
\hline & TRANS FARADAY SOC & 62 & 994 & 1966 & 66014 \\
\hline & TRANS FARADAY SOC & 62 & 987 & 1966 & 66015 \\
\hline & TRANS FARADAY SOC & 62 & 979 & 1966 & 66016 \\
\hline CORRIN ML & $J$ CHEM PHYS & 14 & 216 & 1946 & 46002 \\
\hline & $\mathrm{J}$ CHEM PHYS & 14 & 640 & 1946 & 46004 \\
\hline & $\mathrm{J}$ CHEM PHYS & 14 & 641 & 1946 & 46005 \\
\hline & $\mathrm{J}$ CHEM PHYS & 14 & 480 & 1946 & 46010 \\
\hline & $J$ AM CHEM SOC & 68 & 220 & 1946 & 46013 \\
\hline & $\mathrm{J}$ COLLOID SCI & 1 & 105 & 1946 & 46014 \\
\hline & $\mathrm{J}$ COLLOID SCI & 1 & 469 & 1946 & 46015 \\
\hline & $J$ AM CHEM SOC & 69 & 679 & 1947 & 47006 \\
\hline & $J$ AM CHEM SOC & 69 & 683 & 1947 & 47010 \\
\hline & $\mathrm{J}$ COLLOID SCI & 3 & 333 & 1948 & 48013 \\
\hline & $\mathrm{J}$ PHYS COLLOID CHEM & 53 & 1350 & 1949 & 49006 \\
\hline & J PHYS COLLOID CHEM & 54 & 271 & 1950 & 50008 \\
\hline & J COLLOID SCI & 6 & 576 & 1951 & 51010 \\
\hline COURCHENE $W$ & $J$ PHYS CHEM & 70 & 2909 & 1966 & 66039 \\
\hline CRESCENZI V & NATURE & 184 & 1482 & 1959 & 59014 \\
\hline & PROC INTERN CONGR SURFACE ACTIVITY & 3RD & 302 & 1960 & 60024 \\
\hline CRESCENZI VL & TRANS FARADAY SOC & 55 & 1975 & 1959 & 59015 \\
\hline & $J$ PHYS CHEM & 63 & 650 & 1959 & 59016 \\
\hline CROOK EH & $J$ PHYS CHEM & 67 & 1987 & 1963 & 63017 \\
\hline & $J$ AM OIL CHEMISTS SOC & 41 & 231 & 1964 & 64013 \\
\hline & $J$ PHYS CHEM & 68 & 3592 & 1964 & 64014 \\
\hline & $\mathrm{J}$ COLLOID SCI & 20 & 191 & 1965 & 65001 \\
\hline CUSHMAN A & $\mathrm{J}$ COLLOID SCI & 3 & 425 & 1948 & 48009 \\
\hline CZERNIATSKI M & ROCZN CHEM & 39 & 1059 & 1965 & 65031 \\
\hline & ROCZN CHEM & 39 & 1469 & 1965 & 65032 \\
\hline & ROCZN CHEM & 39 & 1275 & 1965 & 65037 \\
\hline & ROCZN CHEM & 40 & 1265 & 1966 & 66029 \\
\hline & ROCZN CHEM & 40 & 1935 & 1966 & 66030 \\
\hline DAGGETT HM JR & $J$ AM CHEM SOC & 73 & 799 & 1951 & 51013 \\
\hline DAINTON FS & ANN REP PROGR CHEM (CH. S. LONDON) & 45 & 5 & 1948 & 48001 \\
\hline DANIELSSON I & ACTA CHEM SCAND & 6 & 440 & 1952 & 52007 \\
\hline DAVIES DG & $\mathrm{J}$ CHEM SOC & & 2263 & 1930 & 30001 \\
\hline DE MARTIIS $F$ & $J$ PHYS CHEM & 68 & 3624 & 1964 & 64032 \\
\hline DEBYE $P$ & $\mathrm{~J}$ COLLOID SCI & 3 & 407 & 1948 & 48006 \\
\hline & ANN N Y ACAD SCI & 51 & 575 & 1949 & 49001 \\
\hline & $\mathrm{J}$ PHYS COLLOID CHEM & 53 & 1 & 1949 & 49002 \\
\hline & AN. R. SOC ESPAN FIS QUIM (MADRID) & 46 & 335 & 1950 & 50001 \\
\hline & J PHYS COLLOID CHEM & 55 & 644 & 1951 & 51001 \\
\hline & J COLLOID SCI & 17 & 220 & 1962 & 62003 \\
\hline DEL VECCHIO AJ & $\mathrm{J}$ PHYS CHEM & 68 & 3511 & 1964 & 64008 \\
\hline DEMCHENKO LG & UKR KHIM ZH & 28 & 611 & 1962 & 62038 \\
\hline DEMCHENKO PA & DOKLADY AKAD NAUK SSSR & 131 & 120 & 1960 & 60032 \\
\hline & MASLOB ZHIR PROM & 26 & 26 & 1960 & 60034 \\
\hline & KOLLOID ZH & 23 & 528 & 1961 & 61030 \\
\hline & DOPOV. AKAD NAUK UKR RSR & & 928 & 1961 & 61031 \\
\hline & UKR KHIM ZH & 27 & 322 & 1961 & 61032 \\
\hline & MASLOB ZHIR PROM & 27 & 19 & 1961 & 61033 \\
\hline & UKR KHIM ZH & 28 & 46 & 1962 & 62037 \\
\hline & UKR KHIM ZH & 28 & 611 & 1962 & 62038 \\
\hline DERVICHIAN DG & PROC INTERN CONGR SURFACE ACTIVITY & 3RD & 182 & 1960 & 60019 \\
\hline DITMARSCH $R$ & NATURE & 208 & 889 & 1965 & 65029 \\
\hline DIXON JK & J COLLOID SCI & 12 & 452 & 1957 & 57009 \\
\hline & J COLLOID SCI & 13 & 411 & 1958 & 58001 \\
\hline DOAN AS & J PHYS CHEM & 61 & 371 & 1957 & 57003 \\
\hline DOERR IL & $J$ PHYS CHEM & 68 & 3494 & 1964 & 64007 \\
\hline DONBROW M & J PHARM PHARMACOL & 15 & 825 & 1963 & 63021 \\
\hline & J PHARM PHARMACOL & 15 & 317 & 1963 & 63030 \\
\hline & J PHARM PHARMACOL & 18 & $92 \mathrm{~S}$ & 1966 & 66019 \\
\hline DORST T & KONINKI NED AKAD RETEN. PROC SER B & 59 & 190 & 1956 & 56002 \\
\hline DREGER EE & IND ENG CHEM & 36 & 610 & 1944 & 44001 \\
\hline
\end{tabular}




\begin{tabular}{|c|c|c|c|c|c|}
\hline Author & Journal & Vol. & Py. & Year & $\begin{array}{l}\text { Refer- } \\
\text { ence } \\
\text { No. }\end{array}$ \\
\hline \multirow[t]{2}{*}{ DU BROW PL } & $J$ AM CHEM SOC & 69 & 2095 & 1947 & 47003 \\
\hline & J AM CHEM SOC & 70 & 977 & 1948 & 48014 \\
\hline DULIN CI & J PHYS CHEM & 62 & 1390 & 1958 & 58013 \\
\hline DUMANSKII AV & DOKLADY AKAD NAUK SSSR & 131 & 120 & 1960 & 60032 \\
\hline \multirow[t]{2}{*}{ DUNNING HN } & J PHYS CHEM & 59 & 362 & 1955 & 55020 \\
\hline & J PHYS CHEM & 60 & 657 & 1956 & 56014 \\
\hline DYE WB & J AM CHEM SOC & 61 & 3210 & 1939 & 39011 \\
\hline \multirow{11}{*}{ EGGENBERGER DN } & J AM CHEM SOC & 69 & 2095 & 1947 & 47003 \\
\hline & J AM CHEM SOC & 70 & 977 & 1948 & 48014 \\
\hline & J AM CHEM SOC & 70 & 980 & 1948 & 48019 \\
\hline & J AM CHEM SOC & 70 & 2918 & 1948 & 48020 \\
\hline & $\mathrm{J}$ AM CHEM SOC & 70 & 983 & 1948 & 48021 \\
\hline & J PHYS COLLOID CHEM & 52 & 1494 & 1948 & 48023 \\
\hline & J AM CHEM SOC & 70 & 436 & 1948 & 48027 \\
\hline & J AM CHEM SOC & 71 & 2145 & 1949 & 49008 \\
\hline & J AM CHEM SOC & 71 & 672 & 1949 & 49009 \\
\hline & J AM CHEM SOC & 73 & 3353 & 1951 & 51006 \\
\hline & J AM CHEM SOC & 74 & 2061 & 1952 & 52001 \\
\hline EIKREM H & ACTA CHEM SCAND & 17 & 111 & 1963 & 63033 \\
\hline EIRICH FR & J PHYS CHEM & 66 & 1326 & 1962 & 62020 \\
\hline \multirow[t]{20}{*}{ EKWALL $P$} & ACTA ACAD ABOENSIS, MATH PHYS & 4 & 1 & 1927 & 27001 \\
\hline & KOLLOID-Z & 45 & 291 & 1928 & 28001 \\
\hline & KOLLOID-Z & 161 & 195 & 1932 & 32001 \\
\hline & ACTA ACAD ABOENSIS, MATH PHYS & 7 & 3 & 1933 & 33001 \\
\hline & ACTA ACAD ABOENSIS, MATH PHYS & 7 & 3 & 1933 & 33002 \\
\hline & KOLLOID-Z & 77 & 320 & 1936 & 36003 \\
\hline & KOLLOID-Z & 80 & 77 & 1937 & 37001 \\
\hline & KOLLOID-Z & 84 & 284 & 1938 & 38002 \\
\hline & FINSKA KEMISTSAMFUNDETS MEDD & 1 & 8 & 1939 & 39004 \\
\hline & KOLLOID-Z & 92 & 141 & 1940 & 40002 \\
\hline & TEK FOREN FINLAND FORH & 10 & 1 & 1940 & 40003 \\
\hline & MEDD. ABO AKAD.FYS. KEM.IN. & SP NO & 3 & 1941 & 41002 \\
\hline & KOLLOID-Z & 97 & 71 & 1941 & 41003 \\
\hline & KOLLOID-Z & 94 & 42 & 1941 & 41004 \\
\hline & KOLLOID-Z & 101 & 135 & 1942 & 42004 \\
\hline & FINSKA KEMISTSAMFUNDETS MEDD & 25 & 257 & 1943 & 43005 \\
\hline & ACTA CHEM SCAND & 6 & 441 & 1952 & 52006 \\
\hline & ACTA CHEM SCAND & 6 & 440 & 1952 & 52007 \\
\hline & ACTA CHEM SCAND & 17 & 111 & 1963 & 63033 \\
\hline & ACTA CHEM SCAND & 19 & 573 & 1965 & 65023 \\
\hline ELDER ME & $\mathrm{J}$ COLLOID SCI & 9 & 382 & 1954 & 54006 \\
\hline \multirow{6}{*}{ ELWORTHY PH } & J PHARM PHARMACOL & 012 & 293 & 1960 & 60027 \\
\hline & J PHARM PHARMACOL & 14 & 100 & 1962 & 62027 \\
\hline & KOLLOID-Z & 195 & 23 & 1964 & 64049 \\
\hline & J PHARM PHARMACOL & 17 & 65 & 1965 & 65016 \\
\hline & J COLLOID SCI & 21 & 331 & 1966 & 66007 \\
\hline & KOLLOID-Z Z POLYMERE & 208 & 157 & 1966 & 66008 \\
\hline EMERSON MF & J PHYS CHEM & 69 & 3718 & 1965 & 65006 \\
\hline EPSTEIN MB & J PHYS CHEM & 67 & 2075 & 1963 & 63001 \\
\hline ERIKSON JA & J COLLOID SCI & 10 & 71 & 1955 & 55025 \\
\hline \multirow[t]{2}{*}{ EVANS HC } & $\mathrm{J}$ CHEM SOC & & 579 & 1956 & 56006 \\
\hline & $\mathrm{J}$ CHEM SOC & & 1701 & 1957 & 57007 \\
\hline EVERS EC & J AM CHEM SOC & 68 & 1137 & 1946 & 46018 \\
\hline & J AM CHEM SOC & 69 & 1835 & 1947 & 47001 \\
\hline & J AM CHEM SOC & 70 & 3049 & 1948 & 48028 \\
\hline EXNER ML & NATURWISSENSCHAFTEN & 35 & 344 & 1948 & 48018 \\
\hline EYRING H & J PHYS CHEM & 60 & 890 & 1956 & 56016 \\
\hline EZAKI H & BULL CHEM SOC JAPAN & 32 & 624 & 1959 & 59017 \\
\hline FAVA A & J PHYS CHEM & 60 & 890 & 1956 & 56016 \\
\hline FEW A & $\mathrm{J}$ CHEM SOC & & 1712 & 1958 & 58031 \\
\hline FINEMAN MN & J PHYS COLLOID CHEM & 52 & 881 & 1948 & 48011 \\
\hline FLOCKHART BD & J COLLOID SCI & 8 & 105 & 1953 & 53004 \\
\hline & J COLLOID SCI & 8 & 424 & 1953 & 53008 \\
\hline & J COLLOID SCI & 12 & 557 & 1957 & 57013 \\
\hline & $\mathrm{J}$ COLLOID SCI & 16 & 484 & 1961 & 61007 \\
\hline FLORENCE AT & KOLLOID-Z & 195 & 23 & 1964 & 64049 \\
\hline & KOLLOID-Z Z POLYMERE & 208 & 157 & 1966 & 66008 \\
\hline & J PHARM PHARMACOL & 18 & 384 & 1966 & 66020 \\
\hline FORD WPJ & J COLLOID INTERFACE SCI & 21 & 522 & 1966 & 66028 \\
\hline FORDYCE DB & J PHYS CHEM & 67 & 1987 & 1963 & 63017 \\
\hline & J AM OIL CHEMISTS SOC & 41 & 231 & 1964 & 64013 \\
\hline & $\mathrm{J}$ PHYS CHEM & 68 & 3592 & 1964 & 64014 \\
\hline & J COLLOID SCI & 20 & 191 & 1965 & 65001 \\
\hline FOSTER JF & J PHYS CHEM & 57 & 628 & 1953 & 53015 \\
\hline FOWKES FM & J PHYS CHEM & 61 & 1062 & 1957 & 57014 \\
\hline & J PHYS CHEM & 62 & 159 & 1958 & 58029 \\
\hline FOX CJ & J AM CHEM SOC & 73 & 2323 & 1951 & 51011 \\
\hline FRIES BA & IND ENG CHEM & 44 & 1636 & 1952 & 52014 \\
\hline FROTSCHER H & KOLLOID-BEIH. & 45 & 303 & 1937 & 37004 \\
\hline
\end{tabular}




\begin{tabular}{|c|c|c|c|c|c|}
\hline Author & Journal & Vol. & $P_{y}$. & Year & $\begin{array}{l}\text { Refer- } \\
\text { ence } \\
\text { No. }\end{array}$ \\
\hline \multirow[t]{3}{*}{ FUERSTENAU DT } & $\mathrm{J}$ ELECTROCHEM SOC & 106 & 336 & 1959 & 59019 \\
\hline & $\mathrm{J}$ PHYS CHEM & 68 & 3562 & 1964 & 64035 \\
\hline & TRANS SME AIME & & 321 & 1964 & 64038 \\
\hline FUJITA H & J SCI HIROSHIMA UNIV, SER A-II & 28 & 41 & 1964 & 64036 \\
\hline FULLER GW & J COLLOID SCI & 10 & 403 & 1955 & 55022 \\
\hline FURMIDGE CGL & $\mathrm{J}$ CHEM SOC & & 03229 & 1956 & 56019 \\
\hline GERSHMAN JW & J PHYS CHEM & 61 & 581 & 1957 & 57012 \\
\hline GETTY R & $\mathrm{J}$ PHYS COLLOID CHEM & 52 & 774 & 1948 & 48024 \\
\hline GHOSE HM & $\mathrm{J}$ PHYS CHEM & 67 & 1713 & 1963 & 63016 \\
\hline GIESE E & KOLLOID $Z$ & 73 & 276 & 1935 & 35002 \\
\hline GIESKES JMTM & CAN J CHEM & 43 & 1004 & 1964 & 64026 \\
\hline GILBERT AH & $\mathrm{J}$ COLLOID SCI & 20 & 464 & 1965 & 65011 \\
\hline GILBY A & $\mathrm{J}$ CHEM SOC & & 1712 & 1958 & 58031 \\
\hline \multirow[t]{6}{*}{ GINN ME } & J PHYS CHEM & 62 & 1554 & 1958 & 58008 \\
\hline & $J$ AM OIL CHEMISTS SOC & 36 & 332 & 1959 & 59009 \\
\hline & $J$ AM OIL CHEMISTS SOC & 37 & 183 & 1960 & 60010 \\
\hline & $\mathrm{J}$ AM OIL CHEMISTS SOC & 38 & 605 & 1961 & 61014 \\
\hline & J AM OIL CHEMISTS SOC & 38 & 138 & 1961 & 61015 \\
\hline & $\mathrm{J}$ AM OIL CHEMISTS SOC & 38 & 361 & 1961 & 61019 \\
\hline \multirow[t]{6}{*}{ GODDARD ED } & TRANS FARADAY SOC & 49 & 980 & 1953 & 53012 \\
\hline & RES CORRESPONDENCE & 8 & 1 & 1955 & 55011 \\
\hline & RES CORRESPONDENCE & 7 & 1 & 1955 & 55018 \\
\hline & $\mathrm{J}$ PHYS CHEM & 61 & 593 & 1957 & 57002 \\
\hline & CAN J CHEM & 35 & 986 & 1957 & 57011 \\
\hline & TRANS FARADAY SOC & 61 & 190 & 1965 & 65007 \\
\hline \multirow[t]{6}{*}{ GONICK E } & $J$ AM CHEM SOC & 67 & 1191 & 1945 & 45001 \\
\hline & J COLLOID SCI & 1 & 127 & 1946 & 46008 \\
\hline & J COLLOID SCI & 1 & 393 & 1946 & 46009 \\
\hline & REC TRAV CHIM & 65 & 601 & 1946 & 46016 \\
\hline & $J$ AM CHEM SOC & 68 & 177 & 1946 & 46019 \\
\hline & $J$ AM CHEM SOC & 69 & 334 & 1947 & 47007 \\
\hline \multirow[t]{13}{*}{ GOODMAN JF } & TRANS FARADAY SOC & 57 & 1627 & 1961 & 61004 \\
\hline & TRANS FARADAY SOC & 58 & 206 & 1962 & 62006 \\
\hline & TRANS FARADAY SOC & 58 & 1661 & 1962 & 62013 \\
\hline & PROC ROY SOC & 273 & 84 & 1963 & 63014 \\
\hline & TRANS FARADAY SOC & 60 & 202 & 1964 & 64003 \\
\hline & TRANS FARADAY SOC & 60 & 986 & 1964 & 64012 \\
\hline & TRANS FARADAY SOC & 60 & 979 & 1964 & 64023 \\
\hline & TRANS FARADAY SOC & 60 & 996 & 1964 & 64027 \\
\hline & TRANS FARADAY SOC & 61 & 589 & 1965 & 65004 \\
\hline & TRANS FARADAY SOC & 61 & 583 & 1965 & 65005 \\
\hline & TRANS FARADAY SOC & 62 & 994 & 1966 & 66014 \\
\hline & TRANS FARADAY SOC & 62 & 987 & 1966 & 66015 \\
\hline & TRANS FARADAY SOC & 62 & 979 & 1966 & 66016 \\
\hline \multirow[t]{3}{*}{ GOTTE E } & KOLLOID - Z & 117 & 42 & 1950 & 50009 \\
\hline & FETTE, SEIFEN, ANSTRICHMI & 56 & 583 & 1954 & 54007 \\
\hline & PROC INTERN CONGR SURFACE ACTIVITY & $3 R D$ & 45 & 1960 & 60018 \\
\hline GRABENSTETT.RJ & $\mathrm{J}$ COLLOID SCI & 18 & 401 & 1963 & 63019 \\
\hline GRAHAM $\mathrm{H}$ & $\mathrm{J}$ COLLOID SCI & 8 & 105 & 1953 & 53004 \\
\hline GREEN AA & $J$ AM CHEM SOC & 68 & 731 & 1946 & 46022 \\
\hline GREENFIELD A & $J$ PHYS CHEM & 61 & 818 & 1957 & 57004 \\
\hline GREGORY NW & $\mathrm{J}$ AM CHEM SOC & 70 & 1992 & 1948 & 48012 \\
\hline GRIEGER PF & $J$ AM CHEM SOC & 68 & 1137 & 1946 & 46018 \\
\hline & $\mathrm{J}$ AM CHEM SOC & 69 & 1835 & 1947 & 47001 \\
\hline & $J$ AM CHEM SOC & 70 & 3803 & 1948 & 48010 \\
\hline & J AM CHEM SOC & 71 & 95 & 1949 & 49014 \\
\hline & $J$ AM CHEM SOC & 71 & 309 & 1949 & 49017 \\
\hline & $\mathrm{J}$ AM CHEM SOC & 71 & 1455 & 1949 & 49018 \\
\hline GRIESS $W$ & FETTE, SEIFEN, ANSTRICHMI & 57 & 236 & 1955 & 55026 \\
\hline & FETTE, SEIFEN, ANSTRICHMI & 57 & 168 & 1955 & 55027 \\
\hline & FETTE, SEIFEN, ANSTRICHMI & 57 & 24 & 1955 & 55028 \\
\hline GRINDLEY J & $\mathrm{J}$ CHEM SOC & & 679 & 1929 & 29001 \\
\hline GUENTHNER RA & $\mathrm{J}$ PHYS CHEM & 57 & 923 & 1953 & 53014 \\
\hline HAFFNER FD & $\mathrm{J}$ PHYS CHEM & 46 & 662 & 1942 & 42003 \\
\hline HALL NA & J PHARM SCI & 54 & 1529 & 1965 & 65027 \\
\hline HAMANN SD & $\mathrm{J}$ PHYS CHEM & 66 & 1359 & 1962 & 62036 \\
\hline HARKINS WD & $J$ AM CHEM SOC & 59 & 2197 & 1937 & 37005 \\
\hline HAKRINS WD & $\mathrm{J}$ AM CHEM SOC & 62 & 1496 & 1940 & 40005 \\
\hline & $J$ CHEM PHYS & 14 & 216 & 1946 & 46002 \\
\hline & $\mathrm{J}$ CHEM PHYS & 14 & 640 & 1946 & 46004 \\
\hline & $\mathrm{J}$ CHEM PHYS & 14 & 641 & 1946 & 46005 \\
\hline & $\mathrm{J}$ CHEM PHYS & 14 & 480 & 1946 & 46010 \\
\hline & $\mathrm{J}$ CHEM PHYS & 14 & 215 & 1946 & 46011 \\
\hline & $\mathrm{J}$ AM CHEM SOC & 68 & 220 & 1946 & 46013 \\
\hline & $\mathrm{J}$ COLLOID SCI & 1 & 105 & 1946 & 46014 \\
\hline & J COLLOID SCI & 1 & 469 & 1946 & 46015 \\
\hline & $\mathrm{J}$ CHEM PHYS & 14 & 214 & 1946 & 46017 \\
\hline & $\mathrm{J}$ AM CHEM SOC & 69 & 679 & 1947 & 47006 \\
\hline & $J$ CHEM PHYS & 15 & 763 & 1947 & 47008 \\
\hline
\end{tabular}




\begin{tabular}{|c|c|c|c|c|c|}
\hline Author & Journal & Vol. & $\mathrm{Pg}$. & Year & $\begin{array}{l}\text { Refer- } \\
\text { ence } \\
\text { No. }\end{array}$ \\
\hline & $\mathrm{J}$ AM CHEM SOC & 69 & 1428 & 1947 & 47009 \\
\hline & $\mathrm{J}$ AM CHEM SOC & 69 & 683 & 1947 & 47010 \\
\hline & $\mathrm{J}$ CHEM PHYS & 15 & 496 & 1947 & 47012 \\
\hline & J PHYS COLLOID CHEM & 53 & 1350 & 1949 & 49006 \\
\hline & $\mathrm{J}$ COLLOID SCI & 4 & 367 & 1949 & 49007 \\
\hline & $\mathrm{J}$ AM CHEM SOC & 71 & 808 & 1949 & 49011 \\
\hline & $\mathrm{J}$ PHYS COLLOID CHEM & 54 & 271 & 1950 & 50008 \\
\hline & SCI MONTHLY & 70 & 220 & 1950 & 50011 \\
\hline & $\mathrm{J}$ COLLOID SCI & 6 & 576 & 1951 & 51010 \\
\hline HARRIMAN LA & J AM CHEM SOC & 74 & 2061 & 1952 & 52001 \\
\hline \multirow[t]{10}{*}{ HARRIS JC } & SOAP CHEM SPECIALTIES & 1958 & & 1958 & 58002 \\
\hline & J AM OIL CHEMISTS SOC & 35 & 670 & 1958 & 58004 \\
\hline & $\mathrm{J}$ PHYS CHEM & 62 & 1554 & 1958 & 58008 \\
\hline & $\mathrm{J}$ AM OIL CHEMISTS SOC & 35 & 428 & 1958 & 58010 \\
\hline & $J$ AM OIL CHEMISTS SOC & 36 & 332 & 1959 & 59009 \\
\hline & $\mathrm{J}$ AM OIL CHEMISTS SOC & 37 & 183 & 1960 & 60010 \\
\hline & $\mathrm{J}$ AM OIL CHEMISTS SOC & 38 & 169 & 1961 & 61006 \\
\hline & $\mathrm{J}$ AM OIL CHEMISTS SOC & 38 & 605 & 1961 & 61014 \\
\hline & $\mathrm{J}$ AM OIL CHEMISTS SOC & 38 & 138 & 1961 & 61015 \\
\hline & $\mathrm{J}$ AM OIL CHEMISTS SOC & 38 & 361 & 1961 & 61019 \\
\hline \multirow[t]{4}{*}{ HARROLD SP } & $\mathrm{J}$ PHYS CHEM & 63 & 317 & 1959 & 59001 \\
\hline & $\mathrm{J}$ COLLOID SCI & 15 & 280 & 1960 & 60004 \\
\hline & TRANS FARADAY SOC & 60 & 202 & 1964 & 64003 \\
\hline & TRANS FARADAY SOC & 62 & 994 & 1966 & 66014 \\
\hline \multirow[t]{14}{*}{ HARTLEY GS } & $\mathrm{Z}$ PHYSIK CHEM (LEIPZIG) & 170 & 321 & 1934 & 34001 \\
\hline & TRANS FARADAY SOC & 31 & 183 & 1935 & 35001 \\
\hline & TRANS FARADAY SOC & 32 & 795 & 1936 & 36001 \\
\hline & J AM CHEM SOC & 58 & 2347 & 1936 & 36002 \\
\hline & $\mathrm{J}$ CHEM SOC & & 1968 & 1938 & 38001 \\
\hline & NATURE & 142 & 161 & 1938 & 38003 \\
\hline & TRANS FARADAY SOC & 34 & 1288 & 1938 & 38005 \\
\hline & TRANS FARADAY SOC & 35 & 1109 & 1939 & 39001 \\
\hline & KOLLOID-Z & 88 & 22 & 1939 & 39005 \\
\hline & $\mathrm{J}$ CHEM SOC & & 1828 & 1939 & 39008 \\
\hline & TRANS FARADAY SOC & 37 & 130 & 1941 & 41001 \\
\hline & ANN REP PROGR CHEM (CH. S. LONDON) & 45 & 33 & 1948 & 48002 \\
\hline & NATURE & 163 & 767 & 1949 & 49010 \\
\hline & CHEM IND (LONDON) & 24 & 1012 & 1964 & 64030 \\
\hline \multirow[t]{3}{*}{ HARVA 0} & FINSKA KEMISTSAMFUNDETS MEDD & 25 & 257 & 1943 & 43005 \\
\hline & TRANS FARADAY SOC & 49 & 980 & 1953 & 53012 \\
\hline & REC TRAV CHIM & 75 & 112 & 1956 & 56018 \\
\hline \multirow[t]{5}{*}{ HARWOOD HJ } & $\mathrm{J}$ AM CHEM SOC & 69 & 2095 & 1947 & 47003 \\
\hline & J AM CHEM SOC & 71 & 672 & 1949 & 49009 \\
\hline & $\mathrm{J}$ AM CHEM SOC & 71 & 671 & 1949 & 49013 \\
\hline & $\mathrm{J}$ AM CHEM SOC & 73 & 3353 & 1951 & 51006 \\
\hline & $\mathrm{J}$ AM CHEM SOC & 74 & 2061 & 1952 & 52001 \\
\hline HASAN A & ACTA CHEM SCAND & 6 & 440 & 1952 & 52007 \\
\hline HATTORI K & BULL CHEM SOC JAPAN & 36 & 1250 & 1963 & 63006 \\
\hline HAYANO $\mathrm{S}$ & KOLLOID-Z & 181 & 139 & 1962 & 62023 \\
\hline \multirow[t]{3}{*}{ HAYDON DA } & TRANS FARADAY SOC & 54 & 698 & 1958 & 58012 \\
\hline & PHIL TRANS ROY SOC LONDON, SER A & 252 & 225 & 1960 & 60011 \\
\hline & TRANS FARADAY SOC & 58 & 1233 & 1962 & 62004 \\
\hline HEALY TW & $\mathrm{J}$ PHYS CHEM & 68 & 3562 & 1964 & 64035 \\
\hline & TRANS SME AIME & & 321 & 1964 & 64038 \\
\hline HENNE AL & $\mathrm{J}$ AM CHEM SOC & 73 & 2323 & 1951 & 51011 \\
\hline HERMANS JJ & KONINKI NED AKAD WETEN. PROC SER B & 58 & 91 & 1955 & 55001 \\
\hline & $\mathrm{J}$ PHYS CHEM & 59 & 576 & 1955 & 55002 \\
\hline & KONINKI NED AKAD WETEN. PROC SER B & 58 & 97 & 1955 & 55009 \\
\hline & KONINKI NED AKAD WETEN. PROC SER B & 59 & 190 & 1956 & 56002 \\
\hline & KONINKI NED AKAD WETEN. PROC SER B & 59 & 162 & 1956 & 56007 \\
\hline & KONINKI NED AKAD WETEN. PROC SER B & 59 & 298 & 1956 & 56011 \\
\hline HERRMANN KW & $\mathrm{J}$ PHYS CHEM & 66 & 295 & 1962 & 62005 \\
\hline & $\mathrm{J}$ PHYS CHEM & 67 & 935 & 1963 & 63018 \\
\hline & $\mathrm{J}$ PHYS CHEM & 68 & 1540 & 1964 & 64006 \\
\hline & $\mathrm{J}$ COLLOID INTERFACE SCI & 22 & 352 & 1966 & 66013 \\
\hline HERRMANNWK & $\mathrm{J}$ PHYS CHEM & 70 & 2909 & 1966 & 66039 \\
\hline HERZFELD SH & $\mathrm{J}$ PHYS COLLOID CHEM & 54 & 271 & 1950 & 50008 \\
\hline & $\mathrm{J}$ PHYS CHEM & 56 & 953 & 1952 & 52015 \\
\hline & $\mathrm{J}$ PHYS CHEM & 56 & 959 & 1952 & 52017 \\
\hline HESS $\mathrm{K}$ & KOLLOID-Z & 88 & 40 & 1939 & 39009 \\
\hline HICKSON J & PROC INTERN CONGR SURFACE ACTIVITY & 2ND & & 1957 & 57024 \\
\hline HIEBERT EN & $\mathrm{J}$ COLLOID SCI & 1 & 385 & 1946 & 46020 \\
\hline HIGHAM EH & RES CORRESPONDENCE & $\overline{7}$ & 1 & 1955 & 55018 \\
\hline HIGUCHI T & $\mathrm{J}$ AM PHARM ASSOC & 43 & 465 & 1954 & 54015 \\
\hline HISKEY CF & $\mathrm{J}$ COLLOID SCI & 9 & 243 & 1954 & 54011 \\
\hline HOERR CW & $J$ AM CHEM SOC & 64 & 2824 & 1942 & 42001 \\
\hline & $\mathrm{J}$ AM CHEM SOC & 64 & 772 & 1942 & 42002 \\
\hline & $\mathrm{J}$ AM CHEM SOC & 64 & 97 & 1942 & 42005 \\
\hline & $\mathrm{J}$ AM CHEM SOC & 65 & 976 & 1943 & 43002 \\
\hline & $\mathrm{J}$ AM CHEM SOC & 65 & 328 & 1943 & 43009 \\
\hline
\end{tabular}




\begin{tabular}{|c|c|c|c|c|c|}
\hline Author & Journal & \multirow{2}{*}{$\frac{\text { Vol. }}{68}$} & \multirow{2}{*}{$\begin{array}{c}\mathrm{Pg} . \\
2460\end{array}$} & \multirow{2}{*}{$\begin{array}{c}\text { Year } \\
1946\end{array}$} & \multirow{2}{*}{$\begin{array}{c}\begin{array}{c}\text { Refer- } \\
\text { ence } \\
\text { No. }\end{array} \\
46001\end{array}$} \\
\hline & $\mathrm{J}$ AM CHEM SOC & & & & \\
\hline & J AM CHEM SOC & 69 & 883 & 1947 & 47002 \\
\hline & J COLLOID SCI & 15 & 427 & 1960 & 60001 \\
\hline HOEVE CAJ & $J$ PHYS CHEM & 61 & 593 & 1957 & 57002 \\
\hline HOFFMAN EJ & J AM CHEM SOC & 64 & 97 & 1942 & 42005 \\
\hline & J AM CHEM SOC & 64 & 2067 & 1942 & 42008 \\
\hline & $\mathrm{J}$ AM CHEM SOC & 64 & 498 & 1942 & 42009 \\
\hline HOLLAHAN JR & $J$ PHYS CHEM & 63 & 757 & 1959 & 59023 \\
\hline HOLMBERG $P$ & ACTA CHEM SCAND & 19 & 573 & 1965 & 65023 \\
\hline HOLTZER A & $J$ AM CHEM SOC & 83 & 4865 & 1961 & 61016 \\
\hline & $\mathrm{J}$ PHYS CHEM & 69 & 3718 & 1965 & 65006 \\
\hline HONIG JG & $\mathrm{J}$ PHYS CHEM & 58 & 201 & 1954 & 54009 \\
\hline & $\mathrm{J}$ PHYS CHEM & 60 & 1108 & 1956 & 56012 \\
\hline & $\mathrm{J}$ PHYS CHEM & 60 & 1114 & 1956 & 56013 \\
\hline HORI R & BULL CHEM SOC JAPAN & 34 & 237 & 1961 & 61008 \\
\hline HOSOKAWA S & BULL CHEM SOC JAPAN & 35 & 1050 & 1962 & 62033 \\
\hline & BULL CHEM SOC JAPAN & 36 & 204 & 1963 & 63034 \\
\hline HOULTON HG & J AM CHEM SOC & 60 & 544 & 1938 & 38008 \\
\hline HOYER HW & J PHYS CHEM & 61 & 818 & 1957 & 57004 \\
\hline & $J$ PHYS CHEM & 65 & 1804 & 1961 & 61001 \\
\hline & $\mathrm{J}$ PHYS CHEM & 65 & 1807 & 1961 & 61002 \\
\hline & $\mathrm{J}$ PHYS CHEM & 68 & 3494 & 1964 & 64007 \\
\hline HSIAO L & $\mathrm{J}$ PHYS CHEM & 59 & 362 & 1955 & 55020 \\
\hline & $J$ PHYS CHEM & 60 & 657 & 1956 & 56014 \\
\hline HUBBARD HM & J AM CHEM SOC & 76 & 4300 & 1954 & 54014 \\
\hline HUBBARD WD & J PHYS CHEM & 57 & 808 & 1953 & 53009 \\
\hline & $\mathrm{J}$ PHYS CHEM & 58 & 1163 & 1954 & 54002 \\
\hline & J COLLOID SCI & 10 & 428 & 1955 & 55003 \\
\hline & $\mathrm{J}$ PHYS CHEM & 61 & 371 & 1957 & 57003 \\
\hline & J RES NAT BUR STD A & 59 & 113 & 1957 & 57006 \\
\hline & $J$ RES NAT BUR STD A & 68 & 359 & 1964 & 64043 \\
\hline HUDSON JB & $\mathrm{J}$ COLLOID SCI & 12 & 523 & 1957 & 57010 \\
\hline HUFF $\mathrm{H}$ & J COLLOID SCI & 3 & 511 & 1948 & 48004 \\
\hline & $J$ PHYS COLLOID CHEM & 55 & 311 & 1951 & 51004 \\
\hline HUGO WB & J PHARM PHARMACOL & 12 & 447 & 1960 & 60026 \\
\hline HUISMAN HF & KONINKI NED AKAD FETEN. PROC SER B & 67 & 367 & 1964 & 64045 \\
\hline & KONINKI NED AKAD WETEN. PROC SER B & 67 & 376 & 1964 & 64046 \\
\hline & KONINKI NED AKAD WETEN. PROC SER B & 67 & 388 & 1964 & 64047 \\
\hline & KONINKI NED AKAD FETEN. PROC SER B & 67 & 407 & 1964 & 64048 \\
\hline HUTCHINSON E & J PHYS CHEM & 58 & 1124 & 1954 & 54012 \\
\hline & Z PHYSIK CHEM (FRANKFURT) & 11 & 165 & 1957 & 57021 \\
\hline & Z PHYSIK CHEM (FRANKFURT) & 21 & 38 & 1959 & 59018 \\
\hline & Z PHYSIK CHEM (FRANKFURT) & 31 & 397 & 1962 & 62016 \\
\hline & J CHEM EDUC & 40 & 472 & 1963 & 63029 \\
\hline & $J$ PHYS CHEM & 68 & 2818 & 1964 & 64037 \\
\hline & $\mathrm{J}$ PHYS CHEM & 70 & 3502 & 1966 & 66026 \\
\hline IDA 0 & J CHEM SOC JAPAN, PURE CHEM SEC & 77 & 905 & 1966 & 66032 \\
\hline IFUKU $\mathrm{N}$ & REV PHYS CHEM JAPAN & 35 & 32 & 1965 & 65036 \\
\hline & J CHEM SOC JAPAN, PURE CHEM SEC & 87 & 329 & 1966 & 66033 \\
\hline IKEDA S & BULL CHEM SOC JAPAN & 34 & 1236 & 1961 & 61012 \\
\hline & BULL CHEM SOC JAPAN & 35 & 240 & 1962 & 62022 \\
\hline INO $\mathrm{T}$ & BULL CH SOC JAPAN & 30 & 760 & 1957 & 57033 \\
\hline INOUE $\mathrm{H}$ & J CHEM SOC JAPAN, PURE CHEM SEC & 78 & 636 & 1957 & 57019 \\
\hline & $J$ CHEM SOC JAPAN, PURE CHEM SEC & 79 & 348 & 1958 & 58018 \\
\hline & $J$ CHEM SOC JAPAN, PURE CHEM SEC & 79 & 345 & 1958 & 58019 \\
\hline & $J$ CHEM SOC JAPAN, PURE CHEM SEC & 79 & 48 & 1958 & 58028 \\
\hline & J COLLOID SCI & 15 & 268 & 1960 & 60006 \\
\hline & KOLLOID-Z & 183 & 68 & 1962 & 62010 \\
\hline & KOLLOID-Z & 196 & 1 & 1964 & 64019 \\
\hline & KOLLOID-Z & 195 & 93 & 1964 & 64021 \\
\hline & PROC INTERN CONGR SURFACE ACTIVITY & $4 \mathrm{TH}$ & $\mathrm{N}$ & 1964 & 64040 \\
\hline & J PHYS CHEM & 70 & 1108 & 1965 & 65033 \\
\hline ISEMURA T & BULL CHEM SOC JAPAN & 34 & 1236 & 1961 & 610 \\
\hline & BULL CHEM SOC JAPAN & 35 & 1737 & 1962 & 62012 \\
\hline & BULL CHEM SOC JAPAN & 35 & 240 & 1962 & 62022 \\
\hline & BULL CHEM SOC JAPAN & 36 & 1250 & 1963 & 63006 \\
\hline I WAMATSU I & BULL CHEM SOC JAPAN & 35 & 1050 & 1962 & 62033 \\
\hline & BULL CHEM SOC JAPAN & 36 & 204 & 1963 & 63034 \\
\hline JACOBS J & J PHARM PHARMACOL & 18 & $92 \mathrm{~S}$ & 1966 & 66019 \\
\hline JAKOB CW & J PHYS CHEM & 67 & 2075 & 1963 & 63001 \\
\hline JAMES JW & PROC INTERN CONGR SURFACE ACTIVITY & 3RD & 227 & 1960 & 60020 \\
\hline JAN ZA & J PHARM PHARMACOL & 15 & 825 & 1963 & 63021 \\
\hline JOHNSON JS & $J$ PHYS CHEM & 68 & 81 & 1964 & 64017 \\
\hline JOHNSON KE & $J$ AM CHEM SOC & 66 & 9 & 1944 & 44003 \\
\hline JOHNSON WF & ¿ PHYS CHEM & 50 & 440 & 1946 & 46006 \\
\hline & $J$ PHYS COLLOID CHEM & 51 & 636 & 1947 & 47013 \\
\hline & J PHYS COLLOID CHEM & 52 & 22 & 1948 & 48025 \\
\hline & J AM CHEM SOC & 73 & 4563 & 1951 & 51002 \\
\hline & $\mathrm{J}$ AM CHEM SOC & 74 & 20 & 1952 & 52002 \\
\hline & $\mathrm{J}$ AM CHEM SOC & 74 & 22 & 19 & 52003 \\
\hline
\end{tabular}




\begin{tabular}{|c|c|c|c|c|c|}
\hline Author & Journal & Vol. & Py. & Year & $\begin{array}{c}\text { Refer- } \\
\text { ence } \\
\text { No. }\end{array}$ \\
\hline JOHNSTON SA & $J$ AM CHEM SOC & 61 & 3210 & 1939 & 39011 \\
\hline JONES E & PHIL MAG & 4 & 841 & 1927 & 27002 \\
\hline \multirow[t]{2}{*}{ JONES TG } & TRANS FARADAY SOC & 49 & 980 & 1953 & 53012 \\
\hline & RES CORRESPONDENCE & 8 & 1 & 1955 & 55011 \\
\hline KAKIUCHI K & BULL CHEM SOC JAPAN & 36 & 1250 & 1963 & 63006 \\
\hline \multirow[t]{2}{*}{ KAPAUAN P } & J COLLOID SCI & 16 & 481 & 1961 & 61005 \\
\hline & $J$ PHYS CHEM & 70 & 783 & 1966 & 66002 \\
\hline KARNAUKH AM & MASLOB ZHIR PROM & 29 & 22 & 1963 & 63039 \\
\hline KART ZMARK EM & CAN J CHEM & 40 & 839 & 1962 & 62034 \\
\hline KASHIWAGI KM & J COLLOID SCI & 13 & 618 & 1958 & 58021 \\
\hline KASHIWAGI M & BULL CHEM SOC JAPAN & 32 & 624 & 1959 & 59017 \\
\hline KATO Y & CHEM PHARM BULL (TOKYO) & 11 & 1202 & 1963 & 63037 \\
\hline KATSURA K & $J$ PHYS CHEM & 68 & 1568 & 1964 & 64011 \\
\hline \multirow[t]{5}{*}{ KAUFMAN S } & $\mathrm{J}$ COLLOID SCI & 12 & 465 & 1957 & 57026 \\
\hline & $J$ PHYS CHEM & 62 & 1257 & 1958 & 58025 \\
\hline & WORLD PETROL CONGR, PROC & $\mathrm{V} 6$ & 18 & 1959 & 59021 \\
\hline & $\mathrm{J}$ COLLOID SCI & 17 & 231 & 1962 & 62025 \\
\hline & $J$ PHYS CHEM & 68 & 2814 & 1964 & 64041 \\
\hline KAWAMURA S & YAKUGAKU ZASSHI & 84 & 246 & 1964 & 64034 \\
\hline KAZUO T & J CHEM SOC JAPAN, PURE CHEM SEC & 79 & 48 & 1958 & 58028 \\
\hline KEIM GI & IND ENG CHEM & 36 & 610 & 1944 & 44001 \\
\hline KIESSIG H & KOLLOID-Z & 88 & 40 & 1939 & 39009 \\
\hline \multirow[t]{3}{*}{ KINNEY FB } & $\mathrm{J}$ AM OIL CHEMISTS SOC & 36 & 332 & 1959 & 59009 \\
\hline & J AM OIL CHEMISTS SOC & 37 & 18 & s1960 & 60010 \\
\hline & $\mathrm{J}$ AM OIL CHEMISTS SOC & 38 & 138 & 1961 & 61015 \\
\hline KINOSHITA K & $J$ PHYS CHEM & 63 & 648 & 1959 & 59013 \\
\hline \multirow[t]{8}{*}{ KITAHARA A } & BULL CHEM SOC JAPAN & 28 & 234 & 1955 & 55019 \\
\hline & BULL CHEM SOC JAPAN & 29 & 1 & S1956 & 56015 \\
\hline & $\mathrm{J}$ COLLOID SCI & 12 & 342 & 1957 & 57027 \\
\hline & BULL CHEM SOC JAPAN & 30 & 586 & 1957 & 57028 \\
\hline & BULL CHEM SOC JAPAN & 31 & 288 & 1957 & 57029 \\
\hline & BULL CHEM SOC JAPAN & 31 & 653 & 1958 & 58022 \\
\hline & $J$ PHYS CHEM & 66 & 363 & 1962 & 62024 \\
\hline & KOGYO KAGAKU ZASSHI & 68 & 2058 & 1965 & 65002 \\
\hline \multirow[t]{2}{*}{ KLAMANN D } & PROC INTERN CONGR SURFACE ACTIVITY & $3 R D$ & 1 & 1960 & 60016 \\
\hline & PROC INTERN CONGR SURFACE ACTIVITY & 3RD & 27 & 1960 & 60017 \\
\hline KLEVENS HB & $J$ CHEM PHYS & 14 & 216 & 1946 & 46002 \\
\hline & $J$ CHEM PHYS & 14 & 742 & 1946 & 46007 \\
\hline & $J$ CHEM PHYS & 14 & 480 & 1946 & 46010 \\
\hline & $J$ CHEM PHYS & 14 & 567 & 1946 & 46012 \\
\hline & $J$ PHYS COLLOID CHEM & 51 & 114 & 1947 & 47004 \\
\hline & $\mathrm{J}$ COLLOID SCI & 2 & 301 & 1947 & 47005 \\
\hline & $\mathrm{J}$ PHYS COLLOID CHEM & 52 & 130 & 1948 & 48005 \\
\hline & J AM OIL CHEMISTS SOC & 26 & 456 & 1949 & 49003 \\
\hline & J PHYS COLLOID CHEM & 54 & 1012 & 1950 & 50003 \\
\hline & $\mathrm{J}$ AM CHEM SOC & 72 & 3780 & 1950 & 50004 \\
\hline & ANAL CHEM & 22 & 1141 & 1950 & 50006 \\
\hline & CHEM REV & 47 & 1 & 1950 & 50007 \\
\hline & MEM SERV CHIM ETAT (PARIS) & 37 & 13 & 1952 & 52004 \\
\hline & J AM CHEM SOC & 74 & 4624 & 1952 & 52005 \\
\hline & KOLLOID-Z & 128 & 61 & 1952 & 52008 \\
\hline & J AM OIL CHEMISTS SOC & 30 & 74 & 1953 & 53010 \\
\hline & PROC INTERN CONGR SURFACE ACTIVITY & $1 S T$ & 1 & 1954 & 54004 \\
\hline & $\mathrm{J}$ CHIM PHYS & 51 & 1 & 1954 & 54010 \\
\hline & NATURE & 176 & 879 & 1955 & 55017 \\
\hline & $J$ PHYS CHEM & 60 & 1245 & 1956 & 56001 \\
\hline & MEM SERV CHIM ETAT (PARIS) & 41 & 363 & 1956 & 56010 \\
\hline & PROC INTERN CONGR SURFACE ACTIVITY & 2ND & 395 & 1957 & 57017 \\
\hline & KOLLOID-Z & 158 & 53 & 1958 & 58011 \\
\hline KLING W & PROC INTERN CONGR SURFACE ACTIVITY & 2ND & 295 & 1957 & 57022 \\
\hline KOBAYASHI $T$ & $J$ PHYS CHEM & 66 & 363 & 1962 & 62024 \\
\hline KOLBEL H & ANGEW CHEM & 71 & 211 & 1959 & 59022 \\
\hline & PROC INTERN CONGR SURFACE ACTIVITY & 3RD & 1 & 1960 & 60016 \\
\hline & PROC INTERN CONGR SURFACE ACTIVITY & $3 R D$ & 27 & 1960 & 60017 \\
\hline KOLTHOFF IM & $\begin{array}{l}J \text { PHYS CHEM } \\
J \text { PHYS COLLOID CHEM }\end{array}$ & $\begin{array}{l}50 \\
51\end{array}$ & $\begin{array}{l}440 \\
636\end{array}$ & $\begin{array}{l}1946 \\
1947\end{array}$ & $\begin{array}{l}46006 \\
47013\end{array}$ \\
\hline & $J$ PHYS COLLOID CHEM & 52 & 915 & 1948 & 48016 \\
\hline & $\mathrm{J}$ PHYS COLLOID CHEM & 52 & 22 & 1948 & 48025 \\
\hline & $J$ PHYS COLLOID CHEM & 53 & 424 & 1949 & 49005 \\
\hline & $J$ POLYMER SCI & 5 & 201 & 1950 & 50002 \\
\hline & J POLYMER SCI & 5 & 191 & 1950 & 50005 \\
\hline & $J$ AM CHEM SOC & 73 & 4563 & 1951 & 51002 \\
\hline & $\mathrm{J}$ AM CHEM SOC & 74 & 20 & 1952 & 52002 \\
\hline & $J$ AM CHEM SOC & 74 & 22 & 1952 & 52003 \\
\hline KOMOR JA & $J$ AM OIL CHEMISTS SOC & 43 & 435 & 1966 & 66022 \\
\hline KONDO A & $J$ CHEM SOC JAPAN, PURE CHEM SEC & 77 & 905 & 1966 & 66032 \\
\hline KONDO T & 3 CHEM SOC JAPAN, PURE CHEM SEC & 77 & 1236 & 1956 & 56020 \\
\hline & BULL CHEM SOC JAPAN & 30 & 905 & 1957 & S7032 \\
\hline & BULL CHEM SOC JAPAN & 30 & 760 & 1957 & 57033 \\
\hline & $J$ CHEM SOC JAPAN, PURE CHEM SEC & 80 & 818 & 1959 & 59024 \\
\hline
\end{tabular}




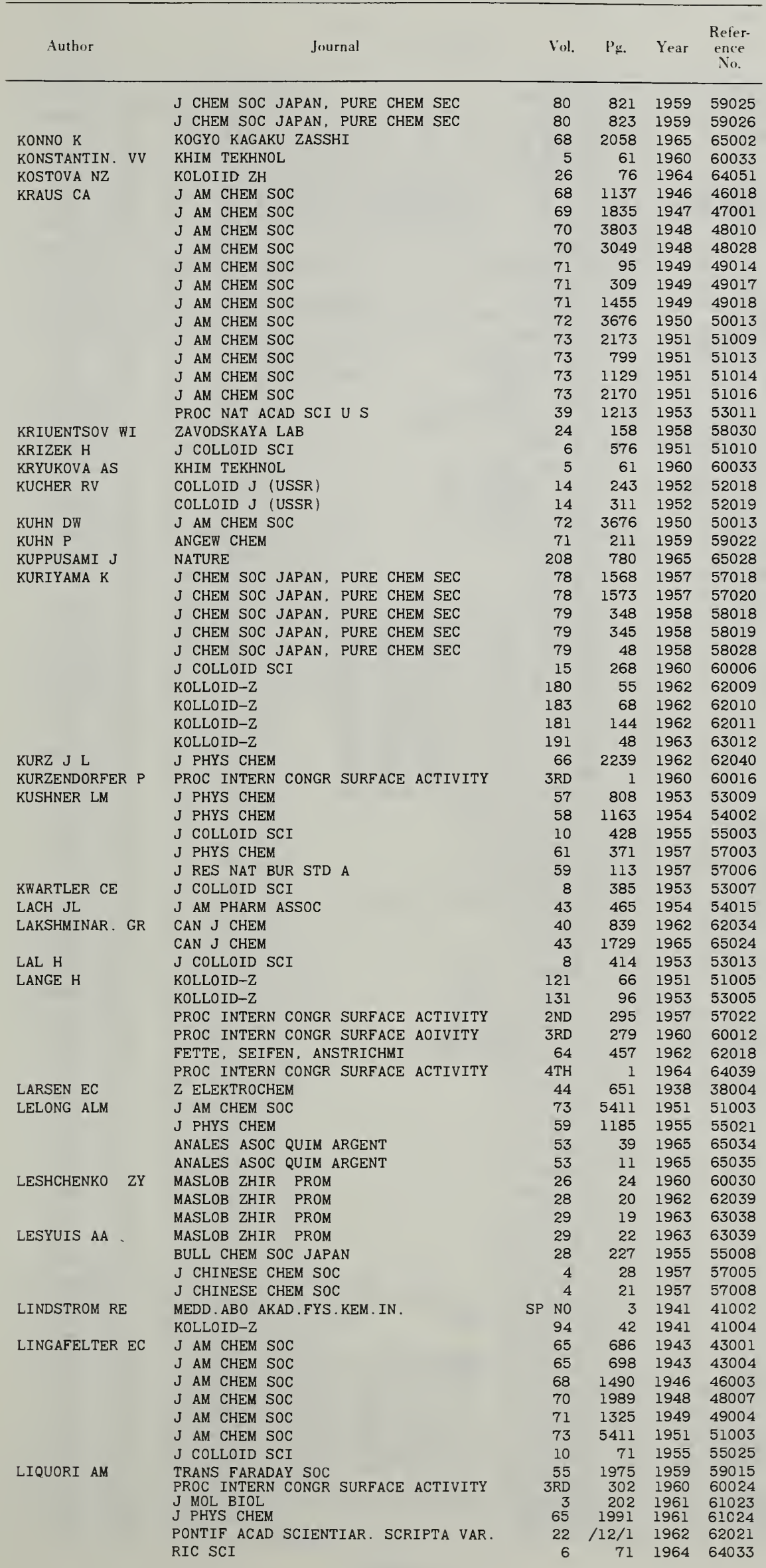




\begin{tabular}{|c|c|c|c|c|c|}
\hline Author & Journal & Vol. & $P_{\mathrm{g}}$. & Year & $\begin{array}{c}\text { Refer- } \\
\text { ence } \\
\text { No. }\end{array}$ \\
\hline \multirow[t]{2}{*}{ LITTLE RC } & J PHYS CHEM & 68 & 3453 & 1964 & 64042 \\
\hline & J PHYS CHEM & 68 & 2709 & 1964 & 64044 \\
\hline \multirow[t]{3}{*}{ LONG FA } & J AM CHEM SOC & 59 & 2197 & 1937 & 37005 \\
\hline & $J$ AM CHEM SOC & 62 & 1496 & 1940 & 40005 \\
\hline & J AM CHEM SOC & 63 & 84 & 1941 & 41006 \\
\hline LORENZ PB & $\mathrm{J}$ PHYS CHEM & 60 & 657 & 1956 & 56014 \\
\hline \multirow[t]{5}{*}{ LOTTERMOSER A } & KOLLOID-Z & 63 & 175 & 1933 & 33003 \\
\hline & KOLLOID-Z & 63 & 49 & 1933 & 33004 \\
\hline & KOLLOID-Z & 73 & 276 & 1935 & 35002 \\
\hline & TRANS FARADAY SOC & 31 & 200 & 1935 & 35004 \\
\hline & KOLLOID-BEIH. & 45 & 303 & 1937 & 37004 \\
\hline LOVELL VM & ANAL CHEM & 38 & 1926 & 1966 & 66024 \\
\hline LUCK W & PROC INTERN CONGR SURFACE ACTIVITY & 3RD & 264 & 1960 & 60023 \\
\hline LUDLUM DB & $\mathrm{J}$ PHYS CHEM & 60 & 1240 & 1956 & 56005 \\
\hline \multirow{2}{*}{ MACFARLANE CB } & J PHARM PHARMACOL & 14 & 100 & 1962 & 62027 \\
\hline & J PHARM PHARMACOL & 17 & 65 & 1965 & 65016 \\
\hline \multirow[t]{2}{*}{ MALIK WU } & INDIAN J CHEM & 3 & 441 & 1965 & 65021 \\
\hline & J AM OIL CHEMISTS SOC & 43 & 446 & 1966 & 66023 \\
\hline MALSCH J & Z PHYSIK CHEM (LEIPZIG) & 170 & 321 & 1934 & 34001 \\
\hline MANCHESTER KE & $\mathrm{J}$ PHYS CHEM & 58 & 1124 & 1954 & 54012 \\
\hline MANDELL L & ACTA CHEM SCAND & 17 & 111 & 1963 & 63033 \\
\hline \multirow[t]{2}{*}{ MANKOWICH AM } & $\mathrm{J}$ AM OIL CHEMISTS SOC & 41 & 449 & 1964 & 64010 \\
\hline & $J$ AM OIL CHEMISTS SOC & 43 & 615 & 1966 & 66021 \\
\hline MANNING DJ & J AM OIL CHEMISTS SOC & 43 & 133 & 1966 & 66001 \\
\hline MARKAN AL & ZAVODSKAYA LAB & 24 & 158 & 1958 & 58030 \\
\hline MARKINA ZN & KOLLOID ZH & 26 & 76 & 1964 & 64051 \\
\hline MARMO A & $\mathrm{J}$ PHYS CHEM & 65 & 1804 & 1961 & 61001 \\
\hline & $\mathrm{J}$ PHYS CHEM & 65 & 1807 & 1961 & 61002 \\
\hline MARON SH & $\mathrm{J}$ COLLOID SCI & 9 & 382 & 1954 & 54006 \\
\hline MARUTA I & J CHEM SOC JAPAN, PURE CHEM SEC & 82 & 1657 & 1961 & 61009 \\
\hline & J CHEM SOC JAPAN, PURE CHEM SEC & 82 & 1512 & 1961 & 61010 \\
\hline & J CHEM SOC JAPAN, PURE CHEM SEC & 82 & 1660 & 1961 & 61011 \\
\hline & J CHEM SOC JAPAN, PURE CHEM SEC & 83 & 732 & 1962 & 62007 \\
\hline & J CHEM SOC JAPAN, PURE CHEM SEC & 83 & 786 & 1962 & 62026 \\
\hline & J CHEM SOC JAPAN, PURE CHEM SEC & 83 & 395 & 1962 & 62028 \\
\hline & J CHEM SOC JAPAN, PURE CHEM SEC & 83 & 782 & 1962 & 62029 \\
\hline & J CHEM SOC JAPAN, PURE CHEM SEC & 83 & 788 & 1962 & 62030 \\
\hline & J CHEM SOC JAPAN, PURE CHEM SEC & 83 & 858 & 1962 & 62031 \\
\hline & J CHEM SOC JAPAN, PURE CHEM SEC & 83 & 861 & 1962 & 62032 \\
\hline & KOGYO KAGAKU ZASSHI & 68 & 1090 & 1965 & 65017 \\
\hline MATHAI $\mathrm{K}$ & TRANS FARADAY SOC & 62 & 759 & 1966 & 66031 \\
\hline & TRANS FARADAY SOC & 62 & 750 & 1966 & 66037 \\
\hline MATIJEVIC E & CROAT CHEM ACTA & 29 & 431 & 1957 & 57001 \\
\hline & TRANS FARADAY SOC & 54 & 587 & 1958 & 58020 \\
\hline MATSUMOTO T & J SCI HIROSHIMA UNIV, SER A-II & 21 & 51 & 1957 & 57025 \\
\hline MATTOON RW & $\mathrm{J}$ AM CHEM SOC & 68 & 220 & 1946 & 46013 \\
\hline & J COLLOID SCI & 1 & 105 & 1946 & 46014 \\
\hline & $\mathrm{J}$ CHEM PHYS & 15 & 763 & 1947 & 47008 \\
\hline MATUURA $\mathrm{R}$ & BULL CHEM SOC JAPAN & 35 & 1050 & 1962 & 62033 \\
\hline & BULL CHEM SOC JAPAN & 36 & 204 & 1963 & 63034 \\
\hline & BULL CHEM SOC JAPAN & 36 & 813 & 1963 & 63035 \\
\hline & BULL CHEM SOC JAPAN & 38 & 373 & 1965 & 65020 \\
\hline MAURER EW & J AM OIL CHEMISTS SOC & 36 & 241 & 1959 & 59004 \\
\hline & $\mathrm{J}$ AM OIL CHEMISTS SOC & 41 & 205 & 1964 & 64002 \\
\hline MCBAIN JW & J AM CHEM SOC & 57 & 1905 & 1935 & 35007 \\
\hline & $\mathrm{J}$ PHYS CHEM & 40 & 493 & 1936 & 36005 \\
\hline & J AM CHEM SOC & 63 & 670 & 1941 & 41005 \\
\hline & $\mathrm{J}$ PHYS CHEM & 46 & 10 & 1942 & 42006 \\
\hline & IND ENG CHEM & 34 & 915 & 1942 & 42007 \\
\hline & $\mathrm{J}$ AM CHEM SOC & 65 & 2072 & 1943 & 43006 \\
\hline & $\mathrm{J}$ PHYS CHEM & 47 & 94 & 1943 & 43008 \\
\hline & $\mathrm{J}$ AM CHEM SOC & 66 & 9 & 1944 & 44003 \\
\hline & $\mathrm{J}$ COLLOID SCI & 1 & 127 & 1946 & 46008 \\
\hline & REC TRAV CHIM & 65 & 601 & 1946 & 46016 \\
\hline & J AM CHEM SOC & 68 & 731 & 1946 & 46022 \\
\hline & $J$ AM CHEM SOC & 69 & 334 & 1947 & 47007 \\
\hline & $\mathrm{J}$ COLLOID SCI & 3 & 425 & 1948 & 48009 \\
\hline & J PHYS COLLOID CHEM & 52 & 881 & 1948 & 48011 \\
\hline & J AM CHEM SOC & 70 & 3838 & 1948 & 48015 \\
\hline & $\mathrm{J}$ PHYS COLLOID CHEM & 52 & 12 & 1948 & 48026 \\
\hline & $\mathrm{J}$ PHYS COLLOID CHEM & 55 & 311 & 1951 & 51004 \\
\hline MCBAIN MEL & $\mathrm{J}$ AM CHEM SOC & 61 & 3210 & 1939 & 39011 \\
\hline & $\mathrm{J}$ COLLOID SCI & 10 & 223 & 1955 & 55023 \\
\hline & $\mathrm{J}$ PHYS CHEM & 47 & 196 & 1943 & 43007 \\
\hline MCCORKLE MR & $\mathrm{J}$ AM CHEM SOC & 65 & 328 & 1943 & 43009 \\
\hline MCDOWELL MJ & $J$ AM CHEM SOC & 73 & 2173 & 1951 & 51009 \\
\hline & $\mathrm{J}$ AM CHEM SOC & 73 & 2170 & 1951 & 51016 \\
\hline MCHAN H & $\mathrm{J}$ AM CHEM SOC & 70 & 3838 & 1948 & 48015 \\
\hline MCNEILL W & $J$ PHYS CHEM & 56 & 701 & 1952 & 52013 \\
\hline MEADER AL & IND ENG CHEM & 44 & 1636 & 1952 & 52014 \\
\hline
\end{tabular}




\begin{tabular}{|c|c|c|c|c|c|}
\hline Author & Journal & Vol. & $\mathrm{Pg}$. & Year & $\begin{array}{c}\text { Refer- } \\
\text { ence } \\
\text { No. }\end{array}$ \\
\hline \multirow[t]{2}{*}{ MEEHAN EJ } & J POLYMER SCI & 5 & 201 & 1950 & 50002 \\
\hline & J POLYMER SCI & 5 & 191 & 1950 & 50005 \\
\hline \multirow{7}{*}{ MEGURO K } & J CHEM SOC JAPAN, PURE CHEM SEC & 77 & 1236 & 1956 & 56020 \\
\hline & BULL CHEM SOC JAPAN & 30 & 905 & 1957 & 57032 \\
\hline & BULL CHEM SOC JAPAN & 30 & 760 & 1957 & 57033 \\
\hline & J CHEM SOC JAPAN, PURE CHEM SEC & 80 & 818 & 1959 & 59024 \\
\hline & J CHEM SOC JAPAN, PURE CHEM SEC & 80 & 821 & 1959 & 59025 \\
\hline & J CHEM SOC JAPAN, PURE CHEM SEC & 80 & 823 & 1959 & 59026 \\
\hline & J CHEM SOC JAPAN, PURE CHEM SEC & 77 & 905 & 1966 & 66032 \\
\hline \multirow[t]{3}{*}{ MELE A } & NATURE & 184 & 1482 & 1959 & 59014 \\
\hline & TRANS FARADAY SOC & 55 & 1975 & 1959 & 59015 \\
\hline & $J$ PHYS CHEM & 63 & 650 & 1959 & 59016 \\
\hline MERRILL RC & J PHYS COLLOID CHEM & 52 & 774 & 1948 & 48024 \\
\hline \multirow[t]{4}{*}{ MERRILL RC JR } & $J$ AM CH M S & 63 & 670 & 1941 & 41005 \\
\hline & J PHYS CHEM & 46 & 10 & 1942 & 42006 \\
\hline & IND ENG CHEM & 34 & 915 & 1942 & 42007 \\
\hline & J PHYS COLLOID CHEM & 52 & 12 & 1948 & 48026 \\
\hline METCALF AD & $\mathrm{J}$ COLLOID SCI & 17 & 523 & $\cdot 1962$ & 62015 \\
\hline MEYER HG & $J$ PHYS CHEM & 70 & 783 & 1966 & 66002 \\
\hline MIJNLIEFF PF & NATURE & 208 & 889 & 1965 & 65029 \\
\hline \multirow[t]{3}{*}{ MILES GD } & IND ENG CHEM & 36 & 610 & 1944 & 44001 \\
\hline & $J$ PHYS CHEM & 48 & 57 & 1944 & 44002 \\
\hline & J PHYS CHEM & 49 & 71 & 1945 & 45002 \\
\hline MILLER ML & J COLLOID SCI & 13 & 411 & 1958 & 58001 \\
\hline MITTELMANN R & $\mathrm{J}$ CHEM PHYS & 15 & 763 & 1947 & 47008 \\
\hline & J PHYS COLLOID CHEM & 53 & 1350 & 1949 & 49006 \\
\hline & $\mathrm{J}$ COLLOID SCI & 4 & 367 & 1949 & 49007 \\
\hline MIURA M & J SCI HIROSHIMA UNIV, SER A-II & 21 & 51 & 1957 & 57025 \\
\hline & J SCI HIROSHIMA UNIV, SER A-II & 22 & 57 & 1958 & 58023 \\
\hline & J SCI HIROSHIMA UNIV, SER A-II & 28 & 41 & 1964 & 64036 \\
\hline MIYAMOTO S & BULL CHEM SOC JAPAN & 33 & 375 & 1960 & 60029 \\
\hline & BULL CHEM SOC JAPAN & 33 & 371 & 1960 & 60035 \\
\hline MODI HJ & J ELECTROCHEM SOC & 106 & 336 & 1959 & 59019 \\
\hline MOLYNEUX P & TRANS FARADAY SOC & 61 & 1043 & 1965 & 65003 \\
\hline MOULE D & TRANS FARADAY SOC & 54 & 1638 & 1958 & 58006 \\
\hline & CAN J CHEM & 37 & 2083 & 1959 & 59007 \\
\hline & CAN J CHEM & 37 & 2086 & 1959 & 59008 \\
\hline MUKERJEE P & $\mathrm{J}$ AM CHEM SOC & 77 & 2937 & 1955 & 55015 \\
\hline & $\mathrm{J}$ PHYS CHEM & 62 & 1390 & 1958 & 58013 \\
\hline & J PHYS CHEM & 62 & 1397 & 1958 & 58014 \\
\hline & J PHYS CHEM & 62 & 1400 & 1958 & 58015 \\
\hline & J PHYS CHEM & 62 & 1404 & 1958 & 58016 \\
\hline & J PHYS CHEM & 67 & 190 & 1963 & 63032 \\
\hline & $\mathrm{J}$ PHYS CHEM & 70 & 783 & 1966 & 66002 \\
\hline & J PHYS CHEM & 70 & 2138 & 1966 & 66004 \\
\hline & J PHYS CHEM & 70 & 2144 & 1966 & 66005 \\
\hline & J PHYS CHEM & 70 & 2150 & 1966 & 66006 \\
\hline MUKHERJI BK & $\mathrm{J}$ PHYS CHEM & 64 & 1 & 1960 & 60028 \\
\hline MULLEY BA & J COLLOID SCI & 17 & 523 & 1962 & 62015 \\
\hline & $\mathrm{J}$ COLLOID SCI & 19 & 201 & 1964 & 64009 \\
\hline MURRAY RC & TRANS FARADAY SOC & 31 & 183 & 1935 & 35001 \\
\hline MYSELS EK & $\mathrm{J}$ COLLOID SCI & 20 & 315 & 1965 & 65018 \\
\hline MYSELS KJ & TRANS FARADAY SOC & 51 & 728 & 1955 & 55005 \\
\hline & J PHYS CHEM & 59 & 325 & 1955 & 55014 \\
\hline & $\mathrm{J}$ AM CHEM SOC & 77 & 2937 & 1955 & 55015 \\
\hline & $\mathrm{J}$ COLLOID SCI & 10 & 507 & 1955 & 55016 \\
\hline & J PHYS CHEM & 62 & 1390 & 1958 & 58013 \\
\hline & J PHYS CHEM & 62 & 1400 & 1958 & 58015 \\
\hline & J PHYS CHEM & 63 & 1696 & 1959 & 59002 \\
\hline & J PHYS CHEM & 63 & 1781 & 1959 & 59003 \\
\hline & J COLLOID SCI & 16 & 481 & 1961 & 61005 \\
\hline & $\mathrm{J}$ COLLOID SCI & 16 & 462 & 1961 & 61017 \\
\hline & J COLLOID SCI & 16 & 474 & 1961 & 61018 \\
\hline & J COLLOID SCI & 20 & 315 & 1965 & 65018 \\
\hline & J PHYS CHEM & 69 & 1466 & 1965 & 65019 \\
\hline & $\mathrm{J}$ COLLOID SCI & 21 & 331 & 1966 & 66007 \\
\hline NAKADATE S & REP INST SCI TECH UNIV TOKYO & 7 & 401 & 1953 & 53001 \\
\hline NAKAGAKI $M$ & J CHEM SOC JAPAN, PURE CHEM SEC & 72 & 113 & 1951 & 51018 \\
\hline & BULL CHEM SOC JAPAN & 37 & 817 & 964 & 64025 \\
\hline & YAKUGAKU ZASSHI & 84 & 246 & 1964 & 64034 \\
\hline NAKAGAWA T & J CHEM SOC JAPAN, PURE CHEM SEC & 78 & 1568 & 1957 & 57018 \\
\hline & J CHEM SOC JAPAN, PURE CHEM SEC & 78 & 636 & 1957 & 57019 \\
\hline & J CHEM SOC JAPAN, PURE CHEM SEC & 78 & 1573 & 1957 & 57020 \\
\hline & SHINOGI KENKYUSHO NEMPO & 8 & 805 & 1958 & 58017 \\
\hline & J CHEM SOC JAPAN, PURE CHEM SEC & 79 & 348 & 1958 & 58018 \\
\hline & J CHEM SOC JAPAN, PURE CHEM SEC & 79 & 345 & 1958 & 58019 \\
\hline & J CHEM SOC JAPAN, PURE CHEM SEC & 79 & 48 & 1958 & 58028 \\
\hline & J COLLOID SCI & 15 & 268 & 1960 & 60006 \\
\hline & PROC INTERN CONGR SURFACE ACTIVITY & 3RD & 636 & 1960 & 60007 \\
\hline & KOLLOID-Z & 168 & 132 & 1960 & 60009 \\
\hline & CHEM IND (LONDON) & 14 & 1135 & 1961 & 61022 \\
\hline
\end{tabular}




\begin{tabular}{|c|c|c|c|c|c|}
\hline Author & Journal & Vol. & $P_{\mu}$ & Year & $\begin{array}{l}\text { Refer- } \\
\text { ence } \\
\text { Nio. }\end{array}$ \\
\hline & KOLLOID-Z & 183 & 68 & 1962 & 62010 \\
\hline & KOLLOID-Z & 189 & 50 & 1963 & 63008 \\
\hline & KOLLOID-Z & 188 & 47 & 1963 & 63009 \\
\hline & KOLLOID-Z & 187 & 44 & 1963 & 63010 \\
\hline & KOLLOID-Z & 191 & 48 & 1963 & 63012 \\
\hline & KOLLOID-Z & 194 & 143 & 1964 & 64015 \\
\hline & KOLLOID-Z & 195 & 93 & 1964 & 64021 \\
\hline & PROC INTERN CONGR SURFACE ACTIVITY & $4 \mathrm{TH}$ & $\mathrm{N}$ & 1964 & 64040 \\
\hline & $\mathrm{J}$ PHYS CHEM & 70 & 1108 & 1965 & 65033 \\
\hline \multirow{2}{*}{ NAKAYAMA $H$} & $\mathrm{~J}$ COLLOID SCI & 18 & 705 & 1963 & 63036 \\
\hline & $J$ PHYS CHEM & 70 & 3502 & 1966 & 66026 \\
\hline NASH $T$ & CHEM IND (LONDON) & & 590 & 1958 & 58027 \\
\hline NATALE I & ANALES ASOC QUIM ARGENT & 53 & 11 & 1965 & 65035 \\
\hline NAUMAN RV & $\mathrm{J}$ PHYS CHEM & 68 & 3498 & 1964 & 64001 \\
\hline NEFF LL & J AM CHEM SOC & 70 & 1989 & 1948 & 48007 \\
\hline NEWTON JM & J PHARM PHARMACOL & 12 & 447 & 1960 & 60026 \\
\hline NICOLESCU A & REV CHIM AC.REP POP.ROUM. & 6 & 309 & 1961 & 61029 \\
\hline NINOMIYA Y & BULL CHEM SOC JAPAN & 37 & 817 & 1964 & 64025 \\
\hline NOEL DR & J AM CHEM SOC & 74 & 2061 & 1952 & 52001 \\
\hline NOGUCHI $\mathrm{J}$ & BULL CHEM SOC JAPAN & 34 & 1236 & 1961 & 61012 \\
\hline \multirow[t]{3}{*}{ NUTTING GC } & J AM CHEM SOC & 59 & 2197 & 1937 & 37005 \\
\hline & $J$ AM CHEM SOC & 62 & 1496 & 1940 & 40005 \\
\hline & $J$ AM CHEM SOC & 63 & 84 & 1941 & 41006 \\
\hline \multirow{2}{*}{ OGDEN CP } & PROC ROY SOC & 273 & 84 & 1963 & 63014 \\
\hline & TRANS FARADAY SOC & 61 & 583 & 1965 & 65005 \\
\hline \multirow{2}{*}{ OHBA N } & BULL CHEM SOC JAPAN & 30 & 905 & 1957 & 57032 \\
\hline & BULL CHEM SOC JAPAN & 30 & 760 & 1957 & 57033 \\
\hline OHKI K & $\mathrm{J}$ PHYS CHEM & 70 & 3437 & 1966 & 66027 \\
\hline \multirow[t]{2}{*}{ OKUYAMA $\mathrm{H}$} & BULL CHEM SOC JAPAN & 27 & 259 & 1954 & 54008 \\
\hline & BULL CHEM SOC JAPAN & 30 & 186 & 1957 & 57023 \\
\hline OLEINIKOVA ZV & MASLOB ZHIR PROM & 26 & 26 & 1960 & 60031 \\
\hline OLIVIER JP & $J$ PHYS CHEM & 63 & 1671 & 1959 & 59020 \\
\hline OLOANE JK & $\mathrm{J}$ AM CH M SOC & 73 & 5411 & 1951 & 51003 \\
\hline \multirow{2}{*}{ OPPENHEIMER H } & $\mathrm{J}$ CHEM PHYS & 15 & 496 & 1947 & 47012 \\
\hline & $J$ AM CHEM SOC & 71 & 808 & 1949 & 49011 \\
\hline OSIPOW L & PROC INTERN CONGR SURFACE ACTIVITY & 2ND & & 1957 & 57024 \\
\hline OSTER G & J COLLOID SCI & 9 & 243 & 1954 & 54011 \\
\hline OSUGI $\mathrm{J}$ & REV PHYS CHEM JAPAN & 35 & 32 & 1965 & 65036 \\
\hline & J CHEM SOC JAPAN, PURE CHEM SEC & 87 & 329 & 1966 & 66033 \\
\hline OTTER RJ & J COLLOID SCI & 16 & 462 & 1961 & 61017 \\
\hline & $J$ COLLOID SCI & 16 & 474 & 1961 & 61018 \\
\hline OTTEWILL RH & TRANS FARADAY SOC & 62 & 750 & 1966 & 66037 \\
\hline & $J$ CHEM SOC & & 1712 & 1958 & 58031 \\
\hline & TRANS FARADAY SOC & 57 & 1627 & 1961 & 61004 \\
\hline & $J$ COLLOID INTERFACE SCI & 21 & 522 & 1966 & 66028 \\
\hline & TRANS FARADAY SOC & 62 & 759 & 1966 & 66031 \\
\hline OYAMA T & $J$ CHEM SOC JAPAN, PURE CHEM SEC & 79 & 348 & 1958 & 58018 \\
\hline & $J$ CHEM SOC JAPAN, PURE CHEM SEC & 79 & 345 & 1958 & 58019 \\
\hline PACKTER A & J PHARM PHARMACOL & 15 & 317 & 1963 & 63030 \\
\hline PANKHURST KGA & TRANS FARADAY SOC & 42 & 523 & 1946 & 46021 \\
\hline PAQUETTE RG & $J$ AM CHEM SOC & 65 & 686 & 1943 & 43001 \\
\hline PARKER RA & $J$ RES NAT BUR STD A & 59 & 113 & 1957 & 57006 \\
\hline PARREIRA HC & ANAIS ACAD BRASIL CIENC & 32 & 207 & 1960 & 60015 \\
\hline & J COLLOID INTERFACE SCI & 21 & 522 & 1966 & 66028 \\
\hline PARRY GA & $J$ CHEM SOC & & 626 & 1935 & 35008 \\
\hline PATEL RM & $J$ PHARM SCI & 55 & 1345 & 1966 & 66017 \\
\hline PATTERSON GD & $J$ PHYS CHEM & 57 & 247 & 1953 & 53003 \\
\hline PETHICA BA & CROAT CHEM ACTA & 29 & 431 & 1957 & 57001 \\
\hline & TRANS FARADAY SOC & 54 & 587 & 1958 & 58020 \\
\hline & PROC INTERN CONGR SURFACE ACTIVITY & 3RD & 227 & 1960 & 60020 \\
\hline PHILIPPOFF $\mathbb{W}$ & KOLLOID-Z & 88 & 40 & 1939 & 39009 \\
\hline PHILLIPS JN & TRANS FARADAY SOC & 51 & 728 & 1955 & 55005 \\
\hline & $J$ PHYS CHEM & 59 & 325 & 1955 & 55014 \\
\hline & TRANS FARADAY SOC & 51 & 561 & 1955 & 55024 \\
\hline & TRANS FARADAY SOC & 54 & 698 & 1958 & 58012 \\
\hline PICCIONE GA & $J$ PHYS CHEM & 46 & 662 & 1942 & 42003 \\
\hline PILPEL $\mathrm{N}$ & $J$ COLLOID SCI & 9 & 285 & 1954 & 54001 \\
\hline & $J$ PHYS CHEM & 60 & 779 & 1956 & 56004 \\
\hline & TRANS FARADAY SOC & 57 & 1426 & 1961 & 61013 \\
\hline & CHEM REV & 63 & 221 & 1963 & 63002 \\
\hline & NATURE & 204 & 378 & 1964 & 64022 \\
\hline POWNEY J & TRANS FARADAY SOC & 31 & 1510 & 1935 & 35005 \\
\hline & TRANS FARADAY SOC & 33 & 851 & 1937 & 37003 \\
\hline & TRANS FARADAY SOC & 34 & 372 & 1938 & 38006 \\
\hline PRINCEN LH & $J$ PHYS CHEM & 63 & 1696 & 1959 & 59002 \\
\hline & $J$ PHYS CHEM & 63 & 1781 & 1959 & 59003 \\
\hline PRINS W & J PHYS CHEM & 59 & 576 & 1955 & 55002 \\
\hline & KONINKI NED AKAD WETEN. PROC SER B & 59 & 190 & 1956 & 56002 \\
\hline & KONINKI NED AKAD WETEN. PROC SER & 59 & 162 & 1956 & 56007 \\
\hline & KONINKI NED AKAD WETEN. PROC SER B & 59 & 298 & 1956 & 56011 \\
\hline
\end{tabular}




\begin{tabular}{|c|c|c|c|c|c|}
\hline Author & Journal & Fol. & $\mathrm{Pg}$. & Year & $\begin{array}{l}\text { Refer- } \\
\text { ence } \\
\text { No. }\end{array}$ \\
\hline PUSCHEL F & KOLLOID-Z & 63 & 175 & 1933 & 33003 \\
\hline \multirow[t]{2}{*}{ PYTASZ G } & ZESZYTY NAUK UNIT JAGIEL & 211 & 199 & 1966 & 66034 \\
\hline & ZESZYTY NAUK UNIT JAGIEL & 211 & 209 & 1966 & 66035 \\
\hline RADLEY JA & PROC INTERN CONGR SURFACE ACTIVITY & $3 R D$ & 246 & 1960 & 60021 \\
\hline \multirow[t]{2}{*}{ RAISON M } & COMPT REND & 235 & 1129 & 1952 & 52016 \\
\hline & $\begin{array}{l}\text { PROC INTERN CONGR SURFACE ACTIVITY } \\
\mathrm{J} \text { CHIM PHYS }\end{array}$ & $\begin{array}{r}1 S T \\
51\end{array}$ & $\begin{array}{l}1 \\
1\end{array}$ & $\begin{array}{l}1954 \\
1954\end{array}$ & $\begin{array}{l}54004 \\
54010\end{array}$ \\
\hline \multirow[t]{19}{*}{ RALSTON AT } & J AM CHEM SOC & 64 & 2824 & 1942 & 42001 \\
\hline & $\mathrm{J}$ AM CHEM SOC & 64 & 772 & 1942 & 42002 \\
\hline & $\mathrm{J}$ AM CHEM SOC & 64 & 97 & 1942 & 42005 \\
\hline & $\mathrm{J}$ AM CHEM SOC & 64 & 2067 & 1942 & 42008 \\
\hline & $J$ AM CHEM SOC & 64 & 498 & 1942 & 42009 \\
\hline & $J$ AM CHEM SOC & 65 & 976 & 1943 & 43002 \\
\hline & $J$ AM CHEM SOC & 65 & 328 & 1943 & 43009 \\
\hline & $J$ AM CHEM SOC & 68 & 2460 & 1946 & 46001 \\
\hline & $J$ AM CHEM SOC & 69 & 883 & 1947 & 47002 \\
\hline & $J$ AM CHEM SOC & 69 & 2095 & 1947 & 47003 \\
\hline & $J$ AM CHEM SOC & 70 & 977 & 1948 & 48014 \\
\hline & $\mathrm{J}$ AM CHEM SOC & 70 & 980 & 1948 & 48019 \\
\hline & $\mathrm{J}$ AM CHEM SOC & 70 & 2918 & 1948 & 48020 \\
\hline & $J$ AM CHEM SOC & 70 & 983 & 1948 & 48021 \\
\hline & $\mathrm{J}$ PHYS COLLOID CHEM & 52 & 1494 & 1948 & 48023 \\
\hline & $\mathrm{J}$ AM CHEM SOC & 70 & 436 & 1948 & 48027 \\
\hline & $\mathrm{J}$ AM CHEM SOC & 71 & 2145 & 1949 & 49008 \\
\hline & $J$ AM CHEM SOC & 71 & 672 & 1949 & 49009 \\
\hline & $\mathrm{J}$ AM CHEM SOC & 71 & 671 & 1949 & 49013 \\
\hline \multirow[t]{4}{*}{ RAY A } & $\mathrm{J}$ PHYS CHEM & 67 & 190 & 1963 & 63032 \\
\hline & $J$ PHYS CHEM & 70 & 2138 & 1966 & 66004 \\
\hline & $\mathrm{J}$ PHYS CHEM & 70 & 2144 & 1966 & 66005 \\
\hline & $J$ PHYS CHEM & 70 & 2150 & 1966 & 66006 \\
\hline \multirow[t]{2}{*}{ REED RM } & $\mathrm{J}$ AM CHEM SOC & 57 & 570 & 1935 & 35006 \\
\hline & $J$ AM CHEM SOC & 58 & 322 & 1936 & 36006 \\
\hline REEVES RL & $J$ PHYS CHEM & 69 & 2357 & 1965 & 65030 \\
\hline REITMIER RE & $J$ AM CHEM SOC & 62 & 2375 & 1940 & 40001 \\
\hline REYNOLDS CA & $\mathrm{J}$ AM CHEM SOC & 76 & 4300 & 1954 & 54014 \\
\hline RHODES CT & TRANS FARADAY SOC & 61 & 1043 & 1965 & 65003 \\
\hline \multirow[t]{2}{*}{ RICCIERI FM } & J PHARM SCI & 52 & 1011 & 1963 & 63024 \\
\hline & J PHARM SCI & 54 & 919 & 1965 & 65010 \\
\hline \multirow[t]{2}{*}{ ROBINS DC } & J PHARM PHARMACOL & 15 & 522 & 1963 & 63005 \\
\hline & J PHARM PHARMACOL & 15 & 157 & 1963 & 63031 \\
\hline ROBINSON RU & $\mathrm{J}$ PHYS CHEM & 56 & 701 & 1952 & 52013 \\
\hline ROBSON P & TRANS FARADAY SOC & 62 & 987 & 1966 & 66015 \\
\hline ROE CP & $\mathrm{J}$ AM CHEM SOC & 76 & 4703 & 1954 & 54013 \\
\hline ROSE GRF & CAN J CHEM ENGR & F28 & 213 & 1950 & 50010 \\
\hline ROSENBLUM C & $\mathrm{J}$ PHYS CHEM & 46 & 662 & 1942 & 42003 \\
\hline ROSS J & IND ENG CHEM & 36 & 610 & 1944 & 44001 \\
\hline ROSS S & $\mathrm{J}$ COLLOID SCI & 8 & 385 & 1953 & 53007 \\
\hline & $\mathrm{J}$ COLLOID SCI & 12 & 523 & 1957 & 57010 \\
\hline & $\mathrm{J}$ PHYS CHEM & 61 & 1261 & 1957 & 57031 \\
\hline & $\mathrm{J}$ PHYS CHEM & 63 & 1671 & 1959 & 59020 \\
\hline RUSH RM & $\mathrm{J}$ PHYS CHEM & 68 & 81 & 1964 & 64017 \\
\hline SAITO $\mathrm{S}$ & BULL CHEM SOC JAPAN & 30 & 186 & 1957 & 57023 \\
\hline SAITO T & J CHEM SOC JAPAN, PURE CHEM SEC & 82 & 1512 & 1961 & 61010 \\
\hline SAKAI T & J CHEM SOC JAPAN, PURE CHEM SEC & 82 & 1512 & 1961 & 61010 \\
\hline SAMIS CS & TRANS FARADAY SOC & 32 & 795 & 1936 & 36001 \\
\hline & TRANS FARADAY SOC & 34 & 1288 & 1938 & 38005 \\
\hline SASAKI H & BULL CHEM SOC JAPAN & 30 & 186 & 1957 & 57023 \\
\hline & BULL CHEM SOC JAPAN & 30 & 326 & 1957 & 57030 \\
\hline SATA N & BULL CHEM SOC JAPAN & 26 & 177 & 1953 & 53006 \\
\hline SATAKE I & BULL CHEM SOC JAPAN & 35 & 1050 & 1962 & 62033 \\
\hline & BULL CHEM SOC JAPAN & 36 & 204 & 1963 & 63034 \\
\hline & BULL CHEM SOC JAPAN & 36 & 813 & 1963 & 63035 \\
\hline SATANEK $\mathrm{J}$ & $J$ AM OIL CHEMISTS SOC & 38 & 169 & 1961 & 61006 \\
\hline SATO M & REV PHYS CHEM JAPAN & 35 & 32 & 1965 & 65036 \\
\hline & J CHEM SOC JAPAN, PURE CHEM SEC & 87 & 329 & 1966 & 66033 \\
\hline SAFYER WM & $\mathrm{J}$ PHYS CHEM & 62 & 159 & 1958 & 58029 \\
\hline SCHICK MJ & $\mathrm{J}$ PHYS CHEM & 61 & 1062 & 1957 & 57014 \\
\hline & $\mathrm{J}$ COLLOID SCI & 17 & 801 & 1962 & 62019 \\
\hline & $\mathrm{J}$ PHYS CHEM & 66 & 1326 & 1962 & 62020 \\
\hline & $\mathrm{J}$ PHYS CHEM & 67 & 1796 & 1963 & 63026 \\
\hline & $J$ AM OIL CHEMISTS SOC & 40 & 680 & 1963 & 63027 \\
\hline & $\mathrm{J}$ COLLOID SCI & 18 & 378 & 1963 & 63028 \\
\hline & $J$ PHYS CHEM & 68 & 3585 & 1964 & 64020 \\
\hline & $\mathrm{J}$ COLLOID SCI & 20 & 464 & 1965 & 65011 \\
\hline & $\mathrm{J}$ AM OIL CHEMISTS SOC & 43 & 133 & 1966 & 66001 \\
\hline & J AM OIL CHEMISTS SOC & 43 & 681 & 1966 & 66025 \\
\hline SCHMID G & Z ELEKTROCHEM & 44 & 651 & 1938 & 38004 \\
\hline SCHOLBERG HM & $\mathrm{J}$ PHYS CHEM & 57 & 923 & 1953 & 53014 \\
\hline SCHOTT H & 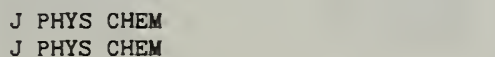 & $\begin{array}{l}68 \\
70\end{array}$ & $\begin{array}{l}3612 \\
2966\end{array}$ & $\begin{array}{l}1964 \\
1966\end{array}$ & $\begin{array}{l}64004 \\
66036\end{array}$ \\
\hline
\end{tabular}




\begin{tabular}{|c|c|c|c|c|c|}
\hline Author & Journal & Vol. & Pg. & Year & $\begin{array}{l}\text { Refer- } \\
\text { ence } \\
\text { No. }\end{array}$ \\
\hline SCIBONA G & ANN CHIM (ROME) & 52 & 1199 & 1962 & 62017 \\
\hline \multirow[t]{2}{*}{ SCOTT AB } & $J$ AM CHEM SOC & 65 & 692 & 1943 & 43003 \\
\hline & $\mathrm{J} A M$ CHEM SOC & 65 & 698 & 1943 & 43004 \\
\hline SCOTT R & NATURE & 167 & 195 & 1951 & 51012 \\
\hline SEARLES $\mathrm{J}$ & $\mathrm{J}$ PHYS CHEM & 40 & 493 & 1936 & 36005 \\
\hline SEBBA $F$ & ANAL CHEM & 38 & 1926 & 1966 & 66024 \\
\hline SHAFFER PM & Z PHYSIK CHEM (FRANKFURT) & 31 & 397 & 1962 & 62016 \\
\hline SHANE N & J PHYS CHEM & 69 & 968 & 1965 & 65025 \\
\hline SHEAFFER VE & $J$ PHYS CHEM & 68 & 2818 & 1964 & 64037 \\
\hline \multirow[t]{3}{*}{ SHEDLOVSKY L } & IND ENG CHEM & 36 & 610 & 1944 & 44001 \\
\hline & $J$ PHYS CHEM & 48 & 57 & 1944 & 44002 \\
\hline & J PHYS CHEM & 67 & 2075 & 1963 & 63001 \\
\hline \multirow[t]{3}{*}{ SHIGEHARA $K$} & BULL CHEM SOC JAPAN & 38 & 1700 & 1965 & 650 \\
\hline & BULL CHEM SOC JAPAN & 39 & 2643 & 1966 & 66009 \\
\hline & BULL CHEM SOC JAPAN & 39 & 2332 & 1966 & 66010 \\
\hline SHIGEHIRO F & KOGYO KAGAKU ZASSHI & 68 & 1090 & 1965 & 65017 \\
\hline \multirow[t]{10}{*}{ SHINODA K } & J PHYS CHEM & 58 & 1136 & 1954 & 54003 \\
\hline & $\mathrm{J}$ PHYS CHEM & 58 & 541 & 1954 & 54005 \\
\hline & BULL FAC ENG, YOKOHAMA NAT UNIV & 4 & 77 & 1955 & 55004 \\
\hline & $\mathrm{J}$ PHYS CHEM & 59 & 432 & 1955 & 55007 \\
\hline & $\mathrm{J}$ PHYS CHEM & 60 & 1439 & 1956 & 56003 \\
\hline & $\mathrm{J}$ PHYS CHEM & 63 & 648 & 1959 & 59013 \\
\hline & BULL CHEM SOC JAPAN & 34 & 237 & 1961 & 61008 \\
\hline & $\mathrm{J}$ COLLOID SCI & 18 & 705 & 1963 & 63036 \\
\hline & $J$ PHYS CHEM & 68 & 1568 & 1964 & 64011 \\
\hline & $\mathrm{J}$ PHYS CHEM & 70 & 3502 & 1966 & 66026 \\
\hline SHIRAHAMA $K$ & BULL CHEM SOC JAPAN & 38 & 373 & 1965 & 65020 \\
\hline \multirow{5}{*}{ SHIRAI M } & BULL CHEM SOC JAPAN & 28 & 545 & 1955 & 55010 \\
\hline & BULL CHEM SOC JAPAN & 29 & 733 & 1956 & 56009 \\
\hline & BULL CHEM SOC JAPAN & 30 & 411 & 1957 & 57015 \\
\hline & BULL CHEM SOC JAPAN & 30 & 542 & 1957 & 57016 \\
\hline & BULL CHEM SOC JAPAN & 31 & 467 & 1958 & 58007 \\
\hline SHISHIDO S & BULL CHEM SOC JAPAN & 24 & 41 & 1951 & 51017 \\
\hline SHOLTES EH & $\mathrm{J}$ COLLOID SCI & 1 & 385 & 1946 & 46020 \\
\hline SHUCK GR & $\mathrm{J}$ AM CHEM SOC & 71 & 1325 & 1949 & 49004 \\
\hline SHUTE HL & TRANS FARADAY SOC & 34 & 758 & 1938 & 38007 \\
\hline SIMON E & $\mathrm{J}$ CHEM PHYS & 15 & 496 & 1947 & 47012 \\
\hline SINGER $K$ & ANN REP PROGR CH M ( HI S. LONDON) & 45 & 51 & 1948 & 48003 \\
\hline SINGLETERRY CR & J AM CHEM SOC & 70 & 3965 & 1948 & 48017 \\
\hline & J COLLOID SCI & 4 & 537 & 1949 & 49012 \\
\hline & $J$ AM CHEM SOC & 73 & 4574 & 1951 & 51007 \\
\hline & $J$ PHYS CHEM & 58 & 201 & 1954 & 54009 \\
\hline & $\mathrm{J}$ PHYS CHEM & 60 & 1108 & 1956 & 56012 \\
\hline & J PHYS CHEM & 60 & 1114 & 1956 & 56013 \\
\hline & $\mathrm{J}$ COLLOID SCI & 12 & 465 & 1957 & 57026 \\
\hline & $\mathrm{J}$ COLLOID SCI & 13 & 569 & 1958 & 58024 \\
\hline & $J$ PHYS CHEM & 62 & 1257 & 1958 & 58025 \\
\hline & WORLD PETROL CONGR, PROC & v 6 & 18 & 1959 & 59021 \\
\hline & $\mathrm{J}$ PHYS CHEM & 68 & 3453 & 1964 & 64042 \\
\hline & $J$ PHYS CH M & 68 & 2709 & 1964 & 64044 \\
\hline SIROIS EH & $J$ PHYS CHEM & 56 & 701 & 1952 & 52013 \\
\hline SIVERTZ V & $\mathrm{J}$ AM CHEM SOC & 61 & 549 & 1939 & 39007 \\
\hline & $J$ AM CHEM SOC & 62 & 2375 & 1940 & 40001 \\
\hline SMEDS $\mathrm{K}$ & ACTA CHEM SCAND & 6 & 441 & 1952 & 52006 \\
\hline SMITH FD & $\mathrm{J}$ AM OIL CHEMISTS SOC & 40 & 538 & 1963 & 63013 \\
\hline SNELL FD & PROC INTERN CONGR SURFACE ACTIVITY & 2ND & & 1957 & 57024 \\
\hline SOLINAS M & BIOCHEM BIOPHYS ACTA & 88 & 415 & 1964 & 64031 \\
\hline & $\mathrm{J}$ PHYS CHEM & 68 & 3624 & 1964 & 64032 \\
\hline SOMASUNDARAN $P$ & J PHYS CHEM & 68 & 3562 & 1964 & 64035 \\
\hline & TRANS SME AIME & & 321 & 1964 & 64038 \\
\hline SPARKS B & TRANS FARADAY SOC & 62 & 3244 & 1966 & 66038 \\
\hline SPINGOLA F & J COLLOID SCI & 20 & 732 & 1965 & 65012 \\
\hline STANLEY JS & PROC INTERN CONGR SURFACE ACTIVITY & 3RD & 246 & 1960 & 60021 \\
\hline STAUFF J & Z PHYSIK CHEM (LEIPZIG) & 183 & 55 & 1939 & 39006 \\
\hline STEARNS RS & $\mathrm{J}$ CHEM PHYS & 14 & 215 & 1946 & 46011 \\
\hline & $\mathrm{J}$ CHEM PHYS & 14 & 214 & 1946 & 46017 \\
\hline & $\mathrm{J}$ CHEM PHYS & 15 & 496 & 1947 & 47012 \\
\hline STEIGMAN $J$ & $\mathrm{~J}$ COLLOID SCI & 20 & 732 & 1965 & 65012 \\
\hline & $J$ PHYS CHEM & 69 & 968 & 1965 & 65025 \\
\hline STERNBERG RJ & J POLYMER SCI & 5 & 191 & 1950 & 50005 \\
\hline STEWART A & TRANS FARADAY SOC & 31 & 208 & 1935 & 35003 \\
\hline STEWART JC & RES CORRESPONDENCE & 7 & 1 & 1955 & 55018 \\
\hline STIRTON AJ & $\mathrm{J}$ PHYS CHEM & 60 & 899 & 1956 & 56008 \\
\hline & $J$ PHYS CHEM & 62 & 1083 & 1958 & 58003 \\
\hline & $\mathrm{J}$ AM OIL CHEMISTS SOC & 36 & 241 & 1959 & 59004 \\
\hline & J AM OIL CHEMISTS SOC & 37 & 679 & 1960 & 60008 \\
\hline & $\mathrm{J}$ AM OIL CHEMISTS SOC & 39 & 55 & 1962 & 62008 \\
\hline & $\mathrm{J}$ AM OIL CHEMISTS SOC & 40 & 538 & 1963 & 63013 \\
\hline & $\mathrm{J}$ AM OIL CHEMISTS SOC & 41 & 205 & 1964 & 64002 \\
\hline & $\mathrm{J}$ AM OIL CHEMISTS SOC & 43 & 157 & 1966 & 66003 \\
\hline STOLL F & KOLLOID-Z & 63 & 49 & 1933 & 33004 \\
\hline
\end{tabular}




\begin{tabular}{|c|c|c|c|c|c|}
\hline Author & Journal & Vol. & Pg. & Year & $\begin{array}{l}\text { Refer- } \\
\text { ence } \\
\text { No. }\end{array}$ \\
\hline \multirow[t]{2}{*}{ STRICKS $\mathrm{W}$} & $\mathrm{J}$ PHYS COLLOID CHEM & 52 & 915 & 1948 & 48016 \\
\hline & $\mathrm{J}$ PHYS COLLOID CHEN & 53 & 424 & 1949 & 49005 \\
\hline SURYANARAY.CV & NATURE & 208 & 780 & 1965 & 65028 \\
\hline SWARBICK J & TRANS FARADAY SOC & 61 & 1043 & 1965 & 65003 \\
\hline \multirow[t]{2}{*}{ SZEGLOWSKI Z } & ZESZYTY NAUK UNIT JAGIEL & 211 & 199 & 1966 & 66034 \\
\hline & ZESZYTY NAUK UNIT JAGIEL & 211 & 209 & 1966 & 66035 \\
\hline TACHIBANA T & $\mathrm{J}$ PHYS CHEM & 66 & 363 & 1962 & 62024 \\
\hline TAMAKI $\mathrm{K}$ & BULL CHEM SOC JAPAN & 31 & 467 & 1958 & 58007 \\
\hline \multirow[t]{6}{*}{ TAMAMUSHI B } & REP INST SCI TECH UNIV TOKYO & 7 & 401 & 1953 & 53001 \\
\hline & BULL CHEM SOC JAPAN & 28 & 545 & 1955 & 55010 \\
\hline & BULL CH M S J & 9 & 733 & 1956 & 56009 \\
\hline & BULL CHEY SOC JAPAN & 30 & 411 & 1957 & 57015 \\
\hline & BULL CHEN SOC JAPAN & 30 & 542 & 1957 & 57016 \\
\hline & BULL CHEM SOC JAPAN & 31 & 467 & 1958 & 58007 \\
\hline \multirow[t]{19}{*}{ TARTAR HV } & J AM CHEM SOC & 57 & 570 & 1935 & 35006 \\
\hline & $\begin{array}{l}J \text { AM CHEM SOC } \\
J \text { AM CHEM SOC }\end{array}$ & $\begin{array}{l}58 \\
60\end{array}$ & $\begin{array}{l}322 \\
544\end{array}$ & $\begin{array}{l}1936 \\
1938\end{array}$ & $\begin{array}{l}36006 \\
38008\end{array}$ \\
\hline & $\mathrm{J}$ AM CHEM SOC & 61 & 539 & 1939 & 39002 \\
\hline & $\mathrm{J}$ AM CHEM SOC & 61 & 549 & 1939 & 39007 \\
\hline & $\mathrm{J}$ AM CHEM SOC & 61 & 544 & 1939 & 39010 \\
\hline & J AM CHEM SOC & 62 & 2375 & 1940 & 40001 \\
\hline & $\mathrm{J}$ AM CHEM SOC & 65 & 686 & 1943 & 43001 \\
\hline & J AM CHEM SOC & 65 & 692 & 1943 & 43003 \\
\hline & J AM CHEM SOC & 65 & 698 & 1943 & 43004 \\
\hline & J AM CHEM SOC & 68 & 1490 & 1946 & 46003 \\
\hline & J AM CHEM SOC & 70 & 1989 & 1948 & 48007 \\
\hline & $\mathrm{J}$ AM CHEM SOC & 70 & 1992 & 1948 & 48012 \\
\hline & $\mathrm{J}$ AM CHEM SOC & 73 & 5411 & 1951 & 51003 \\
\hline & $\mathrm{J}$ PHYS CHEM & 59 & 1190 & 1955 & 55006 \\
\hline & J PHYS CHEM & 59 & 1193 & 1955 & 55012 \\
\hline & $\mathrm{J}$ PHYS CHEM & 59 & 1195 & 1955 & 55013 \\
\hline & $\mathrm{J}$ PHYS CHEM & 59 & 1185 & 1955 & 55021 \\
\hline & J COLLOID SCI & 14 & 115 & 1959 & 59010 \\
\hline & $\mathrm{J}$ COLLOID SCI & 17 & 243 & 1962 & 62014 \\
\hline \multirow[t]{6}{*}{ TATE JR } & PROC ROY SOC & 273 & 84 & 1963 & 63014 \\
\hline & TRANS FARADAY SOC & 60 & 986 & 1964 & 64012 \\
\hline & TRANS FARADAY SOC & 60 & 996 & 1964 & 64027 \\
\hline & TRANS FARADAY SOC & 62 & 994 & 1966 & 66014 \\
\hline & TRANS FARADAY SOC & 62 & 987 & 1966 & 66015 \\
\hline & TRANS FARADAY SOC & 62 & 979 & 1966 & 66016 \\
\hline TAUBMAN AB & KHIM TEKHNOL & 5 & 61 & 1960 & 60033 \\
\hline \multirow[t]{2}{*}{ TAYLOR FH } & PHIL TRANS ROY SOC LONDON, SER A & 252 & 225 & 1960 & 60011 \\
\hline & TRANS FARADAY SOC & 58 & 1233 & 1962 & 62004 \\
\hline TAYLOR H & $\mathrm{J}$ COLLOID SCI & 19 & 495 & 1964 & 64050 \\
\hline THAKKAR AL & J PHARM SCI & 54 & 1529 & 1965 & 65027 \\
\hline THIBAULT HG & $\mathrm{J}$ PHYS CHEM & 56 & 701 & 1952 & 52013 \\
\hline THOMAS IL & J PHARM PHARMACOL & 15 & 522 & 1963 & 63005 \\
\hline & J PHARM PHARMACOL & 15 & 157 & 1963 & 63031 \\
\hline TOFIAS A & J PHYS CHEM & 56 & 701 & 1952 & 52013 \\
\hline TOKIWA F & J CHEM SOC JAPAN, PURE CHEM SEC & 82 & 1657 & 1961 & 61009 \\
\hline & J CHEM SOC JAPAN, PURE CHEM SEC & 82 & 1512 & 1961 & 61010 \\
\hline & J CHEM SOC JAPAN, PURE CHEM SEC & 82 & 1660 & 1961 & 61011 \\
\hline & BULL CHEM SOC JAPAN & 34 & 1236 & 1961 & 61012 \\
\hline & J CHEM SOC JAPAN, PURE CHEM SEC & 83 & 732 & 1962 & 62007 \\
\hline & BULL CHEM SOC JAPAN & 35 & 1737 & 1962 & 62012 \\
\hline & BULL CHEM SOC JAPAN & 35 & 240 & 1962 & 62022 \\
\hline & BULL CHEM SOC JAPAN & 36 & 222 & 1963 & 63003 \\
\hline & BULL CHEM SOC JAPAN & 36 & 1589 & 1963 & 63004 \\
\hline & BULL CHEM SOC JAPAN & 36 & 281 & 1963 & 63007 \\
\hline & BULL CHEM SOC JAPAN & 36 & 1585 & 1963 & 63023 \\
\hline & $J$ CHEM EDUC & 40 & 472 & 1963 & 63029 \\
\hline & BULL CHEM SOC JAPAN & 37 & 1837 & 1964 & 64024 \\
\hline & J PHYS CHEM & 68 & 2818 & 1964 & 64037 \\
\hline & BULL CHEM SOC JAPAN & 38 & 751 & 1965 & 65014 \\
\hline & $\mathrm{J}$ PHYS CHEM & 70 & 3437 & 1966 & 66027 \\
\hline TONG LKJ & $\mathrm{J}$ PHYS CHEM & 69 & 2357 & 1965 & 65030 \\
\hline TORI $\mathrm{K}$ & J CHEM SOC JAPAN, PURE CHEM SEC & 78 & 1573 & 1957 & 57020 \\
\hline & KOLLOID-Z & 168 & 132 & 1960 & 60009 \\
\hline & KOLLOID-Z & 189 & 50 & 1963 & 63008 \\
\hline & KOLLOID-Z & 188 & 47 & 1963 & 63009 \\
\hline & KOLLOID-Z & 187 & 44 & 1963 & 63010 \\
\hline & KOLLOID-Z & 191 & 48 & 1963 & 63012 \\
\hline & KOLLOID-Z & 194 & 143 & 1964 & 64015 \\
\hline TRAP HJL & KONINKI NED AKAD WETEN. PROC SER B & 58 & 97 & 1955 & 55009 \\
\hline TREBBI GF & J PHYS CHEM & 67 & 1987 & 1963 & 63017 \\
\hline & $J$ AM OIL CHEMISTS SOC & 41 & 231 & 1964 & 64013 \\
\hline & $J$ PHYS CHEM & 68 & 3592 & 1964 & 64014 \\
\hline & $\mathrm{J}$ COLLOID SCI & 20 & 191 & 1965 & 65001 \\
\hline TSIKURINA NN & KOLLOID ZH & 26 & 76 & 1964 & 64051 \\
\hline TUDDENHAM RF & J PHYS CHEM & 66 & 1839 & 1962 & 62035 \\
\hline
\end{tabular}




\section{Author Index-Continued}

\begin{tabular}{|c|c|c|c|c|c|}
\hline Author & Journal & Vol. & Pg. & Year & $\begin{array}{c}\text { Refer- } \\
\text { ence } \\
\text { No. }\end{array}$ \\
\hline \multirow[t]{4}{*}{ TYUZYO $\mathrm{K}$} & BULL CHEM SOC JAPAN & 26 & 177 & 1953 & 53006 \\
\hline & BULL CHEM SOC JAPAN & 27 & 259 & 1954 & 54008 \\
\hline & BULL CHEM SOC JAPAN & 31 & 117 & 1958 & 58026 \\
\hline & KOLLOID-Z & 175 & 40 & 1961 & 61025 \\
\hline UBBELOHDE AR & J COLLOID SCI & 8 & 424 & 1953 & 53008 \\
\hline ULEVITCH IN & J COLLOID SCI & 9 & 382 & 1954 & 54006 \\
\hline$\checkmark$ DEN TEMPEL M & PROC INTERN CONGR SURFACE ACTIVITY & 3RD & 248 & 1960 & 60022 \\
\hline \multirow[t]{5}{*}{ V VOORST VA.F } & PROC INTERN CONGR SURFACE ACTIVITY & 3RD & 276 & 1960 & 60013 \\
\hline & TRANS FARADAY SOC & 56 & 1078 & 1960 & 60014 \\
\hline & PROC INTERN CONGR SURFACE ACTIVITY & 3RD & 248 & 1960 & 60022 \\
\hline & TRANS FARADAY SOC & 56 & 1067 & 1960 & 60025 \\
\hline & TRANS FARADAY SOC & 57 & 110 & 1961 & 61026 \\
\hline \multirow[t]{2}{*}{ VASSILIADES T } & $\mathrm{J}$ PHYS CHEM & 65 & 1781 & 1961 & 61027 \\
\hline & $\mathrm{J}$ PHYS CHEM & 65 & 1774 & 1961 & 61028 \\
\hline VEIS A & J COLLOID SCI & 15 & 427 & 1960 & 60001 \\
\hline VENABLE RL & $\mathrm{J}$ PHYS CHEM & 68 & 3498 & 1964 & 64001 \\
\hline \multirow[t]{2}{*}{ VERGNOLLE J } & MEM SERV CHIM ETAT (PARIS) & 41 & 363 & 1956 & 56010 \\
\hline & PROC INTERN CONGR SURFACE ACTIVITY & 2ND & 395 & 1957 & 57017 \\
\hline VERMA SP & INDIAN J CHEM & 3 & 441 & 1965 & 65021 \\
\hline VETTER RJ & $\mathrm{J}$ PHYS COLLOID CHEM & 51 & 263 & 1947 & $\$ 7011$ \\
\hline VINOGRAD JR & $\mathrm{J}$ AM CHEM SOC & 63 & 670 & 1941 & 41005 \\
\hline VOEKS JF & $\mathrm{J}$ PHYS CHEM & 59 & 1190 & 1955 & 55006 \\
\hline WACHS W & KOLLOID-Z & 181 & 139 & 1962 & 62023 \\
\hline WAGNER E & PROC INTERN CONGR SURFACE ACTIVITY & $3 R D$ & 27 & 1960 & 60017 \\
\hline WALKER T & TRANS FARADAY SOC & 61 & 589 & 1965 & 65004 \\
\hline WALTON HF & J COLLOID SCI & 1 & 385 & 1946 & 46020 \\
\hline WAN LSC & J PHARM SCI & 55 & 1395 & 1966 & 66018 \\
\hline \multirow[t]{2}{*}{ WARD AFH } & $\mathrm{J}$ CHEM SOC & & 522 & 1939 & 39003 \\
\hline & PROC ROY SOC & 176 & 412 & 1940 & 40004 \\
\hline WASIK SP & J RES NAT BUR STD A & 68 & 359 & 1964 & 64043 \\
\hline WATARI Y & J SCI HIROSHIMA UNIV, SER A-II & 28 & 41 & 1964 & 64036 \\
\hline WEATHERBURN AS & CAN J CHEM ENGR & F28 & 213 & 1950 & 50010 \\
\hline \multirow[t]{8}{*}{ WEIL JK } & J PHYS CHEM & 60 & 899 & 1956 & 56008 \\
\hline & $\mathrm{J}$ PHYS CHEM & 62 & 1083 & 1958 & 58003 \\
\hline & J AM OIL CHEMISTS SOC & 36 & 241 & 1959 & 59004 \\
\hline & J AM OIL CHEMISTS SOC & 37 & 679 & 1960 & 60008 \\
\hline & J AM OIL CHEMISTS SOC & 39 & 55 & 1962 & 62008 \\
\hline & J AM OIL CHEMISTS SOC & 40 & 538 & 1963 & 63013 \\
\hline & J AM OIL CHEMISTS SOC & 41 & 205 & 1964 & 64002 \\
\hline & J AM OIL CHEMISTS SOC & 43 & 157 & 1966 & 66003 \\
\hline WEINER ND & J PHARM SCI & 54 & 436 & 1965 & 65026 \\
\hline \multirow[t]{2}{*}{ WESTWELL AE } & $J$ PHYS CHEM & 63 & 1022 & 1959 & 59011 \\
\hline & $\mathrm{J}$ PHYS CHEM & 68 & 3490 & 1964 & 64018 \\
\hline WHEELER OL & $\mathrm{J}$ AM CHEM SOC & 68 & 1490 & 1946 & 46003 \\
\hline & $\mathrm{J}$ AM CHEM SOC & 70 & 1989 & 1948 & 48007 \\
\hline WHITE P & $\mathrm{J}$ COLLOID SCI & 13 & 584 & 1958 & 58005 \\
\hline & TRANS FARADAY SOC & 54 & 1638 & 1958 & 58006 \\
\hline & CAN J CHEM & 37 & 2086 & 1959 & 59008 \\
\hline & TRANS FARADAY SOC & 55 & 1025 & 1959 & 59012 \\
\hline & $\mathrm{J}$ PHYS CHEM & 64 & 599 & 1960 & 60002 \\
\hline WILDER AG & $\mathrm{J}$ PHYS COLLOID CHEM & 52 & 12 & 1948 & 48026 \\
\hline WILLIAMS DE & J POLYMER SCI & 5 & 201 & 1950 & 50002 \\
\hline WILLIAMS EF & $\mathrm{J}$ COLLOID SCI & 12 & 452 & 1957 & 57009 \\
\hline WILLIAMS G & ANN REP PROGR CHEM (CH. S. LONDON) & 45 & 51 & 1948 & 48003 \\
\hline WILLIAMS RJ & TRANS FARADAY SOC & 51 & 728 & 1955 & 55005 \\
\hline WINSLOW L & $\mathrm{J}$ PHYS CHEM & 58 & 1124 & 1954 & 54012 \\
\hline & Z PHYSIK CHEM (FRANKFURT) & 11 & 165 & 1957 & 57021 \\
\hline WINSOR PA & TRANS FARADAY SOC & 44 & 463 & 1948 & 48008 \\
\hline & $\mathrm{J}$ PHYS CHEM & 56 & 391 & 1952 & 52009 \\
\hline WOODBERRY $\mathrm{N}$ & $\mathrm{J}$ COLLOID SCI & 12 & 452 & 1957 & 57009 \\
\hline WOODWARD RJ & J PHARM PHARMACOL & 15 & 422 & 1963 & 63015 \\
\hline WRIGHT KA & $\mathrm{J}$ AM CHEM SOC & 61 & 539 & 1939 & 39002 \\
\hline & J AM CHEM SOC & 61 & 549 & 1939 & 39007 \\
\hline & $\mathrm{J}$ AM CHEM SOC & 61 & 544 & 1939 & 39010 \\
\hline YAMAGUCHI T & BULL CHEM SOC JAPAN & 34 & 237 & 1961 & 61008 \\
\hline YAMANAKA $T$ & J PHYS CHEM & 63 & 648 & 1959 & 59013 \\
\hline YANG JT & $\mathrm{J}$ PHYS CHEM & 57 & 628 & 1953 & 53015 \\
\hline YODA 0 & J CHEM SOC JAPAN, PURE CHEM SEC & 77 & 1236 & 1956 & 56020 \\
\hline & BULL CHEM SOC JAPAN & 30 & 760 & 1957 & 57033 \\
\hline YOUNG HS & J AM CHEM SOC & 71 & 309 & 1949 & 49017 \\
\hline YURZHENKO AI & COLLOID J (USSR) & 14 & 243 & 1952 & 52018 \\
\hline & COLLOID J (USSR) & 14 & 311 & 1952 & 52019 \\
\hline ZAKHAROVA NN & UKR KHIM ZH & 28 & 611 & 1962 & 62038 \\
\hline ZOELLNER M & $\mathrm{J}$ PHYS CHEM & 65 & 1804 & 1961 & 61001 \\
\hline ZOGRAFI G & $\mathrm{J}$ PHARM SCI & 54 & 436 & 1965 & 65026 \\
\hline & J PHARM SCI & 55 & 1345 & 1966 & 66017 \\
\hline ZUTRAUEN HA & J CHIM PHYS & 53 & 62 & 1956 & 56017 \\
\hline
\end{tabular}


List of References

\begin{tabular}{|c|c|c|c|c|c|c|c|c|c|}
\hline \multirow{2}{*}{$\begin{array}{c}\begin{array}{c}\text { Refer- } \\
\text { ence } \\
\text { No. }\end{array} \\
27001\end{array}$} & \multicolumn{3}{|c|}{ Authors } & Journal & \multirow{2}{*}{$\begin{array}{r}\text { Vol. } \\
4\end{array}$} & \multirow{2}{*}{$\underbrace{P g}_{1}$} & \multirow{2}{*}{$\begin{array}{l}\text { Year } \\
1927\end{array}$} & \multicolumn{2}{|c|}{$\begin{array}{l}\text { No. of Entries from } \\
\text { Reference }\end{array}$} \\
\hline & EKWALL $P$ & & & ACTA ACAD ABOENSIS, MATH PHYS & & & & YIELDED & 5 ENTRIES \\
\hline 27002 & JONES E & BURY CR & & PHIL MAG & 4 & 841 & 1927 & YIELDED & 2 ENTRIES \\
\hline 28001 & EKWALL $P$ & & & KOLLOID-Z & 45 & 291 & 1928 & NO ENTRIES & \\
\hline 29001 & GRINDLEY $\mathrm{J}$ & BURY CR & & $J$ CHEM SOC & & 679 & 1929 & YIELDED & 5 ENTRIES \\
\hline 30001 & DAVIES DG & BURY CR & & $J$ CHEM SOC & & 2263 & 1930 & Y IELDED & 1 ENTRIES \\
\hline 32001 & EKWALL $P$ & & & KOLLOID-Z & 161 & 195 & 1932 & YIELDED & 5 ENTRIES \\
\hline 33001 & EKWALL $P$ & & & ACTA ACAD ABOENSIS, MATH PHYS & 7 & 3 & 1933 & NO ENTRIES & \\
\hline 33002 & EKWALL $P$ & & & ACTA ACAD ABOENSIS, MATH PHYS & 7 & 3 & 1933 & NO ENTRIES & \\
\hline 33003 & LOTTERMOSER A & PUSCHEL $F$ & & KOLLOID-Z & 63 & 175 & 1933 & NO ENTRIES & \\
\hline 33004 & LOTTERMOSER A & STOLL $\mathrm{F}$ & & KOLLOID-Z & 63 & 49 & 1933 & NO ENTRIES & \\
\hline 34001 & MALSCH J & HARTLEY GS & & Z PHYSIK CHEM (LEIPZIG) & 170 & 321 & 1934 & Y IELDED & 1 ENTRIES \\
\hline 35001 & MURRAY RC & HARTLEY GS & & TRANS FARADAY SOC & 31 & 183 & 1935 & Y IELDED & 1 ENTRIES \\
\hline 35002 & LOTTERMOSER A & GIESE E & & KOLLOID-Z & 73 & 276 & 1935 & NO ENTRIES & \\
\hline 35003 & STEWART A & BUNBURY HM & & TRANS FARADAY SOC & 31 & 208 & 1935 & NO ENTRIES & \\
\hline 35004 & LOTTERMOSER A & & & TRANS FARADAY SOC & 31 & 200 & 1935 & NO ENTRIES & \\
\hline 35005 & POWNEY J & & & TRANS FARADAY SOC & 31 & 1510 & 1935 & NO ENTRIES & \\
\hline 35006 & REED RM & TARTAR HV & & $\mathrm{J}$ AM CHEM SOC & 57 & 570 & 1935 & NO ENTRIES & \\
\hline 35007 & MCBAIN JW & BETZ MD & & J AM CHEM SOC & 57 & 1905 & 1935 & NO ENTRIES & \\
\hline 35008 & BURY CR & PARRY GA & & $\mathrm{J}$ CHEM SOC & & 626 & 1935 & Y IELDED & 2 ENTRIES \\
\hline 36001 & HARTLEY GS & COLLIE B & SAMIS CS & TRANS FARADAY SOC & 32 & 795 & 1936 & Y IELDED & 5 ENTRIES \\
\hline 36002 & HARTLEY GS & & & $\mathrm{J}$ AM CHEM SOC & 58 & 2347 & 1936 & YIELDED & 8 ENTRIES \\
\hline 36003 & EKWALL $P$ & & & KOLLOID-Z & 77 & 320 & 1936 & NO ENTRIES & \\
\hline 36004 & ADAM NK & & & TRANS FARADAY SOC & 32 & 653 & 1936 & NO ENTRIES & \\
\hline 36005 & MCBAIN JW & SEARLES $\mathrm{J}$ & & J PHYS CHEM & 40 & 493 & 1936 & NO ENTRIES & \\
\hline 36006 & REED RM & TARTAR HV & & $\mathrm{J}$ AM CHEM SOC & 58 & 322 & 1936 & NO ENTRIES & \\
\hline 37001 & EKWALL $P$ & & & KOLLOID-Z & 80 & 77 & 1937 & NO ENTRIES & \\
\hline 37003 & POWNEY J & ADDISON CC & & TRANS FARADAY SOC & 33 & 851 & 1937 & NO ENTRIES & \\
\hline 37004 & LOTTERMOSER A & FROTSCHER H & & KOLLOID-BEIH. & 45 & 303 & 1937 & NO ENTRIES & \\
\hline 37005 & LONG FA & NUTTING GC & HARKINS WD & $\mathrm{J}$ AM CHEM SOC & 59 & 2197 & 1937 & NO ENTRIES & \\
\hline 38001 & HARTLEY GS & & & $\mathrm{J}$ CHEM SOC & & 1968 & 1938 & YIELDED & 4 ENTRIES \\
\hline 38002 & EKWALL $P$ & & & KOLLOID Z Z & 84 & 284 & 1938 & NO ENTRIES & \\
\hline 38003 & HARTLEY GS & & & NATURE & 142 & 161 & 1938 & NO ENTRIES & \\
\hline 38004 & SCHMID G & LARSEN EC & & Z ELEKTROCHEM & 44 & 651 & 1938 & NO ENTRIES & \\
\hline 38005 & SAMIS CS & HARTLEY GS & & TRANS FARADAY SOC & 34 & 1288 & 1938 & NO ENTRIES & \\
\hline 38006 & POWNEY J & ADDISON CC & & TRANS FARADAY SOC & 34 & 372 & 1938 & YIELDED & 7 ENTRIES \\
\hline 38007 & ADAM NK & SHUTE HL & & TRANS FARADAY SOC & 34 & 758 & 1938 & NO ENTRIES & \\
\hline 38008 & HOULTON HG & TARTAR HV & & $\mathrm{J}$ AM CHEM SOC & 60 & 544 & 1938 & NO ENTRIES & \\
\hline 39001 & HARTLEY GS & & & TRANS FARADAY SOC & 35 & 1109 & 1939 & NO ENTRIES & \\
\hline 39002 & TARTAR HV & WRIGHT KA & & J AM CHEM SOC & 61 & 539 & 1939 & Y IELDED & 5 ENTRIES \\
\hline 39003 & WARD AFH & & & $\mathrm{J}$ CHEM SOC & & 522 & 1939 & NO ENTRIES & \\
\hline 39004 & EKWALL $P$ & & & FINSKA KEMISTSAMFUNDETS MEDD & 1 & 8 & 1939 & NO ENTRIES & \\
\hline 39005 & HARTLEY GS & & & KOLLOID-Z & 88 & 22 & 1939 & NO ENTRIES & \\
\hline 39006 & STAUFF J & & & Z PHYSIK CHEM (LEIPZIG) & 183 & 55 & 1939 & YIELDED & 5 ENTRIES \\
\hline 39007 & $\begin{array}{l}\text { WRIGHT KA } \\
\text { TARTAR HV }\end{array}$ & ABBOTT AD & SIVERTZ V & J AM CHEM SOC & 61 & 549 & 1939 & YIELDED $1:$ & 12 ENTRIES \\
\hline 39008 & HARTLEY GS & & & $\mathrm{J}$ CHEM SOC & & 1828 & 1939 & NO ENTRIES & \\
\hline 39009 & HESS $\mathrm{K}$ & PHILIPPOFF W & KIESSIG H & KOLLOID-Z & 88 & 40 & 1939 & Y IELDED & 9 ENTRIES \\
\hline 39010 & WRIGHT KA & TARTAR HV & & J AM CHEM SOC & 61 & 544 & 1939 & NO ENTRIES & 5 \\
\hline 39011 & MCBAIN MEL & DYE WB & JOHNSTON SA & J AM CHEM SOC & 61 & 3210 & 1939 & YIELDED & 7 ENTRIES \\
\hline 40001 & TARTAR HV & SIVERTZ V & REITMIER RE & J AM CHEM SOC & 62 & 2375 & 1940 & NO ENTRIES & \\
\hline 40002 & EKWALL $P$ & & & KOLLOID-Z & 92 & 141 & 1940 & NO ENTRIES & \\
\hline 40003 & EKWALL $P$ & & & TEK FOREN FINLAND FORH & 10 & 1 & 1940 & YIELDED & 4 ENTRIES \\
\hline 40004 & WARD AFH & & & PROC ROY SOC & 176 & 412 & 1940 & YIELDED & 8 ENTRIES \\
\hline 40005 & NUTTING GC & LONG FA & HARKINS WD & $\mathrm{J}$ AM CHEM SOC & 62 & 1496 & 1940 & NO ENTRIES & \\
\hline 41001 & HARTLEY GS & & & TRANS FARADAY SOC & 37 & 130 & 1941 & NO ENTRIES & \\
\hline 41002 & EKWALL $P$ & LINDSTROM RE & & MEDD . ABO AKAD . FYS KEM . IN. & SP NO & 3 & 1941 & NO ENTRIES & \\
\hline 41003 & EKWALL $P$ & & & KOLLOID-Z & 97 & 71 & 1941 & YIELDED & 2 ENTRIES \\
\hline 41004 & EKWALL P & LINDSTROM RE & & KOLLOID-Z & 94 & 42 & 1941 & YIELDED & 1 ENTRIES \\
\hline 41005 & MCBAIN JW & MERRILL RC JR & VINOGRAD JR & J AM CHEM SOC & 63 & 670 & 1941 & NO ENTRIES & \\
\hline 41006 & NUTTING GC & LONG FA & & $\mathrm{J}$ AM CHEM SOC & 63 & 84 & 1941 & NO ENTRIES & \\
\hline 42001 & HOERR CW & RALSTON AW & & J AM CHEM SOC & 64 & 2824 & 1942 & NO ENTRIES & \\
\hline 42002 & RALSTON AW & HOERR CW & & J AM CHEM SOC & 64 & 772 & 1942 & Y IELDED & 7 ENTRIES \\
\hline 42003 & HAFFNER FD & PICCIONE GA & ROSENBLUM C & J PHYS CHEM & 46 & 662 & 1942 & Y IELDED & 8 ENTRIES \\
\hline 42004 & EKWALL $P$ & & & KOLLOID-Z & 101 & 135 & 1942 & YIELDED 10 & 10 ENTRIES \\
\hline 42005 & RALSTON AW & HOERR CW & HOFFMAN EJ & J AM CHEM SOC & 64 & 97 & 1942 & NO ENTRIES & \\
\hline 42006 & MERRILL RC JR & MCBAIN JW & & J PHYS CHEM & 46 & 10 & 1942 & NO ENTRIES & \\
\hline 42007 & MCBAIN JW & MERRILL RC JR & & IND ENG CHEM & 34 & 915 & 1942 & NO ENTRIES & \\
\hline 42008 & HOFFMAN EJ & BOYD GE & RALSTON AW & J AM CHEM SOC & 64 & 2067 & 1942 & NO ENTRIES & \\
\hline 42009 & HOFFMAN EJ & BOYD GE & RALSTON AW & J AM CHEM SOC & 64 & 498 & 1942 & NO ENTRIES & \\
\hline 43001 & PAQUETTE RG & LINGAFELTER EC & TARTAR HV & $\mathrm{J}$ AM CHEM SOC & 65 & 686 & 1943 & YIELDED & 8 ENTRIES \\
\hline 43002 & HOERR CW & RALSTON AW & & $\mathrm{J}$ AM CHEM SOC & 65 & 976 & 1943 & NO ENTRIES & \\
\hline 43003 & SCOTT AB & TARTAR HV & & J AM CHEM SOC & 65 & 692 & 1943 & Y IELDED 10 & 10 ENTRIES \\
\hline 43004 & SCOTT AB & TARTAR HV & LINGAFELTER EC & $J$ AM CHEM SOC & 65 & 698 & 1943 & YIELDED & 2 ENTRIES \\
\hline 43005 & EKWALL $P$ & HARVA 0 & & FINSKA KEMISTSAMFUNDETS MEDD & 25 & 257 & 1943 & NO ENTRIES & \\
\hline 43006 & MCBAIN JW & BRADY AP & & J AM CHEM SOC & 65 & 2072 & 1943 & YIELDED & 2 ENTRIES \\
\hline
\end{tabular}




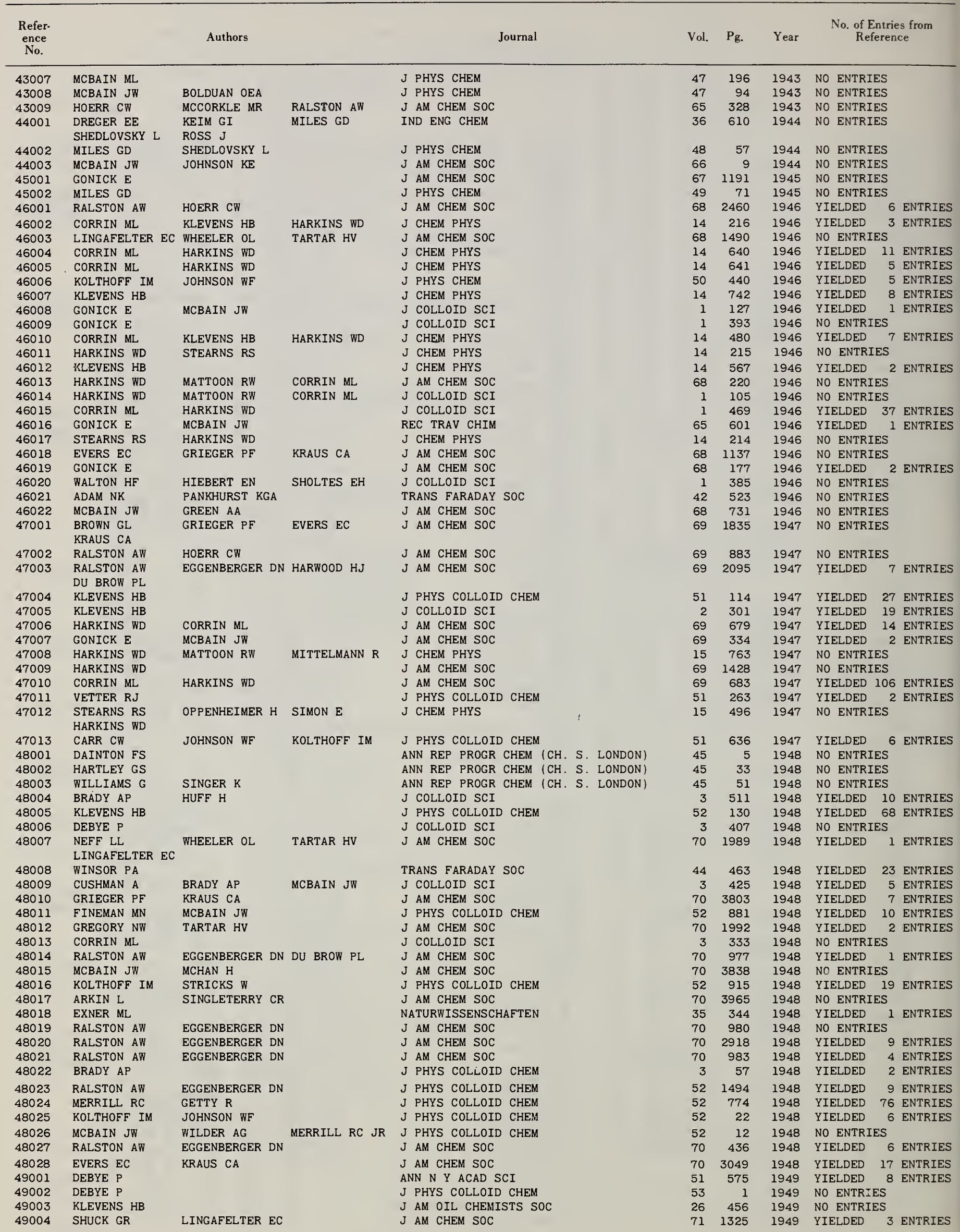




\begin{tabular}{|c|c|c|c|c|c|c|c|c|c|c|}
\hline \multirow{2}{*}{$\begin{array}{c}\begin{array}{c}\text { Refer- } \\
\text { ence } \\
\text { No. }\end{array} \\
49005\end{array}$} & \multicolumn{3}{|c|}{ Authors } & \multirow{2}{*}{$\frac{\text { Journal }}{\text { LD CHEM }}$} & \multirow{2}{*}{$\begin{array}{r}\text { Vol. } \\
53\end{array}$} & \multirow{2}{*}{$\begin{array}{l}\mathrm{Pg} . \\
424\end{array}$} & \multirow{2}{*}{$\begin{array}{c}\text { Year } \\
1949\end{array}$} & $\begin{array}{r}\text { No. of } \\
\text { Re }\end{array}$ & $\begin{array}{l}\text { Entri } \\
\text { feren }\end{array}$ & $\begin{array}{l}\text { ries from } \\
\text { ence }\end{array}$ \\
\hline & KOLTHOFF IM & STRICKS W & & & & & & YIELDED & 49 & ENTRIES \\
\hline 49006 & HARKINS WD & MITTELMANN $\mathrm{R}$ & CORRIN ML & $\mathrm{J}$ PHYS COLLOID CHEM & 53 & 1350 & 1949 & YIELDED & 41 & ENTRIES \\
\hline 49007 & HARKINS WD & MITTELMANN $R$ & & J COLLOID SCI & 4 & 367 & 1949 & NO ENTRIE & & \\
\hline 49008 & RALSTON AW & EGGENBERGER DN & BROOME FK & J AM CHEM SOC & 71 & 2145 & 1949 & YIELDED & 45 & ENTRIES \\
\hline 49009 & RALSTON AW & EGGENBERGER DN & HARWOOD HJ & $\mathrm{J}$ AM CHEM SOC & 71 & 672 & 1949 & YIELDED & 1 & ENTRIES \\
\hline 49010 & HARTLEY GS & & & NATURE & 163 & 767 & 1949 & NO ENTRIE & & \\
\hline 49011 & HARKINS WD & OPPENHEIMER $\mathrm{H}$ & & J AM CHEM SOC & 71 & 808 & 1949 & NO ENTRIE & & \\
\hline 49012 & ARKIN L & SINGLETERRY CR & & J COLLOID SCI & 4 & 537 & 1949 & NO ENTRIE & & \\
\hline 49013 & RALSTON AW & BROOME FK & HARWOOD HJ & J AM CHEM SOC & 71 & 671 & 1949 & YIELDED & 4 & ENTRIES \\
\hline 49014 & BROWN GL & GRIEGER PF & KRAUS CA & J AM CHEM SOC & 71 & 95 & 1949 & YIELDED & 22 & ENTRIES \\
\hline 49015 & ADCOCK WA & COLE RH & & J AM CHEM SOC & 71 & 2835 & 1949 & NO ENTRIE & & \\
\hline 49017 & YOUNG HS & GRIEGER PF & KRAUS CA & $\mathrm{J}$ AM CHEM SOC & 71 & 309 & 1949 & YIELDED & 15 & ENTRIES \\
\hline 49018 & GRIEGER PF & KRAUS CA & & J AM CHEM SOC & 71 & 1455 & 1949 & YIELDED & 2 & ENTRIES \\
\hline 50001 & DEBYE $P$ & & & AN. R. SOC ESPAN FIS QUIM (MADRID) & 46 & 335 & 1950 & NO ENTRIE & & \\
\hline 50002 & $\begin{array}{l}\text { CARR CW } \\
\text { WILLIAMS DE }\end{array}$ & KOLTHOFF IM & MEEHAN EJ & J POLYMER SCI & 5 & 201 & 1950 & NO ENTRIE & & \\
\hline 50003 & KLEVENS HB & & & $\mathrm{J}$ PHYS COLLOID CHEM & 54 & 1012 & 1950 & YIELDED & 30 & ENTRIES \\
\hline 50004 & KLEVENS HB & & & J AM CHEM SOC & 72 & 3780 & 1950 & NO ENTRIE & & \\
\hline 50005 & $\begin{array}{l}\text { CARR CW } \\
\text { STERNBERG RJ }\end{array}$ & KOLTHOFF IM & MEEHAN EJ & J POLYMER SCI & 5 & 191 & 1950 & NO ENTRIE & & \\
\hline 50006 & KLEVENS HB & & & ANAL CHEM & 22 & 1141 & 1950 & NO ENTRIE & & \\
\hline 50007 & KLEVENS HB & & & CHEM REV & 47 & 1 & 1950 & NO ENTRIE & & \\
\hline 50008 & HERZFELD SH & CORRIN ML & HARKINS WD & J PHYS COLLOID CHEM & 54 & 271 & 1950 & YIELDED & 98 & ENTRIES \\
\hline 50009 & GOTTE E & & & KOLLOID-Z & 117 & 42 & 1950 & NO ENTRIE & & \\
\hline 50010 & WEATHERBURN AS & ROSE GRF & BAYLEY CH & CAN J CHEM ENGR & F28 & 213 & 1950 & NO ENTRIE & & \\
\hline 50011 & HARKINS WD & & & SCI MONTHLY & 70 & 220 & 1950 & NO ENTRIE & & \\
\hline 50012 & COLICHMAN EL & & & J AM CHEM SOC & 72 & 4036 & 1950 & YIELDED & 38 & ENTRIES \\
\hline 50013 & KUHN DW & KRAUS CA & & J AM CHEM SOC & 72 & 3676 & 1950 & NO ENTRIE & & \\
\hline 51001 & DEBYE $\mathrm{P}$ & ANACKER EW & & $J$ PHYS COLLOID CHEM & 55 & 644 & 1951 & YIELDED & 1 & ENTRIES \\
\hline 51002 & KOLTHOFF IM & JOHNSON WF & & J AM CHEM SOC & 73 & 4563 & 1951 & NO ENTRIE & & \\
\hline 51003 & $\begin{array}{l}\text { LELONG ALM } \\
\text { OLOANE JK }\end{array}$ & $\begin{array}{l}\text { TARTAR HV } \\
\text { CADLE RD }\end{array}$ & LINGAFELTER EC & J AM CHEM SOC & 73 & 5411 & 1951 & YIELDED & 10 & ENTRIES \\
\hline 51004 & HUFF $\mathrm{H}$ & MCBAIN JW & BRADY AP & $\mathrm{J}$ PHYS COLLOID CHEM & 55 & 311 & 1951 & YIELDED & 29 & ENTRIES \\
\hline 51005 & LANGE $\mathrm{H}$ & & & KOLLOID $-Z$ & 121 & 66 & 1951 & YIELDED & 94 & ENTRIES \\
\hline 51006 & EGGENBERGER DN & HARWOOD HJ & & J AM CHEM SOC & 73 & 3353 & 1951 & YIELDED & 8 & ENTRIES \\
\hline 51007 & SINGLETERRY CR & ARKIN L & & $\mathrm{J}$ AM CHEM SOC & 73 & 4574 & 1951 & NO ENTRIE & & \\
\hline 51008 & COLICHMAN EL & & & J AM CHEM SOC & 73 & 3385 & 1951 & YIELDED & 15 & ENTRIES \\
\hline 51009 & MCDOWELL MJ & KRAUS CA & & J AM CHEM SOC & 73 & 2173 & 1951 & YIELDED & 5 & ENTRIES \\
\hline 51010 & HARKINS WD & KRIZEK H & CORRIN ML & J COLLOID SCI & 6 & 576 & 1951 & YIELDED & 6 & ENTRIES \\
\hline 51011 & HENNE AL & FOX CJ & & $\mathrm{J}$ AM CHEM SOC & 73 & 2323 & 1951 & NO ENTRIE & & \\
\hline 51012 & SCOTT R & BOLAM TR & & NATURE & 167 & 195 & 1951 & NO ENTRIE & & \\
\hline 51013 & DAGGETT HM JR & BAIR EJ & KRAUS CA & $\mathrm{J}$ AM CHEM SOC & 73 & 799 & 1951 & NO ENTRIE & & \\
\hline 51014 & BAIR EJ & KRAUS CA & & J AM CHEM SOC & 73 & 1129 & 1951 & NO ENTRIE & & \\
\hline 51016 & MCDOWELL MJ & KRAUS CA & & $J$ AM CHEM SOC & 73 & 2170 & 1951 & NO ENTRIE & & \\
\hline 51017 & SHISHIDO $\mathrm{S}$ & & & BULL CHEM SOC JAPAN & 24 & 41 & 1951 & NO ENTRIE & & \\
\hline 51018 & NAKAGAKI M & & & J CHEM SOC JAPAN, PURE CHEM SEC & 72 & 113 & 1951 & NO ENTRIE & & \\
\hline 52001 & $\begin{array}{l}\text { CELLA JA } \\
\text { HARRIMAN LA }\end{array}$ & $\begin{array}{l}\text { EGGENBERGER DN } \\
\text { HARWOOD HJ }\end{array}$ & NOEL DR & J AM CHEM SOC & 74 & 2061 & 1952 & YIELDED & 14 & ENTRIES \\
\hline 52002 & KOLTHOFF IM & JOHNSON WF & & $J$ AM CHEM SOC & 74 & 20 & 1952 & NO ENTRIE & & \\
\hline 52003 & JOHNSON WF & KOLTHOFF IM & & J AM CHEM SOC & 74 & 22 & 1952 & NO ENTRIE & & \\
\hline 52004 & KLEVENS HB & & & MEM SERV CHIM ETAT (PARIS) & 37 & 13 & 1952 & NO ENTRIE & & \\
\hline 52005 & KLEVENS HB & & & $J$ AM CHEM SOC & 74 & 4624 & 1952 & NO ENTRIE & & \\
\hline 52006 & EKWALL $P$ & SMEDS K & & ACTA CHEM SCAND & 6 & 441 & 1952 & NO ENTRIE & & \\
\hline 52007 & EKWALL $P$ & HASAN A & DANIELSSON I & ACTA CHEM SCAND & 6 & 440 & 1952 & NO ENTRIE & & \\
\hline 52008 & KLEVENS HB & & & KOLLOID-Z & 128 & 61 & 1952 & NO ENTRIE & & \\
\hline 52009 & WINSOR PA & & & $J$ PHYS CHEM & 56 & 391 & 1952 & NO ENTRIE & & \\
\hline 52011 & BURY CR & BROWNING $\mathrm{J}$ & & TRANS FARADAY SOC & 48 & 209 & 1952 & YIELDED & 3 & ENTRIES \\
\hline 52013 & $\begin{array}{l}\text { BROWN AS } \\
\text { THIBAULT HG }\end{array}$ & $\begin{array}{l}\text { ROBINSON RU } \\
\text { MCNEILL } W\end{array}$ & $\begin{array}{l}\text { SIROIS EH } \\
\text { TOFIAS A }\end{array}$ & $\mathrm{J}$ PHYS CHEM & 56 & 701 & 1952 & YIELDED & 3 & ENTRIES \\
\hline 52014 & MEADER AL & FRIES BA & & IND ENG CHEM & 44 & 1636 & 1952 & NO ENTRIE & & \\
\hline 52015 & HERZFELD SH & & & $J$ PHYS CHEM & 56 & 953 & 1952 & YIELDED & 20 & ENTRIES \\
\hline 52016 & RAISON M & & & COMPT REND & 235 & 1129 & 1952 & YIELDED & 10 & ENTRIES \\
\hline 52017 & HERZFELD SH & & & $\mathrm{J}$ PHYS CHEM & 56 & 959 & 1952 & YIELDED 1 & 108 & ENTRIES \\
\hline 52018 & YURZHENKO AI & KUCHER RV & & COLLOID J (USSR) & 14 & 243 & 1952 & YIELDED & 3 & ENTRIES \\
\hline 52019 & YURZHENKO AI & KUCHER RV & & COLLOID J (USSR) & 14 & 311 & 1952 & NO ENTRIE & & \\
\hline 53001 & TAMAMUSHI B & NAKADATE $S$ & & REP INST SCI TECH UNIV TOKYO & 7 & 401 & 1953 & YIELDED & 2 & ENTRIES \\
\hline 53002 & ANACKER EW & & & $\mathrm{J}$ COLLOID SCI & 8 & 402 & 1953 & YIELDED & 5 & ENTRIES \\
\hline 53003 & ARRINGTON $\mathrm{CH}$ & PATTERSON GD & & $\mathrm{J}$ PHYS CHEM & 57 & 247 & 1953 & Y IELDED & 13 & ENTRIES \\
\hline 53004 & FLOCKHART BD & GRAHAM $\mathrm{H}$ & & $\mathrm{J}$ COLLOID SCI & 8 & 105 & 1953 & YIELDED & 5 & ENTRIES \\
\hline 53005 & LANGE H & & & KOLLOID-Z & 131 & 96 & 1953 & YIELDED & 21 & ENTRIES \\
\hline 53006 & SATA N & TYUZYO K & & BULL CHEM SOC JAPAN & 26 & 177 & 1953 & YIELDED & 11 & ENTRIES \\
\hline 53007 & ROSS S & KWARTLER CE & BAILEY JH & J COLLOID SCI & 8 & 385 & 1953 & YIELDED & 19 & ENTRIES \\
\hline $5 \geq 008$ & FLOCKHART BD & UBBELOHDE AR & & J COLLOID SCI & 8 & 424 & 1953 & YIELDED & 29 & ENTRIES \\
\hline 53009 & KUSHNER LM & HUBBARD WD & & $\mathrm{J}$ PHYS CHEM & 57 & 808 & 1953 & NO ENTRIE & & \\
\hline 53010 & KLEVENS HB & & & $J$ AM OIL CHEMISTS SOC & 30 & 74 & 1953 & YIELDED & 31 & ENTRIES \\
\hline 53011 & KRAUS CA & & & PROC NAT ACAD SCI U S & 39 & 1213 & 1953 & NO ENTRIE & & \\
\hline 53012 & GODDARD ED & HARVA 0 & JONES TG & TRANS FARADAY SOC & 49 & 980 & 1953 & YIELDED & 61 & ENTRIES \\
\hline 53013 & LAL H & & & J COLLOID SCI & 8 & 414 & 1953 & NO ENTRIE & & \\
\hline 53014 & SCHOLBERG HM & GUENTHNER RA & COON RI & $\mathrm{J}$ PHYS CHEM & 57 & 923 & 1953 & NO ENTRIE & & \\
\hline 53015 & YANG JT & FOSTER JF & & $\mathrm{J}$ PHYS CHEM & 57 & 628 & 1953 & YIELDED & 2 & ENTRIES \\
\hline
\end{tabular}




\begin{tabular}{|c|c|c|c|c|c|c|c|c|c|}
\hline $\begin{array}{l}\text { Refer- } \\
\text { ence } \\
\text { No. }\end{array}$ & & Authors & & Journal & Vol. & Pg. & Year & $\begin{array}{l}\text { No. of Entrie } \\
\text { Referen }\end{array}$ & $\begin{array}{l}\text { ies from } \\
\text { nce }\end{array}$ \\
\hline 54001 & PILPEL $\mathrm{N}$ & & & J COLLOID SCI & 9 & 285 & 1954 & NO ENTRIES & \\
\hline 54002 & KUSHNER LM & HUBBARD WD & & J PHYS CHEM & 58 & 1163 & 1954 & NO ENTRIES & \\
\hline 54003 & SHINODA K & & & $J$ PHYS CHEM & 58 & 1136 & 1954 & YIELDED 57 & ENTRIES \\
\hline 54004 & KLEVENS HB & RAISON M & & PROC INTERN CONGR SURFACE ACTIVITY & IST & 1 & 1954 & YIELDED 25 & ENTRIES \\
\hline 54005 & SHINODA K & & & $J$ PHYS CHEM & 58 & 541 & 1954 & YIELDED 139 & ENTRIES \\
\hline 54006 & MARON SH & ELDER ME & ULEVITCH IN & $\mathrm{J}$ COLLOID SCI & 9 & 382 & 1954 & YIELDED 10 & ENTRIES \\
\hline 54007 & GOTTE E & & & FETTE, SEIFEN, ANSTRICHMI & 56 & 583 & 1954 & NO ENTRIES & \\
\hline 54008 & OKUYAMA H & TYUZYO K & & BULL CHEM SOC JAPAN & 27 & 259 & 1954 & YIELDED 3 & 3 ENTRIES \\
\hline 54009 & HONIG JG & SINGLETERRY CR & & $\mathrm{J}$ PHYS CHEM & 58 & 201 & 1954 & NO ENTRIES & \\
\hline 54010 & KLEVENS HB & RAISON M & & $\mathrm{J}$ CHIM PHYS & 51 & 1 & 1954 & YIELDED 25 & ENTRIES \\
\hline 54011 & COHEN I & HISKEY CF & OSTER G & $\mathrm{J}$ COLLOID SCI & 9 & 243 & 1954 & NO ENTRIES & \\
\hline 54012 & HUTCHINSON E & MANCHESTER KE & WINSLOW L & J PHYS CHEM & 58 & 1124 & 1954 & NO ENTRIES & \\
\hline 54013 & ROE CP & BRASS PD & & $J$ AM CHEM SOC & 76 & 4703 & 1954 & YIELDED 4 & 1 ENTRIES \\
\hline 54014 & HUBBARD HM & REYNOLDS CA & & $J$ AM CHEM SOC & 76 & 4300 & 1954 & NO ENTRIES & \\
\hline 54015 & HIGUCHI T & LACH JL & & $\mathrm{J}$ AM PHARM ASSOC & 43 & 465 & 1954 & NO ENTRIES & \\
\hline 55001 & HERMANS JJ & & & KONINKI NED AKAD WETEN. PROC SER B & 58 & 91 & 1955 & NO ENTRIES & \\
\hline 55002 & PRINS W & HERMANS JJ & & J PHYS CHEM & 59 & 576 & 1955 & NO ENTRIES & \\
\hline 55003 & KUSHNER LM & HUBBARD WD & & $\mathrm{J}$ COLLOID SCI & 10 & 428 & 1955 & YIELDED 6 & ENTRIES \\
\hline 55004 & SHINODA K & & & BULL FAC ENG, YOKOHAMA NAT UNIV & 4 & 77 & 1955 & YIELDED 167 & ENTRIES \\
\hline 55005 & WILLIAMS RJ & PHILLIPS JN & MYSELS KJ & TRANS FARADAY SOC & 51 & 728 & 1955 & YIELDED 17 & ENTRIES \\
\hline 55006 & VOEKS JF & TARTAR HV & & J PHYS CHEM & 59 & 1190 & 1955 & YIELDED 2 & ENTRIES \\
\hline 55007 & SHINODA K & & & $J$ PHYS CHEM & 59 & 432 & 1955 & YIELDED 39 & ENTRIES \\
\hline 55008 & LIN $w$ & & & BULL CHEM SOC JAPAN & 28 & 227 & 1955 & YIELDED 20 & ENTRIES \\
\hline 55009 & TRAP HJL & HERMANS JJ & & KONINKI NED AKAD WETEN. PROC SER B & 58 & 97 & 1955 & YIELDED 34 & ENTRIES \\
\hline 55010 & SHIRAI M & TAMAMUSHI B & & BULL CHEM SOC JAPAN & 28 & 545 & 1955 & NO ENTRIES & \\
\hline 55011 & GODDARD ED & JONES TG & & RES CORRESPONDENCE & 8 & 1 & 1955 & NO ENTRIES & \\
\hline 55012 & ABBOTT AD & TARTAR HV & & $J$ PHYS CHEM & 59 & 1193 & 1955 & NO ENTRIES & \\
\hline 55013 & TARTAR HV & & & $J$ PHYS CHEM & 59 & 1195 & 1955 & NO ENTRIES & \\
\hline 55014 & PHILLIPS JN & MYSELS KJ & & $J$ PHYS CHEM & 59 & 325 & 1955 & NO ENTRIES & \\
\hline 55015 & MUKERJEE P & MYSELS KJ & & $\mathrm{J}$ AM CHEM SOC & 77 & 2937 & 1955 & YIELDED 8 & 3 ENTRIES \\
\hline 55016 & MYSELS KJ & & & $\mathrm{J}$ COLLOID SCI & 10 & 507 & 1955 & NO ENTRIES & \\
\hline 55017 & KLEVENS HB & & & NATURE & 176 & 879 & 1955 & NO ENTRIES & \\
\hline 55018 & GODDARD ED & HIGHAM EH & STEWART JC & RES CORRESPONDENCE & 7 & 1 & 1955 & YIELDED 2 & ENTRIES \\
\hline 55019 & KITAHARA A & & & BULL CHEM SOC JAPAN & 28 & 234 & 1955 & NO ENTRIES & \\
\hline 55020 & HSIAO L & DUNNING HN & & J PHYS CHEM & 59 & 362 & 1955 & NO ENTRIES & \\
\hline 55021 & TARTAR HV & LELONG ALM & & $\mathrm{J}$ PHYS CHEM & 59 & 1185 & 1955 & YIELDED 11 & ENTRIES \\
\hline 55022 & FULLER GW & & & $\mathrm{J}$ COLLOID SCI & 10 & 403 & 1955 & NO ENTRIES & \\
\hline 55023 & MCBAIN MEL & & & $\mathrm{J}$ COLLOID SCI & 10 & 223 & 1955 & NO ENTRIES & \\
\hline 55024 & PHILLIPS JN & & & TRANS FARADAY SOC & 51 & 561 & 1955 & NO ENTRIES & \\
\hline 55025 & ERIKSON JA & LINGAFELTER EC & & $\mathrm{J}$ COLLOID SCI & 10 & 71 & 1955 & NO ENTRIES & \\
\hline 55026 & GRIESS W & & & FETTE, SEIFEN, ANSTRICHMI & 57 & 236 & 1955 & NO ENTRIES & \\
\hline 55027 & GRIESS $w$ & & & FETTE, SEIFEN, ANSTRICHMI & 57 & 168 & 1955 & NO ENTRIES & \\
\hline 55028 & GRIESS W & & & FETTE, SEIFEN, ANSTRICHMI & 57 & 24 & 1955 & YIELDED 15 & ENTRIES \\
\hline 56001 & KLEVENS HB & CARR CW & & J PHYS CHEM & 60 & 1245 & 1956 & YIELDED 25 & ENTRIES \\
\hline 56002 & DORST W & PRINS W & HERMANS JJ & KONINKI NED AKAD WETEN. PROC SER B & 59 & 190 & 1956 & YIELDED 20 & ENTRIES \\
\hline 56003 & SHINODA K & & & J PHYS CHEM & 60 & 1439 & 1956 & YIELDED & ENTRIES \\
\hline 56004 & PILPEL N & & & $J$ PHYS CHEM & 60 & 779 & 1956 & NO ENTRIES & \\
\hline 56005 & LUDLUM DB & & & $J$ PHYS CHEM & 60 & 1240 & 1956 & YIELDED & ENTRIES \\
\hline 56006 & EVANS HC & & & $\mathrm{J}$ CHEM SOC & & 579 & 1956 & YIELDED 32 & ENTRIES \\
\hline 56007 & PRINS W & HERMANS JJ & & KONINKI NED AKAD WETEN. PROC SER B & 59 & 162 & 1956 & NO ENTRIES & \\
\hline 56008 & WEIL JK & STIRTON AJ & & $J$ PHYS CHEM & 60 & 899 & 1956 & YIELDED 31 & ENTRIES \\
\hline 56009 & SHIRAI M & TAMAMUSHI B & & BULL CHEM SOC JAPAN & 29 & 733 & 1956 & NO ENTRIES & \\
\hline 56010 & KLEVENS HB & VERGNOLLE J & & MEM SERV CHIM ETAT (PARIS) & 41 & 363 & 1956 & YIELDED & ENTRIES \\
\hline 56011 & PRINS W & HERMANS JJ & & KONINKI NED AKAD WETEN. PROC SER B & 59 & 298 & 1956 & YIELDED 13 & ENTRIES \\
\hline 56012 & HONIG JG & SINGLETERRY CR & & $\mathrm{J}$ PHYS CHEM & 60 & 1108 & 1956 & NO ENTRIES & \\
\hline 56013 & HONIG JG & SINGLETERRY CR & & $\mathrm{J}$ PHYS CHEM & 60 & 1114 & 1956 & NO ENTRIES & \\
\hline 56014 & HSIAO L & DUNNING HN & LORENZ PB & $\mathrm{J}$ PHYS CHEM & 60 & 657 & 1956 & YIELDED 25 & ENTRIES \\
\hline 56015 & KITAHARA A & & & BULL CHEM SOC JAPAN & 29 & 15 & 1956 & NO ENTRIES & \\
\hline 56016 & FAVA A & EYRING $\mathrm{H}$ & & $\mathrm{J}$ PHYS CHEM & 60 & 890 & 1956 & YIELDED & 3 ENTRIES \\
\hline 56017 & ZUTRAUEN HA & & & $\mathrm{J}$ CHIM PHYS & 53 & 62 & 1956 & NO ENTRIES & \\
\hline 56018 & HARVA 0 & & & REC TRAV CHIM & 75 & 112 & 1956 & YIELDED 43 & ENTRIES \\
\hline 56019 & ADDISON CC & FURMIDGE CGL & & $\mathrm{J}$ CHEM SOC & & 3229 & 1956 & YIELDED 13 & ENTRIES \\
\hline 56020 & MEGURO $\mathrm{K}$ & KONDO T & YODA 0 & J CHEM SOC JAPAN, PURE CHEM SEC & 77 & 1236 & 1956 & YIELDED 10 & ENTRIES \\
\hline 57001 & MATIJEVIC E & PETHICA BA & & CROAT CHEM ACTA & 29 & 431 & 1957 & NO ENTRIES & \\
\hline 57002 & GODDARD ED & HOEVE CAJ & BENSON GC & $J$ PHYS CHEM & 61 & 593 & 1957 & NO ENTRIES & \\
\hline 57003 & KUSHNER LM & HUBBARD WD & DOAN AS & $J$ PHYS CHEM & 61 & 371 & 1957 & NO ENTRIES & \\
\hline 57004 & HOYER HW & GREENF IELD A & & $J$ PHYS CHEM & 61 & 818 & 1957 & YIELDED 5 & ENTRIES \\
\hline 57005 & LIN W & & & $\mathrm{J}$ CHINESE CHEM SOC & 4 & 28 & 1957 & YIELDED & ENTRIES \\
\hline 57006 & KUSHNER LM & HUBBARD WD & PARKER RA & J RES NAT BUR STD A & 59 & 113 & 1957 & YIELDED 19 & ENTRIES \\
\hline $\begin{array}{l}57007 \\
57008\end{array}$ & $\begin{array}{l}\text { CARRINGTON RAG } \\
\text { LIN } W\end{array}$ & EVANS HC & & $\begin{array}{l}\mathrm{J} \text { CHEM SOC } \\
\mathrm{J} \text { CHINESE CHEM }\end{array}$ & 4 & 1701 & 1957 & NO ENTRIES & \\
\hline $\begin{array}{l}57008 \\
57009\end{array}$ & $\begin{array}{l}\text { LIN W } \\
\text { WILLIAMS EF }\end{array}$ & WOODBERRY N & DIXON JK & $\begin{array}{l}\mathrm{J} \text { CHINESE CHEM } \\
\mathrm{J} \text { COLLOID SCI }\end{array}$ & $\begin{array}{r}4 \\
12\end{array}$ & $\begin{array}{r}21 \\
452\end{array}$ & 1957 & $\begin{array}{l}\text { NO ENTRIES } \\
\text { YIELDED } 6\end{array}$ & \\
\hline 57010 & $\begin{array}{l}\text { ROSS S } \\
\text { ROS EF }\end{array}$ & HUDSON JB & & $\begin{array}{l}\text { J COLLOID SCI } \\
\text { J COLLOID SCI }\end{array}$ & $\begin{array}{l}12 \\
12\end{array}$ & $\begin{array}{l}452 \\
523\end{array}$ & $\begin{array}{l}1957 \\
1957\end{array}$ & $\begin{array}{ll}\text { YIELDED } & 6 \\
\text { YIELDED } & 1\end{array}$ & $\begin{array}{l}\text { ENTRIES } \\
\text { ENTRIES }\end{array}$ \\
\hline 57011 & GODDARD ED & BENSON GC & & CAN J CHEM & 35 & 986 & 1957 & YIELDED 30 & ENTRIES \\
\hline 57012 & GERSHMAN JW & & & $\mathrm{J}$ PHYS CHEM & 61 & 581 & 1957 & YIELDED 17 & ENTRIES \\
\hline 57013 & FLOCKHART BD & & & J COLLOID SCI & 12 & 557 & 1957 & YIELDED 33 & ENTRIES \\
\hline 57014 & SCHICK MJ & FOWKES FM & & J PHYS CHEM & 61 & 1062 & 1957 & YIELDED 57 & ENTRIES \\
\hline 57015 & SHIRAI M & TAMAMUSHI B & & BULL CHEM SOC JAPAN & 30 & 411 & 1957 & NO ENTRIES & \\
\hline 57016 & SHIRAI M & TAMAMUSHI B & & BULL CHEM SOC JAPAN & 30 & 542 & 1957 & YIELDED 1 & ENTRIES \\
\hline 57017 & KLEVENS HB & VERGNOLLE J & & PROC INTERN CONGR SURFACE ACTIVITY & 2ND & 395 & 1957 & YIELDED 14 & ENTRIES \\
\hline
\end{tabular}




\begin{tabular}{|c|c|c|c|c|c|c|c|c|c|c|}
\hline $\begin{array}{l}\text { Refer- } \\
\text { ence } \\
\text { No. }\end{array}$ & & Authors & & Journal & Vol. & Pg. & Year & \multicolumn{3}{|c|}{$\begin{array}{l}\text { No. of Entries from } \\
\text { Reference }\end{array}$} \\
\hline 57018 & NAKAGAWA T & KURIYAMA K & & J CHEM SOC JAPAN, PURE CHEM SEC & 78 & 1568 & 1957 & NO ENTRIE & & \\
\hline 57019 & NAKAGAWA T & INOUE $\mathrm{H}$ & & J CHEM SOC JAPAN, PURE CHEM SEC & 78 & 636 & 1957 & NO ENTRIE & & \\
\hline 57020 & NAKAGAWA $T$ & KURIYAMA K & TORI K & J CHEM SOC JAPAN, PURE CHEM SEC & 78 & 1573 & 195.7 & YIELDED & 22 & ENTRIES \\
\hline 57021 & HUTCHINSON E & WINSLOW L & & Z PHYSIK CHEM (FRANKFURT) & 11 & 165 & 1957 & YIELDED & 8 & ENTRIES \\
\hline 57022 & LANGE $\mathrm{H}$ & KLING $W$ & & PROC INTERN CONGR SURFACE ACTIVITY & 2ND & 295 & 1957 & YIELDED & 15 & ENTRIES \\
\hline 57023 & SASAKI H & SAITO $\mathrm{S}$ & OKUYAMA $\mathrm{H}$ & BULL CHEM SOC JAPAN & 30 & 186 & 1957 & NO ENTRIE & & \\
\hline 57024 & OSIPOW L & SNELL FD & HICKSON J & PROC INTERN CONGR SURFACE ACTIVITY & 2ND & & 1957 & YIELDED & 2 & ENTRIES \\
\hline 57025 & MIURA M & MATSUMOTO T & & J SCI HIROSHIMA UNIV, SER A-II & 21 & 51 & 1957 & YIELDED & 5 & ENTRIES \\
\hline 57026 & KAUFMAN S & SINGLETERRY CR & & J COLLOID SCI & 12 & 465 & 1957 & NO ENTRIE & & \\
\hline 57027 & KITAHARA A & & & J COLLOID SCI & 12 & 342 & 1957 & NO ENTRIE & & \\
\hline 57028 & KITAHARA A & & & BULL CHEM SOC JAPAN & 30 & 586 & 1957 & NO ENTRIE & & \\
\hline 57029 & KITAHARA A & & & BULL CHEM SOC JAPAN & 31 & 288 & 1957 & NO ENTRIE & & \\
\hline 57030 & SASAKI H & & & BULL CHEM SOC JAPAN & 30 & 326 & 1957 & NO ENTRIE & & \\
\hline 57031 & ROSS S & BRAMFITT TH & & $\mathrm{J}$ PHYS CHEM & 61 & 1261 & 1957 & YIELDED & 18 & ENTRIES \\
\hline 57032 & MEGURO $\mathrm{K}$ & KONDO T & OHBA N & BULL CHEM SOC JAPAN & 30 & 905 & 1957 & NO ENTRIE & & \\
\hline 57033 & $\begin{array}{l}\text { MEGURO K } \\
\text { INO } \mathrm{T}\end{array}$ & $\begin{array}{l}\text { KONDO T } \\
\text { YODA } 0\end{array}$ & OHBA N & BULL CHEM SOC JAPAN & 30 & 760 & 1957 & NO ENTRIE & & \\
\hline 58001 & MILLER ML & DIXON JK & & J COLLOID SCI & 13 & 411 & 1958 & YIELDED & 7 & ENTRIES \\
\hline 58002 & HARRIS JC & & & SOAP CHEM SPECIALTIES & 1958 & & 1958 & NO ENTRIE & & \\
\hline 58003 & WEIL JK & BISTLINE RG & STIRTON AJ & $\mathrm{J}$ PHYS CHEM & 62 & 1083 & 1958 & YIELDED & 20 & ENTRIES \\
\hline 58004 & HARRIS JC & & & J AM OIL CHEMISTS SOC & 35 & 670 & 1958 & YIELDED & 1 & ENTRIES \\
\hline 58005 & WHITE P & BENSON GC & & J COLLOID SCI & 13 & 584 & 1958 & NO ENTRIE & & \\
\hline 58006 & WHITE P & MOULE D & BENSON GC & TRANS FARADAY SOC & 54 & 1638 & 1958 & NO ENTRIE & & \\
\hline 58007 & TAMAMUSHI B & SHIRAI $M$ & TAMAKI K & BULL CHEM SOC JAPAN & 31 & 467 & 1958 & YIELDED & 10 & ENTRIES \\
\hline 58008 & GINN ME & HARRIS JC & & J PHYS CHEM & 62 & 1554 & 1958 & YIELDED & 18 & ENTRIES \\
\hline 58009 & ANACKER EW & & & J PHYS CHEM & 62 & 41 & 1958 & YIELDED & 7 & ENTRIES \\
\hline 58010 & HARRIS JC & & & J AM OIL CHEMISTS SOC & 35 & 428 & 1958 & NO ENTRIE & & \\
\hline 58011 & KLEVENS HB & & & KOLLOID-Z & 158 & 53 & 1958, & YIELDED & 20 & ENTRIES \\
\hline 58012 & HAYDON DA & PHILLIPS JN & & TRANS FARADAY SOC & 54 & 698 & 1958 & YIELDED & 2 & ENTRIES \\
\hline 58013 & MUKERJEE $P$ & MYSELS KJ & DULIN CI & J PHYS CHEM & 62 & 1390 & 1958 & NO ENTRIE & & \\
\hline 58014 & MUKERJEE P & & & $\mathrm{J}$ PHYS CHEM & 62 & 1397 & 1958 & NO ENTRIE & & \\
\hline 58015 & MUKERJEE P & MYSELS KJ & & $\mathrm{J}$ PHYS CHEM & 62 & 1400 & 1958 & NO ENTRIE & & \\
\hline 58016 & MUKERJEE P & & & J PHYS CHEM & 62 & 1404 & 1958 & NO ENTRIE & & \\
\hline 58017 & NAKAGAWA $T$ & & & SHINOGI KENKYUSHO NEMPO & 8 & 805 & 1958 & YIELDED & 9 & ENTRIES \\
\hline 58018 & $\begin{array}{l}\text { NAKAGAWA } T \\
\text { OYAMA T }\end{array}$ & INOUE H & KURIYAMA K & J CHEM SOC JAPAN, PURE CHEM SEC & 79 & 348 & 1958 & NO ENTRIE & & \\
\hline 58019 & $\begin{array}{l}\text { NAKAGAWA T } \\
\text { OYAMA T }\end{array}$ & INOUE $\mathrm{H}$ & KURIYAMA K & J CHEM SOC JAPAN, PURE CHEM SEC & 79 & 345 & 1958 & NO ENTRIE & & \\
\hline 58020 & MATIJEVIC E & PETHICA BA & & TRANS FARADAY SOC & 54 & 587 & 1958 & YIELDED & g & ENTRIES \\
\hline 58021 & KASHIWAGI KM & & & $\mathrm{J}$ COLLOID SCI & 13 & 618 & 1958 & YIELDED & 2 & ENTRIES \\
\hline 58022 & KITAHARA A & & & BULL CHEM SOC JAPAN & 31 & 653 & 1958 & NO ENTRIE & & \\
\hline 58023 & MIURA M & ARISHI S & & J SCI HIROSHIMA UNIV, SER A-II & 22 & 57 & 1958 & YIELDED & 9 & ENTRIES \\
\hline 58024 & BASCOM WD & SINGLETERRY CR & & J COLLOID SCI & 13 & 569 & 1958 & NO ENTRIE & & \\
\hline 58025 & KAUFMAN S & SINGLETERRY CR & & $\mathrm{J}$ PHYS CHEM & 62 & 1257 & 1958 & NO ENTRIE & & \\
\hline 58026 & TYUZYO K & & & BULL CHEM SOC JAPAN & 31 & 117 & 1958 & NO ENTRIE & & \\
\hline 58027 & NASH $\mathrm{T}$ & & & CHEM IND (LONDON) & & 590 & 1958 & NO ENTRIE & & \\
\hline 58028 & $\begin{array}{ll}\text { NAKAGAWA } & T \\
\text { KURIYAMA } & \mathrm{K}\end{array}$ & INOUE $\mathrm{H}$ & KAZUO T & J CHEM SOC JAPAN, PURE CHEM SEC & 79 & 48 & 1958 & YIELDED & 8 & ENTRIES \\
\hline 58029 & SAWYER WM & FOWKES FM & & $J$ PHYS CHEM & 62 & 159 & 1958 & NO ENTRIE & & \\
\hline 58030 & MARKAN AL & KRIUENTSOV WI & & ZAVODSKAYA LAB & 24 & 158 & 1958 & NO ENTRIE & & \\
\hline 58031 & FEW A & GILBY A & OTTEWILL RH & $\mathrm{J}$ CHEM SOC & & 1712 & 1958 & YIELDED & 1 & ENTRIES \\
\hline 59001 & HARROLD SP & & & J PHYS CHEM & 63 & 317 & 1959 & YIELDED & 3 & ENTRIES \\
\hline 59002 & MYSELS KJ & PRINCEN LH & & $\mathrm{J}$ PHYS CHEM & 63 & 1696 & 1959 & YIELDED & 4 & ENTRIES \\
\hline 59003 & PRINCEN LH & MYSELS KJ & & $\mathrm{J}$ PHYS CHEM & 63 & 1781 & 1959 & NO ENTRIE & & \\
\hline 59004 & $\begin{array}{l}\text { WEIL JK } \\
\text { MAURER EW }\end{array}$ & STIRTON AJ & BISTLINE RG & J AM OIL CHEMISTS SOC & 36 & 241 & 1959 & YIELDED & 19 & ENTRIES \\
\hline 59005 & BECHER P & CLIFTON NK & & J COLLÓID SCI & 14 & 519 & 1959 & YIELDED & 1 & ENTRIES \\
\hline 59006 & BECHER $P$ & & & $\mathrm{~J}$ PHYS CHEM & 63 & 1675 & 1959 & YIELDED & 22 & ENTRIES \\
\hline 59007 & MOULE D & BENSON GC & & CAN J CHEM & 37 & 2083 & 1959 & YIELDED & 4 & ENTRIES \\
\hline 59008 & MOULE D & WHITE P & BENSON GC & CAN J CHEM & 37 & 2086 & 1959 & YIELDED & 2 & ENTRIES \\
\hline 59009 & GINN ME & KINNEY FB & HARRIS JC & J AM OIL CHEMISTS SOC & 36 & 332 & 1959 & YIELDED & 23 & ENTRIES \\
\hline 59010 & TARTAR HV & & & J COLLOID SCI & 14 & 115 & 1959 & YIELDED & 2 & ENTRIES \\
\hline 59011 & WESTWELL AE & ANACKER EW & & $\mathrm{J}$ PHYS CHEM & 63 & 1022 & 1959 & NO ENTRIE & & \\
\hline 59012 & WHITE P & BENSON GC & & TRANS FARADAY SOC & 55 & 1025 & 1959 & YIELDED & 9 & ENTRIES \\
\hline 59013 & SHINODA K & YAMANAKA T & KINOSHITA K & J PHYS CHEM & 63 & 648 & 1959 & YIELDED & 3 & ENTRIES \\
\hline 59014 & $\begin{array}{l}\text { ASCOLI F } \\
\text { MELE A }\end{array}$ & BOTRE C & CRESCENZI V & NATURE & 184 & 1482 & 1959 & NO ENTRIE & & \\
\hline 59015 & $\begin{array}{l}\text { BOTRE C } \\
\text { MELE A }\end{array}$ & CRESCENZI VL & LIQUORI AM & TRANS FARADAY SOC & 55 & 1975 & 1959 & YIELDED & 2 & ENTRIES \\
\hline 59016 & BOTRE C & CRESCENZI VL & MELE A & J PHYS CHEM & 63 & 650 & 1959 & YIELDED & 6 & ENTRIES \\
\hline 59017 & KASHIWAGI M & EZAKI H & & BULL CHEM SOC JAPAN & 32 & 624 & 1959 & YIELDED & 4 & ENTRIES \\
\hline 59018 & HUTCHINSON E & & & Z PHYSIK CHEM (FRANKFURT) & 21 & 38 & 1959 & YIELDED & 6 & ENTRIES \\
\hline 59019 & FUERSTENAU DW & MODI HJ & & $\mathrm{J}$ ELECTROCHEM SOC & 106 & 336 & 1959 & NO ENTRIE & & \\
\hline 59020 & ROSS S & OLIVIER JP & & $\mathrm{J}$ PHYS CHEM & 63 & 1671 & 1959 & YIELDED & 7 & ENTRIES \\
\hline 59021 & BASCOM WD & KAUFMAN S & SINGLETERRY CR & WORLD PETROL CONGR, PROC & V 6 & 18 & 1959 & NO ENTRIE & & \\
\hline 59022 & KOLBEL H & KUHN P & & ANGEW CHEM & 71 & 211 & 1959 & NO ENTRIE & & \\
\hline 59023 & HOT.I.AHAN JR & CADY GH & & $\mathrm{J}$ PHYS CHEM & 63 & 757 & 1959 & YIELDED & 1 & ENTRIES \\
\hline 59024 & MEGURO $\overline{\mathrm{K}}$ & KONDO T & & J CHEM SOC JAPAN, PURE CHEM SEC & 80 & 818 & 1959 & YIELDED & 11 & ENTRIES \\
\hline 59025 & MEGURO K & KONDO T & & $J$ CHEM SOC JAPAN, PURE CHEM SEC & 80 & 821 & 1959 & NO ENTRIE & & \\
\hline 59026 & MEGURO $\mathrm{K}$ & KONDO T & & J CHEM SOC JAPAN, PURE CHEM SEC & 80 & 823 & 1959 & YIELDED & 9 & ENTRIES \\
\hline 60001 & VEIS A & HOERR CW & & J COLLOID SCI & 15 & 427 & 1960 & YIELDED & 2 & ENTRIES \\
\hline
\end{tabular}




\begin{tabular}{|c|c|c|c|c|c|c|c|c|c|c|}
\hline \multirow{2}{*}{$\begin{array}{c}\begin{array}{c}\text { Refer- } \\
\text { ence } \\
\text { No. }\end{array} \\
60002\end{array}$} & \multicolumn{3}{|c|}{ Authors } & Journal & \multirow{2}{*}{$\begin{array}{c}\text { Vol. } \\
64\end{array}$} & \multirow{2}{*}{$\begin{array}{l}\mathrm{Pg} \\
599\end{array}$} & Year & $\begin{array}{l}\text { No. of } \\
\operatorname{Re}\end{array}$ & $\begin{array}{l}\text { Entri } \\
\text { eferen }\end{array}$ & $\begin{array}{l}\text { ries from } \\
\text { ence }\end{array}$ \\
\hline & WHITE P & BENSON GC & & $J$ PHYS CHEM & & & & YIELDED & 4 & 4 ENTRIES \\
\hline 60003 & BECHER $P$ & & & $\mathrm{~J}$ PHYS CHEM & 64 & 1221 & 1960 & NO ENTRI & CES & \\
\hline 60004 & HARROLD SP & & & $\mathrm{J}$ COLLOID SCI & 15 & 280 & 1960 & YIELDED & 2 & ENTRIES \\
\hline 60005 & CARTAN $F$ & ANACKER EW & & $\mathrm{J}$ CHEM EDUC & 37 & 36 & 1960 & YIELDED & 2 & ENTRIES \\
\hline 60006 & NAKAGAWA $T$ & KURIYAMA K & INOUE $\mathrm{H}$ & J COLLOID SCI & 15 & 268 & 1960 & YIELDED & 20 & ENTRIES \\
\hline 60007 & NAKAGAWA T & & & PROC INTERN CONGR SURFACE ACTIVITY & $3 R D$ & 636 & 1960 & NO ENTRI & LES & \\
\hline 60008 & $\begin{array}{l}\text { WEIL JK } \\
\text { AULT }\end{array}$ & STIRTON AJ & BISTLINE RG & J AM OIL CHEMISTS SOC & 37 & 679 & 1960 & YIELDED & 9 & ENTRIES \\
\hline 60009 & NAKAGAWA T & TORI K & & KOLLOID-Z & 168 & 132 & 1960 & NO ENTRI & CES & \\
\hline 60010 & GINN ME & KINNEY FB & HARRIS JC & J AM OIL CHEMISTS SOC & 37 & 183 & 1960 & YIELDED & 78 & 3 ENTRIES \\
\hline 60011 & HAYDON DA & TAYLOR FH & & PHIL TRANS ROY SOC LONDON, SER A & 252 & 225 & 1960 & YIELDED & 8 & 3 ENTRIES \\
\hline 60012 & LANGE $\mathrm{H}$ & & & PROC INTERN CONGR SURFACE ACTIVITY & 3RD & 279 & 1960 & YIELDED & 7 & ENTRIES \\
\hline 60013 & V VOORST VA.F & & & PROC INTERN CONGR SURFACE ACTIVITY & $3 R D$ & 276 & 1960 & NO ENTRI & LES & \\
\hline 60014 & V VOORST VA.F & & & TRANS FARADAY SOC & 56 & 1078 & 1960 & NO ENTRI & CES & \\
\hline 60015 & PARREIRA HC & & & ANAIS ACAD BRASIL CIENC & 32 & 207 & 1960 & YIELDED & 4 & 4 ENTRIES \\
\hline 60016 & KOLBEL $\mathrm{H}$ & KLAMANN D & KURZENDORFER P & PROC INTERN CONGR SURFACE ACTIVITY & $3 R D$ & 1 & 1960 & NO ENTRI & IES & \\
\hline 60017 & KOLBEL H & KLAMANN D & WAGNER E & PROC INTERN CONGR SURFACE ACTIVITY & $3 R D$ & 27 & 1960 & YIELDED & 3 & 3 ENTRIES \\
\hline 60018 & GOTTE E & & & PROC INTERN CONGR SURFACE ACTIVITY & 3RD & 45 & 1960 & YIELDED & 20 & ENTRIES \\
\hline 60019 & DERVICHIAN DG & & & PROC INTERN CONGR SURFACE ACTIVITY & $3 R D$ & 182 & 1960 & NO ENTRI & [ES & \\
\hline 60020 & JAMES JW & PETHICA BA & & PROC INTERN CONGR SURFACE ACTIVITY & $3 R D$ & 227 & 1960 & YIELDED & 1 & ENTRIES \\
\hline 60021 & STANLEY JS & RADLEY JA & & PROC INTERN CONGR SURFACE ACTIVITY & 3RD & 246 & 1960 & YIELDED & 3 & ENTRIES \\
\hline 60022 & V VOORST VA.F & $V$ DEN TEMPEL M & & PROC INTERN CONGR SURFACE ACTIVITY & $3 R D$ & 248 & 1960 & NO ENTRI & [ES & \\
\hline 60023 & LUCK W & & & PROC INTERN CONGR SURFACE ACTIVITY & 3RD & 264 & 1960 & NO ENTRI & [ES & \\
\hline 60024 & BOTRE C & CRESCENZI V & LIQUORI AM & PROC INTERN CONGR SURFACE ACTIVITY & 3RD & 302 & 1960 & YIELDED & 3 & ENTRIES \\
\hline 60025 & V VOORST VA.F & & & TRANS FARADAY SOC & 56 & 1067 & 1960 & YIELDED & 9 & ENTRIES \\
\hline 60026 & HUGO WB & NEWTON JM & & $\mathrm{J}$ PHARM PHARMACOL & 12 & 447 & 1960 & YIELDED & 2 & ENTRIES \\
\hline 60027 & ELWORTHY PH & & & $\mathrm{J}$ PHARM PHARMACOL & 012 & 293 & 1960 & YIELDED & 9 & ENTRIES \\
\hline 60028 & BISWAS AK & MUKHERJI BK & & $\mathrm{J}$ PHYS CHEM & 64 & 1 & 1960 & YIELDED & 11 & ENTRIES \\
\hline 60029 & MIYAMOTO $S$ & & & BULL CHEM SOC JAPAN & 33 & 375 & 1960 & YIELDED & 25 & ENTRIES \\
\hline 60030 & BESPYATOV MP & LESHCHENKO $Z Y$ & & MASLOB ZHIR PROM & 26 & 24 & 1960 & NO ENTRI & [ES & \\
\hline 60031 & BESPYATOV MP & OLEINIKOVA ZV & & MASLOB ZHIR PROM & 26 & 26 & 1960 & NO ENTRI & [ES & \\
\hline 60032 & DEMCHENKO PA & DUMANSKII AV & & DOKLADY AKAD NAUK SSSR & 131 & 120 & 1960 & YIELDED & 2 & ENTRIES \\
\hline 60033 & TAUBMAN $A B$ & KONSTANTIN. VV & KRYUKOVA AS & KHIM TEKHNOL & 5 & 61 & 1960 & YIELDED & 28 & 3 ENTRIES \\
\hline 60034 & DEMCHENKO PA & & & MASLOB ZHIR PROM & 26 & 26 & 1960 & YIELDED & 4 & 4 ENTRIES \\
\hline 60035 & MIYAMOTO S & & & BULL CHEM SOC JAPAN & 33 & 371 & 1960 & NO ENTRI & [ES & \\
\hline 61001 & HOYER HW & MARMO A & ZOELLNER M & $J$ PHYS CHEM & 65 & 1804 & 1961 & YIELDED & 2 & ENTRIES \\
\hline 61002 & HOYER HW & MARMO A & & $\mathrm{J}$ PHYS CHEM & 65 & 1807 & 1961 & YIELDED & 8 & ENTRIES \\
\hline 61003 & BECHER P & & & J COLLOID SCI & 16 & 49 & 1961 & YIELDED & 7 & 7 ENTRIES \\
\hline 61004 & CORKILL JM & GOODMAN JF & OTTEWILL RH & TRANS FARADAY SOC & 57 & 1627 & 1961 & YIELDED & 6 & 5 ENTRIES \\
\hline 61005 & MYSELS KJ & KAPAUAN P & & $\mathrm{J}$ COLLOID SCI & 16 & 481 & 1961 & YIELDED & 5 & ENTRIES \\
\hline 61006 & HARRIS JC & SATANEK $\mathrm{J}$ & & J AM OIL CHEMISTS SOC & 38 & 169 & 1961 & NO ENTRI & [ES & \\
\hline 61007 & FLOCKHART BD & & & $\mathrm{J}$ COLLOID SCI & 16 & 484 & 1961 & YIELDED & 83 & ENTRIES \\
\hline 61008 & SHINODA $\mathrm{K}$ & YAMAGUCHI T & HORI $R$ & BULL CHEM SOC JAPAN & 34 & 237 & 1961 & YIELDED & 11 & ENTRIES \\
\hline 61009 & MARUTA I & TOKIWA F & & J CHEM SOC JAPAN, PURE CHEM SEC & 82 & 1657 & 1961 & NO ENTRI & [ES & \\
\hline 61010 & $\begin{array}{l}\text { MARUTA I } \\
\text { SAITO T }\end{array}$ & SAKAI T & TOKIWA F & J CHEM SOC JAPAN, PURE CHEM SEC & 82 & 1512 & 1961 & NO ENTRI & [ES & \\
\hline 61011 & MARUTA I & TOKIWA $F$ & & J CHEM SOC JAPAN, PURE CHEM SEC & 82 & 1660 & 1961 & NO ENTRI & [ES & \\
\hline 61012 & $\begin{array}{l}\text { ISEMURA T } \\
\text { NOGUCHI } \mathrm{J}\end{array}$ & IKEDA S & TOKIWA F & BULL CHEM SOC JAPAN & 34 & 1236 & 1961 & NO ENTRI & [ES & \\
\hline 61013 & PILPEL N & & & TRANS FARADAY SOC & 57 & 1426 & 1961 & NO ENTRI & [ES & \\
\hline 61014 & GINN ME & HARRIS JC & & $\mathrm{J}$ AM OIL CHEMISTS SOC & 38 & 605 & 1961 & YIELDED & 16 & ENTRIES \\
\hline 61015 & GINN ME & KINNEY FB & HARRIS JC & J AM OIL CHEMISTS SOC & 38 & 138 & 1961 & YIELDED & 21 & ENTRIES \\
\hline 61016 & BRUNING $w$ & HOLTZER A & & $\mathrm{J}$ AM CHEM SOC & 83 & 4865 & 1961 & YIELDED & 4 & ENTRIES \\
\hline 61017 & MYSELS KJ & OTTER RJ & & J COLLOID SCI & 16 & 462 & 1961 & YIELDED & 13 & 3 ENTRIES \\
\hline 61018 & MYSELS KJ & OTTER RJ & & $\mathrm{J}$ COLLOID SCI & 16 & 474 & 1961 & NO ENTRI & $E S$ & \\
\hline 61019 & GINN ME & BROWN EL & HARRIS JC & $\mathrm{J}$ AM OIL CHEMISTS SOC & 38 & 361 & 1961 & NO ENTRI & ES & \\
\hline 61022 & NAKAGAWA T & & & CHEM IND (LONDON) & 14 & 1135 & 1961 & NO ENTRI & ES & \\
\hline 61023 & ASCOLI F & BOTRE C & LIQUORI AM & J MOL BIOL & 3 & 202 & 1961 & NO ENTRI & & \\
\hline 61024 & ASCOLI F & BOTRE C & LIQUORI AM & $\mathrm{J}$ PHYS CHEM & 65 & 1991 & 1961 & NO ENTRI & ES & \\
\hline 61025 & TYUZYO K & & & KOLLOID-Z & 175 & 40 & 1961 & YIELDED & 2 & ENTRIES \\
\hline 61026 & V VOORST VA.F & & & TRANS FARADAY SOC & 57 & 110 & 1961 & YIELDED & 5 & ENTRIES \\
\hline 61027 & COHEN I & VASSILIADES T & & $\mathrm{J}$ PHYS CHEM & 65 & 1781 & 1961 & YIELDED & 2 & ENTRIES \\
\hline 61028 & COHEN I & VASSILIADES T & & $\mathrm{J}$ PHYS CHEM & 65 & 1774 & 1961 & NO ENTRI & ES & \\
\hline 61029 & ANGELESCU E. & NICOLESCU A & BARBULESCU EM & REV CHIM AC.REP POP.ROUM. & 6 & 309 & 1961 & Y IELDED & 6 & ENTRIES \\
\hline 61030 & DEMCHENKO PA & & & KOLLOID $\mathrm{ZH}$ & 23 & 528 & 1961 & YIELDED & 4 & ENTRIES \\
\hline 61031 & DEMCHENKO PA & & & DOPOV. AKAD NAUK UKR RSR & & 928 & 1961 & YIELDED & 19 & ENTRIES \\
\hline 61032 & DEMCHENKO PA & & & UKR KHIM $\mathrm{ZH}$ & 27 & 322 & 1961 & NO ENTRI & & \\
\hline 61033 & DEMCHENKO PA & & & MASLOB ZHIR PROM & 27 & 19 & 1961 & NO ENTRI & ES & \\
\hline 62001 & BECHER P & & & $\mathrm{J}$ PHYS CHEM & 66 & 374 & 1962 & YIELDED & 6 & ENTRIES \\
\hline 62002 & BECHER P & & & J COLLOID SCI & 17 & 325 & 1962 & YIELDED & 27 & ENTRIES \\
\hline 62003 & DEBYE $P$ & COLL H & & J COLLOID SCI & 17 & 220 & 1962 & NO ENTRI & & \\
\hline 62004 & HAYDON DA & TAYLOR FH & & TRANS FARADAY SOC & 58 & 1233 & 1962 & YIELDED & 16 & ENTRIES \\
\hline 62005 & HERRMANN KW & & & $\mathrm{J}$ PHYS CHEM & 66 & 295 & 1962 & YIELDED & 12 & ENTRIES \\
\hline 62006 & CORKILL JM & GOODMAN JF & & TRANS FARADAY SOC & 58 & 206 & 1962 & YIELDED & 12 & ENTRIES \\
\hline 62007 & MARUTA I & TOKIWA F & & J CHEM SOC JAPAN, PURE CHEM SEC & 83 & 732 & 1962 & NO ENTRI & & \\
\hline 62008 & $\begin{array}{l}\text { STIRTON AJ } \\
\text { AULT WC }\end{array}$ & BISTLINE RG & WEIL JK & $\mathrm{J}$ AM OIL CHEMISTS SOC & 39 & 55 & 1962 & YIELDED & 13 & ENTRIES \\
\hline 62009 & KURIYAMA K & & & KOLLOID-Z & 180 & 55 & 1962 & YIELDED & 49 & ENTRIES \\
\hline 62010 & KURIYAMA K & INOUE $\mathrm{H}$ & NAKAGAWA $\mathrm{T}$ & KOLLOID-Z & 183 & $\begin{array}{r}68 \\
144\end{array}$ & $\begin{array}{l}1962 \\
1962\end{array}$ & $\begin{array}{l}\text { YIELDED } \\
\text { YIELDED }\end{array}$ & $\begin{array}{l}22 \\
20\end{array}$ & $\begin{array}{l}\text { ENTRIES } \\
\text { ENTRIES }\end{array}$ \\
\hline $\begin{array}{l}62011 \\
62012\end{array}$ & $\begin{array}{l}\text { KURIYAMA K } \\
\text { TOKIWA F }\end{array}$ & ISEMURA T & & $\begin{array}{l}\text { KOLLOID-Z } \\
\text { BULL CHEM SOC JAPAN }\end{array}$ & $\begin{array}{r}181 \\
35\end{array}$ & $\begin{array}{r}144 \\
1737\end{array}$ & $\begin{array}{l}1962 \\
1962\end{array}$ & $\begin{array}{l}\text { YIELDED } \\
\text { NO ENTRIE }\end{array}$ & ES & ENTRIES \\
\hline
\end{tabular}




\begin{tabular}{|c|c|c|c|c|c|c|c|c|c|c|}
\hline $\begin{array}{l}\text { Refer- } \\
\text { ence } \\
\text { No. }\end{array}$ & & Authors & & Journal & Vol. & $\mathrm{Pg}$ & Year & \multicolumn{3}{|c|}{$\begin{array}{l}\text { No. of Entries from } \\
\text { Reference }\end{array}$} \\
\hline 62013 & $\begin{array}{ll}\text { BALMBRA } & R R \\
\text { GOODMAN } & J F\end{array}$ & CLUNIE JS & CORKILL JM & TRANS FARADAY SOC & 58 & 1661 & 1962 & NO ENTRIE & ES & \\
\hline 62014 & TARTAR HV & & & $J$ COLLOID SCI & 17 & 243 & 1962 & NO ENTRIE & & \\
\hline 62015 & MULLEY BA & METCALF AD & & $\mathrm{J}$ COLLOID SCI & 17 & 523 & 1962 & Y IELDED & 4 & ENTRIES \\
\hline 62016 & HUTCHINSON E & SHAFFER PM & & Z PHYSIK CHEM (FRANKFURT) & 31 & 397 & 1962 & NO ENTRIE & ES & \\
\hline 62017 & BOTRE C & SCIBONA G & & ANN CHIM (ROME) & 52 & 1199 & 1962 & NO ENTRIE & & \\
\hline 62018 & LANGE $\mathrm{H}$ & & & FETTE, SEIFEN, ANSTRICHMI & 64 & 457 & 1962 & NO ENTRIE & & \\
\hline 62019 & SCHICK MJ & & & J COLLOID SCI & 17 & 801 & 1962 & Y IELDED & 71 & ENTRIES \\
\hline 62020 & SCHICK MJ & ATLAS SM & EIRICH FR & $J$ PHYS CHEM & 66 & 1326 & 1962 & YIELDED & 14 & ENTRIES \\
\hline 62021 & LIQUORI AM & ASCOLI $\mathbf{F}$ & BOTRE C & PONTIF ACAD SCIENTIAR. SCRIPTA VAR. & 22 & $/ 12 / 1$ & 1962 & NO ENTRIE & & \\
\hline 62022 & ISEMURA T & TOKIWA F & IKEDA S & BULL CHEM SOC JAPAN & 35 & 240 & 1962 & NO ENTRIE & & \\
\hline 62023 & WACHS W & HAYANO S & & KOLLOID-Z & 181 & 139 & 1962 & Y IELDED & 6 & ENTRIES \\
\hline 62024 & KITAHARA A & KOBAYASHI T & TACHIBANA T & J PHYS CHEM & 66 & 363 & 1962 & NO ENTRIE & & \\
\hline 62025 & KAUFMAN S & & & J COLLOID SCI & 17 & 231 & 1962 & NO ENTRIE & & \\
\hline 62026 & MARUTA I & & & J CHEM SOC JAPAN, PURE CHEM SEC & 83 & 786 & 1962 & NO ENTRIE & & \\
\hline 62027 & ELWORTHY PH & MACFARLANE CB & & J PHARM PHARMACOL & 14 & 100 & 1962 & YIELDED & 6 & ENTRIES \\
\hline 62028 & MARUTA I & & & $J$ CHEM SOC JAPAN, PURE CHEM SEC & 83 & 395 & 1962 & NO ENTRIE & & \\
\hline 62029 & MARUTA I & & & J CHEM SOC JAPAN, PURE CHEM SEC & 83 & 782 & 1962 & NO ENTRIE & & \\
\hline 62030 & MARUTA I & & & J CHEM SOC JAPAN, PURE CHEM SEC & 83 & 788 & 1962 & NO ENTRIE & & \\
\hline 62031 & MARUTA I & & & J CHEM SOC JAPAN, PURE CHEM SEC & 83 & 858 & 1962 & NO ENTRIE & & \\
\hline 62032 & MARUTA I & & & J CHEM SOC JAPAN, PURE CHEM SEC & 83 & 861 & 1962 & NO ENTRIE & ES & \\
\hline 62033 & $\begin{array}{l}\text { MATUURA R } \\
\text { HOSOKAWA S }\end{array}$ & SATAKE I & IWAMATSU I & BULL CHEM SOC JAPAN & 35 & 1050 & 1962 & NO ENTRIE & & \\
\hline 62034 & CAMPBELL AN & KARTZMARK EM & LAKSHMINAR. GR & CAN J CHEM & 40 & 839 & 1962 & NO ENTRIE & & \\
\hline 62035 & TUDDENHAM RF & ALEXANDER AE & & $J$ PHYS CHEM & 66 & 1839 & 1962 & YIELDED & 15 & ENTRIES \\
\hline 62036 & HAMANN SD & & & $J$ PHYS CHEM & 66 & 1359 & 1962 & YIELDED & 5 & ENTRIES \\
\hline 62037 & DEMCHENKO PA & & & UKR KHIM ZH & 28 & 46 & 1962 & YIELDED & 6 & ENTRIES \\
\hline 62038 & DEMCHENKO PA & ZAKHAROVA NN & DEMCHENKO LG & UKR KHIM ZH & 28 & 611 & 1962. & YIELDED & 5 & ENTRIES \\
\hline 62039 & BESPYATOV MP & LESHCHENKO ZY & & MASLOB ZHIR PROM & 28 & 20 & 1962 & NO ENTRIE & & \\
\hline 62040 & KURZ J L & & & $\mathrm{J}$ PHYS CHEM & 66 & 2239 & 1962 & Y IELDED & 4 & ENTRIES \\
\hline 63001 & SHEDLOVSKY S & JAKOB CW & EPSTEIN MB & $\mathrm{J}$ PHYS CHEM & 67 & 2075 & 1963 & YIELDED & 12 & ENTRIES \\
\hline 63002 & PILPEL $\mathrm{N}$ & & & CHEM REV & 63 & 221 & 1963 & NO ENTRIE & & \\
\hline 63003 & TOKIWA F & & & BULL CHEM SOC JAPAN & 36 & 222 & 1963 & NO ENTRIE & & \\
\hline 63004 & TOKIWA F & & & BULL CHEM SOC JAPAN & 36 & 1589 & 1963 & NO ENTRIE & & \\
\hline 63005 & ROBINS DC & THOMAS IL & & $J$ PHARM PHARMACOL & 15 & 522 & 1963 & NO ENTRIE & & \\
\hline 63006 & KAKIUCHI K & HATTORI K & ISEMURA $\mathrm{T}$ & BULL CHEM SOC JAPAN & 36 & 1250 & 1963 & NO ENTRIE & & \\
\hline 63007 & TOKIWA F & & & BULL CHEM SOC JAPAN & 36 & 281 & 1963 & NO ENTRIE & & \\
\hline 63008 & TORI K & NAKAGAWA $T$ & & KOLLOID-Z & 189 & 50 & 1963 & YIELDED & 49 & ENTRIES \\
\hline 63009 & TORI K & NAKAGAWA T & & KOLLOID-Z & 188 & 47 & 1963 & YIELDED & 6 & ENTRIES \\
\hline 63010 & TORI K & NAKAGAWA T & & KOLLOID-Z & 187 & 44 & 1963 & YIELDED & 3 & ENTRIES \\
\hline 63012 & TORI K & KURIYAMA K & NAKAGAWA T & KOLLOID-Z & 191 & 48 & 1963 & YIELDED & 4 & ENTRIES \\
\hline 63013 & $\begin{array}{l}\text { WEIL JK } \\
\text { BISTLINE RG }\end{array}$ & SMITH FD & STIRTON AJ & J AM OIL CHEMISTS SOC & 40 & 538 & 1963 & YIELDED & 43 & ENTRIES \\
\hline 63014 & $\begin{array}{l}\text { CORKILL JM } \\
\text { TATE JR }\end{array}$ & GOODMAN JF & OGDEN CP & PROC ROY SOC & 273 & 84 & 1963 & YIELDED & 2 & ENTRIES \\
\hline 63015 & BECKETT AH & WOODWARD RJ & & $J$ PHARM PHARMACOL & 15 & 422 & 1963 & YIELDED & 25 & ENTRIES \\
\hline 63016 & ANACKER EW & GHOSE HM & & $J$ PHYS CHEM & 67 & 1713 & 1963 & YIELDED & 7 & ENTRIES \\
\hline 63017 & CROOK EH & FORDYCE DB & TREBBI GF & $J$ PHYS CHEM & 67 & 1987 & 1963 & YIELDED & 54 & ENTRIES \\
\hline 63018 & CORKILL JM & HERRMANN KW & & $J$ PHYS CHEM & 67 & 935 & 1963 & NO ENTRIE & & \\
\hline 63019 & GRABENSTETT . RJ & CORKILL JM & & J COLLOID SCI & 18 & 401 & 1963 & NO ENTRIE & & \\
\hline 63020 & BECHER P & & & J COLLOID SCI & 18 & 196 & 1963 & YIELDED & 5 & ENTRIES \\
\hline 6.3021 & DONBROW M & JAN ZA & & $J$ PHARM PHARMACOL & 15 & 825 & 1963 & YIELDED & 6 & ENTRIES \\
\hline 63022 & BECHER P & & & J COLLOID SCI & 18 & 665 & 1963 & NO ENTRIE & & \\
\hline 63023 & TOKIWA F & & & BULL CHEM SOC JAPAN & 36 & 1585 & 1963 & NO ENTRIE & & \\
\hline 63024 & BOTRE C & RICCIEKI FM & & $\mathrm{J}$ PHARM SCI & 52 & 1011 & 1963 & NO ENTRIE & & \\
\hline 63025 & ASCOLI F & BOTRE C & & BIOPOLYMERS & 1 & 353 & 1963 & NO ENTRIE & & \\
\hline 63026 & SCHICK MJ & & & $J$ PHYS CHEM & 67 & 1796 & 1963 & YIELDED & 59 & ENTRIES \\
\hline 63027 & SCHICK MJ & & & $J$ AM OIL CHEMISTS SOC & 40 & 680 & 1963 & NO ENTRIE & & \\
\hline 63028 & SCHICK MJ & & & $\mathrm{J}$ COLLOID SCI & 18 & 378 & 1963 & NO ENTRIE & & \\
\hline 63029 & HUTCHINSON E & TOKIWA F & & $J$ CHEM EDUC & 40 & 472 & 1963 & NO ENTRIE & & \\
\hline 63030 & PACKTER A & DONBROW M & & $J$ PHARM PHARMACOL & 15 & 317 & 1963 & YIELDED & 12 & ENTRIES \\
\hline 63031 & ROBINS DC & THOMAS IL & & $J$ PHARM PHARMACOL & 15 & 157 & 1963 & NO ENTRIE & & \\
\hline 63032 & MUKERJEE P & RAY A & & $\mathrm{J}$ PHYS CHEM & 67 & 190 & 1963 & YIELDED & 18 & ENTRIES \\
\hline 63033 & EKWALL $P$ & EIKREM H & MANDELL L & ACTA CHEM SCAND & 17 & 111 & 1963 & NO ENTRIE & & \\
\hline 63034 & $\begin{array}{l}\text { SATAKE I } \\
\text { MATUURA R }\end{array}$ & IWAMATSU I & HOSOKAWA S & BULL CHEM SOC JAPAN & 36 & 204 & 1963 & YIELDED & 14 & ENTRIES \\
\hline 63035 & SATAKE I & MATUURA $R$ & & BULL CHEM SOC JAPAN & 36 & 813 & 1963 & NO ENTRIE & ES & \\
\hline 63036 & SHINODA $K$ & NAKAYAMA H & & $\mathrm{J}$ COLLOID SCI & 18 & 705 & 1963 & NO ENTRIE & & \\
\hline 63037 & КАTO Y & & & CHEM PHARM BULL (TOKYO) & 11 & 1202 & 1963 & YIELDED & 3 & ENTRIES \\
\hline 63038 & BESPYATOV MP & LESHCHENKO ZY & & MASLOB ZHIR PROM & 29 & 19 & 1963 & NO ENTRIE & & \\
\hline 63039 & LESYUIS AA & KARNAUKH AM & & MASLOB ZHIR PROM & 29 & 22 & 1963 & NO ENTRIE & & \\
\hline 64001 & VENABLE RL & NAUMAN RV & & $J$ PHYS CHEM & 68 & 3498 & 1964 & YIELDED & 9 & ENTRIES \\
\hline 64002 & $\begin{array}{l}\text { MAURER EW } \\
\text { WEIL JK }\end{array}$ & STIRTON AJ & AULT WC & $\mathrm{J}$ AM OIL CHEMISTS SOC & 41 & 205 & 1964 & YIELDED & 30 & ENTRIES \\
\hline 64003 & CORKILL JM & GOODMAN JF & HARROLD SP & TRANS FARADAY SOC & 60 & 202 & 1964 & YIELDED & 25 & ENTRIES \\
\hline 64004 & SCHOTT H & & & $\mathrm{J}$ PHYS CHEM & 68 & 3612 & 1964 & YIELDED & 2 & ENTRIES \\
\hline 64005 & BECHER P & & & PROC INTERN CONGR SURFACE ACTIVITY & $4 \mathrm{TH}$ & & 1964 & NO ENTRIE & & \\
\hline 64006 & HERRMANN KW & & & $\mathrm{J}$ PHYS CHEM & 68 & 1540 & 1964 & YIELDED & 4 & ENTRIES \\
\hline 64007 & HOYER HW & DOERR IL & & $J$ PHYS CHEM & 68 & 3494 & 1964 & YIELDED & 1 & ENTRIES \\
\hline 64008 & BECHER P & DEL VECCHIO AJ & & $\mathrm{J}$ PHYS CHEM & 68 & 3511 & 1964 & NO ENTRIE & & \\
\hline 64009 & CARLESS JE & CHALLIS RA & MULLEY BA & J COLLOID SCI & 19 & 201 & 1964 & YIELDED & 16 & ENTRIES \\
\hline
\end{tabular}


List of References-Continued

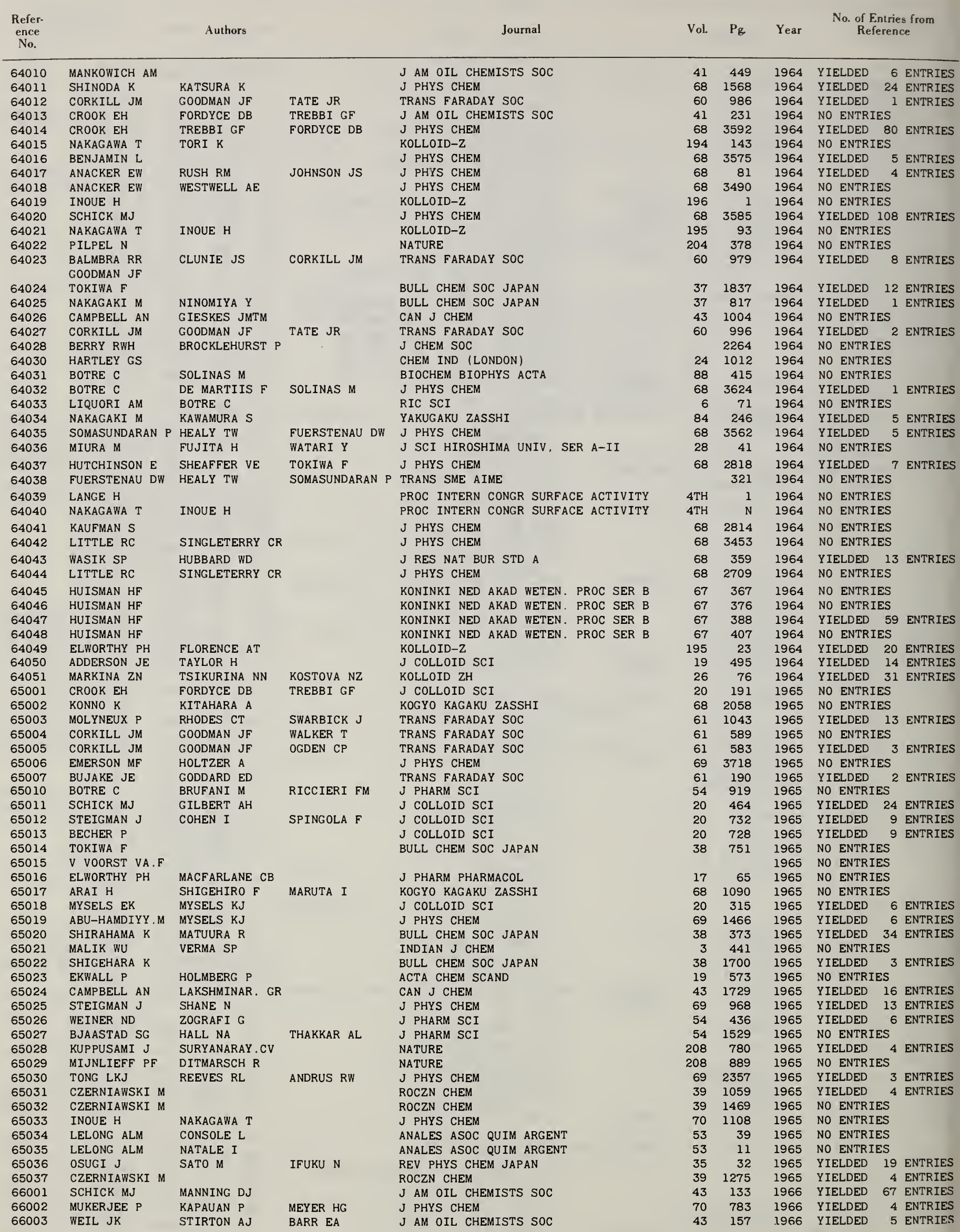


List of References-Continued

\begin{tabular}{|c|c|c|c|c|c|c|c|c|c|c|}
\hline \multirow{2}{*}{$\begin{array}{c}\begin{array}{c}\text { Refer- } \\
\text { ence } \\
\text { No. }\end{array} \\
66004\end{array}$} & \multicolumn{3}{|c|}{ Authors } & Journal & \multirow{2}{*}{$\begin{array}{c}\text { Vol. } \\
70\end{array}$} & \multirow{2}{*}{$\begin{array}{c}\mathrm{Pg} \\
2138\end{array}$} & \multirow{2}{*}{$\begin{array}{c}\text { Year } \\
1966\end{array}$} & \multicolumn{3}{|c|}{$\begin{array}{l}\text { No. of Entries from } \\
\text { Reference }\end{array}$} \\
\hline & MUKERJEE P & RAY A & & J PHYS CHEM & & & & NO ENTRI & ES & \\
\hline 66005 & MUKERJEE P & RAY A & & J PHYS CHEM & 70 & 2144 & 1966 & NO ENTRI & ES & \\
\hline 66006 & MUKERJEE P & RAY A & & J PHYS CHEM & 70 & 2150 & 1966 & YIELDED & 10 & ENTRIES \\
\hline 66007 & ELWORTHY PH & MYSELS KJ & & J COLLOID SCI & 21 & 331 & 1966 & YIELDED & 3 & ENTRIES \\
\hline 66008 & ELWORTHY PH & FLORENCE AT & & KOLLOID-Z Z POLYMERE & 208 & 157 & 1966 & NO ENTRI & ES & \\
\hline 66009 & SHIGEHARA $K$ & & & BULL CHEM SOC JAPAN & 39 & 2643 & 1966 & NO ENTRI & ES & \\
\hline 66010 & SHIGEHARA K & & & BULL CHEM SOC JAPAN & 39 & 2332 & 1966 & YIELDED & 3 & ENTRIES \\
\hline 66011 & CARDWELL PH & & & $\mathrm{J}$ COLLOID INTERFACE SCI & 22 & 430 & 1966 & YIELDED & 6 & ENTRIES \\
\hline 66012 & BENJAMIN L & & & $\mathrm{J}$ COLLOID INTERFACE SCI & 22 & 386 & 1966 & YIELDED & 19 & ENTRIES \\
\hline 66013 & HERRMANN KW & & & J COLLOID INTERFACE SCI & 22 & 352 & 1966 & YIELDED & 12 & ENTRIES \\
\hline 66014 & $\begin{array}{l}\text { CORKILL JM } \\
\text { TATE JR }\end{array}$ & GOODMAN JF & HARROLD SP & TRANS FARADAY SOC & 62 & 994 & 1966 & YIELDED & 10 & ENTRIES \\
\hline 66015 & $\begin{array}{l}\text { CORKILL JM } \\
\text { TATE JR }\end{array}$ & GOODMAN JF & ROBSON P & TRANS FARADAY SOC & 62 & 987 & 1966 & YIELDED & 12 & ENTRIES \\
\hline 66016 & CORKILL JM & GOODMAN JF & TATE JR & TRANS FARADAY SOC & 62 & 979 & 1966 & NO ENTRI & ES & \\
\hline 66017 & PATEL RM & ZOGRAFI G & & J PHARM SCI & 55 & 1345 & 1966 & NO ENTRI & ES & \\
\hline 66018 & WAN LSC & & & $\mathrm{J}$ PHARM SCI & 55 & 1395 & 1966 & YIELDED & 5 & ENTRIES \\
\hline 66019 & DONBROW M & JACOBS J & & J PHARM PHARMACOL & 18 & $92 S$ & 1966 & YIELDED & 2 & ENTRIES \\
\hline 66020 & FLORENCE AT & & & J PHARM PHARMACOL & 18 & 384 & 1966 & YIELDED & 1 & ENTRIES \\
\hline 66021 & MANKOWICH AM & & & $\mathrm{J}$ AM OIL CHEMISTS SOC & 43 & 615 & 1966 & YIELDED & 11 & ENTRIES \\
\hline 66022 & KOMOR JA & BEISWANGE. JPG & & $\mathrm{J}$ AM OIL CHEMISTS SOC & 43 & 435 & 1966 & YIELDED & 12 & ENTRIES \\
\hline 66023 & MALIK WU & CHAND $P$ & & $\mathrm{~J}$ AM OIL CHEMISTS SOC & 43 & 446 & 1966 & YIELDED & 16 & ENTRIES \\
\hline 66024 & LOVELL VM & SEBBA $F$ & & ANAL CHEM & 38 & 1926 & 1966 & NO ENTRI & & \\
\hline 66025 & SCHICK MJ & & & J AM OIL CHEMISTS SOC & 43 & 681 & 1966 & YIELDED & 45 & ENTRIES \\
\hline 66026 & NAKAYAMA H & SHINODA $K$ & HUTCHINSON E & $\mathrm{J}$ PHYS CHEM & 70 & 3502 & 1966 & NO ENTRI & & \\
\hline 66027 & TOKIWA F & OHKI K & & $\mathrm{J}$ PHYS CHEM & 70 & 3437 & 1966 & YIELDED & 6 & ENTRIES \\
\hline 66028 & FORD WPJ & OTTEWILL RH & PARREIRA HC & J COLLOID INTERFACE SCI & 21 & 522 & 1966 & YIELDED & 21 & ENTRIES \\
\hline 66029 & CZERNIAWSKI M & & & ROCZN CHEM & 40 & 1265 & 1966 & NO ENTRI & & \\
\hline 66030 & CZERNIAWSKI M & & & ROCZN CHEM & 40 & 1935 & 1966 & YIELDED & 4 & ENTRIES \\
\hline 66031 & MATHAI $K$ & OTTEWILL RH & & TRANS FARADAY SOC & 62 & 759 & 1966 & NO ENTRI & $E S$ & \\
\hline 66032 & IDA 0 & MEGURO $\mathrm{K}$ & KONDO A & J CHEM SOC JAPAN, PURE CHEM SEC & 77 & 905 & 1966 & NO ENTRI & & \\
\hline 66033 & OSUGI J & SATO M & IFUKU N & J CHEM SOC JAPAN, PURE CHEM SEC & 87 & 329 & 1966 & NO ENTRI & ES & \\
\hline 66034 & PYTASZ G & SZEGLOWSKI Z & & ZESZYTY NAUK UNIW JAGIEL & 211 & 199 & 1966 & NO ENTRI & & \\
\hline 66035 & PYTASZ G & SZEGLOWSKI Z & & ZESZYTY NAUK UNIW JAGIEL & 211 & 209 & 1966 & NO ENTRI & ES & \\
\hline 66036 & SCHOTT H & & & $\mathrm{J}$ PHYS CHEM & 70 & 2966 & 1966 & YIELDED & 8 & ENTRIES \\
\hline 66037 & MATHAI $K$ & OTTEWILL $\mathrm{R}$ & & TRANS FARADAY SOC & 62 & 750 & 1966 & YIELDED & 1 & ENTRIES \\
\hline 66038 & BENTON D & SPARKS B & & TRANS FARADAY SOC & 62 & 3244 & 1966 & YIELDED & 6 & ENTRIES \\
\hline 66039 & HERRMANN WK & BRUSHMILLER J & COURCHENE $W$ & $\mathrm{~J}$ PHYS CHEM & 70 & 2909 & 1966 & YIELDED & 7 & ENTRIES \\
\hline 66040 & BENJAMIN L & & & J PHYS CHEM & 70 & 3790 & 1966 & YIELDED & 11 & ENTRIES \\
\hline
\end{tabular}


Abbreviations: Units of Measure

\begin{tabular}{ll}
\hline Code & \multicolumn{1}{c}{ DesCription } \\
\hline A & MOLES/100 MOLES OF SOLVENT (INCLUDING ADDITIVES) \\
B & VOLUME \% OF SOLVENT \\
C & MOLES/100 MOLES OF SURFACTANT MIXTURE \\
D & W/V \% (GRAMS/IOO MILLILITERS OF SOLUTION) \\
E & \% SATURATION OF SOLUTION BY ADDITIVE \\
H & GRAMS/100 GRAMS OF SOLVENT \\
I & MOLES/100 MOLES OF SURFACTANT \\
K & TOTAL NORMALITY OF COUNTERIONS \\
M & MOLAR (MOLES/LITER OF SOLUTION) \\
N & NORMAL (EQUIVALENTS/LITER OF SOLUTION) \\
P & W/W \% (GRAMS/1OO GRAMS OF SOLUTION) \\
Q & GRAMS/IOO GRAMS OF SURFACTANT \\
R & VALUE VARIED DURING EXPERIMENT \\
S & MOLES/KILOGRAM OF SOLUTION \\
T & GRAMS/IOO GRAMS OF SURFACTANT MIXTURE \\
U & MOLES/LITER OR KILOGRAM OF SOLUTION (UNSPECIFIED) \\
W & MOLAL (MOLES/KILOGRAM OF SOLVENT) \\
Y & PRESSURE IN ATMOSPHERES
\end{tabular}

\section{Abbreviations: Words and Names}

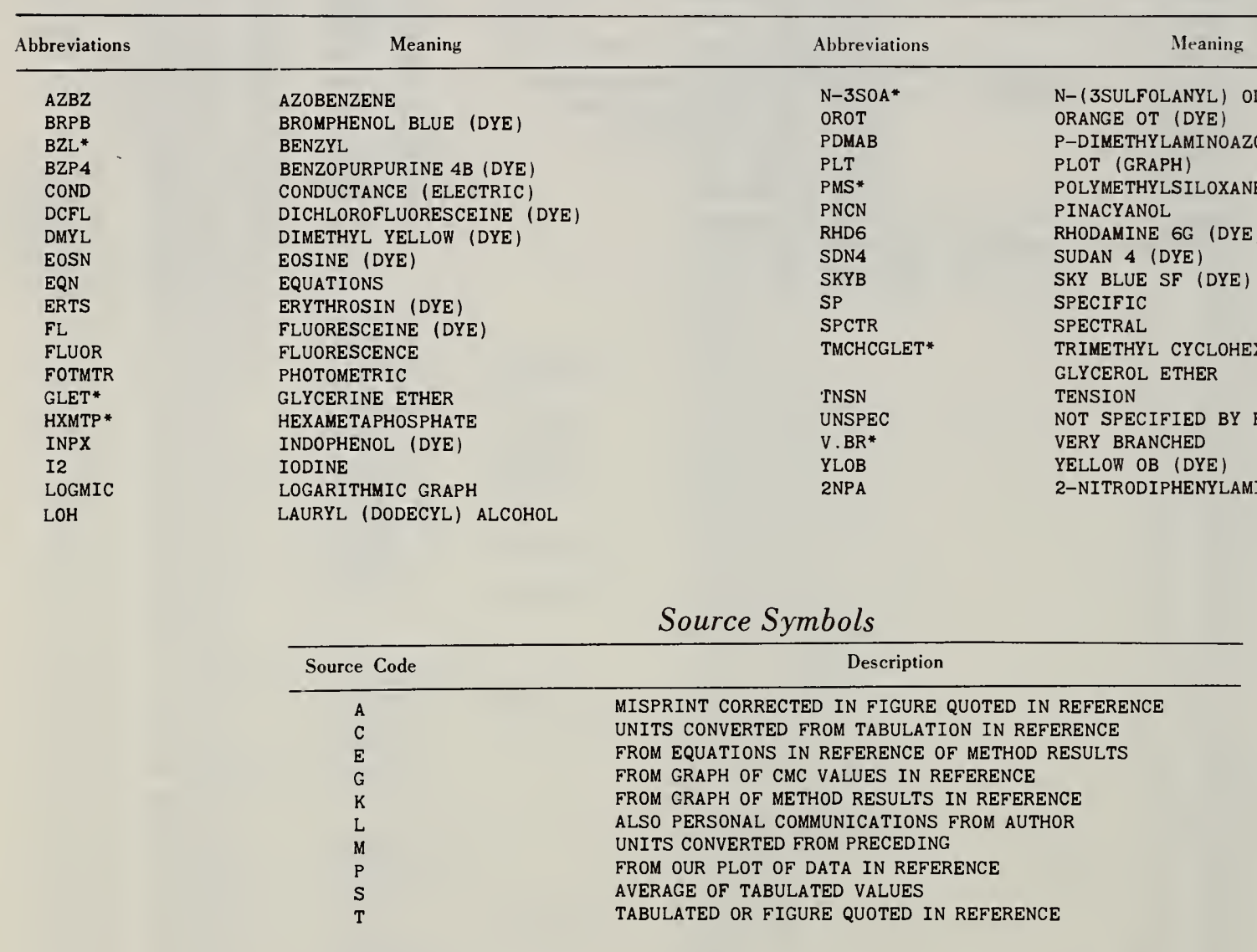

The code $E$ in the numerical value of the concentration in the "Additive" column of the CMC tables stands for "times ten to the power".
Symbols for the quality of materials and methods are discussed on page 6 et seq.

Symbols for methods are discussed on page 8 et seq. 


\section{Announcement of New Publications in National Standard Reference Data Series}

Superintendent of Documents, Government Printing Office, Washington, D.C. 20402

Dear Sir:

Please add my name to the announcement list of new publications to be issued in the series: National Standard Reference Data Series - National Bureau of Standards:

Name

Company

Address

City State Zip Code

(Notification: key N-337) 



\section{Publications in the National Standard Reference Data Series National Bureau of Standards}

You may use this listing as your order form by checking the proper box of the publication(s) you desire or by providing the full identification of the publication you wish to purchase. The full letter symbols with each publications number and full title of the publication and author must be given in your order, e.g. NSRDS-NBS-17, Tables of Molecular Vibrational Frequencies, Part 3, by $\mathrm{T}$. Shimanouchi.

Pay for publications by check, money order, or Superintendent of Documents coupons or deposit account. Make checks and money orders payable to Superintendent of Documents. Foreign remit-

$\square$ NSRDS-NBS 1, National Standard Reference Data SystemPlan of Operation, by E. L. Brady and M. B. Wallenstein, 1964 (15 cents).

$\square$ NSRDS-NBS 2, Thermal Properties of Aqueous Uni-univalent Electrolytes, by V. B. Parker, 1965 (45 cents).

$\square$ NSRDS-NBS 3, Sec. 1, Selected Tables of Atomic Spectra, Atomic Energy Levels and Multiplet Tables, Si II, Si III, Si IV, by C. E. Moore, 1965 (35 cents).

$\square$ NSRDS-NBS 3, Sec. 2, Selected Tables of Atomic Spectra, Atomic Energy Levels and Multiplet Tables, Si I, by C. E. Moore, 1967 ( 20 cents).

$\square$ NSRDS-NBS 3, Sec. 3, Selected Tables of Atomic Spectra, Atomic Energy Levels and Multiplet Tables, C I, C II, C III, C IV, C v, C vI, by C. E. Moore, 1970 (in press).

NSRDS-NBS 4, Atomic Transition Probabilities, Volume 1, Hydrogen Through Neon, by W. L. Wiese, M. W. Smith and B. M. Glennon, $1966(\$ 2.50)$.

NSRDS-NBS 5, The Band Spectrum of Carbon Monoxide, by P. H. Krupenie, 1966 (70 cents).

NSRDS-NBS 6, Tables of Molecular Vibrational Frequencies, Part 1, by T. Shimanouchi, 1967 (40 cents).

$\square$ NSRDS-NBS 7, High Temperature Properties and Decomposition of Inorganic Salts, Part 1, Sulfates, by K. H. Stern and E. L. Weise, 1966 (35 cents).

$\square$ NSRDS-NBS 8, Thermal Conductivity of Selected Materials, by R. W. Powell, C. Y. Ho, and P. E. Liley, 1966 ( $\$ 1.00)$.

$\square$ NSRDS-NBS 9, Bimolecular Gas Phase Reactions (rate coefficients), by A. F. Trotman-Dickenson and G. S. Milne, 1967 (\$2).

$\square$ NSRDS-NBS 10, Selected Values of Electric Dipole Moments for Molecules in the Gas Phase, by R. D. Nelson, Jr., D. R. Lide, Jr., and A. A. Maryott, 1967 (40 cents).

$\square$ NSRDS-NBS 11, Tables of Molecular Vibrational Frequencies, Part 2, by T. Shimanouchi, 1967 (30 cents).

$\square$ NSRDS-NBS 12, Tables for the Rigid Asymmetric Roto: Transformation Coefficients from Symmetric to Asymmetric Bases and Expectation Values of $\mathrm{P}_{z}^{2}, \mathrm{P}_{z}^{4}$ and $\mathrm{P}_{z}^{6}$, by R. H. Schwendeman, 1968 ( 60 cents).

$\square$ NSRDS-NBS 13, Hydrogenation of Ethylene on Metallic Catalysts, by J. Horiuti and K. Miyahara, 1968 (\$1.00).

$\square$ NSRDS-NBS 14, X-Ray Wavelengths and X-Ray Atomic Energy Levels, by J. A. Bearden, 1967 (40 cents).

$\square$ NSRDS-NBS 15, Molten Salts, Vol. 1, Electrical Conductance, Density, and Viscosity Data, by G. Janz, F. W. Dampier, G. R. Lakshminarayanân, P. K. Lorenz, and R. P. T. Tomkins, 1968 (\$3).

$\square$ NSRDS-NBS 16, Thermal Conductivity of Selected Materials, Part 2, by C. Y. Ho, R. W. Powell, and P. E. Liley, 1968 (\$2).

NSRDS-NBS 17, Tables of Molecular Vibration Frequencies, Part 3, by T. Shimanouchi, 1968 (30 cents). tances should be made either by international money order or draft on an American bank. Postage stamps are not acceptable.

No charge is made for postage to destinations in the United States and possessions, Canada, Mexico, and certain Central and South American countries. To other countries, payments for documents must cover postage. Therefore, one-fourth of the price of the publication should be added for postage.

Send your order together with remittance to Superintendent of Documents, Government Printing Office, Washington, D.C. 20402.

NSRDS-NBS 18, Critical Analysis of the Heat-Capacity Data of the Literature and Evaluation of Thermodynamic Properties of Copper, Silver, and Gold From 0 to $300 \mathrm{~K}$, by G. T. Furukawa, W. G. Saba, and M. L. Reilly, 1968 (40 cents).

$\square$ NSRDS-NBS 19, Thermodynamic Properties of Ammonia as an Ideal Gas, by L. Haar, 1968 (20 cents).

NSRDS-NBS 20, Gas Phase Reaction Kinetics of Neutral Oxygen Species, by H. S. Johnson, 1968 (45 cents).

$\square$ NSRDS-NBS 21, Kinetic Data on Gas Phase Unimolecular Reactions, by S. W. Benson and H. E. O'Neal (\$7).

$\square$ NSRDS-NBS 22, Atomic Transition Probabilities, Vol. II, Sodium Through Calcium, A Critical Data Compilation, by W. L. Wiese, M. W. Smith, and B. M. Miles (\$4.50).

$\square$ NSRDS-NBS 23, Partial Grotrian Diagrams of Astrophysical Interest, by C. E. Moore and P. W. Merrill, 1968 (55 cents).

NSRDS-NBS 24, Theoretical Mean Activity Coefficients of Strong Electrolytes in Aqueous Solutions from 0 to $100^{\circ} \mathrm{C}$, by Walter j. Hamer, 1968 ( $\$ 4.25)$.

NSRDS-NBS 25, Electron Impact Excitation of Atoms, by B. L. Moiseiwitsch and S. J. Smith, $1968(\$ 2)$.

$\square$ NSRDS-NBS 26, Ionization Potentials, Appearance Potentials, and Heats of Formation of Positive lons, by J. L. Franklin, J. G. Dillard, H. M. Rosenstock, J. T. Herron, K. Draxl, and F. H. Field (\$4).

NSRDS-NBS 27, Thermodynamic Properties of Argon from the Triple Point to $300 \mathrm{~K}$ at Pressures to 1000 Atmospheres, by A. L. Gosman, R. D. McCarty, and J. G. Hust (\$1.25).

NSRDS-NBS 28, Molten Salts, Vol. 2, Section 1, Electrochemistry of Molten Salts: Gibbs Free Energies and Excess Free Energies From Equilibrium-Type Cells, by G. J. Janz and C. G. M. Dijkhuis. Section 2, Surface Tension Data, by G. J. Janz, G. R. Lakshminarayanan, R. P. T. Tomkins, and J. Wong (\$2.75).

NSRDS-NBS 29, Photon Cross Sections, Attenuation Coefficients and Energy Absorption Coefficients and Energy Absorption Coefficients From $10 \mathrm{keV}$ to $100 \mathrm{GeV}$, by J. H. Hubbell (75 cents).

NSRDS-NBS 30, High Temperature Properties and Decomposition of Inorganic Salts, Part 2, Carbonates, by K. H. Stern and E. L. Weise (45 cents).

$\square$ NSRDS-NBS 31, Bond Dissociation Energies in Simple Molecules, by B. deB. Darwent (55 cents).

$\square$ NSRDS-NBS 32, Phase Behavior in Binary and Multicomponent Systems at Elevated Pressures: $n$ Pentane and Methane $n$ Pentane, by V. M. Berry, 1970 (70 cents).

NSRDS-NBS 33, Electrolytic and Conductance and the Conductances of the Acids in Water, by W. J. Hamer and H. J. DeWane, 1970 ( 50 cents).

NSRDS-NBS 34, Ionization Potentials and Ionization Limits Derived from the Analyses of Optical Spectra, by C. E. Moore, 1970 ( 75 cents). 

Latest developments in the subject area of this publication, as well as in other areas where the National Bureau of Standards is active, are reported in the NBS Technical News Bulletin. See following page. 


\section{HOW TO KEEP ABREAST OF NBS ACTIVITIES}

Your purchase of this publication indicates an interest in the research, development, technology, or service activities of the National Bureau of Standards.

The best source of current awareness in your specific area, as well as in other NBS programs of possible interest, is the TECHNICAL NEWS BULLETIN, a monthly magazine designed for engineers, chemists, physicists, research and product development managers, librarians, and company executives.

If you do not now receive the TECHNICAL NEWS BULLETIN and would like to subscribe, and/or to review some recent issues, please fill out and return the form below.

Mail to: Office of Technical Information and Publications National Bureau of Standards

Washington, D. C. 20234

Name

Affiliation

Address

City State Zip

Please send complimentary past issues of the Technical News Bulletin.

$\square$ Please enter my 1-yr subscription. Enclosed is my check or money order for $\$ 3.00$ (additional $\$ 1.00$ for foreign mailing). Check is made payable to: SUPERINTENDENT OF DOCUMENTS. NSRDS-NBS 36 



